\title{
Development Of Safety Analysis Codes And Experimental Validation For A Very High Temperature Gas-Cooled Reactor
}

Chang $\mathrm{H}$. Oh

Cliff Davis

Larry Siefken

Richard Moore

Hee C. NO

Jong Kim

Goon C. Park

John C. Lee

William R. Martin

March 2006

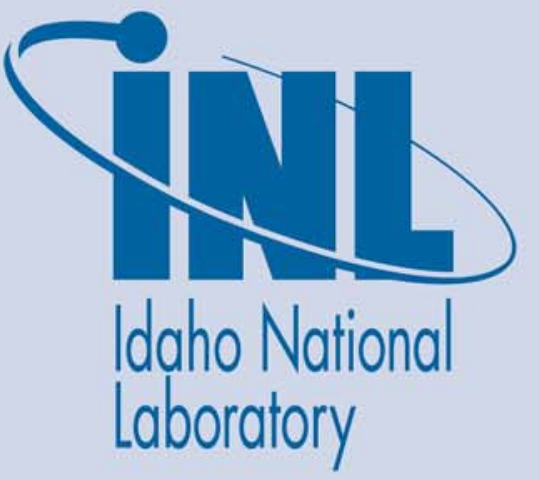

The INL is a U.S. Department of Energy National Laboratory operated by Battelle Energy Alliance 



\title{
Development of Safety Analysis Codes and Experimental Validation for a Very High temperature Gas-Cooled Reactor Final Rep[ort
}

\author{
Chang H. Oh, INL \\ Cliff Davis, INL \\ Larry Siefken, INL \\ Richard Moore, INL \\ Hee C. NO, KAIST \\ Jong Kim, KAIST \\ Goon C. Park, SNU \\ John C. Lee, UM \\ William R. Martin, UM
}

March 2006

Idaho National Laboratory Idaho Falls, Idaho 83415 



\section{INTERNATIONAL NUCLEAR ENERGY RESEARCH INITIATIVE}

\section{Development of Safety Analysis Codes and Experimental Validation for a Very- High-Temperature Gas-Cooled Reactor}

PI (U.S.): C. Oh, Idaho National Laboratory (INL)

PI (Korea): H. NO. and J. Kim Republic of Korea Advanced Institute of Science and Technology (KAIST)

Collaborators: G. Park, Seoul National

University (SNU),

J. Lee and W. Martin, University of Michigan

(UM)

C. Davis, R. Moore and L. Siefken, INL
Project Number: 2003-013-K

Project Start Date: January 1, 2003

Project End Date: December 31, 2005

\section{EXECUTIVE SUMMARY}

The very high-temperature gas-cooled reactor (VHTR) is envisioned as a single- or dual-purpose reactor for electricity and hydrogen generation. The concept has average coolant temperatures above $900^{\circ} \mathrm{C}$ and operational fuel temperatures above $1250^{\circ} \mathrm{C}$. The concept provides the potential for increased energy conversion efficiency and for high-temperature process heat application in addition to power generation. While all the High Temperature Gas Cooled Reactor (HTGR) concepts have sufficiently high temperature to support process heat applications, such as coal gasification, desalination or cogenerative processes, the VHTR's higher temperatures allow broader applications, including thermochemical hydrogen production. However, the very high temperatures of this reactor concept can be detrimental to safety if a loss-of-coolant accident (LOCA) occurs. Following the loss of coolant through the break and coolant depressurization, air will enter the core through the break by molecular diffusion and ultimately by natural convection, leading to oxidation of the in-core graphite structure and fuel. The oxidation will accelerate heatup of the reactor core and the release of toxic gasses $\left(\mathrm{CO}\right.$ and $\left.\mathrm{CO}_{2}\right)$ and fission products. Thus, without any effective countermeasures, a pipe break may lead to significant fuel damage and fission product release.

Prior to the start of this Korean/United States collaboration, no computer codes were available that had been sufficiently developed and validated to reliably simulate a LOCA in the VHTR. Therefore, we have worked for the past three years on developing and validating advanced computational methods for simulating LOCAs in a VHTR.

\section{Research Objectives}

As described above, a pipe break may lead to significant fuel damage and fission product release in the VHTR. The objectives of this Korean/United States collaboration were to develop and validate advanced computational methods for VHTR safety analysis. The methods that have been developed are now available to provide improved understanding of the VHTR during accidents. 


\section{Report Content and Organization}

This report highlights key accomplishments from this project.

Section 1 provides introductory information about the project organization on which this project focused. Detailed information about the objectives and accomplishments from each task completed in this project can be found in Sections 2 through 8. Section 8 highlights results and conclusions that can be drawn from results obtained from each task. In the executive summary, student information and the list of publication supported and produced from this project are listed. This executive summary is provided to highlight accomplishments from this research project.

\section{Project Approach and Organization}

The proposed work has been carried out over a 3-year period. The proposed work plan consists of six major tasks:

- Task 1-Development of benchmark Computational Fluid Dynamic (CFD) code. This code will be used for verification and validation for the system codes, which are basically 1-D codes

- Task 2-RCCS experiment

- Task 3-Air-ingress separate experiment for chemical oxidation model

- Task 4-System code modification; implementation of molecular diffusion to RELAP5/ATHENA code and chemical equilibrium model to MELCOR code

- Task 5-Neutronic physics modeling for power distribution and decay heat

- Task 6-Verification and validation of computer codes.

Figure 1 shows more details of tasks involved in this project. 


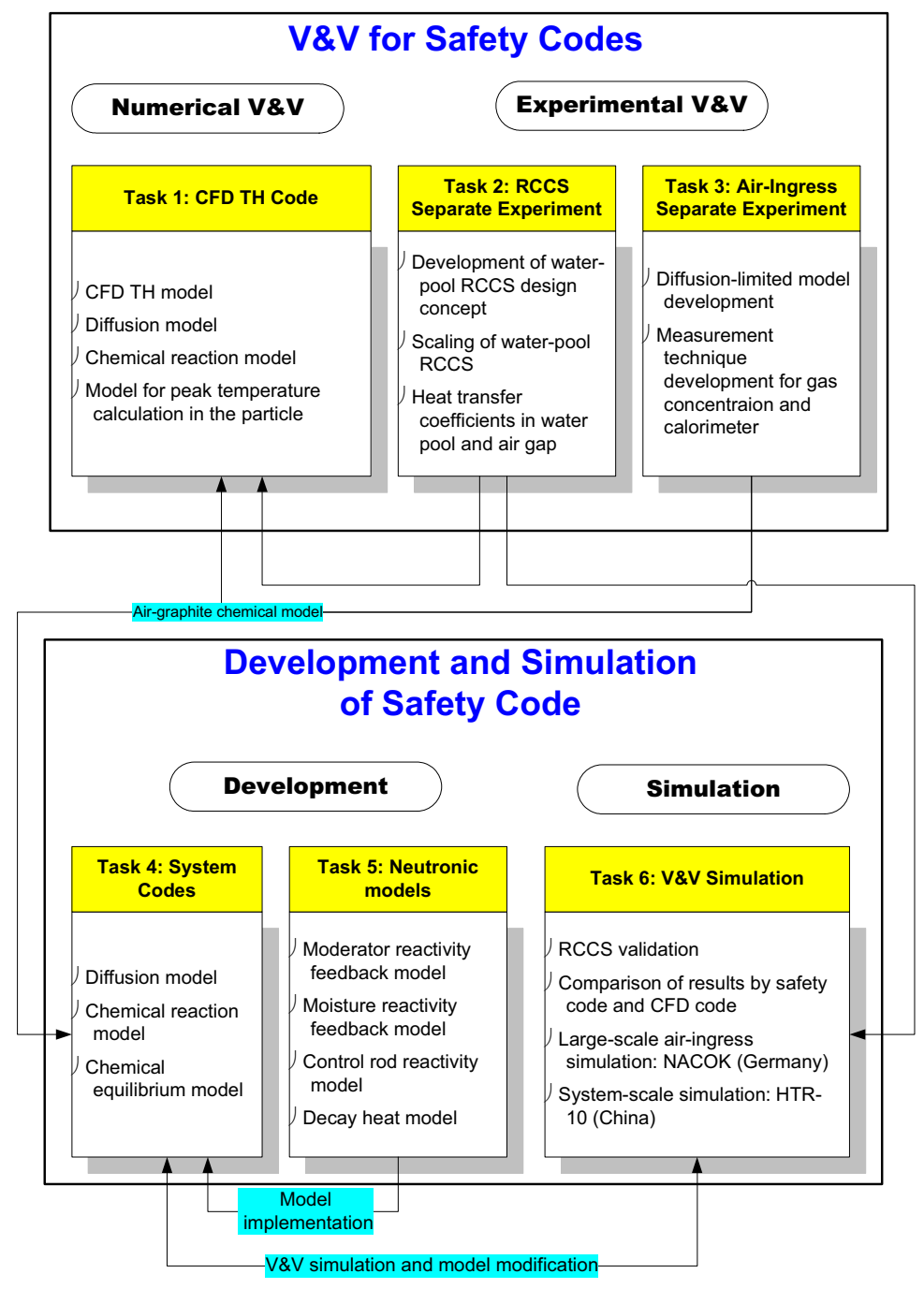

Figure 1. Representative chemical equilibrium.

\section{Key Program Accomplishments}

Key accomplishments from this three year program are highlighted in this section. This project consisted of six tasks for developing, improving, and validating computer codes for analyzing the VHTR. These tasks are to: 1) develop a computational fluid dynamics code for benchmarking, 2) perform a reactor cavity cooling system (RCCS) experiment, 3) perform an air ingress experiment, 4) improve the system analysis codes RELAP5/ATHENA and MELCOR, and 5) develop an advanced neutronic model, and 6) perform verification and validation of the computer codes. The primary activities and key accomplishments for each task are summarized below.

Note that these accomplishments include items required to meet task objectives outlined in the original proposal for this project and items that meet overall I-NERI objectives. As indicated below, this project not 
only advanced the state-of-art in research pertaining to the VHTR, but also helped prepare graduate students to join the nuclear engineering workforce.

\section{Task 1 - CFD thermal hydraulic benchmark code development (KAIST):}

KAIST developed a multi-dimensional gas multi-component mixture analysis code (GAMMA) to predict the thermo-fluid and chemical reaction behaviors in a multi-component mixture system related to an air/water ingress accident in a HTGR. The multi-dimensional governing equations consist of the basic equations for continuity, momentum conservation, energy conservation of the gas mixture, and the mass conservation of each species. GAMMA has the capability to handle the multi-dimensional convection and conduction behaviors as well as heat transfer within the solid components, free and forced convection between a solid and a fluid, and radiative heat transfer between the solid surfaces. Also, the basic equations are formulated with a porous media model to consider a pebble bed-type HTGR.

We performed the code $\mathrm{V} \& \mathrm{~V}$ simulations for the various experiments and benchmark tests for the basic simple problems on the molecular diffusion, graphite oxidation, air ingress, heat transport in a pebble-bed and the reactor cavity cooling system, etc. We performed the chemical reaction test for the VELUNA pebble oxidation experiment in order to select proper reaction models. Finally, the particle model in the pebble is developed and tested against the MIT refined model.

\section{Task 2 - RCCS experiment (SNU):}

SNU proposed a new kind of RCCS, namely the water pool type RCCS, to overcome the disadvantages of the weak cooling ability of air-cooled RCCS and the complex structure of water-cooled RCCS. To estimate the feasibility of the system, a series of experiments were performed simulating the reactor vessel, cavity and RCCS of the HTGR.

At first, separate effect tests for emissivity measurement were carried out to develop the method for the emissivity measurement using infrared thermometer in RCCS environment. Then the separate effect tests for water pool were performed with two different test devices in order. The objectives of this test are to investigate the heat transfer phenomena in both of the water pool and cooling pipe and pressure drop between the inlet and outlet of cooling pipe, especially the multiple U-band type pipes. From the results, it can be concluded that if the total heat transfer area is preserved, reduction of total number of U-band has the advantage in the aspect of pressure drop as well as heat transfer capability.

Finally, the experiments were performed in integral test facility, named SNU-RCCS. Three categories of experiments were conducted in SNU-RCCS, viz. the normal operation tests, the RCCS active cooling failure test and the LOFC test. In both the normal operation test and the active cooling failure tests, it was founded that the maximum temperature of the reactor vessel wall was kept below the design limitation of the PBMR. From the LOFC experiment, it was concluded that the passive heat removal capability of the water pool was not significantly retarded by the increase in the area of the uncovered cavity wall until this area attained approximately $12 \%$ of the total area of the cavity wall.

These experimental results were also used to validate MARS-GCR as well as the thermal hydraulic code developed at KAIST. In addition, code-to-code benchmarks were carried out using CFX and MARS-GCR. As a result, it was founded that MARS-GCR under-predicts the heat transfer coefficient of inner surface of cooling pipe when the helical SG type heat structure was implemented as a convection boundary, but overpredicts the natural circulation of the water in the water pool and the air in the cavity. Therefore it is needed 
to develop new correlation or modify existing correlation of helical SG to precisely simulate heat transfer phenomena of water pool type RCCS.

\section{Task 3 - Air ingress experiment (KAIST):}

KAIST experimentally investigated the graphite oxidation concerned with the HTGR air-ingress. In this research, the effects of kinetics, mass diffusion, combined effect of kinetics and mass diffusion, geometrical effect, burn-off effect and minor chemical reactions were experimentally studied. As a result, the order of reaction (n) and activation energy (Ea) were estimated as $0.75 \pm 0.146$ and $218 \pm 3.76 \mathrm{~kJ} / \mathrm{mol}$ respectively with a $95 \%$ confidence level, and heat/mass transfer analogy was confirmed. The reaction rates and $\mathrm{CO} / \mathrm{CO}_{2}$ were also measured in a temperature range of 700 to $1500{ }^{\circ} \mathrm{C}$ and an oxygen concentration of less than $20 \%$. The empirical correlation was developed for $\mathrm{CO} / \mathrm{CO}_{2}$ ratio and it yields good predictions within $10 \%$ deviation of the experimental data. A Computational Fluid Dynamics (CFD) simulation was conducted and compared to the experimental data using the oxidation parameters and the $\mathrm{CO} / \mathrm{CO}_{2}$ ratio developed here. We derived a graphite oxidation model to cover the chemical reaction and mass transfer over the whole temperature range and validated the model against the data.

The separate experimental facility for the effects of geometry was designed and manufactured. And the concept of internal surface density was introduced into the Arrhenius-type reaction model. Using the 16 different samples of IG-110 graphite, which have different ratios of external surface to volume, the value of internal surface density was obtained as $12760 \mathrm{~m}^{-1}$. It was revealed that the external surface reaction is very small compared to the total reaction for the IG-110 graphite.

The burn-off effect on the rate of reaction was experimentally investigated and the modeling was performed. As a result, the time variation of the reaction rate was well predicted by the suggested numerical simulation.

Finally, the chemical characteristics of $\mathrm{C} / \mathrm{CO}_{2}$ reaction were investigated. As a result, its activation energy was $295 \pm 8 \mathrm{~kJ} / \mathrm{mol}$ and the order of reaction was 0.9 . It turns out that the rate of $\mathrm{C} / \mathrm{CO}_{2}$ reaction is much smaller than the rate of the $\mathrm{C} / \mathrm{O}_{2}$ reaction, which is dominant in $\mathrm{HTGR}$ air-ingress below $1400^{\circ} \mathrm{C}$. A correlation of the reaction rate was developed.

\section{Task 4 - Improvement of system codes (INL):}

Two systems codes, RELAP5/ATHENA and MELCOR, were improved to simulate the thermal-hydraulic behavior of the VHTR. The improvements were focused on the ability to represent air ingress following a LOCA. Molecular diffusion models and graphite oxidation models that account for the chemical equilibrium between $\mathrm{CO}_{2}$ and $\mathrm{CO}$ were added to both codes. The chemical equilibrium of $\mathrm{CO}, \mathrm{CO}_{2}$, and $\mathrm{O}_{2}$ is temperature-dependent as shown in Figure 2. The equimolar fraction of $\mathrm{CO}$ and $\mathrm{CO}_{2}$ appears at $950 \mathrm{~K}$ temperature and Figure 2 indicates that the chemical reaction of graphite oxidation proceeds favorably towards the generation of $\mathrm{CO}$ gas at temperatures higher than $950 \mathrm{~K}\left(677^{\circ} \mathrm{C}\right)$ as opposed to temperatures less than $950 \mathrm{~K}$ where $\mathrm{CO}_{2}$ is more dominant. 


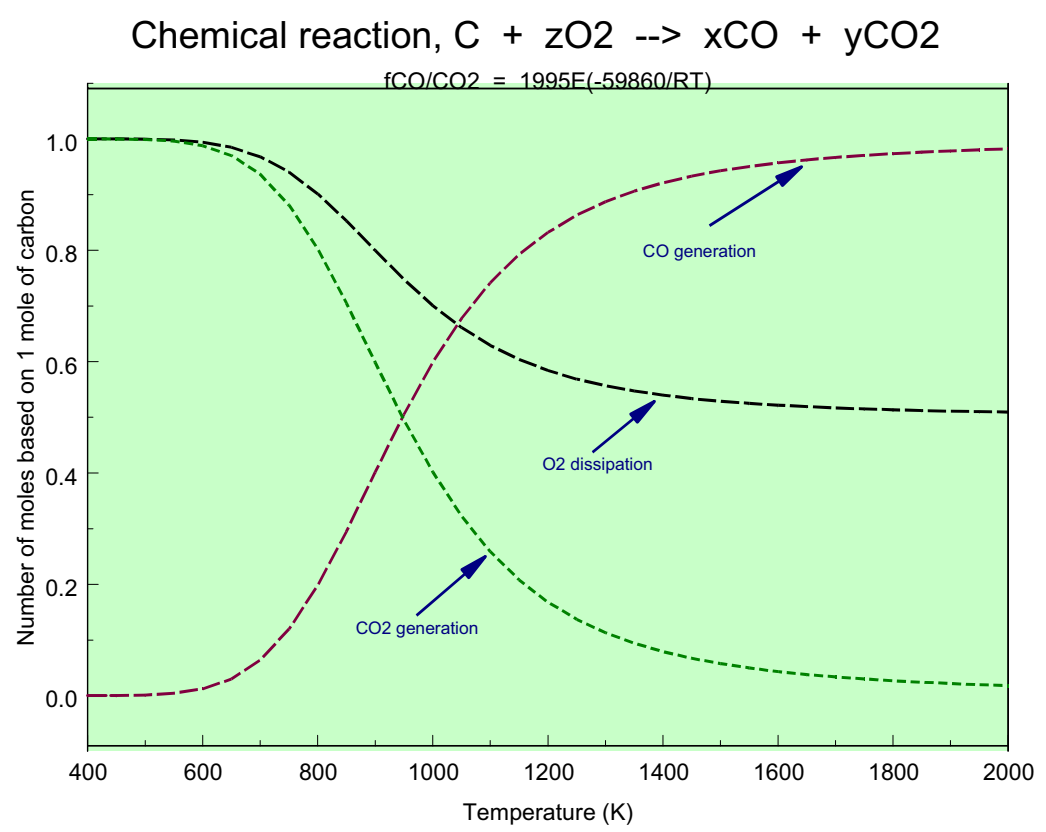

Figure 2. Representative chemical equilibrium.

Depending on the mole fraction of each chemical component, the total energy deposit to graphites varies, which will affect the graphite temperature. The chemical equilibrium was implemented to both RELAP5 and MELCOR codes.

Thermodynamic and transport properties for three gases involved in graphite oxidation $\left(\mathrm{O}_{2}, \mathrm{CO}_{2}\right.$, and $\left.\mathrm{CO}\right)$ were added to RELAP5/ATHENA as illustrated in Figures 3 and 4.

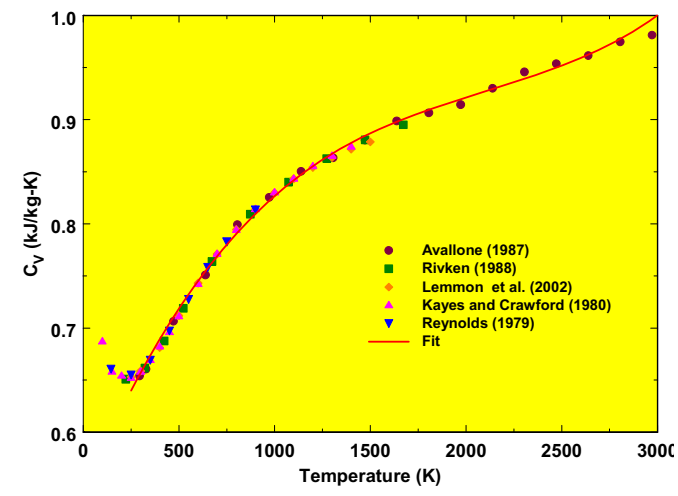

Figure $3 . \mathrm{O}_{2}$ specific heat capacity.

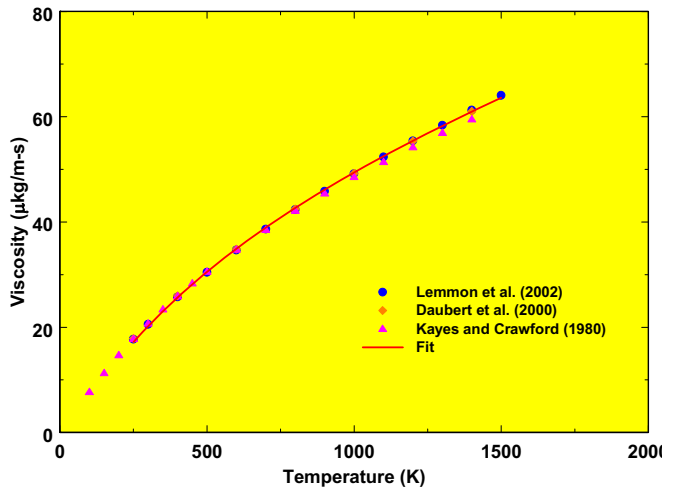

Figure 4. $\mathrm{O}_{2}$ viscosity.

The detailed description and calculations are shown in the section 5 .

\section{Task 5 - Neutronic modeling (UM):}

The following are the key accomplishments for Task 5: 
Analysis of Double Heterogeneity. A neutronic model for particle fuel has been created and tested that accounts for the double heterogeneity posed by the particle fuel and is valid at all levels of analysis, from a microsphere cell to full core.

Development of Two-region Model for Fuel Microspheres. We have developed a two-region model of the six-region microsphere cell and demonstrated that it is an excellent model for all levels of analysis from a single microsphere to full core depletion.

Elimination of Clipped Cells. Clipped cells are an artifact of the geometry processing routines in MCNP5 that arises when a lattice of fuel particles is inserted in a fuel compact cylinder. We have developed a method that eliminates clipping while preserving both the packing fraction and a simple cubic lattice within the fuel region.

Accounting for Stochastic Fuel. We have shown that the random distribution of fuel particles in the TRISO fuel mixture can be modeled by placing the fuel particles on a simple cubic lattice. This has been shown by comparison of MCNP5 simulations with randomly placed fuel particles in the fuel region (using a stochastic geometry option in MCNP5) versus fuel particles that are placed on a simple cubic lattice. These comparisons were performed for single microsphere geometries, fuel compact geometries, and full core geometries. In all cases, the $\mathrm{k}_{\mathrm{eff}}$ difference was less than $0.15 \%$. The stochastic geometry option has recently been made a regular option in the production version of MCNP5.

Development of Pseudo-material Model in MCNP5. A capability has been developed to allow interpolation of cross sections at arbitrary temperatures using cross sections libraries that were generated at lower and higher temperatures. This feature allows temperature feedback to be accommodated easily and accurately by MCNP5.

Coupling of Athena and MCNP5. The thermal/hydraulic code RELAP/Athena has been successfully coupled with the Monte Carlo code MCNP5, resulting in a capability to account for nuclear-thermalhydraulic feedback, using the pseudo-material model mentioned above. This capability has been used to obtain a converged flux-power distribution for a full-core VHTR configuration at BOC.

Full Core Depletion with Particle Fuel. A full-core Monte Carlo depletion of the VHTR core with 30 depletion zones (10 axial zones and three radial rings) has been performed with an explicit representation of the particle fuel and a non-uniform temperature distribution.

\section{Task 6-- Validation and Verification - (All)}

KAIST applied the GAMMA code to assess the system behaviors during the air ingress accident following the complete break of main pipes. And then we performed the analysis of the air ingress accident for PBMR 268MWt. In the GAMMA analysis, the significant rise in pebble temperature was observed in the bottom of the core due to graphite oxidation. Since the air ingress process depends on the vault conditions, further analysis coupled with more detailed vault or containment modeling would be necessary as future study. As a further plant application of GAMMA code, we conducted two analyses: IAEA GT-MHR benchmark calculation for LPCC and air ingress analysis for PMR 600MWt. The GAMMA code shows comparable peak fuel temperature trend to those of other country codes. The analysis result for air ingress shows much different trend from that of previous PBR analysis: later onset of natural circulation and less significant rise in graphite temperature. 
In INL, the RELAP5/ATHENA and MELCOR system codes were validated using molecular diffusion data from inverted U-tube and two-bulb experiments. The codes were also validated using experiments that simulated natural circulation flow through a pebble bed. RELAP5/ATHENA was also validated using heat transfer data from SNU's RCCS experiment. Selected results are shown below.

Results from both codes are compared with the NACOK natural circulation experiments in Figure 5. These experiments measured the mass flow rate of air through a pebble bed as a function of the temperature in the experimental channel for a given temperature of the return tube. Values for return tube temperatures of 200 and $400^{\circ} \mathrm{C}$ are shown. The trends were similar in the calculations and the experiment. The mass flow rate initially increased sharply with increasing experimental channel temperature, reached a maximum value, and then gradually decreased. The volumetric flow increased monotonically with experimental channel temperature because the increased temperature difference between the channel and the supply tube caused an increased driving head for natural circulation. However, the mass flow decreased at higher temperatures because the density decreased at a faster rate than the volumetric flow increased, and, to a lesser extent, because the Reynolds number was decreasing, which caused increased hydraulic resistance. The calculated results with both codes were in reasonable agreement with the experiment.

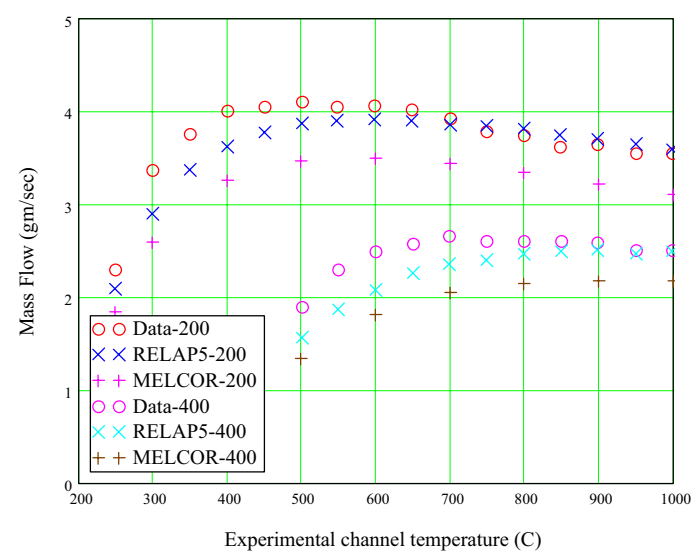

Figure 5. Measured and calculated air flow rates for the NACOK natural circulation experiments.

MELCOR calculations of coupled molecular diffusion and graphite oxidation were compared with data from the Takeda (1996) inverted U-tube experiment containing a 450-mm long graphite sleeve. Both ends of the tube were attached to the top of a tank containing helium and air. The tube was initially isolated from the tank by valves located at the ends of the tube. One side of the U-shaped tube was heated and the other side is cooled. Figure 6 shows calculated and measured mixture density at selected locations in the experiment. The MELCOR results are in good agreement with the experiment data. 


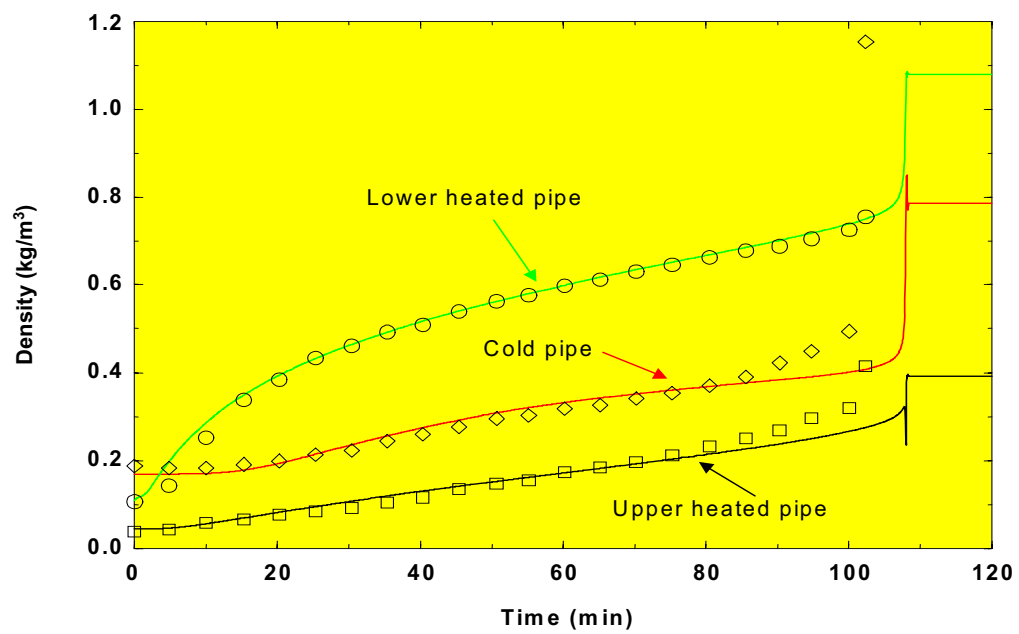

Figure 6. Comparison of MELCOR-predicted (solid lines) and the inverted U-tube experiment mixture densities (symbols).

\section{Student Participation}

\section{$\underline{\text { List of Student (ROK) }}$}

\section{KAIST}

Hyun Min Kim (Ph. D)

Young Min Moon (Ph. D)

Sang Ik Lee (Ph. D)

Kyung Won Lee (Ph. D Candidate)

Eung Soo Kim (Ph. D Candidate)

Ji Hwan Kim (Ph. D Candidate)

Eu Hwak Lee (Ph. D Candidate)

Ho Jun Yoon (M.S. Candidate)

Sung Ju Song (M.S. Candidate)

Seung Hun Yu (M.S. Candidate)

\section{Seoul National University (SNU)}

Moon O Kim (Ph. D)

Hyuk Kweon (Ph. D Candidate)

Song Won Kim (Ph. D Candidate)

Dong Woon Seo (M.S. Candidate)

Yun Jei Cho (M.S. Candidate)

\section{University of Michigan (UM)}

Wei Ji, (Ph.D Candidate)

Gokhan Yesilyurt, (Ph.D Candidate)

Jeremy Conlin, (M.S. Candidate) 


\section{Publications}

\section{Task 1: KAIST}

HS Lim and HC NO, "Transient Multicomponent Mixture Analysis Based on ICE Numerical Technique for Simulation of an Air Ingress Accident in a HTGR," Spring Meeting of the Korean Nuclear Society, Gyeongju, May 2003.

HS Lim and HC NO, "Transient Multicomponent Mixture Analysis Based on ICE Numerical Technique for Simulation of an Air Ingress Accident in a HTGR," The 10th International Topical Meeting on Nuclear Reactor Thermal Hydraulics (NURETH-10), Seoul, Korea, Oct. 5-10, 2003.

HS Lim and HC NO, "Development of 3-D Muliticomponent Mixture Analysis (GAMMA) Code for Pebblebed Safety Analysis in a HTGR," Proc. of KNS Spring Meeting, Gyeongju, Korea, May 2004.

HS Lim and HC NO, "Validation of GAMMA-3D Using Inverse U-tube and HTTR-Simulated Air Ingress Experiments," Spring Meeting of the Korean Nuclear Society, Gyeongju, Korea, May 2004.

H.S. Lim and H.C. NO, "Development and Validation of 3-D Multi-component Mixture Analysis Tool (GAMMA) for Air Ingress in HTGRs", the 2nd International Topical Meeting on High Temperature Reactor Technology (HTR), Beijing, China, September 2004

Hee Cheon NO, Eung Soo Kim and Hong Sik Lim, "Multi-Dimensional Multicomponent Mixture Air-Ingress Analysis and Air-Ingress Experiment in a HTGR," 6th International Topical Meeting on Nuclear Reactor Thermal Hydraulics, Operations and Safety (NUTHOS-6), Nara, Japan, Oct. 4-8, 2004.

Won-Jae Lee, Hong-Sik Lim, Jong-Hwa Chang, Hee-Cheon No* and Gun-Cheorl Park, "Progress of Accident Analysis Codes Development for Gas-Cooled Reactors", ANS 2004 Winter Meeting, OMNI Shoreham Hotel, November 14-18, 2004, Washington, DC

Hong Sik Lim and Hee Cheon NO, "GAMMA Validation with the HTTR RCCS Mockup Test for the Assessment of Decay Heat Removal of GCRs under Accident Conditions", Proc. of KNS Autumn Meeting, Yongpyong, Korea, Oct 2004.

Hong Sik Lim, Hee Cheon NO, "Transient Multicomponent Mixture Analysis Based on an ICE Numerical Technique for the Simulation of an Air Ingress Accident in an HTGR", Journal of the Korea Nuclear Society, Vol. 36, No. 5, pp. 375 387 (2004)

Hee-Cheon NO, Chang H. Oh, Jong H. Kim, Goon-Cherl Park, John C. Lee, William R. Martin, Hong Sik Lim, Eung Soo Kim, M.O. Kim, Jeremy L. Conlin, Wei Ji, "Very High Temperature Gas-cooled Reactors: Progress and Challenges," ICAPP'05, Seoul, Korea, 2005.

Hong Sik Lim and Hee Cheon NO, "GAMMA Air Ingress Analysis for a Pebble-Bed Gas-Cooled Reactor", Proc. of KNS Spring Meeting, Jeju, Korea, May 2005.

\section{Task 2: SNU}

MO Kim, DU Seo, BU Bae, and GC Park, "Introduction to SNU-RCCS for MHTGR," Autumn Meeting of the Korean Nuclear Society, Yong-pyeong, Korea, May 2003. 
Y.J. Cho, M. O. Kim, G. C. Park, "Measuring Characteristics on Emissivity Using Infrared Thermometer for RCCS," NTHAS4: Fourth Japan-Korea Symposium on Nuclear Thermal Hydraulics and Safety, Sapporo, Japan, November 28-December 1, 2004

D.U. Seo, M.O. Kim, H.K. Cho, T.W. Kim, G.C. Park, "Numerical Study on the Heat Transfer Phenomena in Water Pool Type-Reactor Cavity Cooling System of Very High Temperature Gas-cooled Reactor ", 18th International Conference on Structural Mechanics in Reactor Technology (SMiRT-18), Beijing, China, Aug. $7-12,2005$.

H.K.Cho, D.U. Seo, M.O.Kim, G.C.Park, "Study on The Heat Transfer In The Water Pool Type Reactor Cavity Cooling System Of The Very High Temperature Gas Cooled Reactor," 2005 ASME Summer Heat Transfer Conference, July 17-22, 2005, San Francisco, California, USA

H.K.Cho, M.O.Kim, H.K.Ahn, G.C.Park, "Experimental Study for the Water Pool Type Reactor Cavity Cooling System in the Very High Temperature Gas Cooled Reactor", 5th International Symposium on Multiphase Flow, Heat Mass Transfer and Energy Conversion, 3-6 July, 2005, Xi'an, China

Y.J. Cho, M. O. Kim, G. C. Park, "Emissivity Measurement and Evaluation for Analysis of SNU-RCCS," The Second Korea-China Workshop on Nuclear Reactor Thermal Hydraulics, International Center, Cheju National University, Jeju, KOREA, May 24-25, 2005

Y. J. Cho, M. O. Kim and G. C. Park, "Experimental Study on Measurement of Emissivity for Analysis of SNU-RCCS," Journal of the Korean Nuclear Society, Vol. 38, 1, February, 2006

G.C. Park, Y.J. Cho, H.K. Cho, "Assessment of a New Design for a Reactor Cavity Cooling System in a Very High Temperature Gas-cooled Reactor," Journal of the Korean Nuclear Society, Vol. 38, 1, February, 2006

\section{Task 3: KAIST}

Eung Soo Kim and Hee Cheon NO, "Measurement of Kinetic Parameters of Nuclear Graphite IG-110 for HTGR Air-ingress," Proceedings of Korean Nuclear Society Spring Meeting, Gyeongju, Korea, May 2004

Eung Soo Kim and Hee Cheon NO, "Oxidation Parameters for Nuclear Graphite IG-110," 6th International Topical Meeting on Nuclear Reactor Thermal Hydraulics, Operations and Safety (NUTHOS-6), Nara, Japan, Oct. 4-8, 2004.

Eung Soo Kim*, Kyung Won Lee and Hee Cheon NO, Analysis of geometrical effects on graphite oxidation through measurement of internal surface area, Journal of Nuclear Materials, 2006, Vol. 348, pp. 174-180.

Eung Soo Kim* and Hee Cheon NO, Experimental study on the oxidation of nuclear graphite and development of an oxidation model, Journal of Nuclear Materials, 2006, Vol. 349, pp. 182-194.

Eung Soo Kim* and Hee Cheon NO, Experimental study on the reaction between nuclear graphite IG-110 and carbon dioxide, Journal of Nuclear Materials, 2006, vol. 350, pp. 96-100. 


\section{Task 4: INL}

H. No, C. Oh, J. Kim, G. Park, J. Lee, W. Martin, M. Kim, J. Conlin, and W. Ji, "Very-HighTemperature Gas-Cooled Reactors: Progress and Challenges," Proc. International Congress on Advances in Nuclear Power Plants, ICAPP 2005, Seoul, Korea, May 15-19, 2005.

R. Moore and C. Oh, "Assessment of a Molecular Diffusion Model in Melcor," 2005 ANS Transactions, Vol. 92. pp.215-216,Annual Meeting, San Diego, CA. June 5-9, 2005

C. Davis, L. Siefken, and C. Oh, "Assessment of RELAP5-3D for Analysis of Very High Temperature Gas-Cooled Reactors," NURETH-11, Avignon, France, October 1-8, 2005.

C. Davis, L. Siefken, and C. Oh, "Assessment of a Molecular Diffusion Model in RELAP5," submitted to 2005 ANS Winter Meeting, Washington DC, November 13-17, 2005.

C. Davis, L. Siefken, and C.H. Oh, Assessment of a Molecular Diffusion Model in RELAP5-3D, ANS Winter Annual Meeting, Washington DC, Nov.14-18 ,2004

C.H. Oh, R.L. Moore, and B.J. Merrill, Addition of Diffusion Model to MELCOR and Comparison with Data, ANS National Meeting, Pittsburgh, PA, June 13-17, 2004.

C. Davis, C.H. Oh, The Addition of Noncondensable Gases into RELAP5-3D for Analysis of High temperature Gas-Cooled Reactors, the 10th International Topical Meeting on Nuclear Reactor Thermal Hydraulics, Seoul, Korea, October 5-9, 2003.

C. Davis, C.H. Oh, Modification of RELAP5-3D for noncondensable Gases for Analysis of High temperature Gas-Cooled Reactors, RELAP5 Workshop, West Yellowstone, 2003.

\section{Task 5: UM}

F. B. Brown and W. R. Martin, "Stochastic Geometry for MCNP5," Trans. Am. Nucl. Soc. 91, 171-173, Washington, DC (November 2004).

F. B. Brown and W. R. Martin, "Stochastic Geometry Capability in MCNP5 for the Analysis of Particle Fuel," Annals of Nuclear Energy 31, (November 2004).

W. Ji, J. L. Conlin, W. R. Martin, and J. C. Lee, "Reactor Physics Analysis of the VHTGR Core," Trans. Am. Nucl. Soc. 91, 556-558, Washington, DC (November 2004).

F. B. Brown, W. R. Martin, W. Ji, J. L. Conlin, and J. C. Lee, "Stochastic Geometry and HTGR Modeling with MCNP5," Proc. Monte Carlo 2005 Topical Meeting, American Nuclear Society, Chattanooga, TN, April 17-21, 2005, on CD-ROM, American Nuclear Society, LaGrange Park, IL (2005).

W. Ji, J. L. Conlin, W. R. Martin, J. C. Lee, and F. B. Brown, "Explicit Modeling of Particle Fuel for the Very-High Temperature Gas-Cooled Reactor," Trans. Am. Nucl. Soc. 92, 236-238, San Diego, CA (2005). 
J. L. Conlin, W. Ji, J. C. Lee, and W. R. Martin, "Pseudo Material Construct for Coupled Neutronic-ThermalHydraulic Analysis of VHTGR," Trans. Am. Nucl. Soc. 92, 225-227, San Diego, CA (2005).

H.-C. No, C. H. Oh, J. H. Kim, G.-C. Park, J. C. Lee, W. R. Martin, M. O. Kim, J. L. Conlin, and W. Ji, "Very-High-Temperature Gas-Cooled Reactors: Progress and Challenges," Proc. International Congress on Advances in Nuclear Power Plants, ICAPP 2005, Seoul, Korea.

W. Ji, J. L. Conlin, G. Yesilyurt, W. R. Martin, J. C. Lee, and F. B. Brown, "Neutronic Analysis to Support Validation of Safety Analysis Codes for the VHTR," Trans. Am. Nucl. Soc. 93, 931-933, Washington, DC (November 2005). 


\section{Table of Content}

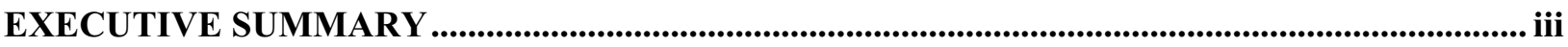

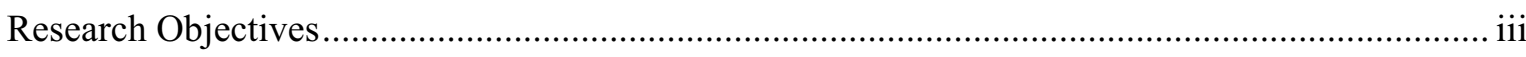

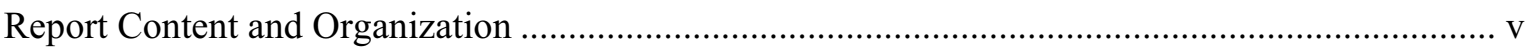

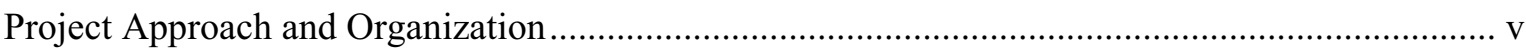

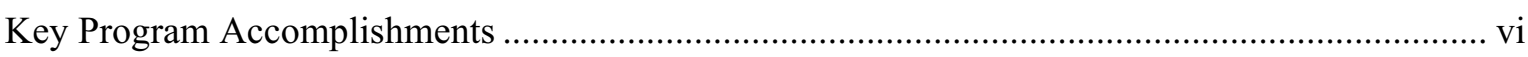

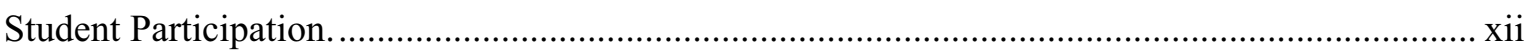

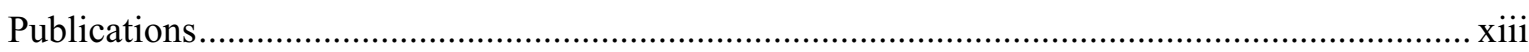

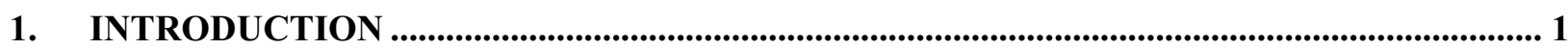

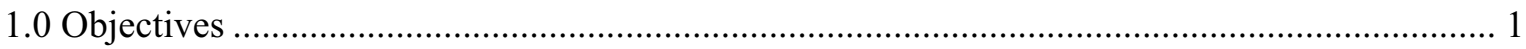

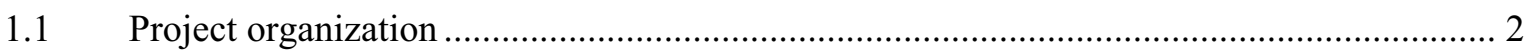

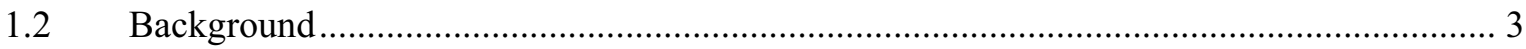

2. TASK 1: CFD thermal hydraulic benchmark code development (KAIST)........................................ 6

2.1. Governing Equations and Numerical Method .............................................................. 6

2.2. Development and V\&V of Physical Models............................................................. 11

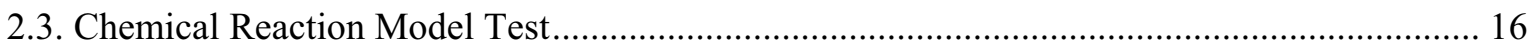

2.4. Validation with Air Ingress Experiments: Inverse U-tube Air Ingress Experiment ................ 21

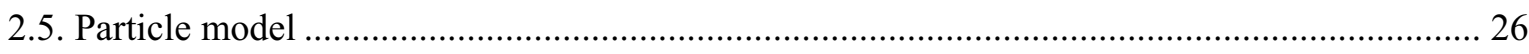

3. TASK 2: RCCS experiment (SNU) .................................................................................................... 32

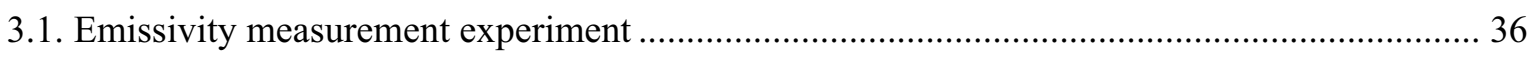

3.2. Separate effect test for the water pool of the RCCS-SNU - Part 1 ...................................... 44

3.3. Separate effect test for the water pool of the RCCS-SNU -Part 2 ....................................... 57

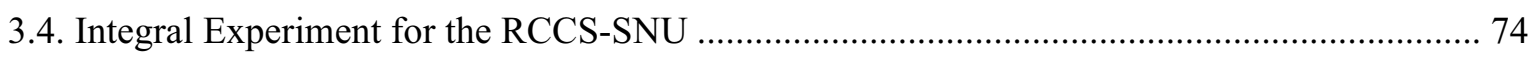

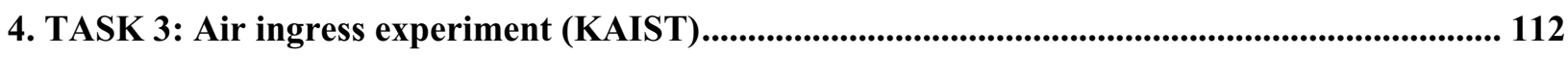

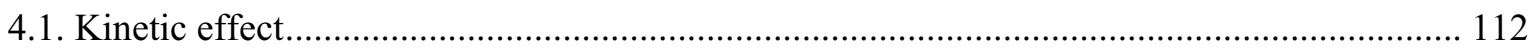

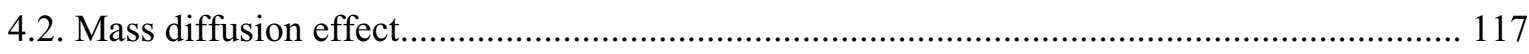




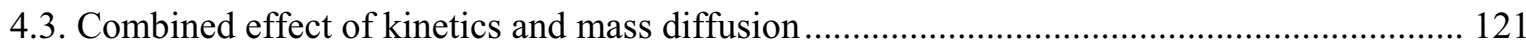

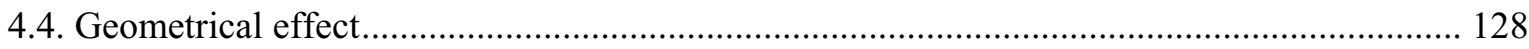

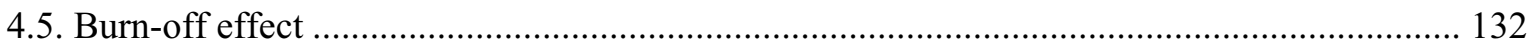

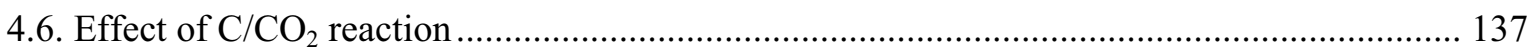

4.7. Overview on the improvement in oxidation resistance for nuclear graphite ....................... 140

5. TASK 4: Improvement of System Codes (INL) ................................................................... 142

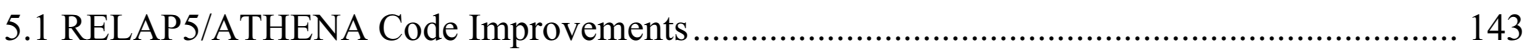

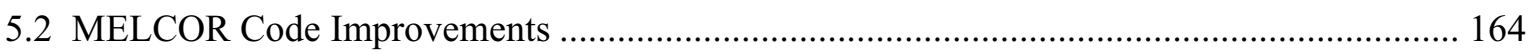

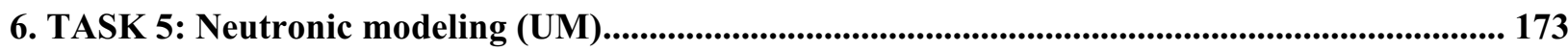

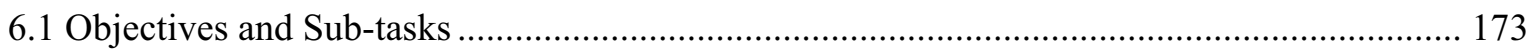

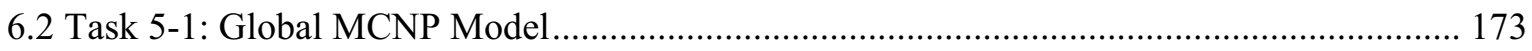

6.3 Task 5-2: Coupled Nuclear-Thermal-Hydraulic Calculations .............................................. 186

7. Task 6: Code Validation and Verification (V\&V) (All) .................................................................... 194

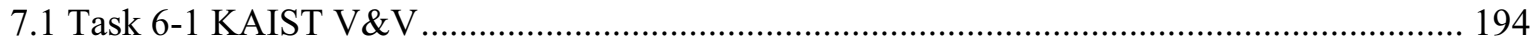

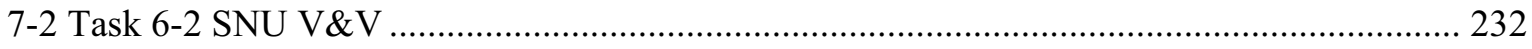

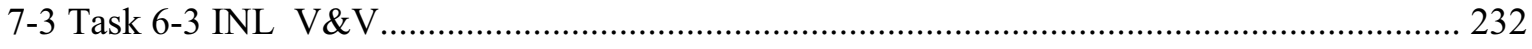

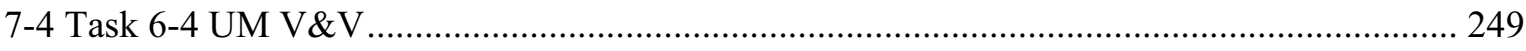

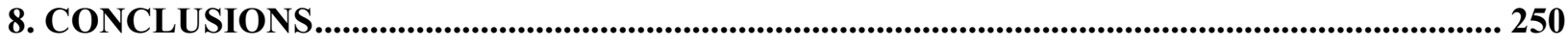

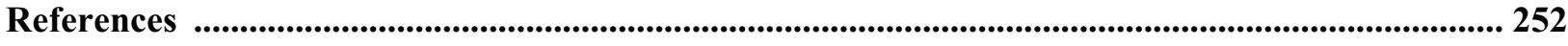




\section{INTRODUCTION}

A loss-of-coolant accident (LOCA) has been considered a critical event for very high temperature gas-cooled reactor (VHTR). Following helium depressurization, it is anticipated that unless countermeasures are taken, air will enter the core through the break by molecular diffusion and ultimately by natural convection leading to oxidation of the in-core graphite structure. Thus, without any mitigating features, a LOCA will lead to an air ingress event, which will lead to exothermic chemical reactions of graphite with oxygen, potentially resulting in significant increases of the core temperature.

New and safer nuclear reactors (Generation IV) are now in the early planning stages in many countries throughout the world. One of the reactor concepts being seriously considered is the VHTR. To achieve public acceptance, these reactor concepts must show an increased level of inherent safety over current reactor designs (i.e., a system must be designed to eliminate any concerns of large radiological releases outside the site boundary).

The objective of the proposed program is to demonstrate how a VHTR can achieve a higher reactor safety margin. Given the level of maturity associated with today's nuclear designs, this level of safety feature is a challenge and will never be overcome by marginal improvements. Rather, aggressive technical approaches, which recognize constraints of passive safety, are required to meet the world challenge of the power industry. Without adequate numerical tools, the technical challenge of reactor safety cannot be met.

\subsection{Objectives}

The following are the major objectives and specific goals of the proposed program:

\subsubsection{Objective 1-Development of the benchmark CFD tool}

We will develop a thermal hydraulics safety code for analyzing air ingress, reactivity cavity cooling, and other safety-related issues. This code will handle (1) molecular diffusion, (2) multi-dimensional convective mass, momentum, energy transport, (3) energy transport through radiation, (4) energy production by fission and decay heat removal, (5) chemical kinetics, (6) wall-gas convective heat transfer, (7) graphite depletion due to oxidation, (8) oxygen depletion in the reactor cavity, and (9) $\mathrm{CO}$ accumulation in the reactor vessel and reactor cavity.

\subsubsection{Objective 2-Improvement of two world famous system codes}

We will improve two system codes (RELAP5/ATHENA and MELCOR) for more detailed accident analyses of the VHTR. The improvements to RELAP5 include the addition of thermodynamic and transport properties for $\mathrm{O}_{2}, \mathrm{CO}_{2}$, and $\mathrm{CO}$ noncondensable gases. Models for molecular diffusion and graphite oxidation that account for the chemical equilibrium between $\mathrm{CO}_{2}$ and $\mathrm{CO}$ will be added to both codes.

\subsubsection{Objective 3-Verification and Validation of Computer Codes and Models}

Verification and validation of the computer codes and models will be performed with the neutronic and safety analysis in performance of the VHTR concept, particularly for steady-state operation and air ingress event. At present, verification and validation are very limited in the U.S. and nothing has been reported for VHTR or other HTGRs. This is essential in nuclear plant licensing. 


\subsection{Project organization}

This NERI project has been organized and managed by Dr. Chang Oh at the INEEL with the help of Professor Hee Cheon NO at the Korea Advanced Institute of Science and Technology (KAIST). The project has been organized into the code developmental work and experimental work, which includes two separate tests. The organization of this project is described in the following organizations and principal investigators working on each task:

\begin{tabular}{|l|c|c|c|}
\hline \multicolumn{1}{|c|}{ Task } & Lead Responsibility & $\begin{array}{c}\text { Collaborating } \\
\text { Organization(s) }\end{array}$ & Principal Investigator(s) \\
\hline $\begin{array}{l}\text { 1. Development of } \\
\text { Benchmark CFD } \\
\text { Code }\end{array}$ & KAIST & INEEL & $\begin{array}{c}\text { Prof. Hee Chun NO and } \\
\text { Jong Kim }\end{array}$ \\
\hline $\begin{array}{l}\text { 2.RCCS Experiment } \\
\text { Seoul National } \\
\text { University }\end{array}$ & KAIST & Prof. Goon Cherl Park \\
\hline $\begin{array}{l}\text { Experiment } \\
\text { System Improvement of } \\
\text { Systed }\end{array}$ & INEEL & INEEL & Prof. Hee Chun NO \\
\hline 5. Neutronic Models & University of Michigan & INEEL/KAIST & Dr. Chang Oh \\
\hline $\begin{array}{l}\text { 6. Verification and } \\
\text { Validation } \\
\text { Simulation }\end{array}$ & INEEL & KAIST/UM/SNU & $\begin{array}{c}\text { Pr. Oh and Professors NO, } \\
\text { Park, and Lee }\end{array}$ \\
\hline
\end{tabular}

Figure 1-1 illustrates the allocations of all the project tasks. 


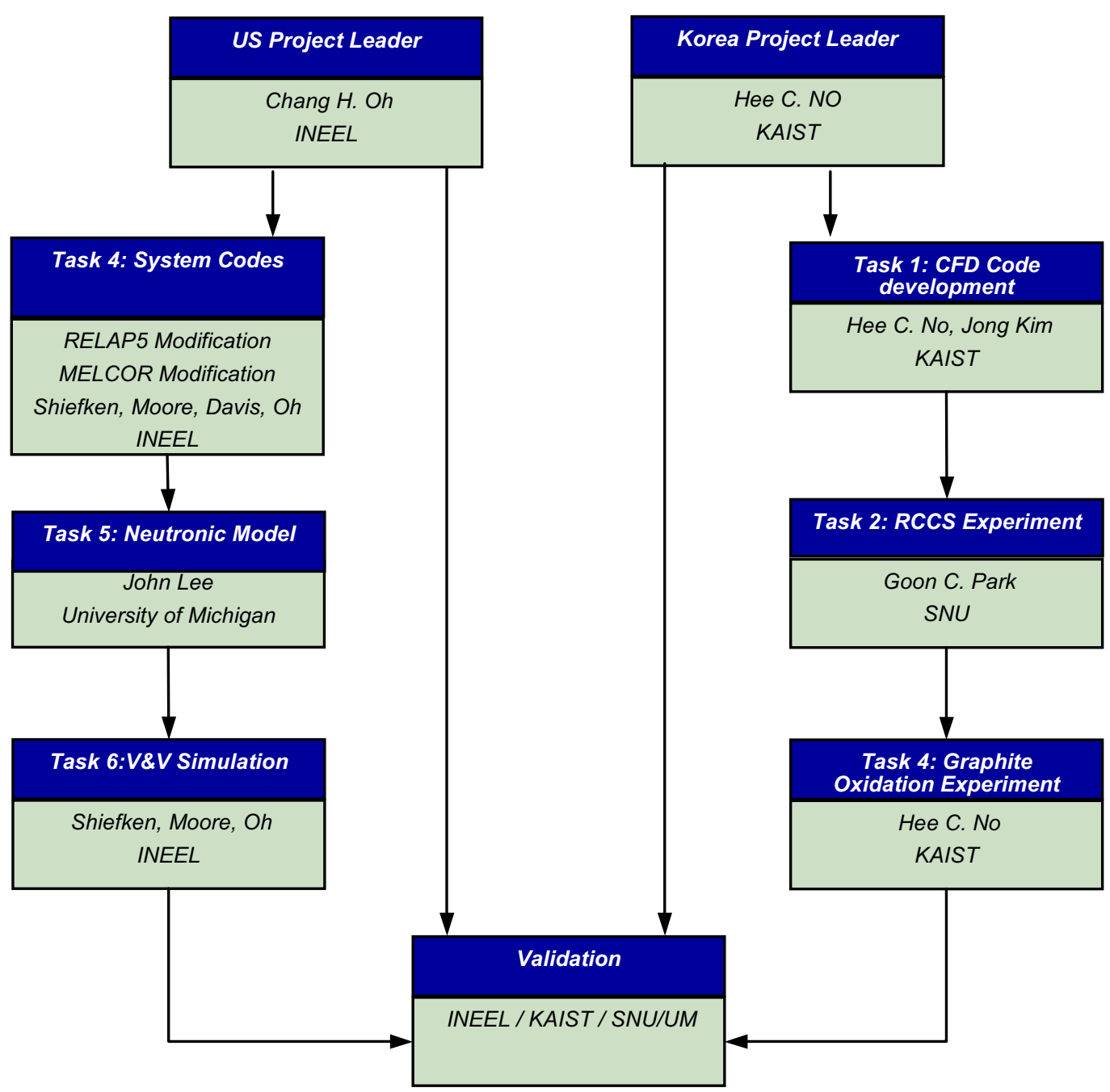

Figure 1-1. Project organization.

\subsection{Background}

The VHTR is a uranium-fueled, graphite-moderated, helium-cooled reactor using a direct or indirect gas cycle to convert the heat generated by nuclear fission into electrical energy by means of a helium turbogenerator. The HTGR technology has been researched and built since the 1950s. The VHTR produces a higher outlet temperature than the HTGR. The HTGRs work on the principle of passing a cooling gas through the core and then running the heated gas directly to a steam generator or a gas turbine. The VHTRs have been built in Japan and China for their nuclear research.

The VHTR is one of the survivors among five other reactor concepts such as the supercritical water reactor, gas-cooled fast reactor, lead-bismuth batter reactor, sodium-cooled liquid metal reactor, and molten salt reactor. In the U.S., the INEEL is trying to build one prototype gas-cooled reactor, either a very high temperature reactor or gas-cooled fast reactor. The popularity of the VHTR has been growing and this will ultimately change American's perception on nuclear reactors if the energy crisis and ozone layer depletion due to $\mathrm{CO}_{2}$ emissions from fossil power plants get more serious. 
There are several advantages of the VHTR over the light-water reactors (LWRs). These include fuel integrity, proliferation resistance, a relatively simple fuel cycle, online fuel maintenance, and modularity to supply electricity to remote areas and energy-starved underdeveloped countries with little power generation infrastructure. The characteristics of the VHTR are (1) helium coolant, (2) higher than $900^{\circ} \mathrm{C}$ outlet temperature, (3) a modularity of $600 \mathrm{MWe}$, and (4) solid graphite block core based on GT-MHR (Figure 1-1). Benefits of the VHTR concept (Figure 1-2) are (1) high thermal efficiency compared to other concepts, (2) hydrogen production, (3) process heat applications, and (4) high degree of passive safety.

However, the VHTR faces some technical and economical challenges, particularly reactor safety and costs. Our concerns about the VHTR are an air ingress event following a LOCA. A LOCA is one of the design-basis accidents for VHTR. Following the

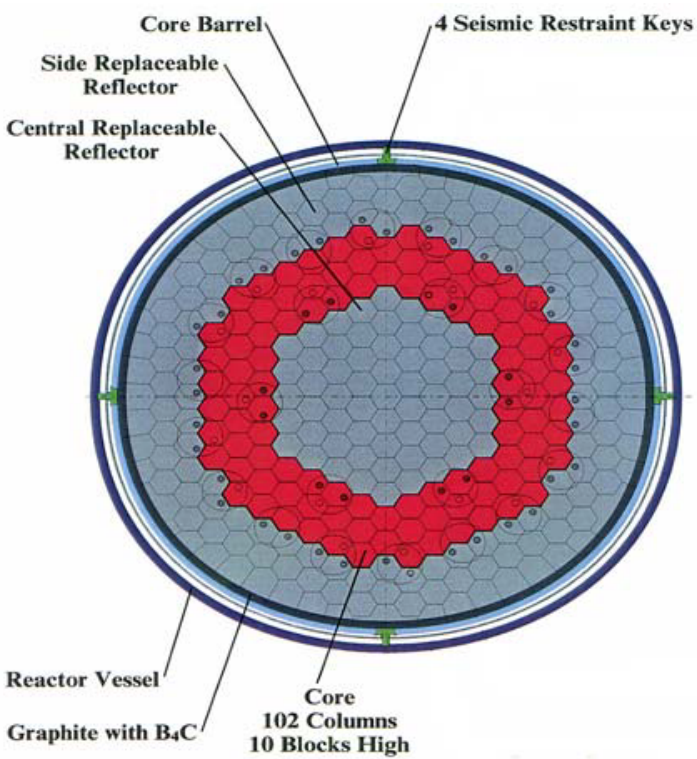

Figure 1-2. The GT-MHR core. depressurization of helium in the core, if the accident is not mitigated, there exists the potential for air to enter the core through the break and oxidize the in-core graphite structure in the VHTR. We all remember that the Chernobyl accident in 1986 was a result of a flawed reactor design that was operated with inadequately trained personnel and without proper regard for safety. On April 25, 1986, prior to a routine shutdown, the reactor crew at Chernobyl-4 began preparing for a test to determine how long turbines would spin and supply power following a loss of main electrical power supply. Similar tests had already been carried out at Chernobyl and at other plants, despite the fact that these reactors were known to be very unstable at low power settings. A series of operator actions, including the disabling of automatic shutdown mechanisms, preceded the attempted test early on April 26. As flow of coolant water diminished, power output increased. When the operator moved to shut down the reactor from its unstable condition arising from previous errors, a peculiarity of the design caused a dramatic power surge. The fuel elements ruptured and the resultant explosive force of steam lifted off the cover plate of the reactor, releasing fission products to the atmosphere. A second explosion threw out fragments of burning fuel and graphite from the core and allowed air to rush in, causing the graphite moderator to burst into flames.

There are still some disputes among experts about the character of the second explosion. When the graphite reflector was exposed to air from the first explosion, air ingress could have led to the second explosion, which is one scenario for this accident. However, the fact is that this accident led to graphite burning for 9 days, causing the main release of radioactivity into the environment. About $12 \times 10^{18} \mathrm{~Bq}$ of radiation was released. 


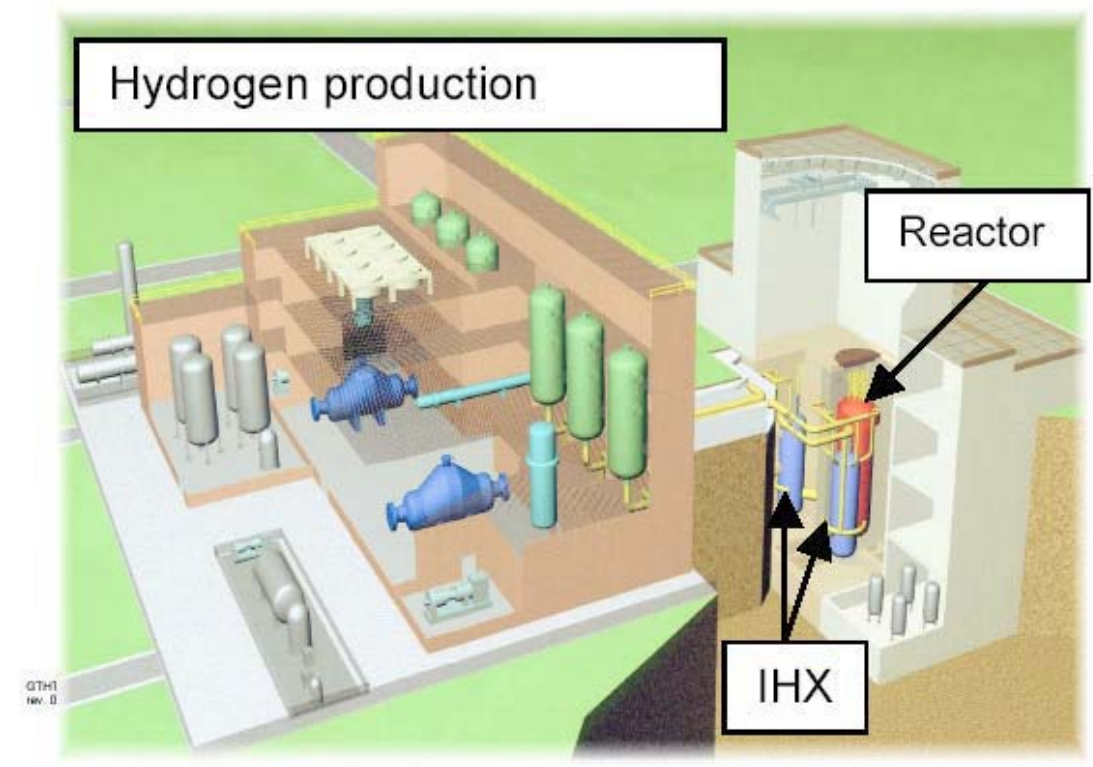

Figure 1-3. Conceptual VHTR schematic with hydrogen production.

Therefore, an air ingress event should be considered a very important technical issue on VHTR. The INEEL has studied this event for 3 years (INEEL 1999, INEEL 2000, INEEL 2001) as part of the INEEL Generation IV Initiative; preliminary results indicate that without analyzing the accident scenarios using the validated numerical tools and without proper design for preventing this type of accident, a second Chernobyl accident could happen in the future if this type of reactor is built without mitigating this event with reactor safety, which can be analyzed by validated numerical tools before the plant licensing.

Again, the VHTR provides an alternative approach to the commercialization of nuclear power as compared to other fission-power-producing systems such as LWRs and liquid metal-cooled fast breeder reactors. Gas cooling for nuclear reactors had been considered in the United States. The fundamental design of the VHTR is aimed at achieving a system without any physical process that could cause an internally or externally induced radiation hazard outside the site boundary. The thermal hydraulic stabilization is provided by modularizing the core with a relatively low power density $\left(<4.5 \mathrm{MW} / \mathrm{m}^{3}\right)$ such that the integrated heat loss capability from the reactor exceeds the decay heat production of the core under all conceivable accident conditions. The use of helium as a coolant, which is both chemically and radiologically inert, combined with the high-temperature integrity of the fuel and structural graphite, allows for the use of high primary coolant temperature (up to $1200^{\circ} \mathrm{C}$ ) that yields high thermal efficiencies.

Conclusively, the plant design should be streamlined to be technically sound, robust, proliferation-resistant, and low cost. Although gas reactors have been developed in the past with limited success, the innovations of modularity and integrated state-of-the-art safety systems make the VHTR design potentially very attractive from a technical and economic perspective. 


\section{TASK 1: CFD thermal hydraulic benchmark code development (KAIST)}

The objective of this task is to develop a multidimensional system analysis tool for the thermo-fluid transport processes in VHTGRs.

\subsection{Governing Equations and Numerical Method}

For the system analysis code developed to predict the important phenomena expected to occur during the thermo-fluid transients including an air ingress accident in HTGRs, the following requirements should be considered:

Fluid transport and material properties

Multi-dimensional heat conduction

Multi-dimensional fluid flow

Chemical reactions

Multi-component molecular diffusion

Fluid heat transfer and pressure drop

Heat generation and dissipation

Radiation heat transfer

\section{$\underline{\text { 2.1-1 Governing Equations }}$}

The multi-dimensional governing equations for a chemically reacting flow (Poinsot and Veynante 1999) consist of the basic equations for continuity, momentum conservation, energy conservation of the gas mixture, and the mass conservation of each species. Six gas species $\left(\mathrm{He}, \mathrm{N}_{2}, \mathrm{O}_{2}, \mathrm{CO}, \mathrm{CO}_{2}\right.$, and $\left.\mathrm{H}_{2} \mathrm{O}\right)$ are considered in the present analytical model, and it is assumed that each gas species and the gas mixture follow the equation of state for an ideal gas. The GAMMA code has the capability to handle the thermo-fluid and chemical reaction behaviors in a multi-component mixture system as well as heat transfer within the solid components, free and forced convection between a solid and a fluid, and radiative heat transfer between the solid surfaces. Also, the basic equations are formulated with a porous media model ((Neild and Bajan 1999) to consider heat transport in a pebble-bed core) as well as solid-fluid mixed components.

The equation of continuity for the gas mixture:

$\varphi \frac{\partial \rho}{\partial t}+\nabla \cdot(\rho \mathbf{u})=\varphi \sum_{s} R_{s}$

The equation of momentum conservation:

$$
\rho\left(\frac{1}{\varphi} \frac{\partial \mathbf{u}}{\partial t}+\frac{1}{\varphi^{2}} \mathbf{u} \cdot \nabla \mathbf{u}\right)=-\nabla P+\frac{1}{\varphi} \nabla \cdot(\mu \nabla \mathbf{u})-\frac{\mu}{K} \mathbf{u}-\frac{C_{F} \rho}{\sqrt{K}}|\mathbf{u}| \mathbf{u}+\rho \mathbf{g}
$$

The equation of sensible energy conservation:

$$
\begin{aligned}
\varphi \frac{\partial}{\partial t}(\rho H) & +\nabla \cdot(\rho \mathbf{u} H)=\nabla \cdot\left[\left(\varphi \lambda_{f}+\lambda_{\text {disp }}\right) \nabla T_{f}\right]-\nabla \cdot\left(\varphi \sum_{s=1}^{m} H_{s} \mathbf{J}_{s}\right) \\
& -\varphi \sum_{s} \Delta h_{f_{s}}^{o} R_{s}+h_{s f} a_{s f}\left(T_{p}-T_{f}\right)
\end{aligned}
$$

The conservation equation of each species, $s$ :

$\varphi \frac{\partial}{\partial t}\left(\rho Y_{s}\right)+\nabla \cdot\left(\rho \mathbf{u} Y_{s}\right)=-\nabla \cdot\left(\varphi \mathbf{J}_{s}\right)+\varphi R_{s}$

and for He, $Y_{m}=1-\sum_{s=1}^{m-1} Y_{s}$ 
The equation of state for an ideal gas:

$\rho=\frac{P}{\bar{R} T}\left(\sum_{s=1}^{m} Y_{s} / W_{s}\right)^{-1}$

For a solid and a pebble bed, the same heat conduction equation is used. A thermal non-equilibrium model for porous media is used to consider the heat exchange between the fluid and the pebbles as follows:

$\left[(1-\varphi)(\rho C)_{p}\right] \frac{\partial T_{p}}{\partial t}=\nabla \cdot\left(\lambda_{e f f} \nabla T_{p}\right)+q^{\prime \prime \prime}-h_{s f} a_{s f}\left(T_{p}-T_{f}\right)$

Radiative heat transfer in the enclosure is well-modeled by using an irradiation/radiosity method (Holaman 1986) which assumes that the fluid is non-participating and the radiation exchange between surfaces is gray and diffuse. The net radiative flux from agglomerated surface $\mathrm{k}$, which consists of $\mathrm{N}_{\mathrm{k}}$ faces of the original mesh, is given by

$$
\begin{aligned}
& q_{r k}^{\prime \prime}=\left[\left(\sum_{j \neq k}^{M} F_{k j}\right) \varepsilon_{k} \bar{T}_{k}^{4}-\varepsilon_{k} \sum_{j \neq k}^{M} F_{k j} J_{j}\right]\left[\varepsilon_{k}+\left(1-\varepsilon_{k}\right) \sum_{j \neq k}^{M} F_{k j}\right]^{-1} \\
& J_{j}=\varepsilon_{j} C_{j} \sigma \bar{T}_{j}^{4}+\left(1-\varepsilon_{j}\right) C_{j} \sum_{i \neq j}^{M} F_{j i} J_{i}
\end{aligned}
$$

The ordinary diffusion flux $\left(\mathrm{J}_{\mathrm{s}}\right)$ is given in two forms, the full multi-component diffusion (Hirschfelder et al. 1964) and the effective diffusion (Walker et al. 1960) by the assumption that a dilute species, s, diffuses through a homogeneous mixture:

$$
\begin{aligned}
& \mathbf{J}_{s}=\rho \frac{W_{s}}{W^{2}} \sum_{k=1, k \neq s}^{m}\left[D_{s k} \nabla\left(Y_{k} W\right)\right] \text { and } \\
& \mathbf{J}_{s}=-\rho D_{s-m i x} \nabla Y_{s} \text { where } D_{s-m i x}=\left(\sum_{k=1, k \neq s}^{m} X_{k} / \mathbf{D}_{s k}\right)^{-1} \quad(\mathrm{~m} \geq 3)
\end{aligned}
$$

Although Eq. (2-8) predicts the accurate diffusion behaviors of species in a multi-component mixture, Eq. (29) is generally used in numerical calculation because of its computational efficiency and its accuracy close to that of Eq. (2-8). Physical properties, such as molar weight, viscosity, thermal conductivity, and sensible enthalpy, for each gas component and gas mixtures, are obtained from the handbooks of gas properties (Poling et al. 2001, Raznjevic 1976).

\section{$\underline{\text { 2.1-2 Numerical Method }}$}

The governing equations are discretized in a semi-implicit manner in the staggered mesh layout and then dependent variables are linearized by the Newton method. For a fast computation, the Implicit Continuous Eulerian (ICE) technique (Harlow and Amsden 1971) is adopted to reduce a $10 \mathrm{~N} \times 10 \mathrm{~N}$ whole system matrix to a $\mathrm{N} \times \mathrm{N}$ pressure difference matrix.

All the conservation equations, Eqs. (2-1)-(2-4), are discretized as follows:

$$
\begin{aligned}
\varphi_{i} \frac{\rho_{i}^{n+1}-\rho_{i}^{n}}{\Delta t} & +\nabla_{i} \cdot\left(\dot{\rho}^{n} \mathbf{u}^{n+1}\right)=\varphi_{i} \sum_{s} R_{s i}^{n+1}, \\
\frac{1}{\varphi_{j}} \frac{\mathbf{u}_{j}^{n+1}-\mathbf{u}_{j}^{n}}{\Delta t} & +\frac{1}{\varphi_{j}^{2}} \mathbf{u}_{j}^{n} \cdot \nabla_{j}\left(\mathbf{u}^{n}\right)=-\frac{1}{\bar{\rho}_{j}^{n}} \nabla_{j}\left(P^{n+1}\right)+\frac{1}{\bar{\rho}_{j}^{n} \varphi_{j}} \nabla_{j} \cdot\left[\mu^{n} \nabla\left(\mathbf{u}^{n}\right)\right] \\
& -\frac{\bar{\mu}_{j}^{n}}{K_{j}} \mathbf{u}_{j}^{n+1}-\frac{C_{F j} \bar{\rho}_{j}^{n}}{\sqrt{K_{j}}}\left|\mathbf{u}_{j}^{n+1}\right| \mathbf{u}_{j}^{n+1}+\mathbf{g}_{j}^{n}
\end{aligned}
$$




$$
\begin{gathered}
\varphi_{i} \frac{(\rho H)_{i}^{n+1}-(\rho H)_{i}^{n}}{\Delta t}+\nabla_{i} \cdot\left(\dot{\rho}^{n} \dot{H}^{n} \mathbf{u}^{n+1}\right)=\nabla_{i} \cdot\left[\left(\varphi \lambda_{f}+\lambda_{\text {disp }}\right)^{n} \nabla\left(T_{f}{ }^{n}\right)\right] \\
-\nabla_{i} \cdot\left[\sum_{s=1}^{m}\left(\varphi \bar{H}_{s}^{n} \mathbf{J}_{s}{ }^{n}\right)\right]-\varphi_{i} \sum_{s} \Delta h_{f_{s}}^{o} R_{s i}^{n+1} \\
+h_{s f_{i}} a_{s f}\left(T_{p}-T_{f}\right)_{i}^{n} \\
\varphi_{i} \frac{\left(\rho Y_{s}\right)_{i}^{n+1}-\left(\rho Y_{s}\right)_{i}^{n}}{\Delta t}+\nabla_{i} \cdot\left(\dot{\rho}^{n} \dot{Y}_{s}^{n} \mathbf{u}^{n+1}\right)=-\nabla_{i} \cdot\left(\varphi \mathbf{J}_{s}^{n}\right)+\varphi_{i} R_{s i}{ }^{n+1}
\end{gathered}
$$

where a bar (-) indicates average property and a dot (.) indicates donor property ( $1^{\text {st }}$-order upwind) which depends on flow direction. In the staggered mesh, $i$ is the index of a scalar cell and $j$ is the index of a momentum cell.

Using the Newton method, pressure is linearized as $P^{n+1} \rightarrow P^{k}+\delta P$ and then inserted into Eq. (2-11), resulting in the following form:

$\mathbf{u}_{j}{ }^{n+1}=\mathbf{u}_{j}{ }^{k}+\left(\frac{d \mathbf{u}}{d P}\right)_{j} \nabla_{j}(\delta P)$

where $\left(\frac{d \mathbf{u}}{d P}\right)_{j}$ is the partial derivative of velocity component with respect to pressure. Other dependent variables and the source terms treated implicitly also are linearized as follows:

$$
\begin{aligned}
& Y_{s}^{n+1} \rightarrow Y_{s}^{k}+\delta Y_{s}, \quad T^{n+1} \rightarrow T^{k}+\delta T \\
& \rho^{n+1} \rightarrow \rho^{k}+\left(\frac{\partial \rho}{\partial P}\right)^{k} \delta P+\left(\frac{\partial \rho}{\partial T}\right)^{k} \delta T+\sum_{s}\left(\frac{\partial \rho}{\partial Y_{s}}\right)^{k} \delta Y_{s} \\
& H^{n+1} \rightarrow H^{k}+\left(\frac{\partial H}{\partial T}\right)^{k} \delta T+\sum_{s}\left(\frac{\partial H}{\partial Y_{s}}\right)^{k} \delta Y_{s} \\
& R_{s}^{n+1} \rightarrow R_{s}^{k}+\sum_{s}\left(\frac{\partial R_{s}}{\partial Y_{s}}\right)^{k} \delta Y_{s}
\end{aligned}
$$

By inserting $\mathbf{u}_{j}^{n+1}$ of Eq. (2-14) and linearized variables $\left(\rho_{i}^{n+1}, Y_{s i}^{n+1}, T_{f_{i}}^{n+1}, H_{i}^{n+1}, R_{s i}^{n+1}\right)$ into the discretized scalar equations, Eqs. (2-10), (2-12), and (2-13), and then combining all the resulting equations together into a linear algebraic form, a $7 \times 7$ square matrix is obtained:

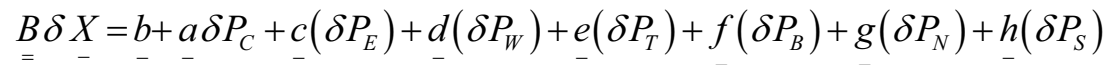

where $[\delta \underline{X}]^{T}=\left[\begin{array}{lllllll}\delta P & \delta T_{f} & \delta Y_{\mathrm{N}_{2}} & \delta Y_{\mathrm{O}_{2}} & \delta Y_{\mathrm{CO}} & \delta Y_{\mathrm{CO}_{2}} & \delta Y_{\mathrm{H}_{2} \mathrm{O}}\end{array}\right]^{T}$

Multiplying Eq. (2-16) by the inverse matrix of $\underset{=}{B}$, the solution vector is expressed as

$$
\delta X_{-}=b_{-}^{\prime}+a_{-}^{\prime} \delta P_{C}+c_{-}^{\prime}\left(\delta P_{E}\right)+d_{-}^{\prime}\left(\delta P_{W}\right)+e_{-}^{\prime}\left(\delta P_{T}\right)+f^{\prime}\left(\delta P_{B}\right)+g^{\prime}\left(\delta P_{N}\right)+h_{-}^{\prime}\left(\delta P_{S}\right)
$$

As a result, the first row in Eq. (2-17) becomes the $\mathrm{N} \times \mathrm{N}$ pressure matrix. The remaining rows, the fluid temperature and the mass fraction of each species, are expressed as a function of pressure only. The above calculation process is repeated until the convergence criterion, $\varepsilon=\max \left(\delta P_{i} / P_{i}^{k}\right)$, is satisfied. According to whether the convergence succeeds or fails, the time step size is controlled but restricted by the maximum time step limit, $\Delta t_{\max } \leq \min \left(\Delta t_{\text {convective }}, \Delta t_{\text {viscous }}, \Delta t_{\text {conductive }}, \Delta t_{\text {diffusive }}\right)$, due to explicit treatment of the second-order terms. 
The heat conduction equation, Eq. (2-6), is solved by the Crank-Nicolson method and coupled with the thermo-fluid calculation explicitly or implicitly.

$$
\begin{aligned}
& {\left[(1-\varphi)\left(\rho_{p} C_{p}\right)_{i}^{n}\right] \frac{\left(T_{p}\right)_{i}^{n+1}-\left(T_{p}\right)_{i}^{n}}{\Delta t}=q_{N_{i}}^{{ }^{\prime \prime}}-h_{s f_{i}}{ }^{n}\left(T_{p_{i}}^{n}-T_{i}^{n}\right)} \\
& +\frac{\theta}{V^{n} l_{i}} \nabla_{i} \cdot\left(\bar{\lambda}_{\text {eff }} \nabla T_{p}^{n+1}\right)++\frac{1-\theta}{V o l_{i}} \nabla_{i} \cdot\left(\bar{\lambda}_{\text {eff }} \nabla T_{p}{ }^{n}\right) \\
& a_{c} T_{p}^{c}+a_{w} T_{p}{ }^{w}+a_{e} T_{p}^{e}+a_{b} T_{p}^{b}+a_{t} T_{p}^{t}+a_{s} T_{p}{ }^{s}+a_{n} T_{p}^{n}=b_{c}
\end{aligned}
$$

The above solution scheme is applicable to a 1-D piping flow network with simple structure re-arrangement. Therefore, a concerned system can be configured by the linkage of a 1-D calculation module and a 2/3-D calculation module.

\section{1-3 Calculation Procedure}

The calculation procedure of the program follows eight major steps, as shown in Figure 2-1.

Step 1: By using the geometry input and the initial conditions supplied by a user, the mesh configuration (cell and face numbering) and the connection information for the linkage of 1-D and 2/3-D modules are set up and the computational mesh data are generated for the fluid and solid equations. Also, the initial fluid and material properties are calculated and then used to get the undetermined variables.

Step 2: In this step, the donored and averaged properties at a junction are defined first, and then the model and correlation parameters for friction, mass and heat transfer, and porous media, etc., are calculated. The radiation heat flux and the source terms without respect to time step, convective and viscous terms for the momentum equation, are calculated.

Step 3: The time step control loop starts here. This step solves the heat conduction equation, and calculates the explicitly-treated source terms, the species diffusion term in the species equation and the heat conduction and inter-molecular energy diffusion terms in the energy equation, and the velocity derivatives.

Step 4: The outer iteration loop starts here. After calculating the velocity components, the coefficient matrix and the source vector of the linearized fluid equations are obtained. In this step, the chemical reaction source terms treated implicitly are added.

Step 5: The pressure matrix is solved by an efficient sparse matrix solver. Thereafter, the temperature and the species mass fractions are calculated directly from the obtained pressures and then the primary variables are updated for the next iteration.

Step 6: If the convergence succeeds, the calculation continues to next step. Otherwise, go to step 4 for more outer iteration.

Step 7: If the maximum iteration number is exceeded, the time step size is reduced by half, and then goes to step 3 for new calculation. If the convergence succeeds and the number of iterations is less then the maximum iteration number, the calculation continues to next step.

Step 8: The time step size is limited by convective, conductive, viscous, and diffusive transport times. If the simulation time is not reached, go to step 2 for new time advance after the properties and variables are updated. 


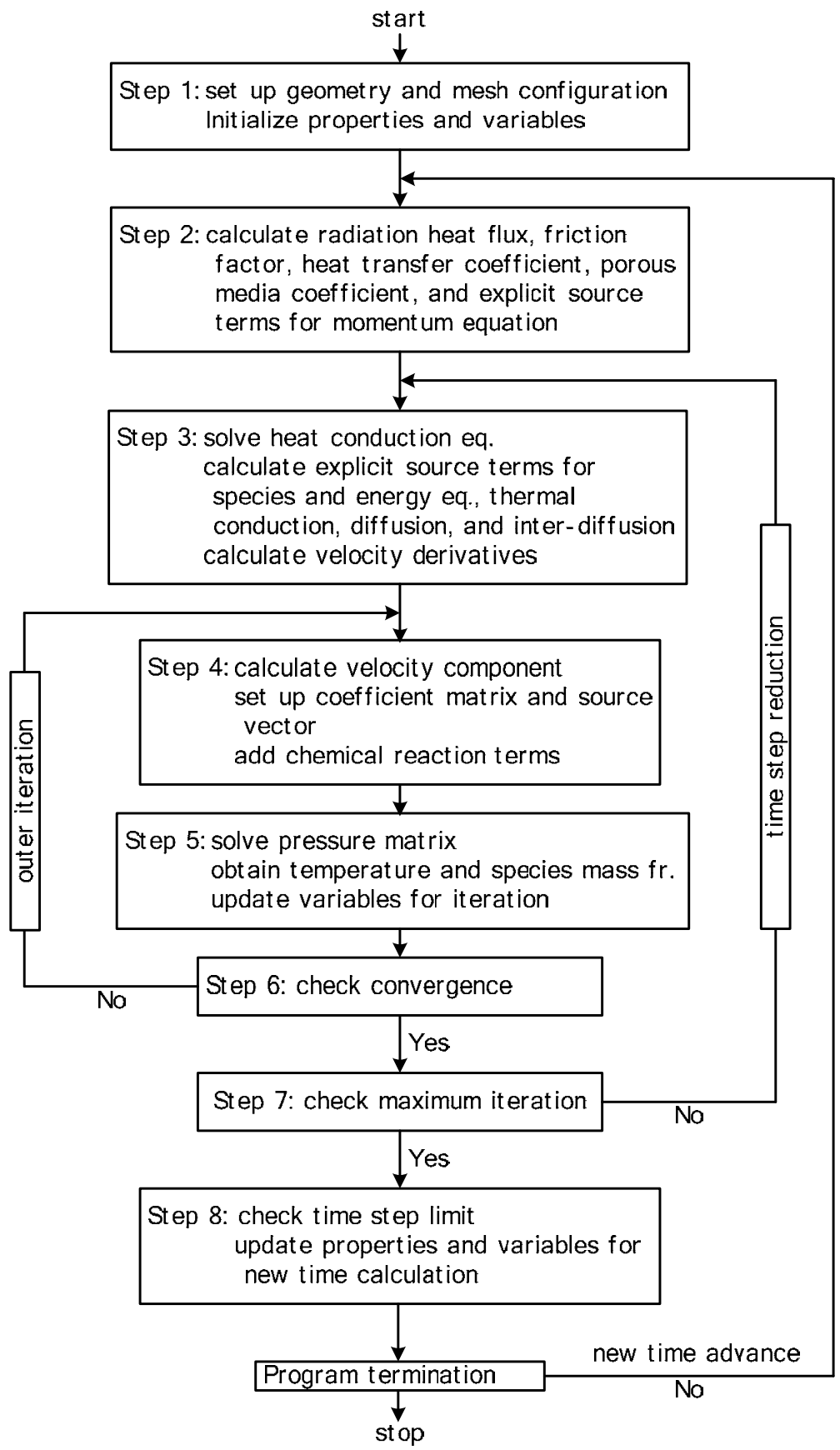

Figure 2-1 Calculation procedure of the analysis program 


\subsection{Development and V\&V of Physical Models}

Table 2-1 lists all the test cases collected for the development and the verification and validation of the physical models of the GAMMA code. Among those, this section will describe the representative cases (marked by an asterisk in the table) to ensure the GAMMA capability to predict the basic physical phenomena expected to occur during the transients in a HTGR.

Table 2-1 Assessment matrix for development and V\&V of physical models of GAMMA

\begin{tabular}{||l|l|l||}
\hline No. & Test Facility & Physical models/phenomena \\
\hline 1 & Pipe Network, NWU & Flow balancing in a complex pipe network \\
\hline 2 & Blowdown, NWU & Pressure transient and critical flow \\
\hline $3^{*}$ & Duncan \& Toor's experiment & Multi-component molecular diffusion \\
\hline $4^{*}$ & $\begin{array}{l}\text { Inverse U-tube single/multiple } \\
\text { channel test }\end{array}$ & $\begin{array}{l}\text { Binary molecular diffusion and natural } \\
\text { convection }\end{array}$ \\
\hline 6 & Ogawa's circular tube test & Chemical reactions in a IG-110 \\
\hline $7^{*}$ & Takahashi's annular tube test & Chemical reactions in a IG-110 \\
\hline $8^{*}$ & VELUNA pebble bed test & Chemical reactions in a pebble bed \\
\hline $9^{*}$ & $\begin{array}{l}\text { HTTR-simulated air ingress } \\
\text { experiment }\end{array}$ & $\begin{array}{l}\text { Molecular diffusion, natural convection, and } \\
\text { chemical reactions }\end{array}$ \\
\hline 10 & Vertical slot experiment & $\begin{array}{l}\text { Molecular diffusion, natural convection, and } \\
\text { chemical reactions Multi-D effect on air ingress } \\
\text { process }\end{array}$ \\
\hline 11 & NACOK natural convection test & Local circulation effect on molecular diffusion \\
\hline $12^{*}$ & SANA-1 afterheat removal test & $\begin{array}{l}\text { Patural convection in a pebble bed } \\
\text { tests and power ramp-down and step-up tests }\end{array}$ \\
\hline $13^{*}$ & HTTR RCCS mockup test & $\begin{array}{l}\text { Free convection and radiation inside the vessel } \\
\text { Air convection and radiation in a reactor cavity }\end{array}$ \\
\hline $14^{*}$ & SNU RCCS test & Air convection and radiation in a reactor cavity \\
\hline $15^{*}$ & MIT particle model & Particle model in a pebble \\
\hline
\end{tabular}

\section{$\underline{\text { 2.2-1 Multi-component Molecular Diffusion Model Test }}$}

Since at least five species must be considered in an air ingress accident and the concentrations of each species determine the chemical reaction rate as well as the buoyancy force driven by the density difference, it is essential to understand the diffusion behavior in the multi-component mixture system.

\section{Duncan and Toor's Two Bulbs Experiment}

The apparatus of the "two bulbs" experiment (Duncan and Toor 1962) conducted by Duncan and Toor consisted of two bulbs and a small capillary tube. The bulbs had volumes of $77.99 \mathrm{~cm}^{3}$ and $78.63 \mathrm{~cm}^{3}$, respectively. The capillary tube joining them was $85.9 \mathrm{~mm}$ long and $2.08 \mathrm{~mm}$ in diameter. Initially the bulbs were isolated by a stopcock installed at the center of the capillary tube. The entire device was maintained at a temperature of $35.2^{\circ} \mathrm{C}$ in the atmospheric pressure. The initial concentrations of the filling gases and the nodalization diagram for the simulation are given in Figure 2-2. 
Bu b \# 1

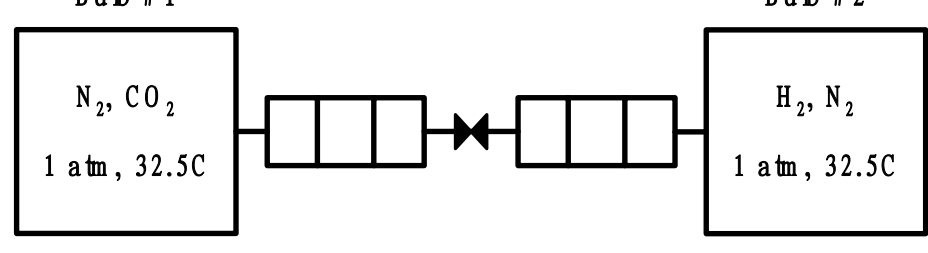

Bulb \#1: $X_{\mathrm{H}_{2}}=0 ., \quad X_{\mathrm{N}_{2}}=0.50086, \quad X_{\mathrm{CO}_{2}}=0.49914$

Bulb \#2: $X_{\mathrm{H}_{2}}=0.50121, X_{\mathrm{N}_{2}}=0.49879, \quad X_{\mathrm{CO}_{2}}=0$.

(a) Initial conditions at each bulb

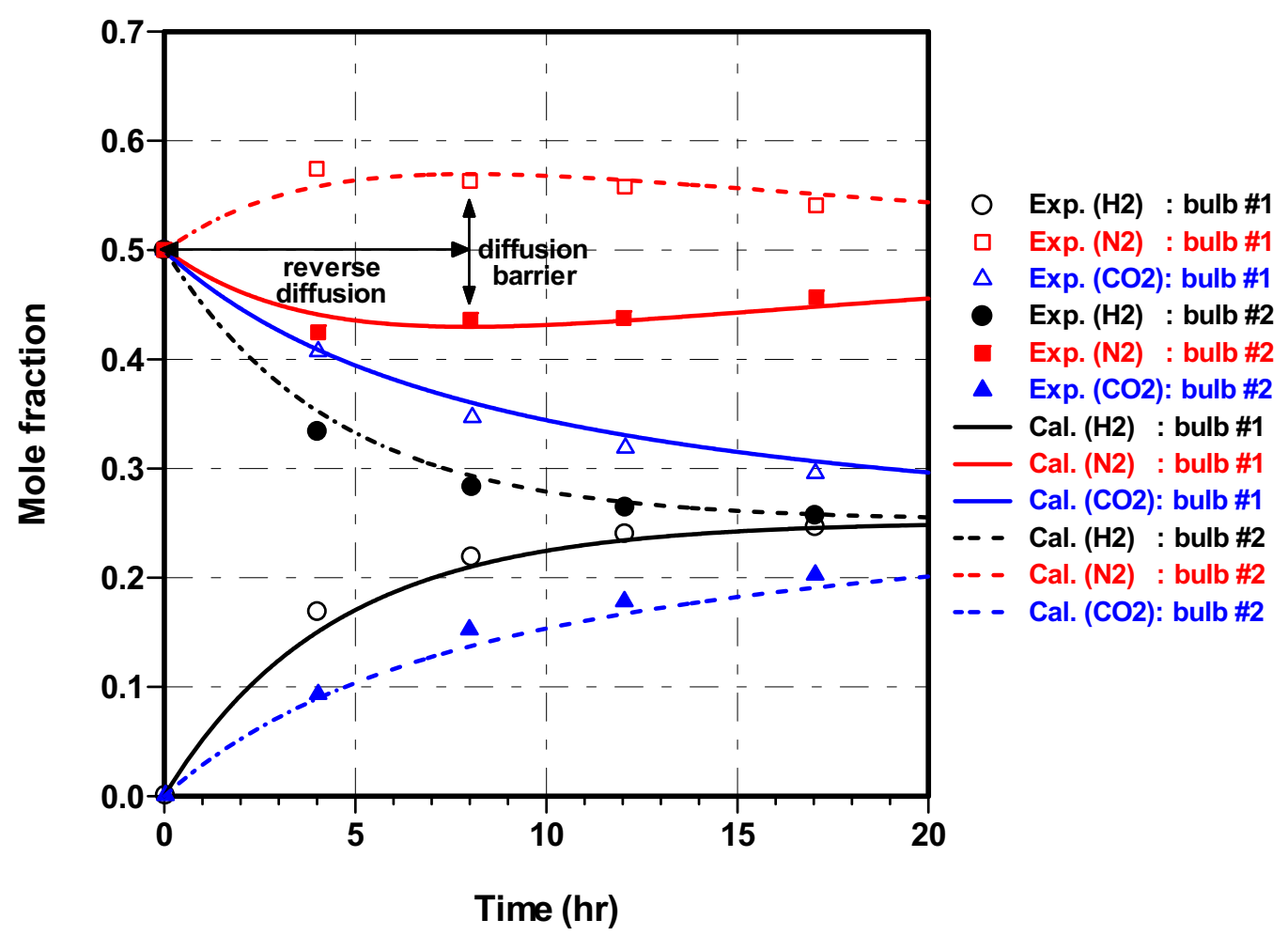

(b) Predicted mole fractions of each species

Figure 2-2 Calculation results for the "two bulbs" experiment

As shown in Figure 2-2, the calculation results using the full multi-component diffusion form, Eq. (2-8), follow almost exactly the data measured at each bulb. Particularly, the exact prediction of $\mathrm{N}_{2}$ gas behavior demonstrates the typical diffusion phenomena observed in the multi-component system: reverse diffusion in which a species moves against its own concentration gradient, osmotic diffusion in which a species diffuses even though its concentration gradient is zero, and diffusion barrier when a species does not diffuse even though its concentration gradient is nonzero.

\section{Inverse U-tube Molecular Diffusion Experiment}

Figures 2-3 and 2-4 show the inverse U-tube test apparatus (Hishida and Takeda 1991) used to investigate the molecular diffusion behavior in the binary mixture and the nodalization diagram for the GAMMA simulation, respectively. The apparatus consisted of an inverse U-shaped tube having an inner diameter of $52.7 \mathrm{~mm}$ connected to a gas tank. The ball valves between the reverse U-tube and the gas tank were closed and the tube 
was evacuated by a vacuum pump. Helium and nitrogen are filled in the tube and the gas tank, respectively. Then, the high temperature side and connecting pipes were heated to the elevated temperatures. When the temperature of the gas and the pipe wall reached a steady state condition, the gas pressure in the reverse U-tube was equalized to the atmosphere pressure by opening a small release valve.

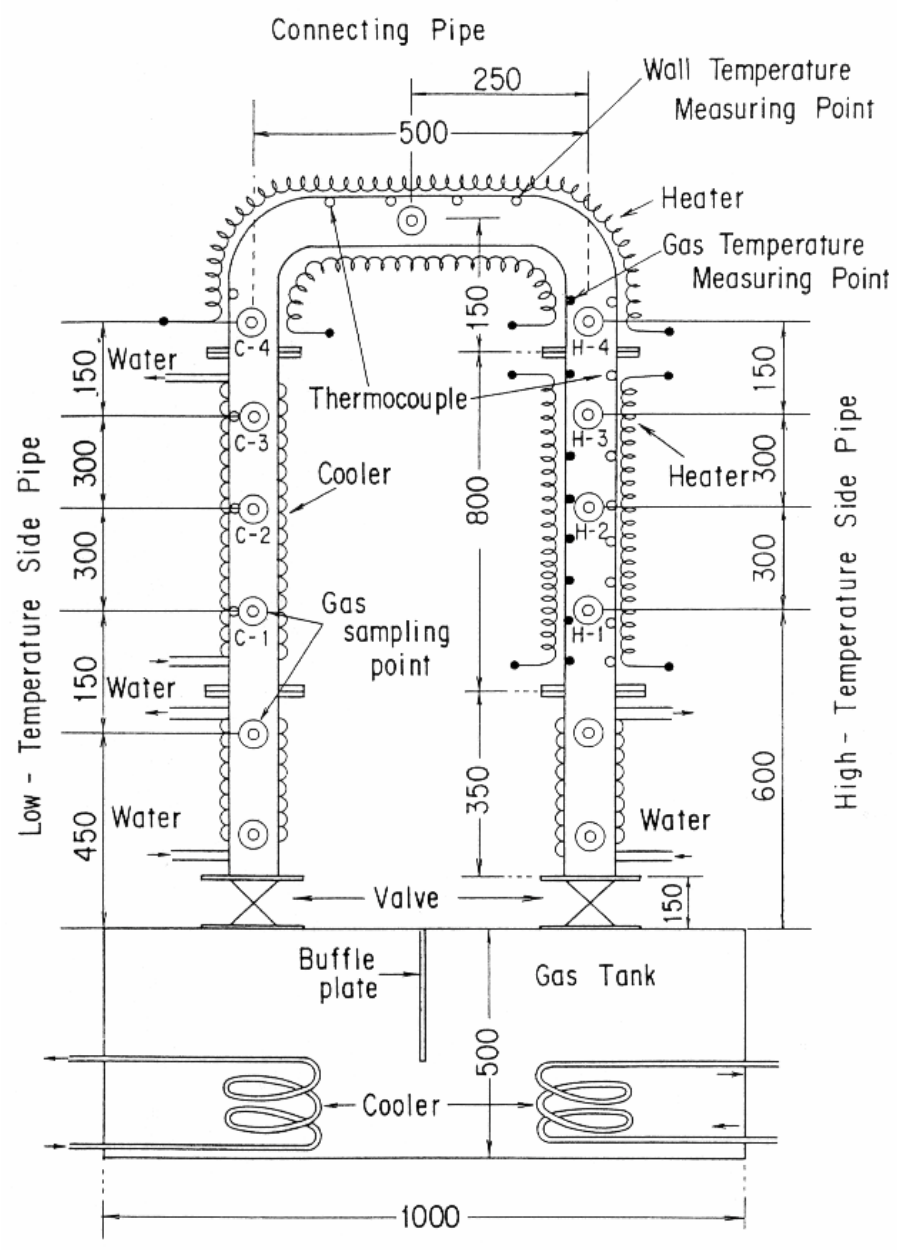

Figure 2-3 Schematic diagram of the inverse U-tube system

When the valves open simultaneously, $\mathrm{N}_{2}$ gas in the bottom tank starts to diffuse into both sides of the inverse tube. Two kinds of experiments were performed: the isothermal test and non-isothermal test. In the isothermal test, the inverse U-tube is kept at room temperature $\left(18^{\circ} \mathrm{C}\right)$. In the non-isothermal test, the inverse U-tube has a non-uniform temperature distribution along the tube as shown in Figure 2-4. The mole fraction of $\mathrm{N}_{2}$ was obtained at eight sampling points (C-1 through $\mathrm{C}-4$ and $\mathrm{H}-1$ through $\mathrm{H}-4$ ) shown in Figure 2-3 by measuring the sound velocity of the gas mixture. 


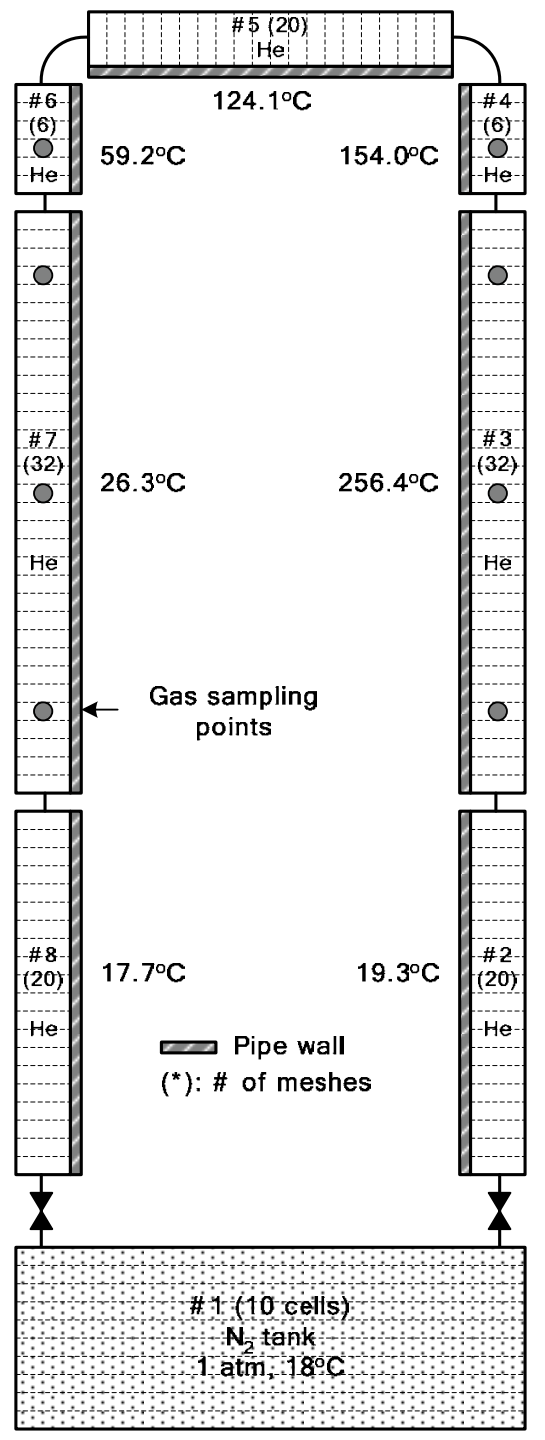

Figure 2-4 Nodalization diagram of the inverse U-tube system

In the isothermal test, $\mathrm{N}_{2}$ in a gas tank begins to move into the inverse tube filled with helium by pure molecular diffusion. As the mole fraction distribution of $\mathrm{N}_{2}$ is the same between the hot and cold pipes, the natural convection of the gas mixture does not occur. As shown in Figure 2-5, the calculated results well follow the experimental data.

The calculated results using a non-uniform temperature distribution are shown in Figure 2-6. As the mole fraction of $\mathrm{N}_{2}$ in the tube gradually increases faster in the hot side than in the cold side, the buoyancy force induced by the distribution of the gas mixture density increases. Around 220 minutes after the valves open, the buoyancy force becomes large enough to initiate global natural circulation throughout the inverse Utube. The calculated velocities by very weak and global natural convection are about $<10^{-4} \mathrm{~m} / \mathrm{s}$ in the early stage and around $0.1 \mathrm{~m} / \mathrm{s}\left(\operatorname{Re}_{d}=500\right)$ in the later stage, respectively. The predicted onset time of natural convection is in well accordance with the data.

In the both isothermal and non-isothermal tests, the predicted results agree well within a $10 \%$ deviation with the experimental values measured at six sampling locations. The slight discrepancy of the mole fractions of 
$\mathrm{N}_{2}$ is attributed to the entrance effect between the tube inlet and the gas tank, that is, a non-uniform concentration distribution, and the use of a rough temperature distribution along the tube.

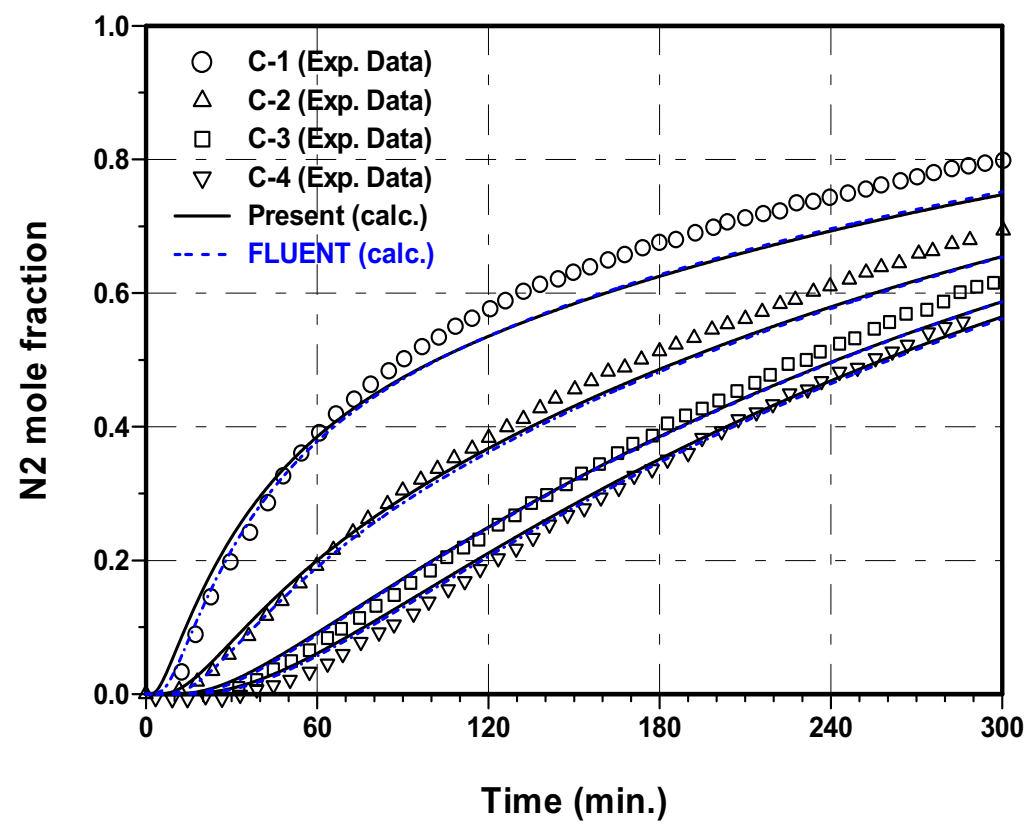

Figure 2-5 Calculation results of the inverse U-tube system: isothermal test

Figures 2-5 and 2-6 also show the comparative results between the present calculations and the FLUENT simulations. The FLUENT 6 (Fluent 6.0 User's Guide 2002) simulations are performed with the SIMPLE algorithm using the 3-D mesh layout shown in Figure 2-7. To reduce the computation time, the original circular geometry of the test apparatus was transformed into the rectangular shape for generating coarse meshes by preserving the volumes of the bottom tank and the connecting pipe. The predicted trends are very similar in both the FLUENT and the GAMMA simulation. The remarkable point is the comparison of their computing times given in Table 2-2 when a $900 \mathrm{MHz}$ Pentium III PC is used for the simulation. Even though the number of mesh used in the FLUENT calculation is about twice that in the present calculation, it takes a very long time for the FLUENT calculation, almost 40 times that of the present calculation. In addition, the maximum time step size in the FLUENT calculation is restricted to be lower than that of the present solution scheme. 


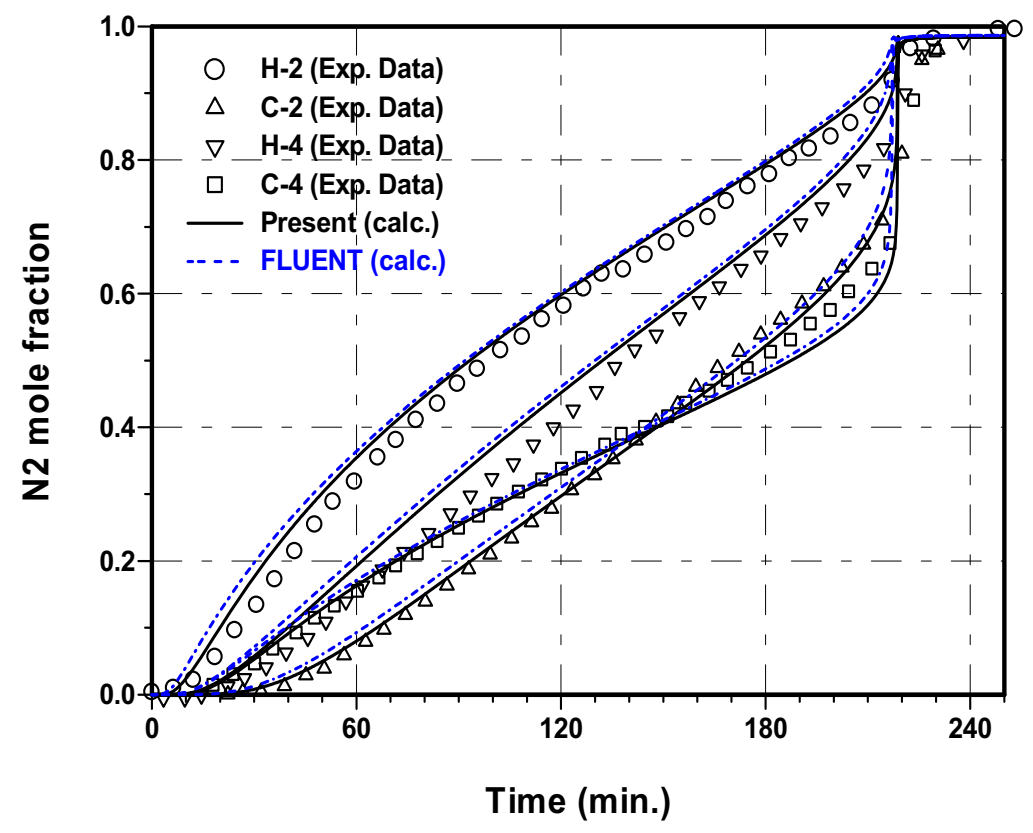

Figure 2-6 Calculation results of the inverse U-tube system: non-isothermal test

Table 2-2 Computation times and time steps for ICE and FLUENT

\begin{tabular}{|c|c|c|c|c|c|}
\hline Test cases & \multicolumn{2}{|c|}{ Maximum time step } & \multicolumn{2}{|c|}{ Computation time } & Time step limit \\
\hline & ICE & FLUENT & ICE & FLUENT & - \\
\hline Isothermal & $0.5 \mathrm{sec}$ & $0.2 \mathrm{sec}$ & $32 \mathrm{~min}$. & $20 \mathrm{hrs}$ & $4.7 \mathrm{sec}$ (diffusion) \\
\hline $\begin{array}{c}\text { Non- } \\
\text { isothermal }\end{array}$ & $0.5 \mathrm{sec}$ & $0.2 \mathrm{sec}$ & $36 \mathrm{~min}$. & $22 \mathrm{hrs}$ & $\begin{array}{c}0.6-1.7 \mathrm{sec} \\
\text { (conduction) }\end{array}$ \\
\hline
\end{tabular}

\subsection{Chemical Reaction Model Test}

\section{3-1 Chemical Reaction Models}

To analyze the consequence of air ingress, the chemical reaction rates for the graphite oxidation and $\mathrm{CO}$ combustion must be determined first. The most important chemical reactions between oxygen and graphite are the following homogeneous reaction
A. $\mathrm{CO}+(1 / 2) \mathrm{O}_{2}$
$\rightarrow \mathrm{CO}_{2}+2.830 \times 10^{5}(\mathrm{~J} / \mathrm{mole})$
and the heterogeneous reactions
B. $\mathrm{C}+\mathrm{O}_{2} \rightarrow \mathrm{CO}_{2}+3.935 \times 10^{5}(\mathrm{~J} / \mathrm{mole})$
C. $\mathrm{C}+(1 / 2) \mathrm{O}_{2} \rightarrow \mathrm{CO}+1.105 \times 10^{5}(\mathrm{~J} / \mathrm{mole})$
D. $\mathrm{C}+\mathrm{CO}_{2} \rightarrow 2 \mathrm{CO}-1.725 \times 10^{5}(\mathrm{~J} / \mathrm{mole})$ 


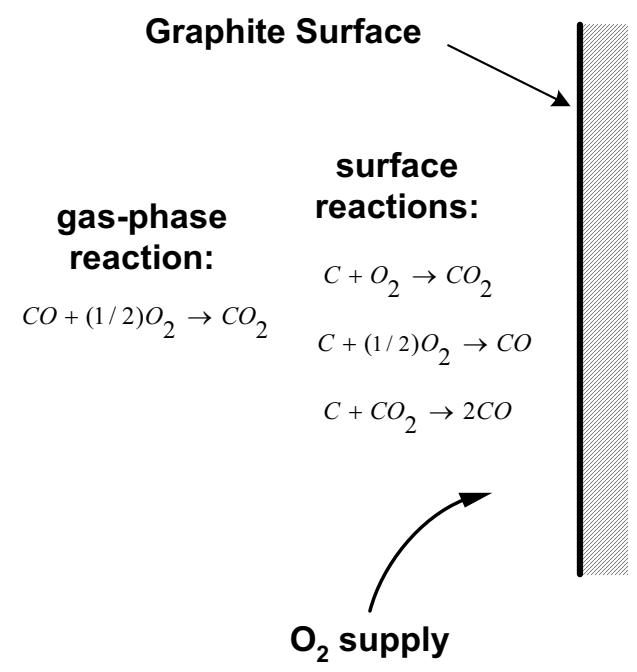

A Dryer and Glassman's correlation (1973) is used for the CO combustion (A) in the bulk fluid region.

$$
\begin{aligned}
& R_{\mathrm{CO}}=-r_{\mathrm{CO}-\mathrm{O}_{2}} \rho\left(\frac{\rho}{W_{\mathrm{O}_{2}}}\right)^{0.25}\left(\frac{\rho}{W}\right)^{0.5} Y_{\mathrm{CO}} Y_{\mathrm{O}_{2}}^{0.25} X_{\mathrm{H}_{2} \mathrm{O}}^{0.5} \quad\left[\mathrm{~kg} / \mathrm{m}^{3}-\mathrm{s}\right] \\
& R_{\mathrm{O}_{2}}=0.5\left(R_{\mathrm{CO}}\right) \frac{W_{\mathrm{O}_{2}}}{W_{\mathrm{CO}}} \\
& R_{\mathrm{CO}_{2}}=-\left(R_{\mathrm{CO}}\right) \frac{W_{\mathrm{CO}_{2}}}{W_{\mathrm{CO}}}
\end{aligned}
$$

where the reaction rate is expressed as $r_{\mathrm{CO}_{-} \mathrm{O}_{2}}=2.24 \times 10^{12} \exp (-167400 / \bar{R} T)$.

For the IG-110 graphite oxidation (B and C) at the graphite surface, the Kim and NO's empirical correlations (NO et al. 2004) are used.

Reaction rate $\quad: r_{C-O_{2}}=7500 \exp (-218000 / \bar{R} T)\left(P_{O_{2}}\right)^{0.75}$

Production ratio of $\mathrm{CO}$ and $\mathrm{CO}_{2}: f_{\mathrm{CO} / C O_{2}}=7396 \exp (-69604 / \bar{R} T)$

And then the dissipation/generation rates for each species are expressed as

$$
\begin{aligned}
& R_{\mathrm{O}_{2}}{ }^{w}=-\left(\frac{f_{\mathrm{CO} / \mathrm{CO}_{2}}+2}{2 f_{\mathrm{CO} / \mathrm{CO}_{2}}+2}\right) r_{\mathrm{C}-\mathrm{O}_{2}} \frac{W_{\mathrm{O}_{2}}}{W_{\mathrm{C}}} \quad\left[\mathrm{kg} / \mathrm{m}^{2}-\mathrm{s}\right] \\
& R_{\mathrm{CO}}{ }^{w}=\left(\frac{f_{\mathrm{CO} / \mathrm{CO}_{2}}}{f_{\mathrm{CO} / \mathrm{CO}_{2}}+1}\right) r_{\mathrm{C}-\mathrm{O}_{2}} \frac{W_{\mathrm{CO}}}{W_{\mathrm{C}}} \\
& {R_{\mathrm{CO}}{ }^{w}}^{w}=\left(\frac{1}{f_{\mathrm{CO} / \mathrm{CO}_{2}}+1}\right) r_{\mathrm{C}-\mathrm{O}_{2}} \frac{W_{\mathrm{CO}_{2}}}{W_{\mathrm{C}}}
\end{aligned}
$$

For the Boudouard surface reaction (D), since the reaction rate for IG-110 is unavailable, we selected the correlation for the graphite A3-3 (Moorman 1984) having fine grains like IG-110.

$$
R_{C}{ }^{w}=\frac{0.145 \exp (-25000 / T) P_{\mathrm{CO}_{2}}}{1+3.4 \times 10^{-5} \exp (7000 / T) P_{\mathrm{CO}_{2}}{ }^{0.5}} \quad\left[\mathrm{~kg} / \mathrm{m}^{2}-\mathrm{s}\right]
$$


The heat generated due to the $\mathrm{CO}$ combustion (A) and due to the blowing effect from the $\mathrm{C}-\mathrm{O}_{2}$ graphite oxidation ( $\mathrm{B}$ and $\mathrm{C}$ ) is added to the fluid. The net heat, which is produced from the $\mathrm{C}-\mathrm{O}_{2}$ exothermic reaction and absorbed by the $\mathrm{C}-\mathrm{CO}_{2}$ endothermic reaction (D), is added to the graphite wall.

\section{3-2 Ogawa's Circular Tube Specimen Experiment}

Ogawa (Ogawa 1993) investigated the IG-110 graphite oxidation behavior in the temperature range from 600 to $1030^{\circ} \mathrm{C}$ for an oxygen concentration of $20 \mathrm{wt} \%$ and an inlet Reynolds number of 80 . Figure $2-8$ shows the axi-symmetric calculation layout for a circular graphite tube, $200 \mathrm{~mm}$ in height and $21 \mathrm{~mm}$ in inner diameter, and the boundary conditions for the simulation. Since the test specimen has a relatively long entrance length $(500 \mathrm{~mm})$ below the graphite tube, the parabolic velocity profile can be used as an inlet boundary condition. In both the FLUENT and GAMMA simulations, the species concentration at the reacting surface is calculated by balancing the convection and diffusion of each species to (or from) the surface and the rate at which it is consumed (or produced) at the surface:

$$
-(\rho V)_{w} Y_{s}^{w}-\left(\frac{\rho W_{s}}{W^{2}}\right)_{w} \sum_{p=1}^{m}\left[D_{s p} \frac{\partial}{\partial r}\left(W Y_{p}\right)\right]_{w}=R_{s}^{w}
$$

When a test starts, oxygen at the tube inlet is transported upward and then consumed by chemical reaction with the graphite. The $\mathrm{CO}$ and $\mathrm{CO}_{2}$ produced from the graphite oxidation are transported away by convection and diffusion from the graphite wall. At the same time, the $\mathrm{CO}$ diffused into the interior chemically reacts with the oxygen transported from the inlet, producing $\mathrm{CO}_{2}$ as well as heat. During this experiment, the graphite temperature was controlled at constant value.

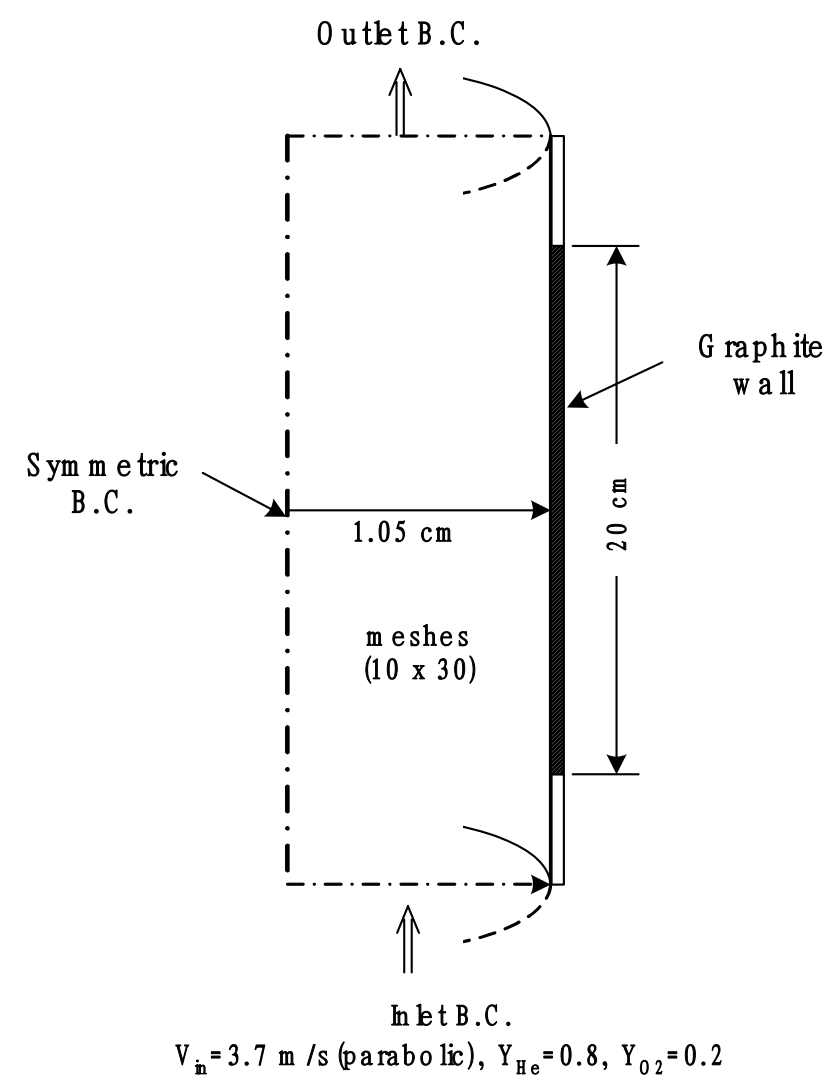

Figure 2-8 Axi-symmetric calculation layout for a graphite tube specimen 
The calculation results at the middle plane of the graphite tube for concentration and temperature profiles at $900^{\circ} \mathrm{C}$ are shown in Figure 2-9. Even with different numerical approach, the predictions of GAMMA are comparable to those of FLUENT 6 User's Guide 2002. The predicted mean mass fractions of species at the tube outlet as the graphite temperature increases are shown in Figure 2-10. More oxygen is consumed as the graphite temperature increases, producing more $\mathrm{CO}$ and $\mathrm{CO}_{2}$. Over $850^{\circ} \mathrm{C}$, the effect of $\mathrm{CO}$ combustion becomes large and furthermore the produced amount of $\mathrm{CO}$ and $\mathrm{CO}_{2}$ is highly sensitive to the moisture fraction. The turn-around trend of $\mathrm{CO}$ around $950^{\circ} \mathrm{C}$ is predicted well by a Dryer and Glassman's correlation for the $\mathrm{CO}$ combustion because of its higher activation energy.

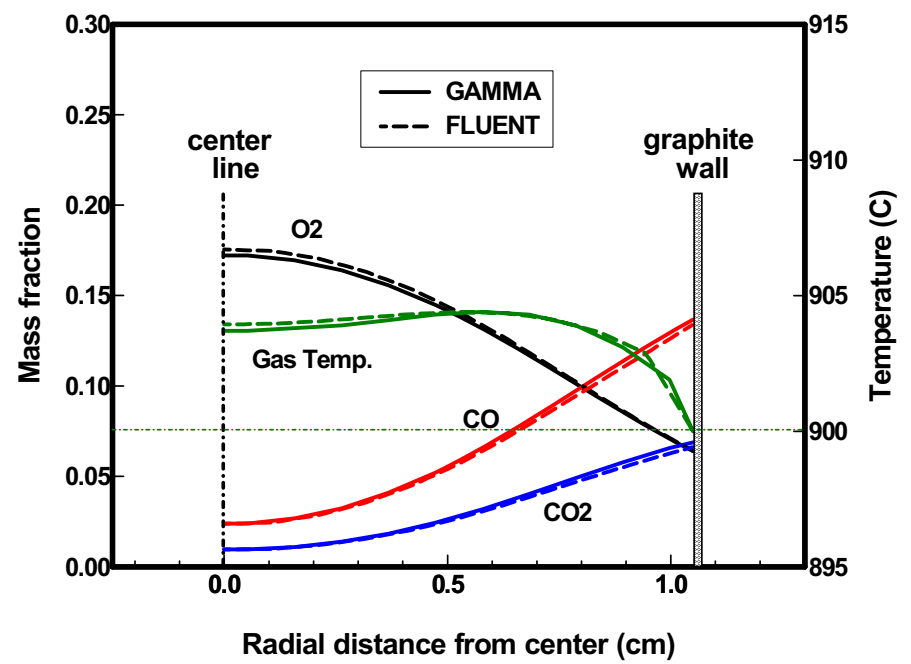

Figure 2-9 Predicted concentration and temperature profiles at the mid-plane of a graphite tube specimen

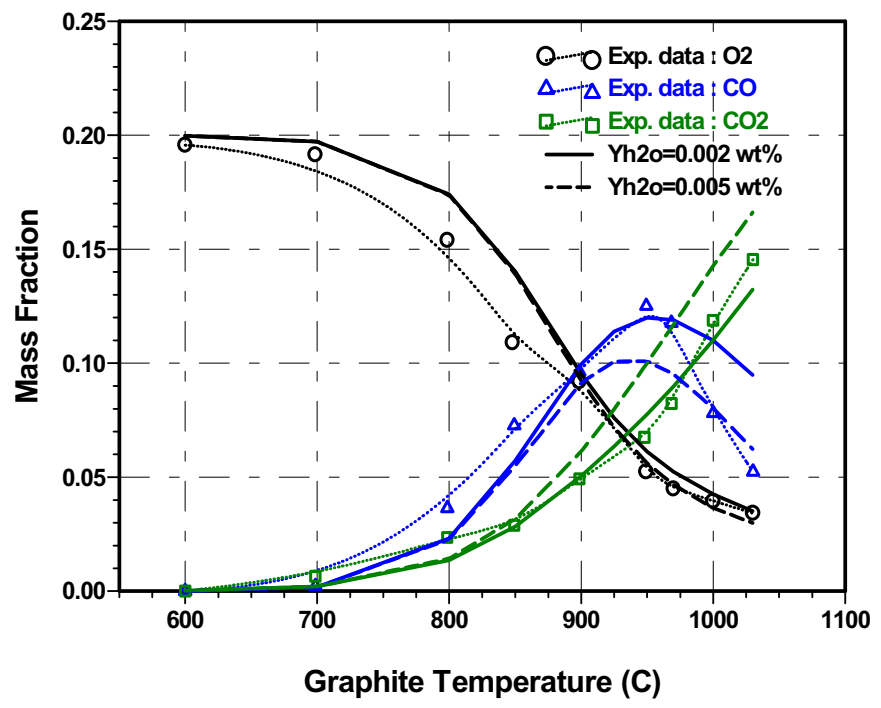

Figure 2-10 Predicted mass fractions of oxygen, monoxide, and dioxide at the tube outlet as a function of graphite temperature 


\section{3-3 VELUNA Pebble-Bed Oxidation Experiment}

In order to investigate the chemical reactions in a pebble-bed during the air ingress process, the pebble oxidation experiment conducted in the VELUNA test facility (Roes 1994) has been selected. The facility consisted of a bottom reflector having 25 small flow holes where no chemical reactions occurred and a long pebble column $(2.8 \mathrm{~m})$. The pebbles with a diameter of $6 \mathrm{~cm}$ were stacked by $4 \times 4$ arrays in the pebble column section. At the condition which the test section temperature was controlled at constant value, the graphite pebbles were attacked with air and then did undergo the oxidation reaction. The following chemical reaction models have been selected for the simulation:

A. $\mathrm{CO}-\mathrm{O}_{2}$ exothermic bulk reaction: Dryer and Glassman 1973

$$
\begin{aligned}
R_{C O}\left(\mathrm{~kg} / \mathrm{m}^{3}-\mathrm{s}\right) & =-2.24 \times 10^{12} \exp (-167400 / \bar{R} T) \\
& * \rho\left(\rho / W_{\mathrm{O}_{2}}\right)^{1 / 4}(\rho / W)^{1 / 2} Y_{\mathrm{CO}} Y_{\mathrm{O}_{2}}^{1 / 4} X_{\mathrm{H}_{2} \mathrm{O}}^{1 / 2}
\end{aligned}
$$

B. C-O $\mathrm{O}_{2}$ exothermic surface reaction: Roes 1994

$$
R_{C}{ }^{w}\left(\mathrm{~kg} / \mathrm{m}^{2}-\mathrm{hr}\right)=720 \exp (-16140 / T) P_{O_{2}}
$$

C. $\mathrm{CO}_{2}-\mathrm{C}$ endothermic surface reaction: Moorman 1984

$$
R_{C}{ }^{w}\left(\mathrm{~kg} / \mathrm{m}^{2}-\mathrm{s}\right)=\frac{0.145 \exp (-25000 / T) P_{\mathrm{CO}_{2}}}{1+3.4 \times 10^{-5} \exp (7000 / T) P_{\mathrm{CO}_{2}}{ }^{0.5}}
$$

Figure 2-11 shows the calculated mole fractions of oxygen, $\mathrm{CO}$, and $\mathrm{CO}_{2}$ at the outlet mixing section. As pebble temperature increases, more oxygen is consumed, producing the heavy gases, $\mathrm{CO}$ and $\mathrm{CO}_{2}$. At middle temperature, the produced $\mathrm{CO}_{2}$ is balanced with the depleted $\mathrm{CO}_{2}$ due to the depletion reaction $(\mathrm{C})$. At high temperature larger than $800^{\circ} \mathrm{C}$, the $\mathrm{CO}$ production reactions ( $\mathrm{A}$ and $\mathrm{C}$ ) become dominant.
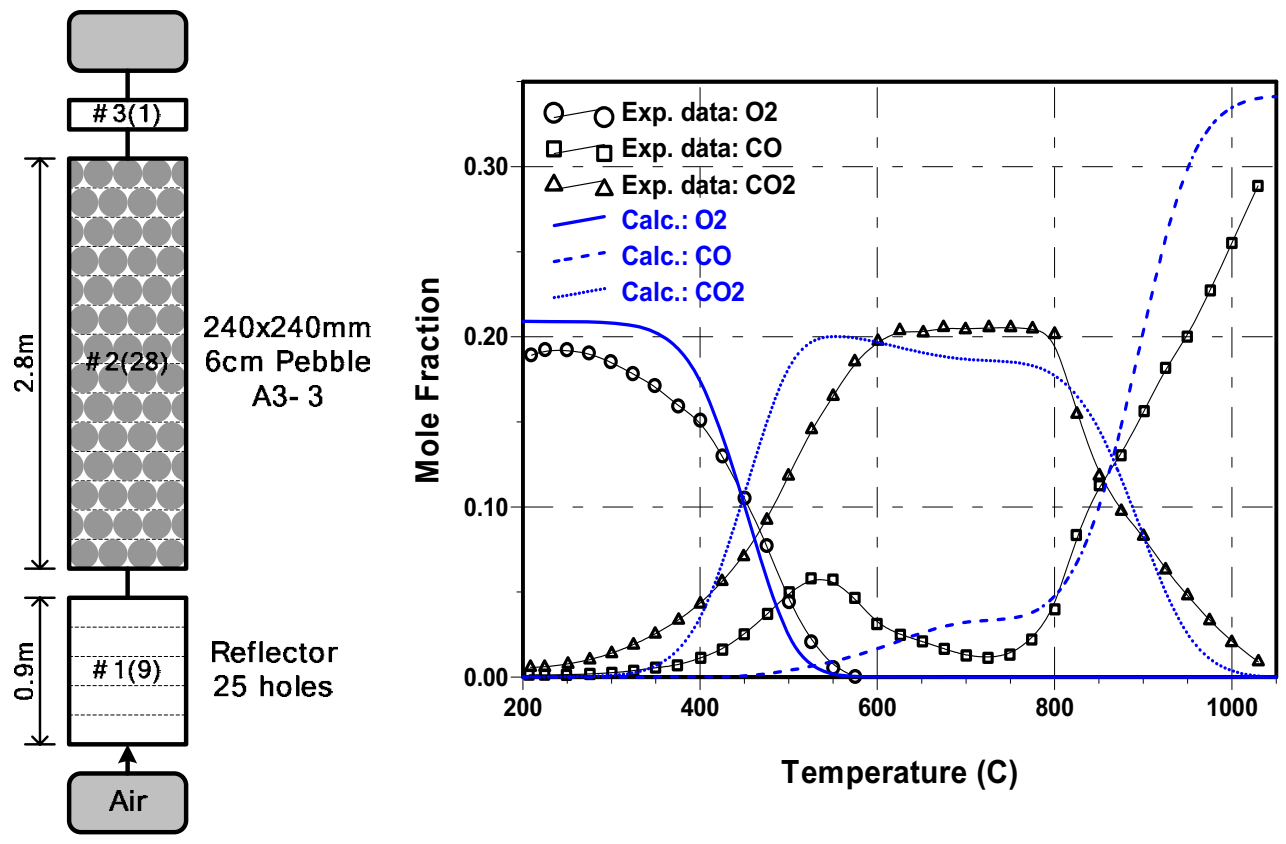

Figure 2-11 Predicted mole fractions of oxygen, monoxide, and dioxide at the outlet as a function of pebble temperature (VELUNA experiment) 


\subsection{Validation with Air Ingress Experiments: Inverse U-tube Air Ingress Experiment}

Figure 2-12 shows the schematic diagram of the inverse U-tube test apparatus (Takeda 1997), which consisted of a gas tank and an inverse U-shaped tube with a graphite (IG-110) tube inserted in the middle of the hot side. Figure 2-13 shows the GAMMA nodal scheme of the present model using a total of 94 control volumes. For the surface reaction in the graphite tubes, the 1-D stream tube approach equivalent to Eq. (2-24) is used as follows:

$$
\rho k_{s}\left(Y_{s}^{w}-Y_{s}\right)=R_{s}^{w}, \quad \text { for } s=O_{2}, C O, \mathrm{CO}_{2}
$$

where the mass transfer coefficient $\left(k_{s}\right)$ is obtained by means of heat-mass transfer analogy, $S h=k_{s}\left(d / D_{s-m i x}\right)=3.66(S c / P r)^{1 / 3}$, for the laminar flow.

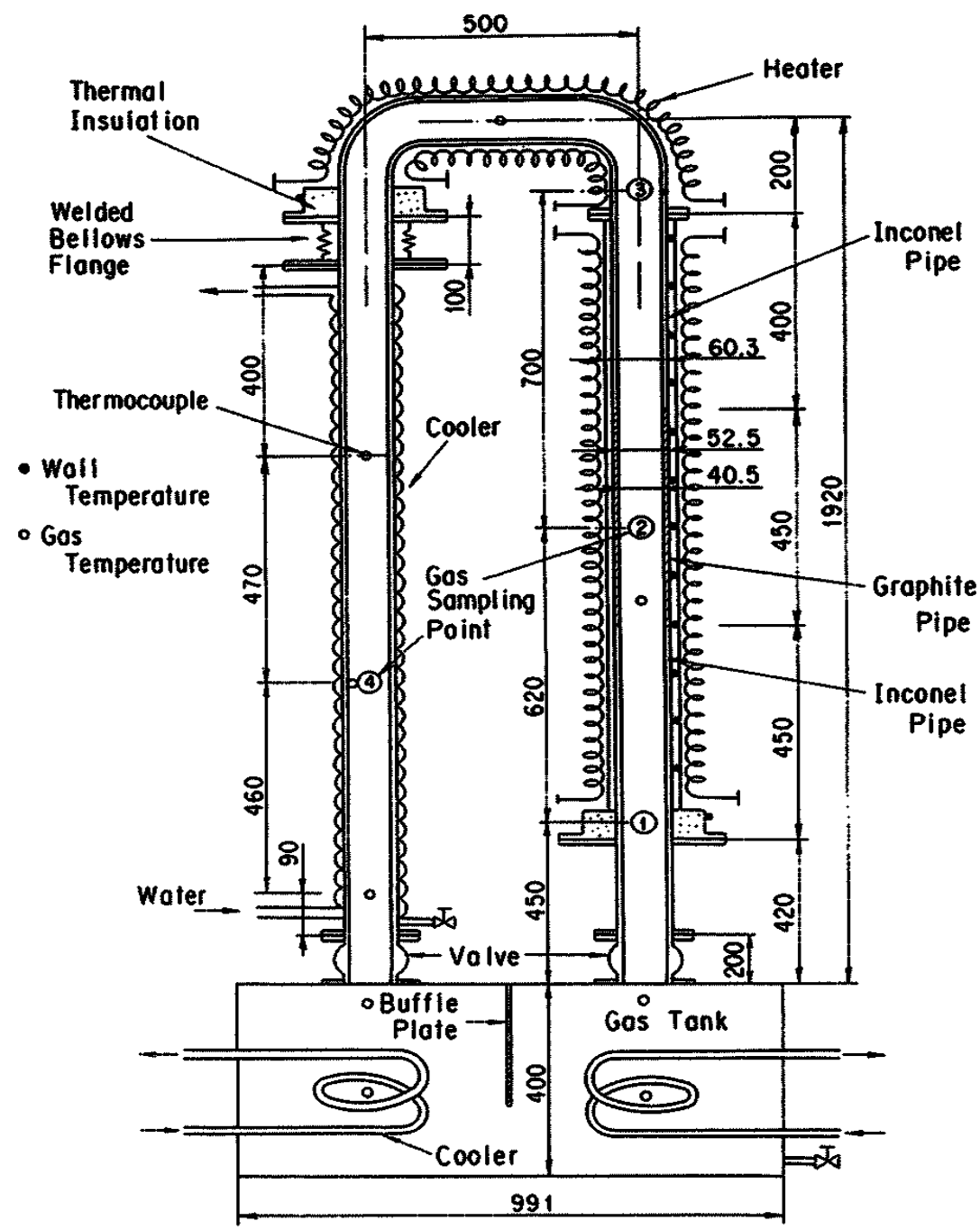

Figure 2-12 Nodalization diagram for the inverse U-tube system with a graphite tube 


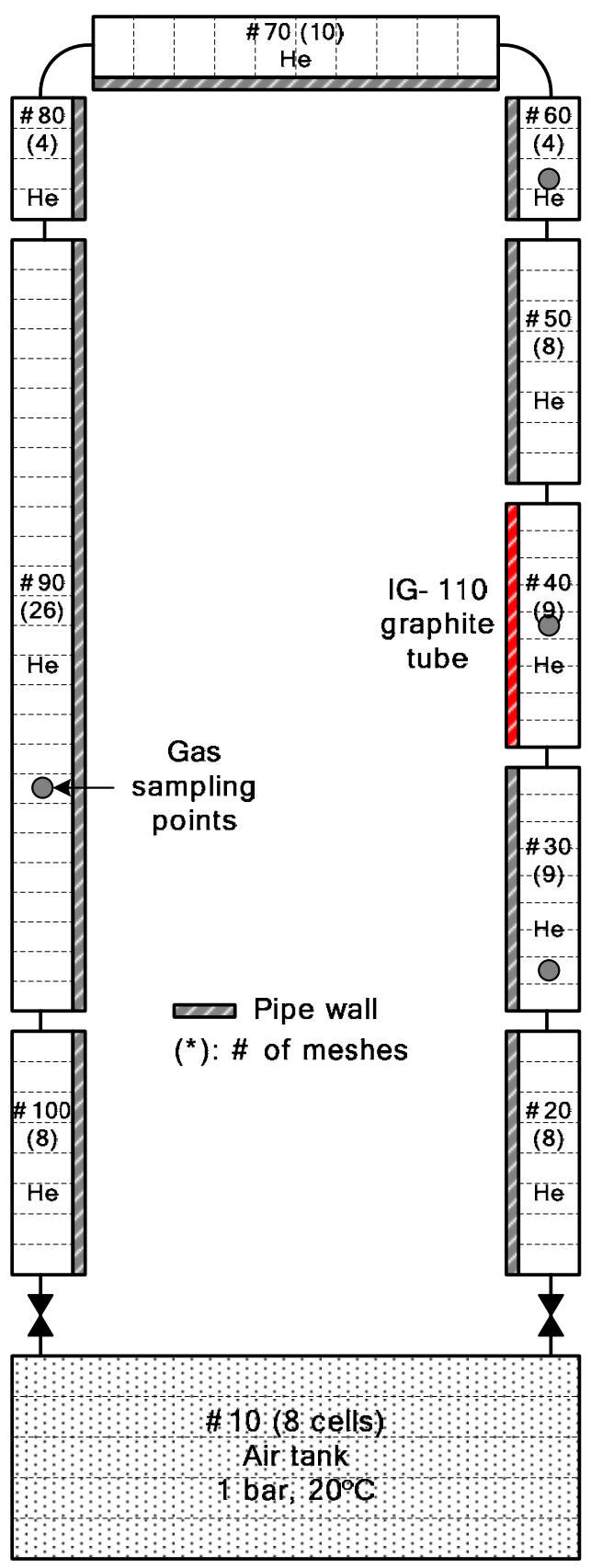

Figure 2-13 Nodalization diagram for the inverse U-tube system with a graphite tube

When the ball valves installed at the inlets of an inverse U-shaped pipe open, nitrogen or air enters the vertical pipe by molecular diffusion and a very weak natural convection. As time passes, the density of the gas mixture in the hot side gradually increases but at a faster rate than in the cold pipe. Eventually a global natural convection is initiated. In the air-filled case, the air transported from the tank chemically reacts with the graphite. As the graphite is oxidized by a chemical reaction with oxygen, $\mathrm{CO}$ and $\mathrm{CO}_{2}$ are produced and transported both upward and downward by reverse diffusion. A part of the $\mathrm{CO}$ produced dissipates by a homogeneous reaction with oxygen, thus further producing an amount of $\mathrm{CO}_{2}$. 
Table 2-3 Experimental cases conducted in the inverse U-tube test facility with a graphite tube

\begin{tabular}{|c|c|c|c|}
\hline \multicolumn{2}{|c|}{$\mathrm{N}_{2}$-filled condition } & \multicolumn{2}{c|}{ Air-filled condition } \\
\hline Run No. & $\begin{array}{c}\text { Average wall temperature } \\
\text { of the heated pipe }\left({ }^{\circ} \mathrm{C}\right)\end{array}$ & Run No. & $\begin{array}{c}\text { Average wall temperature } \\
\text { of the heated pipe }\left({ }^{\circ} \mathrm{C}\right)\end{array}$ \\
\hline NS1 & 379.7 & AS1 & 569.1 \\
\hline NS2 & 462.8 & AS2 & 661.8 \\
\hline NS3 & 555.3 & AS3 & 767.7 \\
\hline NS4 & 659.4 & AS4 & 715.4 \\
\hline NS5 & 758.0 & AS5 & 618.1 \\
\hline NS6 & 707.4 & AS6 & 466.2 \\
\hline NS7 & 618.9 & AS7 & 376.6 \\
\hline NS8 & 508.7 & AS8 & 523.5 \\
\hline NS9 & 425.2 & AS9 & 423.8 \\
\hline- & & AS10 & 16.3 (isothermal) \\
\hline- & & AS12 & 811.0 \\
\hline
\end{tabular}

All the test cases listed at Table 2-3 have been simulated: $9 \mathrm{~N}_{2}$-filled tests and 10 air-filled tests. Among those, the simulation results for the case AS3 are described in detail. During the calculation, the non-uniform wall temperature distribution along the pipe shown in Figure 2-14 is assumed to be kept constant.

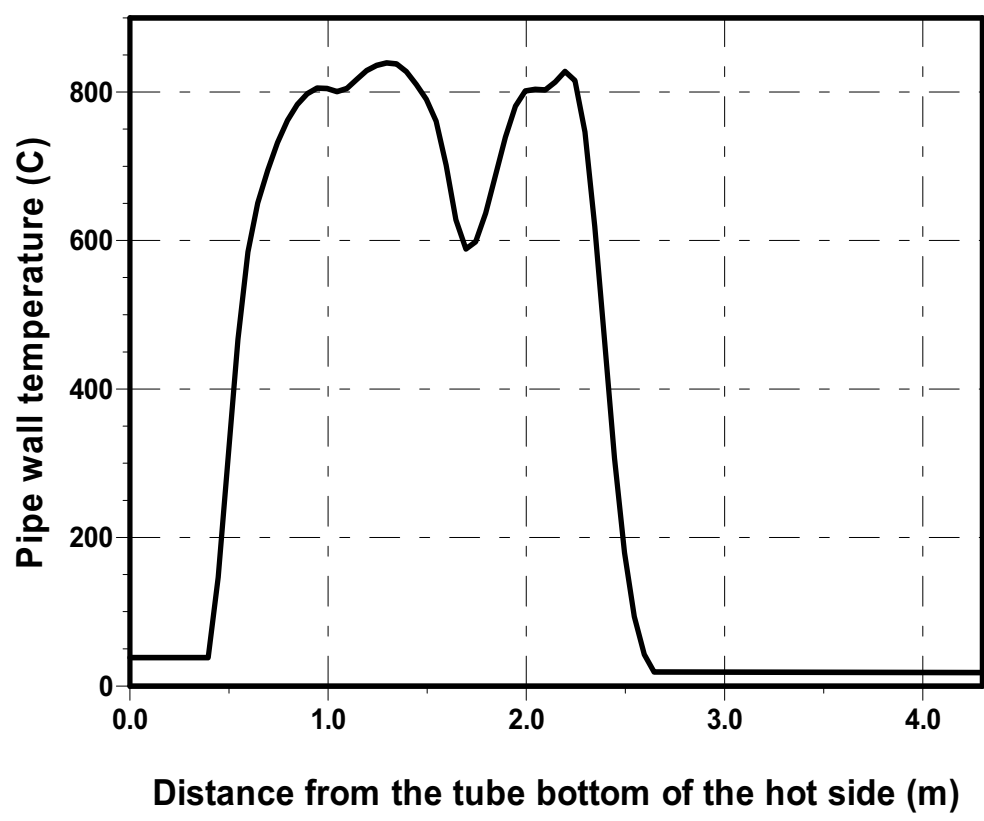

Figure 2-14 Non-uniform temperature distribution along the inverse U-tube for the case AS3

Figures 2-15 2-18 show the predicted results of the velocities of the gas mixture at the tube inlets and the mole fractions of $\mathrm{O}_{2}, \mathrm{CO}$, and $\mathrm{CO}_{2}$ at different sampling locations. As shown in Figure 2-16, as $\mathrm{O}_{2}$ in the gas tank is transported into the tube, and as $\mathrm{CO}$ and $\mathrm{CO}_{2}$ are produced by a chemical reaction with the graphite, the buoyancy force induced by the distribution of the gas mixture density gradually increases. Approximately 95 minutes after the valves open, the buoyancy force becomes large enough to initiate a global natural 
circulation throughout the inverse U-tube. Figure 2-15 shows the velocities caused by a very weak and global natural convection calculated to be roughly $<3 \times 10^{-4} \mathrm{~m} / \mathrm{s}$ in the early stage and $0.2 \mathrm{~m} / \mathrm{s}\left(\operatorname{Re}_{d}=400\right)$ in the later stage. The range of the Rayleigh number, which is calculated based on the height of the inverse U-tube, is about $1 \times 10^{9}<R a_{H}<5 \times 10^{10}$. As shown in Figures 2-15 2-18, although the calculated mole fractions of $\mathrm{O}_{2}$, $\mathrm{CO}$, and $\mathrm{CO}_{2}$ are slightly different from the measured mole factions, the calculated onset time of natural convection agrees well with that of the experiment, because the density change of the gas mixture is less sensitive to the concentrations of each gas species.

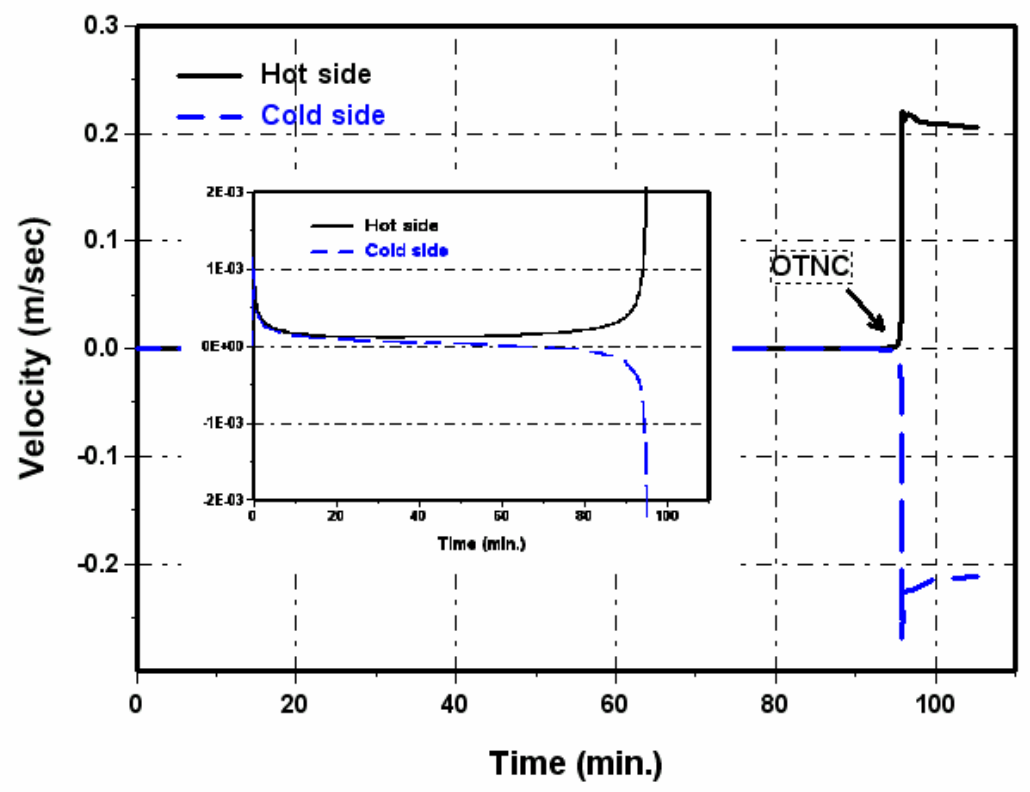

Figure 2-15 Predicted velocities at the tube inlets for the case AS3

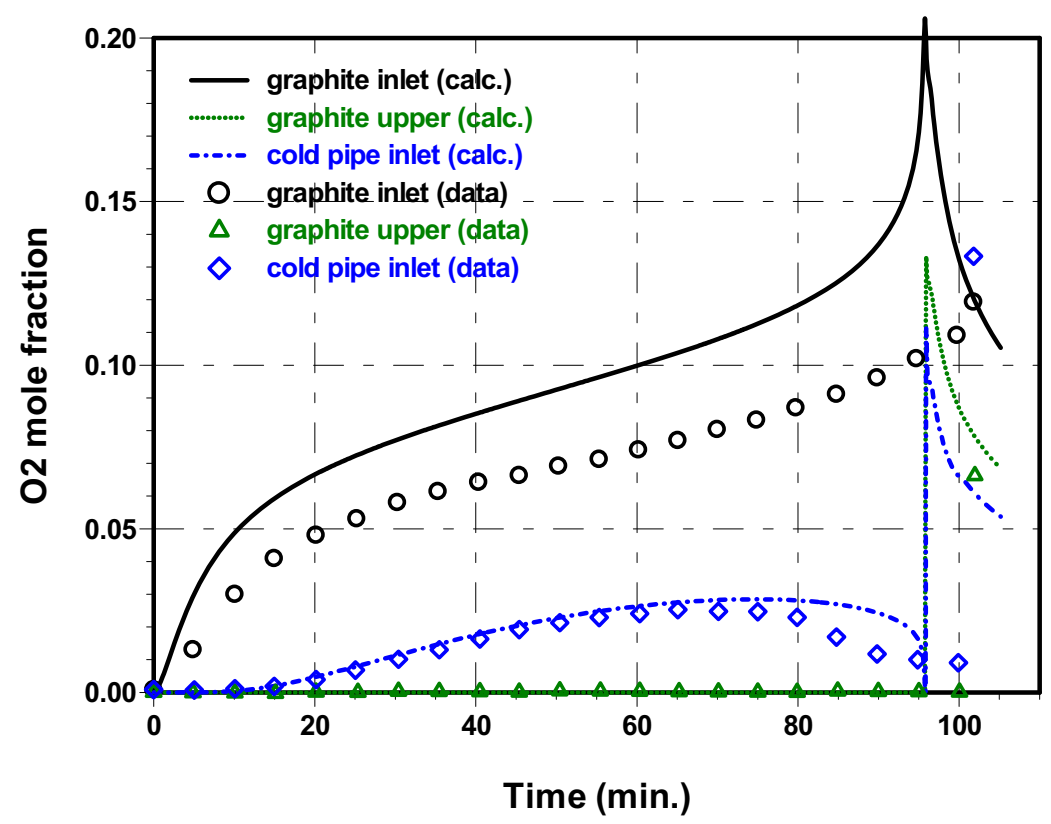

Figure 2-16 Predicted mole fractions of $\mathrm{O}_{2}$ for the case AS3 


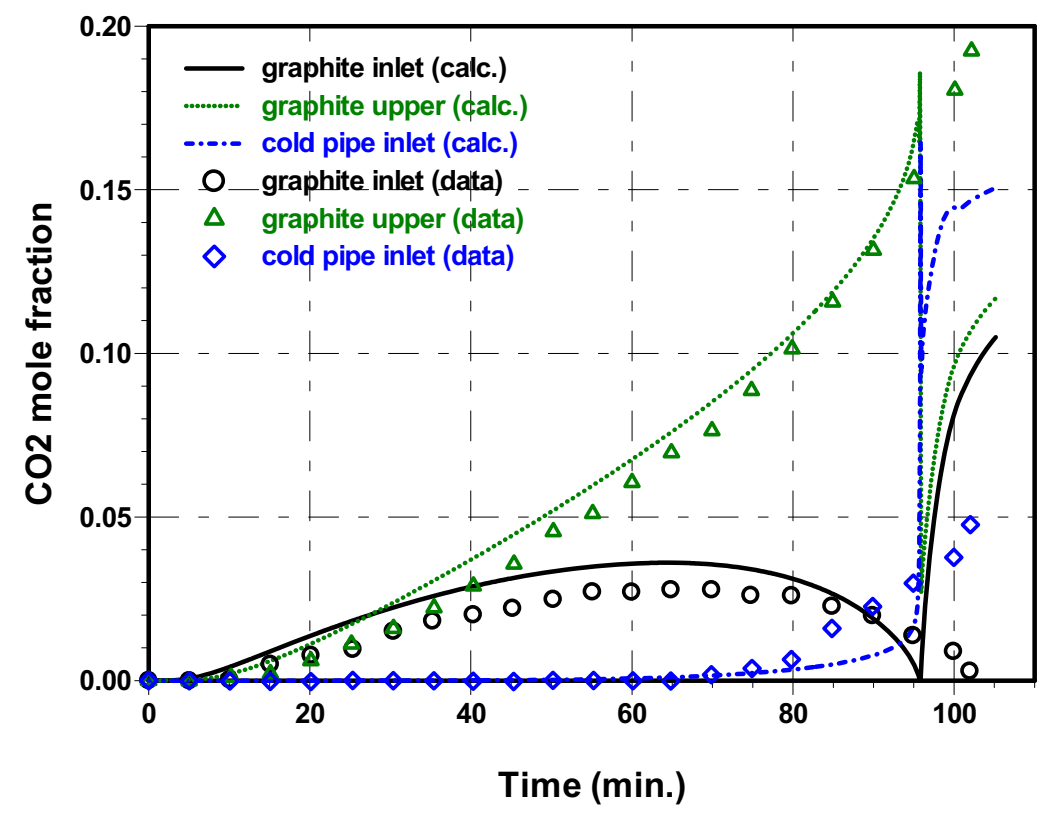

Figure 2-17 Predicted mole fractions of $\mathrm{CO}_{2}$ for the case AS3

Even with some discrepancies in the concentrations of the species, as shown in Figure 2-19, the onset times of a natural convection agree closely with those of the experiments, with a $3 \%$ deviation for the $\mathrm{N}_{2}$-filled condition and about $6 \%$ for the air-filled condition, respectively. The initiation time of a global natural convection is slightly reduced in the air-filled case due to the production of heavier gases by a chemical reaction, when compared to the $\mathrm{N}_{2}$-filled case.

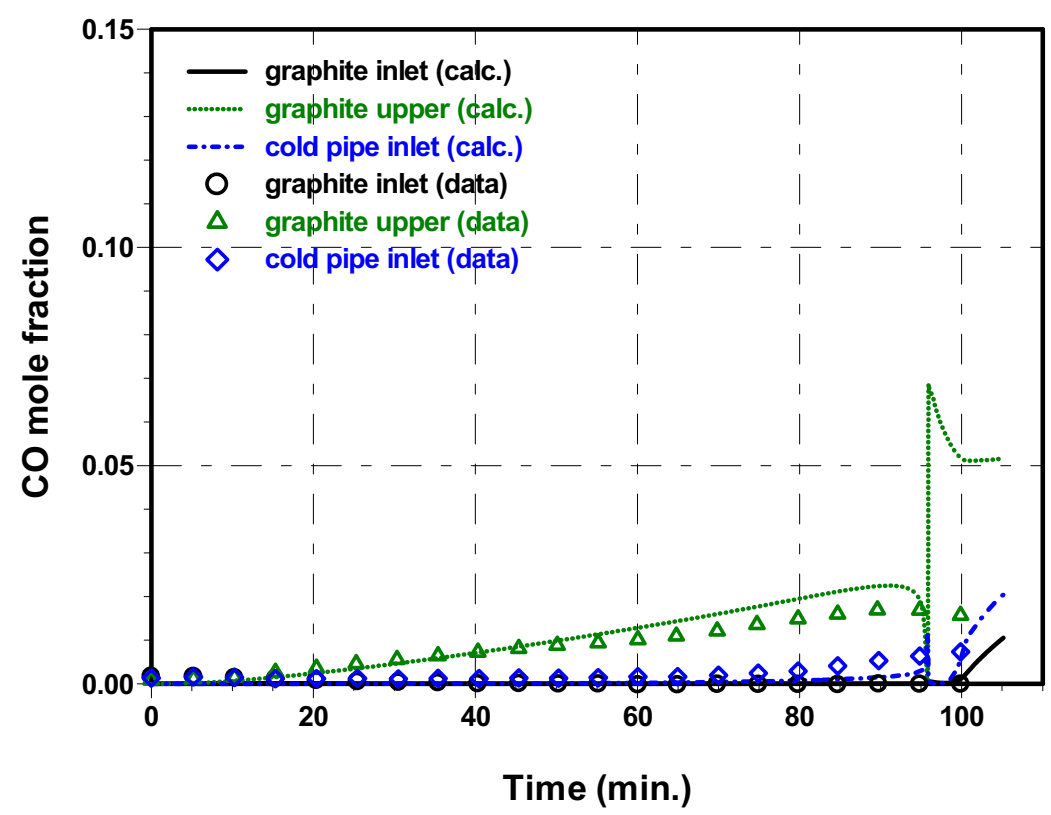

Figure 2-18 Predicted mole fractions of CO for the case AS3 


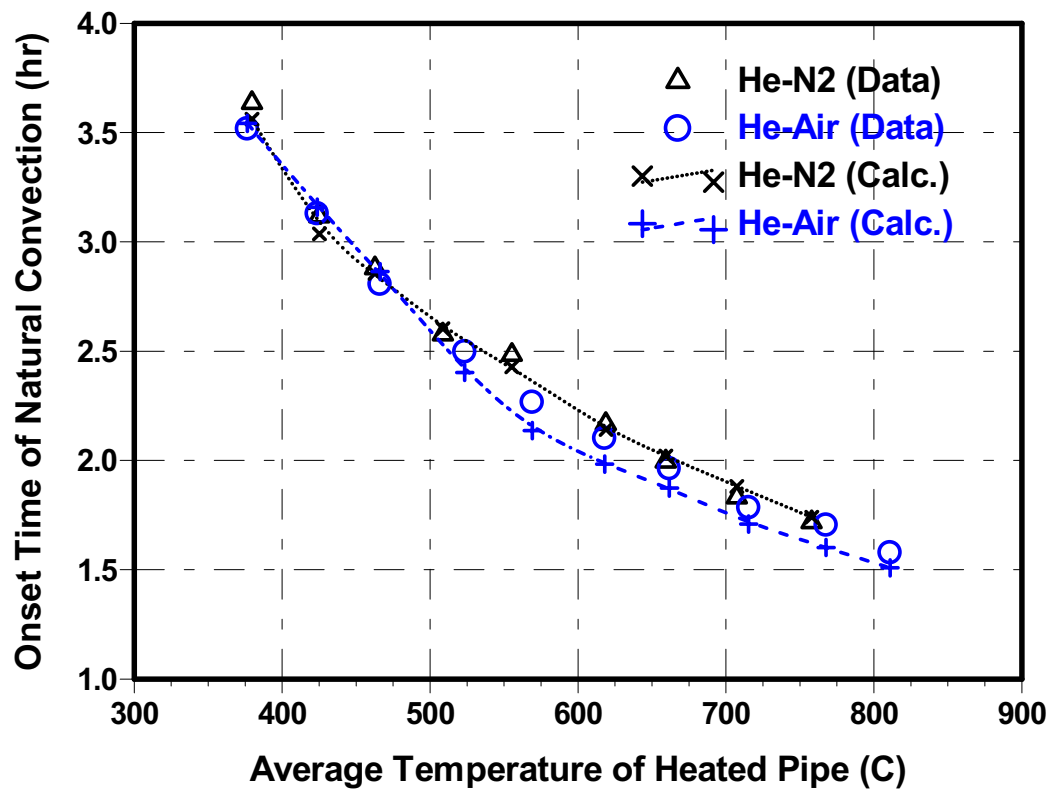

Figure 2-19 Predicted onset times of natural convection for the inverse U-tube system with a graphite tube

\subsection{Particle model}

Starting from the spherical conduction equation and boundary conditions, we have

(i) Spherical conduction equation:

$k_{f} \frac{d}{d R}\left(R^{2} \frac{d T}{d R}\right)+\dot{q} R^{2}=0$

$R^{2} \frac{d T}{d R}=-\frac{\dot{q} R^{3}}{3 k_{f}}+C 1$

$T(R)=-\frac{\dot{q} R^{2}}{6 k_{f}}-\frac{C 1}{R}+C 2$

(ii) Boundary conditions:

$\left.\frac{d T}{d r}\right|_{r=0}=0$ and $T(r, i)=T_{f, i}$ and $\mathrm{C} 1=0$ and $C 2=T_{s, i}+\frac{\dot{q} r_{i}^{2}}{6 k_{f}}$

From the above equations, we can obtain the following fuel temperatures:

(i) Fuel outer surface temperature:

$T_{\text {s.o }}=T_{H e}+\frac{q R_{i}^{3}}{3 h R_{o}^{3}}$

(ii) Fuel inner surface temperature:

$T_{S, i}=T_{S, o}+\frac{h}{k_{\text {graphite }}}\left(\frac{R_{o}}{R_{i}}-1\right) R_{o}\left(T_{s . o}-T_{H e}\right)$

Fuel center temperature in homogenized fuel:

$T(0)=T_{s, i}+\frac{q R_{i}^{2}}{6 k_{f}}$ 
To obtain the heat transfer coefficient on the surface of the pebble, we have

$h=\frac{\lambda}{d} N u$

where

$N u=1.27 \frac{\operatorname{Pr}^{0.333}}{\varepsilon^{1.18}} \operatorname{Re}^{0.36}+0.033 \frac{\operatorname{Pr}^{0.5}}{\varepsilon^{1.07}} \operatorname{Re}^{0.86}$

$\operatorname{Pr}=\frac{\eta c_{p}}{\lambda}, \operatorname{Re}=\frac{\dot{m} R_{f, i}}{A \eta}$

After generating the temperature of the center in the homogenized fuel, we need to obtain the temperature of the particle located at the center of pebble:

$T=T_{H e}+\frac{Q_{f}}{4 \pi R_{f, o}{ }^{2}}+\frac{Q_{f}}{4 \pi k_{c}}\left(\frac{1}{R_{f, i}}-\frac{1}{R_{f . o}}\right)+\frac{\dot{q} R_{f, i}{ }^{2}}{6 k_{f}}$.

$+\frac{Q_{p}}{4 \pi}\left\{\frac{1}{k_{o}}\left(\frac{1}{R_{p, s}}-\frac{1}{R_{p, o}}\right)+\frac{1}{k_{s}}\left(\frac{1}{R_{p, i}}-\frac{1}{R_{p, s}}\right)+\frac{1}{k_{i}}\left(\frac{1}{R_{p, b}}-\frac{1}{R_{p, i}}\right)+\frac{1}{k_{b}}\left(\frac{1}{R_{p, f}}-\frac{1}{R_{p, b}}\right)\right\}+\frac{\dot{q} R_{p, f}^{2}}{6 k_{p, f}}$

We need homogenized thermal conductivity in the fuel region of the pebble:

$\bar{k}=\frac{\sum V_{i} k_{i}}{V_{\text {total }}}$

The thermal conductivity of each material is as follows:

(i) Property of Pyc

\begin{tabular}{|c|c|}
\hline Temperature(K) & Property $(\mathrm{W} / \mathrm{mK})$ \\
\hline 350 & 9.2 \\
\hline 390 & 7.5 \\
\hline 490 & 6.7 \\
\hline 600 & 5.9 \\
\hline 730 & 5.4 \\
\hline 830 & 5.2 \\
\hline
\end{tabular}

(ii) Property of SiC

\begin{tabular}{|c|c|}
\hline Temperature & Property(W/mK) \\
\hline 773 & 19.9 \\
\hline 1173 & 18.3 \\
\hline 1373 & 17.9 \\
\hline 1573 & 17.8 \\
\hline
\end{tabular}

(iii) Property of Buffer

$$
\begin{array}{l|l}
\text { Temperature(K) } & \text { Property }(\mathrm{W} / \mathrm{mK})
\end{array}
$$




\begin{tabular}{|l|l|}
\hline 350 & 2.30 \\
\hline 390 & 1.88 \\
\hline 490 & 1.68 \\
\hline 600 & 1.48 \\
\hline 730 & 1.35 \\
\hline 830 & 1.30 \\
\hline
\end{tabular}

(iv) Property of UO2

\begin{tabular}{|c|c|}
\hline Temperature(K) & Property $(\mathrm{W} / \mathrm{mK})$ \\
\hline 1000 & 3.81 \\
\hline 1200 & 3.46 \\
\hline 1400 & 2.77 \\
\hline 1600 & 2.60 \\
\hline 1800 & 2.42 \\
\hline 2000 & 2.25 \\
\hline
\end{tabular}

Using the following particle data,

Data of particle

\begin{tabular}{|c|c|c|}
\hline Description & Unit & Value \\
\hline Uranium mass per fuel sphere & $\mathrm{g}$ & \multicolumn{1}{|c|}{} \\
\hline U-245 mass per fuel sphere & $\mathrm{g}$ & 193.5 \\
\hline Carbon mass per fuel sphere & $\mathrm{g}$ & 25 \\
\hline Radius of fuel zone & $\mathrm{mm}$ & 30 \\
\hline Fuel sphere outer radius & $\mathrm{mm}$ & 95 \\
\hline Radius of fuel kernels & $\mu \mathrm{m}$ & 40 \\
\hline Thickness of C particle buffer coatings & $\mu \mathrm{m}$ & 35 \\
\hline Thickness of particle inner Pyc coatings & $\mu \mathrm{m}$ & 40 \\
\hline Thickness of particle SiC coatings & $\mu \mathrm{m}$ & 2.5 \\
\hline Thickness of particle outer PyC coatings & $\mu \mathrm{m}$ & 1006 \\
\hline Power Peak Factor $\left(\mathrm{F}_{\mathrm{q}}\right)$ & & $\mathrm{W} / \mathrm{m}^{2} \mathrm{k}$ \\
\hline Heat Transfer Coefficient & & \\
\hline
\end{tabular}


We can obtain the temperature according to the variation of power peaking factor, $\mathrm{F}_{\mathrm{q}}$, as shown in Figure 220. The temperature of the central particle is highly influenced by $F_{q}$ and there is a big jump in the film of the pebble due to the He heat transfer coefficient relatively low compared with the thermal conductivity of graphite.

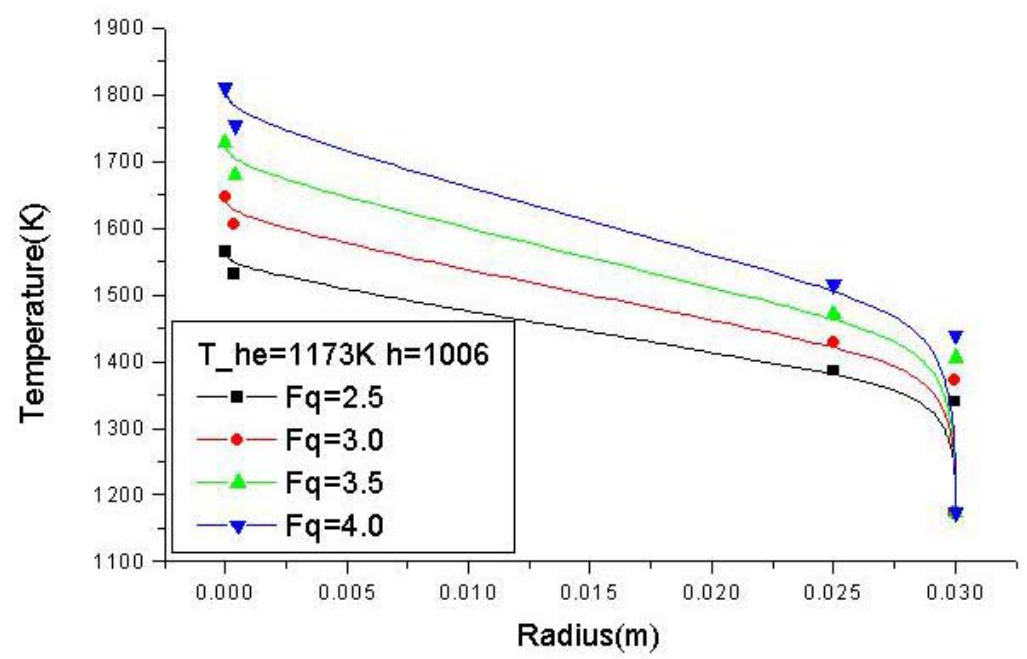

Figure 2-20 Temperature variation in the pebble with power peaking factor, $\mathrm{Fq}$

To validate the particle model, we compare the results from the present particle model with those from the MIT detailed particle model as shown in Figure 2-21. The present model is in very good agreement with the MIT model.

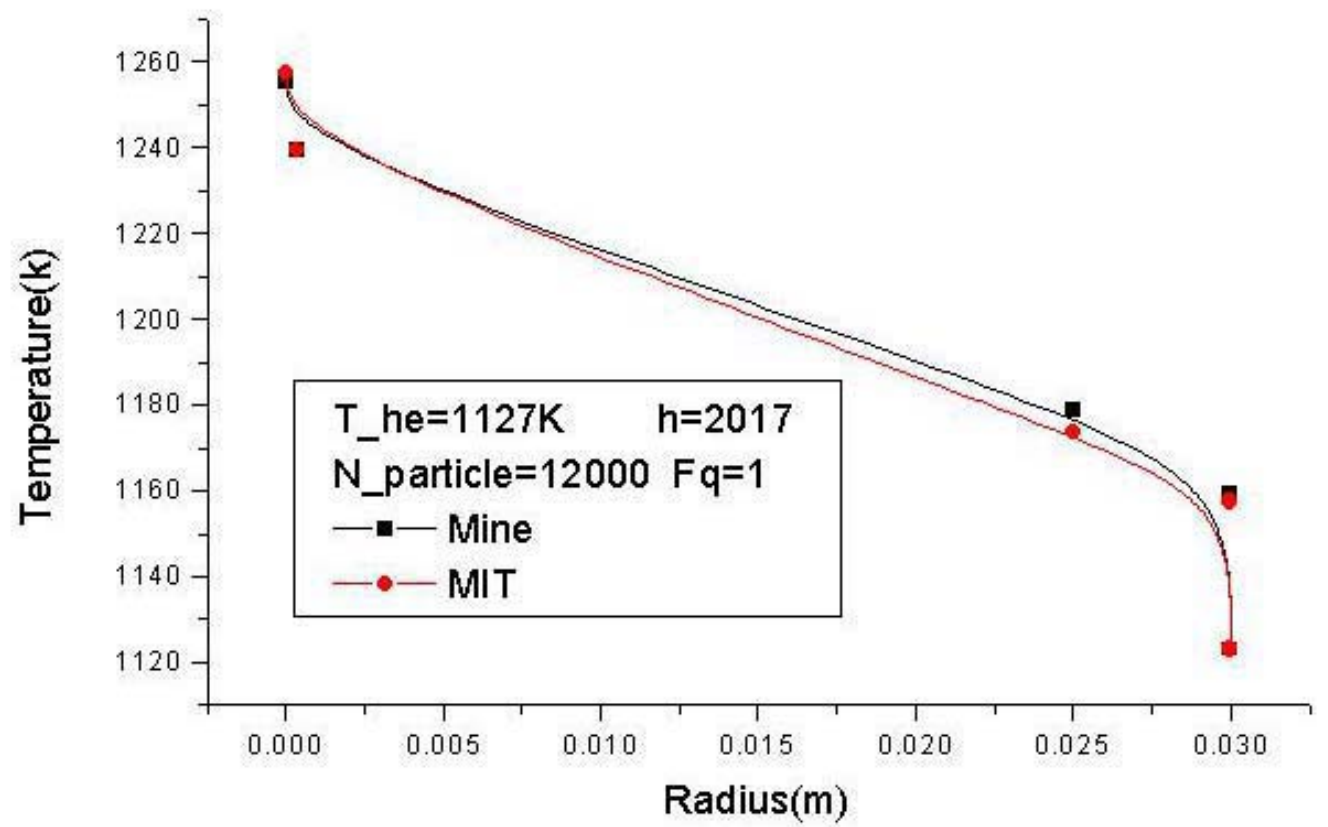

Figure 2-21 comparison of the results between the present particle model and the MIT detailed particle model 


\section{Nomenclatures for Task 1}

\section{English}

$a_{s f} \quad$ specific solid-to-fluid interfacial area $\left(\mathrm{m}^{-1}\right)$

$C_{F} \quad$ drag coefficient in a pebble bed

$d_{p} \quad$ pebble diameter $(\mathrm{m})$

$D_{s k} \quad$ multicomponent diffusion coefficient $\left(\mathrm{m}^{2} / \mathrm{s}\right)$

D $_{s k} \quad$ binary diffusion coefficient $\left(\mathrm{m}^{2} / \mathrm{s}\right)$

$D_{s-m i x}$ effective diffusion coefficient $\left(\mathrm{m}^{2} / \mathrm{s}\right)$

$F_{i j} \quad$ geometric view factor

$f_{\mathrm{CO} / \mathrm{CO}_{2}}$ production ratio of $\mathrm{CO}$ and $\mathrm{CO}_{2}$ for graphite oxidation

g gravitational constant $\left(\mathrm{m} / \mathrm{s}^{2}\right)$

$h_{s f} \quad$ interfacial heat transfer coefficient $\left(\mathrm{W} / \mathrm{m}^{2}-\mathrm{K}\right)$

$\Delta h_{f}^{o} \quad$ latent heat of formation for chemical reaction $(\mathrm{J} / \mathrm{kg})$

$H \quad$ sensible enthalpy of gas mixture $(\mathrm{J} / \mathrm{kg})$

$H_{s} \quad$ sensible enthalpy of species, $\mathrm{s}(\mathrm{J} / \mathrm{kg})$

$J_{i} \quad$ radiosity of the radiating surface, $\mathrm{i}\left(\mathrm{W} / \mathrm{m}^{2}\right)$

$\mathbf{J}_{s} \quad$ total diffusion flux with respect to mass average velocity $\left(\mathrm{kg} / \mathrm{m}^{2}-\mathrm{s}\right)$

$K \quad$ permeability

$k_{s} \quad$ mass transfer coefficient of species, $\mathrm{s}(\mathrm{m} / \mathrm{s})$

$m$ total number of species

$N \quad$ total number of computational cells

$P \quad$ total pressure $(\mathrm{Pa})$

$P_{s} \quad$ partial pressure of species, $\mathrm{s}(\mathrm{Pa})$

$P_{r} \quad$ Prandtl number

$q^{\prime \prime \prime} \quad$ volumetric heat source at the wall $\left(\mathrm{W} / \mathrm{m}^{3}\right)$

$q_{r}^{\prime \prime} \quad$ net radiative heat flux $\left(\mathrm{W} / \mathrm{m}^{2}\right)$

$\bar{R} \quad$ universal gas constant

$\mathrm{Ra} \quad$ Rayleigh number

$R e_{p} \quad$ Reynolds number based on pebble diameter

$R_{s} \quad$ generation/dissipation of species, $\mathrm{s}$, by chemical reaction $\left(\mathrm{kg} / \mathrm{m}^{3}-\mathrm{s}\right)$

Sc Schmidt number

$\mathrm{Sh} \quad$ Sherwood number

$T_{f} \quad$ temperature of gas mixture $(\mathrm{K})$

$\bar{T}_{k} \quad$ temperature of agglomerated surface, $\mathrm{k}(\mathrm{K})$

$T_{p} \quad$ solid or pebble temperature $(\mathrm{K})$

u mass average velocity of gas mixture $(\mathrm{m} / \mathrm{s})$

$W \quad$ molar weight of gas mixture $(\mathrm{g} / \mathrm{mol})$

$W_{s} \quad$ molar weight of species, $\mathrm{s}(\mathrm{g} / \mathrm{mol})$

$W_{C} \quad$ molar weight of graphite $(\mathrm{g} / \mathrm{mol})$

$X_{s} \quad$ mole fraction of species, $\mathrm{s}$ 
$Y_{s} \quad$ mass fraction of species, $\mathrm{s}$, in the bulk, and $Y_{s}^{w}$ at the surface of the wall

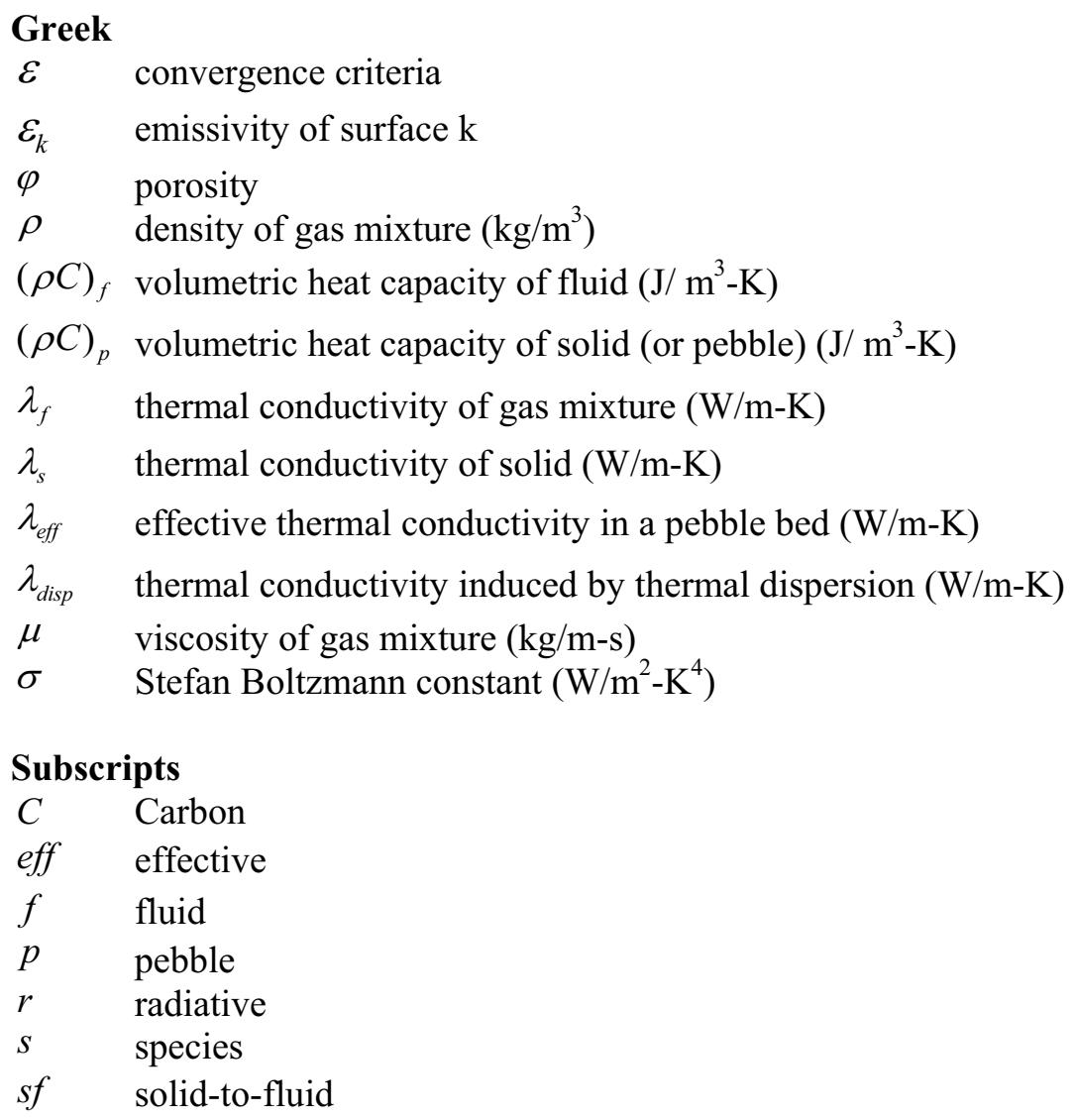

\section{Superscripts}

$w \quad$ wall

$n \quad$ old time level

$n+1$ new time level

$k$ or ${ }^{\prime}$ iteration step

\section{Acronyms}

GCR Gas Cooled Reactor

GT-MHR Gas-Turbine Modular Helium Reactor

HTGR High Temperature Gas-cooled Reactor

HTTR High Temperature Engineering Test Reactor

IAEA International Atomic Energy Agency

PBMR Pebble Bed Modular Reactor

OTNC Onset Time of Natural Convection

RCCS Reactor Cavity Cooling System

RPV Reactor Pressure Vessel 


\section{TASK 2: RCCS experiment (SNU)}

In the cavity of the VHTGR, which is the space between the reactor vessel and containment, a reactor cavity cooling system (RCCS) is equipped to remove the heat transferred from the reactor vessel to the structure of the containment. The RCCS are typically safety grade systems, either with passive or with high reliable, redundant forced-convection cooling system, designed to remove the entire core afterheat in the unlikely case of failure or unavailability of the main and all other shutdown cooling systems. The performance and reliability of the RCCS, therefore, are considered as the critical factors in determining maximum design power level related to afterheat removal. The over-designed capacity of the system, however, would not be acceptable for the RCCS because during normal operation, and in some cases for normal shutdowns, excessive parasitic heat losses are undesirable. Also the fact that heat load distribution during long term loss of forced convection (LOFC) accidents can vary considerably with the accident characteristics would make the design of the RCCS difficult. Due to this difficulty of the optimization of the RCCS capacity, experimental studies for the code validation and numerical studies using the validated codes are necessary to determine the adequacy of the design (IAEA-TECDOC-1163 2000).

The characteristics of the RCCS in the HTGR under development are summarized in Table 3-1. In the high temperature engineering test reactor (HTTR) which is the first HTGR in Japan, the reactor cavity cooling is provided by forced convection of water along the cooling panel (S. Saito et al.1989). Two independent RCCSs named vessel cooling system (VCS) were equipped in the cavity. The heated water in the VCS is cooled by forced convection of water at the secondary side of the

Table 3-1 RCCS types in the HTGRs

\begin{tabular}{|l||l||l||}
\hline Reactor & RCCS Coolant Type & Secondary Coolant Type \\
\hline \hline HTTR & Water Forced Convection & Water Forced Convection \\
\hline HTR-10 & Water Natural Convection & Air Natural Convection \\
\hline \hline PBMR & Water Natural Convection & Air Natural Convection \\
\hline \hline GT-MHR & $\begin{array}{l}\text { Air } \\
\text { Natural Convection }\end{array}$ & No Secondary cooling \\
\hline \hline MHTGR & $\begin{array}{l}\text { Air } \\
\text { Natural Convection }\end{array}$ & No Secondary cooling \\
\hline \hline
\end{tabular}

RCCS. The 10 MW High Temperature Gas-cooled Test Reactor (HTR-10) developed in China adopts two independent water cooled RCCSs which remove the after heat by natural circulation of water (Wu et al. 2002). The water coolers are connected with the air coolers on the top of the reactor building which are located in the two chimneys. The air flow supplied by the chimneys removes the after heat to atmosphere. The RCCS of the $265 \mathrm{MW}$ pebble bed modular reactor (PBMR) developed in South Africa includes three 
independent systems each consisting of a natural convection driven water cooling system with passive external water-to-air heat exchanger (IAEA-TECDOC-1198 2001). In the case where all the cooling units are failed, the heat of the reactor is absorbed by heating up and then boiling off the water in the system. The systems are sized to provide this cooling function for up to three days. The gas turbine modular helium reactor (GT-MHR) has $600 \mathrm{MW}$ thermal power and is planned for construction in Russia (IAEA-TECDOC1198 2001). The RCCS of the GT-MHR removes heat by conduction through the graphite reflector and by radiation and natural convection from the uninsulated vessel. The system, which receives the heat transferred from the vessel, includes a cooling panel placed around the reactor vessel. Heat is removed from the reactor cavity by natural circulation of outside air through the cooling panel. The $450 \mathrm{MW}$ modular high temperature gas-cooled reactor (MHTGR) developed in the United States has an air cooled RCCS which rejects the after heat to the atmosphere by buoyancy-driven natural circulation of outside air through the cooling panels (D.A.Dilling et al. 1982). The system should function during all period of normal operation and accidents and does not rely on any active component or operation action.

As summarized in Table 3-1, the cooling capability of the RCCS in the developed HTGR is provided by forced convection of water, natural circulation of water or natural circulation of air. It was reported that the active water cooling RCCS has efficient cooling capability and is easy to design comparing with the others. However it has the possibility of over-cooling as well as the very complex features to provide the same level of reliability as the passive cooling scheme. The passive water cooling RCCS can reject the after heat efficiently with high reliability but needs complicated structures and affiliated systems such as secondary side cooling system and water purification system. Also it was reported that there is significant uncertainty and complexity associated with two-phase phenomena in the boiling mode for the water cooling scheme (D.A.Dilling et al. 1982). The air cooling scheme has fewer failure modes and is more passive than the others but it was known to be difficult to design because the air flow around the RPV is deviated due to the effects of nozzle locations (IAEA-TECDOC-1163 2000). Also due to poor cooling capability of natural circulating air, a very high chimney is necessary to supply enough air flow to remove the after heat.

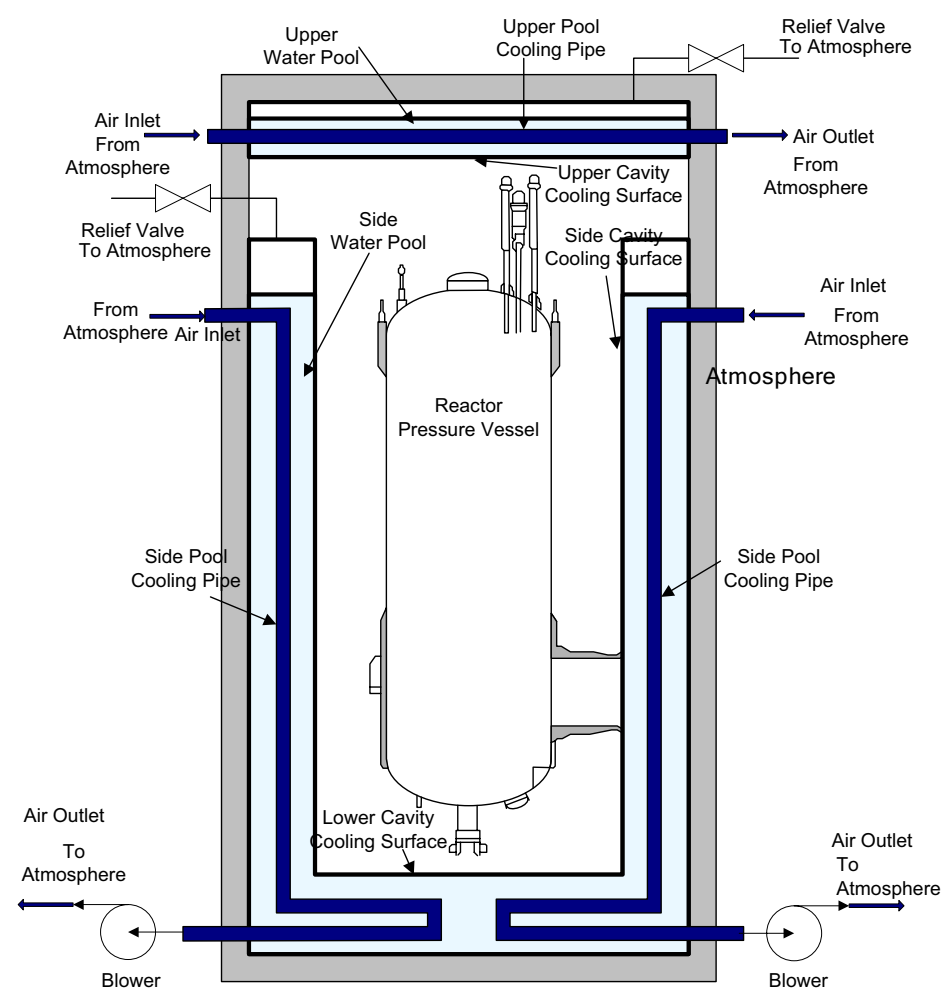

(a) System configuration 


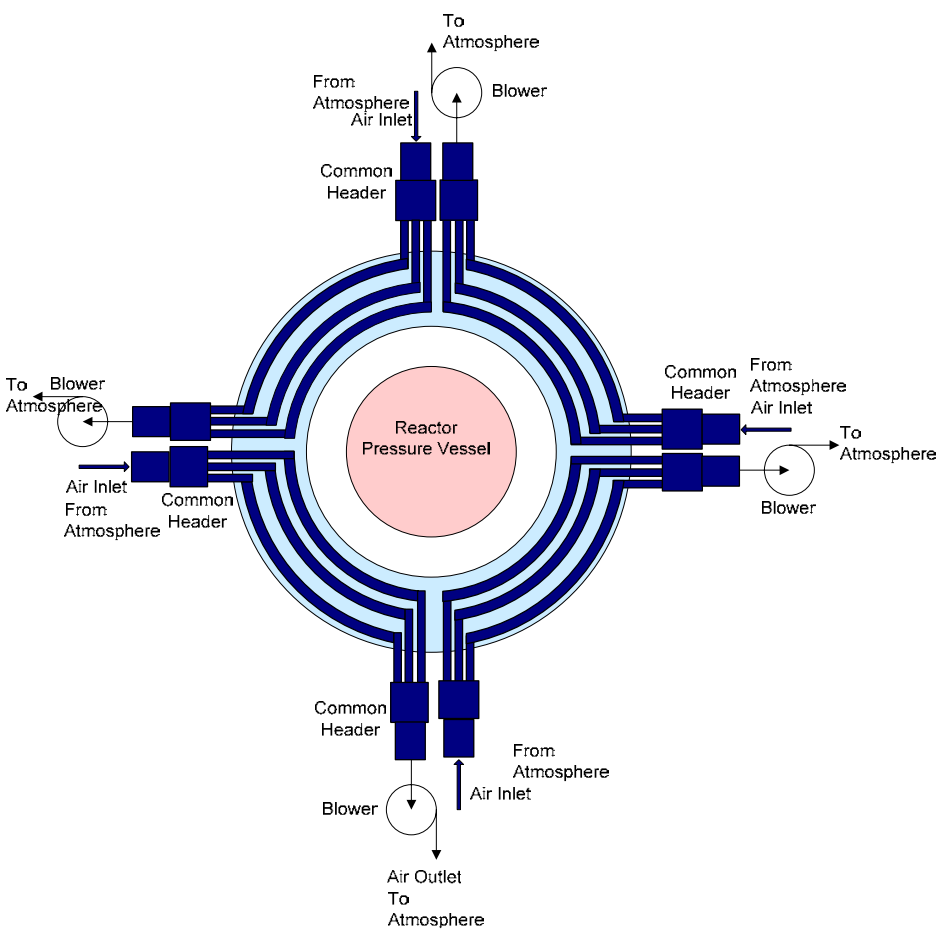

(b) Top view of the side water pool

Figure 3-1 Schematic diagram of the water pool type RCCS 
Herein, we propose a new kind of RCCS, called RCCS-SNU, in order to overcome the disadvantages of the weak cooling ability of the air-cooled RCCS and the complex structures of the water-cooled RCCS. Unlike the common water cooled RCCSs, which use the natural circulation of water, the proposed system uses a water pool as a heat sink for the transferred heat which occurs during normal operation and the afterheat generated during accident conditions. A schematic diagram of the water pool type RCCS is presented in Figure 3-1. The system consists of three main parts, the side water pool located between the containment and reactor vessels, the upper water pool located above the reactor vessel and the five trains of the air cooling systems installed in the water pools. During normal operation, the heat loss from the reactor vessel is transferred into the water pools via the cavity and then released to the atmosphere by the forced convection of air flowing through the cooling pipes. Ambient air is utilized for this purpose. For the redundancy of the RCCS, five trains of active cooling systems are installed independently and it is able to continue its normal operation, even if one of the five active cooling systems fails. In the case of a LOFC accident with the failures of all the active cooling systems, the afterheat is passively absorbed by heating up and then boiling off the water in the water pools. The steam generated in the water pools is released to the atmosphere. We would like to make the capacity of the water pool sufficient to allow the passive cooling of the afterheat to continue for three days.

This new kind of RCCS is similar to the common water cooling systems presently in operation, but is expected to be easier to design and analyze, because of the simple geometry of the cavity cooling surface. It uses ambient air to release the afterheat to the atmosphere, so that no additional cooling systems are necessary. Also, it is expected to have less uncertainty and complexity in the boiling situation than the other water cooling systems, since typical pool boiling occurs in the water pool of the system. The poor cooling capability of air, however, may result in the air supply system having an undesirably large capacity. Also, the size of the water pool needs to be optimized due to the limited space available inside the cavity. For this reason, we performed a series of experiments to evaluate the feasibility of the water pool type RCCS and assess the cooling capability of the device.

The major objectives of this task are to carry out the experiment for the RCCS-SNU, which is a water pool type RCCS constructed in Seoul National University (SNU), to provide the experimental data for the validation of the thermal hydraulic code being developed at KAIST and to evaluate the feasibility of the system using the experimental results and system analysis codes validated by code-to-experiment and codeto-code benchmarks. To achieve these objectives, we planned three categories of experiments; 1) separate effect test for emissivity measurement, 2) separate effect test for the RCCS-SNU water tank and 3) integral test (IET) for the RCCS-SNU. In this report, the results of these experiments are presented. Also, the code-toexperiment validation for the system analysis codes, MARS-GCR, and CFX5.7 calculation were conducted for the separate effect test results. Finally the assessment of MARS-GCR for RCCS-SNU will be examined by prediction of IET experimental data. 


\subsection{Emissivity measurement experiment}

In the RCCS, heat loss and the decay heat can be transferred from the reactor vessel to the atmosphere through several heat transfer processes such as conduction, radiation, and convection. Among these processes, radiation heat transfer was reported to be the most important process, particularly under accident conditions, because the radiation heat transfer rate becomes the main heat transfer mechanism as the vessel temperature increases. That is, under accident conditions, the radiation heat transfer is known to be more than $70 \%$ of the total heat transferred from the reactor vessel to the RCCS through the cavity (IAEA-TECDOC$11632000)$.

Because the rate of radiation heat transfer is determined by the emissivity, the temperature of the materials and the geometry of apparatus, it is very important to know the exact emissivity of each material as well as the temperature in order to calculate the amount of heat removal by the RCCS. Previous studies were reported to be carried out using a constant emissivity, for example 0.80 (IAEA-TECDOC-1163 2000). However, the emissivity depends on many factors such as temperature, surface roughness, oxidation level, wavelength, direction, atmosphere condition and so on. Therefore, information on the emissivity for a given material in the real circumstance of the RCCS is essential for analyzing the heat transfer in the HTGR.

Several methods have been developed to measure the emissivity using various detectors such as an IR spectrometer, radiometer, and an infrared thermometer (L. Chen, B. T. Yang 1990). In this study, a single band infrared thermometer was selected because of its simplicity in operation as well as economical advantage. The single band infrared thermometer is commonly used to measure the temperature of the object using the information of the radiation emitted from the target material whose emissivity is known. Inversely, the emissivity can also be estimated with the true temperature of the target material.

As a preliminary process of measuring the emissivity in RCCS-SNU, the experiments with separate emissivity measurement device were performed to verify the methodology for measuring the emissivity using an infrared thermometer as well as determining the uncertainty factors.

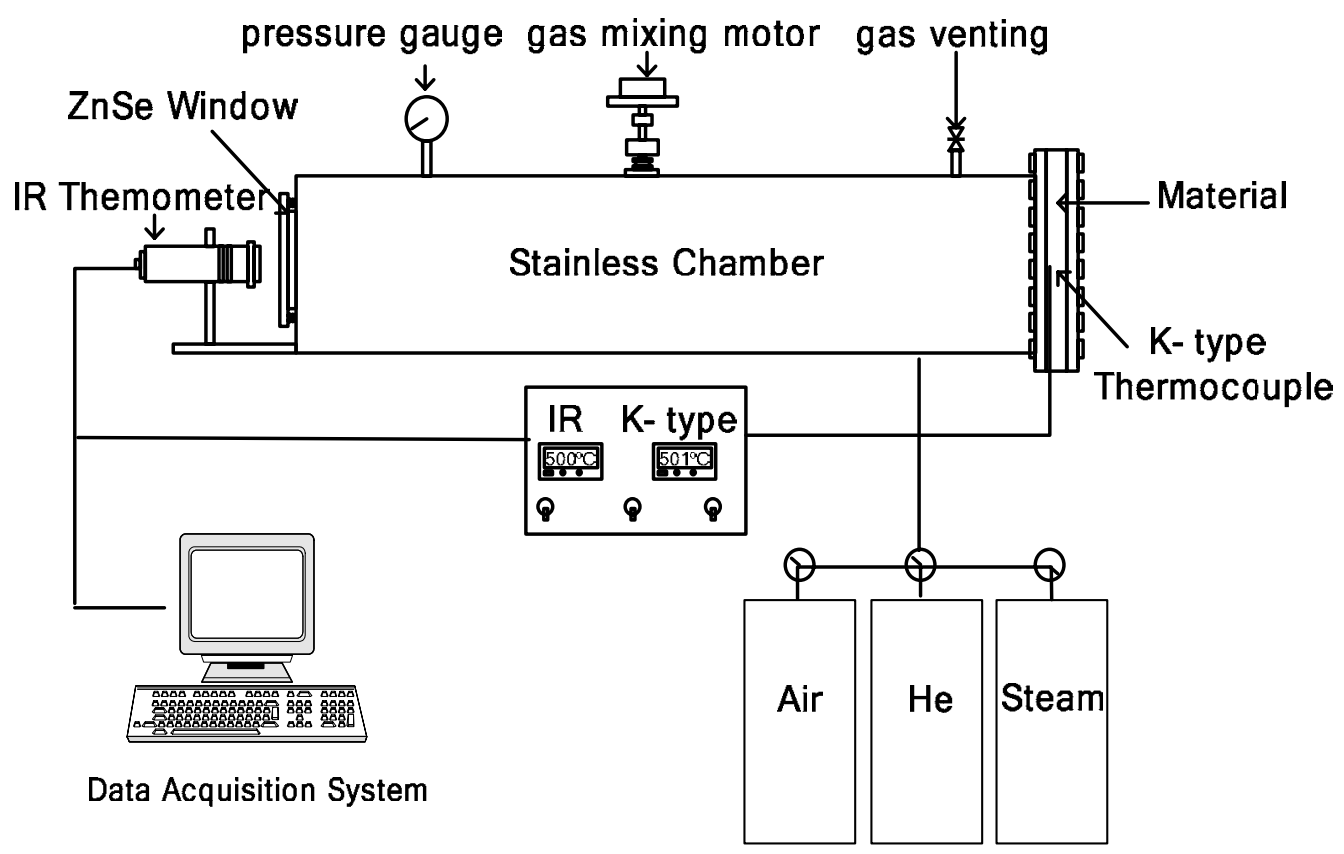

Figure 3-2 Schematic diagram of the separate emissivity measurement device

The test device is shown in Figure 3-2. A stainless steel chamber was composed mainly of an infrared thermometer, a target material, and a thermocouple. The infrared thermometer is a single band type and has a 
measuring temperature range of $-32 \sim 900{ }^{\circ} \mathrm{C}$, a wavelength range of $8 \sim 14$, an accuracy of $1 \%$, and a spot size of $16 \mathrm{~mm}$ at a distance of $800 \mathrm{~mm}$. A thermocouple was installed at the center of the target

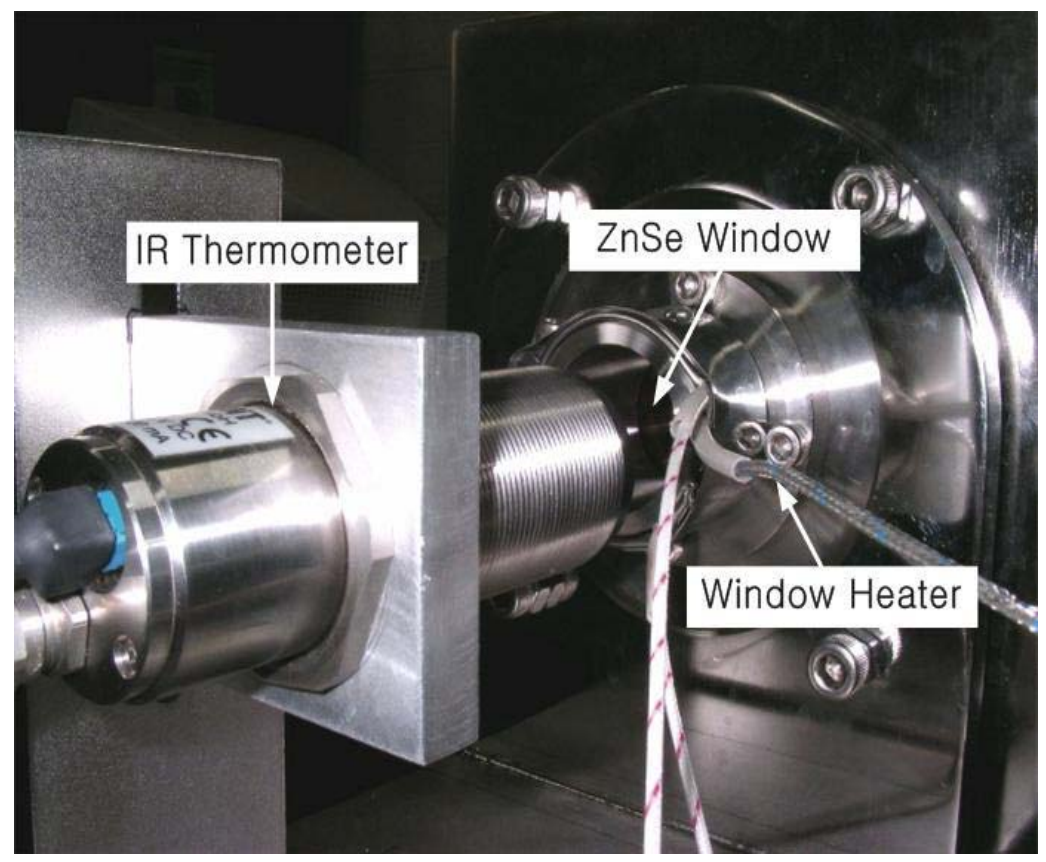

Figure 3-3 ZnSe window and window heater

material to compare this result with temperature measured by the infrared thermometer. The target material was made from carbon steel and it was oxidized sufficiently before the experiments. Although the target material in the separate emissivity measurement device was different from the reactor vessel material of the RCCS-SNU, the attenuation effect of the window and filling gas could be investigated independently on the type of target material. For a closed system, a $50 \mathrm{~mm}$ diameter and $5 \mathrm{~mm}$ thick Zinc Selenide (ZnSe) window was placed at the left sidewall of the chamber, as shown in Figure 3-3. Because the steam condensation on the inner window surface can cause many erroneous results as a result of absorption and reflection in the test case of steam, a window heater was added in order to prevent steam condensation on the window surface.

The gas concentration was controlled by a vacuum pump and the maximum degree of the vacuum was $20 \%$. Steam and helium, which was supplied from the steam generator and a helium tank respectively, were mixed well using a gas mixer. For the emissivity measurements, the temperature was measured within the temperature range of 100 to $500{ }^{\circ} \mathrm{C}$ with both an infrared thermometer and a thermocouple. The temperature range covers the temperature of the reactor vessel under both normal and accident conditions.

\section{1-1 Effect of Window}

The $\mathrm{ZnSe}$ window has a transmittance of approximately $72 \sim 76 \%$ at wavelength range of $0.6 \sim 20$. That is, infrared light through the $\mathrm{ZnSe}$ window undergoes an attenuation of $24 \sim 28 \%$. Therefore the quantity of the radiation decreases after passing through the $\mathrm{ZnSe}$ window, which decreases the radiation reaching the infrared thermometer and causes a decrease in measured emissivity. Hence, this attenuation should be compensated for in order to obtain the accurate emissivity.

Figure 3-4 shows the effect of the $\mathrm{ZnSe}$ window, which was estimated by experiments performed with and without the window in air. The transmittance of the window was estimated to be 74.7 to $76.7 \%$ by dividing 
the experimental value with the window by that without the window. The results were within the maximum deviation range of $1.5 \%$ from the average value as shown in Figure 3-5. The result satisfies the range of the reference transmittance over the wavelength range of $8 \sim 14$, i.e. 72 to $76 \%$. The reference value was obtained from the specifications of the window. Although the measured transmittance of the $\mathrm{ZnSe}$ window was higher than the reference value, approximately $1.5 \%$ in this wavelength range, it agrees fairly well within an acceptable error. Thereafter, the final compensated emissivity could be acquired by dividing the measured emissivity by the transmittance.

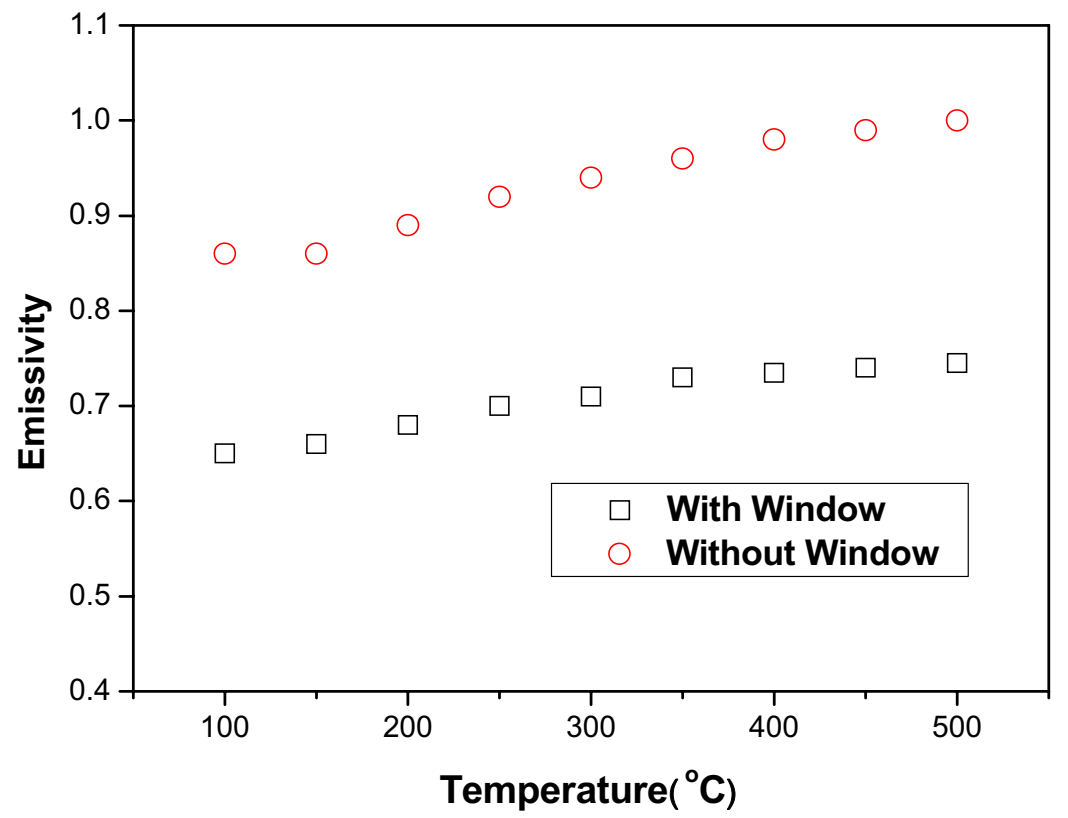

Figure 3-4 Temperature variation of the emissivity with or without the window

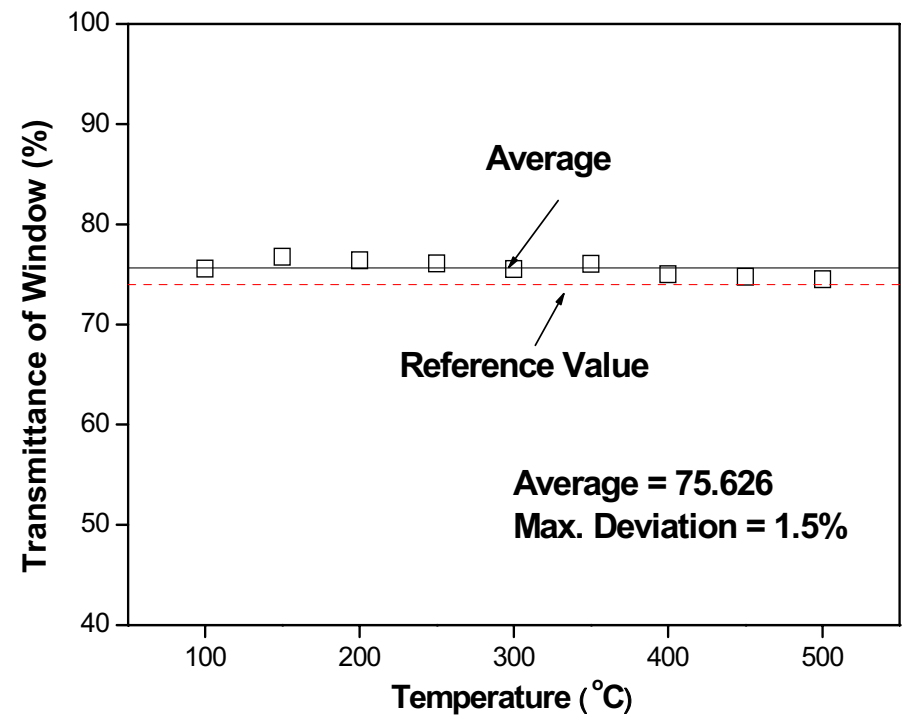

Figure 3-5 Transmittance of the ZnSe window 
Table 3-2 Test matrix of emissivity measurement

\begin{tabular}{|c|c|c|c|c|c|}
\hline & & $\operatorname{Air}(\%)$ & Helium (\%) & Steam (\%) & Pressure (atm) \\
\hline \multirow{4}{*}{ Case A } & 1 & 100 & 0 & 0 & 0.2 \\
\hline & 2 & 100 & 0 & 0 & 0.5 \\
\hline & 3 & 100 & 0 & 0 & 0.8 \\
\hline & 4 & 100 & 0 & 0 & 1 \\
\hline \multirow{3}{*}{ Case $\mathrm{H}$} & 1 & 20 & 80 & 0 & 1 \\
\hline & 2 & 50 & 50 & 0 & 1 \\
\hline & 3 & 80 & 20 & 0 & 1 \\
\hline \multirow{7}{*}{ Case $\mathrm{S}$} & 1 & 20 & 0 & 80 & 1 \\
\hline & 2 & 30 & 0 & 70 & 1 \\
\hline & 3 & 40 & 0 & 60 & 1 \\
\hline & 4 & 50 & 0 & 50 & 1 \\
\hline & 5 & 60 & 0 & 40 & 1 \\
\hline & 6 & 70 & 0 & 30 & 1 \\
\hline & 7 & 80 & 0 & 20 & 1 \\
\hline
\end{tabular}

\section{$\underline{\text { 3.1-2 Effect of Gases }}$}

The type of filling gas in the cavity as well as its concentration can affect the emissivity measurements. Under normal operation conditions of the VHTR, the cavity would be filled with air. However, if the vessel is broken, a coolant such as helium can be released into the cavity. In addition, it is possible that water spills into the cavity and evaporates when the inner surface of the water tank is broken. For these reasons, the experiments were carried out with the various gaseous concentrations in order to evaluate the effects of air and helium concentration on the measurement of emissivity. Table 3-2 shows a test matrix. 
First, experiments were performed at various air pressures, $0.2,0.5,0.8$, and $1.0 \mathrm{~atm}$ in order to evaluate the effects of the air concentration on the emissivity measurements. Figure 3-6 shows the measured emissivity as a function of pressure. In the figure, the compensated emissivity means that the attenuation effect of the window was eliminated by dividing the measured emissivity value by transmittance.

The compensated emissivity increased with temperature, varying from 0.85 to 1.0 . This value was slightly larger than the reference emissivity for oxidized carbon steel that ranges 0.80 0.95 (Aleksander Sala 1986), which was attributed to background radiation as discussed later.

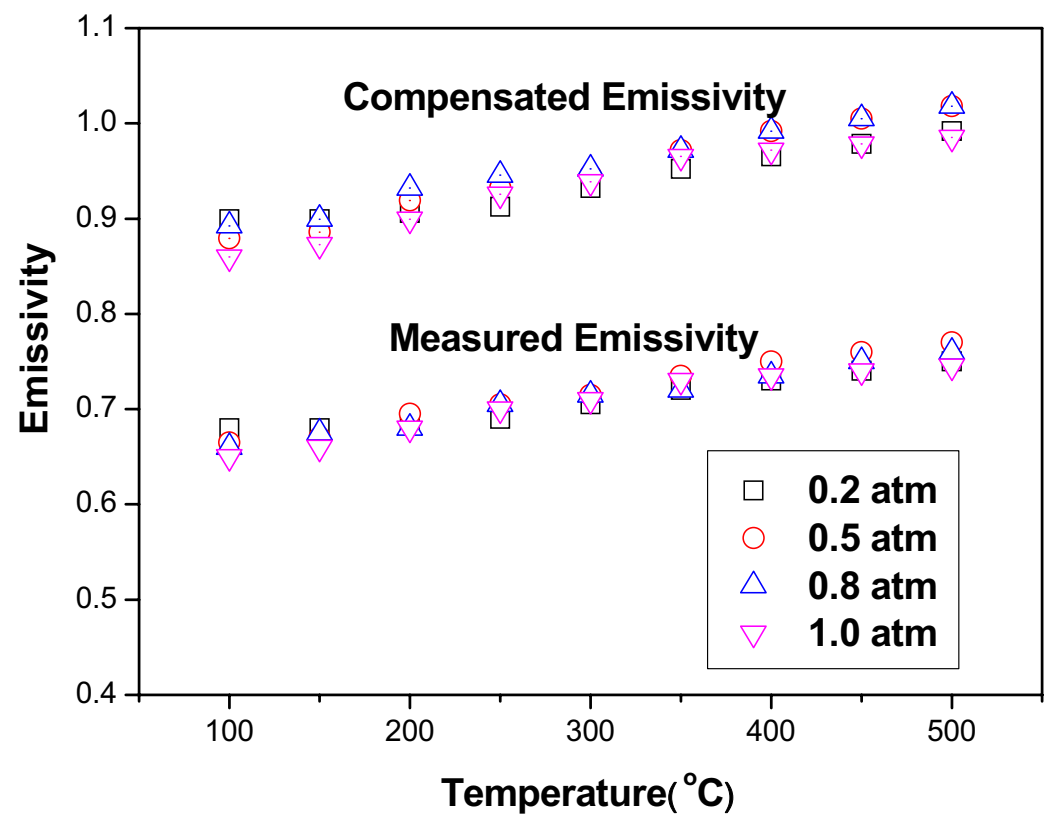

Figure 3-6 Temperature variation of the emissivity with different pressure 


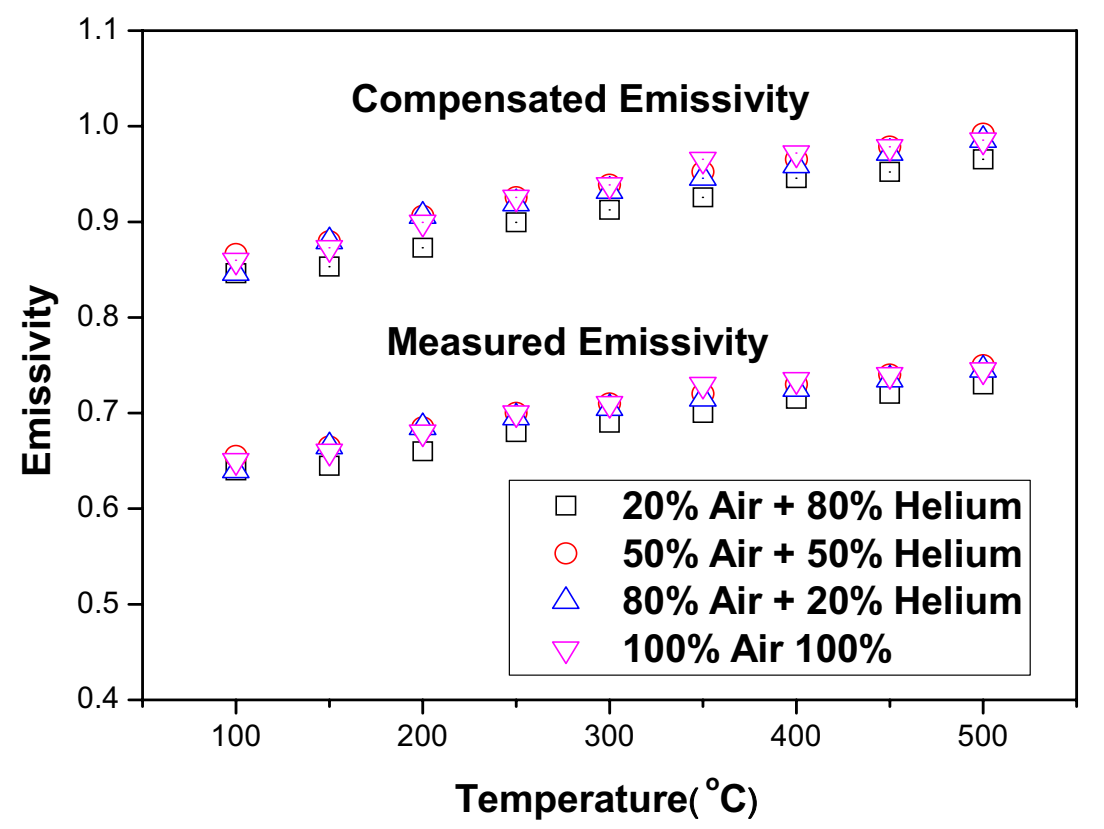

Figure 3-7 Temperature variation of emissivity at different helium concentrations

The variations in the measured emissivity with the air concentration were quite small, within $3 \%$ of the average value. The calculated transmittance of atmosphere by LOWTRAN7 code, which is commercial code to calculate the transmittance of atmosphere, was about $96 \%$ in our experimental condition. However this result was not clearly presented in the experiment because the absorption is not significant comparing with the uncertainty of the measurement. Therefore, in the main experiment, the effect of the atmosphere absorption was compensated using the calculated transmittance and then the deviation between calculated transmittance and experimental transmittance was considered as the uncertainty of infrared thermometer.

Using a similar method, the effect of the helium concentration on the emissivity measurements was examined. The total pressure in the chamber was maintained at $1 \mathrm{~atm}$. The air partial pressure was the same as the case of the air experiment, i.e., 0.2, 0.5, 0.8, and 1.0, and the remaining part was filled with helium instead of a vacuum. Similarly in the case of air, variations in the measured emissivity according to the helium concentration were within $3 \%$ of the average value, as shown in Figure 3-7. In addition, the compensated emissivity showed a similar range to that in the air experiment, $0.84 \sim 0.99$ with temperature. These results show that the air and helium concentration had a minor effect on the emissivity measurement. This means that, when the pass length is in order of meters, air and helium do not substantially absorb or reflect radiation at the wavelength range from 8 to 14 , and the pressure range of $0.2 \sim 1.0 \mathrm{~atm}$. 


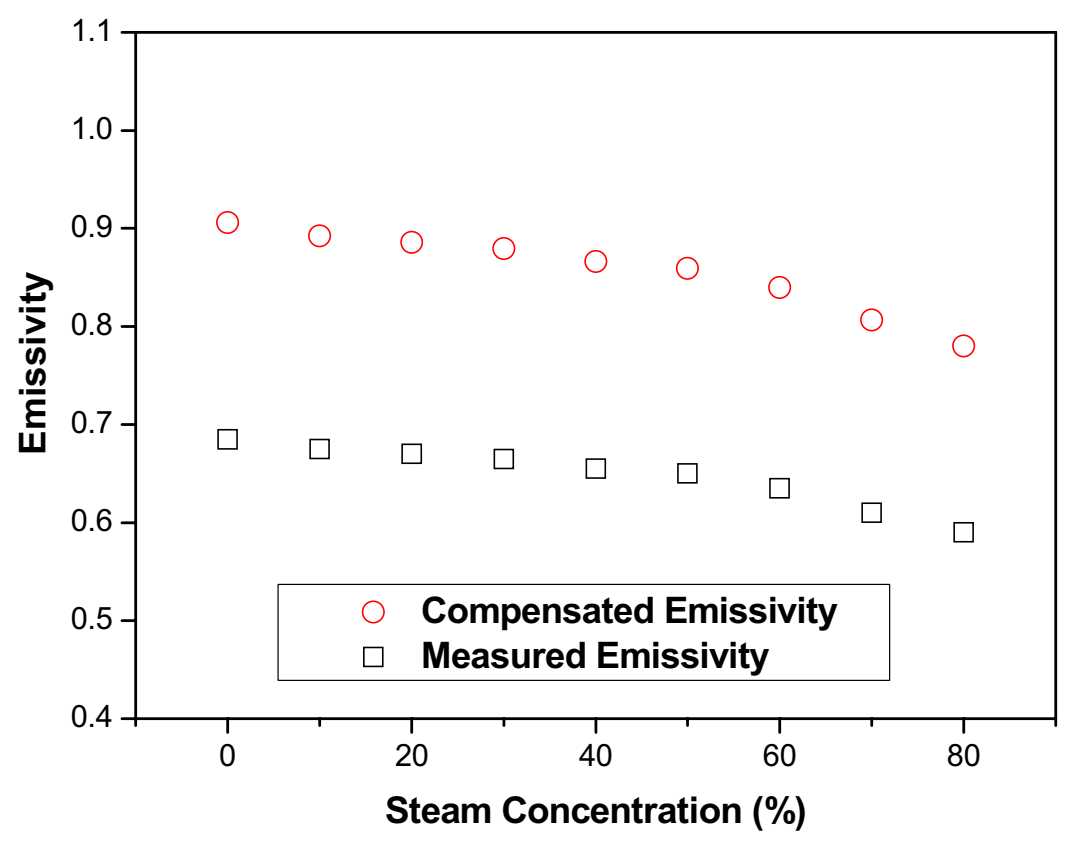

Figure 3-8 Effect of steam on the measured emissivity

The effect of steam on the emissivity measurements was estimated. At low temperatures the steam injected into the chamber was condensed at the inner surface of the chamber so that the proportion of steam could not be precisely controlled. Thus, the effect of the steam concentration was examined at $400{ }^{\circ} \mathrm{C}$ to prevent condensation. During the experiments, the total pressure was maintained as $1 \mathrm{~atm}$ and the steam concentration was varied from $0 \%$ to $80 \%$. The steam concentration was determined by the partial pressure. As the steam concentration was increased, the measured emissivity decreased gradually because of the absorption or reflection effect of steam, as shown in Figure 3-8.

\section{1-3 Effect of Background Radiation}

The most important factor affecting the emissivity measurement is the background radiation. The results of the two experiments for the air and the helium show that the measured emissivity increases with the temperature, which is believed to be caused by background radiation. In order to verify this phenomenon clearly the experiments to measure the emissivity were carried out inside and outside stainless steel chamber. The measured emissivity inside chamber was higher than that of outside chamber as shown in Figure 3-9. The additional radiation due to the chamber has the ratio about $4 \sim 18 \%$ with the temperature. In order to compensate the effect of background radiation the qualitative analysis of radiation is needed. It is expected that it could be solved by commercial code such as NEVADA (TAC Technologies 2000). The result from the code will be compared with the experimental results. With this methodology the actual emissivity will be measured in RCCS-SNU and the radiative heat transfer in the cavity will be analyzed. 


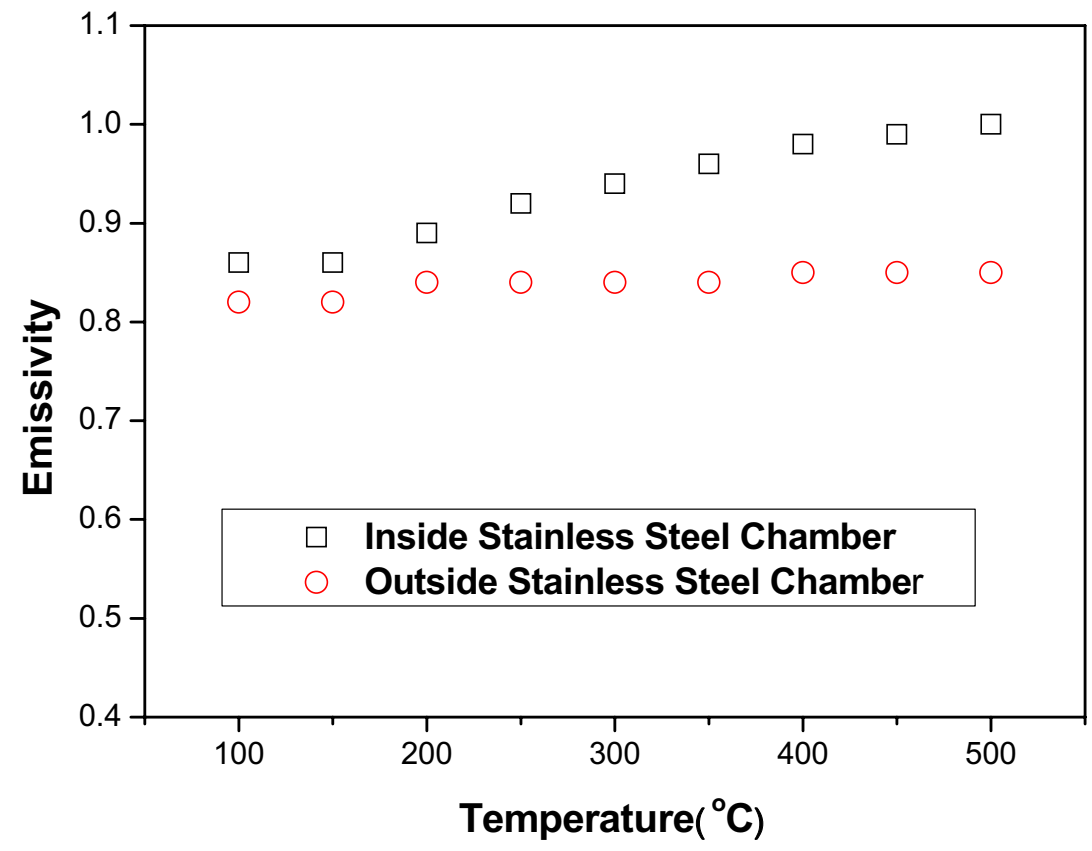

Figure 3-9 Effect of background radiation on the measured emissivity

Table 3-3 Measuring parameters and instrumentations

\begin{tabular}{||l||l||l||}
\hline Measuring Parameters & Measuring Location & Instrumentations \\
\hline \hline Flow Rate & Cooling Pipe Inlet & Bi-directional Flow Tube \\
\hline \hline Temperature & & \\
& $\begin{array}{l}\text { Cooling Pipe Axis: 9 Points } \\
\text { Cooling Pipe Surface: 7 Points } \\
\text { Liquid Temperature: 6 Points }\end{array}$ & Thermocouples \\
\hline \hline Differential Pressure & Between Inlet and Outlet & DP Transmitter \\
\hline
\end{tabular}


Table 3-4 experimental cases

\begin{tabular}{||l||l||}
\hline Gas Velocity & Total Heat Power (kW) \\
\hline \hline $32 \mathrm{~m} / \mathrm{s}$ & $2.7,4.0,5.0$ \\
\hline \hline $40 \mathrm{~m} / \mathrm{s}$ & $2.7,4.0,5.0,6.0$ \\
\hline \hline $48 \mathrm{~m} / \mathrm{s}$ & $2.7,4.0,5.0,6,0,7.0$ \\
\hline \hline $52 \mathrm{~m} / \mathrm{s}$ & $2.7,4.0,5.0,6,0,7.0$ \\
\hline \hline $57 \mathrm{~m} / \mathrm{s}$ & $2.7,4.0,5.0,6.0,7.0,8.0$ \\
\hline
\end{tabular}

\subsection{Separate effect test for the water pool of the RCCS-SNU - Part 1}

We have performed separate effect tests for the heat transfer phenomena in the water pool of the RCCS-SNU to investigate the natural convection of water in the water pool and the forced convection of air in the cooling pipe. From the measurement of local bulk temperature distributions in the tank water, cooling pipe surface and cooling pipe center, we would like to derive heat transfer coefficients of the cooling pipe and evaluate heat transfer coefficient correlations of previous studies. In addition we have estimated the correlations of the friction pressure drop and form loss friction drop as measuring the pressure drop between inlet and outlet of the cooling pipe.

Figure 3-10 shows the schematic diagram of the test facility and the measuring parameters. Instrumentations are summarized in Table 3-3. The major measuring parameters are the air flow rates, pressure drops along the cooling pipes, water level in the water pool and temperatures at the cooling pipe surface, cooling pipe center and the water pool. The test section simulates a quarter section of the water pool and it has six U-bend heaters in the vicinity of the inner wall to reproduce the heat coming from the cavity wall. The total power of the heaters was calculated considering the scaling ratio of the RCCS-SNU test facility (1/100 power ratio). To remove the heat, a cooling pipe is equipped in the test section which has fifteen U-bends as shown in Figure 3-10. The outlet of the cooling pipe is connected to the suction of a blower and ambient air flows through the cooling pipe. The experimental cases are tabulated in Table 3-4.

Figure 3-11 shows the experimental results of the pressure drop measurement between the inlet and outlet of the cooling pipe. The frictional pressure drop was computed from Equation (3-1) applying the friction factor correlation of Nikuradse (1966) for a smooth circular duct.

$\Delta P_{\text {fric }}=\frac{4 f L}{D_{h}} \frac{\rho_{g} v_{g}^{2}}{2}$

$f=0.0008+0.0553 \mathrm{Re}^{-0.237}$

The form loss pressure drop was calculated subtracting the friction loss from the total pressure drop as shown in Figure 3-11 and a comparison among the calculated values and predicted values using the form loss 

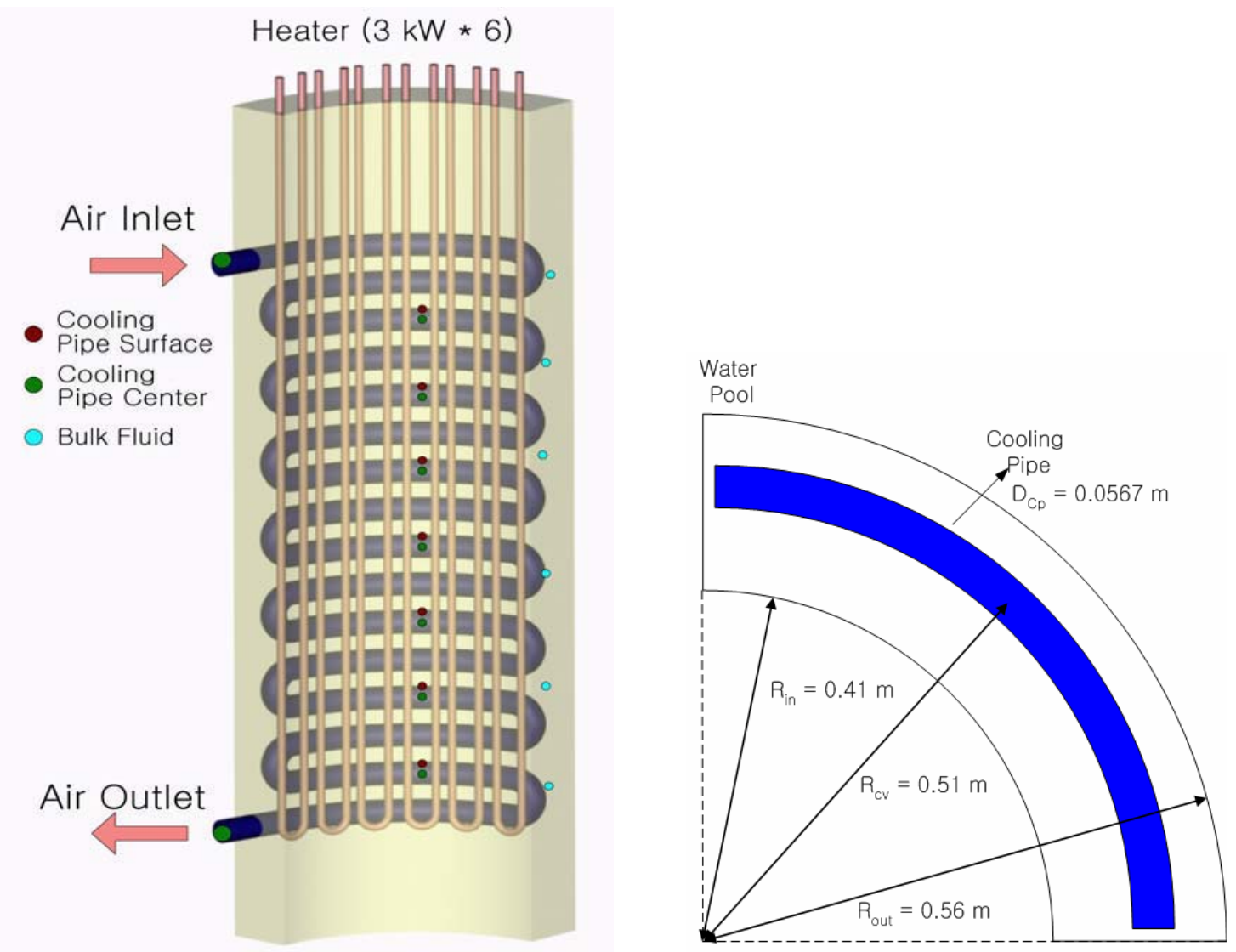

Figure 3-10 Schematic diagram of the separate effect test facility for the RCCS-SNU water tank 


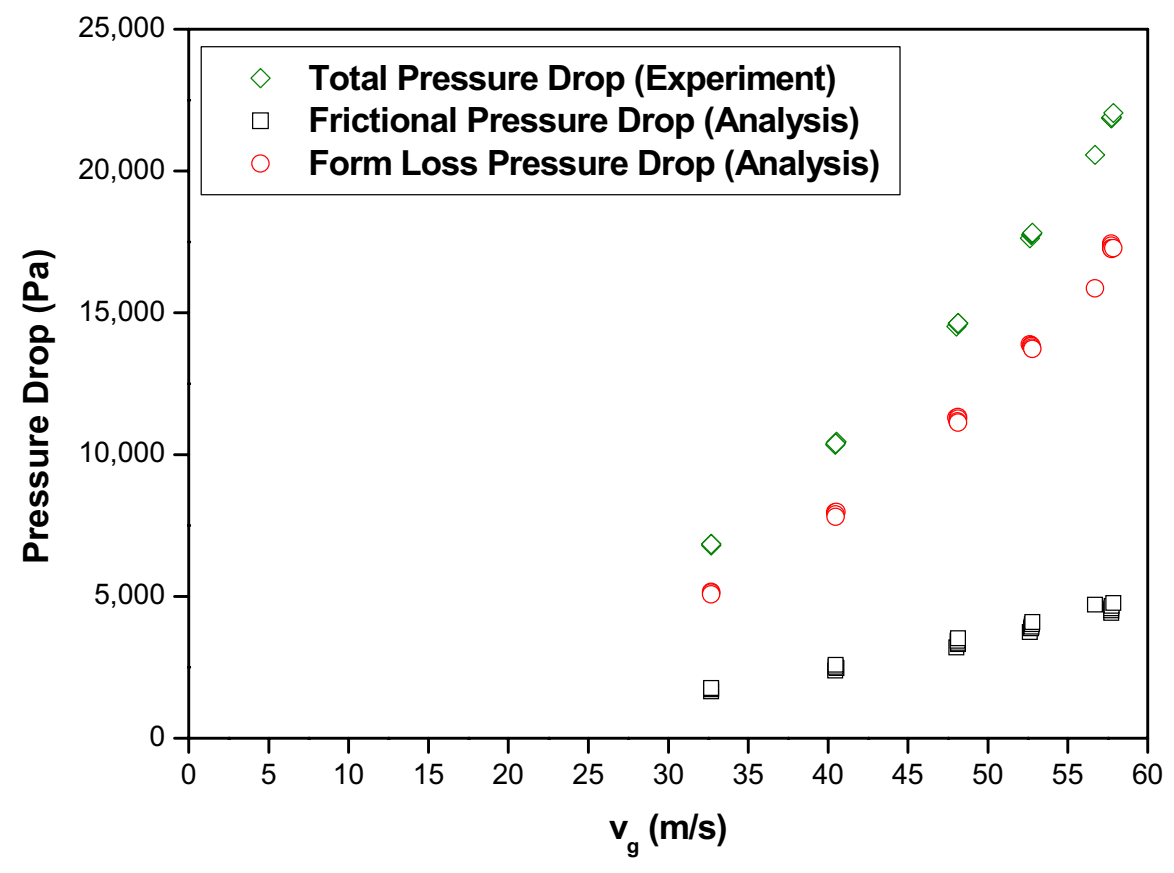

Figure 3-11 Pressure drop vs velocity

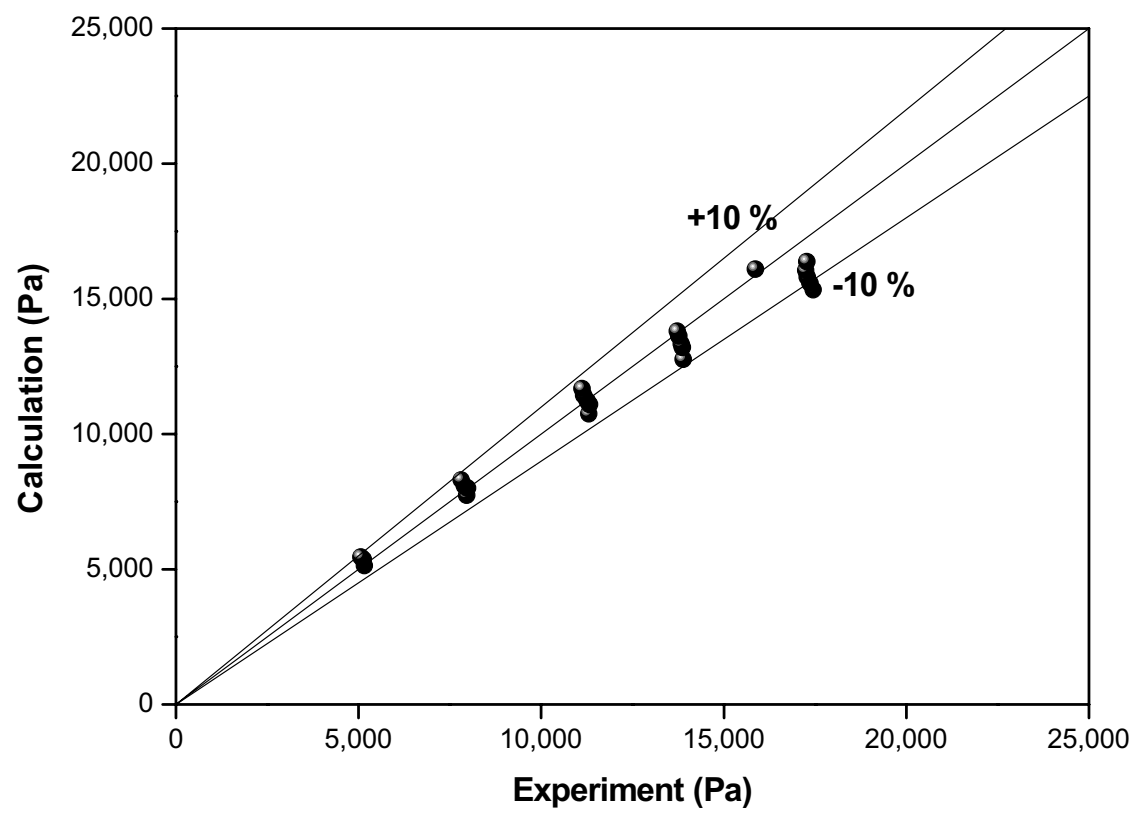

Figure 3-12 Comparison of measured pressure drop with prediction 
coefficient of a standard U-bend, Equation (3-3), is provided in Figure 3-12.

$K=\frac{K_{1}}{\operatorname{Re}}+K_{\infty}\left(1+0.5 D^{*}\right)$

(Hooper, 1981)

where, $K_{1}=1000, K_{\infty}=0.35$ in U-bend,

The figure shows reasonable agreement among the experimental data and the predicted form loss pressure drops using the above correlation and it was found that the loss coefficient of a standard U-bend is available for the cooling pipe of the RCCS-SNU. These experimental results of the pressure drop and loss coefficient will be used for the validation of the CFD code and the preparation of the input deck for system analysis codes, MARS-GCR (KAERI 2004, W.J. Lee et al. 2003).

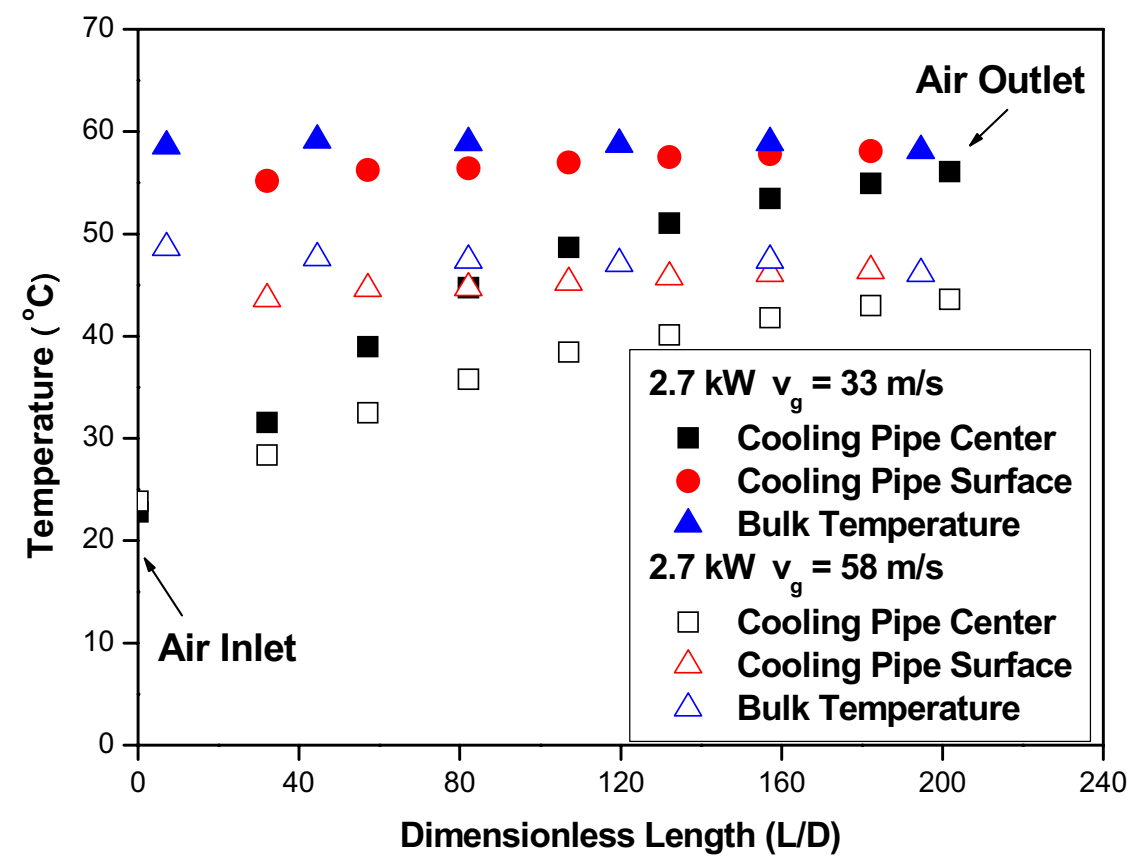

Figure 3-13 Axial distribution of bulk and air temperature

Figure 3-13 indicates the experimental results of the temperature distributions at the water pool, cooling pipe surface and axis of the cooling pipe. As air flows through the cooling pipe, its temperature increases markedly. However the temperature of the cooling pipe surface shows little variation along the pipe axis. The axial temperature distributions are therefore similar to those for heat transfer in a tube with constant surface temperature condition. The bulk liquid temperature is higher at the top part of the tank than at the bottom part by $1 \sim 2{ }^{\circ} \mathrm{C}$ only. This means that a thermal stratification does not occur in the water pool since large portion of the heat is removed at the top part wherein the water temperature is largely different from the inhaled air temperature.

\section{2-1 Analysis for Heat Transfer Coefficient}

From the experimental results the heat transfer coefficient at inner surface of cooling pipe for the forced convection was calculated. 


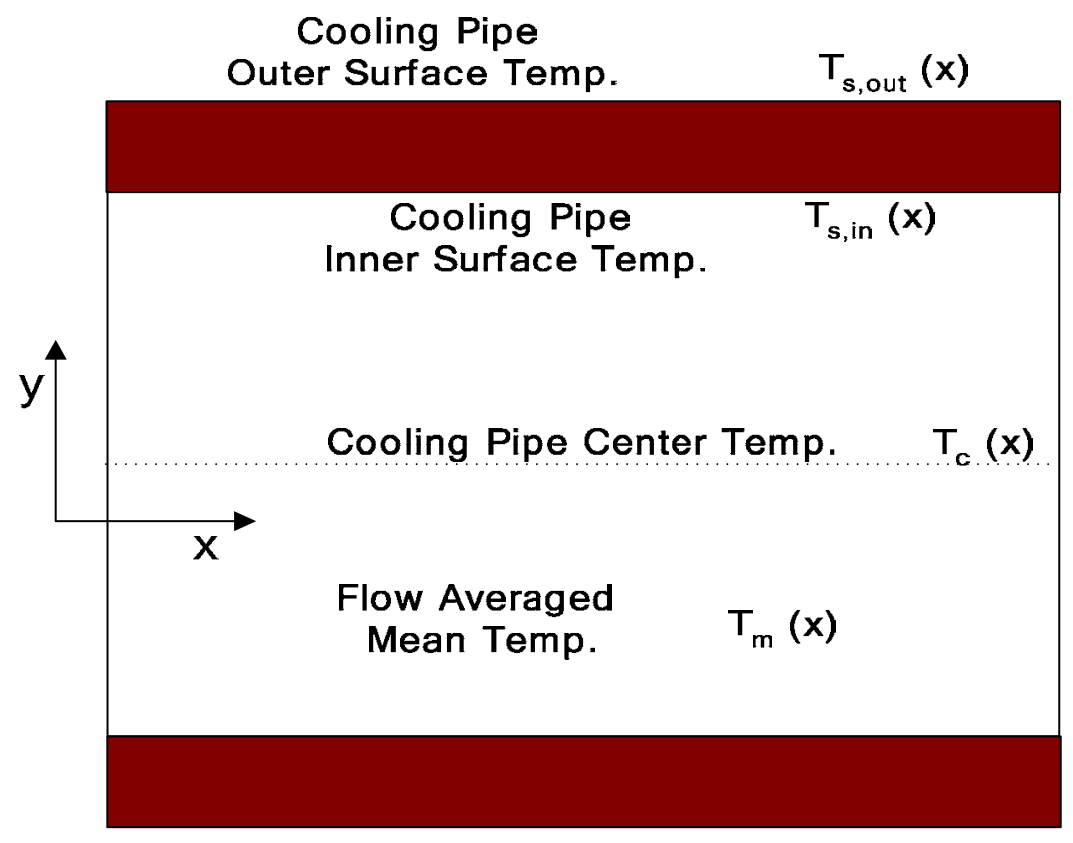

Figure 3-14 Control volume of the cooling pipe

Applying conservation of energy, Equation (3-4), to the differential control volume of Figure 3-14, we obtain

$$
d \dot{q}_{c o n v}=\dot{m}_{g} c_{p} d T_{m}=2 \pi h r_{i n} d x\left(T_{s, i n}-T_{m}\right) \quad \text { or } \quad h=\frac{\dot{m}_{g} c_{p}}{2 \pi r_{i n}} \frac{d T_{m}}{d x} \frac{1}{T_{s, i n}-T_{m}}
$$

The flow averaged mean temperature and pipe inner surface temperature in above equation should be substituted by the temperature of the cooling pipe center and outer surface that were measured in the experiments to calculate the heat transfer coefficient. Since information for the velocity and temperature fields of the cooling pipe is necessary to obtain the mean temperature, we assumed that those are the same with the velocity and temperature profiles of the fully developed turbulent flow under the constant temperature condition. The appropriateness of this assumption will be discussed with a calculation result using the CFX5.7 code later. Applying the assumption, the flow averaged mean temperature can be expressed as follows.

$T_{m}=\frac{3}{2(n+2)} T_{s, i n}+\frac{2 n+1}{2(n+2)} T_{c}$

(where, $n=7$ ) (Kakac et al., 1987)

The heat transfer in the cooling pipe by conduction can be expressed as,

$d \dot{q}_{\text {cond }}=\frac{2 \pi d x k\left[T_{s, \text { out }}-T_{s, \text { in }}\right]}{\ln \left(r_{\text {out }} / r_{\text {in }}\right)}$

Equating Equations (3-4) and (3-6) it follows that the derivative of the mean temperature is related to the temperatures at the cooling pipe inner and outer surface.

$\frac{d T_{m}}{d x}=\frac{2 \pi k}{\dot{m}_{g} c_{p}} \frac{\left[T_{s, \text { out }}-T_{s, \text { in }}\right]}{\ln \left(r_{\text {out }} / r_{\text {in }}\right)}=\frac{2 \pi h r_{\text {in }}}{\dot{m}_{g} c_{p}}\left[T_{s, \text { in }}-T_{m}\right]=\frac{2 \pi h r_{\text {in }}}{\dot{m}_{g} c_{p}}\left[T_{s, \text { in }}-T_{c}\right] \frac{2 n+1}{2(n+2)}$

Rearranging Equation (3-7), 


$$
\frac{k\left(T_{s, \text { out }}-T_{s, \text { in }}\right)}{\ln \left(r_{\text {out }} / r_{\text {in }}\right)}=h r_{\text {in }}\left(T_{s, \text { in }}-T_{c}\right) \frac{2 n+1}{2(n+2)} \text { or } \quad T_{s, \text { in }}=\frac{\frac{k T_{s, \text { out }}}{\ln \left(r_{\text {out }} / r_{\text {in }}\right)}+h r_{\text {in }} T_{c} \frac{2 n+1}{2(n+2)}}{h r_{\text {in }} \frac{2 n+1}{2(n+2)}+\frac{k}{\ln \left(r_{\text {out }} / r_{\text {in }}\right)}}
$$

Substituting from Equation (3-8), Equations (3-4) and (3-7) can be expressed as follows,

$$
\begin{aligned}
& h=\frac{\dot{m}_{g} c_{p}}{2 \pi r_{\text {in }}} \frac{d T_{m}}{d x} \frac{2(n+2)}{2 n+1} \frac{1}{\frac{k T_{s, \text { out }}}{\ln \left(r_{\text {out }} / r_{\text {in }}\right)}+h r_{\text {in }} T_{c} \frac{2 n+1}{2(n+2)}}-T_{c} \\
& \frac{h r_{\text {in }} \frac{2 n+1}{2(n+2)}+\frac{k}{\ln \left(r_{\text {out }} / r_{\text {in }}\right)}}{d x}=\frac{3}{2(n+2)} \frac{d T_{s, \text { in }}}{d x}+\frac{2 n+1}{2(n+2)} \frac{d T_{c}}{d x}
\end{aligned}
$$

$=\frac{2 n+1}{2(n+2)} \frac{d T_{c}}{d x}+\frac{3}{2(n+2)}\left[\frac{\frac{k}{\ln \left(r_{\text {out }} / r_{\text {in }}\right)} \frac{d T_{s, o}}{d x}+r_{\text {in }} \frac{2 n+1}{2(n+2)}\left[\frac{T_{c} d h}{d x}+\frac{h d T_{c}}{d x}\right]}{\frac{k}{\ln \left(r_{\text {out }} / r_{\text {in }}\right)}+h r_{\text {in }} \frac{2 n+1}{2(n+2)}}-\frac{\left(\frac{k T_{s, o}}{\ln \left(r_{\text {out }} / r_{\text {in }}\right)}+h r_{\text {in }} T_{c} \frac{2 n+1}{2(n+2)}\right) \cdot \frac{2 n+1}{2(n+2)} r_{\text {in }} \frac{d h}{d x}}{\left(\frac{k}{\ln \left(r_{\text {out }} / r_{\text {in }}\right)}+h r_{\text {in }} \frac{2 n+1}{2(n+2)}\right)^{2}}\right]$

spatial derivatives of the temperature at the outer surface and axis of the pipe in Equation (3-10) can be obtained from the experimental data with the multiple regression method. Assuming the spatial distribution of the heat transfer coefficient to a third order polynomial, we can solve Equations (3-9) and (3-10) at each measuring location. The calculated local heat transfer coefficients are presented in Figure 3-15. To consider the effect of the conduction through the cooling pipe, the heat transfer coefficients were recalculated with an assumption, $T_{s, \text { in }}=T_{s, o u t}$ and compared with the previously calculated values as shown in Figure 3-16. As the result it was found that the effect of the conduction is negligible for the calculation of the heat transfer coefficient. Excluding the effect of conduction, Equation (3-9) can be reduced as follows,

$$
h=\frac{\dot{m} c_{p}}{2 \pi r_{i n}} \frac{1}{T_{s}-T_{c}} \frac{2(n+2)}{2 n+1} \frac{d T_{m}}{d x}=\frac{\dot{m} c_{p}}{2 \pi r_{i n}} \frac{1}{T_{s}-T_{c}} \frac{2(n+2)}{2 n+1}\left[\frac{3}{2(n+2)} \frac{d T_{s}}{d x}+\frac{2 n+1}{2(n+2)} \frac{d T_{c}}{d x}\right]
$$

Integrating above equation along the axis, we can obtain averaged heat transfer coefficients.

$$
\bar{h}=\frac{1}{L} \int_{0}^{L} h d x=\frac{\dot{m} c_{p}}{2 \pi r L} \frac{2(n+2)}{2 n+1} \int_{0}^{L} \frac{1}{T_{s}-T_{c}}\left[\frac{3}{2(n+2)} \frac{d T_{s}}{d x}+\frac{2 n+1}{2(n+2)} \frac{d T_{c}}{d x}\right] d x
$$




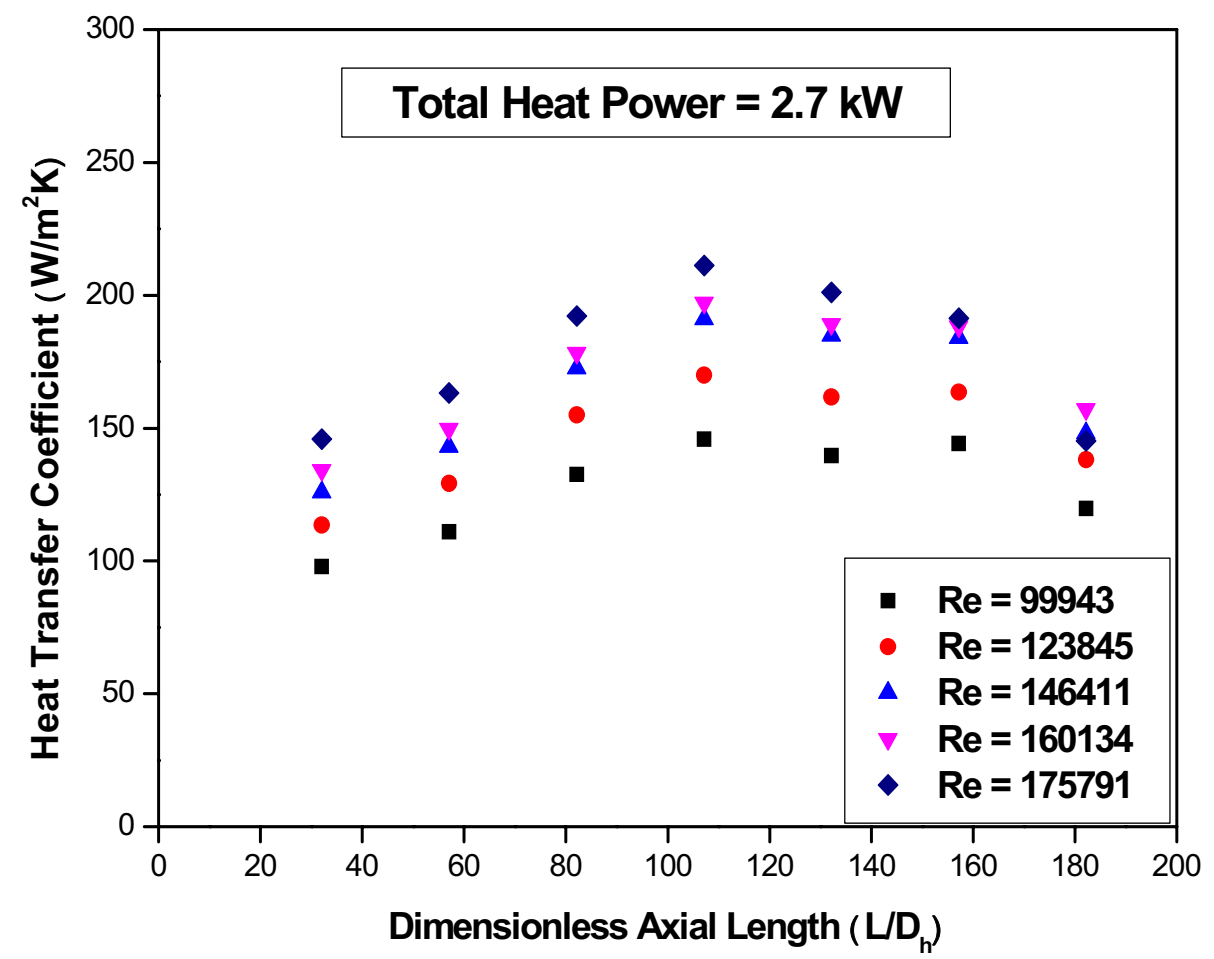

Figure 3-15 Local heat transfer coefficients

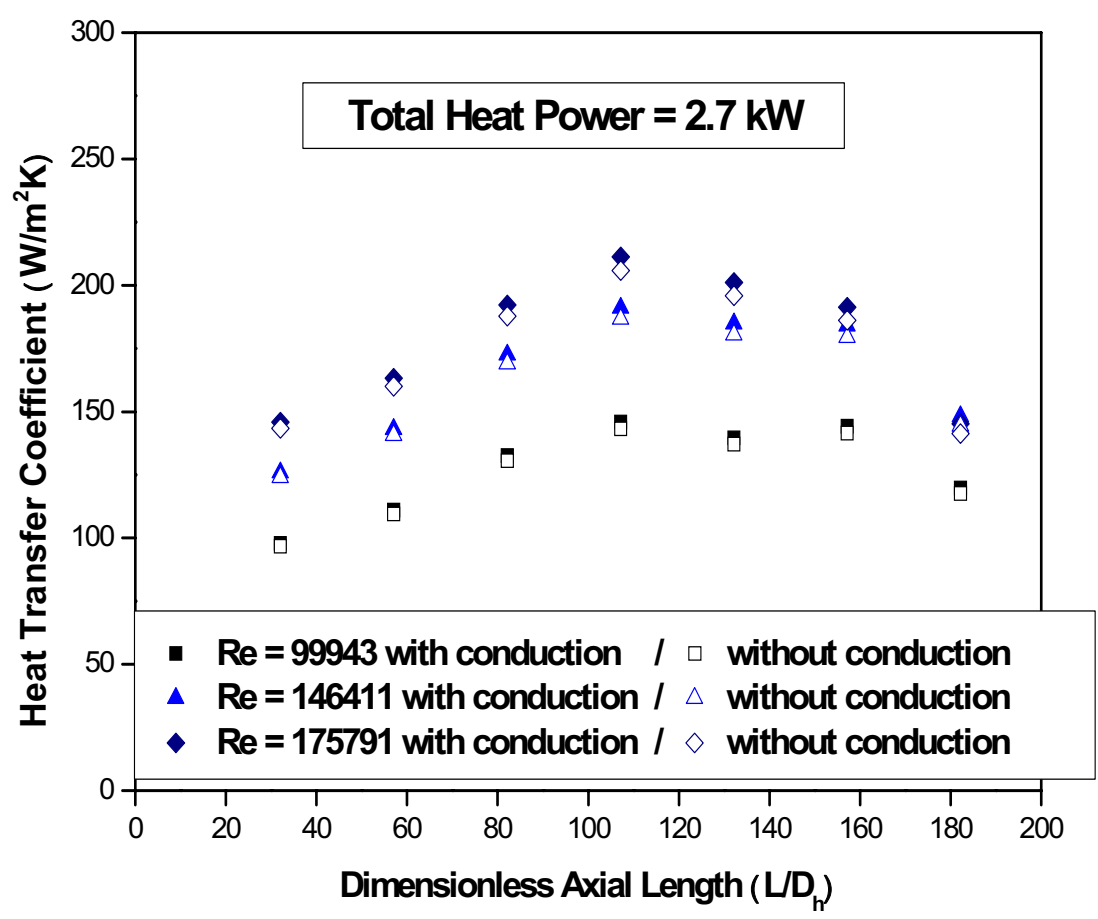

Figure 3-16 Effect of conduction on heat transfer coefficients 


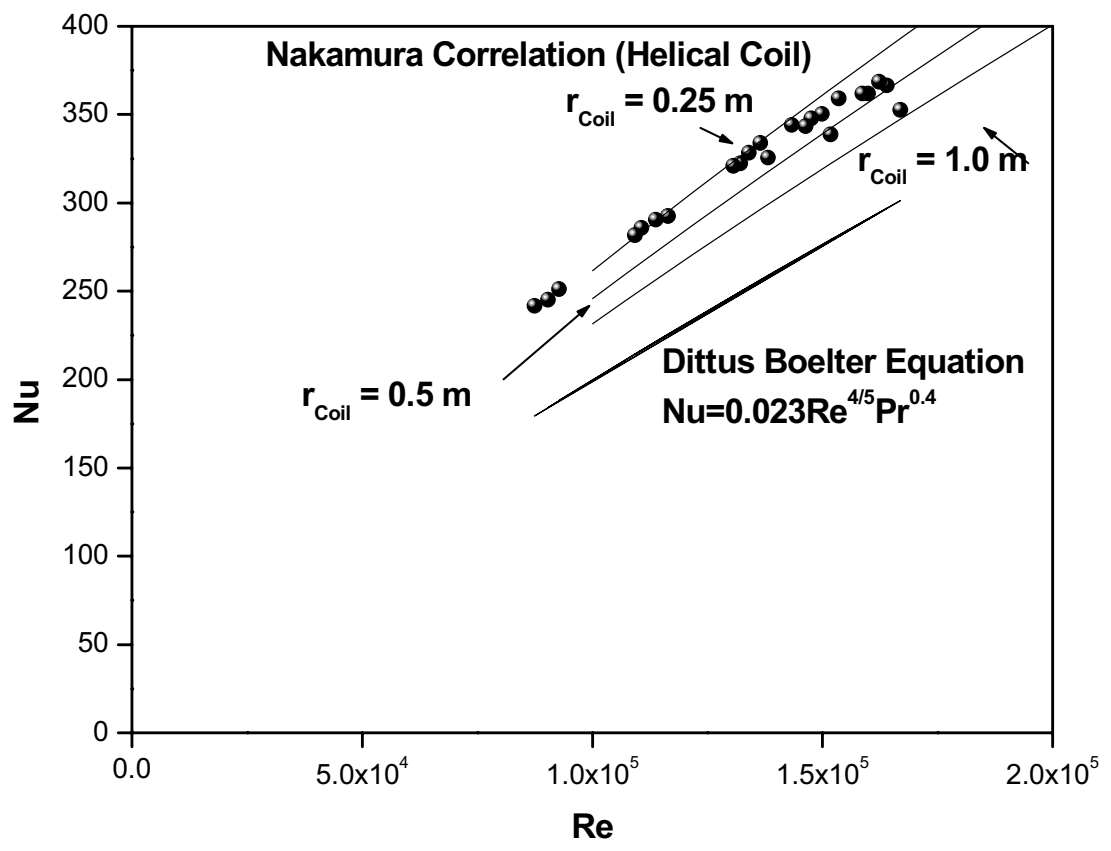

Figure 3-17 Averaged heat transfer coefficient

In Figure 3-17, the calculation results of the Nusselt number in the cooling pipe were indicated and compared with correlations of previous studies to evaluate the correlations. The Dittus-Boelter correlation (Dittus and Boelter 1930) for fully developed turbulent flow and the Mori-Nakayama (Mori and Nakayama 1967) correlation for a helical coil were used for the evaluation. As a comparison result, our experimental data found to be $30 \%$ higher than the predicted curve by Dittus-Boelter for a straight pipe because of the effect of secondary flow in curved pipes. Also the Nusselt numbers are about $10 \%$ larger for the cooling pipe than for a helical coil tube which has the same radius $\left(\mathrm{r}_{\text {coil }}=0.5 \mathrm{~m}\right)$ with our test facility. This underestimation is seemed to be caused by the presence of the U-bend. Tailby and Staddon (S. R. Tailby and P.W. Staddon 1970) reported Nusselt number increments for air cooling in $180^{\circ}$ bend. They explained that in a bend secondary flow pushes heavier fluid particles toward the outer wall and lighter ones toward the inner wall and thus the bend augments the secondary flow resulting in significantly higher heat transfer coefficients at the outer wall. Also Moshfeghhian and Bell (M. Moshfeghian and K.J. Bell 1979) observed higher heat transfer coefficient in the downstream of the bend as well as in the bend. Therefore it is needed to develop a new correlation or modify the correlation of helical coil in order to simulate multiple U-band shape pipe installed in our facility.

\section{2-2 CFX 5.7 Calculations}

From this characteristic of the heat transfer phenomena in the cooling pipe, a CFX 5.7 calculation was carried out to obtain detailed information of the fluid velocity and temperature. The calculation simulates the cooling pipe and the water pool respectively as the experimental results of the cooling pipe surface temperature were applied as a boundary condition. The k- $\varepsilon$ model was used for turbulent modeling. 


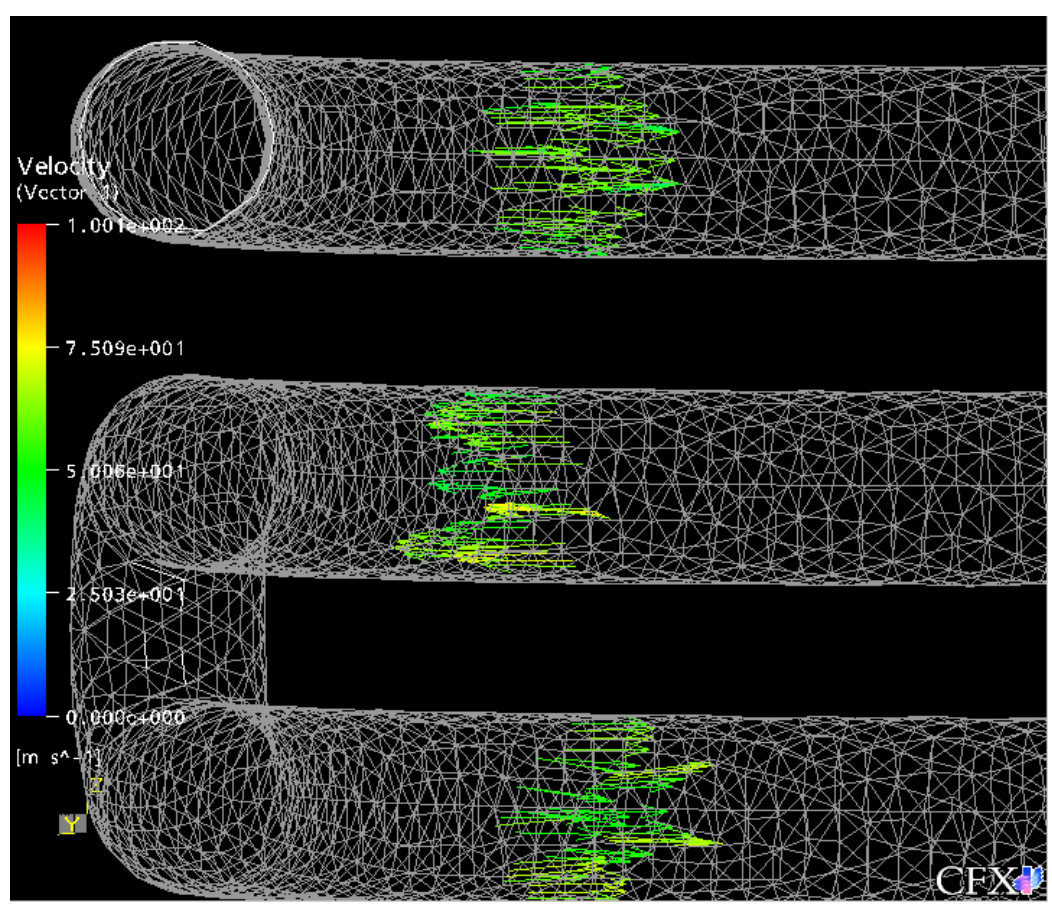

Figure 3-18 Grid and velocity vectors in the cooling pipe

Figure 3-18 shows the grid and calculated velocity vectors of the cooling pipe. Figures 3-19 and 3-20 show the calculation results of the velocity and temperature profiles at the temperature measuring locations of the cooling pipe.

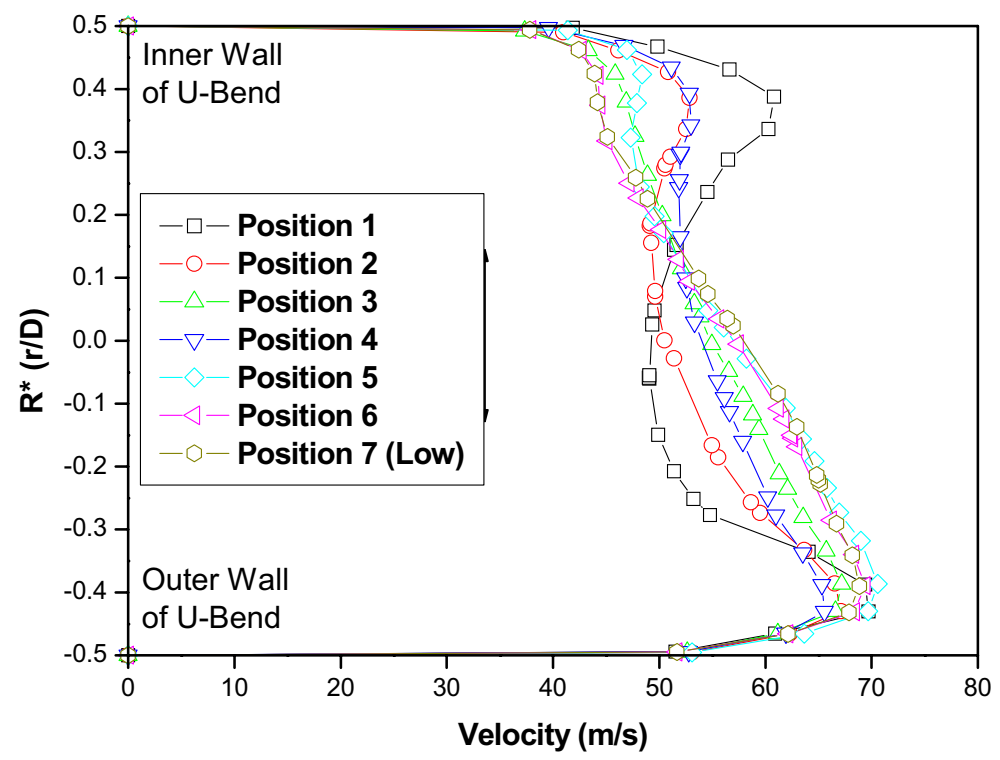

Figure 3-19 Velocity profiles in the cooling pipe 


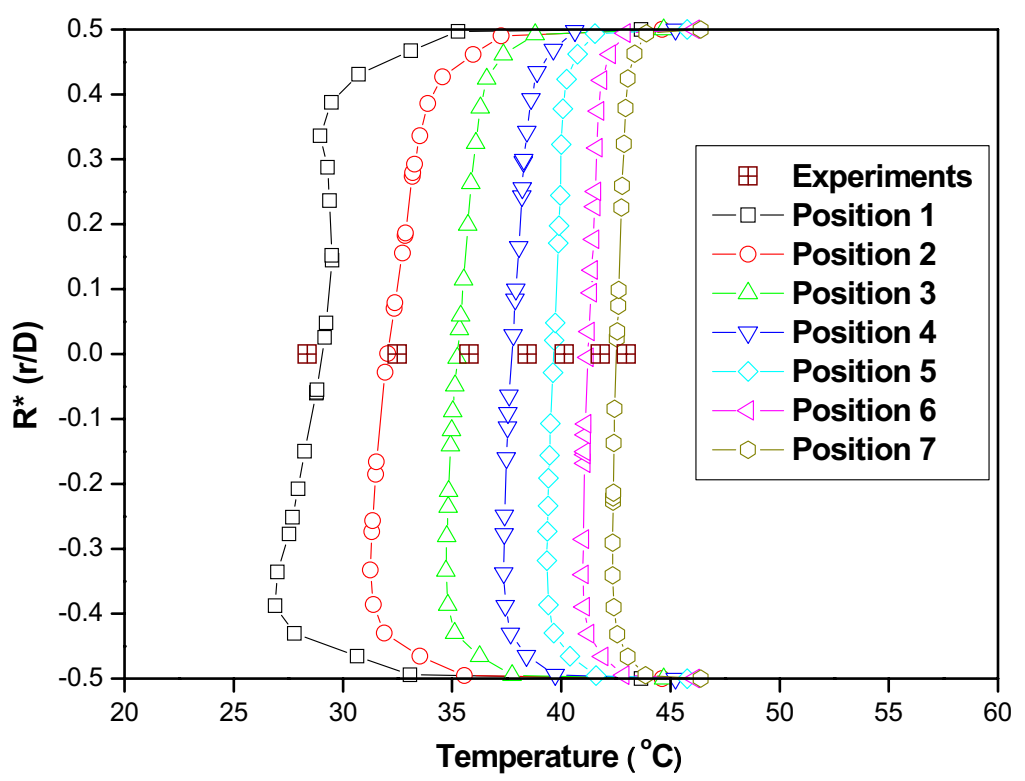

Figure 3-20 Temperature profiles in the cooling pipe

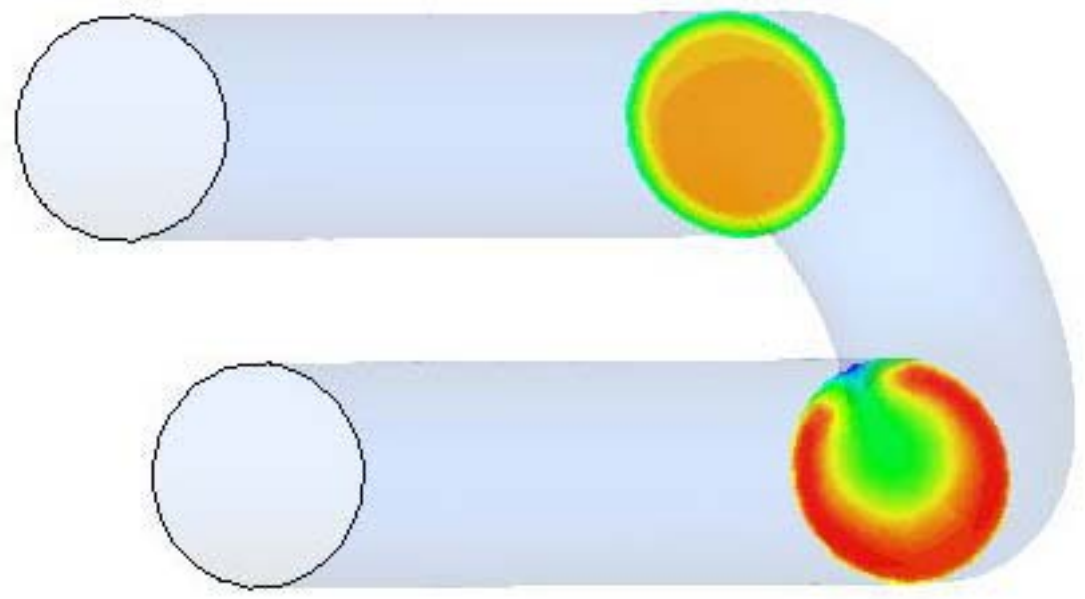

Figure 3-21 Velocity at the inlet and outlet in the U-band 


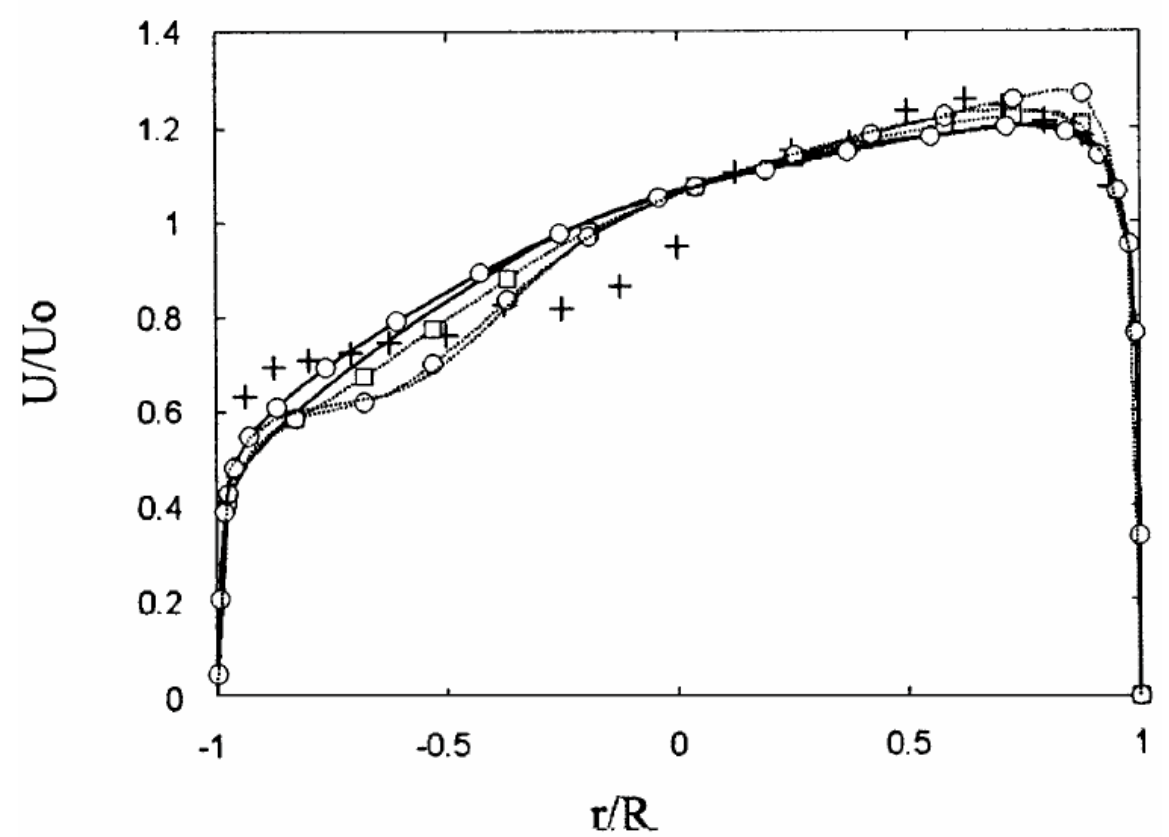

Figure 3-22 Velocity profiles in the U-band

The centrifugal effect of the bend, which shifts the maximum of the axial velocity toward the outer wall, is well represented in our calculation as shown in Figure 3-21. This is the same trend with the results of Pruvost et. al. (2004) who investigated the flow structure in U-bend using the FLUENT code as shown in Figure 3-22. Also our calculation result for the air temperature at the axis showed a reasonably good agreement with the experimental data. From this calculation results, we concluded that the CFX 5.7 code can simulate the heat transfer phenomena in the cooling pipe of RCCS-SNU.

As stated above, the assumption for the temperature profile of fully developed turbulent flow was applied for the calculation of the flow averaged mean temperature. To evaluate the assumption, we computed the flow averaged temperatures from the CFX 5.7 results and compared it with the temperatures calculated by Equation (3-5) in Figure 3-23. 


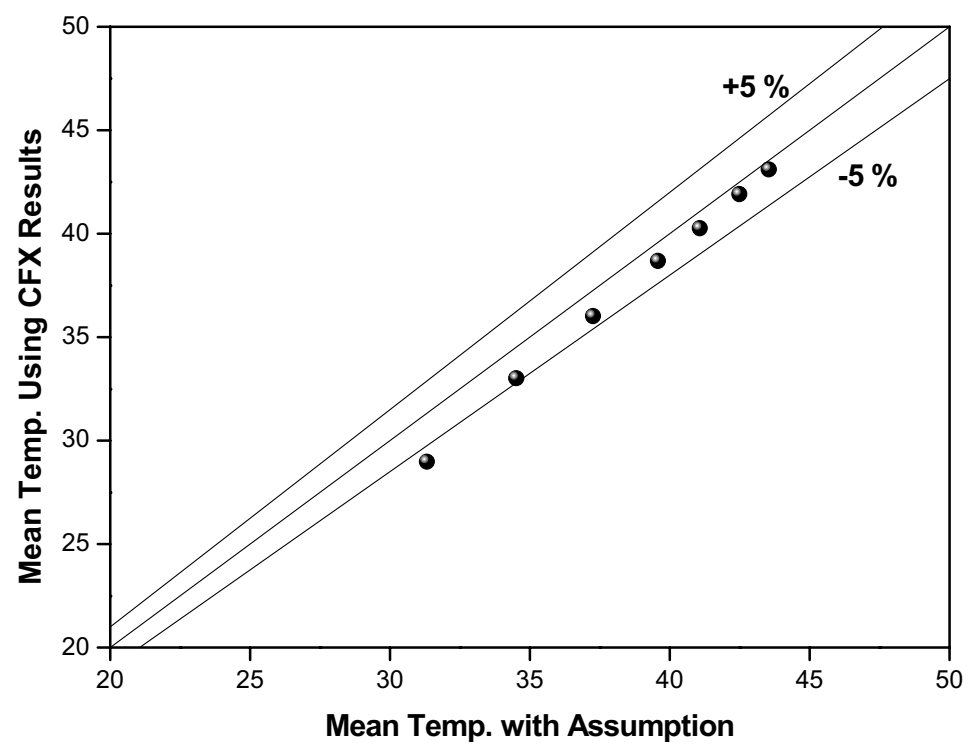

Figure 3-23 Comparison of mean temperature

The mean temperature shows about 5\% larger values with the assumption than with the CFX 5.7 predicted results. Even though the assumption does not cause significant distortion of the mean temperature, we will recalculate the heat transfer coefficient of the experimental data applying the velocity and temperature profile of the calculation result. Also more evaluation for other correlations will be carried out and an appropriate correlation will be implemented in the system analysis code of MARS-GCR to simulate the thermal hydraulic behaviors of the RCCS-SNU.
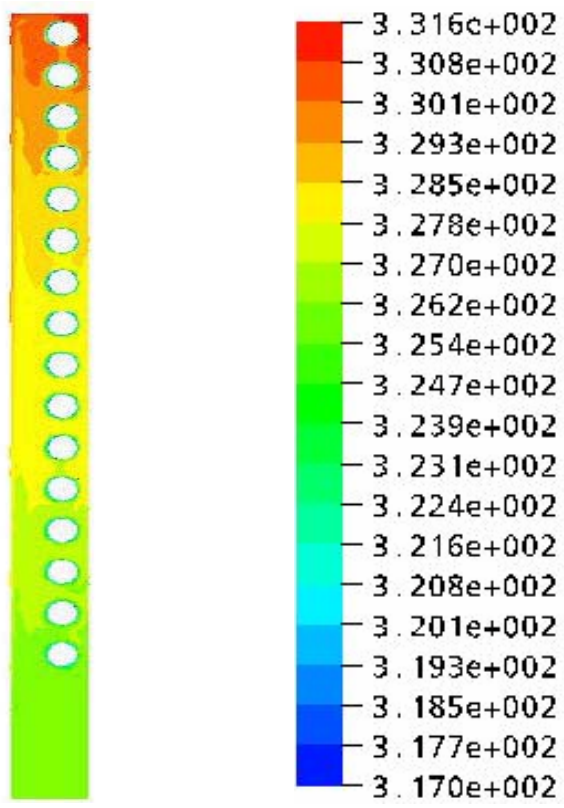

Figure 3-24 CFX 5.7 calculation result: temperature distribution 
In addition, the calculation was performed to simulate the heat transfer phenomena from the water pool to the cooler and the liquid behaviors in the water tank. Figure 3-24 shows the water temperature distribution calculated by the CFX 5.7 code for the separate effect test. The temperature field and velocity vector near the cooling pipe are indicated in the Figure 3-25. The natural circulation of the water, the upward flow near the hot wall and downward flow near the cooling pipe, was observed in the calculation. The liquid temperature difference between the top and bottom of the test section is about $6^{\circ} \mathrm{C}$ which is $4.5^{\circ} \mathrm{C}$ larger than the experimental data. Also the CFX 5.7 code over-predicts the liquid bulk temperature by $7 \sim 10^{\circ} \mathrm{C}$ as shown in Figure 3-26. It is likely that this difference is affected by the mesh size at the boundary layer near the cooling surface that has the sharp gradient of the liquid temperature as shown in Figure 3-25. The present nodes near the cooling surface will be divided to make the node size smaller than the boundary layer of the natural convection.
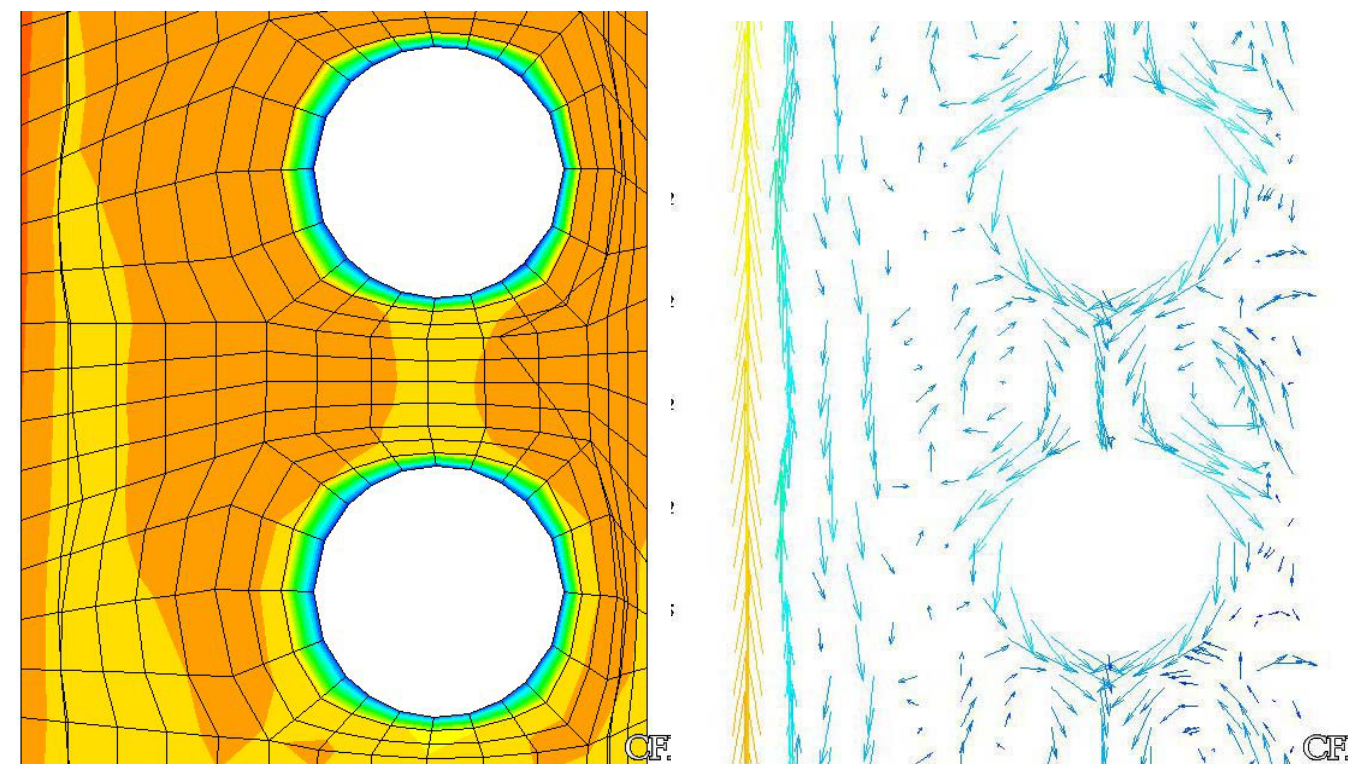

Figure 3-25 CFX 5.7 calculation result: temperature and velocity vector distribution near the cooling surface 


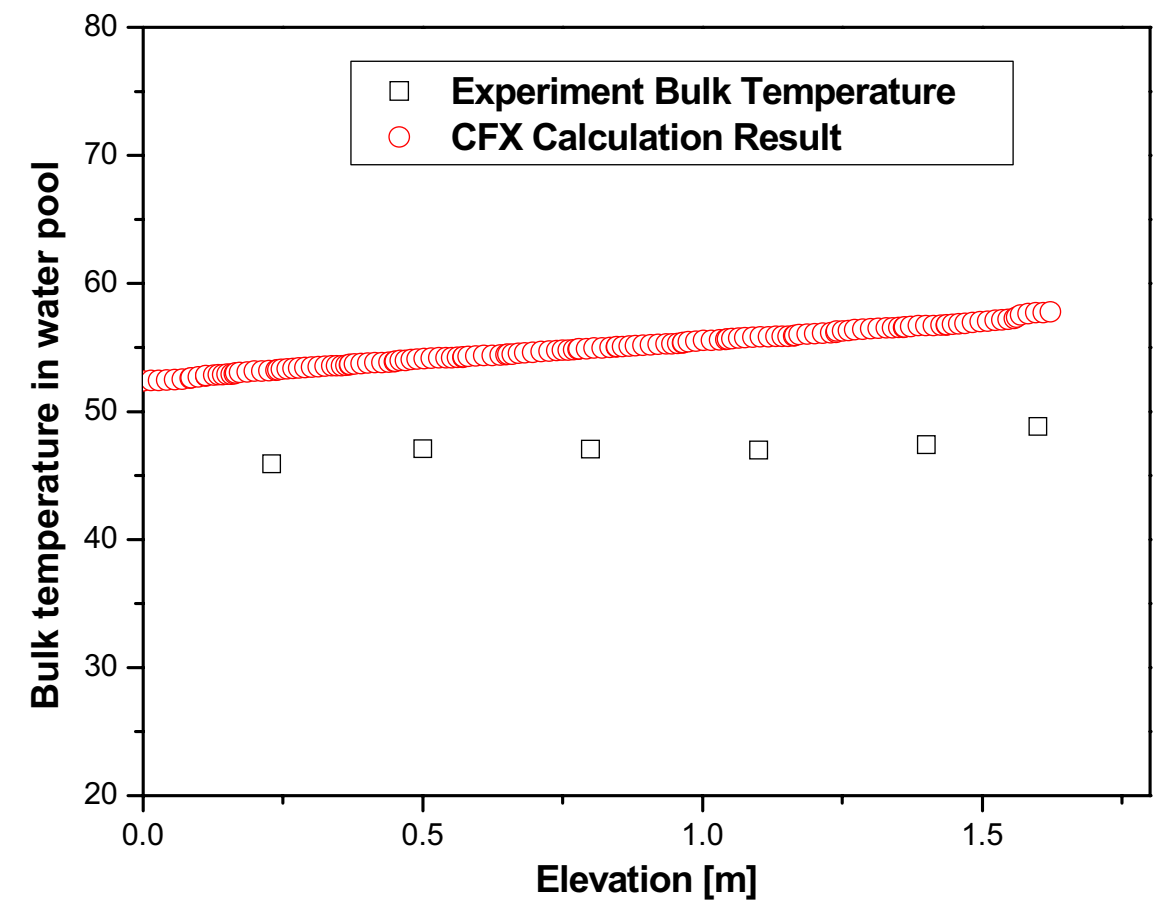

Figure 3-26 Comparison of bulk temperature between CFX 5.7 calculation and experiment

\subsection{Separate effect test for the water pool of the RCCS-SNU -Part 2}

We have constructed new separate effect test device and performed additional separate effect tests to investigate the geometric effect of the cooling pipe such as a number of U-bends and diameter. Moreover, a detailed temperature distribution fields on the pipe surface as well as inside pipe were obtained by additional measurement. New separate effect test device simulated a quarter of the water pool of RCCS-SNU in azimuthal direction and one third in radius direction as shown in Figure 3-27. 


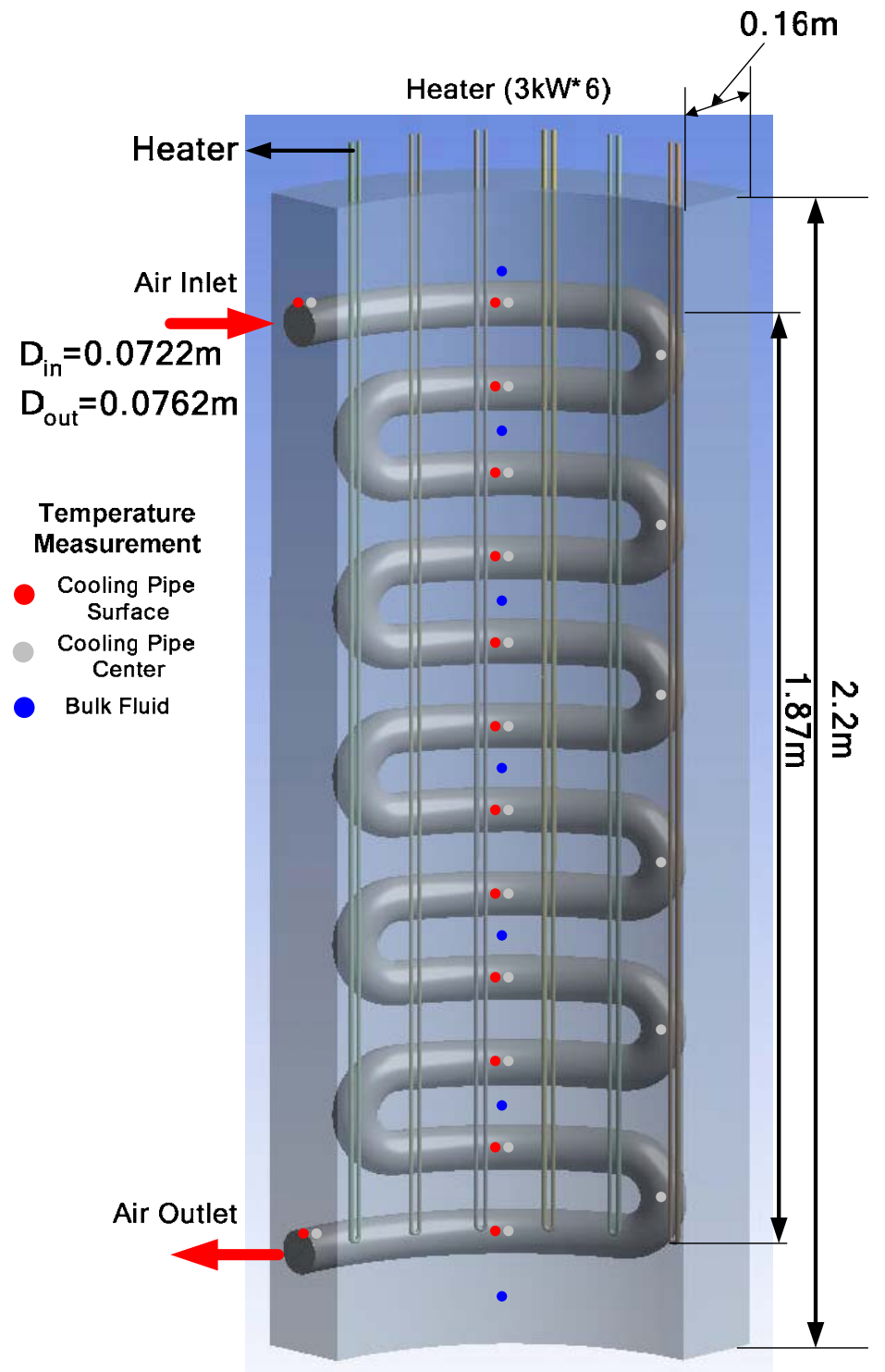

Figure 3-27 Schematic diagram of the new separate effect test device 
Table 3-5 Test matrix

\begin{tabular}{|l||l||}
\hline Gas Velocity & Total Heat Power $(\mathbf{k W})$ \\
\hline \hline $28 \mathrm{~m} / \mathrm{s}$ & $3.0,4.0,5.0,6.0$ \\
\hline \hline $33 \mathrm{~m} / \mathrm{s}$ & $3.0,4.0,5.0,6.0,7.0$ \\
\hline \hline $40 \mathrm{~m} / \mathrm{s}$ & $3.0,4.0,5.0,6.0,7.0$ \\
\hline $48 \mathrm{~m} / \mathrm{s}$ & $3.0,4.0,5.0,6.0,7.0$ \\
\hline \hline $52 \mathrm{~m} / \mathrm{s}$ & $3.0,4.0,5.0,6.0,7.0$ \\
\hline \hline
\end{tabular}

Table 3-6 Measuring parameters and instrumentations for the new separate effect test

\begin{tabular}{|c|c|c|c|}
\hline $\begin{array}{l}\text { Measuring } \\
\text { Parameters }\end{array}$ & Measuring Location & Instrumentations & Uncertainty \\
\hline Flow Rate & Cooling Pipe Inlet & $\begin{array}{l}\text { Bi-directional } \\
\text { Flow Tube }\end{array}$ & $0.9 \%$ \\
\hline Temperature & $\begin{array}{l}\text { Center : } 12 \\
\text { Surface : } 14 \text { Liquid : } 8\end{array}$ & Thermocouples & $1.5{ }^{\circ} \mathrm{C}$ \\
\hline $\begin{array}{l}\text { Differential } \\
\text { Pressure }\end{array}$ & $\begin{array}{l}\text { Between Inlet } \\
\text { and Outlet }\end{array}$ & DP Transmitter & $0.6 \%$ \\
\hline
\end{tabular}

Compared to previous device, the total number of U-bands of air cooling pipe was decreased from 16 to 11. In addition the diameter of air-cooling pipe was enlarged from $63.5 \mathrm{~mm}$ to $76.6 \mathrm{~mm}$ maintaining total heat transfer area as constant. As a result of the design modification, the pressure drop between the inlet and outlet of cooling pipe was reduced and the heat transfer coefficient was enhanced. Moreover, the total volume of cooling pipe was slightly reduced so that the water capacity of water pool was increased about $22.3 \%$ when other geometry such as height and width of water pool was preserved. The experimental cases are tabulated in Table 3-5, and the instrumentations, measuring locations and their uncertainties are summarized in Table 3-6. 


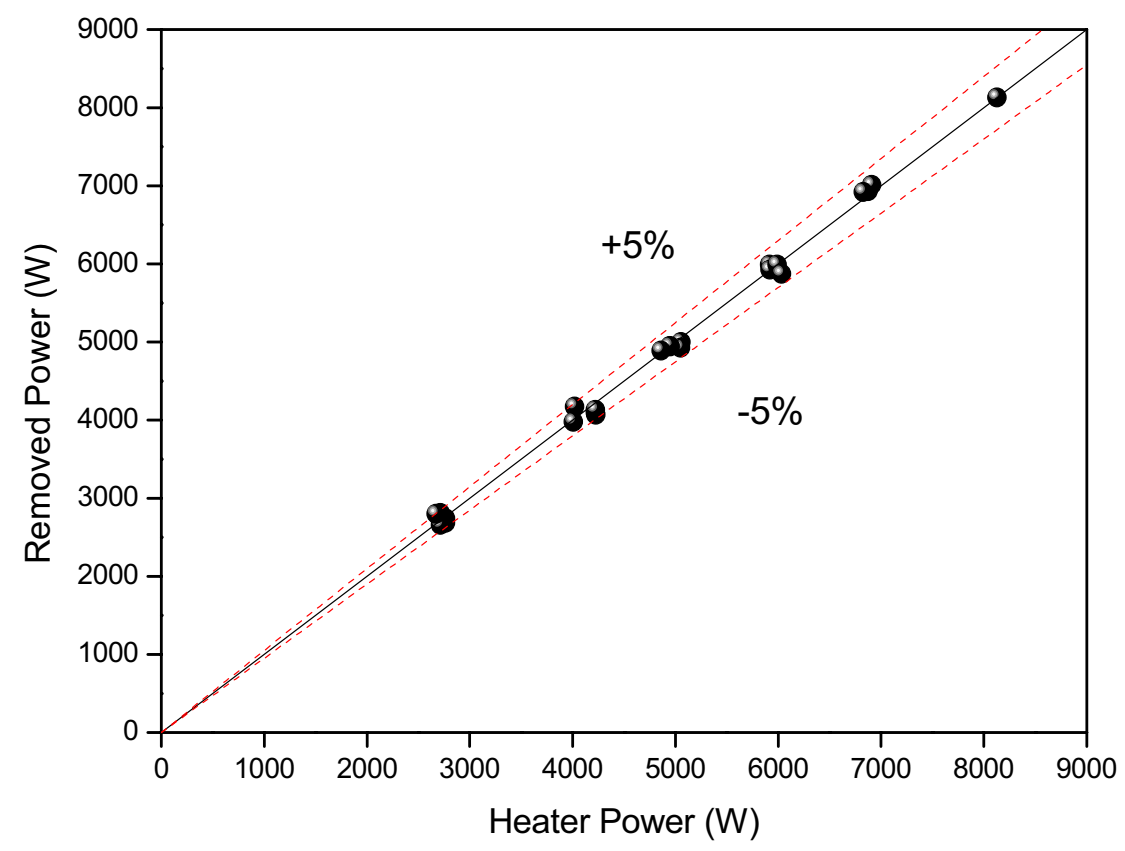

Figure 3-28 Energy balance of the separate effect tests

The separate effect tests are steady state experiments, and the steady state was confirmed by the comparison of the heating power and the heat removal rate. The heat removal rate by the cooling pipe was calculated from the measurement of the flow rate and the temperatures of the inlet and outlet. Discrepancy of heat balances in the experiments was less than 5\% as shown in Figure 3-28.

Figure 3-29 shows the measure pressure drops and its components between the inlet and outlet of the cooling pipe. The frictional pressure drop and the form loss pressure drop were calculated by the identical correlation used for previous separate effect test. Figure 3-30 shows reasonable agreement among the experimental data and the predicted total pressure drops. These results support that the loss coefficient of a standard U-bend is available for the cooling pipe of the water pool type RCCS. 


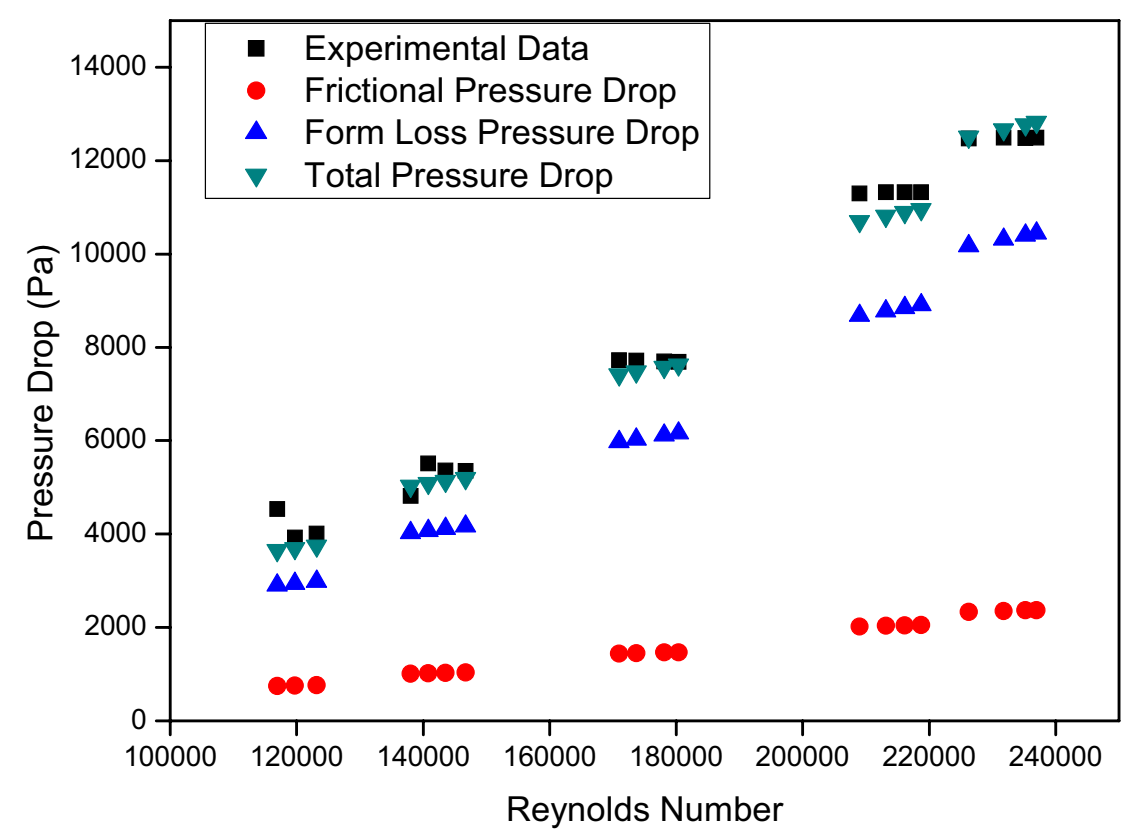

Figure 3-29 Pressure drop vs re.

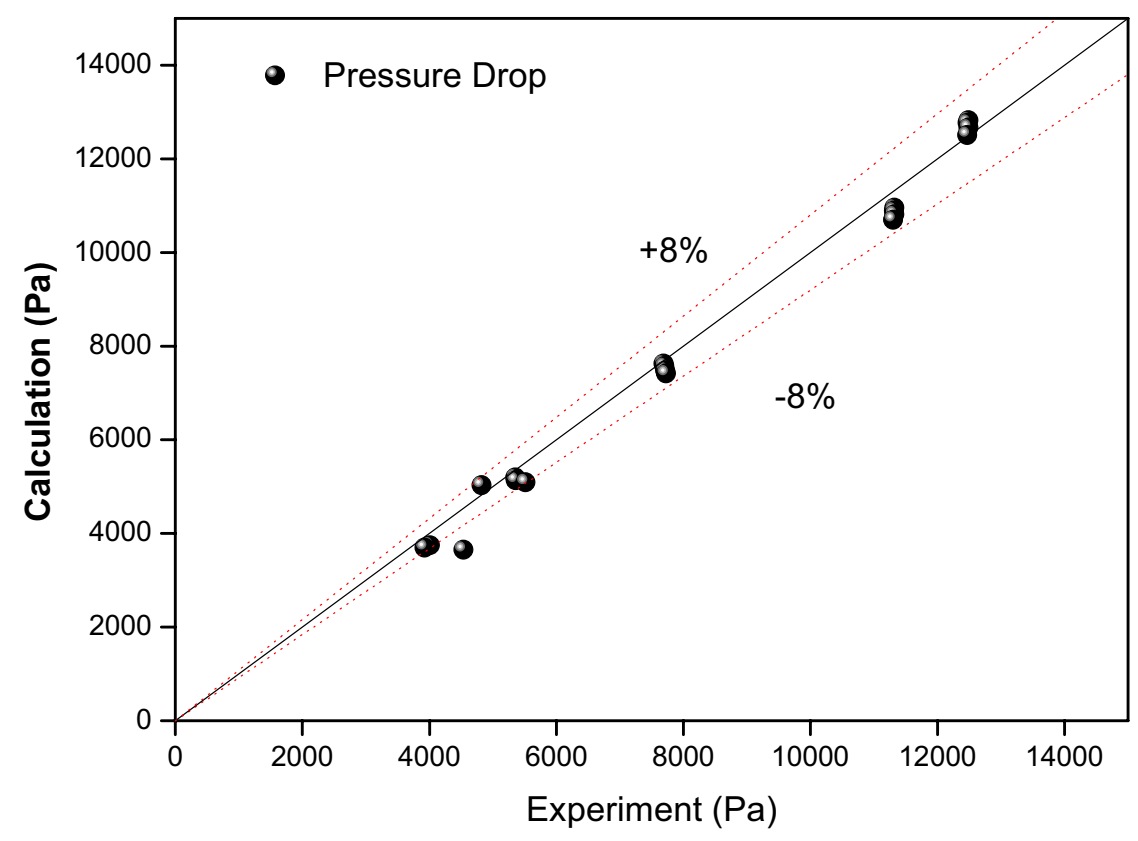

Figure 3-30 Comparison of measured pressure drop with prediction 


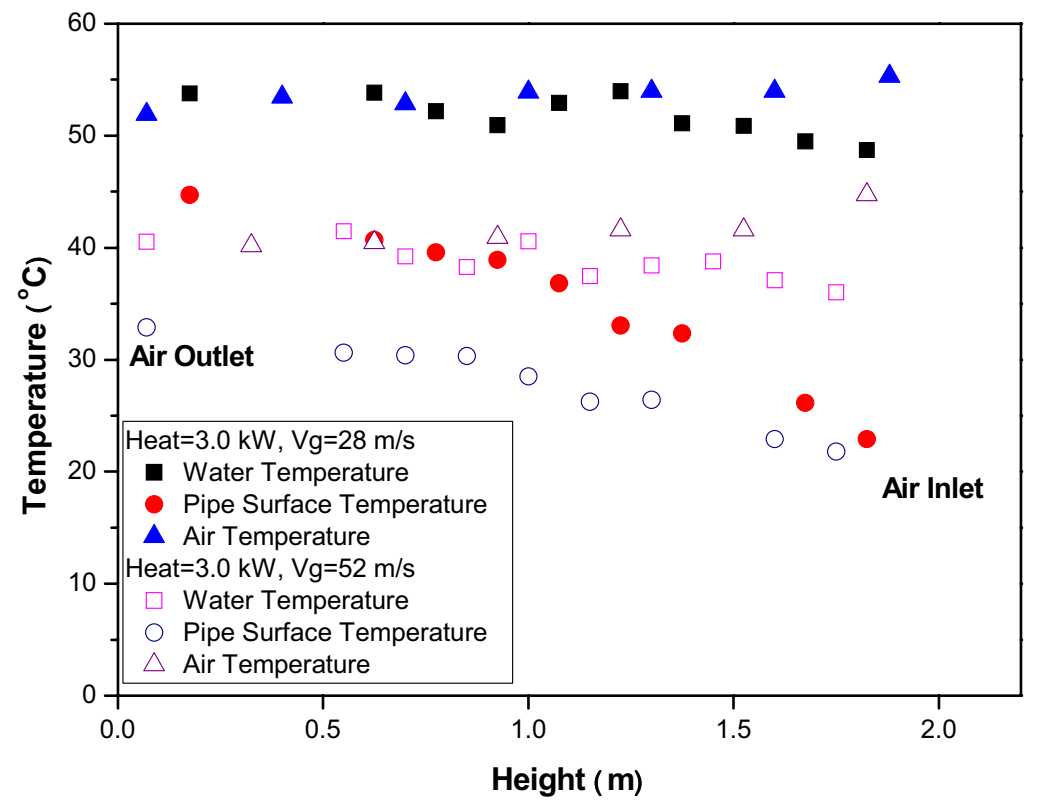

Figure 3-31 Temperature distribution

Figure 3-31 indicates the experimental results of the axial temperature distributions in the water pool, and at the cooling pipe surface and the center of the cooling pipe for different inlet air flow rates. The air temperature at the center of the cooling pipe gradually increased from atmosphere temperature up to near the bulk temperature as air flows through the cooling pipe. Bulk temperature of upper part of water pool was higher than bottom part about $3 \sim 4{ }^{\circ} \mathrm{C}$. The surface temperature of cooling pipe was lower than the bulk temperature at inlet by $6 \sim 8{ }^{\circ} \mathrm{C}$, and it closed to bulk temperature near the outlet. These results show good agreement with experimental results for previous separated effect test.

Table 3-7 Correlations of heat transfer coefficient

\begin{tabular}{|l|l|}
\hline & Correlation \\
\hline $\begin{array}{l}\text { Dittus and } \\
\text { Boelter }\end{array}$ & $N u=0.023 \operatorname{Re}^{4 / 5} \operatorname{Pr}^{0.4}$ \\
\hline $\begin{array}{l}\text { Mori and } \\
\text { Nakayama }\end{array}$ & $N u=\frac{\operatorname{Pr}}{26.2\left(\operatorname{Pr}^{2 / 3}-0.074\right)} \operatorname{Re}^{4 / 5}\left(\frac{D}{D_{\text {coil }}}\right)^{1 / 10}\left[1+\frac{0.098}{\left\{\operatorname{Re}\left(D / D_{\text {coil }}\right)^{2}\right\}^{1 / 5}}\right]$ \\
\hline $\begin{array}{l}\text { Moshfeghian } \\
\text { and Bell }\end{array}$ & $N u_{x}=0.0931\left(\frac{D}{L}\right)^{-0.116} \operatorname{Re}^{0.825} \operatorname{Pr}^{0.4}\left(\frac{\mu_{m}}{\mu_{w}}\right)^{0.14}\left(\frac{R}{D}\right)^{0.884}\left[\left(1+\frac{L}{\pi R}\right)^{0.884}-1\right]$ \\
\hline $\begin{array}{l}\text { Tailby and } \\
\text { Staddon }\end{array}$ & $N u_{x}=0.0987\left(\frac{D}{L}\right) \operatorname{Re}^{0.82} \operatorname{Pr}^{0.4}\left(\frac{R}{L}\right)^{0.85}\left[\left(1+\frac{L}{\pi R}\right)^{0.96}-1\right]$ \\
\hline
\end{tabular}


Local heat transfer coefficients of cooling pipe of new SET were calculated by the identical method used for previous separate effect test. The air inlet and outlet temperatures from experiments were used, and the surface temperature was obtained from overall averaged

temperature of the entire surface in experiments. These heat transfer coefficients in the experimental results were compared with the well-known correlations for the heat transfer coefficient of a straight pipe, a helical coil and a U-bend as shown in Figure 3-32.

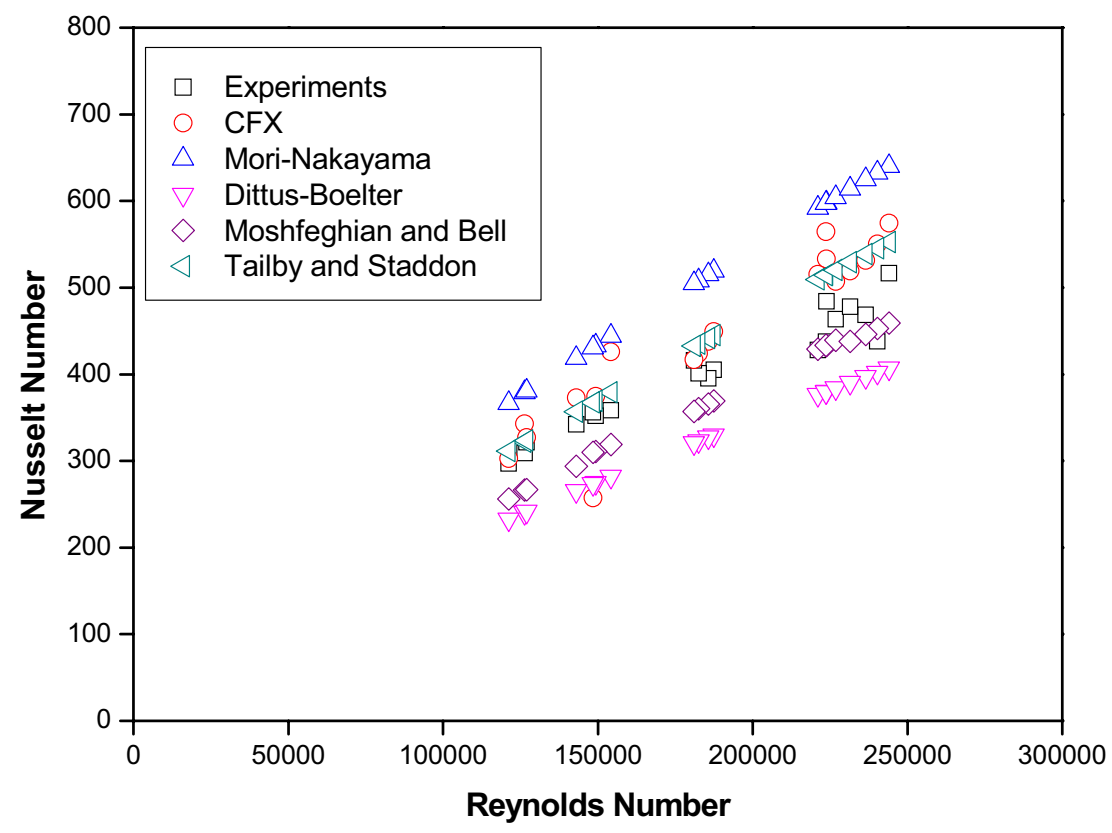

Figure 3-32 Comparison of local heat transfer coefficient of cooling pipe

The Dittus-Boelter correlation (Frank P. Incopera 1996) for a straight tube in fully developed turbulent flow, the Mori-Nakayama correlation (Y. Mori and W. Nakayama 1967), the Tailby-Staddon correlation (S. R. Tailby and P.W. Staddon 1970) and the Moshfeghian-Bell correlation (M. Moshfeghian and K.J. Bell 1979) for a U-bend were used for the evaluation. These correlations are summarized in Table 3-7.

The experimental data are found to be about $20 \%$ higher than the predicted value by Dittus-Boelter. It can be seen that the heat transfer coefficient in curved pipe is always large than in straight tube (G. W. Hogg 1968). Nusselt numbers of experiments are about $20 \%$ lower than those of Mori-Nakayama correlation. The correlations in U-bend, which are developed by Tailby and Staddon, Moshfeghian and Bell, are predicted in good agreement with the experimental data due to the characteristics of heat transfer phenomena by the similarity of geometric structure.

The power for operating air blower was reduced more than $30 \%$ because the pressure drop was decreased due to the enlarged diameter of cooling pipe as well as reduction of the total number of U-bends. It means if the diameter of cooling pipe increase, we can get more advantage in aspect of active cooling power. In this case, however, the capacity of water in the water pool was decreased and it can influence passive long term cooling of RCCS. Thus, the diameter and total number of U-bands of cooling pipe and water capacity should be considered synthetically for design optimization. 


\section{$\underline{\text { 3.3-1 CFX 5.7 Calculation }}$}

CFX 5.7 calculations were carried to investigate the characteristics of the heat transfer phenomena in the cooling pipe and to obtain detailed information of the fluid velocity and temperature for the new separate effect test. For simple calculation, the heat transfers in air inside the cooling pipe and water outside the cooling pipe were simulated independently.

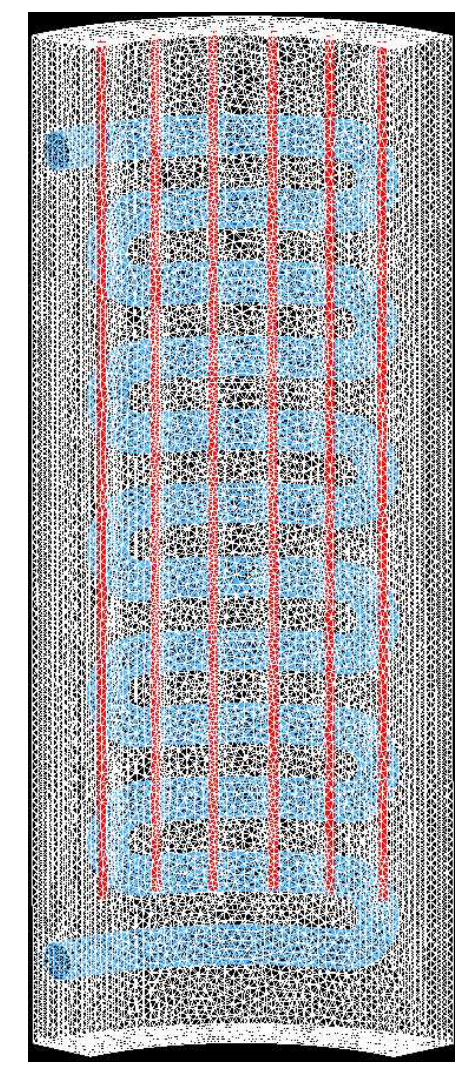

Figure 3-33 Nodalization for the CFX 5.7 calculation

First, the calculation results inside the cooling pipe were described. For the calculation, the experimental data of the cooling pipe surface temperature were adopted as wall boundary conditions. Figure 3-33 shows the grid in the calculation and the standard k- $\varepsilon$ model was used for turbulence modeling. 177,540 nodes in total were used in cooling pipe by structured mesh. Figures 3-34 and 3-35 show the calculated results of the velocity profile at various axial locations, and temperature profiles including the measured temperatures at the center of the cooling pipe. The present calculation results for the air temperature at the pipe center were compared with the experimental results in Figure 3-36. Although CFX 5.7 slightly under-predicted the experimental data, the calculated temperatures showed a reasonably good agreement with the experimental data. 


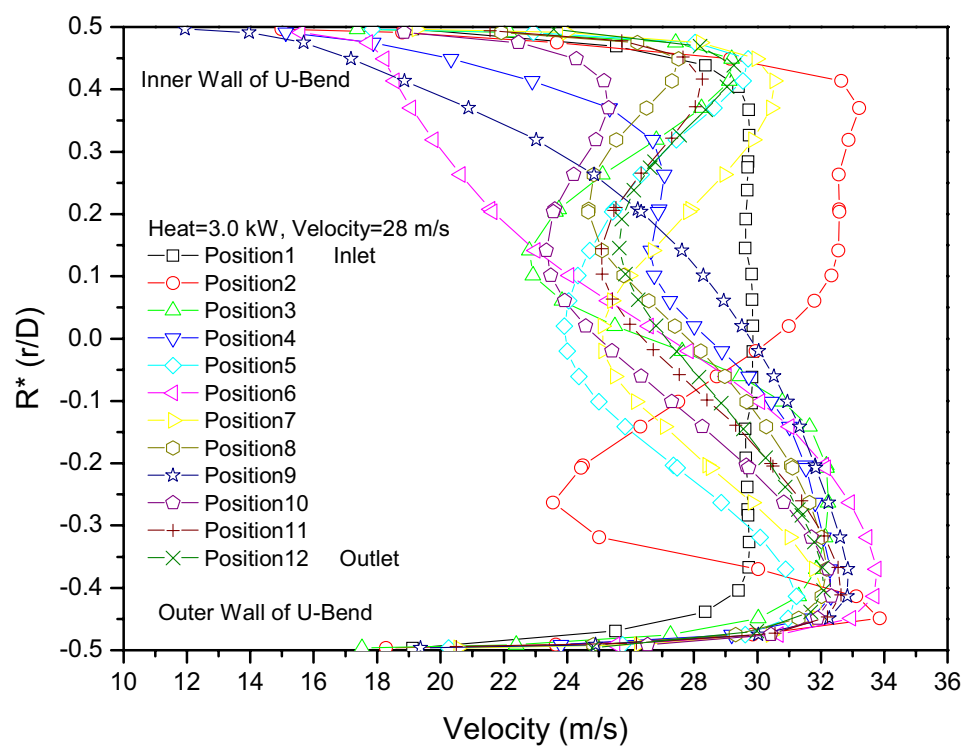

Figure 3-34 Air Velocity profiles in the cooling pipe

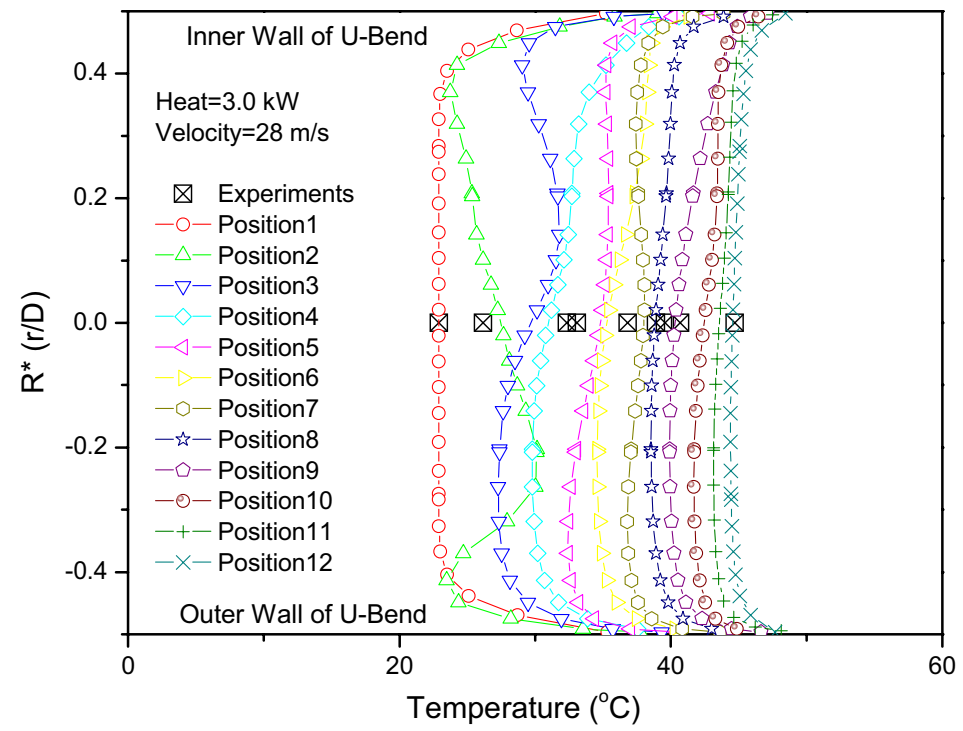

Figure 3-35 Air temperature profiles in the cooling pipe 


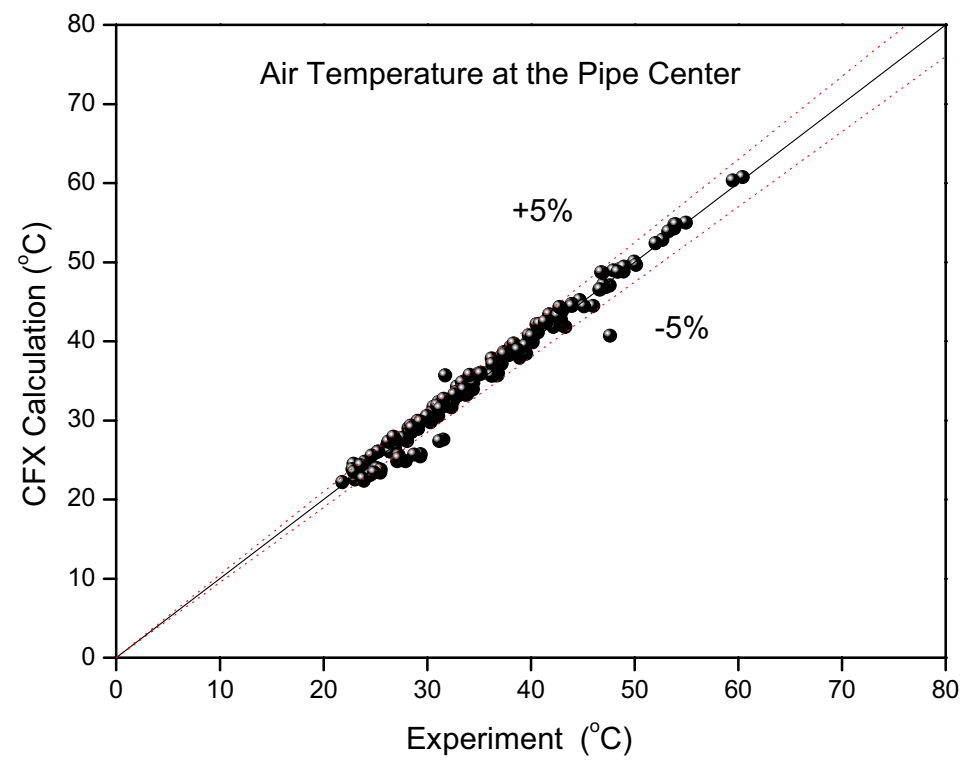

Figure 3-36 Comparison of air temperatures

Second, the calculation results for the water outside the cooling pipe are described. As same as the above calculation, the experimental data of the cooling pipe surface temperature were implemented as wall boundary conditions. 1,189,466 elements in total by unstructured mesh were formed to model the water pool. Figures 3-37 and 3-38 show the typical calculation results of the velocity and temperature profiles in the water pool. Driven by buoyancy force, the heated fluid adjacent to the heaters begins to move upward at the bottom part of heater and to move downward at the cooling pipe with the maximum velocity of about 1 2 $\mathrm{cm} / \mathrm{s}$. However, the velocity is much lower at the bottom region of water pool, which indicates that fluid is actually stagnant there. Also the calculated results for the water temperature in the pool were compared with the experimental results in Figure 3-39. As shown in the figure, the predicted water temperatures by CFX 5.7 corresponded well with those measured in experiments. 


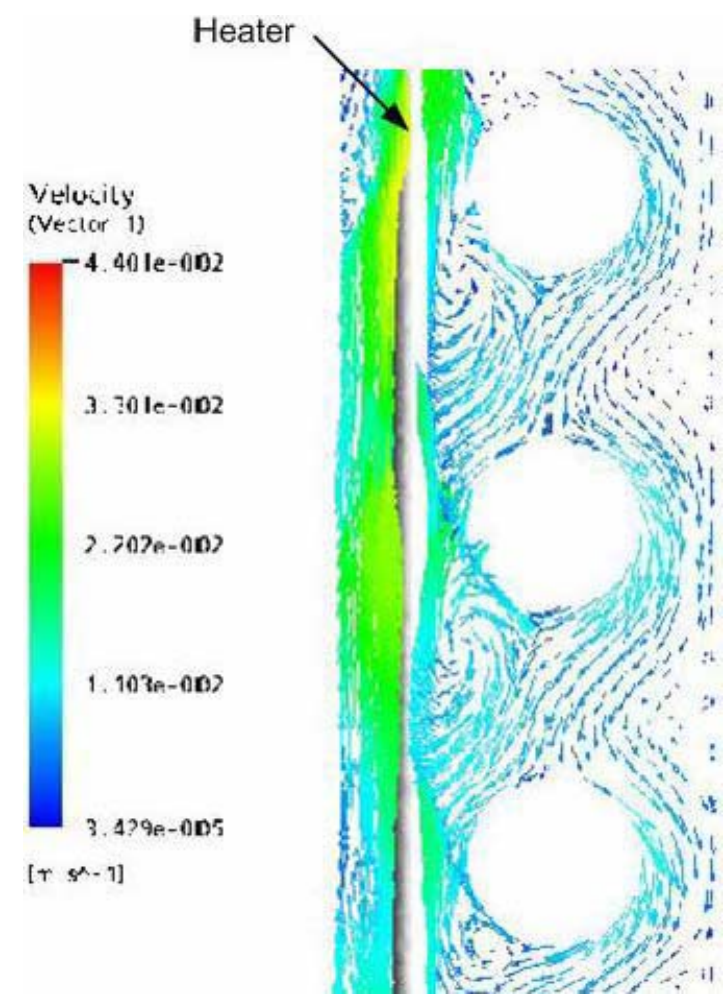

Figure 3-37 Water velocity distribution in the water pool

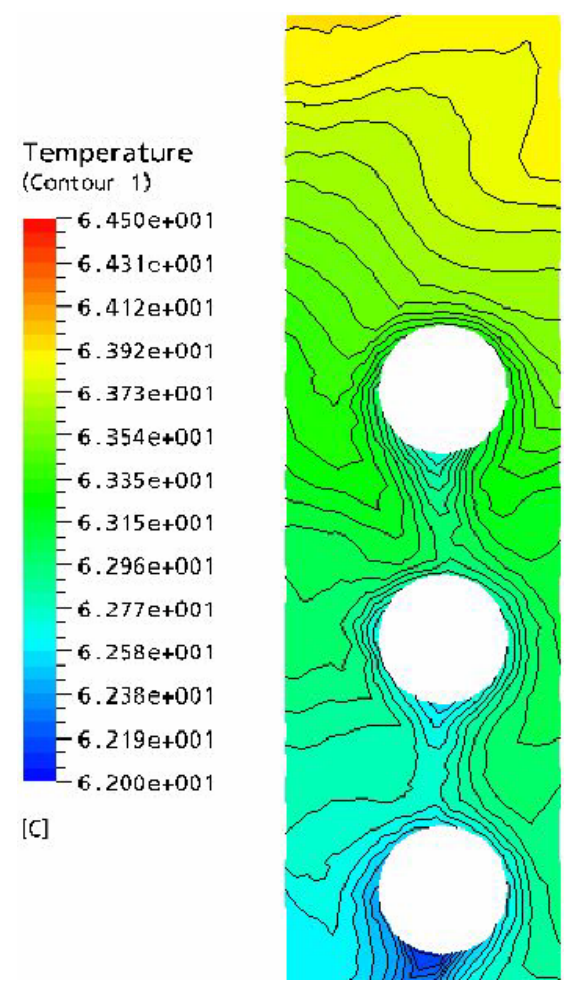

Figure 3-38 Water temperature distribution in the water pool 


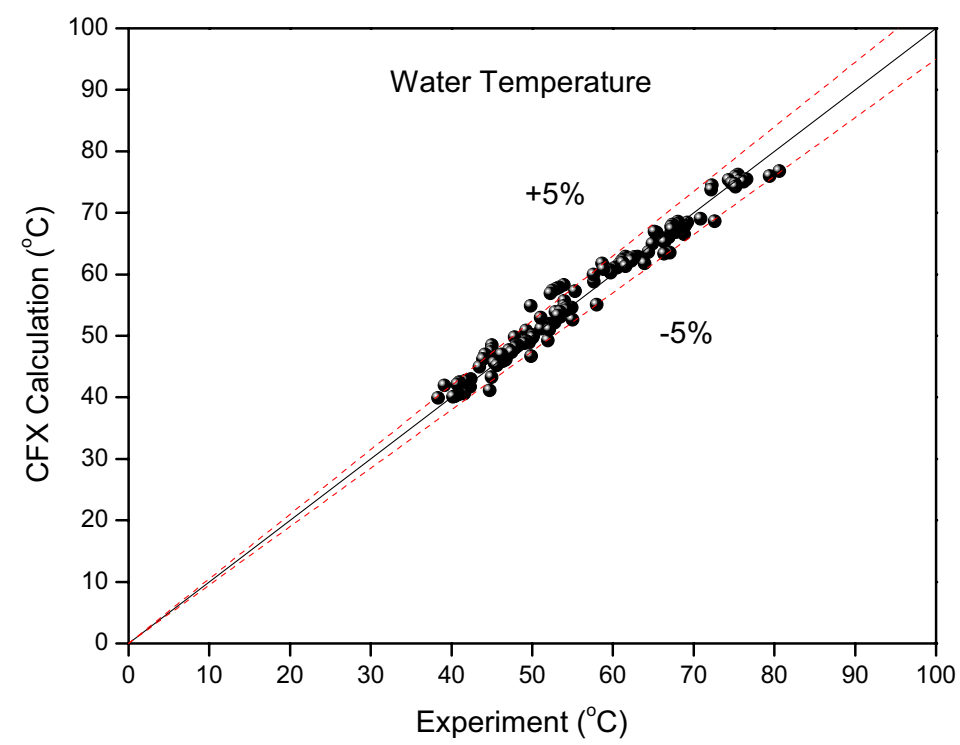

Figure 3-39 Comparison of water temperature in the water pool

As comparing results of SET-part 1, new SET was simulated with the smaller mesh size at the boundary layer near the cooling surface and with density variation with temperature in water pool. Thus, the sharp gradient of the water temperature did not occur in near cooling pipe and the water in water pool was well mixed by natural convection due to the density difference between top and bottom region in water pool.

\section{3-2 MARS Calculation}

For the MARS-GCR calculation, the new separate effect test facility was nodalized with 29 volumes, 33 junctions and 31 heat structures, as shown in Figure 3-40. The water pool was modeled using the multidimensional component which consists of $2 \times 1 \times 7(\mathrm{r}-\theta-\mathrm{z})$ cylindrical coordinates. The cooling pipe was modeled as pipe component 211 with 12 volumes connected by 11 junctions. The water pool was radially divided by two nodes. Each node shares the half of air cooling pipe, 


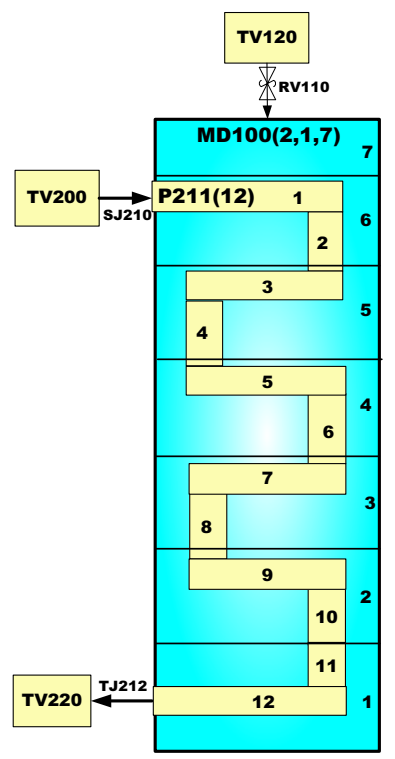

(a) Front View

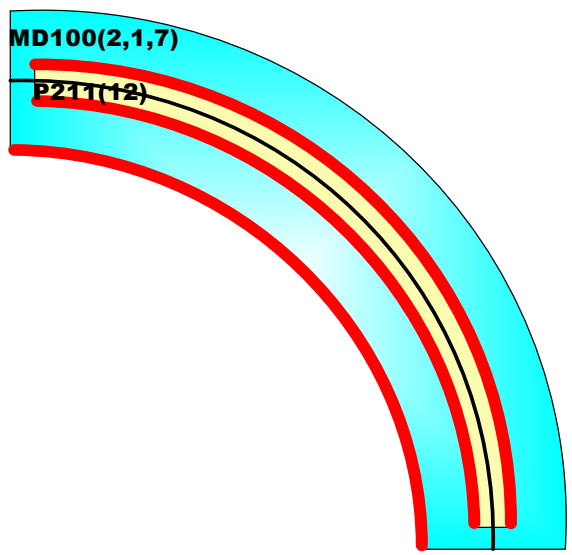

(b) Top View

Figure 3-40 Nodalization of new SET facility for the MARS-GCR calculation

which means each node has a preserved water volume with a heat structure of a half of heat transfer area of cooling pipe. The electrical power from six U-bend heaters was modeled as constant heat flux from 7 heat structures connected to the inner wall of the water pool. Air flows in the cooling pipe with constant mass flows through a time dependent junction 212 and a single junction 210 connected to the outlet and the inlet of cooling pipe, respectively. Time dependent volumes 120, 200, 220 were connected to trip valve 110, single junction 210, time dependent junction 212 respectively to simulate the ambient air.

The cooling pipe surface of water side and the heated wall in the water pool was represented by straight pipes when the water convection boundary condition was imposed. Inside the cooling pipe, the conditions of helical tube geometry was implemented for air convection. MARS-GCR code uses the Churchill-Chu correlation for the heat transfer coefficient for the water natural convection and the Dittus-Boelter

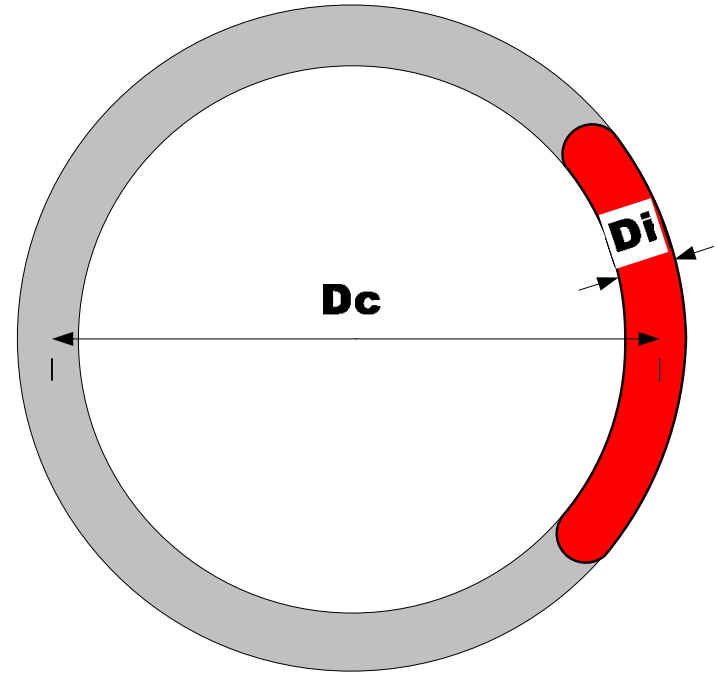

Figure 3-41 Helical circle diameter-to-tube inner diameter ratio. 
correlation for the air convection in the straight pipe (S. W. Churchill and H. H. S. Chu 1975). The MoriNakayama correlation for the air convection is used in the helical tube (Y. Mori and W. Nakayama 1967). Since the Mori-Nakayama correlation uses the ratio between helical circle diameter and tube inner diameter, the radius of curvature of the cooling pipe was used as the helical circle diameter as shown in Figure 3-41.

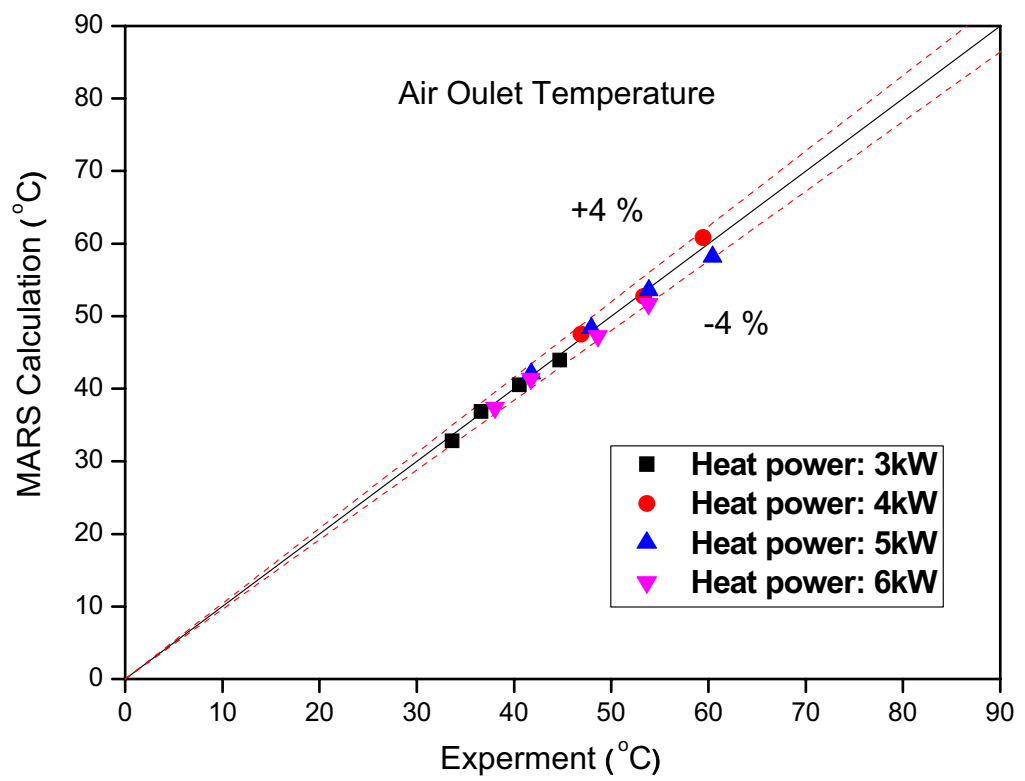

Figure 3-42 Comparisons of air outlet temperature

MARS-GCR calculation results for the air temperature at the outlet of the cooling pipe were compared with the experimental data as shown in Figure 3-42, which showed also good agreement as like in experiment.

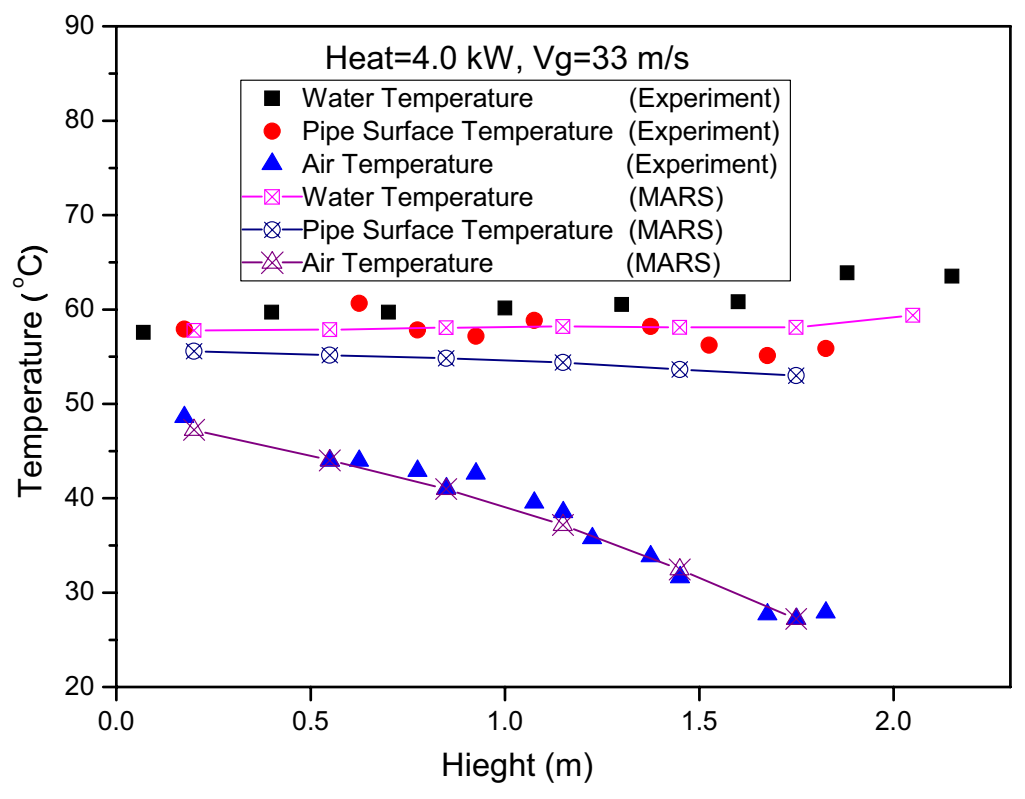

Figure 3-43 Comparison of temperature distribution 
Figure 3-43 shows calculation results for the axial temperature distributions of bulk temperature in the water pool, pipe surface temperature and air temperature at the pipe center. Although MARS-GCR code wellpredicted the air temperature, pipe surface temperature and bulk water temperature were slightly underpredicted, which is summarized in Figures 3-44 and 3-45. Under-predictions of the pipe surface temperature as well as the bulk temperature in the water pool are estimated to be caused by the inadequacy of heat transfer coefficient correlation inside the cooling pipe, which was shown in CFX 5.7 calculation.

The fluid adjacent nodes to the heated wall in the water pool moves upward and downward at the radially outer nodes with the maximum velocity of $1.5 \mathrm{~cm} / \mathrm{s}$. This result shows a good agreement with CFX 5.7 result and means the natural circulation is not significant in the water pool.

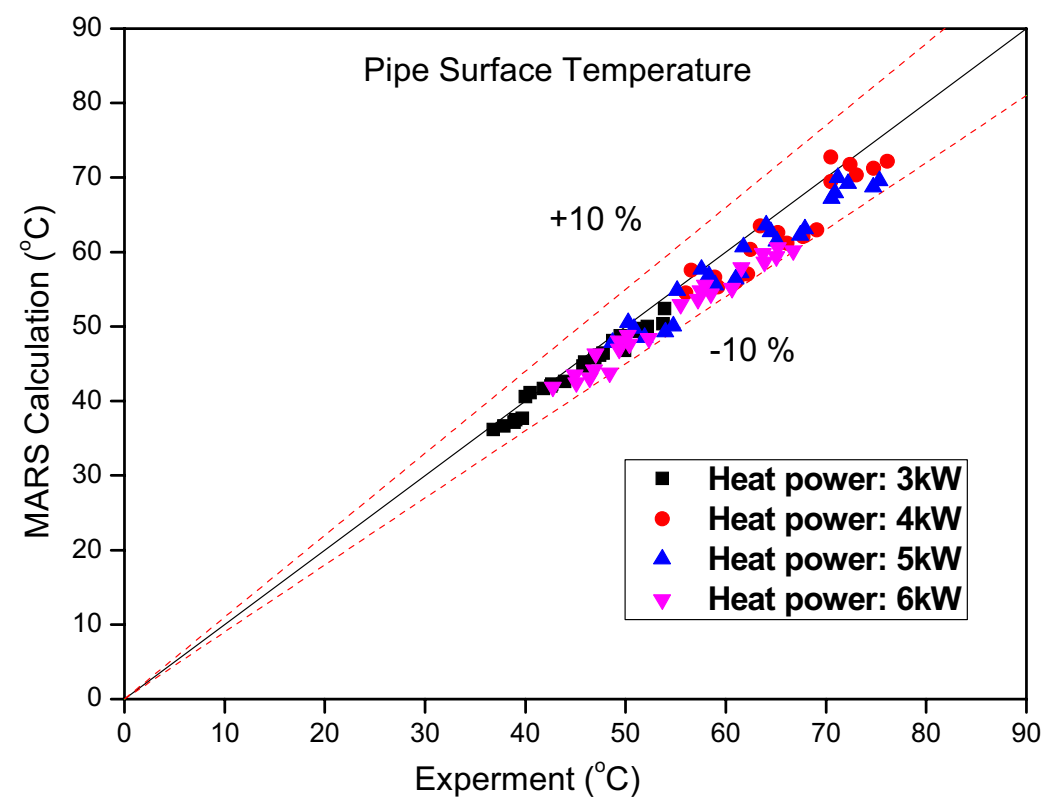

Figure 3-44 Comparisons of pipe surface temperature 


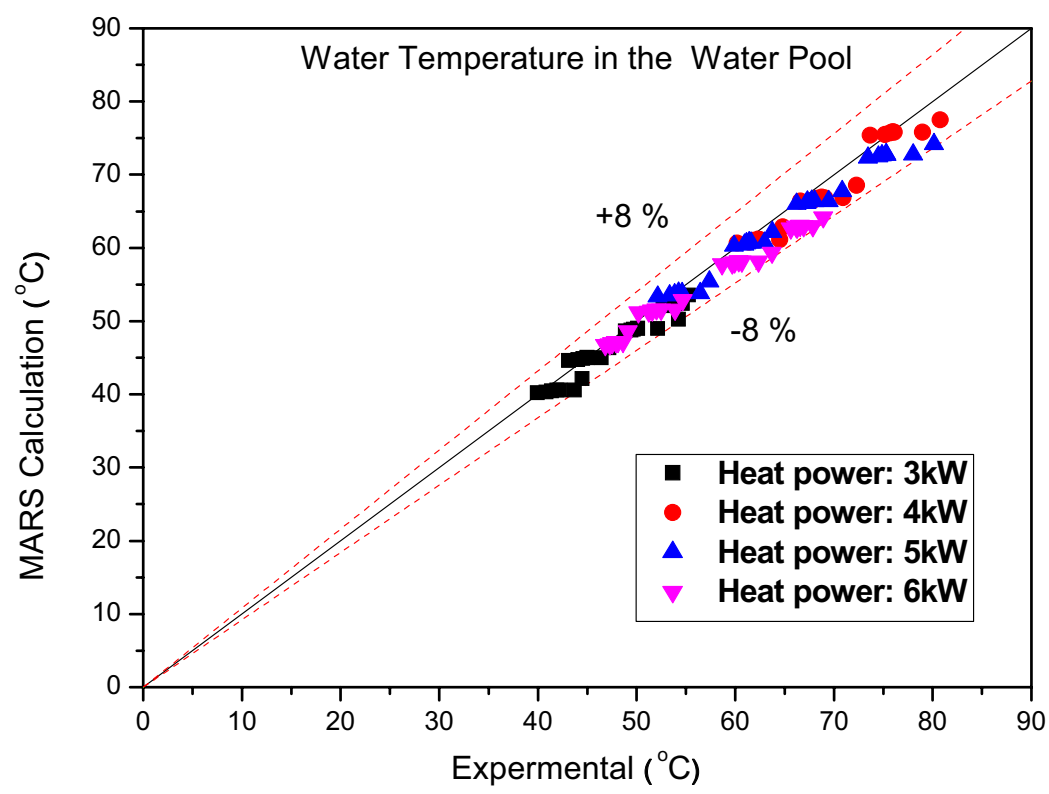

Figure 3-45 Comparisons of bulk temperature in the water pool

Figures 3-46 and 3-47 show the calculation results when inherent correlations in MARS-GCR for air convection were implemented inside cooling pipe.

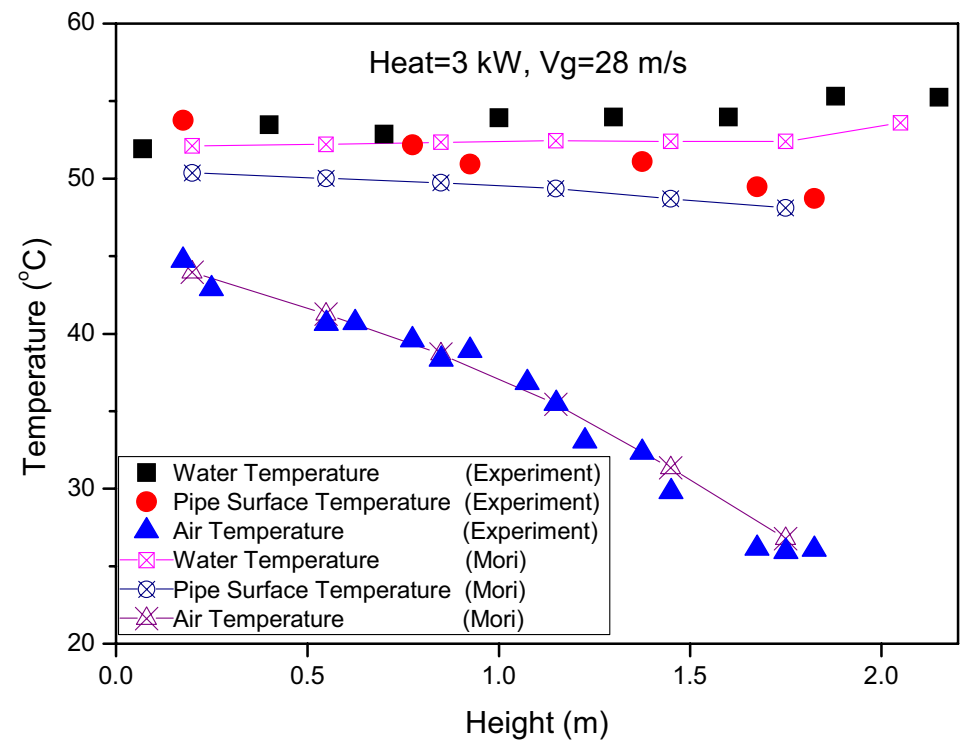

Figure 3-46 Comparisons of temperature distribution with the Mori-Nakayama correlation 


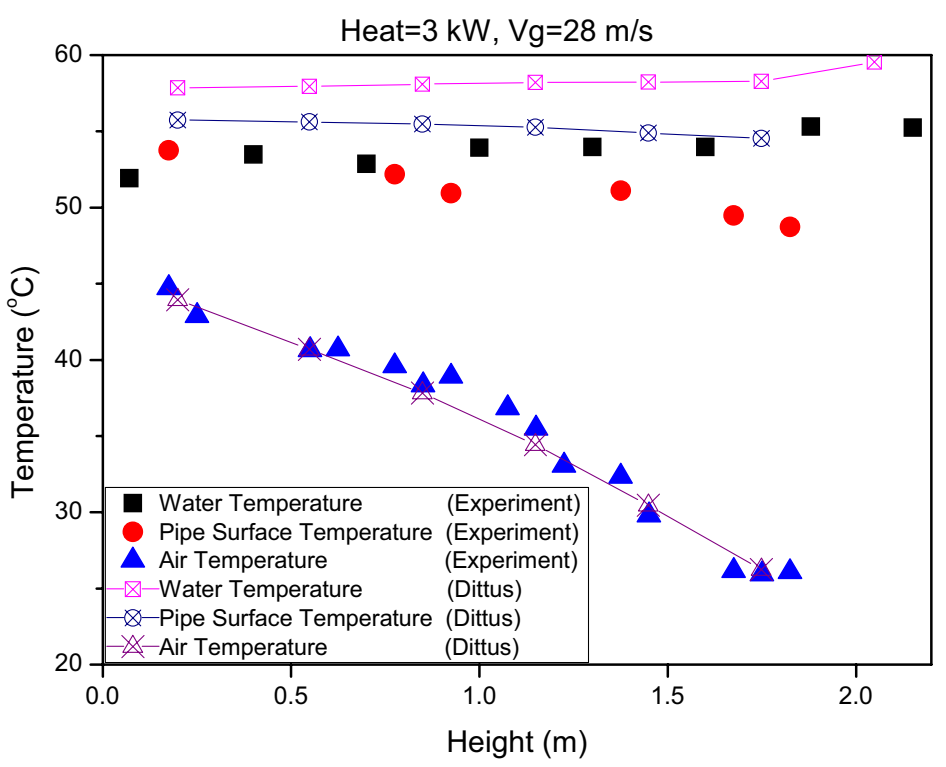

Figure 3-47 Comparisons of temperature distribution with the Dittus-Boelter correlation

Air temperatures at the pipe center show good agreement with measured temperatures independently from the correlations because of the heat balance. However, water and pipe surface temperatures were under-predicted when the Mori-Nakayama correlation was implemented, and over-predicted when the Dittus-Boelter correlation was implemented. These discrepancies are because the Mori-Nakayama correlation over-predicts the heat transfer coefficient and the Dittus-Boelter correlation under-predict the heat transfer coefficient inside the cooling pipe as proved in CFX 5.7 calculation.

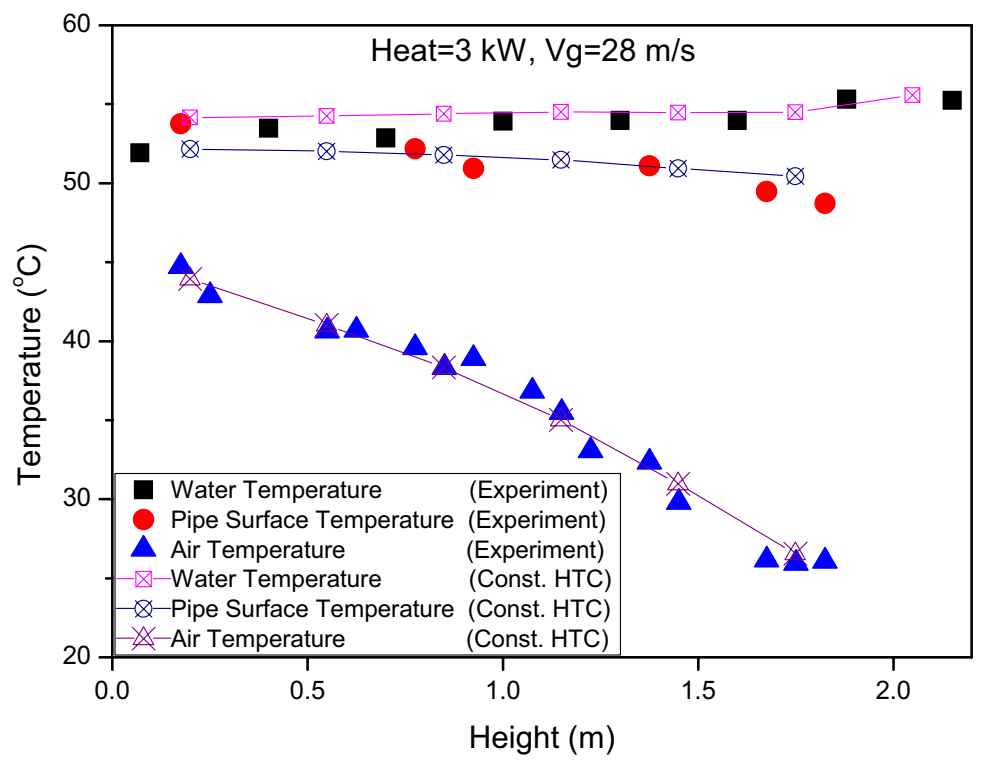

Figure 3-48 Comparisons of temperature distribution with constant heat transfer coefficient from SET 
In conclusion, MARS-GCR predicts well the heat transfer phenomena by natural convection inside water pool. On the other hand, the heat transfer by forced convection of air inside the cooling pipe shows the discrepancies from experimental data in both cases when the Mori-Nakayama correlation and the DittusBoelter correlation was implemented. However, MARS-GCR calculation results with a heat transfer coefficient from experimental data inside pipe show good agreement with the experimental results of the temperature distribution of pipe surface, water in water pool as well as air at the pipe center, as shown in Figure 3-48.

\subsection{Integral Experiment for the RCCS-SNU}

An experimental apparatus was constructed to investigate the various heat transfer phenomena in the water pool type RCCS, such as the natural convection of air inside the cavity, radiation in the cavity, the natural convection of water in the water pool and the forced convection of air in the cooling pipe. The schematic diagram and the photos of the experimental apparatus are shown in Figures 3-39 and 3-40.

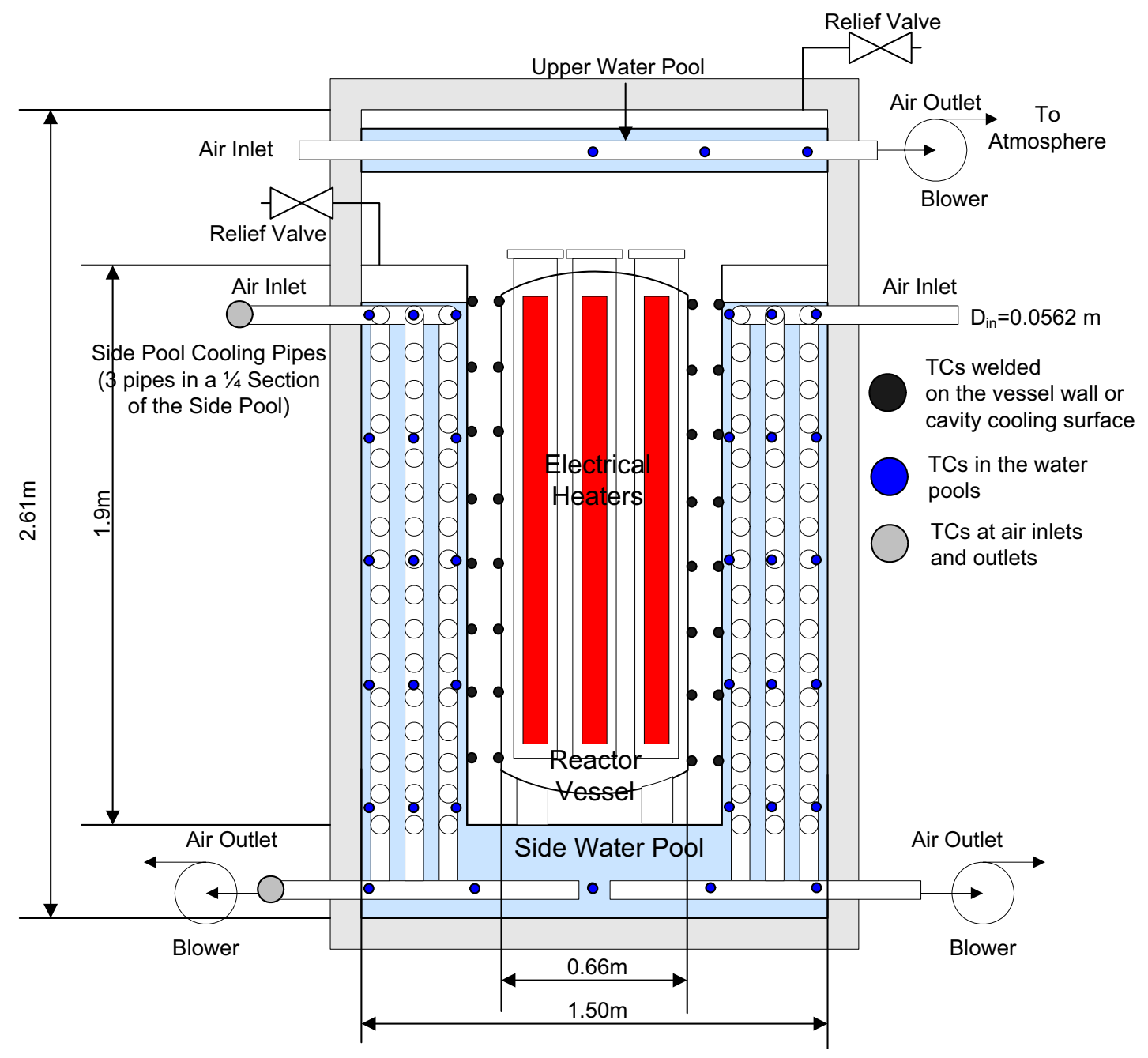

Figure 3-39 Schematic diagram of the test facility 


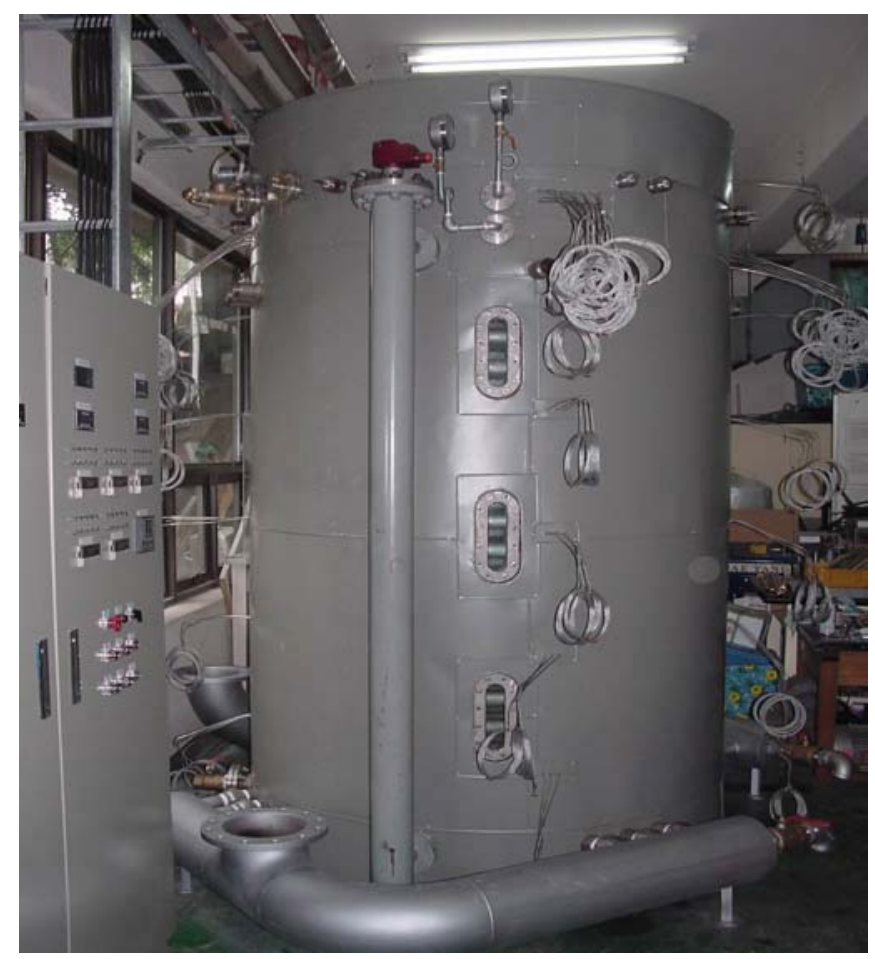

Figure 3-40 Photograph of the test facility

The apparatus mainly consists of a reactor vessel, a side water pool surrounding the vessel, an upper water pool, cooling pipes in the water pools and air supply systems. The reactor vessel of the test facility is a $1 / 10$ linear scaled model of the PBMR (265 MW). In the reactor vessel, six heaters were installed to simulate the heat loss which occurs during normal operation and the afterheat during an LOFC accident. The heat released from the reactor vessel is transferred to the side pool and the upper pool through the cavity by radiative and natural convective heat transfer. To remove the heat, thirteen cooling pipes are installed in the side pool and the upper pool (12 in the side pool, one in the upper pool) as shown in Figures 3-41(a) and 3-41(b). Three of the cooling pipes within the same quarter section of the side pool are contained in one train of the RCCS. The outlets of the cooling pipes are connected to

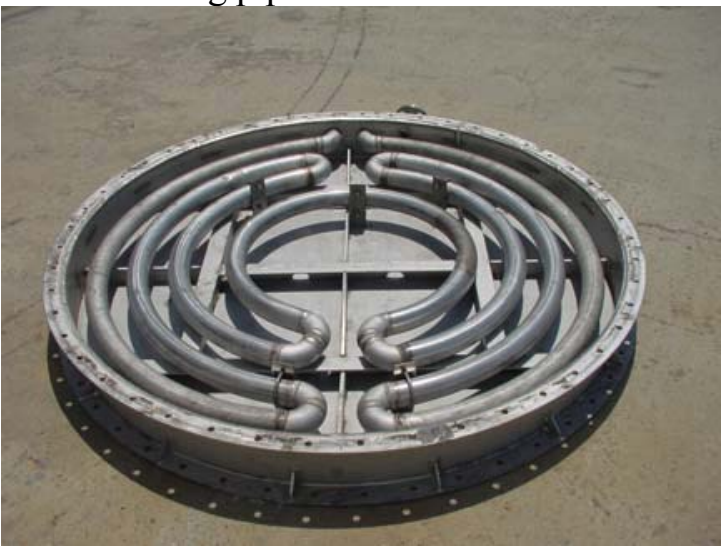

(a) Upper tank

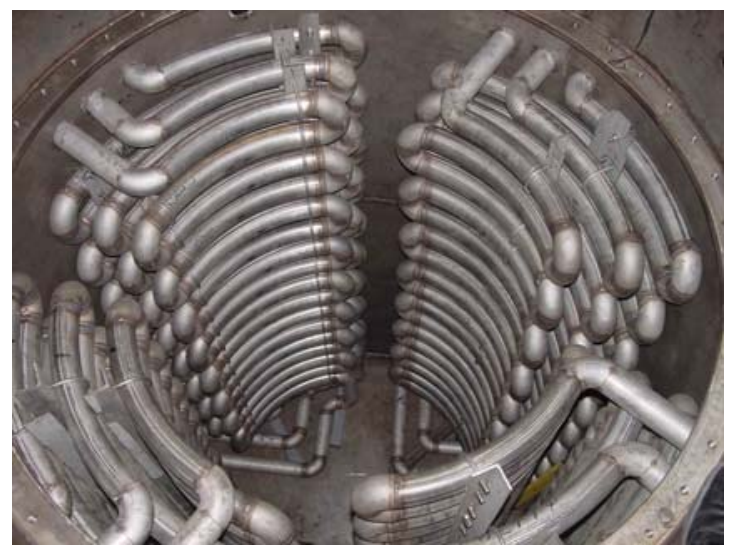

(b) Water tank

Figure 3-41 Configuration of the cooling pipe 


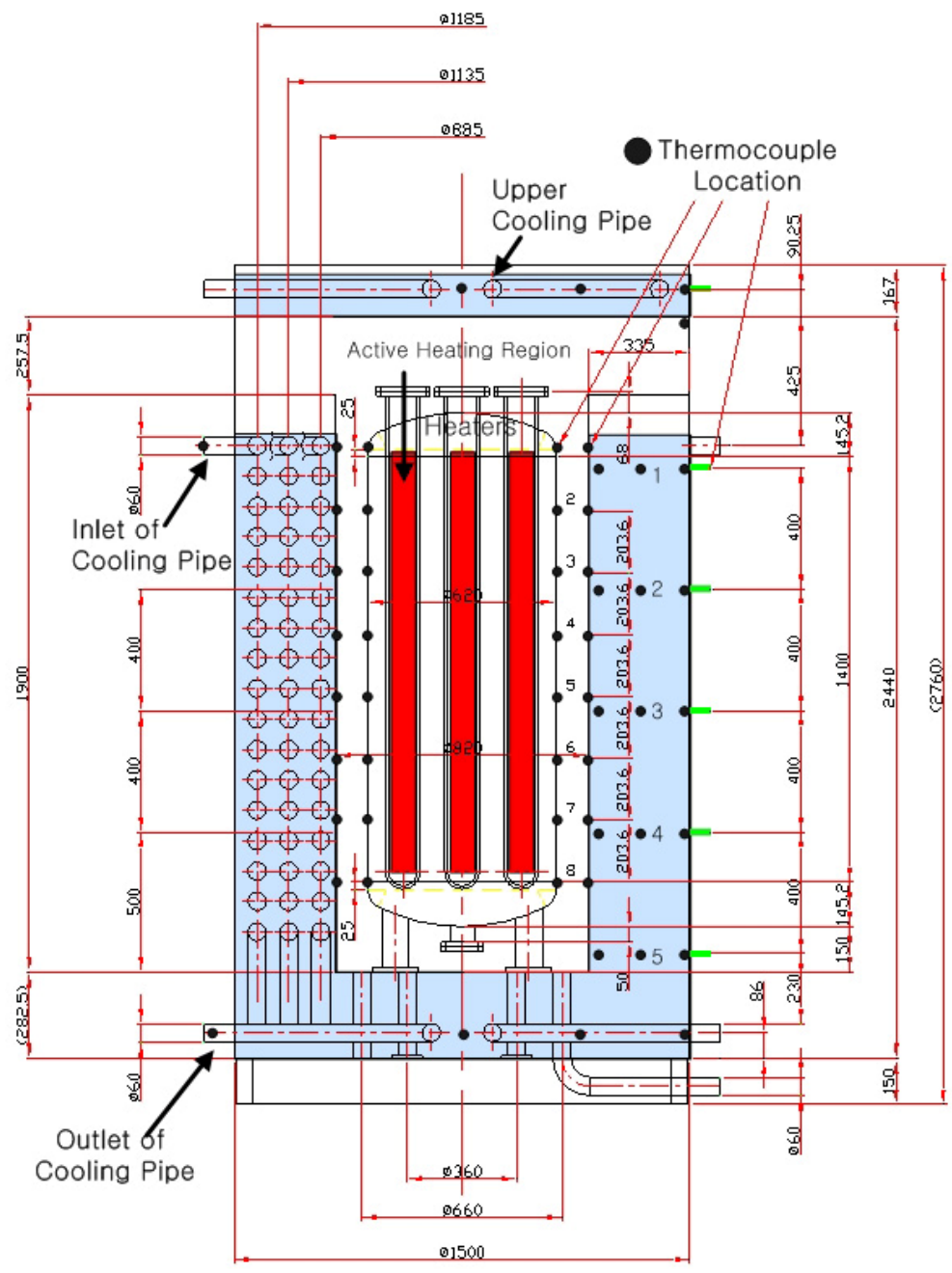

Figure 3-42 Detail dimensions of the test facility

common headers, and each common header is connected to a suction of the blowers. Ambient air enters into the inlet of the cooling pipes and enables the heat from the side pool and the upper pool to be released to the atmosphere. During both normal operation and accident conditions, the steam generated by evaporation or boiling in the water pools is vented to the atmosphere through the relief valves which are opened at 1.5 bars. The detailed dimensions of each component are indicated in Figure 3-42.

The major measurement parameters are the air flow rates, the pressure drops along the cooling pipes, the water level in the side pool, the pressures in the water pools, reactor vessel and cavity and the temperatures. The instrumentation, measuring locations and quantity of the measurements are summarized in Table 3-8 and the location of the thermocouples is presented in Figure 3-42. The temperatures of the water pool were measured at three radial positions, five axial positions and three azimuthal positions, and four additional thermocouples were installed at the bottom of the water pool and at the water level elevation. A total of 48 thermocouples were welded to the wall of the reactor vessel and the cavity. The flow rates of air were measured by means of thirteen average bi-directional flow tubes located at each inlet of the cooling pipes (B. J. Yun et al. 2004) and the water level in the side pool was measured by means of a differential pressure transmitter. 
Table 3-8 Measuring parameters and instrumentations

\begin{tabular}{|c|c|c|c|}
\hline Parameters & Instrumentation & Measuring Location & Quantity \\
\hline Air Flow Rate & Average BDFT & Air Inlet & 13 \\
\hline Pressure Drop & DP Transmitter & Air In and Out & 1 \\
\hline Pressure & Pressure Transducer & Vessel, Cavity Water pools & 4 \\
\hline Water Level & DP Transmitter & Water Tank & 1 \\
\hline \multirow{9}{*}{ Temperature } & \multirow{9}{*}{ K-type TC (132) } & Upper Tank & 3 \\
\hline & & Water Tank & 49 \\
\hline & & Reactor Vessel & 24 \\
\hline & & Cavity Wall & 24 \\
\hline & & Reactor Vessel For Emissivity & 3 \\
\hline & & Cavity Top & 1 \\
\hline & & Air In & 8 \\
\hline & & Air Out & 8 \\
\hline & & Cooling Pipe Surface & \\
\hline
\end{tabular}

In the present work, three categories of experiments were performed, namely the normal operation tests, the RCCS active cooling failure tests and the LOFC accidents tests with the accompanying failure of all the active cooling systems. Test matrix is shown in Table 3-9

During the normal operation of the PBMR, the heat released from the reactor vessel is transferred to the RCCS and the amount of heat loss was reported to be $0.2 \sim 0.3 \%$ of the reactor's thermal power. To be on the conservative side, the normal operation experiments were carried out with a heat loss of $0.35 \%$. The air velocity of this experiment is $20 \mathrm{~m} / \mathrm{s}$. The objective of the normal operation test was to evaluate the cooling capability of the RCCS, by verifying whether the maximum temperature of the reactor vessel was kept below the design temperature of the PBMR, namely $300^{\circ} \mathrm{C}$. Also, additional experiments with heat losses of $0.94 \%$ and $1.51 \%$ and velocities of $20 \mathrm{~m} / \mathrm{s}, 30 \mathrm{~m} / \mathrm{s}$ and $40 \mathrm{~m} / \mathrm{s}$ were carried out, in order to investigate the heat transfer phenomena in the water pools and the cavity. 
Table 3-9 Test matrix for IET

\begin{tabular}{|c|c|c|}
\hline & Heat power & Air velocity \\
\hline \multirow{9}{*}{ Normal Operation } & $10 \mathrm{~kW}$ & $20 \mathrm{~m} / \mathrm{s}$ \\
\hline & $25 \mathrm{~kW}$ & $20 \mathrm{~m} / \mathrm{s}$ \\
\hline & $40 \mathrm{~kW}$ & $20 \mathrm{~m} / \mathrm{s}$ \\
\hline & $10 \mathrm{~kW}$ & $30 \mathrm{~m} / \mathrm{s}$ \\
\hline & $25 \mathrm{~kW}$ & $30 \mathrm{~m} / \mathrm{s}$ \\
\hline & $40 \mathrm{~kW}$ & $30 \mathrm{~m} / \mathrm{s}$ \\
\hline & $10 \mathrm{~kW}$ & $40 \mathrm{~m} / \mathrm{s}$ \\
\hline & $25 \mathrm{~kW}$ & $40 \mathrm{~m} / \mathrm{s}$ \\
\hline & $40 \mathrm{~kW}$ & $40 \mathrm{~m} / \mathrm{s}$ \\
\hline \multirow{2}{*}{ 1/4 Train Trip } & $10 \mathrm{~kW}$ & $20 \mathrm{~m} / \mathrm{s}$ \\
\hline & $10 \mathrm{~kW}$ & $30 \mathrm{~m} / \mathrm{s}$ \\
\hline LOFC & $10 \mathrm{~kW}$ & $30 \mathrm{~m} / \mathrm{s}$ \\
\hline
\end{tabular}

As stated previously, normal operation should be able to continue even if one of the five active cooling systems of the RCCS fails. In the case of the partial active cooling failure of the RCCS, however, the local temperature increase in the failed part may induce an undesirable local increase in the reactor vessel temperature. To investigate the transient of the temperature distribution in this situation, partial RCCS active cooling failure tests were conducted for two different cases, viz. a 1/4 side cooler failure and an upper cooler failure.

In the LOFC experiments, the performance of the RCCS was investigated when it was operated in the passive heat removal mode. The analysis results for the 450 MW MHTGR were used to determine the experimental conditions of the afterheat released from the reactor vessel to the RCCS, as shown in Figures 3-43 and 3-44 shows an example of the transient of the controlled heater power in the LOFC experiments. The goal of this experiment was to verify whether the maximum reactor vessel temperature was kept at less than the design limit, namely $350^{\circ} \mathrm{C}$, during the accident condition. 


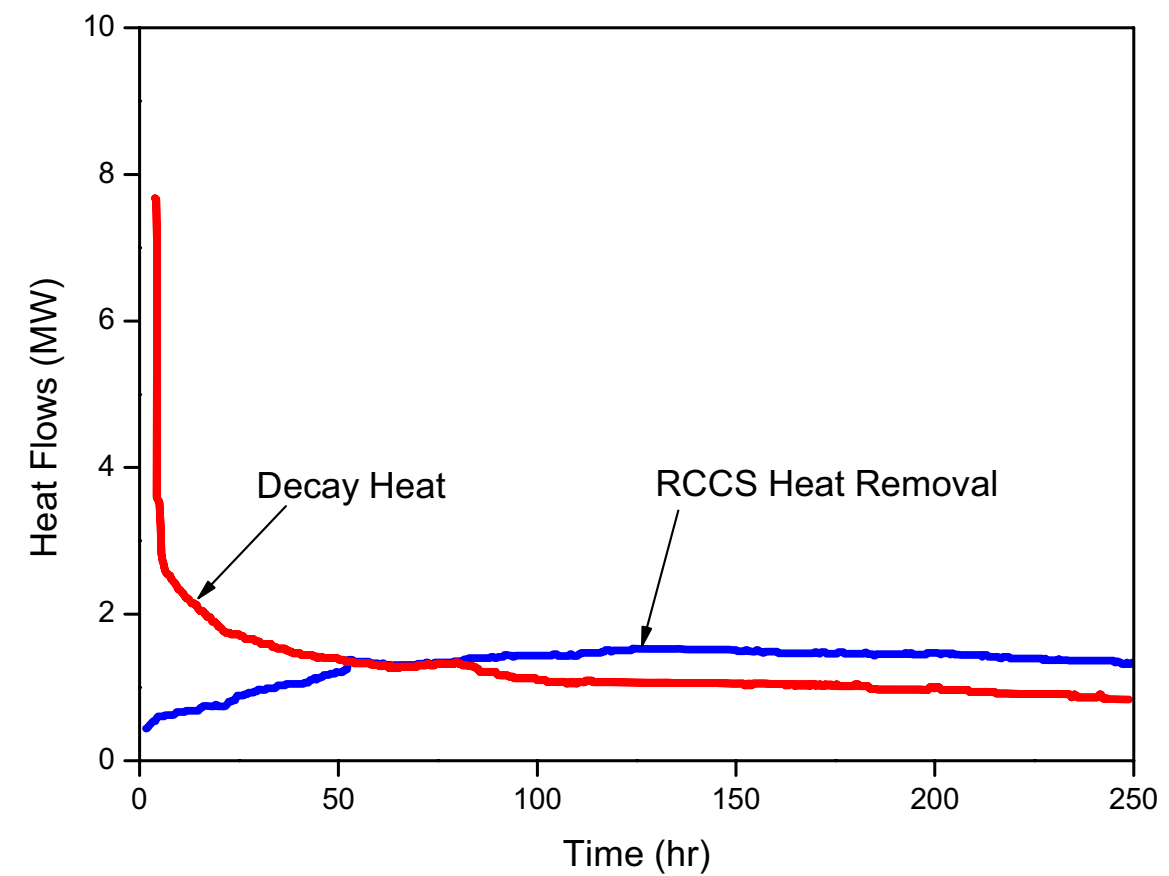

Figure 3-43 Analysis result for the MHTGR

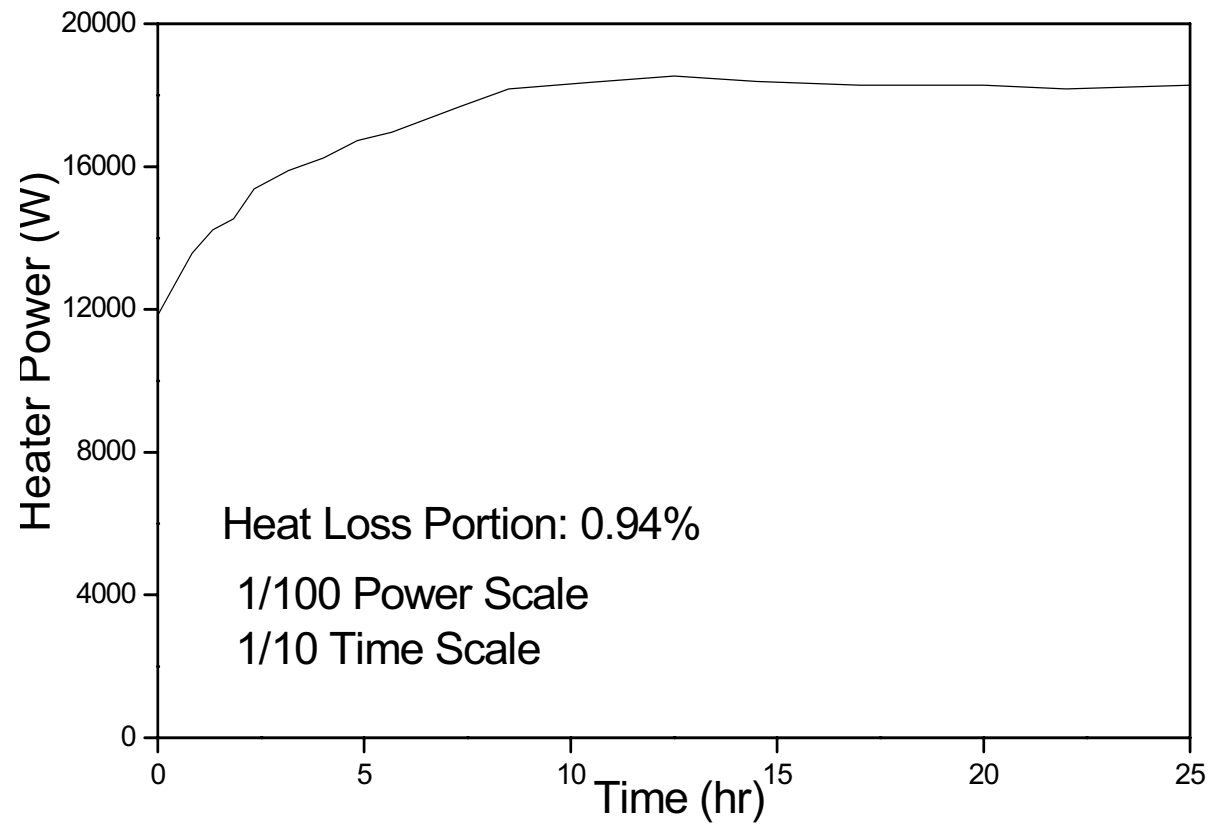

Figure 3-44 Power transient during the LOFC experiment

\section{$\underline{\text { 3.4-1 Scaling Analvsis }}$}

The present test facility is the $1 / 10$ linear scaled model of the PBMR and it has $1 / 100$ power scale, $1 / 1000$ volume scale and 1/100 area scale with applying the linear scaling methodology (W.A. Carbiener and R.A. Cudnik 1969). So the heat flux is preserved in the proto and model and the time scale is reduced to 1/10. Even though there is no prototype water pool, scaling analysis for the temperature in the water pool type 
RCCS was carried out to derive the similarity criteria of the reactor vessel temperature. For the simple scaling analysis, the axial distribution of temperatures was not considered.

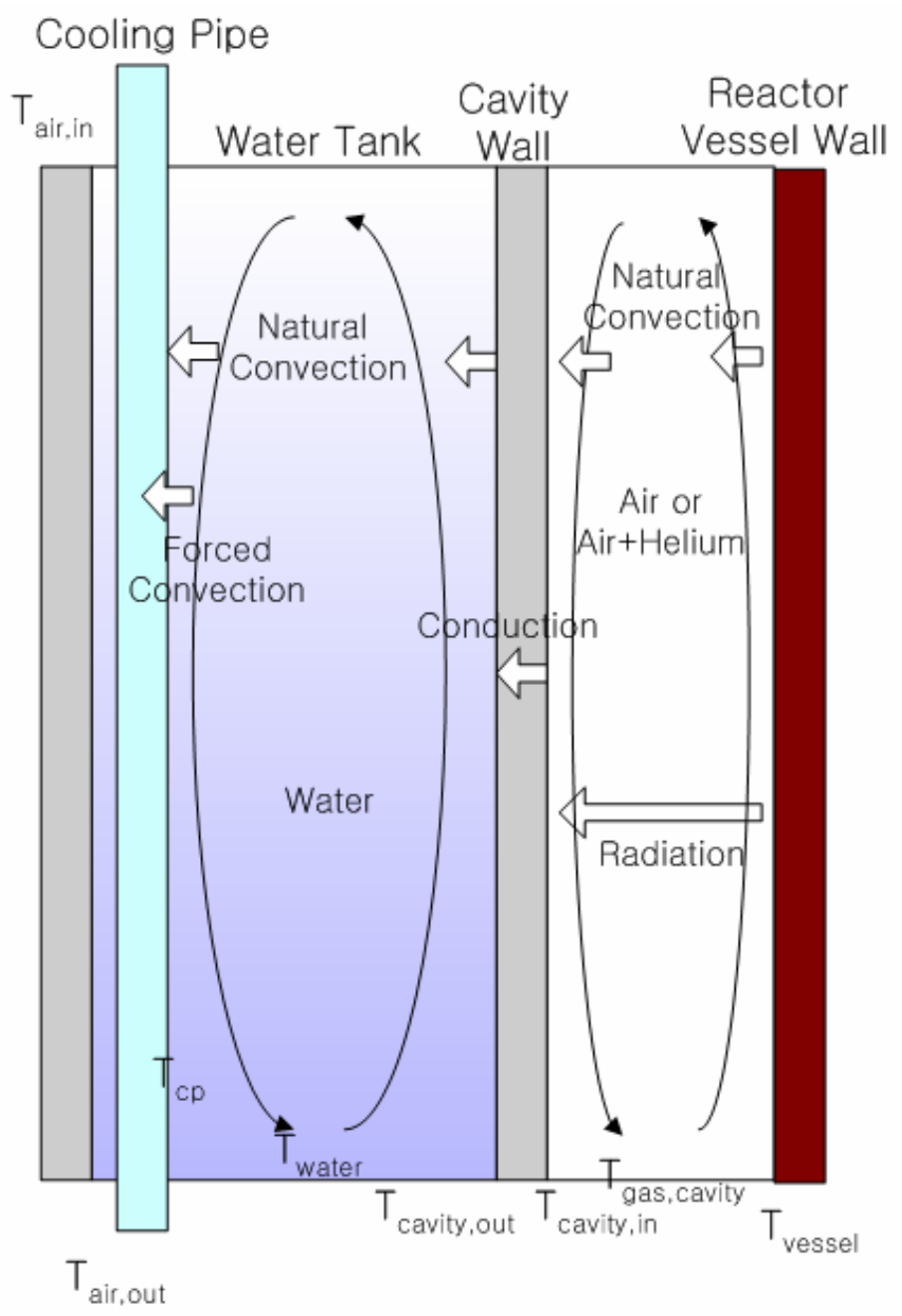

Figure 3-45 Heat transfer process in the water pool type RCCS

The heat transfer procedure in the water pool type RCCS is illustrated in Figure 3-35 and energy balance equations of each process are as follows.

$$
\begin{aligned}
& =\frac{2 \pi L_{\text {cavity }} k_{\text {cavity }}\left(T_{\text {cavity, in }}-T_{\text {cavity, out }}\right)}{\ln \left(R_{\text {cavity, out }} / R_{\text {cavity, in }}\right)} \\
& =h_{\text {gas, cavity }} A_{\text {cavity }}\left(T_{\text {gas, cavity }}-T_{\text {cavity, in }}\right)+\frac{\sigma A_{\text {vessel }}}{\left(\frac{1-\varepsilon_{\text {cavity }}}{\varepsilon_{\text {cavity }}}+1+\frac{1-\varepsilon_{\text {vessel }}}{\varepsilon_{\text {vessel }}}\right)}\left(T_{\text {vessel }}^{4}-T_{\text {cavity, in }}^{4}\right) \\
& =h_{\text {vessel }} A_{\text {vessel }}\left(T_{\text {vessel }}-T_{\text {gas,cavity }}\right)+\frac{\sigma A_{\text {vessel }}}{\left(\frac{1-\varepsilon_{\text {cavity }}}{\varepsilon_{\text {cavity }}}+1+\frac{1-\varepsilon_{\text {vessel }}}{\varepsilon_{\text {vessel }}}\right)}\left(T_{\text {vessel }}^{4}-T_{\text {cavity, in }}^{4}\right)
\end{aligned}
$$

Above equations can be normalized by following non-dimensional parameters. 


$$
\begin{aligned}
& \theta_{c p, \text { in }}^{*}=\frac{T_{c p}-T_{\text {air }, \text { in }}}{\Delta T_{\text {air }}} \quad \theta_{c p, \text { out }}^{*}=\frac{T_{c p}-T_{\text {air }, \text { out }}}{\Delta T_{\text {air }}} \quad \theta_{\text {water }}^{*}=\frac{T_{\text {water }}-T_{\text {air }, \text { in }}}{\Delta T_{\text {air }}} \quad \theta_{\text {cavity,in }}^{*}=\frac{T_{\text {cavity, in }}-T_{\text {air }, \text { in }}}{\Delta T_{\text {air }}} \\
& \theta_{\text {cavity }, \text { out }}^{*}=\frac{T_{\text {cavity }, \text { out }}-T_{\text {air }, \text { in }}}{\Delta T_{\text {air }}} \\
& \theta_{\text {gas }, \text { cavity }}^{*}=\frac{T_{\text {gas }, \text { cavity }}}{T_{\text {cavity }, \text { in }}} \quad \theta_{\text {vessel }}^{*}=\frac{T_{\text {vessel }}}{T_{\text {cavity }, \text { in }}}
\end{aligned}
$$

The normalized equations are expressed as

$$
\begin{aligned}
& \frac{\theta_{c p, \text { out }}^{*}-\theta_{c p, \text { in }}^{*}}{\ln \left(\theta_{c p, \text { out }}^{*} / \theta_{c p, \text { in }}^{*}\right)}=\frac{\operatorname{Re}_{\text {air }} \operatorname{Pr}_{\text {air }}}{4 N u_{c p}} \frac{D_{c p}}{L_{c p}}=\frac{\operatorname{Re}_{\text {air }} \operatorname{Pr}_{\text {air }}}{4 \cdot 0.328 \operatorname{Re}_{\text {air }}^{0.58} \operatorname{Pr}_{\text {air }}^{0.4}} \frac{D_{c p}}{L_{c p}}=\Pi_{1} \\
& \theta_{\text {water }}^{*}-\theta_{c p}^{*}=\frac{\dot{q}}{0.046 \pi D_{c p} L_{c p} \Delta T_{\text {air }}^{4 / 3} k_{\text {water }}}\left(\frac{g \beta\left(\theta_{\text {water }}^{*}-\theta_{c p}^{*}\right)}{v \alpha}\right)^{-1 / 3} \\
& =\Pi_{2}\left(\theta_{\text {water }}^{*}-\theta_{c p}^{*}\right)^{-1 / 3} \\
& \theta_{\text {cavity, out }}^{*}-\theta_{\text {water }}^{*}=\frac{\dot{q}}{0.046 k_{\text {water }}} \frac{1}{D_{\text {cavity }} L_{\text {cavity }}}\left(\frac{g \beta \Delta T_{\text {air }}}{v \alpha}\right)^{-1 / 3} \\
& \cdot\left(\theta_{\text {cavity }, \text { out }}^{*}-\theta_{\text {water }}^{*}\right)^{-1 / 3}=\Pi_{3}\left(\theta_{\text {cavity }, \text { out }}^{*}-\theta_{\text {water }}^{*}\right)^{-1 / 3} \\
& \left(\theta_{\text {cavity, in }}^{*}-\theta_{\text {cavity,out }}^{*}\right)=\frac{\dot{q} \ln \left(R_{\text {cavity, out }} / R_{\text {cavity }, \text { in }}\right)}{2 \pi L k \Delta T_{\text {air }}}=\Pi_{4} \\
& \frac{h_{\text {gas, cavity }} A_{\text {cavity }} T_{\text {cavity, in }}}{\dot{q}}\left(\theta_{\text {gas,cavity }}^{*}-1\right)+\frac{\sigma A_{\text {vessel }} T_{\text {cavity, in }}}{\dot{q}\left(\frac{1-\varepsilon_{\text {vessel }}}{\varepsilon_{\text {cavity }}}+1+\frac{1-\varepsilon_{\text {vessel }}}{\varepsilon_{\text {cavity }}}\right)}\left(\theta_{\text {vessel }}^{* 4}-1\right) \\
& =\Pi_{5}\left(\theta_{\text {gas }, \text { cavity }}^{*}-1\right)+\Pi_{6}\left(\theta_{\text {vessel }}^{* 4}-1\right)=1 \\
& \frac{h_{\text {vessel }} A_{\text {vessel }} T_{\text {cavity, in }}}{\dot{q}}\left(\theta_{\text {vessel }}^{*}-\theta_{\text {gas,cavity }}^{*}\right)+\frac{\sigma A_{\text {vessel }} T_{\text {cavity, in }}}{\dot{q}\left(\frac{1-\varepsilon_{\text {vessel }}}{\varepsilon_{\text {cavity }}}+1+\frac{1-\varepsilon_{\text {vessel }}}{\varepsilon_{\text {cavity }}}\right)}\left(\theta_{\text {vessel }}^{* 4}-1\right) \\
& =\Pi_{7}\left(\theta_{\text {vessel }}^{*}-\theta_{\text {gas, cavity }}^{*}\right)+\Pi_{6}\left(\theta_{\text {vessel }}^{* 4}-1\right)=1
\end{aligned}
$$

Where, the heat transfer coefficient correlations of Seban (R.A. Seban and E.F. Mclaughlin 1963) for the forced convection in the helical coil and MacGregor (R.K. MacGregor et al. 1969) for the natural circulation in enclosures were introduced. The applied correlations are as follows.

$$
\begin{aligned}
& N u=0.328 \operatorname{Re}^{0.58} \operatorname{Pr}^{0.4} \\
& N u=0.046 R a_{L}^{1 / 3}
\end{aligned}
$$

From the algebraic analysis for the each pi parameter, we can obtain following similarity criteria for the reactor vessel temperature. 


$$
\frac{L_{c p, m}}{L_{c p, p}}=l_{R}^{1.42}, \frac{\ln \left(R_{\text {cavity }, \text { out }} / R_{\text {cavity }, \text { in }}\right)_{m}}{\ln \left(R_{\text {cavity }, \text { out }} / R_{\text {cavity, } \text { in }}\right)_{p}}=l_{R}^{-1}=10
$$

\section{4-2 Normal Operation Experiment}

Temperature distributions at the reactor vessel wall and cavity wall were measured in the experiments as shown in Figures 3-46. The temperature of the reactor vessel wall markedly increases along the axis from the bottom to the elevation of the third lowest thermocouple, because of the thermal stratification of air inside the vessel. Above the elevation of the third lowest thermocouple, however, the temperature of

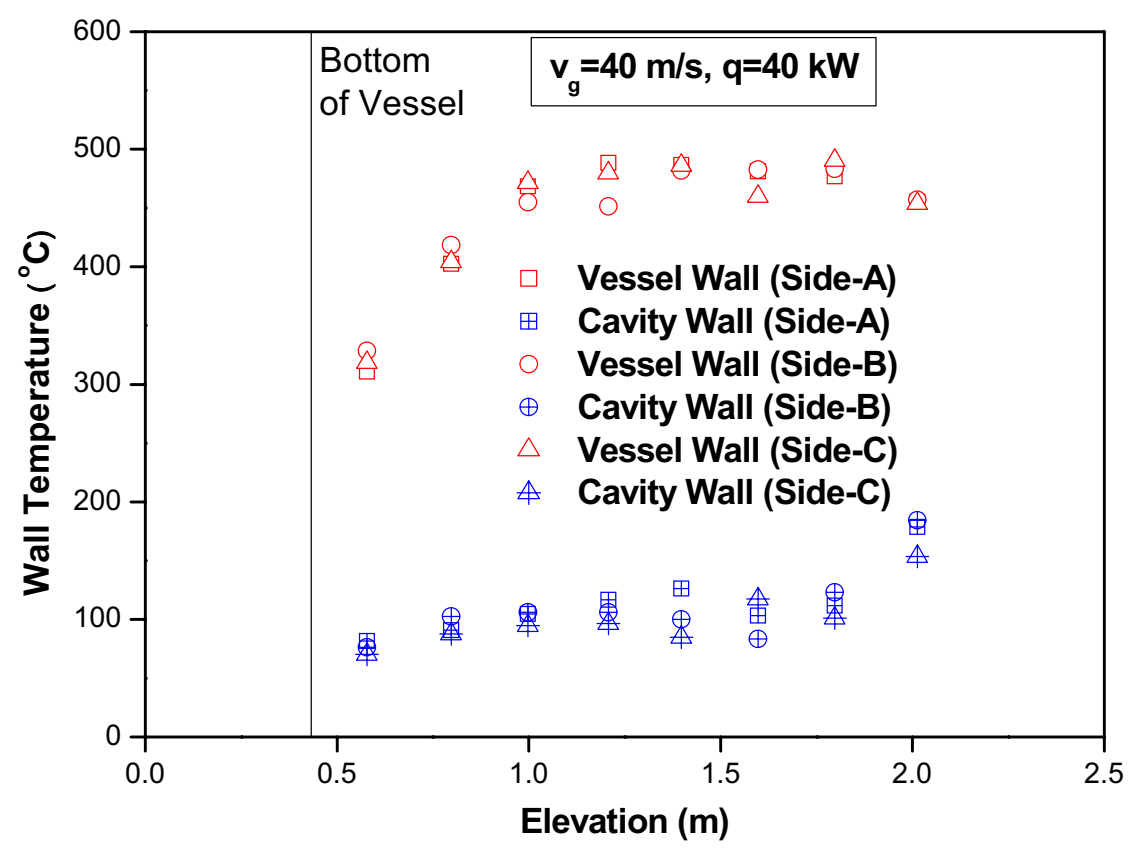

(a) $\mathrm{v}_{\mathrm{g}}=40 \mathrm{~m} / \mathrm{s}, \mathrm{Q}=40 \mathrm{~kW}(2 \%$ Heat Loss $)$ 


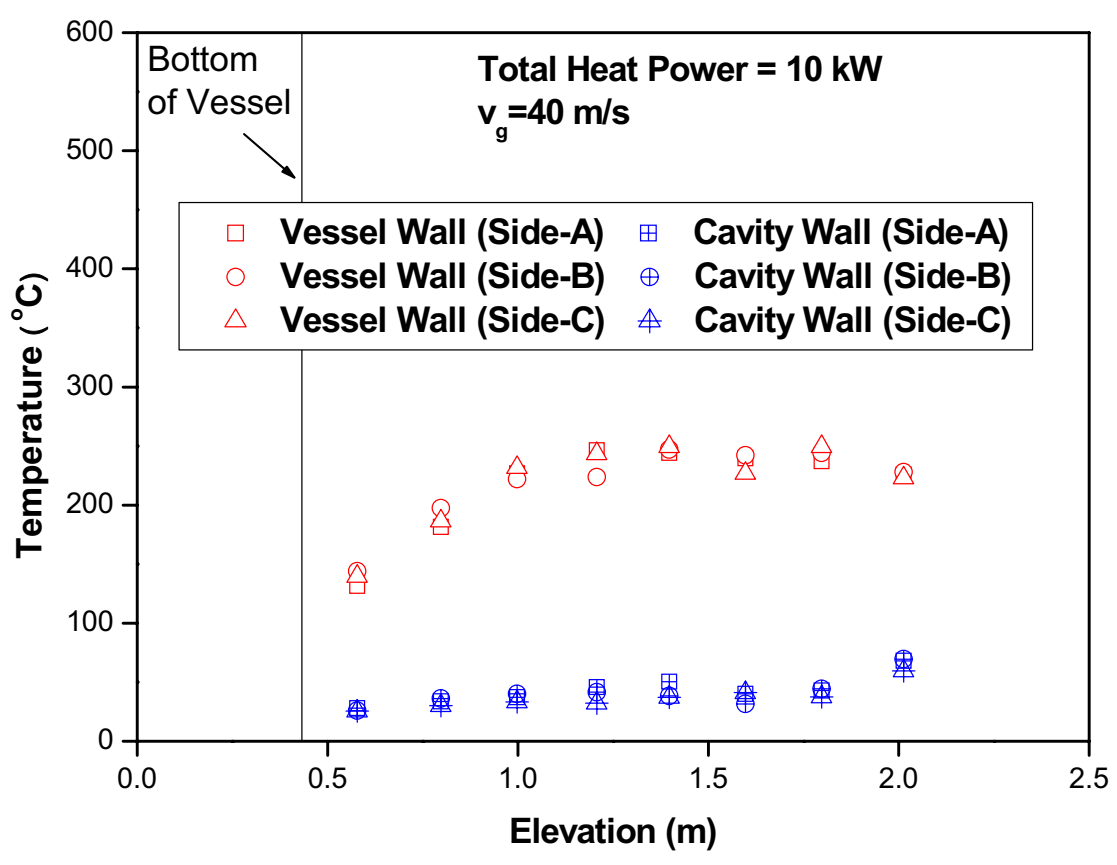

(b) $\mathrm{v}_{\mathrm{g}}=40 \mathrm{~m} / \mathrm{s}, \mathrm{Q}=10 \mathrm{~kW}(0.5 \%$ Heat Loss $)$

Figure 3-46 Axial distribution of the reactor vessel and cavity temperatures

the vessel wall remains nearly constant along the vertical axis. At the top of the reactor vessel, where the elevation is higher than that at the top of the active heater, the temperature of the wall decreases slightly because of the decrease in the radiative heat transfer from the heater. The temperature of the cavity wall, however, has a different profile from that of the vessel wall. Even though the heat source has a large temperature variation along the vertical direction of about $150{ }^{\circ} \mathrm{C}$, the temperature of the cavity wall does not show a rapid increase, but rather increases slightly along the vertical axis, except for the top part of the cavity wall where there is no water to cool the cavity wall. It is estimated that there exists a large gradient of the cavity wall temperature across the water level and the uncovered part of the cavity by the water may remain uncooled in the system. However, the existence of this uncooled section does not seem to significantly affect the radiative heat transfer from the reactor vessel, because the view factor of the uncooled region is very small when compared with that of the other region of the cavity wall. Although the effect of the uncooled region is not significant, it would be advisable for the area of the uncooled region to be reduced as much as possible for the optimization of the system.

It should be noted that the maximum temperature of the reactor vessel wall was kept below the design limitation of $300^{\circ} \mathrm{C}$. The observed maximum temperature was $265^{\circ} \mathrm{C}$ and this means that the water pool type RCCS removes the heat released from the reactor vessel sufficiently well. However, it would not be acceptable for the capacity of the system to be over-designed, because excessive parasitic heat losses are undesirable during normal operation and, consequently, more effort needs to be made to optimize some important parameters, such as the water pool temperature and air velocity. 


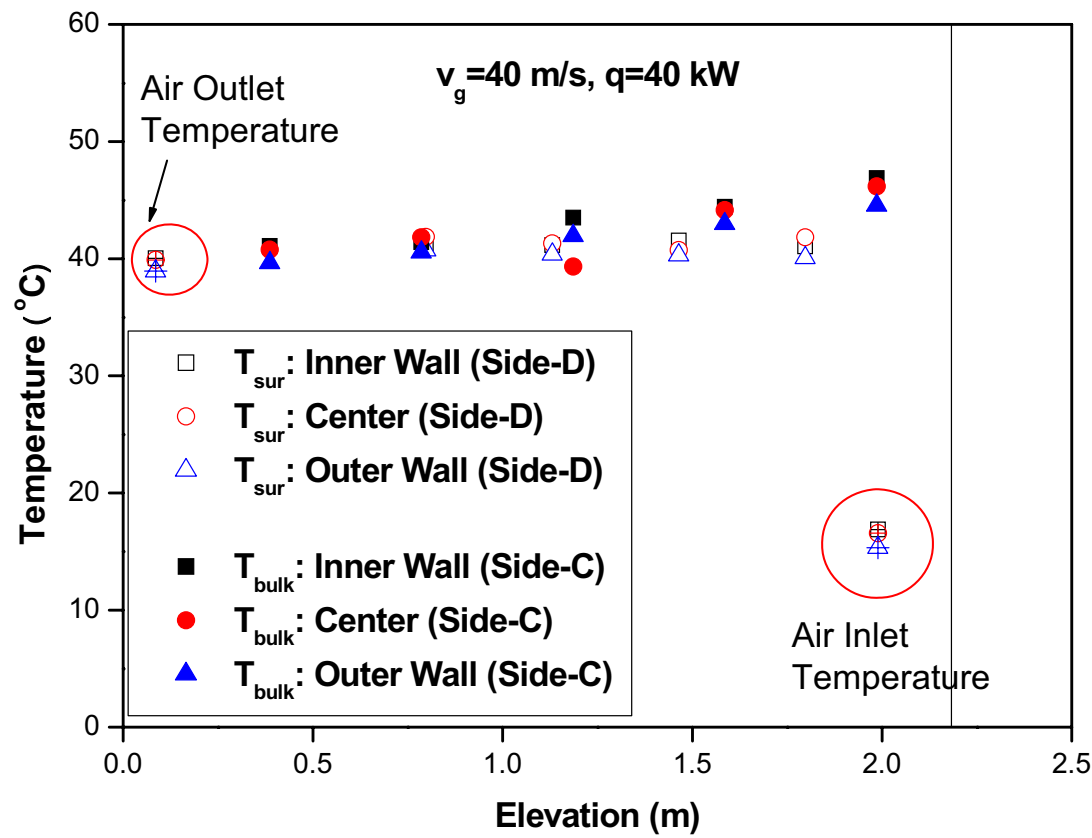

(a) $\mathrm{v}_{\mathrm{g}}=40 \mathrm{~m} / \mathrm{s}, \mathrm{Q}=40 \mathrm{~kW}(2 \%$ Heat Loss $)$

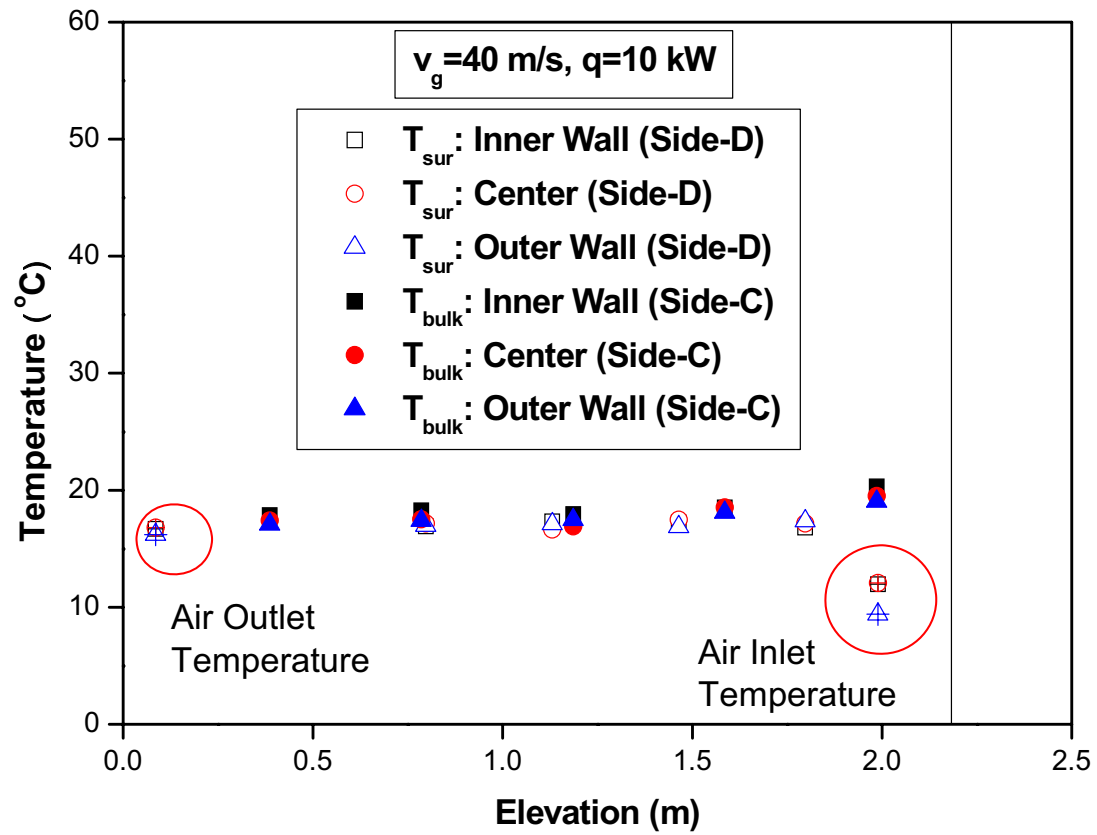

(b) $\mathrm{v}_{\mathrm{g}}=40 \mathrm{~m} / \mathrm{s}, \mathrm{Q}=10 \mathrm{~kW}(0.5 \%$ Heat Loss $)$

Figure 3-47 Axial distribution of the bulk and cooling pipe surface temperature 
In Figure 3-47, the temperature distributions along the elevation in the side pool and those at the surfaces of the cooling pipes are presented. The former were measured at three different radial positions, viz. the inner part (10 $\mathrm{mm}$ from the inner wall of the side pool), middle part (at the center of the side pool) and outer part (10 $\mathrm{mm}$ from the outer wall). The latter were measured at the surfaces of the three cooling pipes located in the inner part, middle part and outer part, respectively. The difference in the bulk liquid temperature between the top and bottom of the water pool is about $2 \sim 4^{\circ} \mathrm{C}$ and no thermal stratification of the water was observed in the present experiments. Also, the deviation of the liquid temperature between the inner part and outer part of the water pool is less than $2^{\circ} \mathrm{C}$. These results imply that a large portion of the heat is removed at the top part, where the water temperature varies significantly from the air temperature. From these experimental results, it was concluded that the characteristics of the system, in which relatively cold ambient air is inhaled from the top part of the water pool, allow effective natural circulation to be achieved in the water pool. The cooling pipe surface temperatures are nearly constant along the elevation and in the radial direction and, consequently, the assumption of a constant surface temperature boundary condition is reasonable for the analysis of the cooling system.

Comparing the temperatures between the cavity wall and bulk liquid temperature near the water tank inner surface, it seems that large gradients exist as shown in Figure 3-48. The narrow gap between the cooling pipe and heating surface makes the effect of viscous force significant and it reduces the heat transfer of natural convection in that region as same as in the enclosures which have narrow gap and large aspect ratio (Incropera and Dewitt, 1996). Because of the low heat transfer coefficient, the water temperature decreases rapidly at the vicinity of the heating surface.

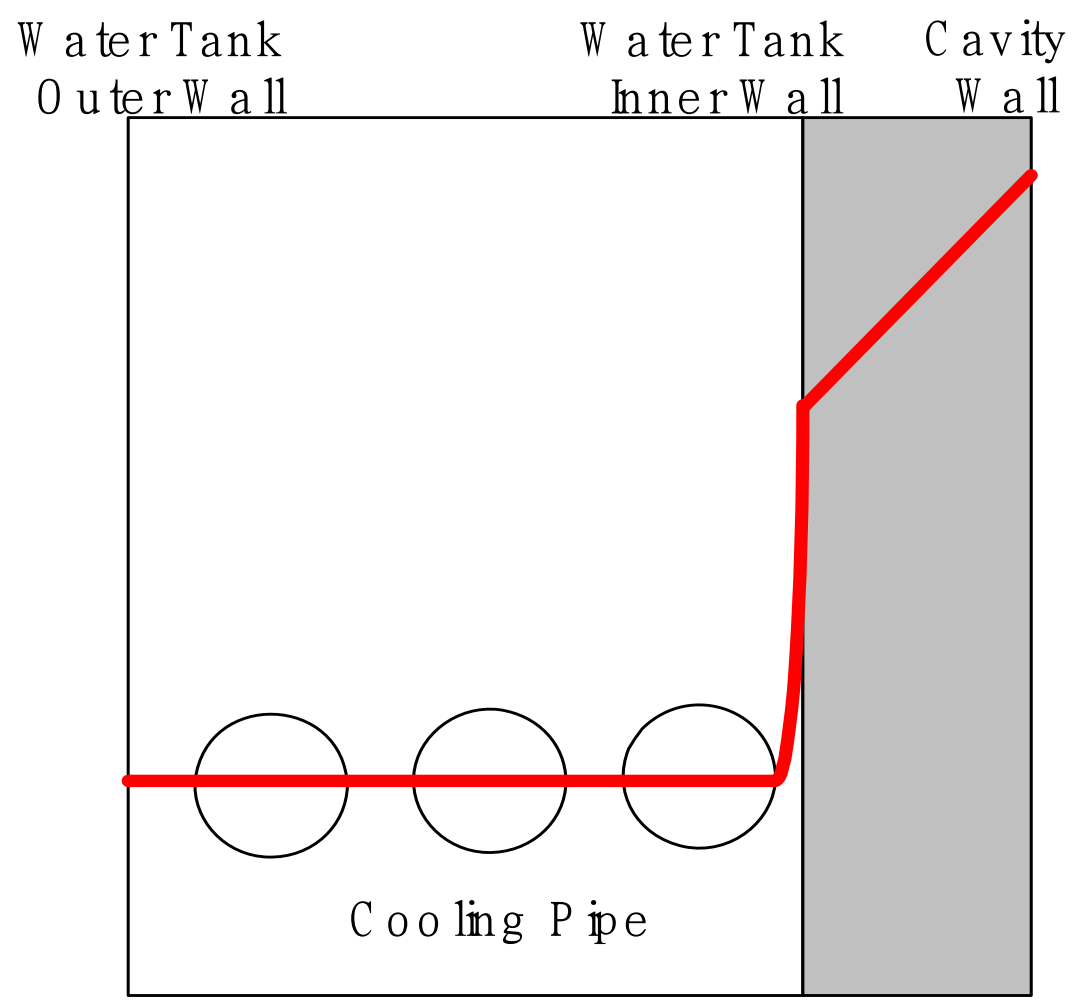

Figure 3-48 Temperature profile near the water tank inner wall

In the current design of the RCCS-SNU, the upper pool was installed separately from the side water pool. In order to optimize the capacity of the upper water pool, it is necessary to know the proportion of the released heat that is transferred to the upper pool. For this reason, the proportion of heat removed by the upper pool 
was observed under various power and gas velocity conditions, and the results are presented in Figure 3-49. The experimental results showed that $13 \sim 23 \%$ of the released heat was removed by the upper pool, depending on the heater power. Since, in the cavity, the heated air at the reactor vessel wall flows upward to the upper part of the cavity by natural circulation, the amount of heat removed by the upper pool is proportional to that removed by natural convective heat transfer. The temperature of the reactor vessel wall rises in proportion to the heating power and subsequently augments the effect of the radiation on the total heat transfer. As the proportion of radiation in the total heat transfer increases, that of the natural convective heat transfer is reduced and, consequently, the proportion of the heat removed by the upper water pool decreases with increasing heating power, as shown in Figure 3-49.

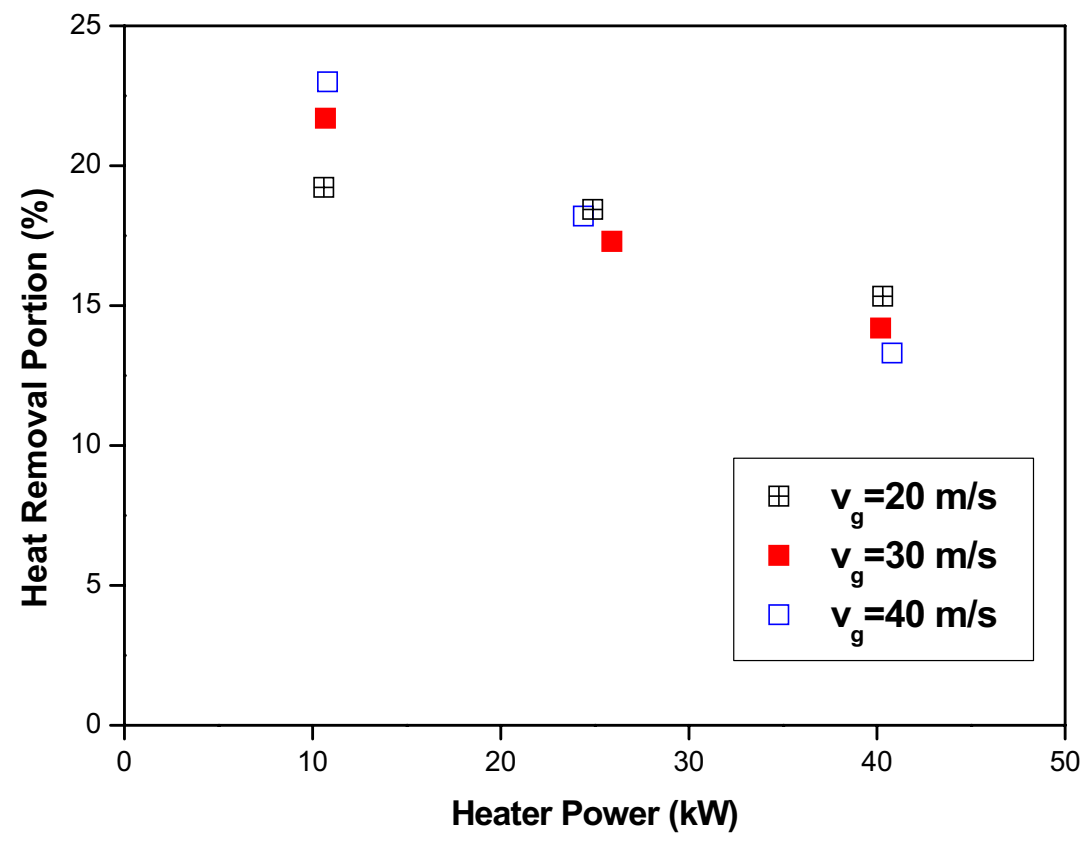

Figure 3-49 Heat removal portion of the upper tank

From these experimental results, we can obtain detailed information on the proportion of heat removed by the upper water pool and these experimental results form the basis of the design guidelines for the upper pool cooling system. In the optimized design, the cooling capacity of the upper water pool should be sufficient to remove about $23 \%$ of the heat released from the reactor vessel.

\section{4-3 Side Pool 1/4 Cooling Failure Experiment}

For the sake of redundancy, the RCCS should be designed to have enough cooling capability for normal operation, even in the case where one of the four trains of the air cooling systems in the side pool is unavailable. To investigate the temperature distribution in the RCCS under these conditions, 1/4 train failure tests were carried out. As soon as one active cooling system is stopped, the overall temperature of the water pool increases gradually towards a new steady state condition. The experiment was continued until all of the measuring parameters reached this new steady state. 


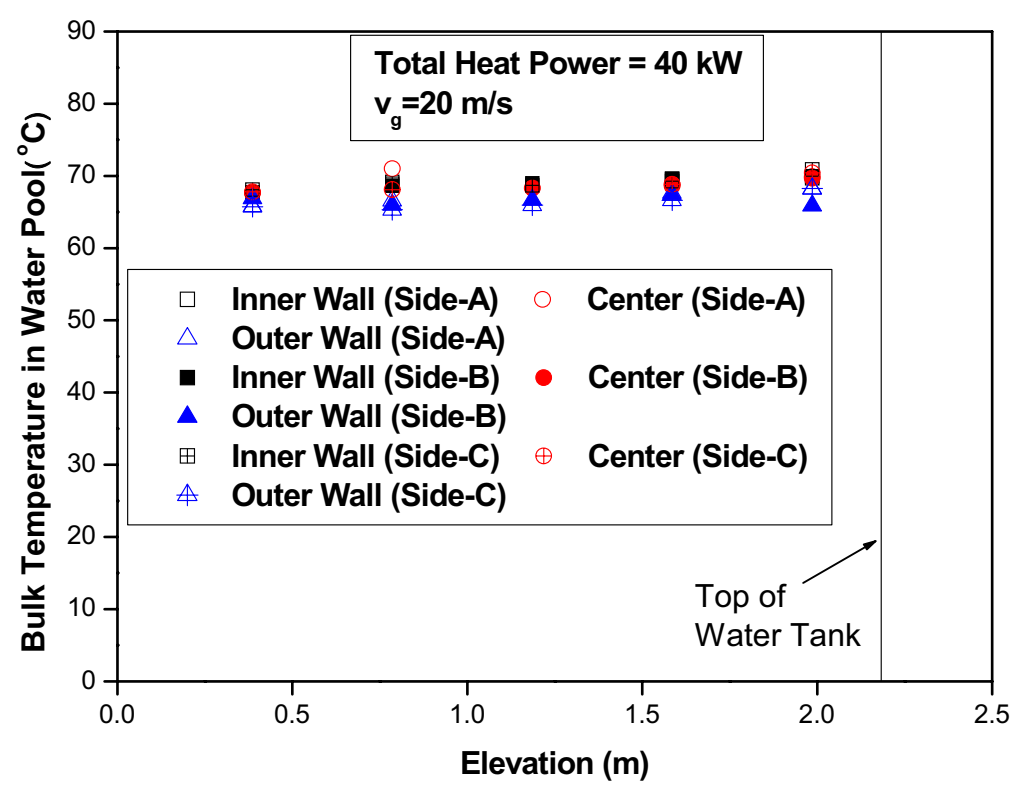

(a) Normal case

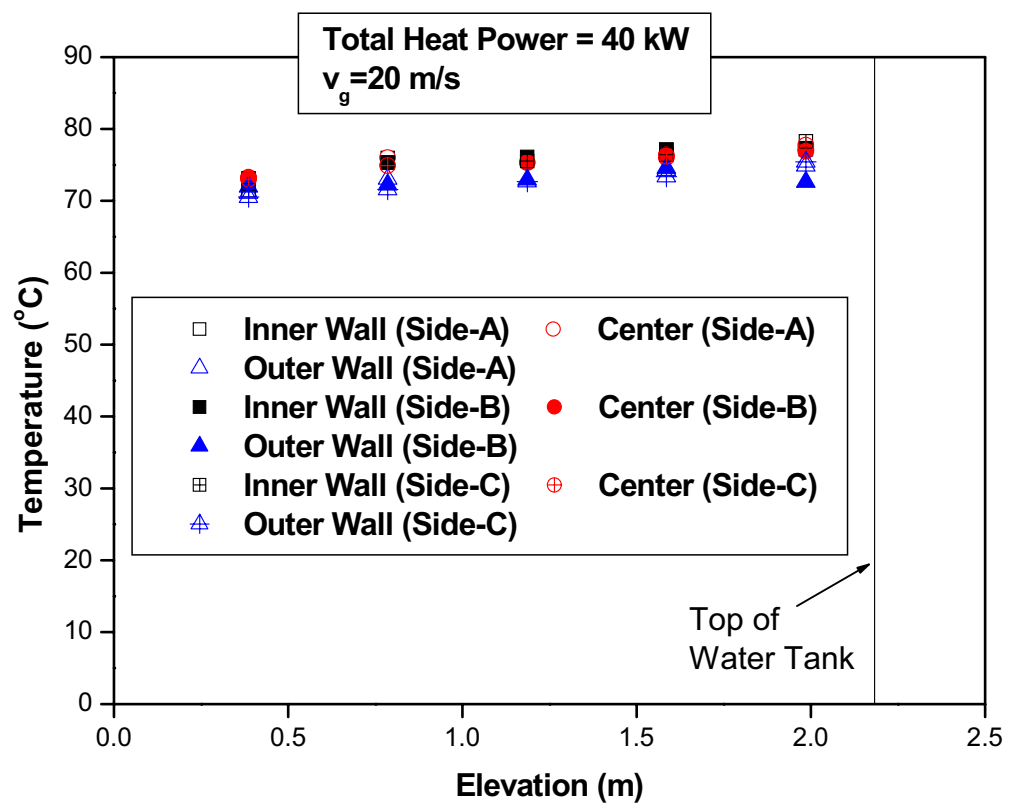

(b) $1 / 4$ train trip

Figure 3-50 Axial distribution of the bulk temperature in the water pool

In Figure 3-50, the experimental results of the normal operation and 1/4 failure cases were compared. As shown in this figure, the temperature in the water tank increases by about $5 \sim 6{ }^{\circ} \mathrm{C}$ in the failure test. However, no local increase of the water temperature in the water pool was observed on the failure side, and the differences in temperature between the active sides and the failure side were observed to be less than $2{ }^{\circ} \mathrm{C}$. This result means that the natural circulation in azimuthal direction was more driven in the $1 / 4$ failure cases. 
As a result of the increase of the water temperature in the case of the 1/4 air cooling system failure, the cavity and reactor vessel temperatures were raised by about $4 \sim 5^{\circ} \mathrm{C}$, but this is found to have a negligible effect, as shown in Figure 3-51.

From these results, we concluded that no undesirable local increase of the reactor vessel temperature is likely to occur as a result of a local failure of the water pool cooling system, since effective natural circulation is established in the water pool even in the case of a local cooling failure.

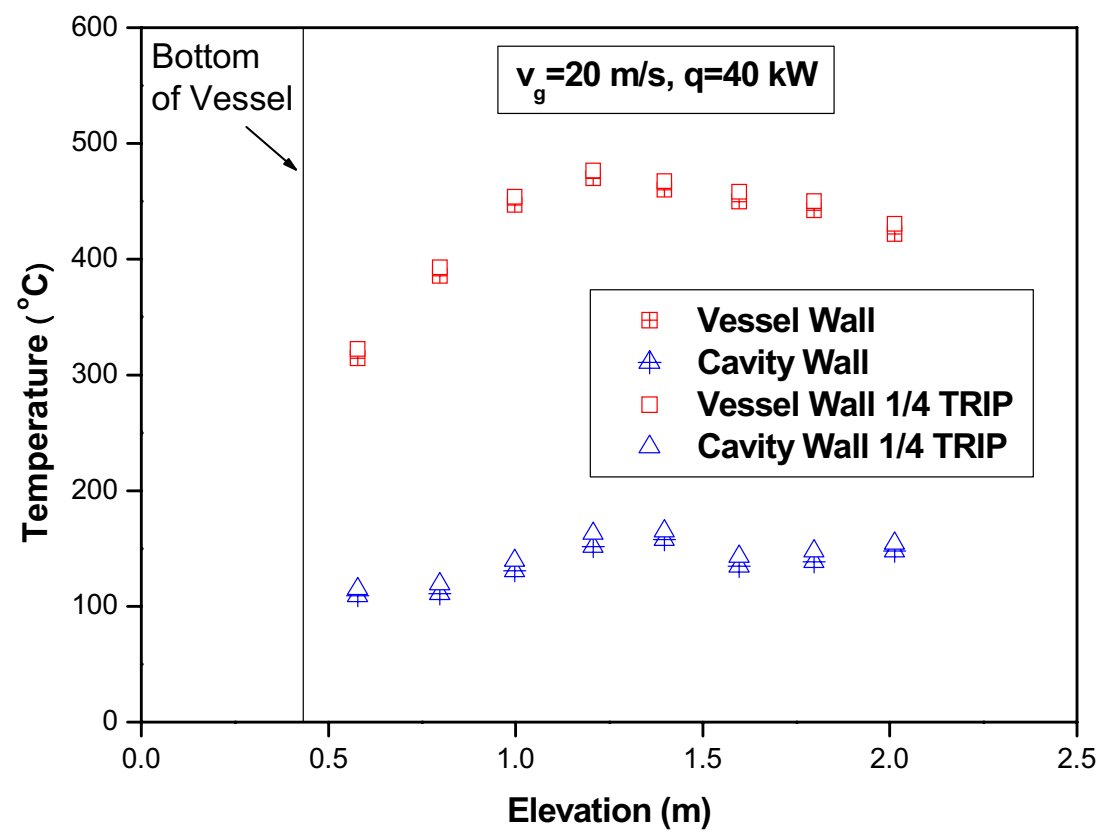

Figure 3-51 Axial distribution of the vessel and cavity temperatures: normal case and 1/4 train trip

\section{4-4 Upper Pool Cooling Failure Experiment}

To investigate the variation in temperature of the reactor vessel wall when the upper RCCS cooling system is unavailable, an upper pool cooling failure experiment was carried out. The pressure in the upper tank was maintained at atmospheric pressure and the relief valve installed at the upper pool was manually opened in order to accelerate the depletion of the water inventory by boiling off. Figure 3-52 shows the temperature transient of the cavity wall and the water in the upper pool. When the active cooling was stopped, the water temperature of the upper pool increased, and reached to the saturation temperature of $100{ }^{\circ} \mathrm{C}$ at about 10 hours and remains constant at this temperature until the entire reserve of water was depleted. The temperature measured by the highest elevation thermocouple near the water level showed the same 


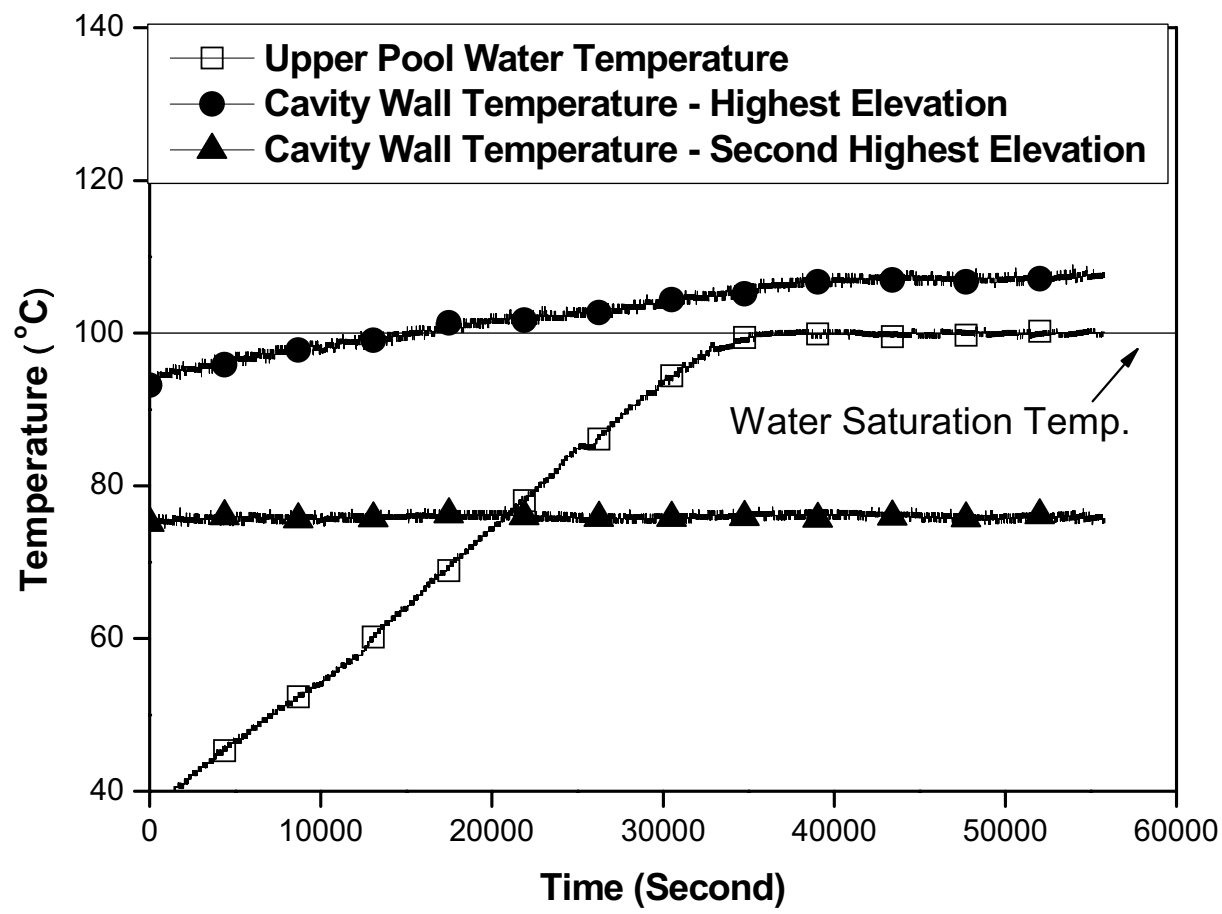

Figure 3-52 Temperature Transient during the Upper Cooling Trip Test

trend as that of the upper pool temperature, and remains constant after the water in the upper pool reaches the saturation temperature. The temperature below the elevation of the second highest thermocouple did not change during the experiment.

The transient of the axial temperature distribution of the reactor vessel wall is presented in Figure 3-53. The temperature near the top of the reactor vessel wall increases when the upper cooling is stopped. However, it does not increase any more once the cavity wall temperature reaches a steady state. These experimental results showed that the heat transferred to the upper pool can be removed to a sufficient extent by the sensible and latent heat transfer until the entire reserve of water is depleted, even in the case where the active cooling provided by the upper pool is unavailable.

However, if the entire contents of the upper water pool are depleted by boiling off, it can be surmised that the top part of the cavity wall and the reactor vessel wall temperature would likely increase and, therefore, it is recommended that an additional train be added to the upper pool cooling system in order to optimize the design of the water pool type RCCS. 


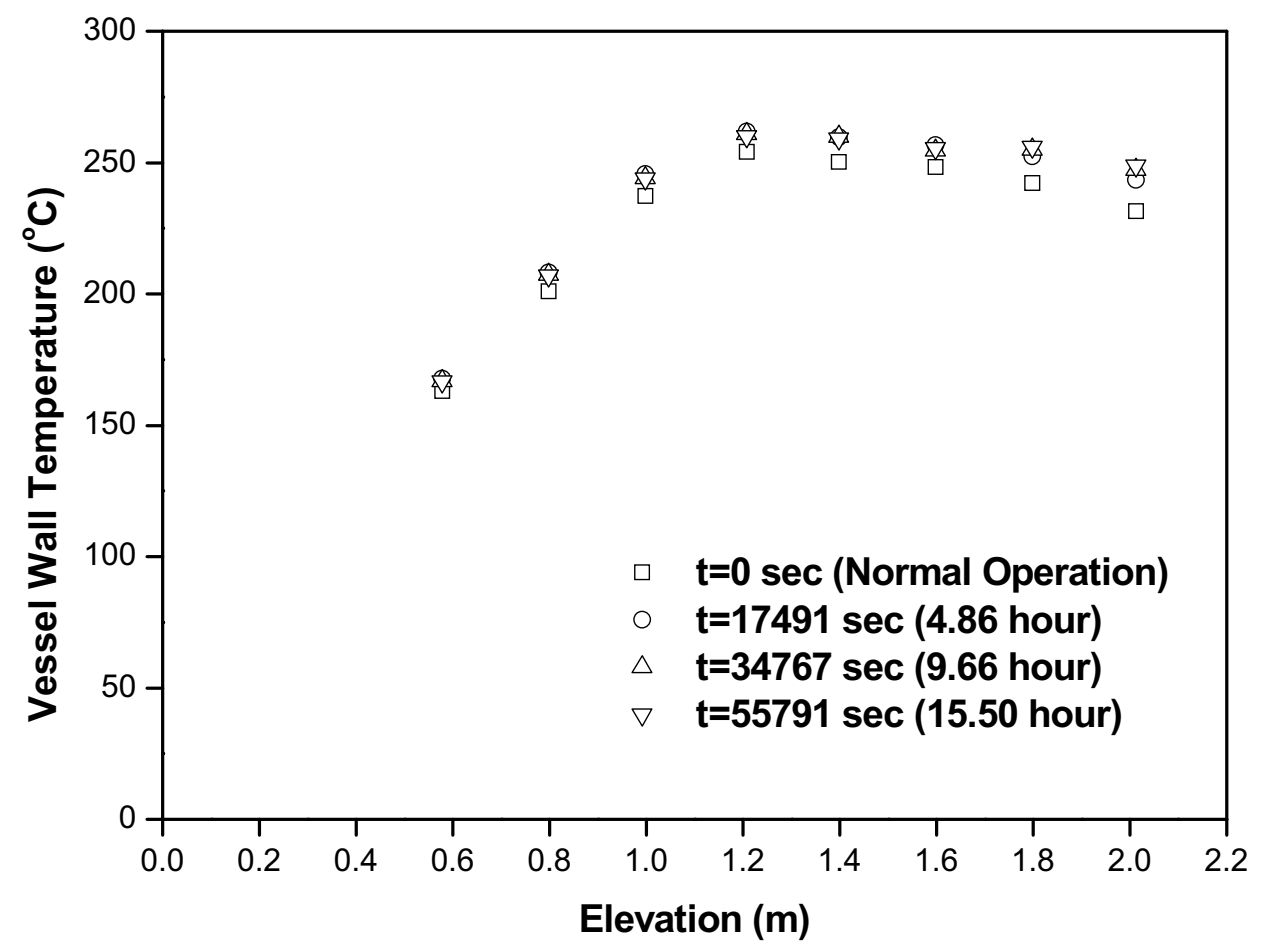

Figure 3-53 Vessel Wall Temperature Transient during Upper Cooling Trip Test

\section{4-5 LOFC Experiment}

An experiment was carried out to simulate an LOFC accident of the HTGR, which corresponds to the case where the failure of the forced convection of the main cooling system of the reactor is accompanied by the loss of all the other active cooling systems, including the RCCS air coolers. According to our design criteria, the RCCS-SNU should be able to remove the core afterheat passively for three days, which is equivalent to 7.2 hours $(25,920 \mathrm{sec})$ in the $1 / 10$ time reduced test facility. The present LOFC experiment, however, was continued for 25 hours, in order to ascertain what would happen when the water reserve was so depleted that the heat released from the reactor vessel could no longer be removed. The power transient during the LOFC accident is shown in Figure 3-43. To accentuate the depletion of the side pool and upper pool, in the present experiment it was assumed that the relief valve was stuck in the open position when the water in the water pools reached the saturation temperature. Then the relief valve installed at the water pool was manually opened at the moment. 


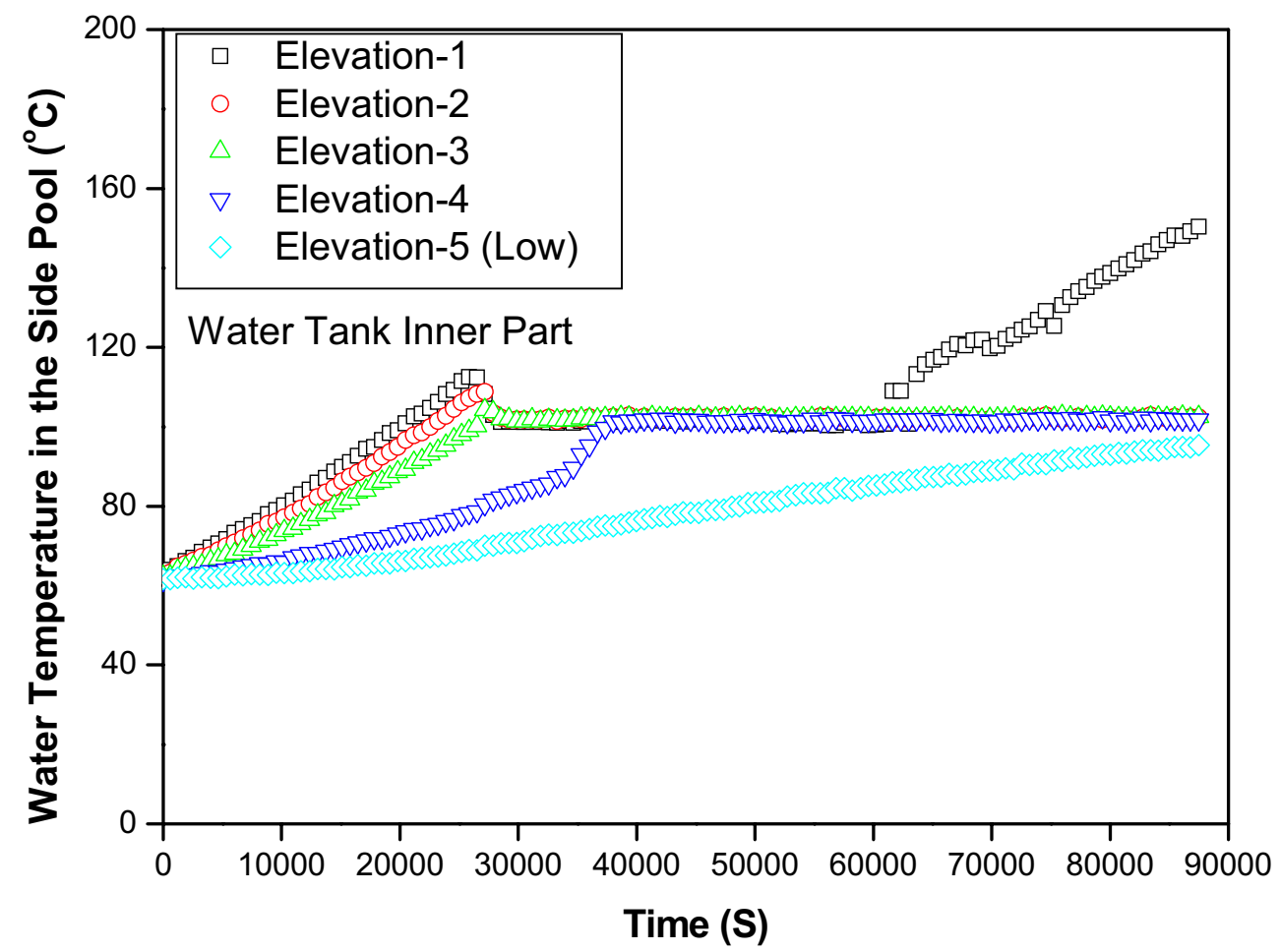

Figure 3-54 Temperature transients of the water during the LOFC Experiment

The temperature transient in the water pool is presented in Figure 3-54. During normal operation, the temperatures of the water pool were nearly constant along the vertical axis. However, following the failure of the entire RCCS active cooling system, and the increase in the amount of heat released from the reactor vessel, thermal stratification occurs within the water pool and, consequently, the temperature of the top part of the water pool increases rapidly. The relief valves were manually opened after about 25,000 seconds when the upper region water reached the saturation temperature at 1.5 bars. Then, the temperature of the upper part of the water pool gradually decreased towards $100^{\circ} \mathrm{C}$ and subsequently remained constant at this temperature. A lot of steam was generated in the water pool after the opening of the relief valve and the water level decreased, as shown in Figure 3-55. The slight increase in the water level which occurred before the relief valve was opened was caused by the swelling of the water. When the thermocouple in the top of water pool was uncovered due to the decrease in the water level after about $60,000 \mathrm{sec}$, the temperature measured by the thermocouple increased sharply because of the presence of superheated steam. 


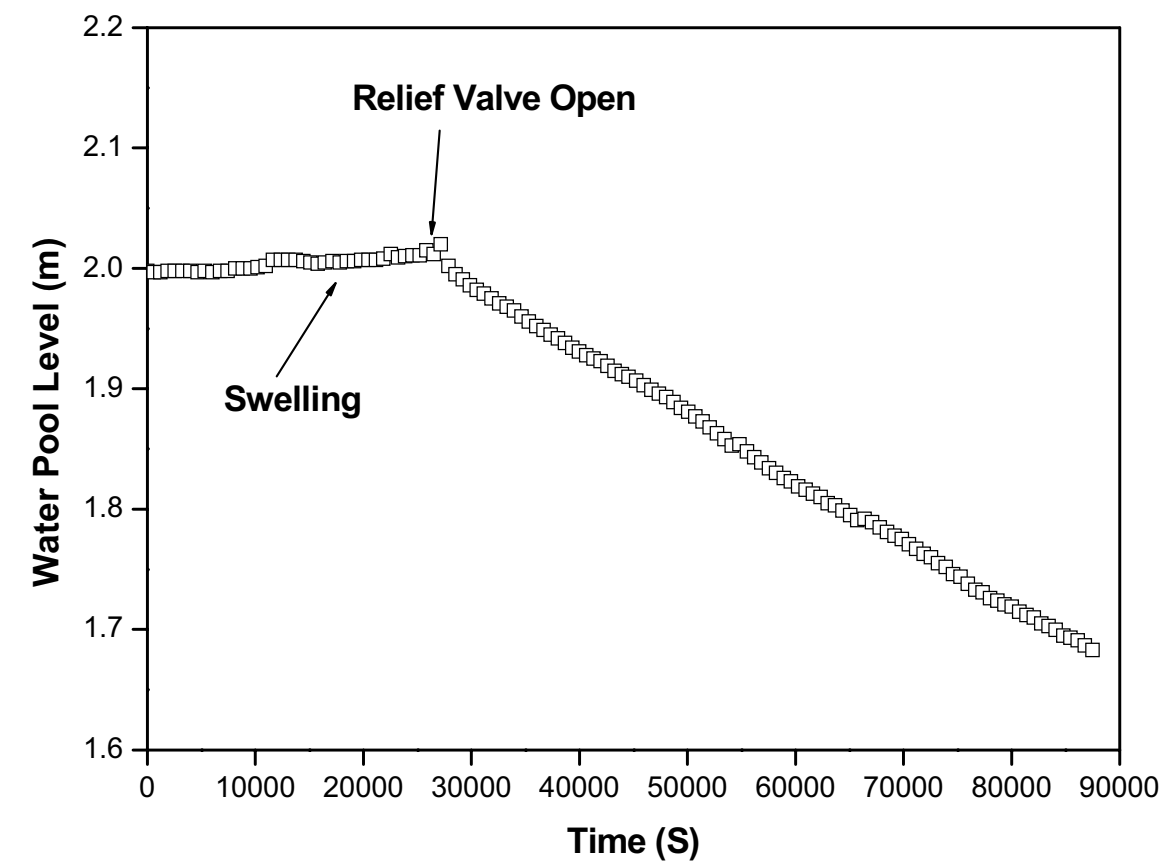

Figure 3-55 Water level transients in the side pool during the LOFC experiment

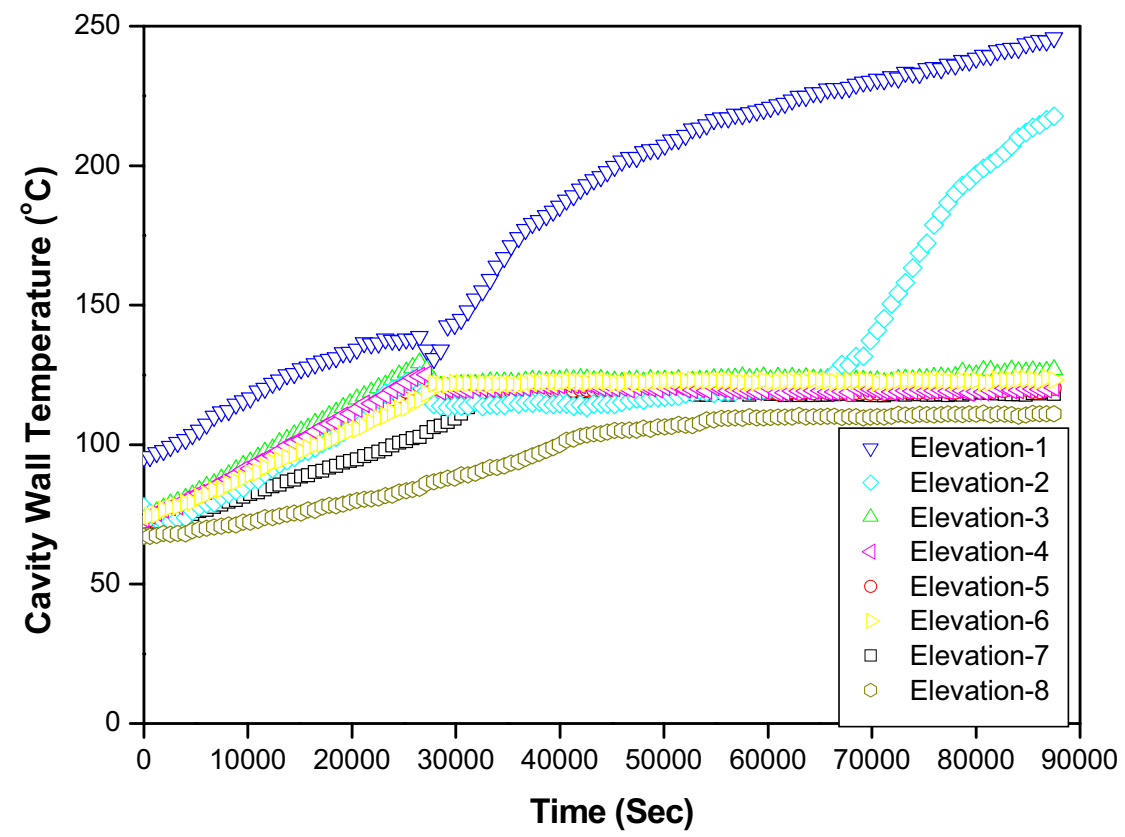

Figure 3-56 Temperature transients of the cavity wall during the LOFC experiment

Figure 3-56 shows the results for the temperature of the cavity wall during the LOFC experiment. The temperature of the cavity wall during normal operation increased slightly along the vertical axis, with the exception of the top part of the cavity. Once the LOFC accident began, however, it was affected by the thermal stratification of the water pool and the temperature at each elevation increased at a different rate. After about 25,000 seconds, the temperature of the cavity wall was held nearly constant except in the region that was not covered by water since the temperature of the heat sink, the water in the side pool, was fixed at 
the saturation point. In the dried out region, the temperature of the cavity wall increased rapidly, because the incoming heat could not be sufficiently dissipated.

Figure 3-57 shows the temperature transient of the reactor vessel wall. The temperature increased gradually when the amount of heat released

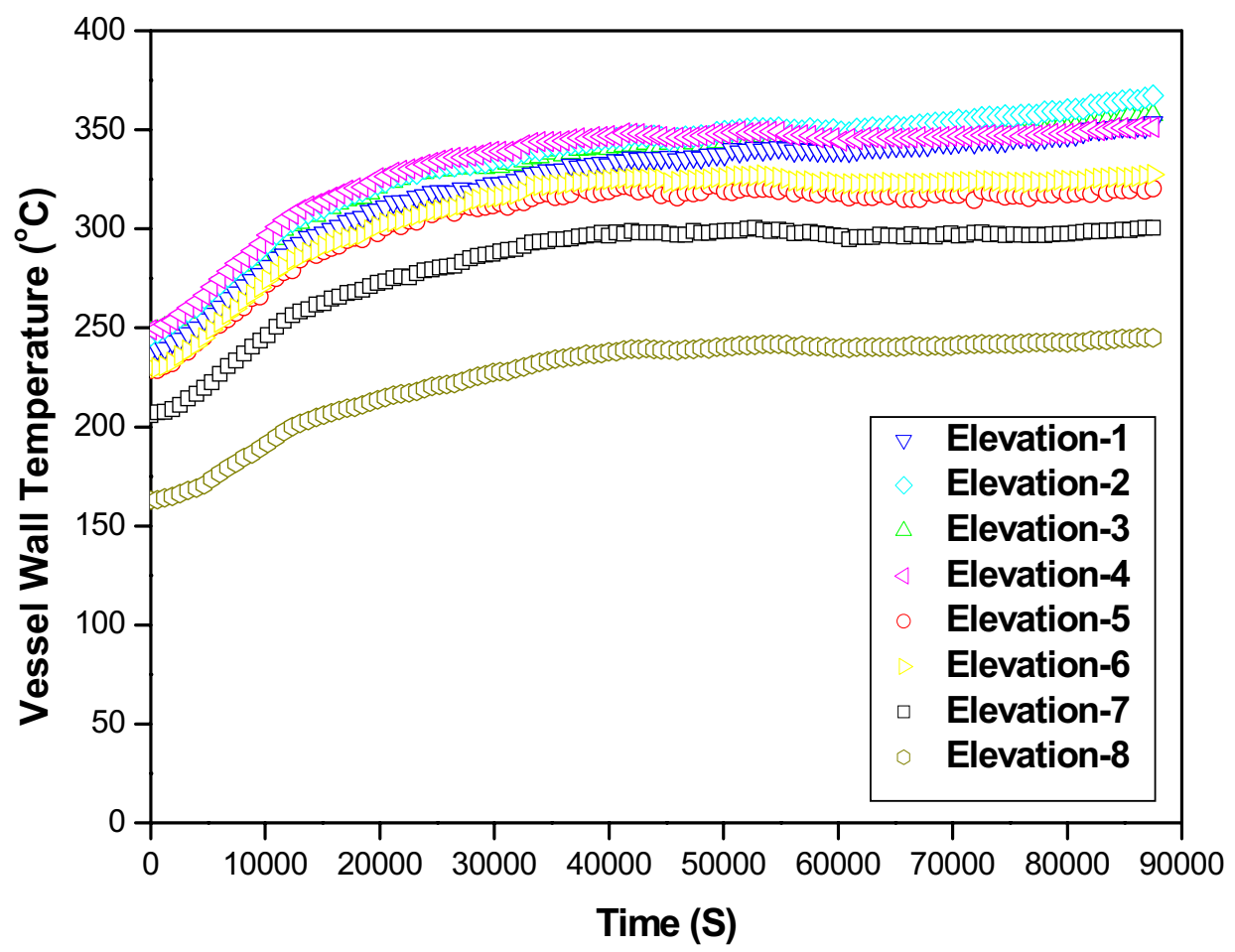

Figure 3-57 Temperature transients of the vessel wall during the LOFC experiment

from the heater increased as shown in Figure 3-43. After 40,000 seconds, the heating power remained constant until the end of the experiment and, therefore, the temperature of the wall of the reactor vessel remained almost constant except in the upper parts. The sharp increase in the cavity wall temperature in the dried out region does not seem to affect the reactor vessel temperature before 60,000 seconds, due to the much smaller view factor of the uncovered region as compared with that of the cooling region. However, the temperatures in the upper part of the reactor vessel began to increase after about 60,000 seconds. This means that the increase in the area of the uncovered cavity wall caused by the decrease in the water level began to affect the radiative heat transfer from the upper part of the reactor vessel. At this moment, the water level was reduced by $10 \%$, and about $12 \%$ of the total cavity wall length was no longer covered by the water. From this experimental result, we can conclude that a reduction in the water level of $10 \%$ due to boiling off would be allowed in the water pool type RCCS and this criterion will be used for the optimization of the system.

One of the design criteria of the current water pool type RCCS is that it should have a passive cooling capability of three days during an LOFC accident, which is equivalent to 7.2 hours $(25,920 \mathrm{sec})$ in the $1 / 10$ time reduced test facility. It should be noted that the maximum temperature of the vessel wall did not exceed the design limitation of the PBMR $\left(350^{\circ} \mathrm{C}\right.$ during the LOFC accident), as shown in Figure 3-57. From these experimental results, it can be concluded that the current design of the RCCS allows the afterheat to be sufficiently removed during an LOFC accident. 


\section{4-6 Emissivity Measurement Experiment}

The emissivity of the vessel surface of RCCS-SNU was measured via sight tube by the infrared thermometer. The sight tube was installed through the water pool and cavity to simplify geometry and facilitate temperature measurement for the calculation. Figure 3-58 depicts the process of emissivity measurement and calculation. First, the measured emissivity was compensated by the transmittance of window and the effect of air concentration was ignored from the result of SET device. Then the effect of background radiation was removed via solving a series of radiative heat transfer equations. This result was compared with the emissivity of the same material with the reactor vessel of the RCCS-SNU, named true-emissivity in this study, which was measured without any surrounding-surface near the target material in the SET device.

\section{Schematic Diagram of Experiments}

SNU-RCCS

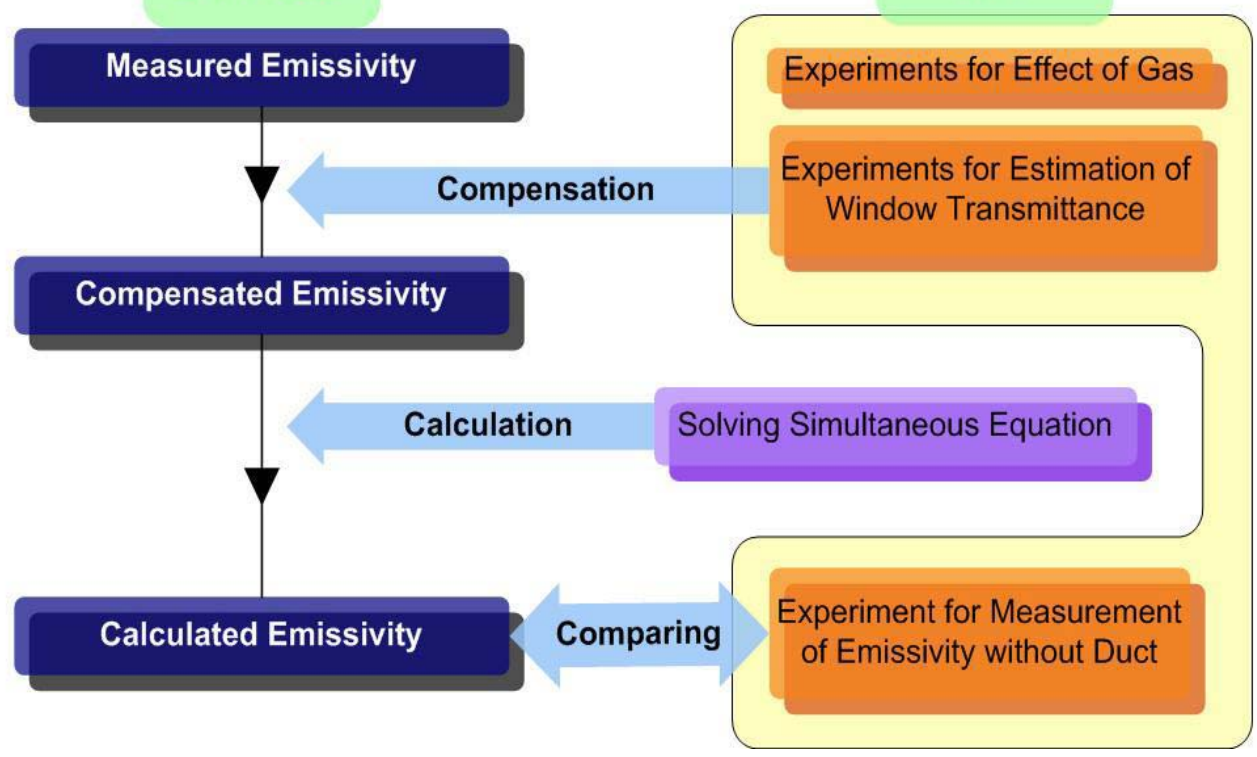

Figure 3-58 Process of emissivity measurement and calculation 


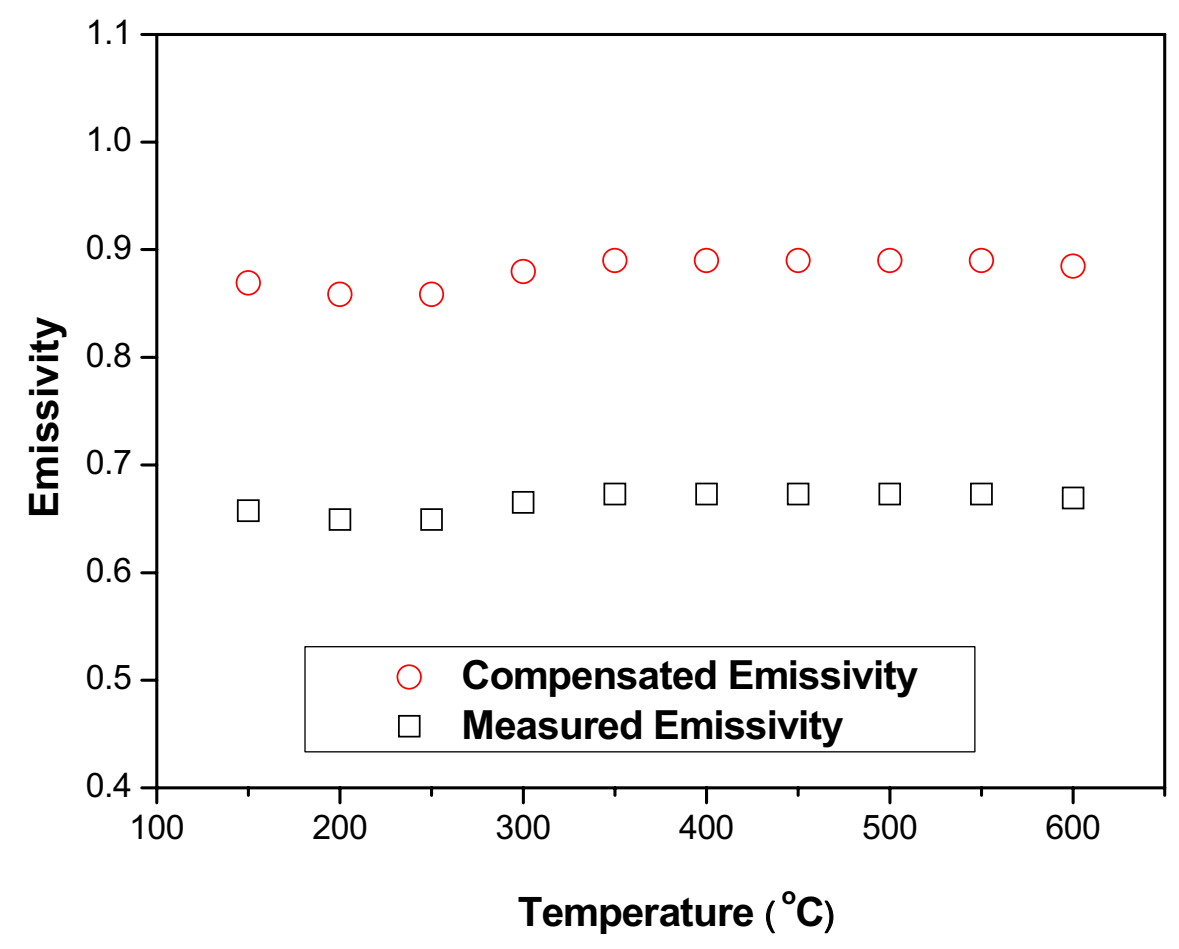

Figure 3-59 Temperature variation of measured emissivity with the sight tube

Figure 3-59 shows the measured emissivity at the middle height of the vessel surface with the sight tube in the RCCS-SNU. As the results of separate emissivity measurement device, the compensated emissivity was founded considering the transmittance of window and atmosphere.

The measured emissivity showed increasing trend with the temperatures. The increase in emissivity with temperature was due to both the inherent dependency of the emissivity on temperature and the background radiation by other surfaces. Therefore, the effect of background radiation needs to be removed from the analysis in order to find the true-emissivity as well as the temperature dependence of the emissivity.

The measured emissivity in the RCCS-SNU means the emissivity including background radiation. However, the true-emissivity which does not include the background radiation is required to analyze RCCS-SNU using MARS code. Therefore, in order to remove the effect of background radiation on the measured emissivity, the analysis was carried out with a simplified geometry furnished by the sight tube, as shown in Figure 3-60. The total number of divided surfaces was 8 and temperature was measured at each surface.

At first, the compensated emissivity was founded as mentioned before and then the calculation was carried out by solving a simultaneous equation, as shown in Equation (3-24). In this equation, $\mathrm{i}$ and $\mathrm{j}$ are indicial notation of surfaces: the target surface, the sight tube surface from 1 to 6 and the detector surface.

$$
\frac{E_{B, i}-J_{i}}{\left(1-\varepsilon_{i}\right) / \varepsilon_{i} A_{i}}=\sum_{j}^{N} A_{i} F_{i-j}\left(J_{i}-J_{j}\right) \quad(i \neq j)
$$

In these equations, the radiosity of the target material was obtained with the measured emissivity using Equation (3-25).

$$
J_{i}=E_{i}+\rho_{i} G=\varepsilon_{i} E_{B i}+\left(1-\varepsilon_{i}\right) G_{i}=\varepsilon_{m i} E_{B i}
$$

The view factors were calculated using commercial code, NEVADA (Net Energy Verification and Determination Analyzer). The emissivity of the sight tube $(1-\varepsilon)$ was substituted by a constant emissivity of 
stainless steel which is the material of the sight tube. It is because that the emissivity of stainless steel is not significantly affected by the oxidation level at the temperature range used in these experiments (Aleksander, 1986). The emissivity and reflectivity of the infrared thermometer were assumed to be those of ZnS (Zinc Sulfide), which is the lens of an infrared thermometer. In other cases, the reflectivity was substituted by because the sum of the emissivity and reflectivity is the unit for an opaque material.

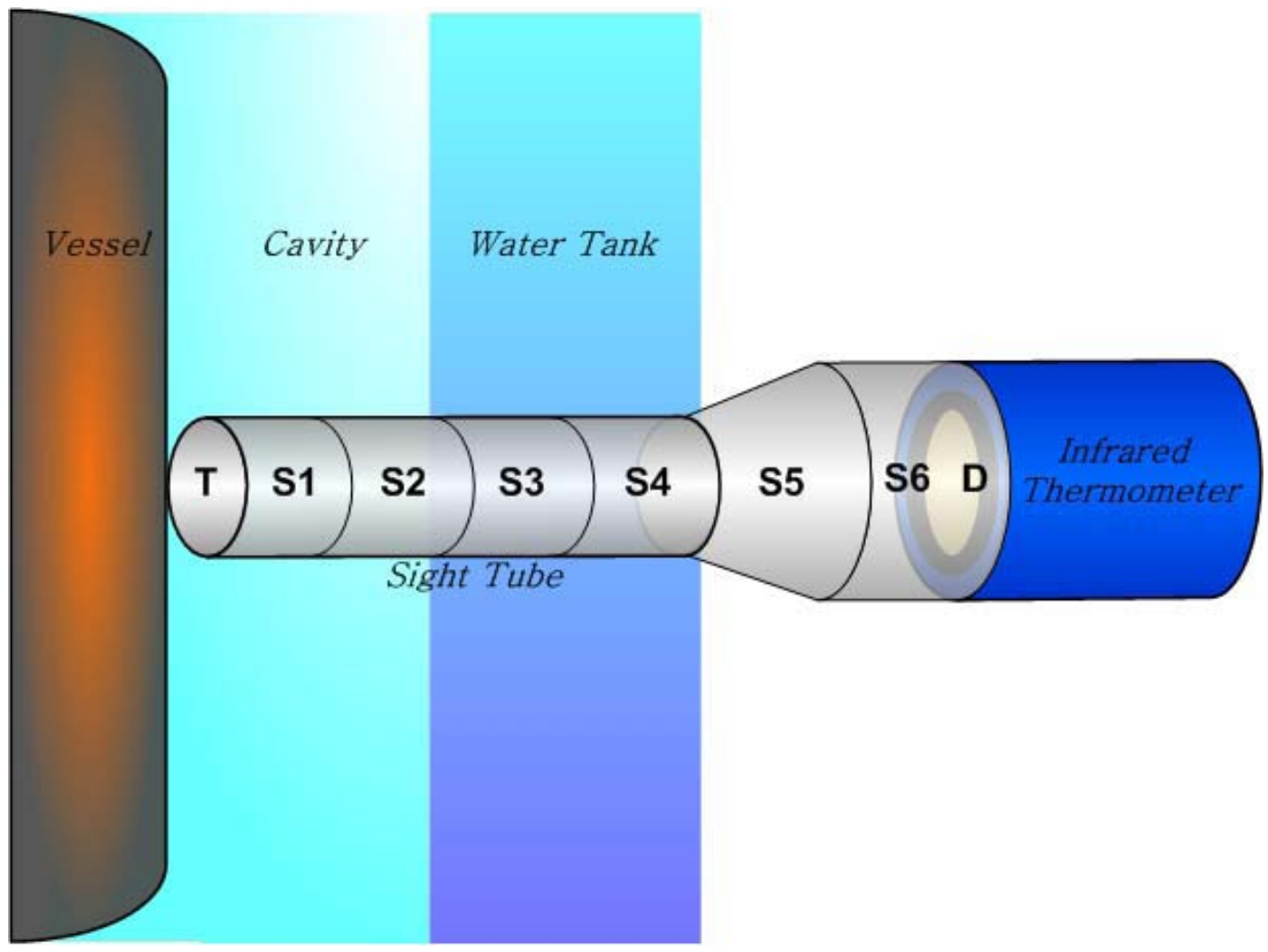

Figure 3-60 Simplified geometry for the estimation of the true-emissivity

The calculated emissivity was compared with the compensated emissivity and the true-emissivity, as shown in Figure 3-61. The calculated emissivity was in good agreement with the true-emissivity and its trend. Therefore, it is believed that the effect of background radiation was as much as the difference between the calculated and the measured emissivity, and the background radiation was removed properly by the calculation. The calculated emissivity and true-emissivity increased gradually with increasing temperature at the low temperature range. Finally, both emissivities reached an almost constant value as 0.88 around $400{ }^{\circ} \mathrm{C}$. 


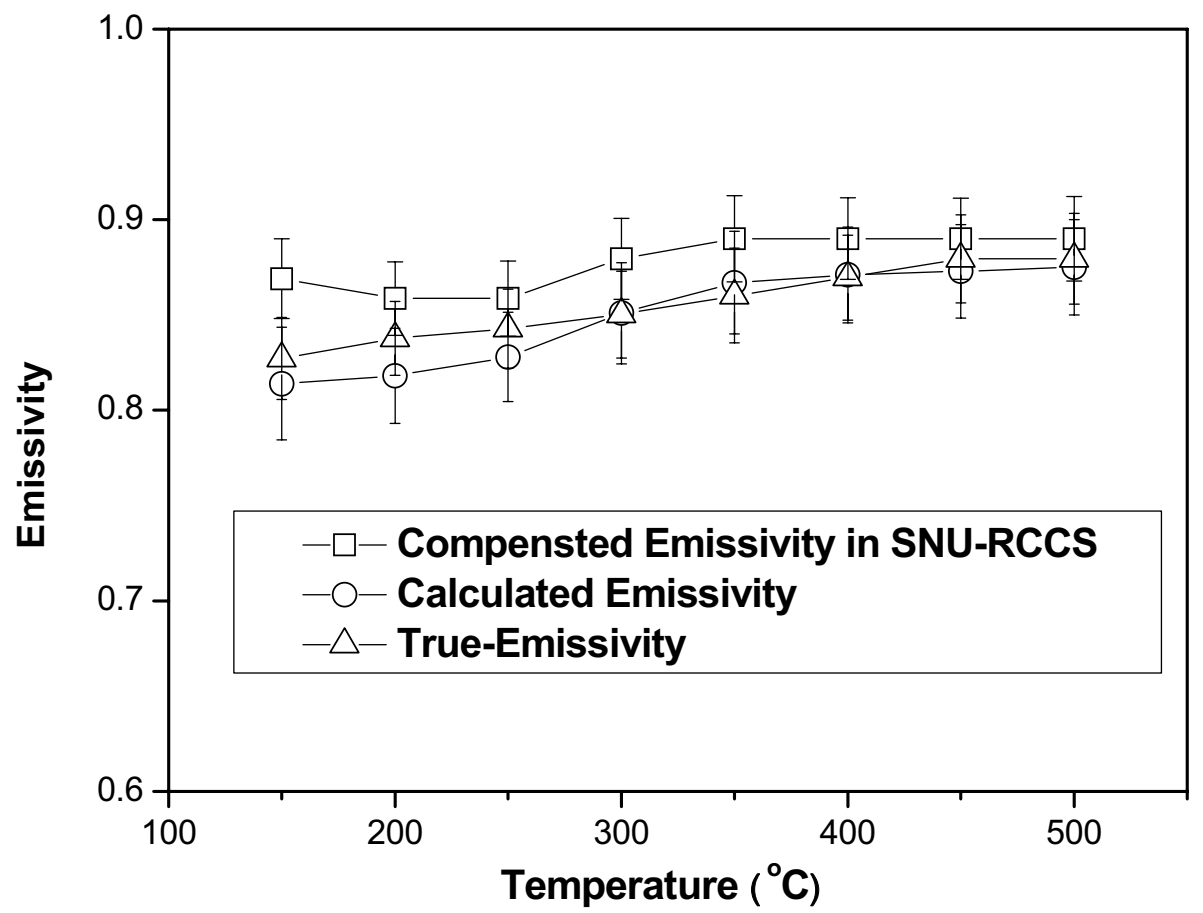

Figure 3-61 Comparison of calculated emissivity with true-emissivity in RCCS-SNU

In order to verify the method used to remove the effect of background radiation, additional experiments were performed with different material made from S45 steel in the independent emissivity measurement device. The emissivity was measured through the sight tube and without surrounding surface, and then compensation was performed using the identical method. Although the emissivity of S45 was different from that of ASTM A36 Steel, it was shown that the calculation process properly estimated the effect of background radiation on emissivity measurement in both cases, as shown in Figure 3-61 and 62. It was founded that the calculated emissivity was smaller than the true emissivity at low temperature due to the over-estimation of background radiation in both cases. It is caused by uncertainty of measurement as well as calculation. The result of uncertainty analysis shows this trend as shown in Figure 3-65. 


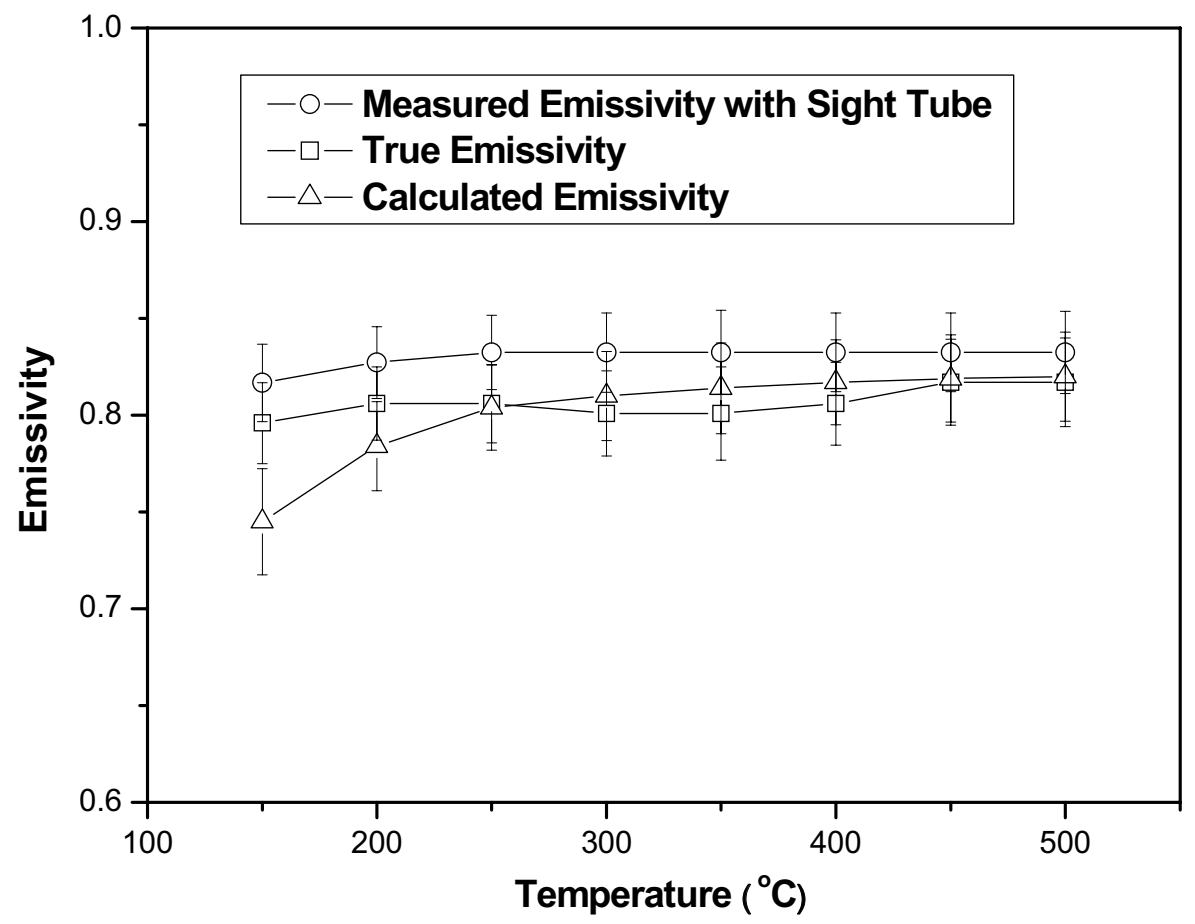

Figure 3-62 Comparison of calculated emissivity with true-emissivity in separate emissivity measurement device

Figure 3-63 shows the validation result comparing between the calculated and true emissivity. The calculated emissivity showed good agreement with the true emissivity within $3 \%$. This means that the calculated process is sufficient to predict the effect of background radiation.

In the estimation of the calculated emissivity, the emissivity of the sight tube was assumed to be constant at 0.60 . This value was obtained by an experiment performed at $150{ }^{\circ} \mathrm{C}$ using stainless steel, which is the same material found in the sight tube. Because the assumption did not consider the emissivity variation of stainless steel as a function of temperature, there might be some error in the calculations. The validity of this assumption was tested by carrying out sensitivity analysis with various stainless steel emissivities using the same calculation process. 


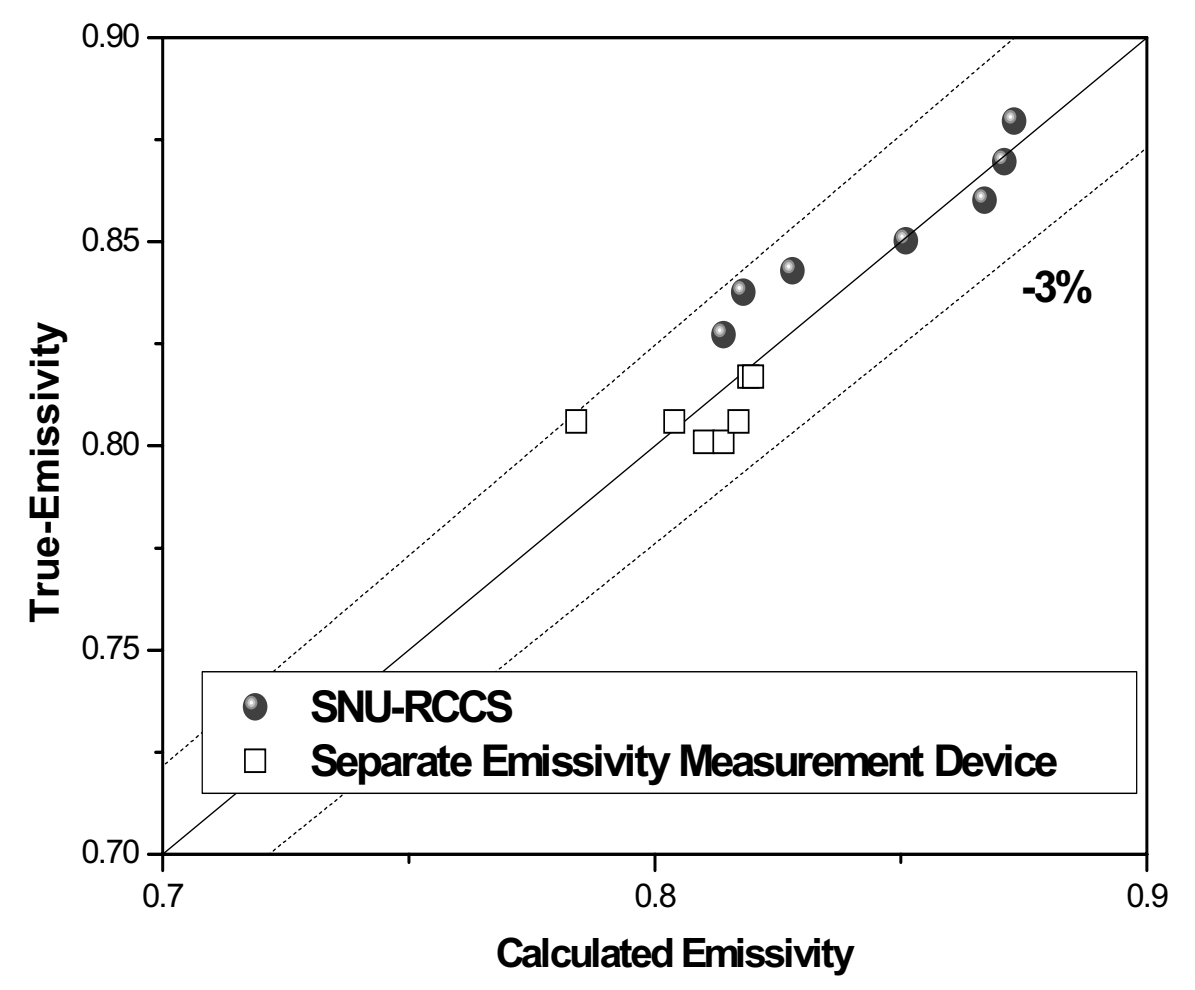

Figure 3-63 Validation of the calculated emissivity

As shown in Figure 3-64, the calculated emissivities resulted in a similar value even if we used different stainless steel emissivity ranging from 0.4 to 0.8 . The maximum difference in the calculated emissivity was 0.016 at $300{ }^{\circ} \mathrm{C}$. Therefore, the error caused by the assumption of stainless steel emissivity had little effected on the analysis of the calculated emissivity. Likewise, the emissivity and reflectivity of the detector also were presumed to be constant. The temperature of the detector was so low compared with those of the other surfaces that the emissivity variation of the detector had almost no influence on the calculation results. 


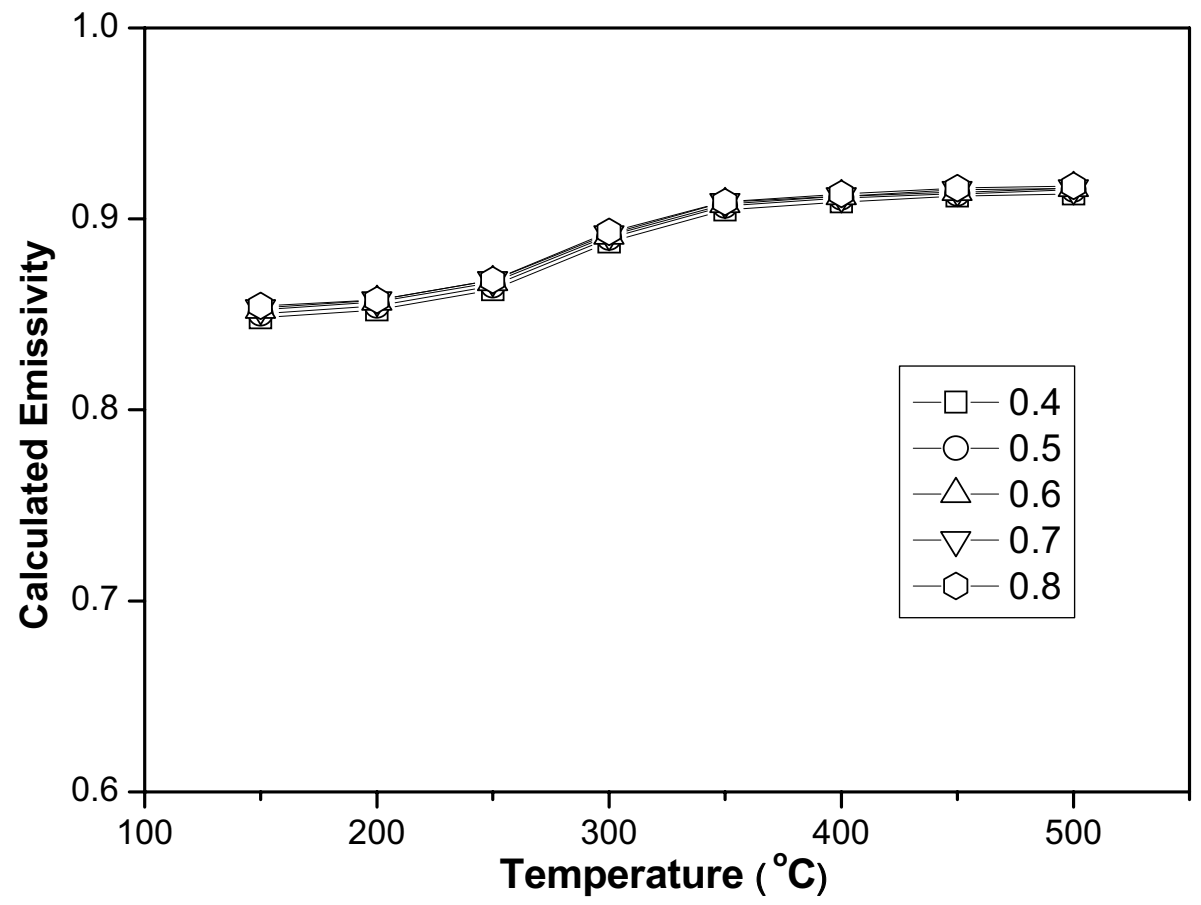

Figure 3-64 Calculated emissivity with the emissivity of various stainless steels

Uncertainty in emissivity measurement was assessed considering uncertainties of thermocouple, infrared thermometer. In addition, uncertainties due to the detecting angle and position of infrared thermometer were also considered. Level of oxidation was considered constant after 50 hours heating at $500{ }^{\circ} \mathrm{C}$ because we confirmed that

Table 3-10 Averaged uncertainty

\begin{tabular}{||l||l||l||}
\hline & RCCS-SNU & $\begin{array}{l}\text { Separate emissivity } \\
\text { measurement device }\end{array}$ \\
\hline \hline Measurement of true emissivity & $2.6 \%$ & $2.7 \%$ \\
\hline \hline Measurement of emissivity with sight tube & $2.4 \%$ & $2.4 \%$ \\
\hline \hline Calculation of true emissivity & $3.0 \%$ & $2.9 \%$ \\
\hline
\end{tabular}

emissivity does not change at the time. The effect of surface roughness was included in uncertainty of position and oxidation level.

K-Type thermocouples were calibrated using calibration curve from $0{ }^{\circ} \mathrm{C}$ to $500{ }^{\circ} \mathrm{C}$ within $0.5{ }^{\circ} \mathrm{C}$ error band. Although the accuracy of infrared thermometer was given by manufacturer as $1 \%$, we considered $2 \%$ the accuracy of infrared thermometer including the error in compensation of atmosphere transmittance. In addition, sensitivity of emissivity measurement on detecting angle and position was tested with traversing 
system installed in the infrared thermometer. The uncertainties of calculated emissivity at each temperature are shown in Figure 3-65, and averaged uncertainties are summarized in Table 3-10. This result was used to plot error bar in Figures 3-61 and 62.

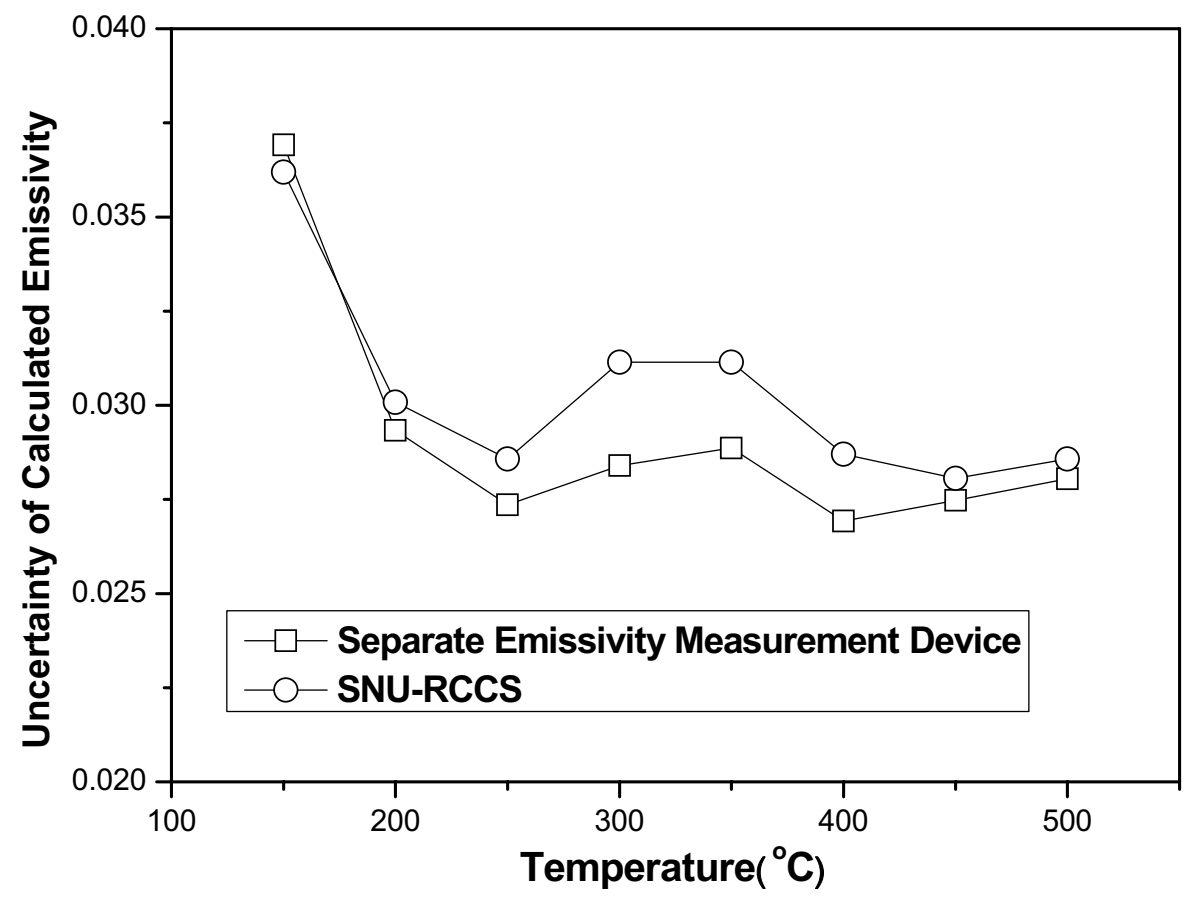

Figure 3-65 Uncertainty of calculated emissivity

\section{4-7 MARS Calculation}

From the point of view of the analysis of IET the interesting phenomena are the radiative heat transfer in the cavity, multi-dimensional motions of water in the water pool and two-phase behavior in case of LOFC accident. Since MARS-GCR code has the capability to simulate the radiative heat transfer as well as twophase behavior and CFX 5.7 code needs extremely large computational time to simulate entire integral test facility, the code-to-experiment validation was performed only using MARS-GCR code.

The nodalization of IET facility for MARS-GCR is shown in Figure 3-66. The facility is modeled with 4 independent systems; a side and lower water pools, upper water pool, a cavity, and air cooling pipes. The reactor vessel was modeled as boundary conditions. The side and the 


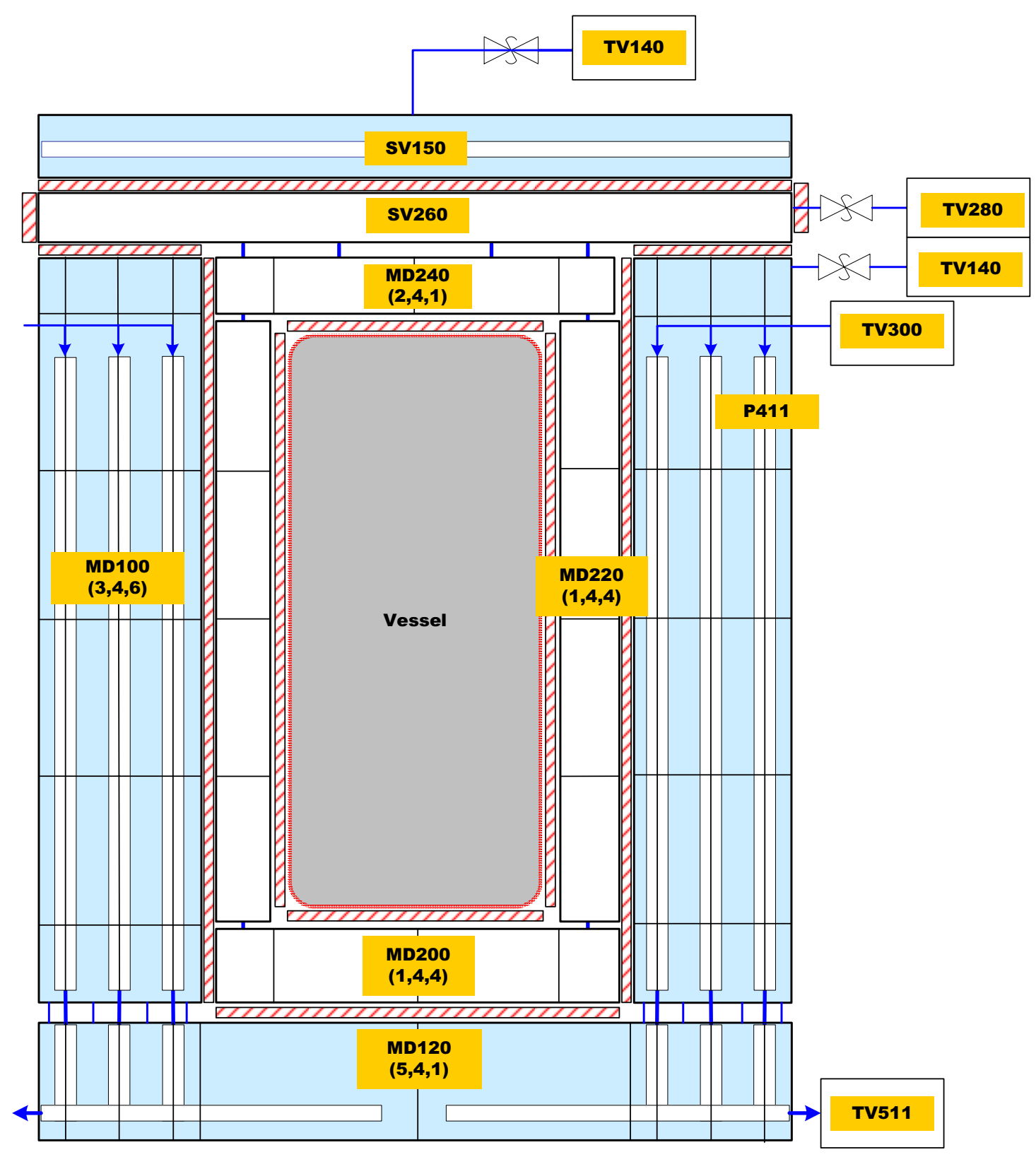

Figure 3-66 Nodalization of IET Facility for MARS-GCR Calculation

lower water pools were modeled with the multi-dimensional components 100 and 120 which consist of $4 \times 4 \times 6$ and $5 \times 4 \times 1(\mathrm{r}-\theta-\mathrm{z})$ cylindrical coordinates, respectively. The upper water pool was modeled with a single volume 150 . The cavity was modeled with 3 multi-dimensional components 200, 220, 240 and a single volume 260. Pipe components were used to model the 12 air cooling pipes in the side water pool and an air cooling pipe in the upper water pool. To provide boundary condition, time dependent volumes were attached to the each system. 


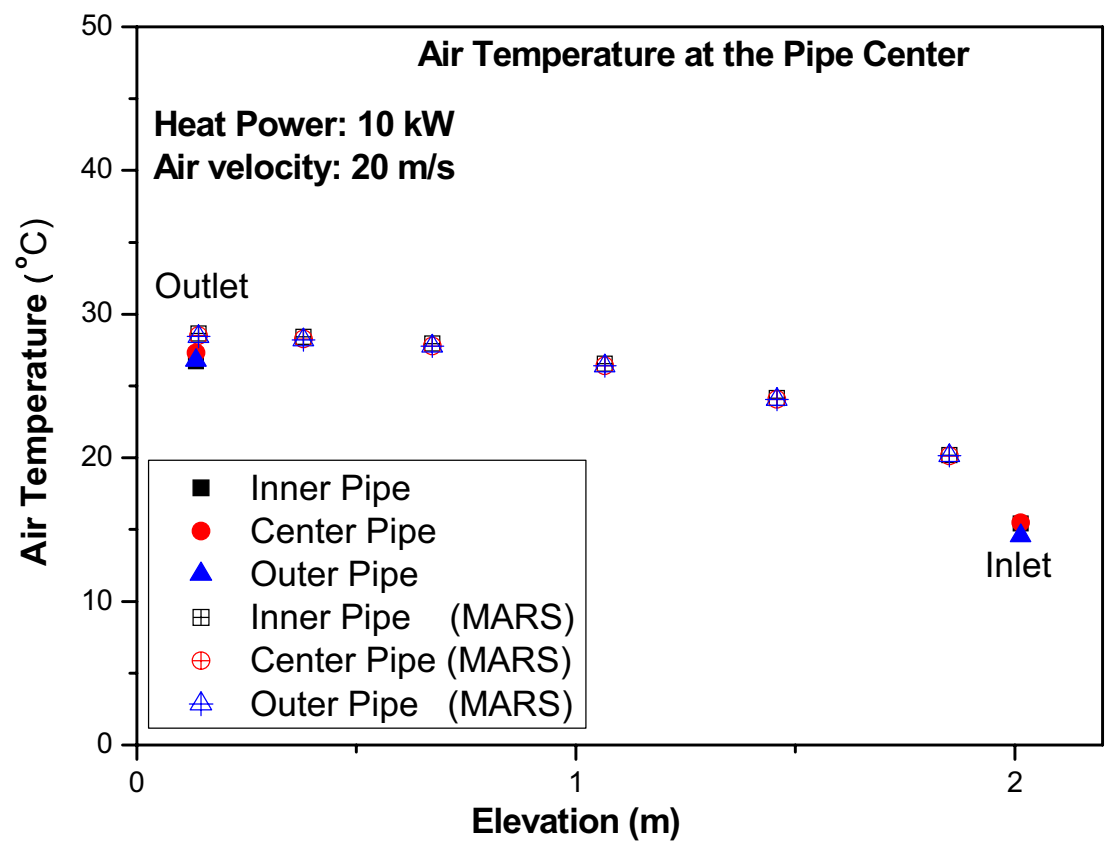

Figure 3-67 Comparison of air temperature distribution

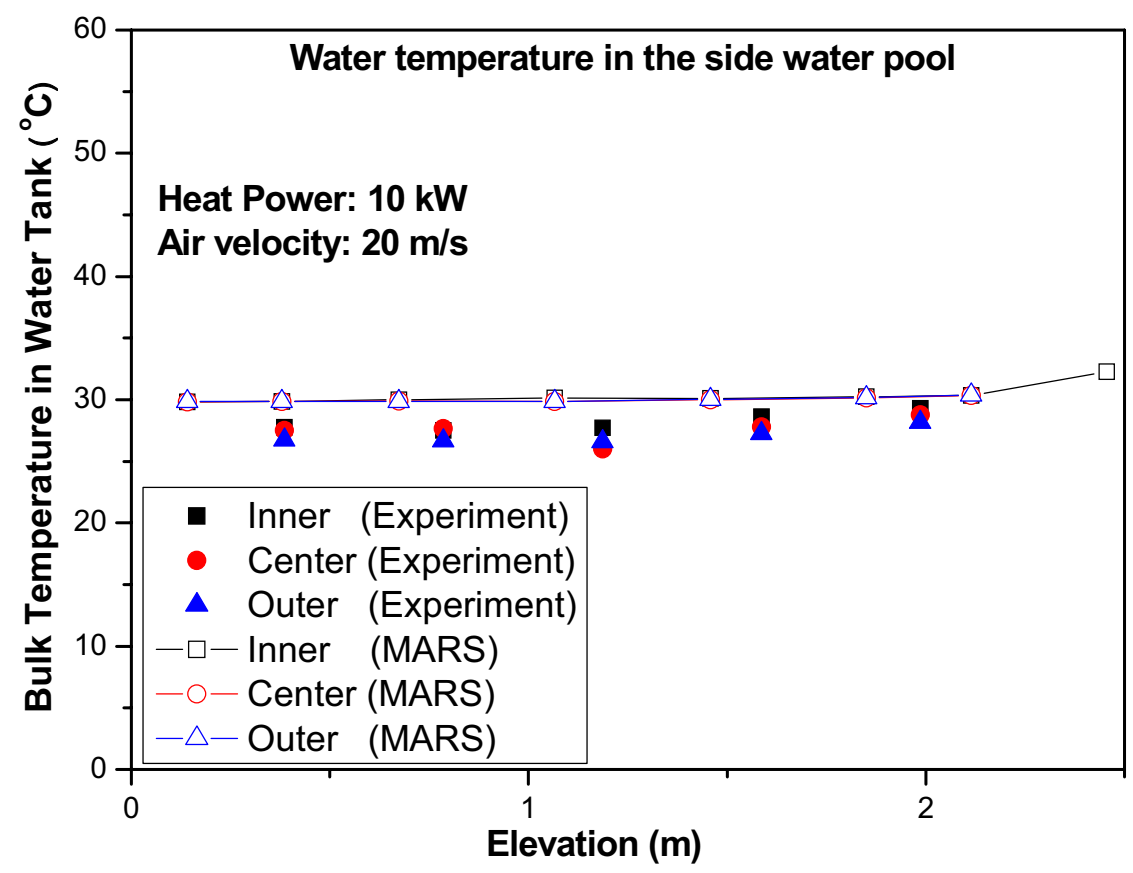

Figure 3-68 Comparison of water temperature distribution 
To simulate the heat transfer in the cavity, 49 heat structures were modeled on the cavity wall and 24 on the vessel wall. Thus total $73 \times 73$ view factors were needed and calculated by commercial code, NEVADA (Net Energy Verification and Determination Analyzer), for the analysis of radiation heat transfer. Emissivity of the reactor vessel wall, 0.85 , was measured using the infrared thermometer as described previous section. The emissivity of cavity wall was assumed as 0.60 , emissivity of SUS304 measured by the infrared thermometer.

\section{$\underline{\text { 3.4-8 Normal Operation }}$}

Figures 3-67 and 3-68 show typical calculation results for the air temperature at the cooling pipe center and the water temperature in the side water pool. Because MARS-GCR over-predicts heat removal fraction of the side water pool, air outlet temperature and water temperature in the side water pool are predicted higher than experiments about 2 3 ${ }^{\circ} \mathrm{C}$. Figures 3-69 and 3-70 show the over-prediction trends of the air temperature at the pipe center and water temperature in the side water pool compared to experiments.

The experimental results showed that $13 \sim 23 \%$ of the released heat was removed by the upper pool depending on the heater power. Since, in the cavity, the heated air at the reactor vessel wall flows upward to the upper part of the cavity by natural circulation, the amount of heat removed by the upper pool is proportional to that removed by natural convective heat transfer. The calculation results, however, showed only $8 \sim 12 \%$ of the released heat was removed by the upper pool. This is estimated to be caused by underprediction of air temperature and natural convective heat transfer in the upper part of cavity.

MARS-GCR under-predicts the cavity wall temperature as shown in Figure 3-71 because MARS-GCR estimates the heat transfer by natural circulation in the narrow gap between the cooling pipe and heating surface, as described in experiment results, that means higher heat transfer than experiments .

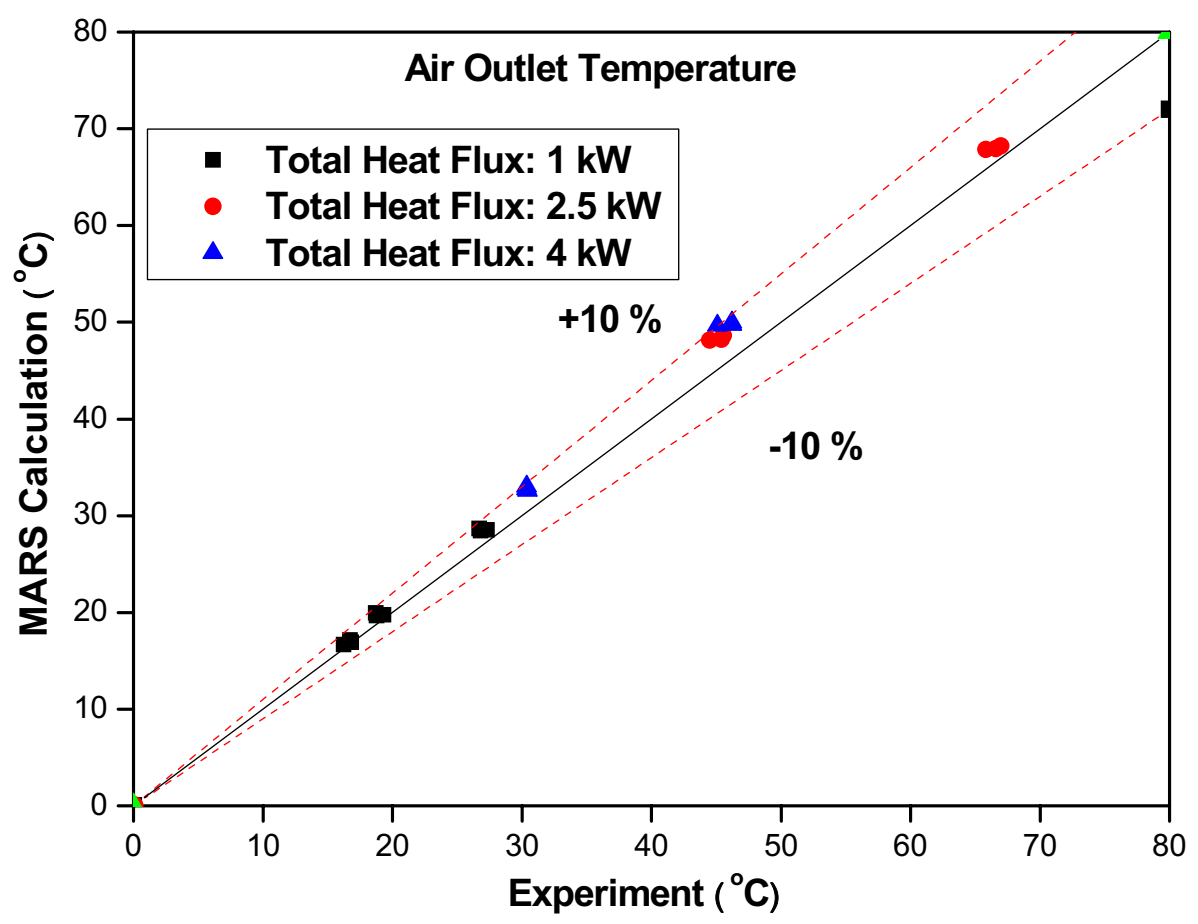

Figure 3-69 Comparisons of air outlet temperature 


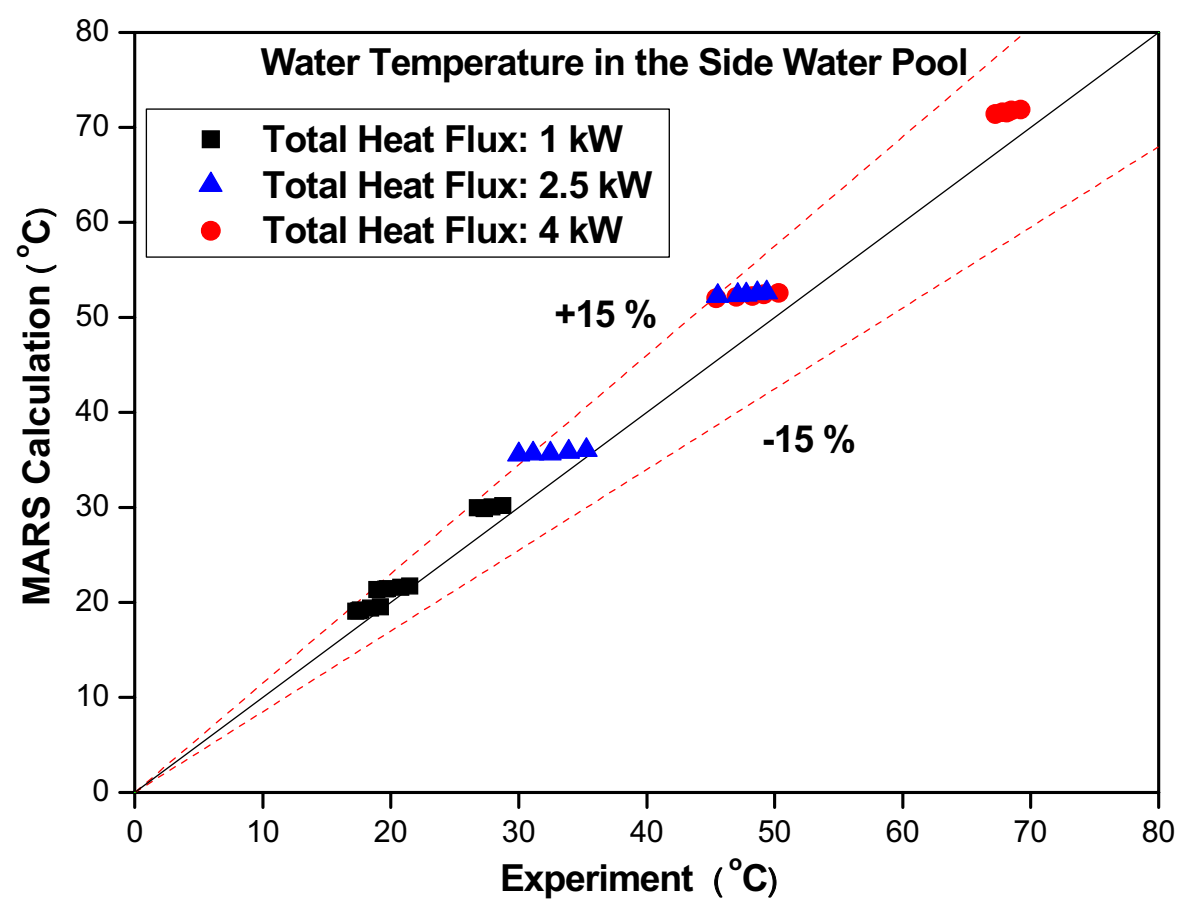

Figure 3-70 Comparisons of water temperature

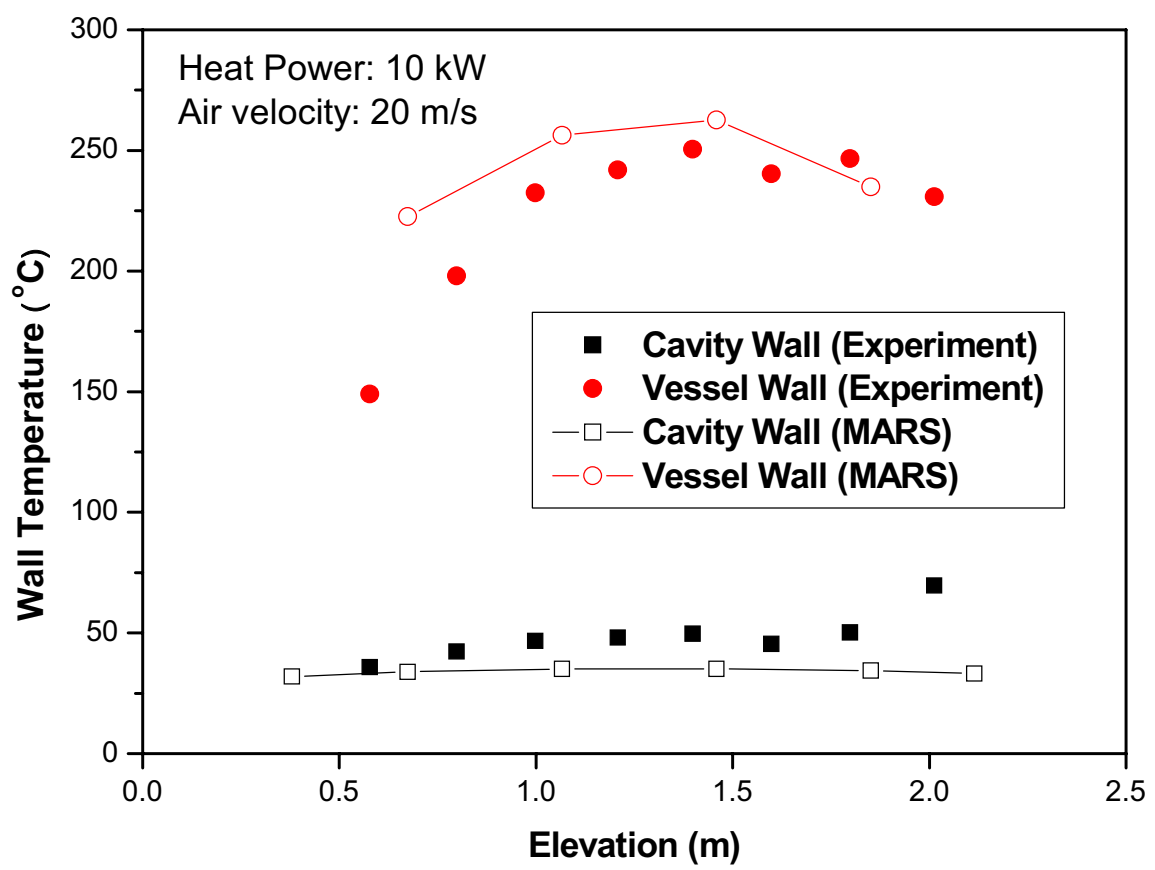

Figure 3-71 Comparison of wall temperature distributions 


\section{4-9 Side Pool 1/4 Cooling Failure Experiment}

In Figure 3-72, the comparison results of the water temperature distribution in the side water pool is plotted when $1 / 4$ portion of the air cooling in the side pool was failed. Although the water temperature calculated by MARS-GCR is higher than the experiments as described in the comparison results for normal operation, the temperature in the water tank increases with time by about $4 \sim 5^{\circ} \mathrm{C}$ in the calculation result as well as the experiment. Also, no local increase of the water temperature in the water pool was observed on the failure side, and the differences in temperature between the active sides and the failure side were observed to be less than $0.5^{\circ} \mathrm{C}$.

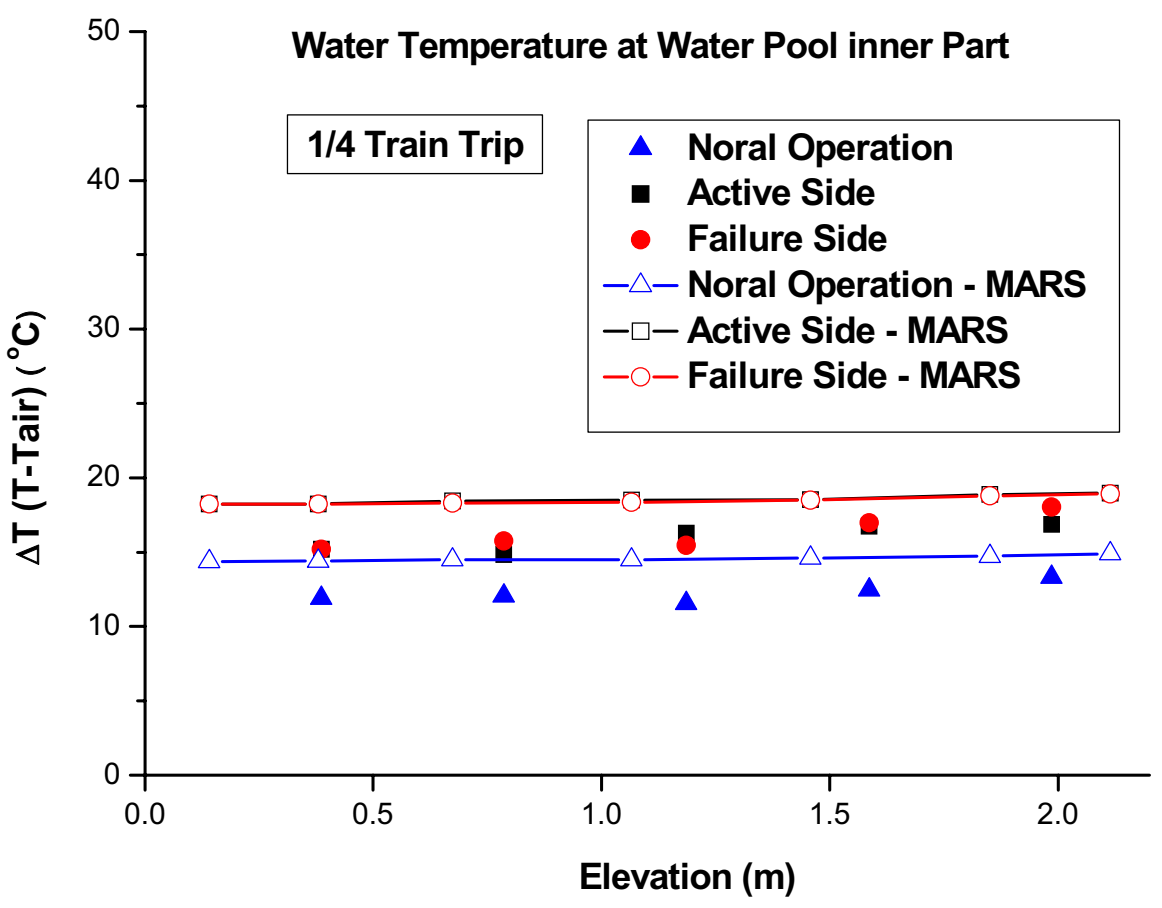

Figure 3-72 Comparison of water temperature distributions (1/4 train trip) 


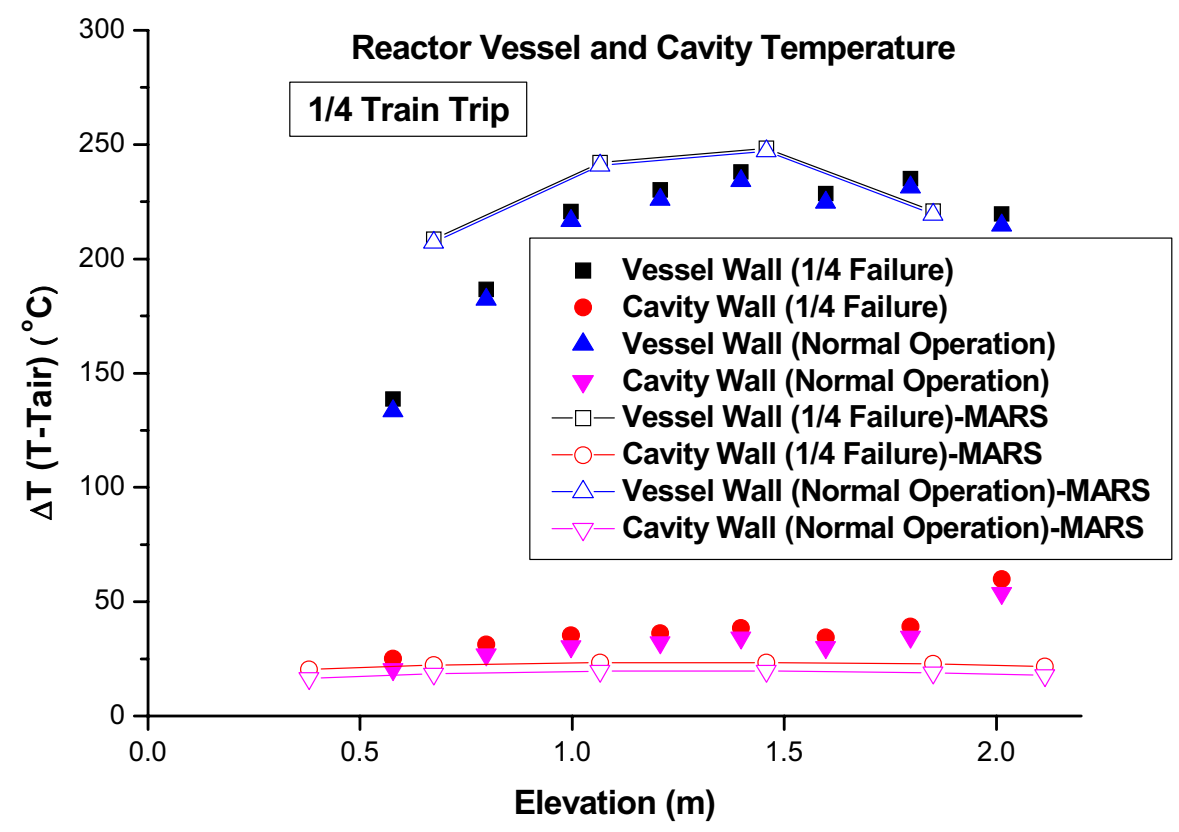

Figure 3-73 Comparison of wall temperature distributions (1/4 train trip)

This result is smaller than the experimental result, $2{ }^{\circ} \mathrm{C}$, and means that MARS-GCR over-predicts the natural circulation also in the $1 / 4$ train trip case. The cavity wall temperature are also increased slightly about $4 \sim 5^{\circ} \mathrm{C}$ as a result of the increase of the water temperature in the case of the $1 / 4$ air cooling system failure as shown in Figure 3-73.

\section{4-10 LOFC Experiment}

MARS-GCR calculation to simulate the LOFC experiment was performed implementing the vessel wall temperature measured in the experiment as boundary condition with time. Figure 3-74 shows the transient of water level in the side water pool. The slight increase in the water level was occurred, which is caused by the swelling of the water, before the relief valve was opened. The swelling of the water was also observed in experiment. 


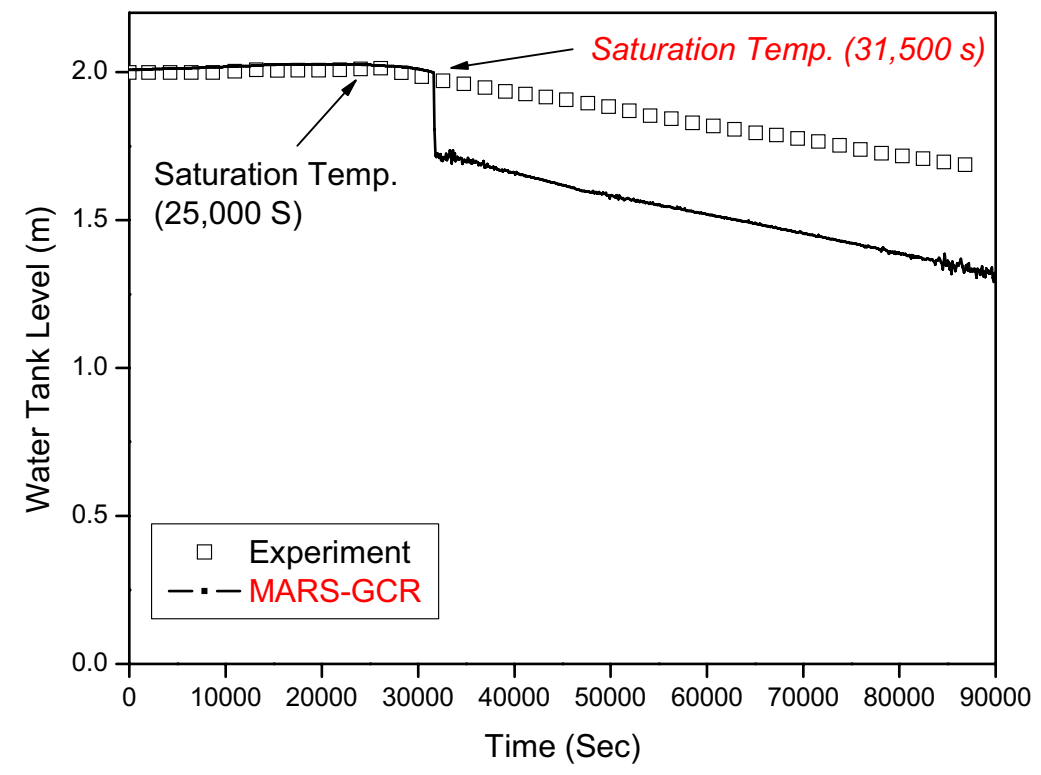

Figure 3-74 Comparison of water level transient (LOFC test)

The relief valve was manually opened when the water temperature in upper region of the side water pool reached the saturation temperature at 1.5 bars. Since MARS-GCR predicts that the thermal stratification in the side water pool is not significant comparing to experimental results, valve manual opening time was delayed by about 6,500 second as shown in Figure 3-75.

In the experiment, the water level was decreased gradually by the heat transfer from the cavity wall after valve opening. In the calculation, however, the water temperature was saturated in upper two nodes because MARS-CGR under-predicts the effect of thermal stratification. Thus, when the valve was opened manually, the water in upper two nodes was simultaneously flashed with the depressurization during 200 seconds.

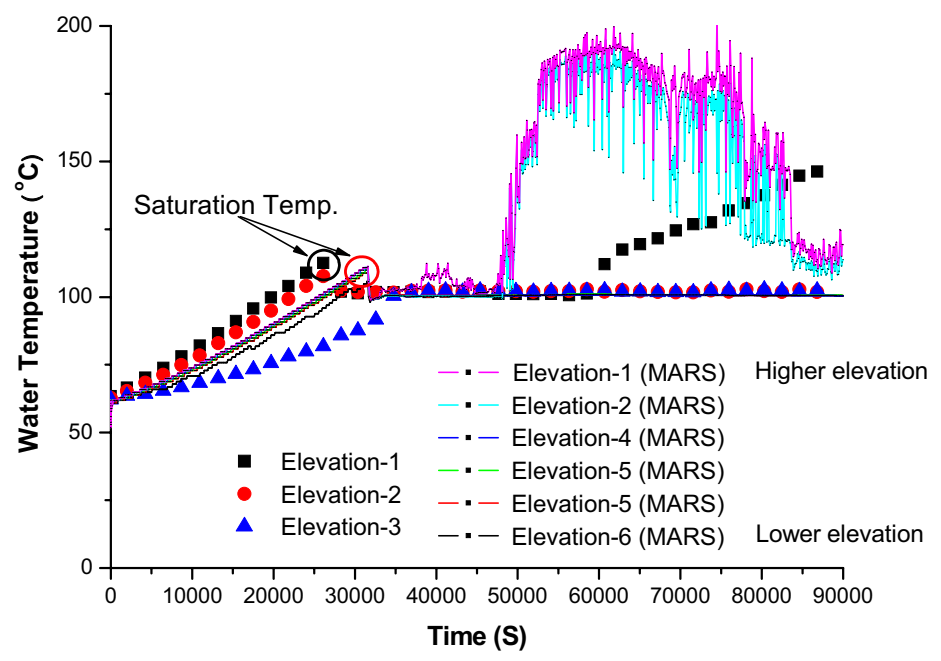

Figure 3-75 Temperature transient of water in the side pool during the (LOFC test) 
Figure 3-76 shows the comparing results of transient of the cavity wall temperature. The temperature of the cavity wall increased gradually with time before the valve manual opening. After the valve opening, the temperature of the cavity wall at the node where the water was deplete $\mathrm{d}$ began to rise sharply and reached near the temperature of superheated steam inside the node.

MARS-GCR has the capability to simulate the radiative heat transfer as well as multi-dimensional behavior such as the natural convection in the water pool and the cavity. In the calculation for the IET, however, the results of MARS-GCR calculation show the under-prediction trends of thermal stratification of water in the side water pool and air in the cavity as described in the calculation results.

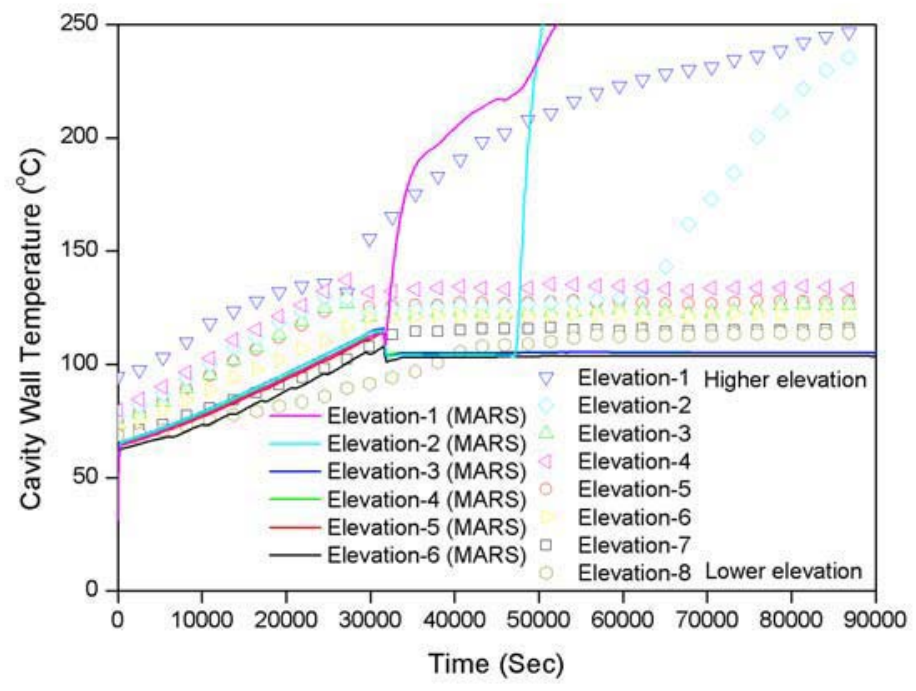

Figure 3-76 Temperature transient of the cavity wall (LOFC test) 


\section{Nomenclatures for Task 2}

\section{English}

A Area [m2]

$c_{p} \quad$ Specific heat $[\mathrm{J} / \mathrm{kg} \mathrm{K}]$

$D \quad$ Diameter [m]

E Emissive radiation

F View factor

G Irradiation

$g \quad$ Gravity acceleration $\left[\mathrm{m} / \mathrm{sec}^{2}\right]$

$h \quad$ Heat transfer coefficient $\left[\mathrm{W} / \mathrm{m}^{2} \mathrm{~K}\right]$

J Radiosity

K Loss coefficient

$k \quad$ Thermal conductivity [W/mK]

$L \quad$ Length [m]

$\dot{m} \quad$ Mass flow rate $[\mathrm{kg} / \mathrm{s}]$

$\mathrm{Nu} \quad$ Nusselt number

$\Delta \mathrm{P} \quad$ Pressure drop

Pr Prandtl Number

$\dot{q} \quad$ Heat [W]

$R \quad$ Radius [m]

Re Reynolds Number

$T$ Temperature [K]

\section{Greek Symbol}

$\alpha \quad$ Thermal diffusivity

$\beta \quad$ Thermal expansion coefficient

$\varepsilon \quad$ Emissivity

$v \quad$ Dynamic viscosity

$\rho \quad$ Density, Reflectivity

$\theta^{*} \quad$ Dimensionless temperature

$\sigma \quad$ Stenfan-Boltzmann constant

\section{Subscript}

$\begin{array}{ll}\text { air } & \text { Air } \\ \text { B } & \text { Blackbody } \\ \text { cavity } & \text { Cavity } \\ \text { cp } & \text { Cooling pipe } \\ \text { D } & \text { Detector } \\ \text { fric } & \text { Frictional } \\ \text { g } & \text { Gas } \\ \text { gas } & \text { Gas } \\ \text { heat loss } & \text { Heat loss } \\ \text { i, k } & \text { Surface index }\end{array}$




$\begin{array}{ll}\mathrm{m} & \text { Measured } \\ \text { in } & \text { Inlet } \\ \text { out } & \text { Outlet } \\ \text { S1 S6 } & \text { Surface index of sight tube } \\ \mathrm{T} & \text { Target } \\ \text { vessel } & \text { Vessel } \\ \text { water } & \text { Water }\end{array}$




\section{TASK 3: Air ingress experiment (KAIST)}

The objective of this task is to carry out the graphite oxidation experiment to determine the oxidation-limited model (chemical kinetics-limited, diffusion-limited or in-pore diffusion-limited model), and to develop measurement techniques of the concentration of each species.

The present study investigates the graphite oxidation reaction, which is one of the most serious problems during an air-ingress accident in a high temperature gas-cooled reactor (HTGR). There are many factors concerned with the rate of graphite oxidation. Among them, the followings were investigated in this study. Kinetic effect

Mass diffusion effect

Combined effect of kinetics and mass diffusion

Geometrical effect

Effect of burn-off

Other reactions (Boudouard reaction)

\subsection{Kinetic effect}

In order to investigate kinetics (chemical effects) on the graphite during an air-ingress accident in HTGR, the kinetic tests were performed in Zone 1, where the kinetic effect controls the rate of reaction. The main variables related to the kinetic effect are temperature, oxygen partial pressure and each effect was experimentally investigated.

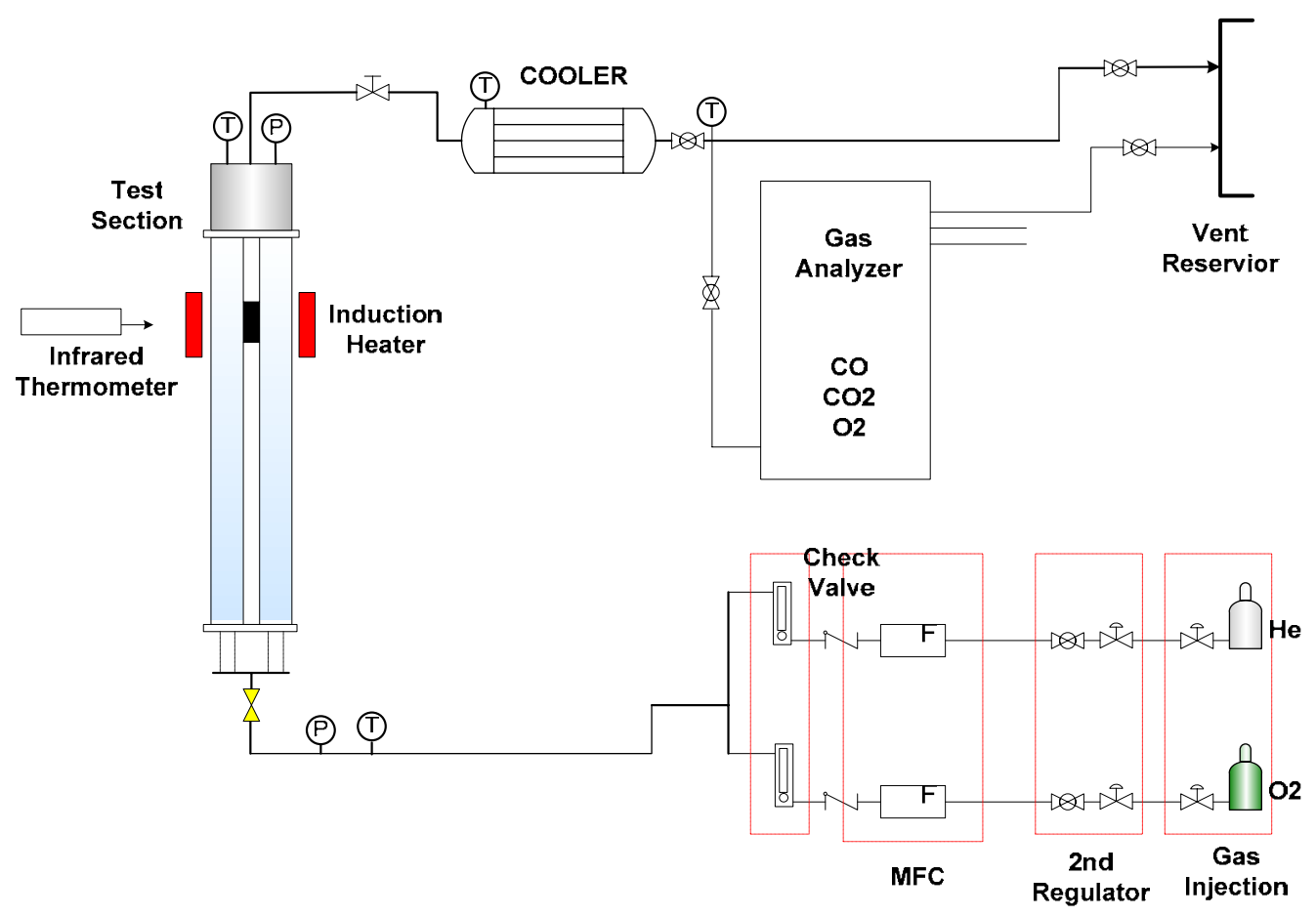

Figure 4-1. Schematics of Experimental Facility

We measured the oxidation rates by using gas concentration analysis (see Figure 4-1). This method offers two advantages: (1) faster and more precise response than a general thermogravimetric analysis (TGA); and (2) availability of direct analysis for gases such as $\mathrm{CO}$ and $\mathrm{CO}_{2}$. A He/O $/ \mathrm{O}_{2}$ mixture gas was injected into the test section with a concentration that is controlled by a mass flow controller (Brooks), and a $15 \mathrm{~kW}$ induction 
heater heated a graphite sample. The reaction rate was calculated by a gas components analysis through two non-dispersive infrared (NDIR) analyzers (Rosemount NGA2000, Yokogawa IR100)

Table 4-1. Properties of IG-110 Graphite

\begin{tabular}{|c|c|}
\hline Material & IG-110 \\
\hline Producer & Toyo Tanso \\
\hline Bulk Density (g/cm3) & 1.75 \\
\hline Young Modulus (GPa) & 9.6 \\
\hline Compressive Strength (MPa) & 70.5 \\
\hline Rockwell Hardness (MPa) & 74.2 \\
\hline Fracture Toughness (MPa) & 0.82 \\
\hline Thermal Conductivity (W/mK) & 116 \\
\hline Porosity (vol \%) & 21.6 \\
\hline Impurities (ppm) & $<20$ \\
\hline
\end{tabular}

The test section was made of a cylindrical quartz tube. In order to maintain a hydraulically fully developed flow, a long entry length was designed. This gave us a well-known flow field (Hagen-Poiseuille flow) around the surface of the test specimen. The specimen was installed at the center of the test section and a ceramic rod supported it. The diameter and height of the specimen were $2.1 \mathrm{~cm}$ and $3 \mathrm{~cm}$ and the diameter of the testsection was $7.6 \mathrm{~cm}$. An induction heater was used for heating the sample and its temperature was measured by a non-contact technique using two infrared thermometers (IRtex Raymatic 10, Raytec Ranger 3i). Setting temperature could be achieved within $30 \mathrm{sec}$ by the induction heating method without disturbing the gas flow field.

As a test material, isotropic fine-grained IG-110 graphite was selected in this study. Its properties are summarized in Table 4-1. Temperature, oxygen concentration, and flow rate were selected as the main experimental variables and the conditions are summarized in Table 4-2.

Table 4-2. Experimental Conditions

\begin{tabular}{|c|c|c|}
\hline & Pretest & Main test \\
\hline Temperature $\left({ }^{\circ} \mathrm{C}\right)$ & $540 \sim 600$ & $700 \sim 1500$ \\
\hline
\end{tabular}




\begin{tabular}{|c|c|c|}
\hline Flow rate (SLPM) & $\sim 18$ SLPM $(0.072 \mathrm{~m} / \mathrm{s})$ & 40 SLPM $(0.16 \mathrm{~m} / \mathrm{s})$ \\
\hline Inlet Oxygen Fraction (\%) & $\sim 32 \%$ & $\sim 20 \%$ \\
\hline
\end{tabular}

Figure 4-2 shows an example of the results measured at 5.05\% oxygen mole fraction. Our result shows good agreement with Arrhenius-type model. We have performed a total of 33 tests at various conditions, and we illustrated them in Figure 4-3. Each symbol denotes a different flow rates. This figure shows that the activation energy Ea is not sensitive to flow rate and it confirms that the chemical effects are only ratedetermining process in our experimental conditions. Our results can be summarized as follows:

(a) $\mathrm{Ea}=218 \pm 4 \mathrm{~kJ} / \mathrm{mol}$ within a $95 \%$ level of confidence.

(b) Ea is not affected by oxygen concentration, as Hinsen et al. 1983 showed.

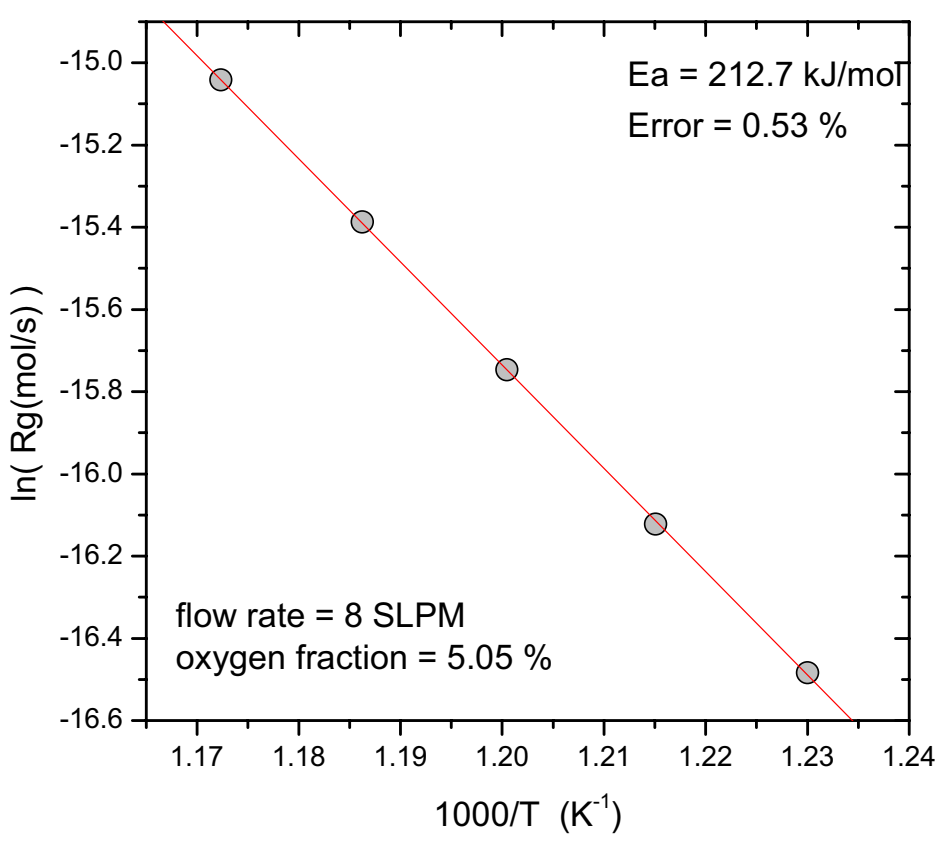

Figure 4-2. Effect of Temperature on Oxidation Rate 


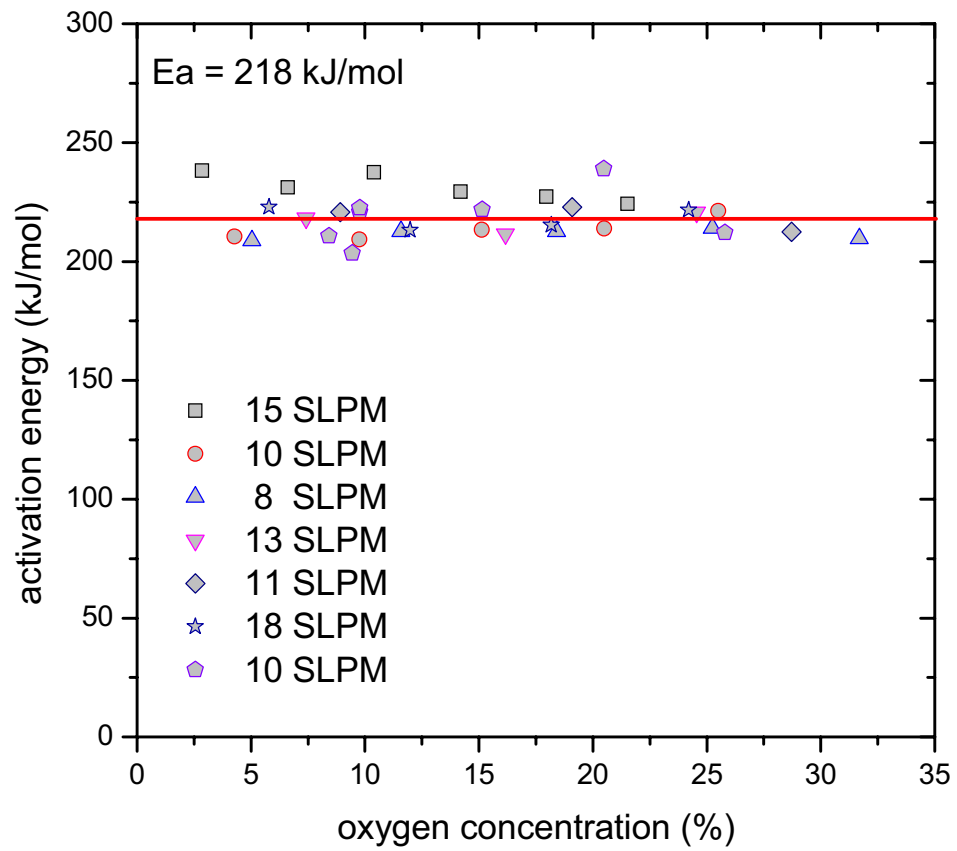

Figure 4-3. Results of Activation Energy

Table 4-3 summarizes the previous and our activation energies measured for the same graphite material. This table shows that the activation energies of Fuller 1997, Ogawa 1993 and Kawakami 1986 are very close to ours and it confirms that $200 \sim 220 \mathrm{~kJ} / \mathrm{mol}$ is a reasonable value for the activation energy of IG-110 graphite.

Table 4-3. Previous and Present Experimental Results on Kinetic Parameters

\begin{tabular}{|c|c|c|c|c|c|c|}
\hline Author & $\mathrm{T}\left({ }^{\circ} \mathrm{C}\right)$ & $\begin{array}{l}\text { Oxygen mole } \\
\text { fraction }\end{array}$ & $\begin{array}{c}\text { Flow rate } \\
\text { (SLPM) }\end{array}$ & $\begin{array}{c}\mathrm{E}_{\mathrm{a}} \\
(\mathrm{kJ} / \mathrm{mol})\end{array}$ & $\mathrm{n}$ & Method \\
\hline $\begin{array}{c}\text { Fuller } \\
\text { [3] }\end{array}$ & $450 \sim 750$ & 0.2 & 0.496 & 201 & - & TGA \\
\hline $\begin{array}{c}\text { Kawakami } \\
{[5]}\end{array}$ & $550 \sim 650$ & 0.2 & - & 210 & $\begin{array}{l}0.76 \sim 1 \\
.06\end{array}$ & $\begin{array}{c}\text { Gas } \\
\text { Analysis }\end{array}$ \\
\hline $\begin{array}{c}\text { Ogawa } \\
{[4]}\end{array}$ & $700 \sim 1500$ & $0.05 \sim 0.19$ & $0.2 \sim 4.5$ & 200 & - & $\begin{array}{c}\text { Gas } \\
\text { Analysis }\end{array}$ \\
\hline $\begin{array}{l}\text { KAIST } \\
\text { (present } \\
\text { study) }\end{array}$ & $540 \sim 630$ & $0.03 \sim 0.32$ & $7 \sim 18$ & 218 & $\begin{array}{c}0.75 \\
\pm 0.15\end{array}$ & $\begin{array}{c}\text { Gas } \\
\text { Analysis }\end{array}$ \\
\hline
\end{tabular}

To obtain the order of reaction, we measured the reaction rate at different oxygen concentrations and illustrated them in Figure 4-4. 


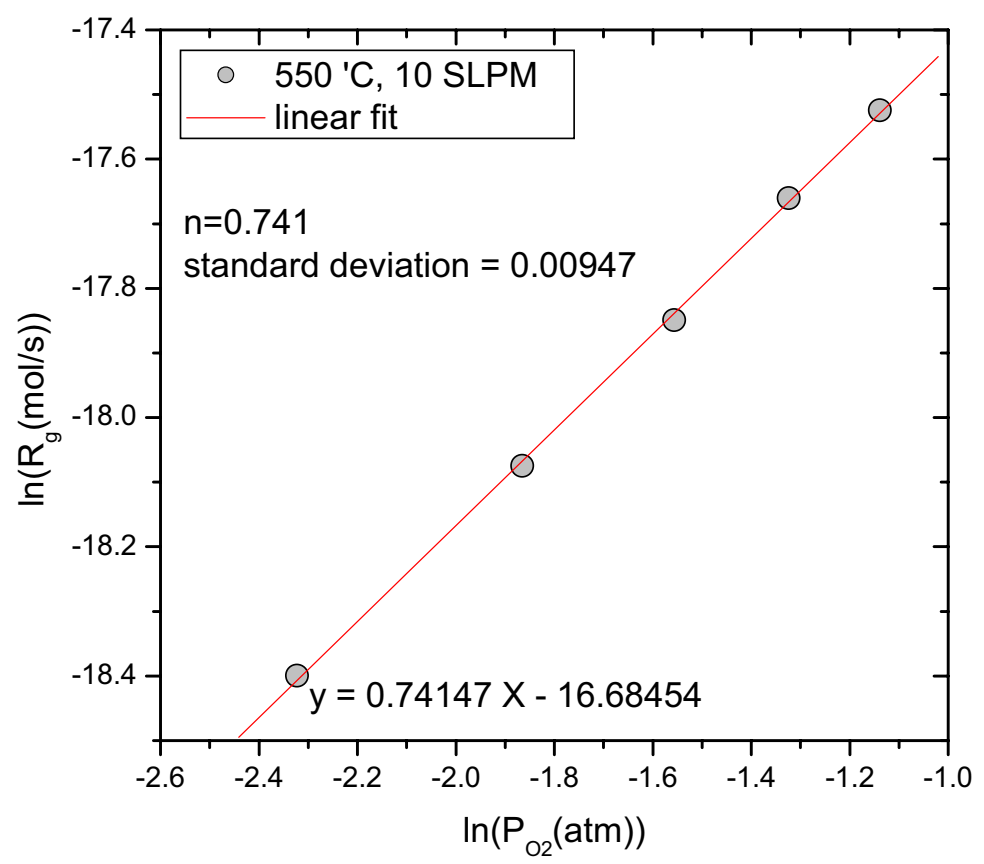

Figure 4-4. Effect of Oxygen Concentration on Oxidation Rate

This graph shows that the $\mathrm{n}$ values ranged from 0.6 to 0.9 . To increase the statistical reliability, we conducted tests at several conditions and obtained 66 sets of data. Figure 4-5 illustrates the results. As shown in figure 4-4, the distribution of $\mathrm{n}$ value does not show any trend with temperature, burn-off, and sample or flow rate. Especially, burn-off independencies confirm that the chemical characteristics do not change with oxidation history even though the internal structure and geometry of graphite change. So we analyzed the data by general descriptive statistics. As a result, $0.75 \pm 0.146$ was obtained within $95 \%$ level of confidence. 


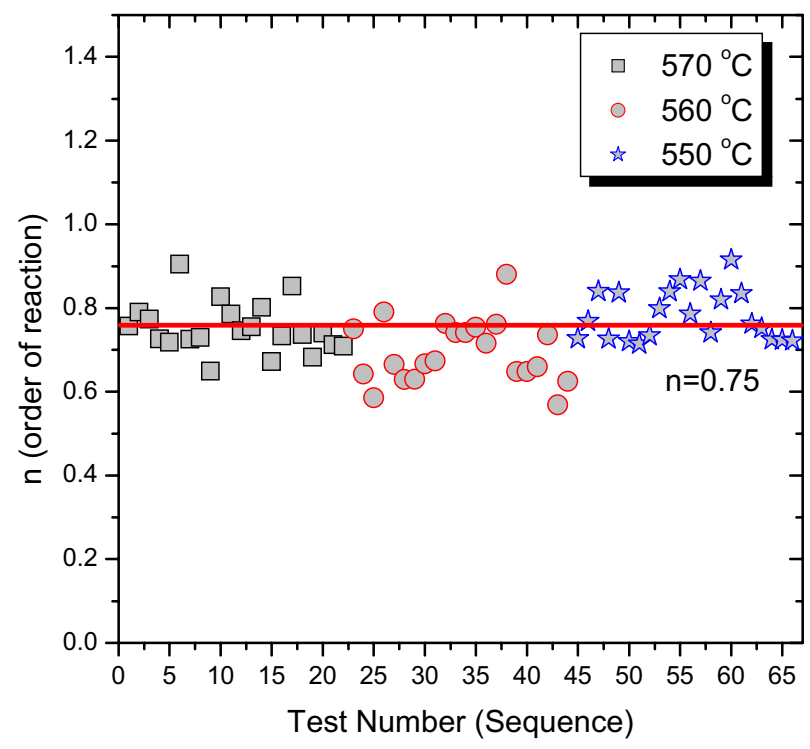

Figure 4-5. The Results of the Order of Reaction

\subsection{Mass diffusion effect}

While the rate of reaction is controlled by only chemical parameters at low temperature, the rate of reaction is limited by mass diffusion process at high temperature. In the previous studies, though the researchers have been applied heat/mass transfer analogy for predicting the rate of mass diffusion, it has not been sufficiently investigated yet. So, this study tried to confirm it by experiments. We compared the experimental results to CFD simulation and analytic correlations.
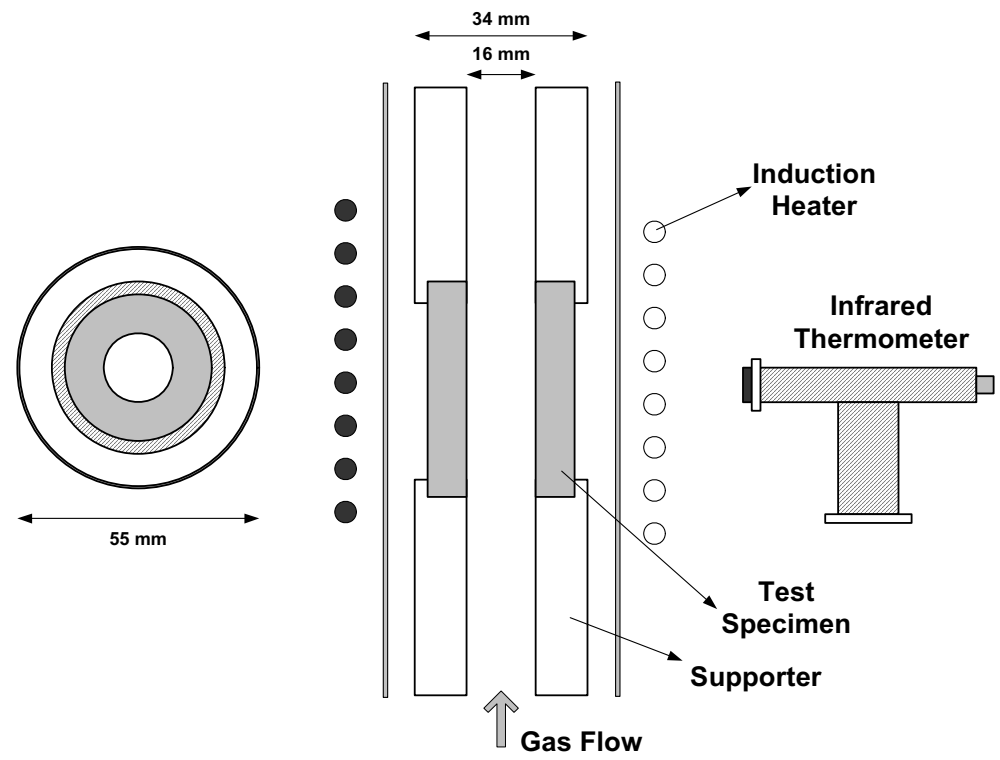

Figure 4-6. Test Section of Mass Transfer Experiment 
The same test loop used in Task 4-1 was also used here, but the different test-section was installed as shown in Figure 4-6. The $\mathrm{He} / \mathrm{O} 2$ gas mixture was introduced into the inside of the tube channel. The 5 graphite channels with different lengths; 3, 5, 7, 9, $12 \mathrm{~cm}$ were tested. As in the Task 4-1, the graphite was heated by the induction heater and controlled by the IR thermometer and controller. Each test was performed at $1070{ }^{\circ} \mathrm{C}$ and this temperature level is high enough to be considered as Zone 3 . The velocity of mixture gas ranges between $0.8 \mathrm{~m} / \mathrm{s}$ and $4.0 \mathrm{~m} / \mathrm{s}$. Figure $4-7$ shows the picture of the test-section.
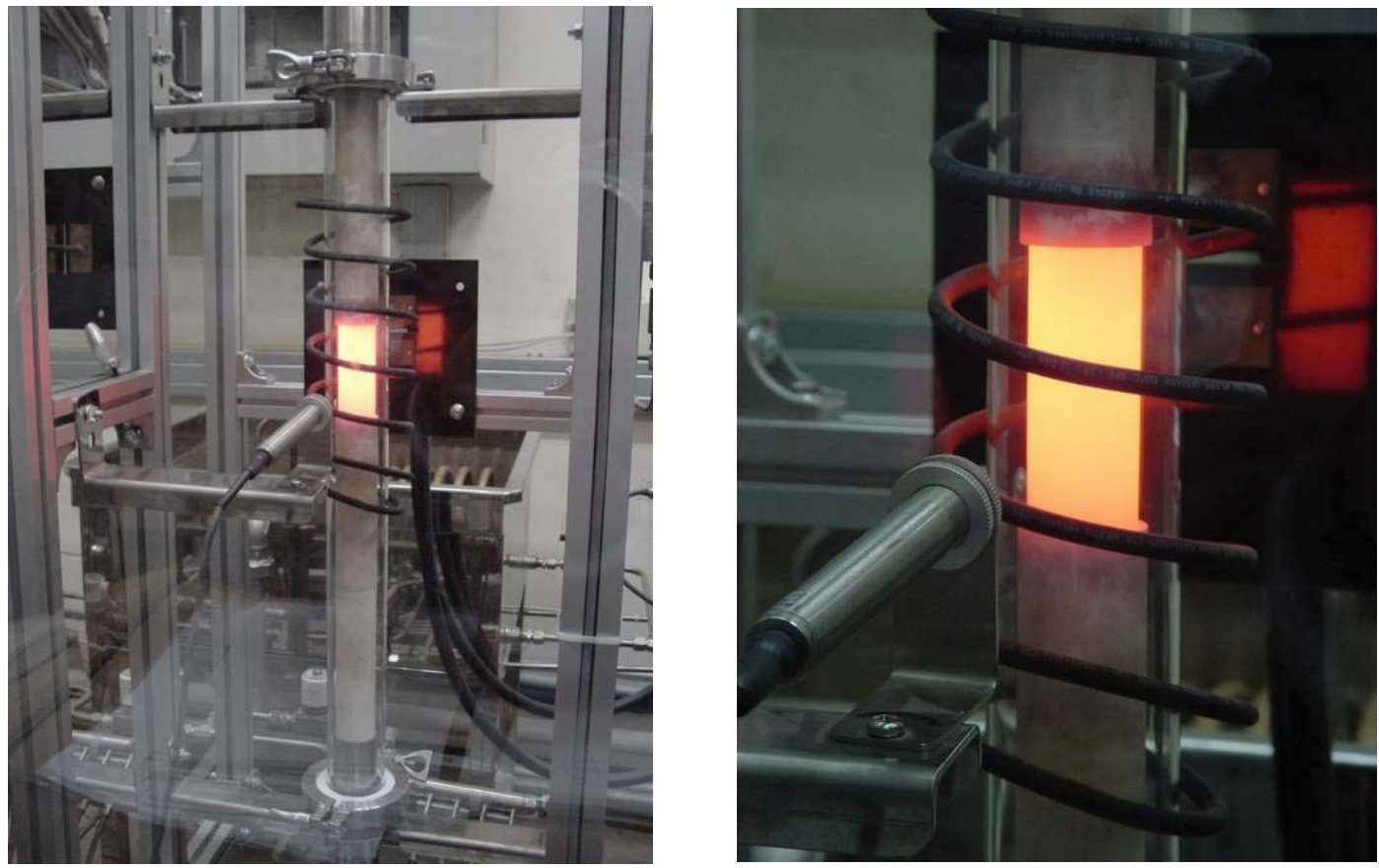

Figure 4-7. Picture of the Test-Section

To investigate the mass diffusion effect on the graphite oxidation, we compared the experimental data to the CFD simulation. Fluent 6.1 was selected for this calculation and Figure 4-8 shows one example of the calculation. Two-dimensional axi-symmetric calculation was applied here since the geometry of the testsection was a simple cylinder. Figure 4-9 illustrates the compared result on the rate of reaction between calculation and the experiment. This figure shows that the CFD code predicts well the experimental data and it confirms that the secondary reactions in this experiment can be considered as negligible. To confirm the Heat/Mass transfer analogy, we compared the experimental data to the following analytical correlation produced by heat/mass transfer analogy.

$K_{m}=0.664 \frac{D_{A B}}{L} \operatorname{Re}_{L}^{1 / 2} S c^{1 / 3}$

This correlation is made by conversion of the heat transfer correlation, which was developed for heat transfer through the laminar boundary layer and averaged through the whole length (Welty et al. 1984). Figure 4-10 illustrates the comparisons on the mass transfer rate between correlation and experiment. As shown in this figure, the calculation results are in good agreement with the experimental data. Figure 4-11 is another comparison results between calculation and experiment. All experimental data ranges within $\pm 20 \%$ of calculated results. From this agreement, we can conclude that the heat/mass transfer analogy can be applicable for predicting the mass diffusion rate in Zone 3. 
$3.000+02$

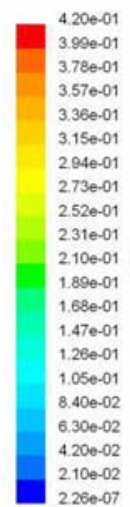

Contours of Static Temperature (k) FLUENT 6.1 (axi, segregated, spe5, 2005

Figure 4-8. Sample Result of CFD Simulation (Top: Temperature, Bottom: Mole Fraction of Oxygen)

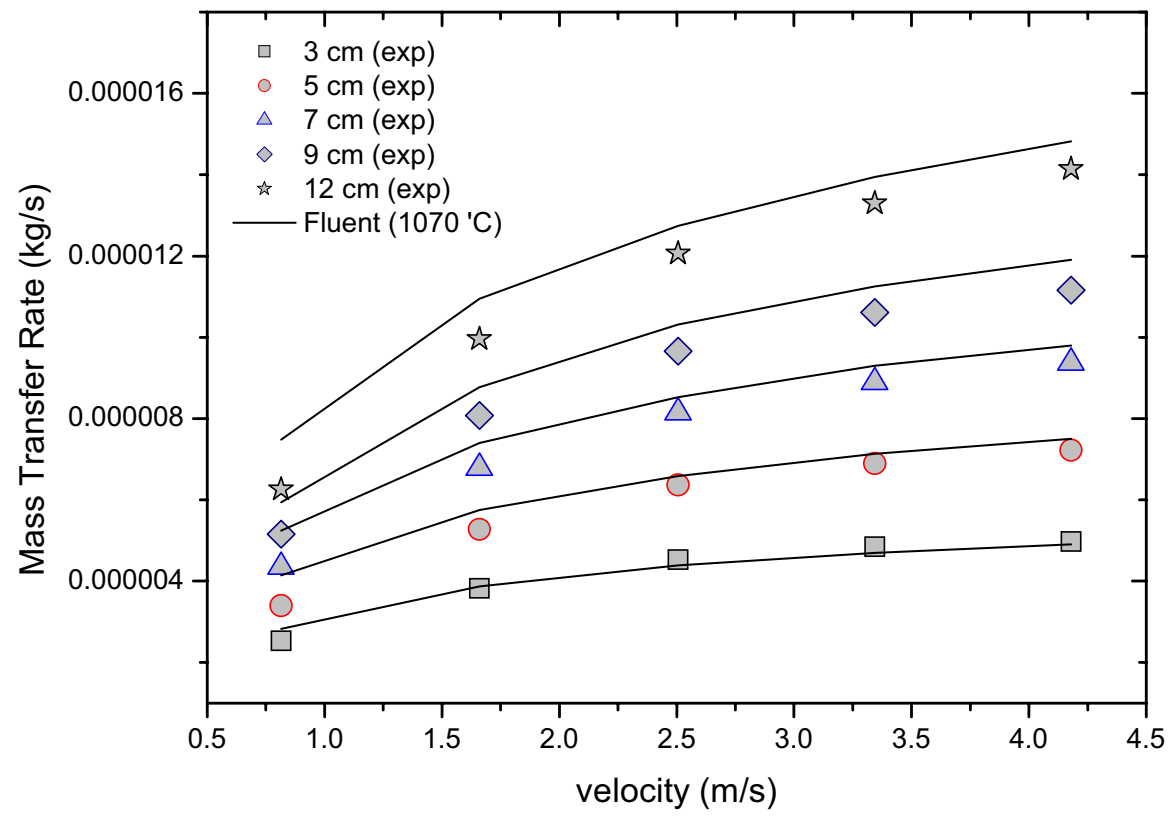

Figure 4-9. Comparison of the Mass Transfer Rate between CFD Predictions and Experimental Data 


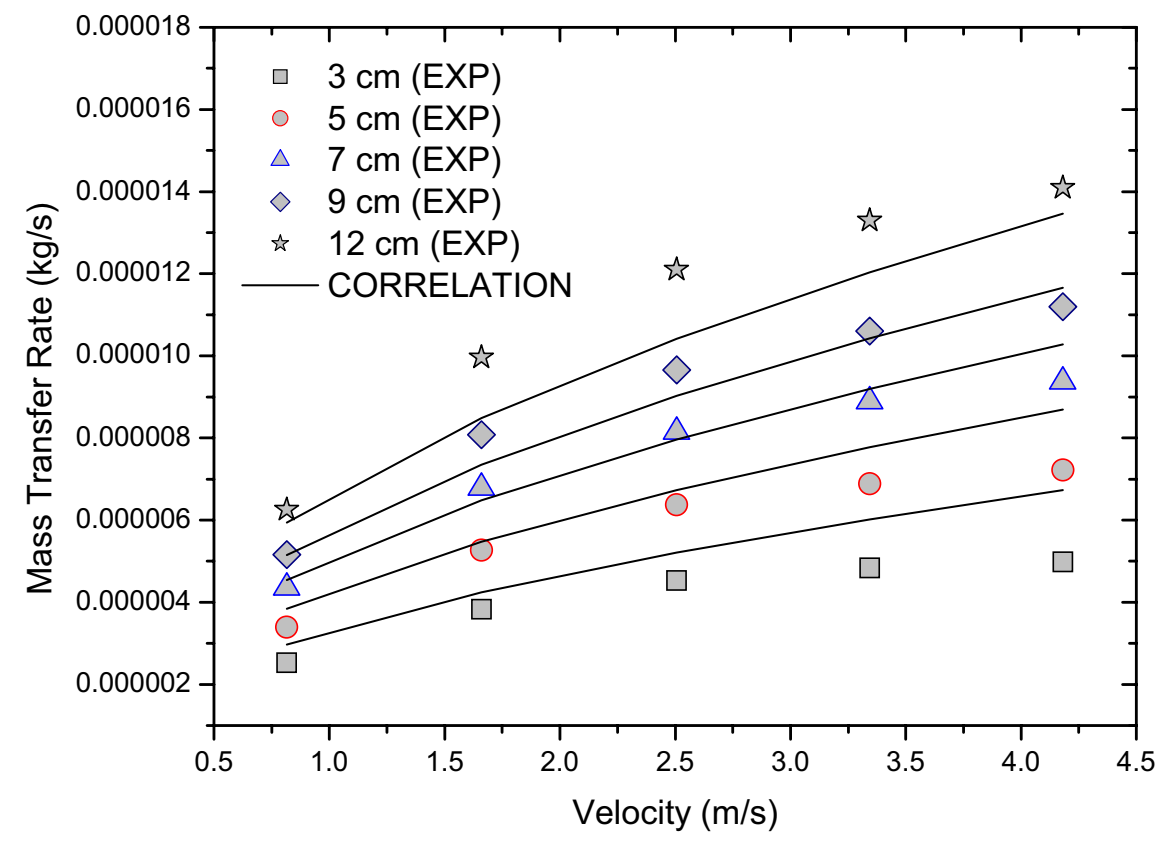

Figure 4-10. Comparison of the Mass Transfer Rate between Predictions by Correlation and Experimental Data

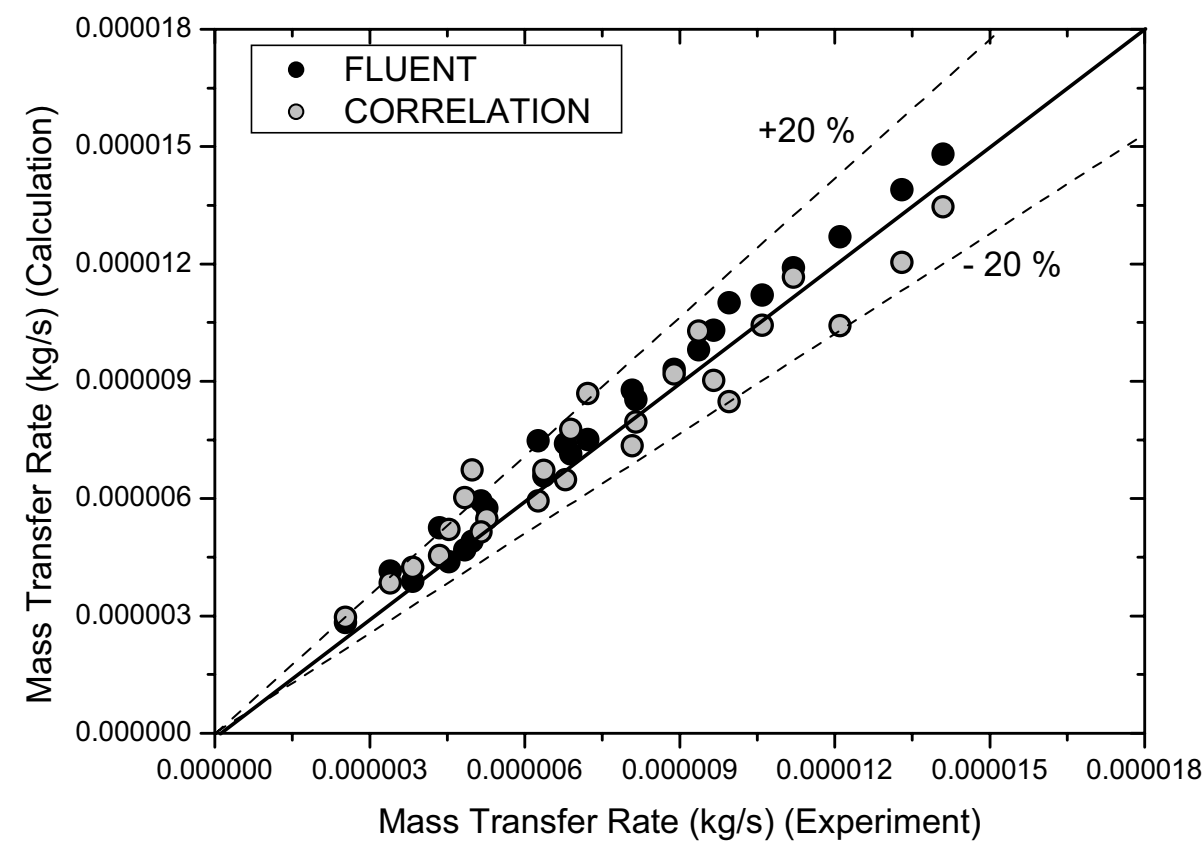

Figure 4-11. Comparison of the Mass Transfer Rate between Predictions and Experimental Data 


\subsection{Combined effect of kinetics and mass diffusion}

In order to investigate the combined effect of kinetics and mass diffusion, we performed the experiment at wide range of temperature from 700 to $1500^{\circ} \mathrm{C}$. Then we incorporated the kinetic parameters in Task 4-1 into a computational fluid dynamics (CFD) code, and subsequently compared the calculations to the experimental data. Finally, we developed and validated a correlation applicable for a system analysis tool.

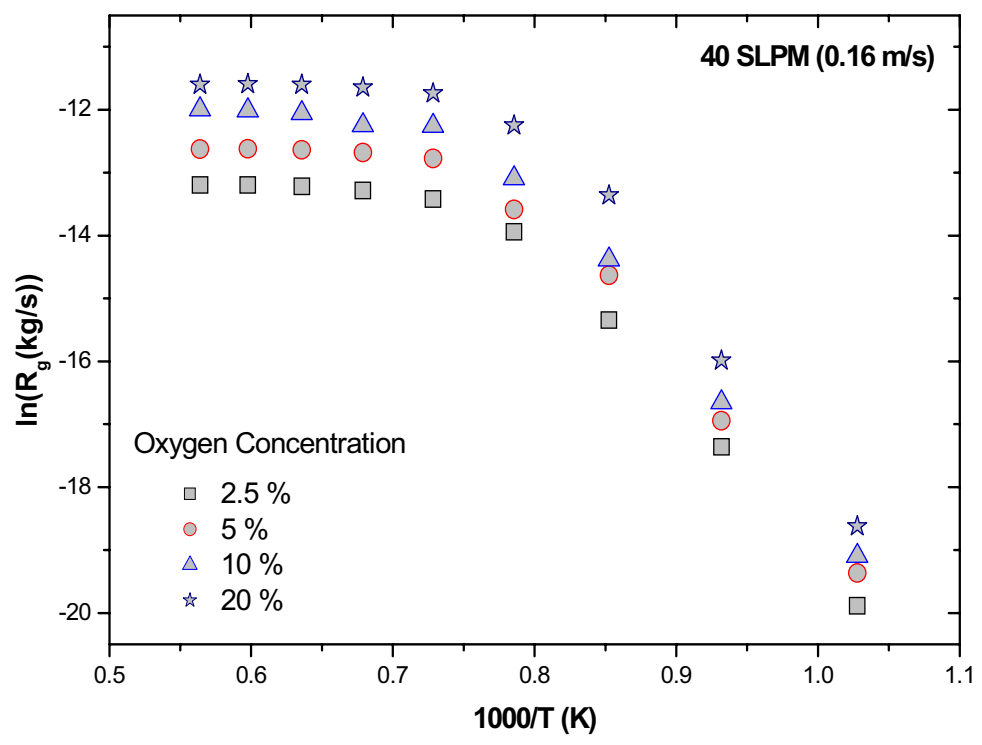

Figure 4-12. Rate of Oxidation

This experiment was also performed by the same test loop used in Task 4-1 at the similar conditions to the air-ingress accident of a HTGR. From this test, we obtained the reaction rate and relative fractions of $\mathrm{CO}$ and $\mathrm{CO}_{2}$. Figure 4-12 shows the initial oxidation rates, which becomes saturated at higher temperatures. It is because mss transfer is a main rate-determining step at high temperature. In the other hand, at low temperature, the trend well follows the general Arrhenius curve.

Figure 4-13 shows the $\mathrm{CO} / \mathrm{CO}_{2}$ ratio. In this graph, our data and those of Takahashi et al. 1994 are included. The latter experiment (Takahashi et al. 1994) was also performed with the same graphite material as ours.

The $\mathrm{CO} / \mathrm{CO}_{2}$ ratio data was compared with the previous Arthur's and Rossberg's correlations.

Arthur 1951:

$$
f_{\mathrm{CO} / \mathrm{CO} 2}=10^{3.4} \exp \left(-\frac{51900}{R T}\right)
$$

Rossberg 1956:

$$
f_{\mathrm{CO} / \mathrm{CO} 2}=10^{3.27} \exp \left(-\frac{59900}{R T}\right)
$$




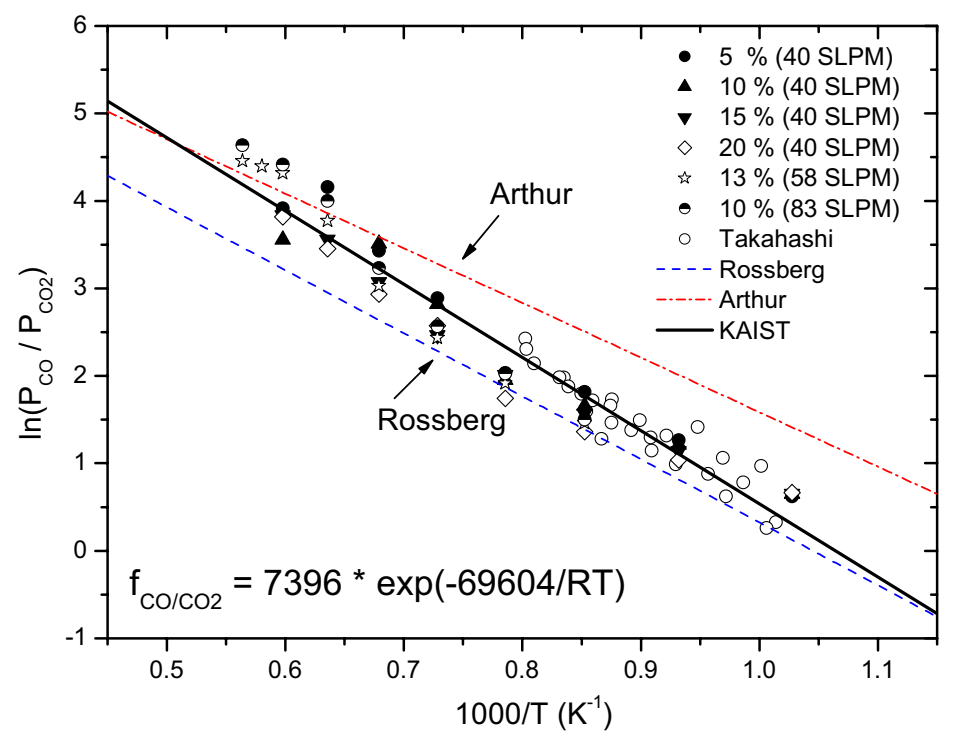

Figure 4-13. $\mathrm{CO} / \mathrm{CO}_{2}$ Ratio

Rossberg (1956) obtained the product ratio for the oxidation of two electrode carbons in the temperature range of $520^{\circ} \mathrm{C}$ to $1420^{\circ} \mathrm{C}$. Arthur (1951) derived the above expression for coal char and natural graphite. As shown in Figure 4-13, the data was distributed between Arthur's and Rossberg's correlations. The 30\% overprediction for Authur's and the $20 \%$ underpredction for Rossberg's were observed here. Especially the latter shows quiet good agreements with our experimental data even though different material may be expected to have different $\mathrm{CO} / \mathrm{CO}_{2}$ ratios. Most of data lie between their predictions. Here, for the better prediction for IG-110, an empirical correlation was suggested.

$f_{\mathrm{CO} / \mathrm{CO} 2}=7396 \exp \left(-\frac{69604}{R T}\right)$

We used a CFD simulation to calculate a theoretical reaction rate. This approach was selected for the following reason. In an air-ingress accident, the temperature of the reactor core increases to nearly $1600^{\circ} \mathrm{C}$. In this situation, the graphite oxidation reaction is largely affected not only by reaction chemistry but also by mass transfer by local distribution of flow, temperature, pressure, and gas components. The CFD simulation allows one to estimate the distribution of local parameters without any further assumption regarding wall friction or heat transfer, as long as the chemical kinetic parameters and the $\mathrm{CO} / \mathrm{CO}_{2}$ ratio are well defined.

Figure 4-14 shows the grid of this simulation. Fluent 6.1 software was selected as the CFD tool to simulate the main test. The geometry was hexagonally meshed to 77274 nodes. We used the oxidation parameters (Ea $=218 \mathrm{~kJ} / \mathrm{mol}, \mathrm{n}=0.75$ ) developed here and the $\mathrm{CO} / \mathrm{CO}_{2}$ ratio correlation (eq. (4-4)).

The Total of 8 transport equations was solved here for each node: 1 mass conservation, 3 momentum conservation, 1 energy conservation, and 3 species conservation equations. Since the experimental conditions are in the laminar flow regime, turbulent effects were neglected. Inlet velocity and outlet pressure were given as boundary conditions. During the simulation, the constant wall temperature condition was imposed at the graphite wall. Figure 4-14 also shows the cross sectional grid scheme. In this figure, the center region is a solid part and the outer region is a fluid part. To reduce the numerical error, the fluid part was symmetrically meshed. In the fluid region, all transport equations were solved while in the solid region, only energy equation was. 


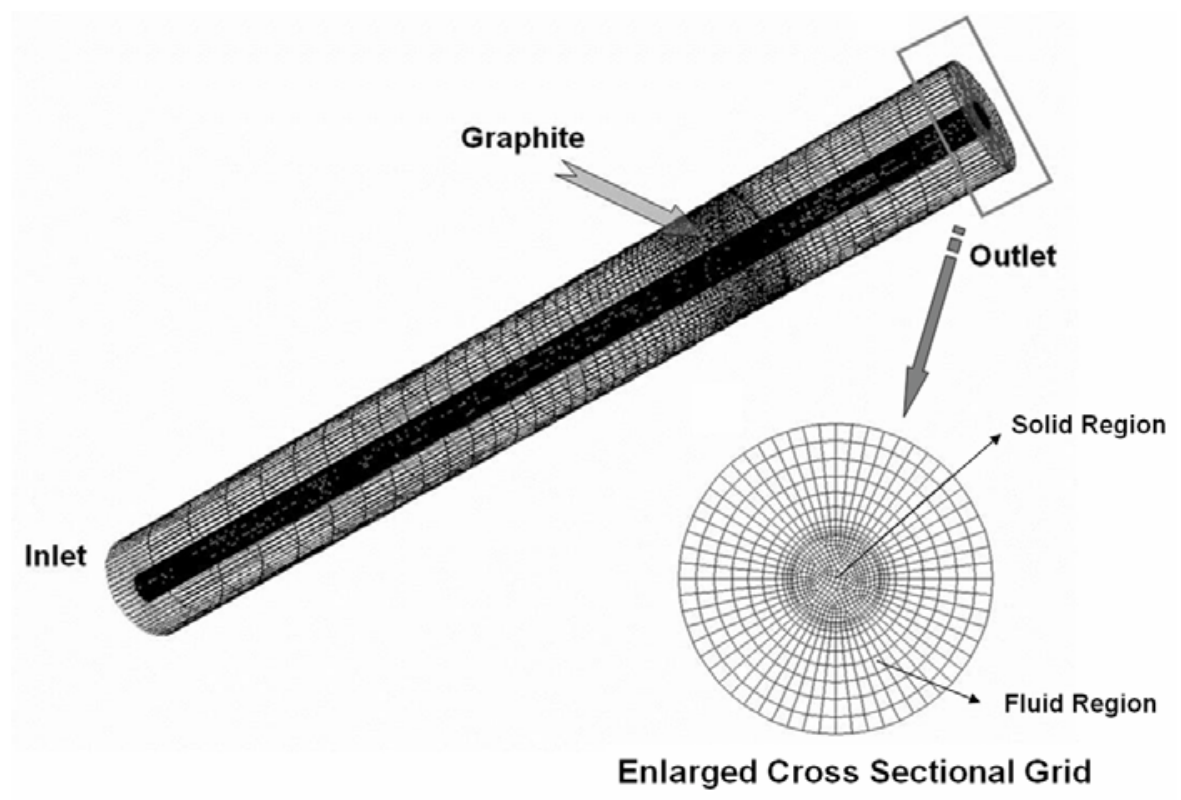

Figure 4-14. Geometry and Mesh of CFD Simulation

Density was calculated by ideal gas law, and viscosity was calculated by general multicomponent model. The thermal conductivity and heat capacity for each species were calculated by polynomial functions and mass averaged values were used for mixture values. Species diffusion was calculated by full multicomponent diffusion model, so a single value of mixture diffusion coefficient was not calculated here. The example of calculated gas properties were illustrated in Figure 4-15.

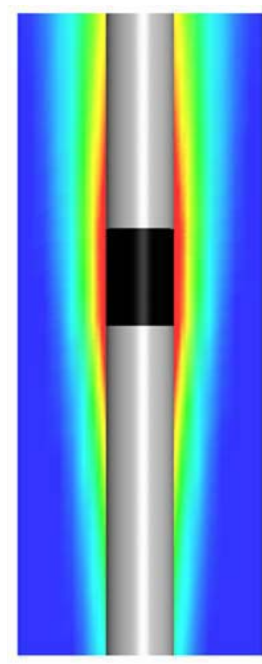

Temperature (K)

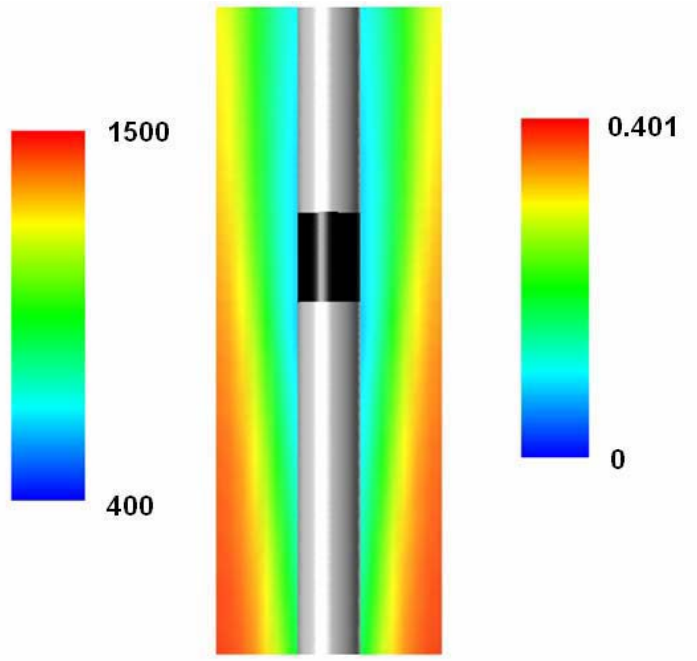

Density $\left(\mathrm{kg} / \mathrm{m}^{3}\right)$

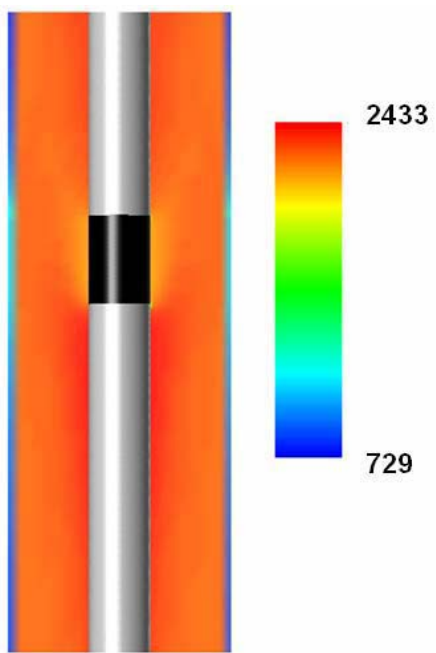

Heat Capacity (J/kg K)

Figure 4-15. Calculated Temperature and Gas Properties (1500 C, 0.16 m/s, $20 \%$ of oxygen)

Figure 4-16 shows a comparison of reaction rate between the simulation and experiment. Good agreement was observed in the comparison. The CFD simulation showed quantitatively good agreement, but it can also give us the following qualitatively important information. 


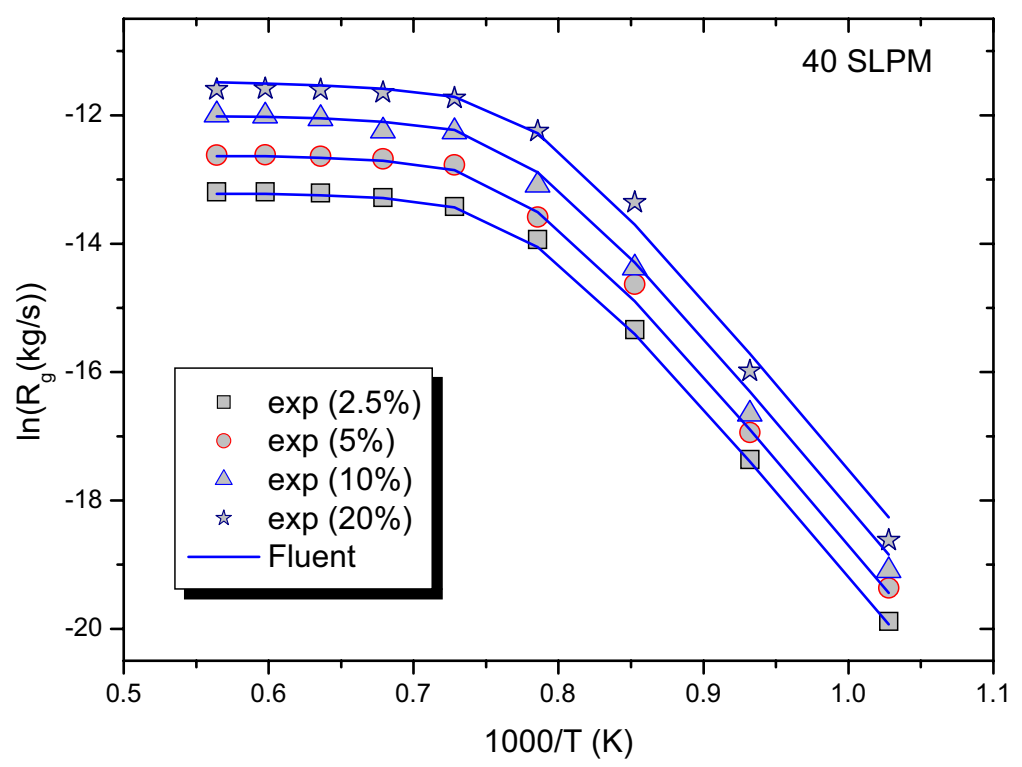

Figure 4-16. Comparison of the Oxidation Rate between FLUENT Predictions and Experimental Data

Figure 4-17 shows the velocity field around the reacting graphite material. There is a large distortion of the field around the surface by the large amount of gas produced on the graphite wall. Figure 4-18 shows the gas concentration pattern around the graphite surface. Note that the $\mathrm{CO}$ concentration near the surface is much higher than in the bulk flow.

A CFD simulation shows very good performance, but it always requires a large amount of computation time and big memory size due to a large number of grids. In actual applications such as nuclear system analysis or safety analysis, generating fine meshes for the whole complicated system is unrealistic due to its limited memory size and computation time. For this reason, system analysis codes, which use course grids, are more generally used. As the course grid does not calculate detail distributions for temperatures, velocities or properties as done in the CFD simulation, a lot of constitutive models and correction methods are frequently required. 


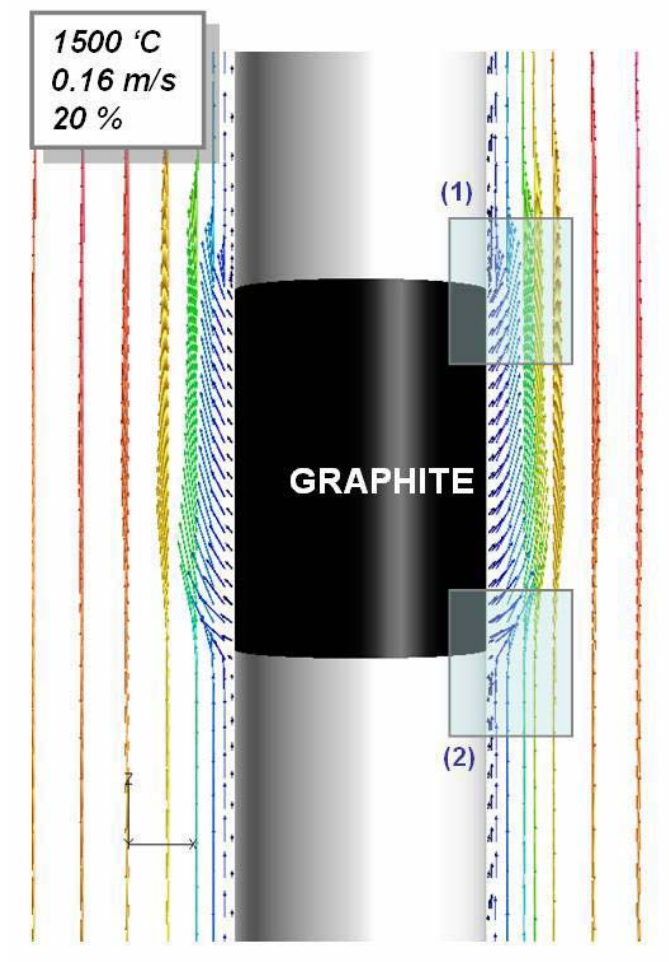

(1)

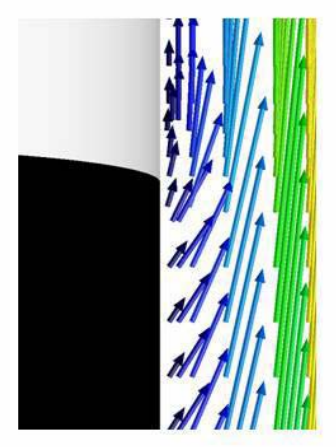

(2)

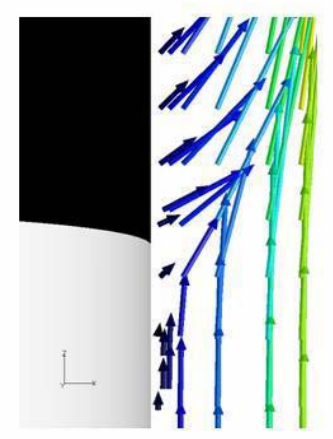

Figure 4-17. Simulation Results of Velocity Vectors

$\mathrm{O}_{2}$

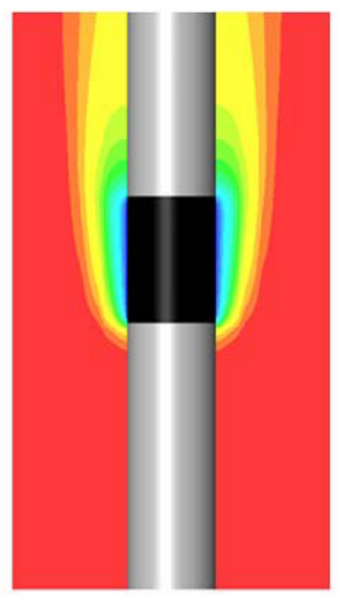

CO

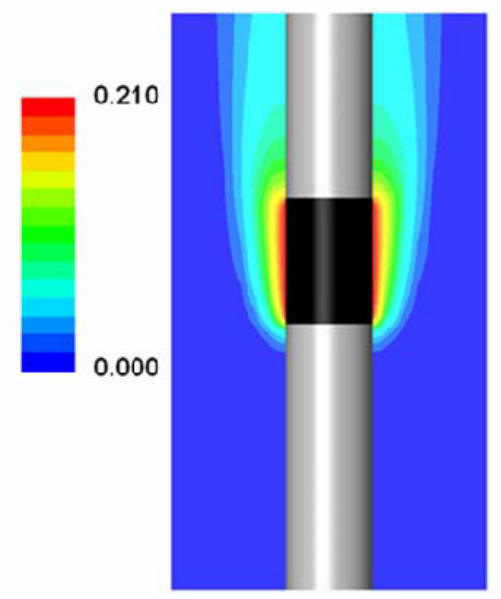

$\mathrm{CO}_{2}$

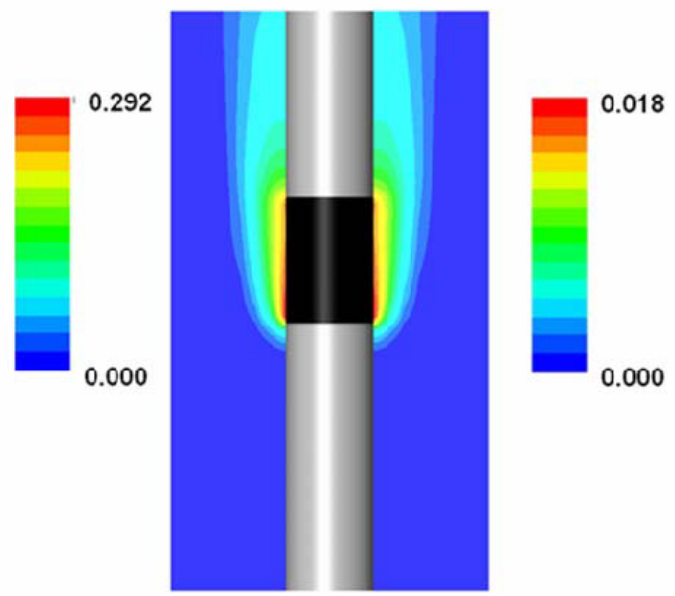

Figure 4-18. Calculated Gas Concentrations (1500 C, $0.16 \mathrm{~m} / \mathrm{s}, 20 \%$ of Oxygen)

In this study, a semi-empirical constitutive model, which is applicable for the wide ranges of graphite oxidation, was developed from the following balance equation.

$$
k \cdot C_{s, o 2}{ }^{n}=K_{m} \cdot\left(C_{b, O 2}-C_{s, O 2}\right)
$$

where the left and right terms mean chemical reaction rate and external mass transfer rate. Since eq. (4-5) is an analytically unsolvable equation besides $n=1$ or 2 , numerical method should be used for a general $n$. However, to avoid numerical calculations, a semi-empirical solution was developed by the following slight modification of the well-known solution for $n=1$. The solution of $n=1$ can be easily derived as follows (Mills 2001). 
$r_{g}=k \cdot C_{s, O 2} \quad(4-6)$

$m^{\prime \prime}\left(\mathrm{kg} / \mathrm{m}^{2} \mathrm{~s}\right)=K_{m} \cdot\left(C_{b, O 2}-C_{s, O 2}\right)$

Balancing the above two equations, that is, $r_{g}=m^{\prime \prime}$, yields $C_{s, O 2}$ :

$C_{s, O 2}=C_{b, O 2} \cdot \frac{K_{m}}{K_{m}+k}$

Note that, as $\mathrm{T}$ approaches zero and an infinite value, $C_{s, O 2}=C_{b, O 2}, 0$, respectively, as expected. Then, the chemical reaction flux becomes

$r_{g}=\frac{\left(K_{m} \cdot C_{b, O 2}\right) \cdot k}{K_{m}+k}$

This is the general form of the solution for $\mathrm{n}=1$. This equation can be rearranged as follows:

$$
r_{g}=\frac{\left(K_{m} \cdot C_{b, O 2}\right) \cdot\left(k \cdot C_{b, O 2}\right)}{\left(K_{m} \cdot C_{b, O 2}\right)+\left(k \cdot C_{b, O 2}\right)}
$$

Then, it is changed into the following.

$$
\frac{1}{r_{g}}=\frac{1}{r_{m b}}+\frac{1}{r_{c b}}
$$

where $r_{g}$ is an overall reaction rate, $r_{m b}$ is an asymptotic mass transfer flux and $r_{c b}$ is an asymptotic chemical reaction flux. In this solution, all terms are separated by independent simple flux terms. The physical meaning of this solution is that the overall reaction rate $r_{c b}$ approaches as the chemical reaction becomes slower, and conversely, it approaches $r_{m b}$ as the chemical reaction becomes faster. Then, we can assume that replacing the above chemical reaction term, $r_{c b}$ with the following general form (eq. (4-12)) will not change its original physical meaning.

$\frac{1}{R_{g}}=\frac{1}{R_{m b}}+\frac{1}{R_{c b}}$

where

$R_{m b}=K_{m} \cdot C_{b, O 2} \cdot A$

$R_{c b}=K_{0} \cdot e^{-\frac{E a}{R \cdot T}} \cdot P_{O 2, b}{ }^{n} \cdot A(n \neq 0)$

This semi-empirical model becomes exactly the same equation as the original one, eq. (4-8), for $n=1$.

We compared the calculated results with the experimental data for assessment of this correlation. To calculate the mass transfer coefficient, $K_{m}$, we used heat/mass transfer analogy (Welty et al. 1969) and the following Graetz solution (Kakac and Yener 1994), which includes the effect of the entrance effect.

$S h=\frac{k_{m} \cdot d}{D}=3.66+\frac{0.0668(d / x) \cdot(\operatorname{Re} \cdot S c)}{1+0.04 \cdot[(d / x) \cdot(\operatorname{Re} \cdot S c)]^{2 / 3}}$

In the reaction entrance region, since the diffusion boundary layer is not fully developed, the boundary layer thickness is much smaller than the fully developed flow. Since the boundary layer thickness is inversely proportional to the mass transfer rate, the mass transfer rate will be underestimated by the normal correlations assuming the fully developed flow. In fully developed region, the Graetz solution shows the same results as the normal correlations. 


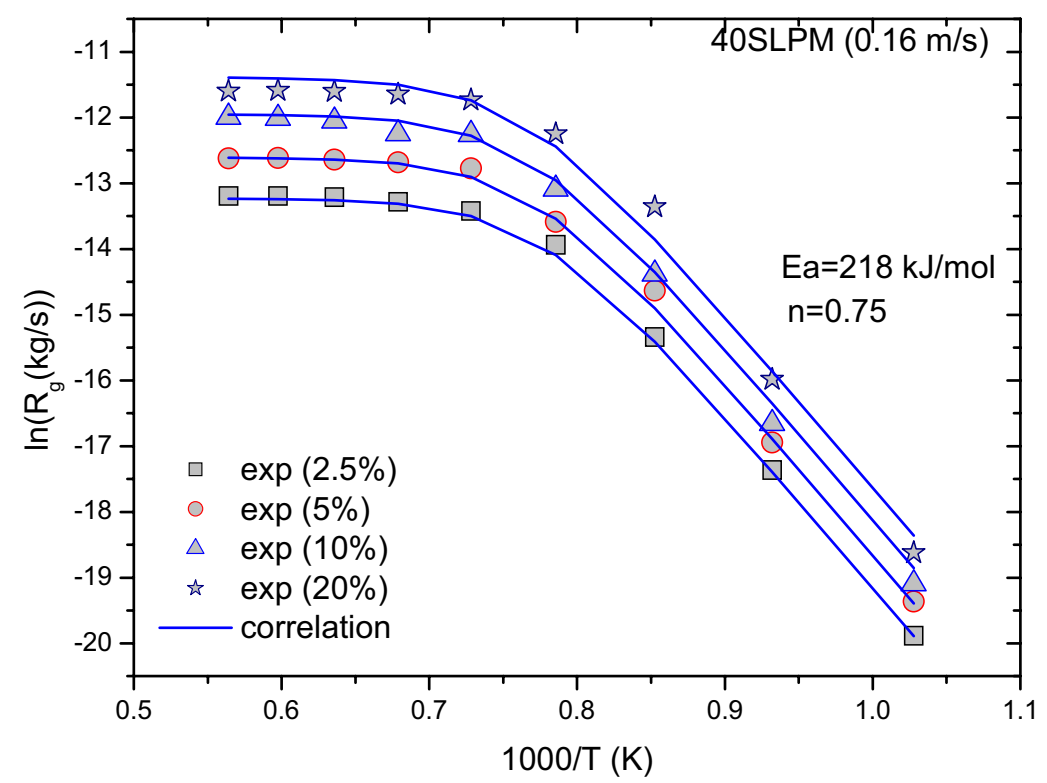

Figure 4-19. Comparison of Reaction Rates between Experimental Data and Predictions by Analytical Model

Generally, most of the mass transfer coefficient correlations are derived by well-known velocity fields, which are assumed for no mass transfer. This is a good assumption for low reaction rate condition. However, it is not suitable for high reaction rate, because such high mass transfer will cause the large velocity distortion. Therefore, for more accurate calculations, a correction is required. In this study, the following correction was performed (Mills 2001).

$k_{m}{ }^{\text {corrected }}=\theta \cdot k_{m}$

where

$\theta=\frac{\ln \left(B_{m}+1\right)}{B_{m}}$

$B_{m}=\frac{X_{O 2, b}-X_{O 2,0}}{X_{O 2, b}-1}$

To calculate the mass transfer coefficient from eq. (4-13), diffusion coefficient should be decided. A binary diffusion coefficient is generally the most frequent selection. But at high reaction rate, it is not suitable because the gases produced at the reacting surface will affect the gas properties, especially diffusion coefficients. For an exact calculation, muticomponent diffusion model should be used as in the CFD simulation. But because of its complexity, the following effective diffusion coefficient, which means an averaged diffusion coefficient for gas mixture was used here (Mills 2001).

$$
D_{O 2, m}=\frac{\left(1-X_{1}\right)}{\sum_{i=2}^{n}\left(X_{i} / D_{O 2, i}\right)}
$$

Figure 4-19 illustrates the comparisons on the rate of reaction between the experiment and the analytic model. As shown in this figure, this model is in good agreement with the experimental data from low temperature to high temperature. 


\subsection{Geometrical effect}

To investigate the geometrical effect on the graphite oxidation, we developed a new method of analysis. The details are as follows. Since graphite is a porous material, the oxidation reaction occurs not only on the external surface but also in the internal pores. Therefore, the reaction rate of graphite oxidation $\left(\mathrm{R}_{\mathrm{g}}\right)$ can be expressed as follows:

$R_{g}=A_{0} \cdot \exp \left(-\frac{E a}{R \cdot T}\right) \cdot P_{O 2}{ }^{n} \cdot\left(A_{s}+A_{v}\right)$,

where $\mathrm{A}_{0}$ is a pre-exponent factor, $\mathrm{Ea}$ is an activation energy, $\mathrm{R}$ is a gas constant, $\mathrm{T}$ is the temperature, $\mathrm{P}_{\mathrm{O} 2}$ is the partial pressure of the oxygen, $n$ is the order of reaction, $A_{s}$ is the external surface area, and $A_{v}$ is the internal surface area available for reaction. In order to determine $A_{v}$, we assume that $A_{v}$ is proportional to the volume $(\mathrm{V})$ of the graphite because the internal pores are uniformly distributed:

$A_{v}=\theta \cdot V$,

where $\theta$ is an internal surface density, which physically means an internal surface area in unit volume. If we put Eq. (4-21) into Eq. (4-20), we can obtain the following equation:

$\frac{R_{g}}{A_{s}}=A_{0} \cdot \exp \left(-\frac{E a}{R \cdot T}\right) \cdot P_{O 2}{ }^{n} \cdot\left(1+\frac{\theta \cdot V}{A_{s}}\right)$.

If the temperature and oxygen pressure are fixed in Eq. (4-3), the value of $A_{0} \cdot \exp \left(-\frac{E a}{R \cdot T}\right) \cdot P_{O 2}{ }^{n}$ is also fixed. Therefore, we can rewrite Eq. (4-22) as

$\frac{R_{g}}{A_{s}}=C \cdot\left(1+\theta \cdot \frac{V}{A_{s}}\right)$,

where

$C=A_{0} \cdot \exp \left(-\frac{E a}{R \cdot T}\right) \cdot P_{O 2}{ }^{n}$

In Eq. (4-24), $\mathrm{R}_{\mathrm{g}} / \mathrm{A}_{\mathrm{s}}$ is linearly related to $\mathrm{V} / \mathrm{A}_{\mathrm{s}}$ under constant temperature and oxygen pressure conditions. As illustrated in Figure 4-1, $C \cdot \theta$ represents a slope and $C$ represents a y-axis intercept of the linear graph.

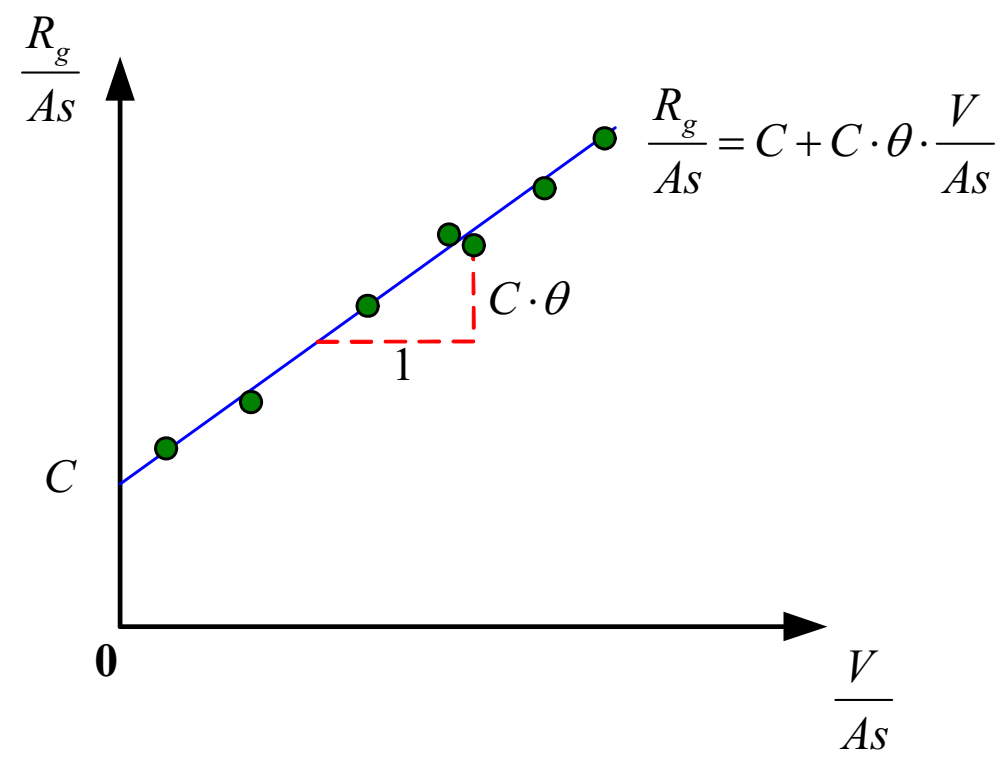


Figure 4-20 Analytical method for geometrical effect

In this study, to determine the value of $\theta$, we measured the reaction rates for various graphite samples with different surface-to-volume ratios, and obtained the relation graph shown in Figure 4-20. We then calculated $\theta$ by analyzing its gradient and y-interception.

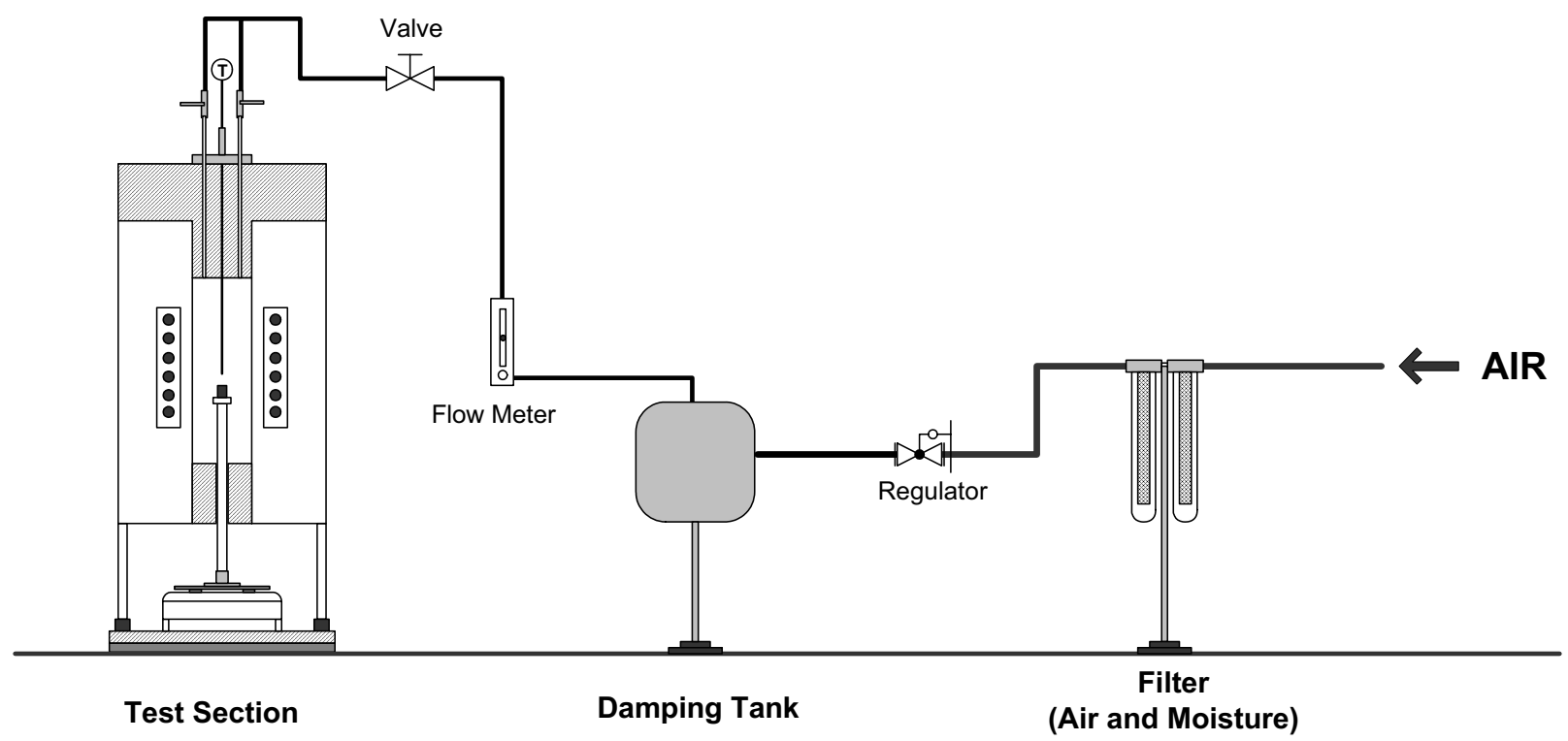

Figure 4-21 Experimental facility for geometrical effect

To measure the graphite oxidation velocity, we manufactured the experimental facility, as shown in Figure 421. We injected dehumidified natural air into the test section through a damping tank, and controlled the flow rate with a mass flow controller within $\pm 1 \%$ accuracy. In our experiment, the temperature was constantly maintained at $600^{\circ} \mathrm{C}$ within $\pm 1^{\circ} \mathrm{C}$ accuracy. The test was carried out under the condition, where the chemical reaction is a rate-controlling process. We placed graphite samples on the beam at the center of the furnace, and connected the support beam to the balance. Weights were measured with a precision of $\pm 1 \mathrm{mg}$. 


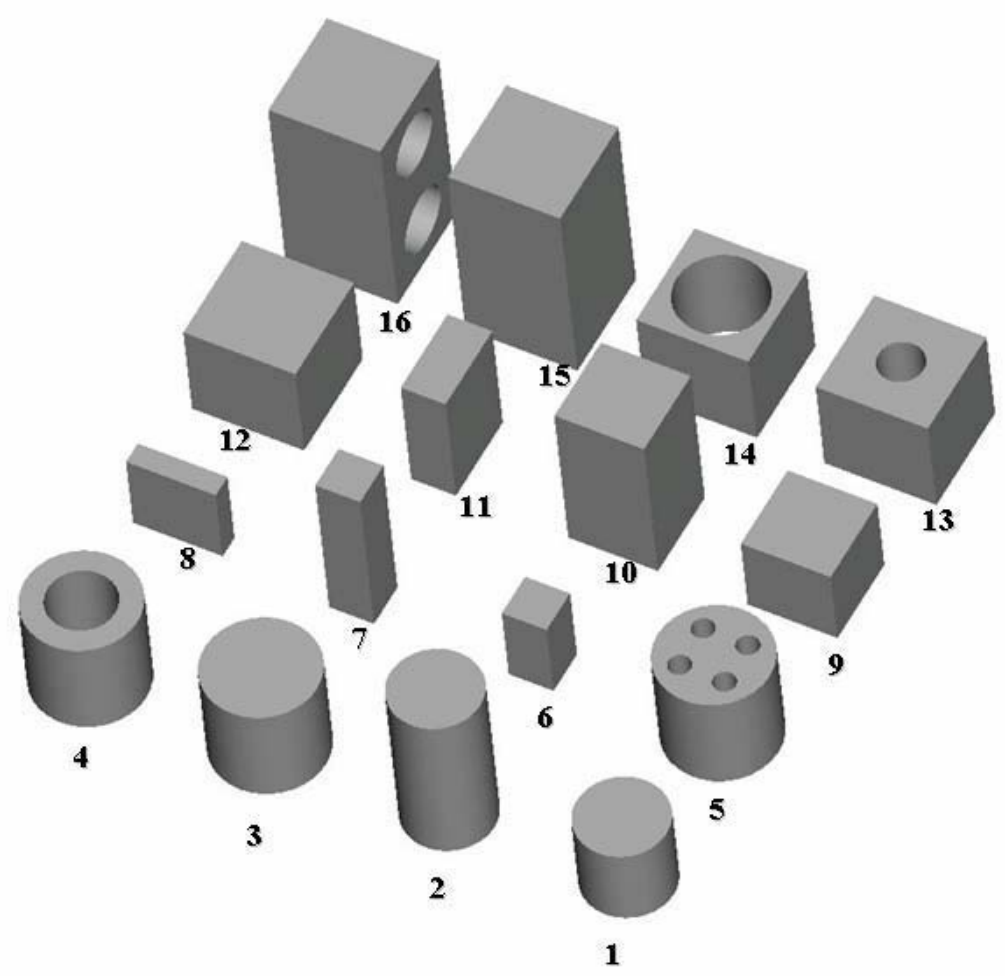

Figure 4-22 Graphite specimens used in the geometrical effect test

Figure 4-22 illustrates the graphite specimens used in the geometrical effect test and Table 4-4 summarizes the shapes and sizes.

Figure 4-23 shows the relation between $\mathrm{R} / \mathrm{A}_{\mathrm{s}}$ and $\mathrm{V} / \mathrm{A}_{\mathrm{s}}$. By analyzing the slope and $\mathrm{y}$-axis intercept of this graph, we deduced the internal surface density as follows:

$\theta=12760 \mathrm{~m}^{-1}$.

Physically, it means that a unit volume of graphite includes a $12760 \mathrm{~m}^{2}$ internal surface area.

With this result, we calculated the proportion (I) of the external surface reaction among the total reaction for each sample. Since the reaction rate is proportional to the surface area, the proportion of external reaction can be calculated by the following equation:

$I(\%)=\frac{A_{s}}{A_{s}+A_{v}} \times 100=\frac{A_{s}}{A_{s}+\theta \cdot V} \times 100$,

where I $(\%)$ means the percentage of the external surface reaction in the total reaction. As a result, the proportion of the external reaction was below 5 percent of the total reaction, which means that the external surface reaction is negligible.

Finally, we propose the following reaction equation for this material:

$r^{\prime \prime \prime}\left(\mathrm{kg} / \mathrm{m}^{3} \mathrm{~s}\right) \approx 2552000 \cdot \exp \left(-\frac{218000}{R \cdot T}\right) \cdot P_{O 2}{ }^{0.75}$ 


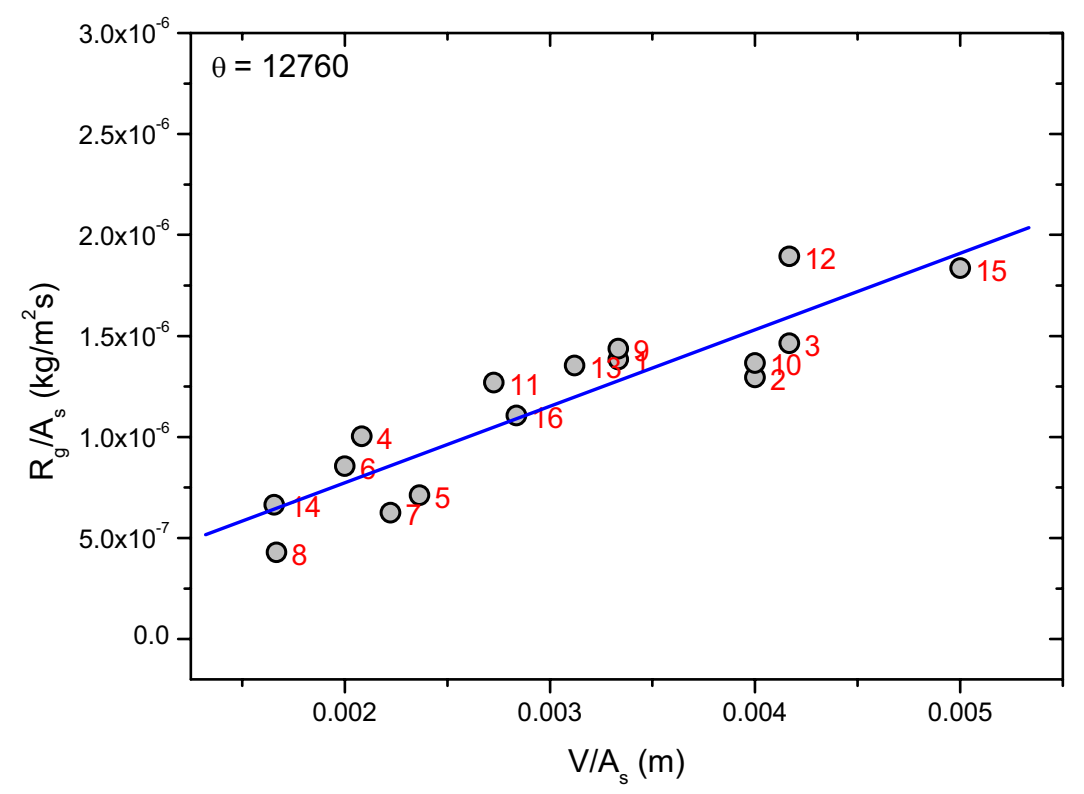

Figure 4-23 Relation between $\mathrm{V} / \mathrm{A}_{\mathrm{s}}$ vs. $\mathrm{R}_{\mathrm{g}} / \mathrm{A}_{\mathrm{s}}$

Table 4-4 Specifications of the graphite specimens used in the geometrical effect test

\begin{tabular}{|c|c|c|c|c|c|}
\hline & Type & Size (mm) & $\begin{array}{l}\text { Volume } \\
\left(\mathbf{m m}^{3}\right)\end{array}$ & Area $\left(\mathrm{mm}^{2}\right)$ & $\mathbf{V} / \mathbf{A}_{\mathbf{s}}(\mathbf{m})$ \\
\hline 1 & Cylinder & D20 $\times$ L20 & 6283 & 1885 & 0.0033 \\
\hline 2 & Cylinder & $\mathrm{D} 20 \times \mathrm{L} 40$ & 12566 & 3142 & 0.0040 \\
\hline 3 & Cylinder & $\mathrm{D} 25 \times \mathrm{L} 25$ & 12272 & 2945 & 0.0042 \\
\hline 4 & Cylinder & $\mathrm{D} 25 \times \mathrm{L} 25(15 \mathrm{~mm}$ 1hole $)$ & 7854 & 3770 & 0.0021 \\
\hline 5 & Cylinder & D25 × L25 (5mm 4holes) & 10308 & 4359 & 0.0024 \\
\hline 6 & Rectangular & $10 \times 10 \times 20$ & 2000 & 1000 & 0.002 \\
\hline 7 & Rectangular & $10 \times 10 \times 40$ & 4000 & 1800 & 0.0022 \\
\hline 8 & Rectangular & $5 \times 20 \times 20$ & 2000 & 1200 & 0.0017 \\
\hline 9 & Rectangular & $20 \times 20 \times 20$ & 8000 & 2400 & 0.0033 \\
\hline 10 & Rectangular & $20 \times 20 \times 40$ & 16000 & 4000 & 0.004 \\
\hline 11 & Rectangular & $10 \times 20 \times 30$ & 6000 & 2200 & 0.0027 \\
\hline 12 & Rectangular & $25 \times 25 \times 25$ & 15625 & 3750 & 0.0042 \\
\hline 13 & Rectangular & $25 \times 25 \times 25(10 \mathrm{~mm}$ 1hole $)$ & 13662 & 4278 & 0.0031 \\
\hline 14 & Rectangular & $25 \times 25 \times 25(20 \mathrm{~mm} 1$ hole $)$ & 7771 & 4693 & 0.0017 \\
\hline 15 & Rectangular & $25 \times 25 \times 50$ & 31250 & 6250 & 0.005 \\
\hline 16 & Rectangular & $25 \times 25 \times 50(15 \mathrm{~mm} 2$ holes $)$ & 22414 & 7899 & 0.0028 \\
\hline
\end{tabular}




\subsection{Burn-off effect}

The rate of reaction between graphite and oxygen is dependent on the level of burn-off although it looks constant in a small time scale. In this study, the effect of burn-off was experimentally investigated and the modeling was performed. The same facility and specimens used in Task 4-4 were also used here.

At first, we measured the rates of reaction for various geometries at the same temperature $600{ }^{0} \mathrm{C}$ where the chemical reaction is the rate-controlling process. At this temperature, we can assume that the reaction is uniform inside of the graphite since the rate of reaction is very slow. Figure 4-24 shows the results of burn-off variation with time. As shown in this figure, the geometries and sizes do not affects the reaction history, and it confirms that the internal reaction occurred uniformly in the graphite. Therefore, we expect that the same pattern of the reaction history will happen irrespective of the geometries or sizes.

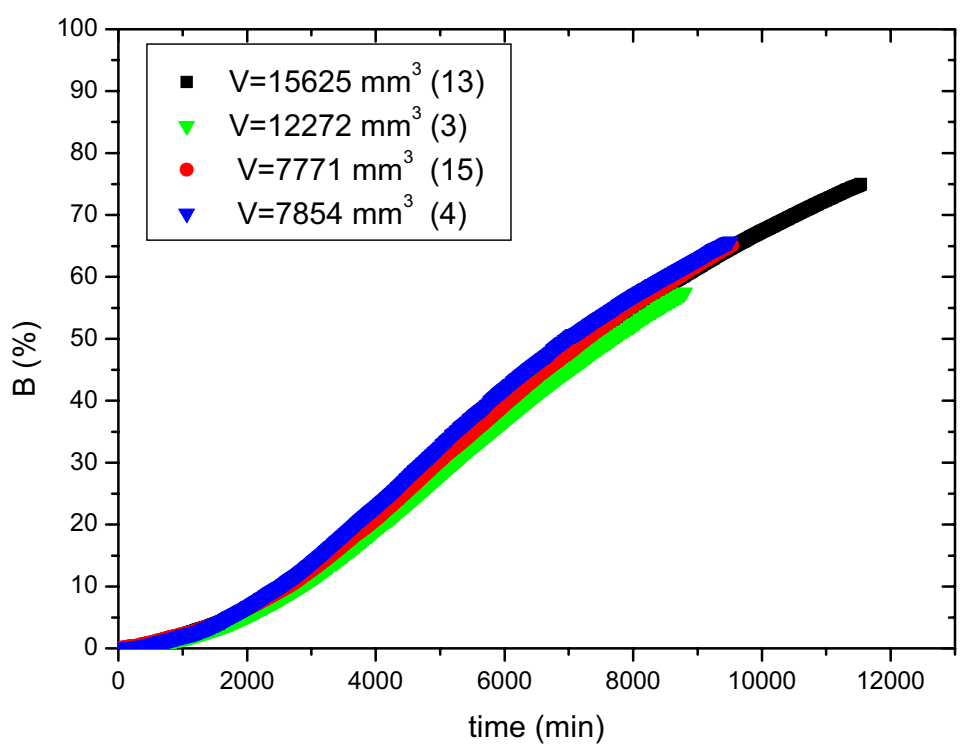

Figure 4-24 Rate of reaction for various geometries

From Figure 4-24, we obtained the relation between the burn-off and the relative reaction rate as shown in Figure 4-25.

We estimated the change of shape and size during the test for sample 4. Figure 4-26 shows the graphite sample tested up to $65 \%$ of burn-off level. As shown in this figure, the shape and size changes are negligible compared to the mass variation.

We also carried out the same test at different temperatures where the diffusion effect can not be ignored any more. Figure 4-27 shows the results of mass variation with time at different temperatures and it shows that the trends are different for different temperatures. At low temperature, since the internal reaction increases the pore size inside, the reaction increases with time. On the other hand, at high temperature, since the reaction is concentrated on the external surface, it changes the bulk shapes and sizes with time and, as a result, the reaction decreases with time. 


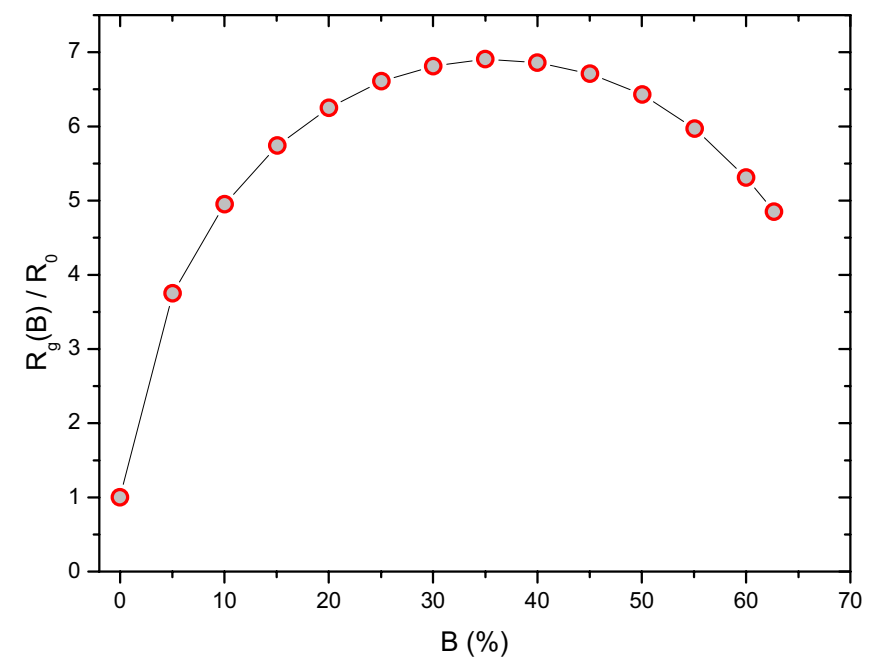

Figure 4-25 Relation between burn-off and oxidation rate
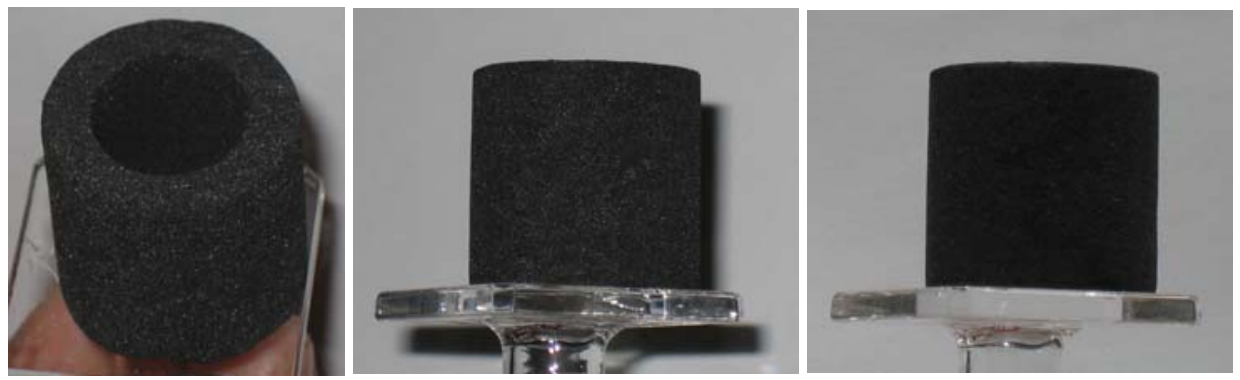

Figure 4-26 shape of the tested graphite sample (sample 4)

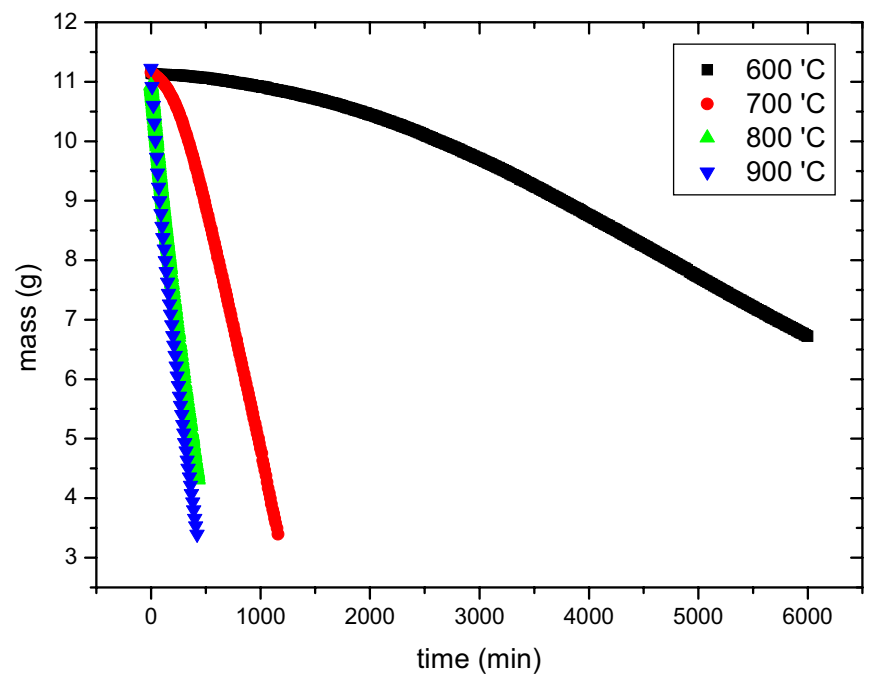

Figure 4-27 Mass change of the tested graphite sample (sample 4) 
To predict the variation of the graphite reaction with time, a modeling and simulation were performed. The following assumptions were used in this modeling.

- The variation of the external surface roughness with time is negligible.

- The variation of the diffusion coefficient with time is negligible.

- The level of burn-off does not affect the chemical characteristics of the graphite.

- The variation of the internal structure of the graphite is only dependent on the level of burn-off.

The following equations were selected for modeling.

- 2 dimensional diffusion equation (cylindrical coordinate)

$\frac{\partial C_{O 2}}{\partial t}=\frac{1}{r} \frac{\partial}{\partial r}\left(r D_{e} \frac{\partial C_{O 2}}{\partial r}\right)+\frac{\partial}{\partial z}\left(D_{e} \frac{\partial C_{O 2}}{\partial z}\right)+S_{O 2}$

- mass change

$\frac{\partial \rho_{g}}{\partial t}=-r^{\prime \prime \prime}(B)$

- burn-off

$B=\frac{\rho_{\text {initial }}-\rho}{\rho_{\text {initial }}} \times 100$

- graphite oxidation

Bulk reaction: $r^{\prime \prime \prime}(B)=200 \cdot \exp (-218000 / R \cdot T) \cdot p_{O 2}{ }^{0.75} \cdot \theta(B)$

Surface reaction: $r^{\prime \prime}=200 \cdot \exp (-218000 / R \cdot T) \cdot p_{O 2}{ }^{0.75}$

- diffusion coefficient

$D_{e}=D_{T} \times($ void fraction $) /($ tortousity $)$

$1 / D_{T}=1 / D_{\text {binary }}+1 / D_{\text {knussent }}$

The finite volume method was used as a discretization method. And implicit scheme and Gauss-Siedal method were applied here. Figure 4-28 shows the calculation procedure for this simulation.

Figure 4-29 shows the comparison results between the calculation and the experimental results. As shown in this figure, the simulation results are in good agreement with the experimental data. Figures 4-30 through 433 illustrate the simulation results for the density variation of the graphite with time. At low temperatures, the internal density change is main reaction mechanism. However, at high temperatures, internal density changes are very small and the size change is the main reaction mechanism. 


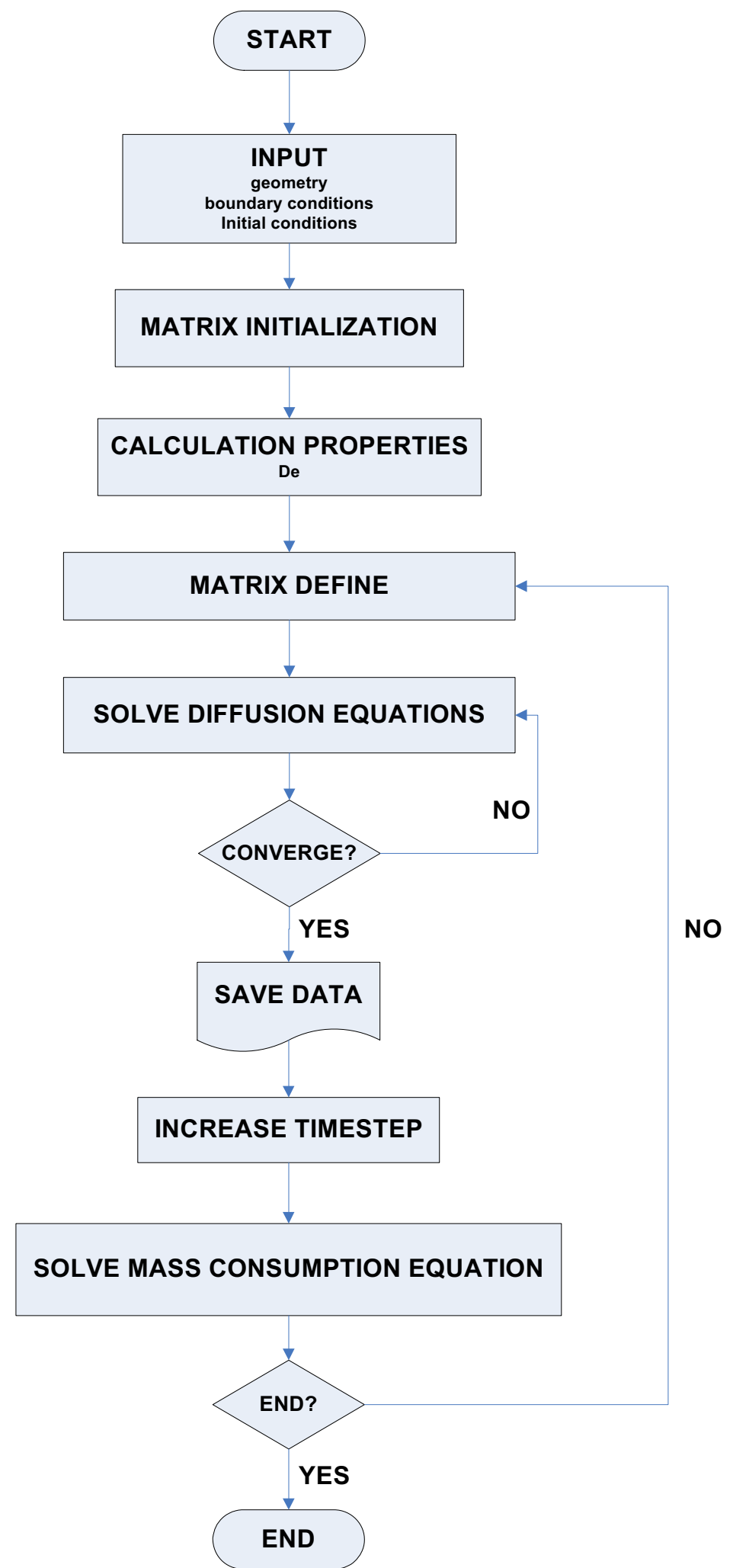

Figure 4-28 Calculation procedure for burn-off effect modeling 


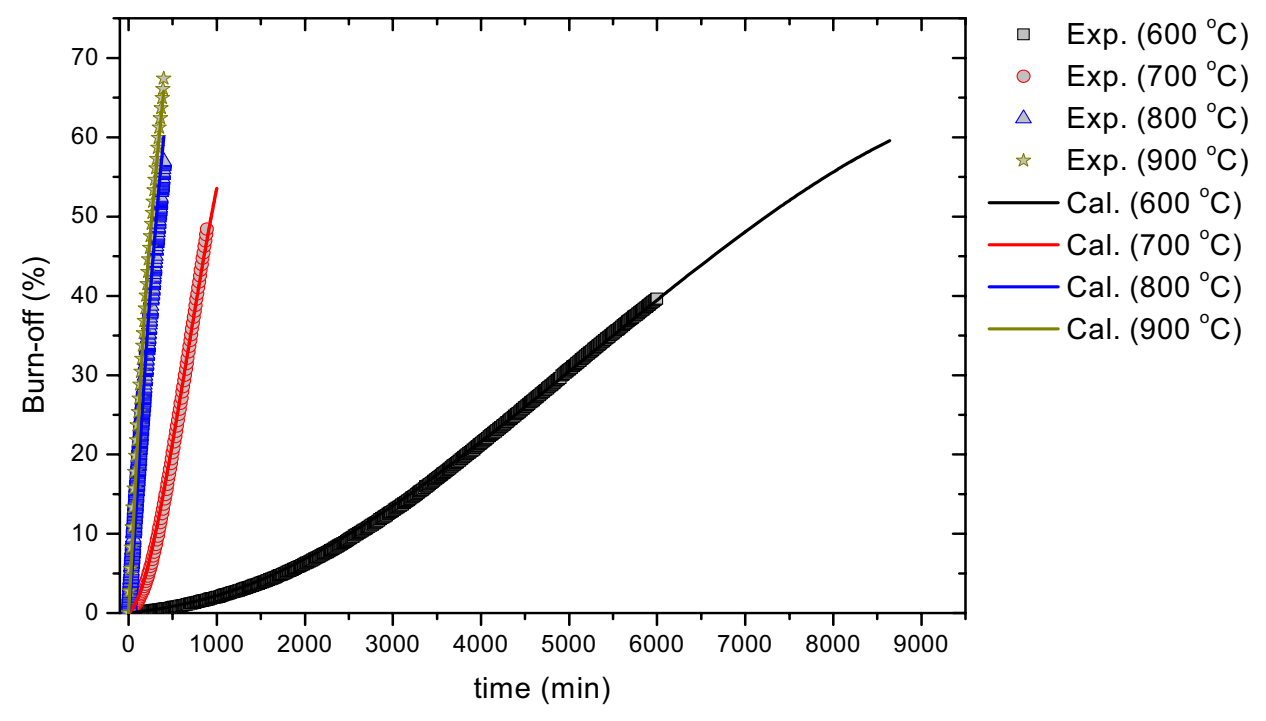

Figure 4-29 Comparison of Burn-off between experimental data and calculation results
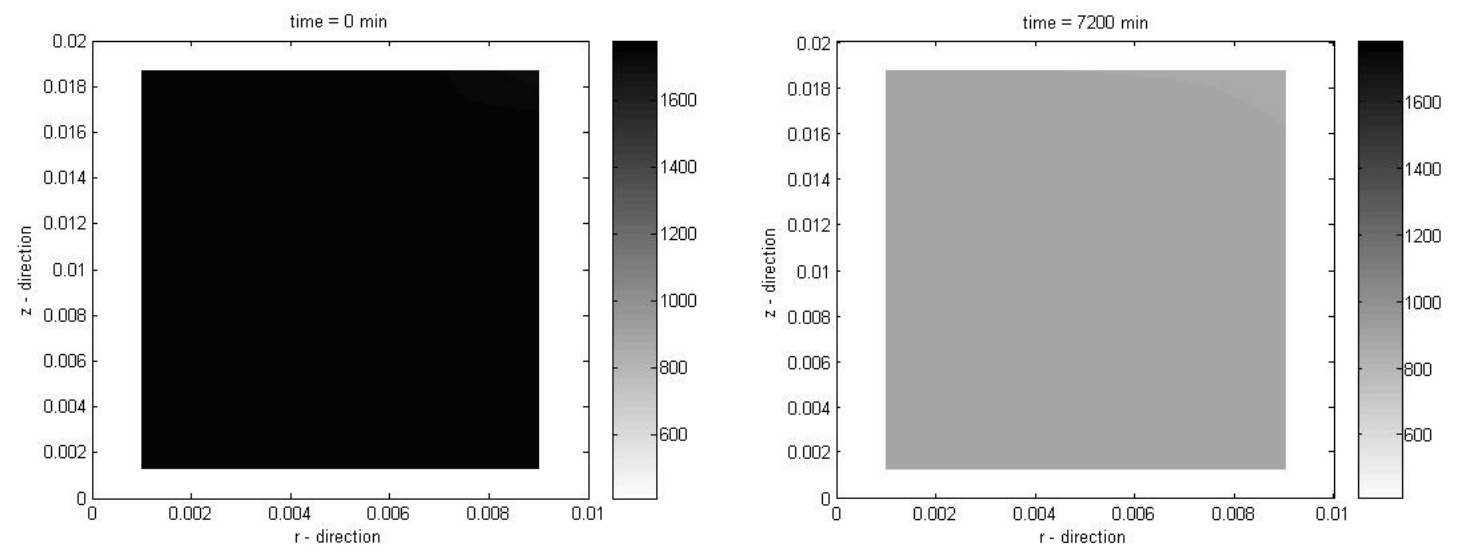

Figure 4-30 Simulation results at $600{ }^{\circ} \mathrm{C}$ (density)
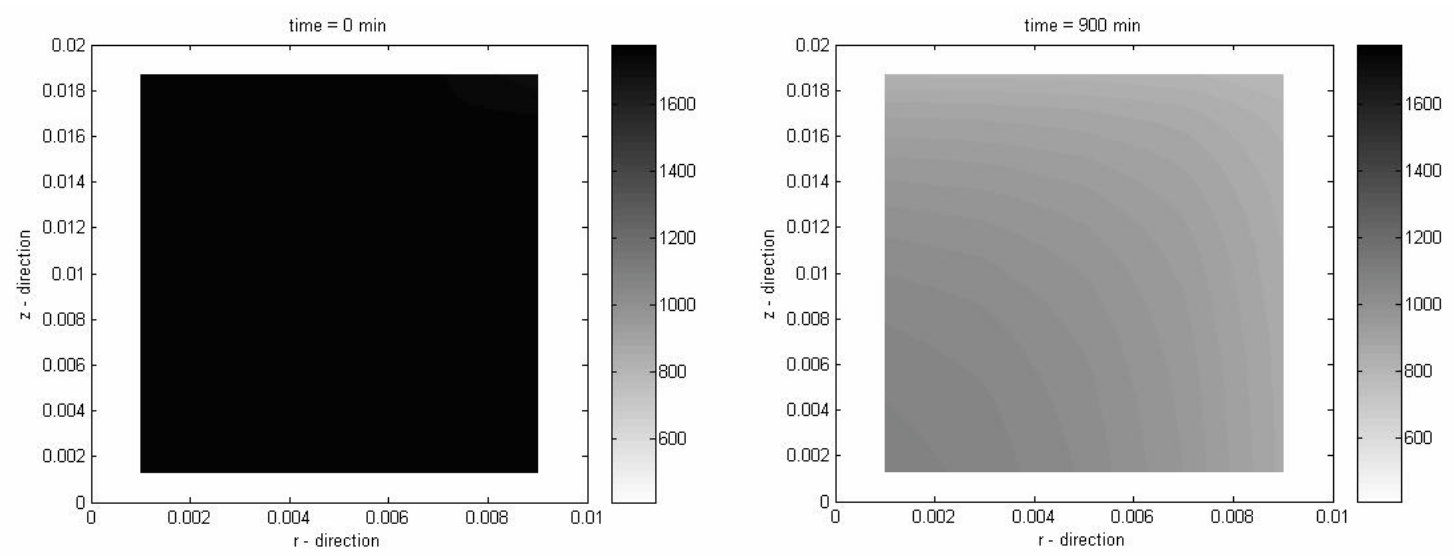

Figure 4-31 Simulation results at $700{ }^{\circ} \mathrm{C}$ (density) 

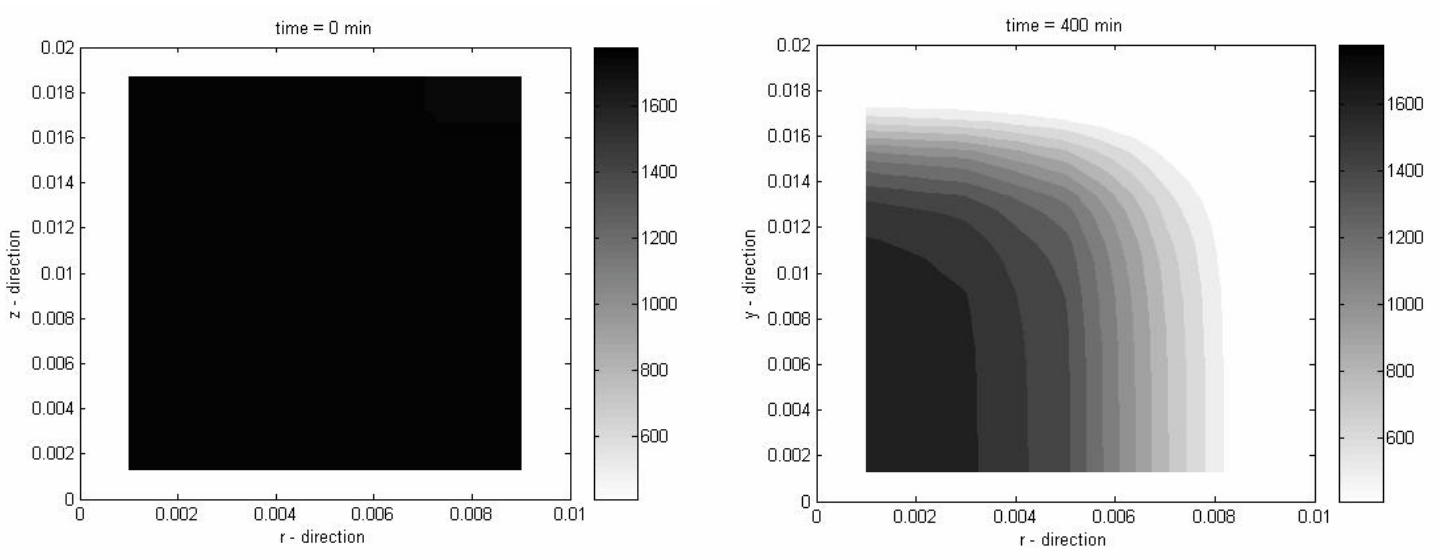

Figure 4-32 Simulation results at $800{ }^{\circ} \mathrm{C}$ (density)
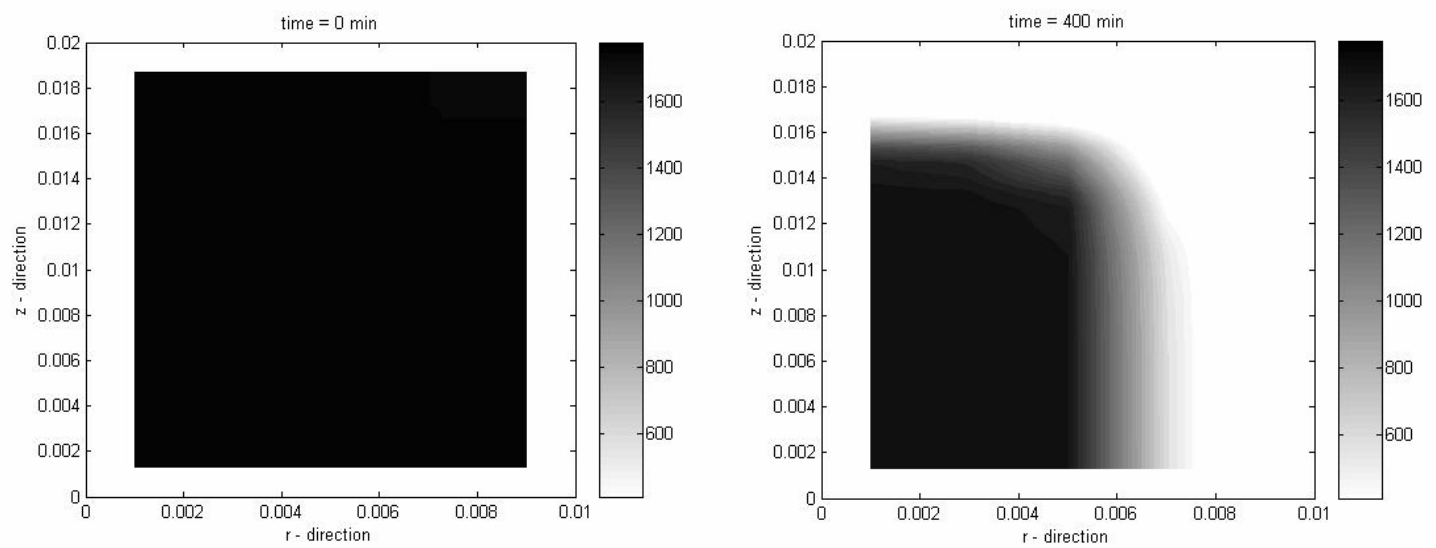

Figure 4-33 Simulation results at $900{ }^{\circ} \mathrm{C}$ (density)

\subsection{Effect of $\mathrm{C} / \mathrm{CO}_{2}$ reaction}

Until recently, many researchers have studied the reaction of $\mathrm{C} / \mathrm{O}_{2}$ and obtained excellent results. However, relatively, little attention has been given to other reactions. The reaction of graphite and $\mathrm{CO}_{2}$ gas $\left(\mathrm{C} / \mathrm{CO}_{2}\right.$ reaction) is among those neglected reactions. The reaction of the $\mathrm{C} / \mathrm{CO}_{2}$ is written as follows: $\mathrm{C}+\mathrm{CO}_{2} \rightarrow 2 \mathrm{CO}$.

This equation shows that the $\mathrm{C} / \mathrm{CO}_{2}$ reaction produces $\mathrm{CO}$ gas as a main product, which is known toxic and explosive. Furthermore, since this reaction can damage the structural integrity, investigation on this reaction is necessary for better analysis of the air-ingress.

Figure 4-34 shows our experimental facility. First, we obtained the reaction rate by analysis on the concentrations of $\mathrm{O}_{2}, \mathrm{CO}$ and $\mathrm{CO}_{2}$ species. The specimen was supported by a ceramic rod and heated by an induction heater. We then measured its surface temperature with an infrared thermometer. The test specimens made of IG-110 graphite, which is an isostatically molded, isotropic fine-grained and halogen purified, were machined to $2.1 \mathrm{~cm}$ in diameter and $3 \mathrm{~cm}$ in length. The mixture gas of Helium and $\mathrm{CO}_{2}$ was used as a reacting gas and injected at the bottom of the test section, which was made of a quartz tube. This experiment was conducted in the temperature range between $600^{\circ} \mathrm{Cand} 1400^{\circ} \mathrm{C}$, and in the mole fraction of $\mathrm{CO}_{2}$ between 5 percent and 20 percent. 
To investigate the reaction of $\mathrm{C} / \mathrm{CO}_{2}$, we measured kinetic parameters: an activation energy (Ea) and an order of reaction (n). Figure 4-35 illustrates the effect of temperature on the reaction rate. In this graph, which is generally called as Arrhenius plot, the $\mathrm{x}$-axis represents 1000/T and $\mathrm{y}$-axis represents the logarithm of the reaction rate. This figure shows a linear trend of the data between the two main parameters: 1000/T and a log of the reaction rates and it confirms that the Arrhenius model globally well represents the reaction of $\mathrm{C} / \mathrm{CO}_{2}$ in our conditions. The activation energy can be obtained from the slope of this graph and we repeated the same tests 7 times for more confidence. By applying a statistical method, we determined the value of activation energy as $295 \pm 8 \mathrm{~kJ} / \mathrm{mol}$ within $95 \%$ confidence level.

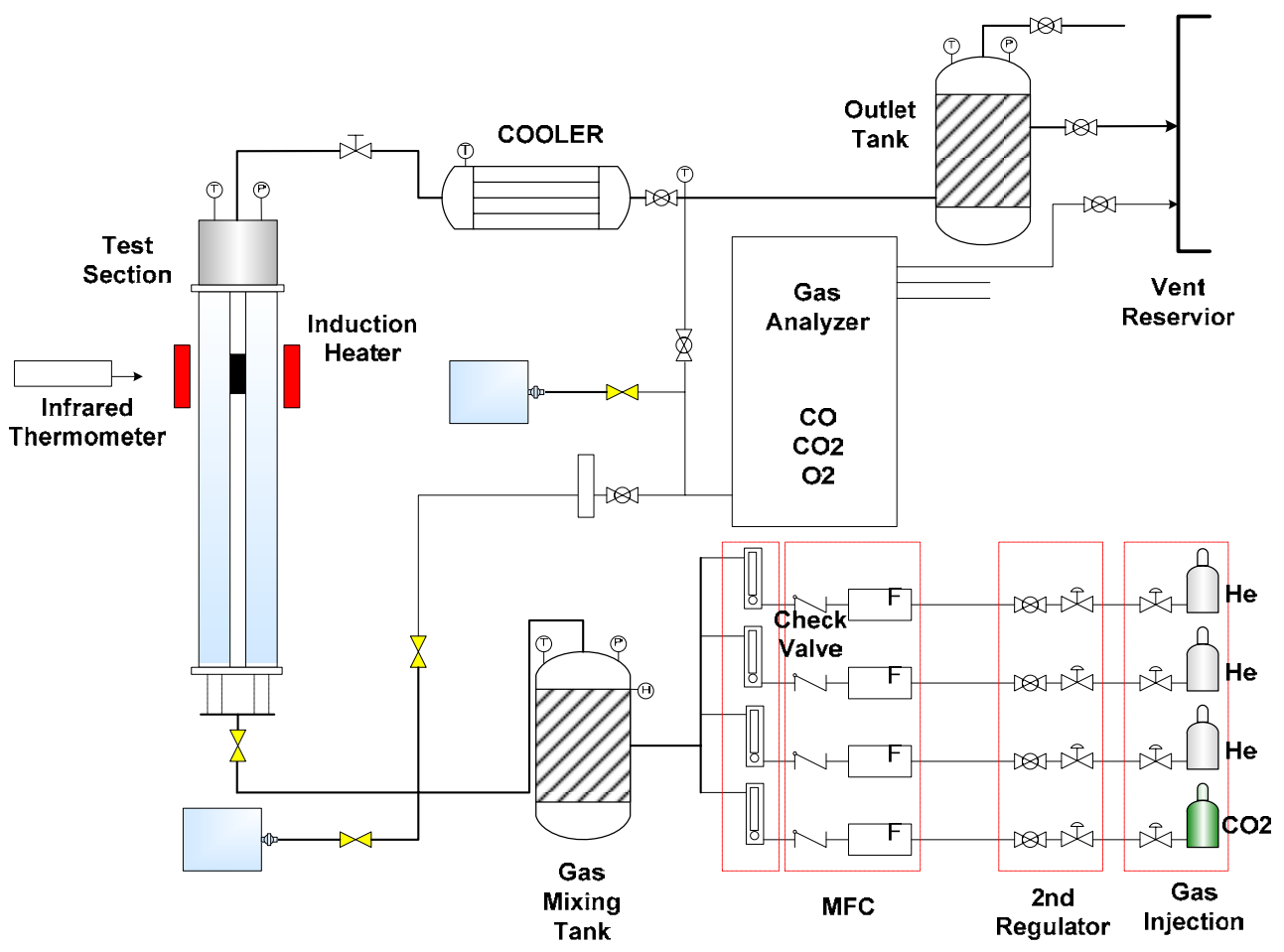

Figure 4-34 Test facility for $\mathrm{C} / \mathrm{CO}_{2}$ reaction

Although we tried to measure the reaction from $600{ }^{\circ} \mathrm{C}$, we could not detect it below $1000^{\circ} \mathrm{C}$ due to its slow rate of reaction. Figure 4-36 illustrates the rates of reaction at the temperature between $1000^{\circ} \mathrm{C}$ and $1400^{\circ} \mathrm{C}$ at the mole fraction of $\mathrm{CO}_{2}$ between 5 and 15 percent. This figure shows that the effect of $\mathrm{CO}_{2}$ concentration is much smaller than the effect of temperature. On the basis of the experimental data, the value of the order of reaction was calculated as 0.9 . Figure 4-36 also shows that there is no transition in the reaction rate data, and it confirms that the rate of the $\mathrm{C} / \mathrm{CO}_{2}$ reaction is not affected by mass diffusion in our experimental conditions. We expect that the mass diffusion effect would occur at higher temperature. 


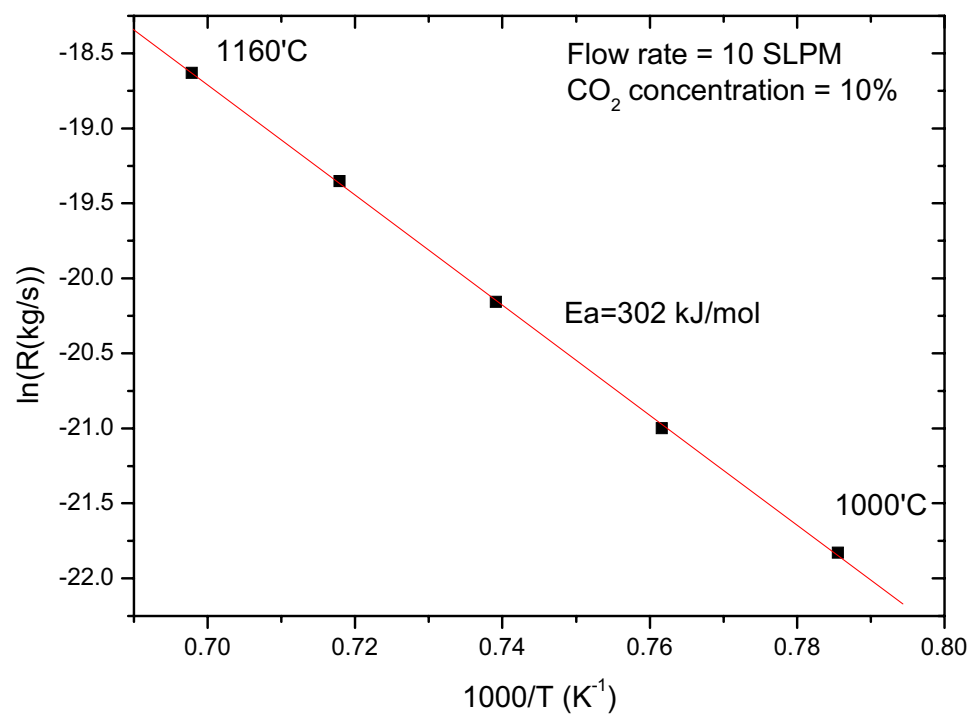

Figure 4-35 Arrehinus curve for $\mathrm{C} / \mathrm{CO}_{2}$ reaction

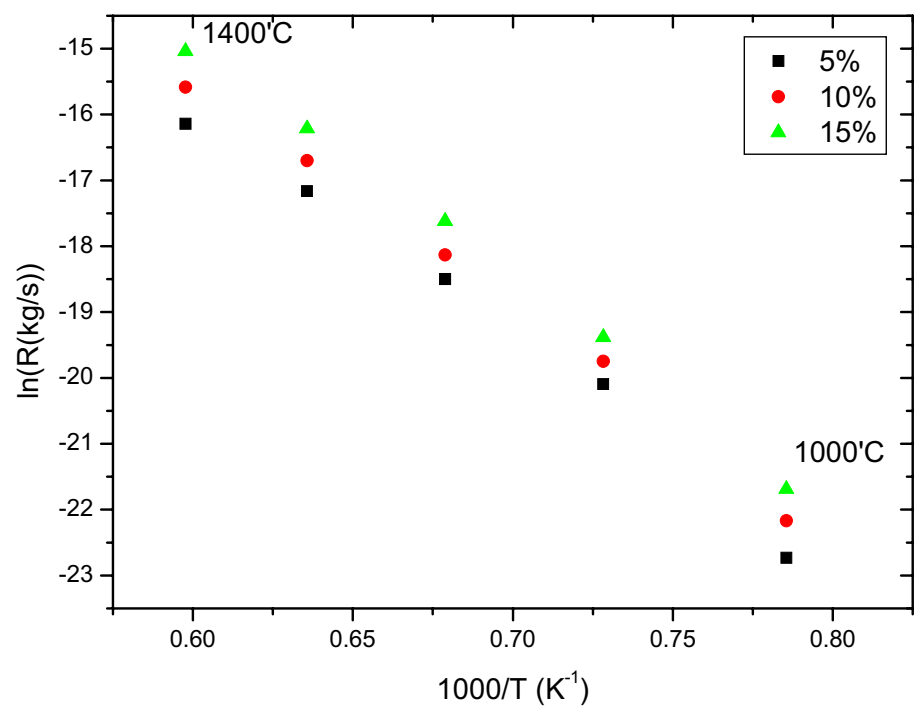

Figure 4-36 Experimental results for the rate of $\mathrm{C} / \mathrm{CO}_{2}$ reaction

Figure 4-37 compares the rates of reactions between the $\mathrm{C} / \mathrm{CO}_{2}$ and the $\mathrm{C} / \mathrm{O}_{2}$, which is the dominant reaction in HTGR air-ingress. The experimental temperature ranged between $700^{\circ} \mathrm{C}$ and $1500^{\circ} \mathrm{C}$, and the $\mathrm{CO}_{2}$ mole fraction was 2.5 percent to 20 percent. This figure shows that the rate of the $\mathrm{C} / \mathrm{CO}_{2}$ reaction is much smaller than that of the $\mathrm{C} / \mathrm{O}_{2}$ reaction. The differences between them are very large at low temperature, but the differences are reduced as the temperature increases due to the limitation of the $\mathrm{C} / \mathrm{O}_{2}$ reaction by mass diffusion effect. Based on the trend of Figure 4-17, we deduce that both of the reaction rates would be comparable around 2000C. For the situation where the portion of $\mathrm{O}_{2}$ gas is very small, on the basis of our experimental data, we propose the following rate equation: 
$r_{g}\left(\mathrm{~kg} / \mathrm{m}^{3} \mathrm{~s}\right)=3.95 \times 10^{3} \exp \left(-\frac{295000}{R \cdot T}\right) \cdot\left(p_{\mathrm{CO} 2}\right)^{0.9}$,

where $r_{g}$ is a volumetric rate of $\mathrm{C} / \mathrm{CO}_{2}$ reaction, $\mathrm{R}$ is a gas constant, $\mathrm{T}$ is temperature $(\mathrm{K})$, and $\mathrm{P}_{\mathrm{CO} 2}$ is a partial pressure $(\mathrm{Pa})$ of $\mathrm{CO}_{2}$. This equation is a general type of Arrhenius equation and it agrees well with our experimental data with RMS error of $\pm 5 \%$.

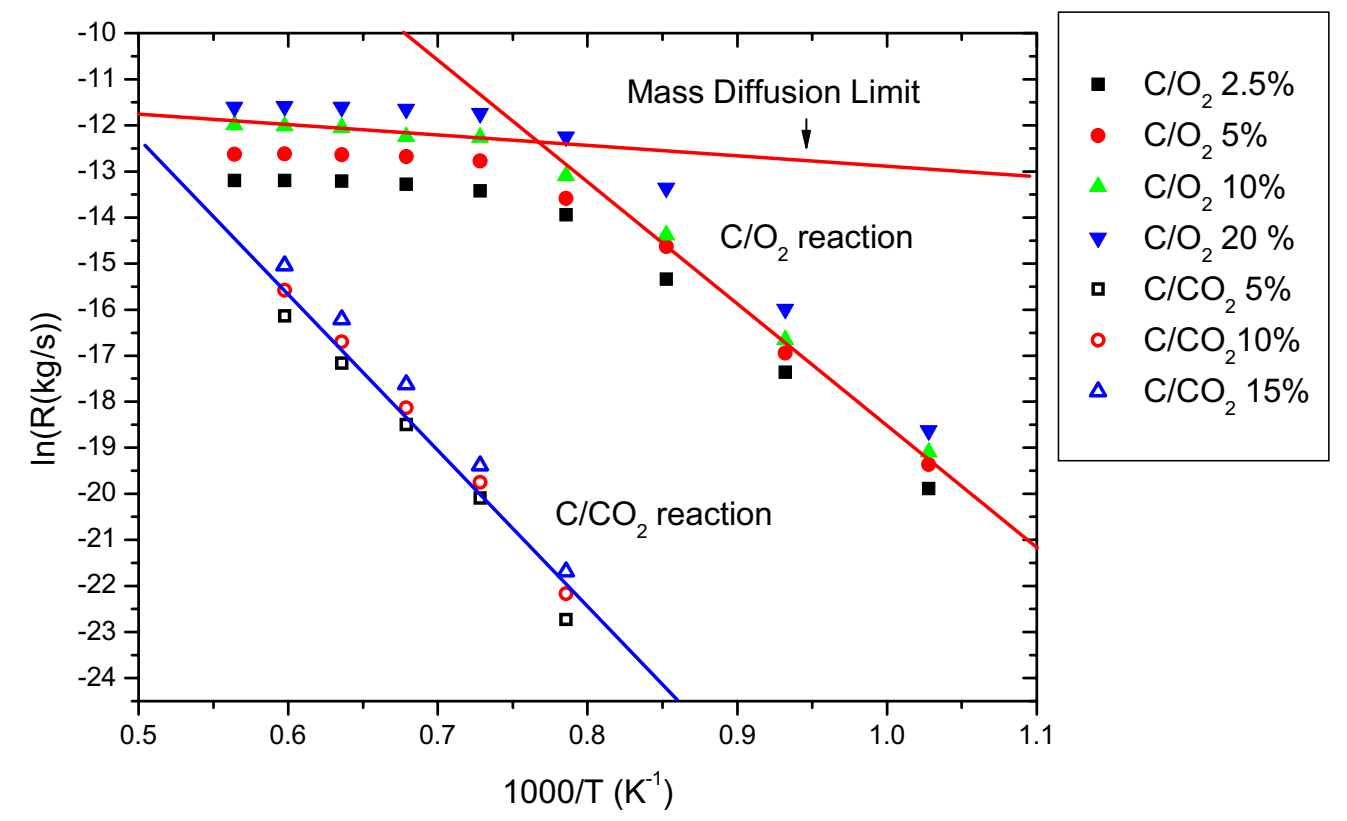

Figure 4-37 Comparison of the rates of reaction between $\mathrm{C} / \mathrm{O}_{2}$ reaction and $\mathrm{C} / \mathrm{CO}_{2}$ reaction

\subsection{Overview on the improvement in oxidation resistance for nuclear graphite}

Carbon and graphite are attractive materials for high temperature applications due to their high strength, high modulus, excellent thermal shock resistance and light weight. However, as mentioned in the above sections, the use of carbon materials has been greatly restricted due to the poor oxidation resistance at high temperature in an oxidizing atmosphere. Achieving good oxidation resistance is crucial to utilize their full potential as high-temperature materials (Zhu et al. '1999).

Oxidation protection for carbon materials has been extensively studied in the past 60 years. Ceramic coatings are commonly employed to protect carbon materials from oxidation. Although several coating systems have been developed, $\mathrm{SiC}$ is considered to be the best coating material due to its good mechanical properties, low density, good physical-chemical compatibility with carbon and excellent oxidation resistance below $1800^{\circ} \mathrm{C}$ (Tang and Guan 1995). For this reason, SiC coating is regarded as the best candidate in advanced HTGRs to improve the oxidation resistance of graphite ( $\mathrm{Zhu}$ et al. '1998). The $\mathrm{SiC}$ coating can be formed by the following three methods.

(1) Chemical vapor deposition (CVD) (Popper 1970, Nino et al. 1985)

(2) Slip/packing coating (Hurtado et al. 1993)

(3) Reaction-formed process (Shuford and Prairie 1984) 
Among the above methods, reaction-formed process, in which molten silicon reacts with the substrate on the surface to form a SiC coating, is one of the most effective ways to form a SiC coating. Chunhe et al. (1995) estimated this coating method for nuclear graphite. Their oxidation experiments showed that when the graphite sample was heated at $1000 \mathrm{C}$ in static air for $8 \mathrm{~h}$, was burnt off $68 \mathrm{wt} \%$, whereas for the SiC-coated graphite only $1.7 \mathrm{wt} \%$, and $1.0 \mathrm{wt} \%$ at $1500 \mathrm{C}$ in air for $2 \mathrm{~h}$.

Reaction-formed method is very effective way to form a SiC coating, however, this coating often causes many defects (pores, pinholes or cracks), and oxidation resistance of the coating is not sufficient at high temperature, because oxygen can attack the substrate through these defects. To overcome these limitations, several multilayer coating systems has been investigated and the most effective multilayer coating system has been a combination of a $\mathrm{SiC}$ outer layer with a boron-containing inner layer (Buchanan and Little 1994).

These additional processes have been improved the resistance of oxidation; however, they are generally complicated and required longer time than the original process (Yamamoto et al. 1995). Another problem is that the coating formation behavior can be quite different when different carbon materials are used; sometimes it is difficult to form a SiC coating. Zhu et al. (1999) developed a new process, which was aimed at making a dense $\mathrm{SiC}$ coating directly by $\mathrm{SiC}$ coating. In this process, large pores in the coating were eliminated by application of a primary coating, and pinholes were filled with free silicon. They reported that the $\mathrm{SiC}$ coating is totally intact after oxidizing for $1000 \mathrm{~h}$ together with 100 thermal cycles at $1200 \mathrm{C}$ and $200 \mathrm{~h}$ with 20 thermal cycles at $1400^{\circ} \mathrm{C}$.

For the air-ingress accident, the oxidation resistive coating is very important issue. Therefore the coating material and method should be selected including the effect of neutron, chemical compatibility and stability in the system lifetime. 


\section{TASK 4: Improvement of System Codes (INL)}

The objective of this task (Oh et al., 2002) is to improve the RELAP5/ATHENA (INEEL 2005) and MELCOR computer codes (MELCOR 1997) for analysis of VHTR systems. A LOCA has been considered a critical event for the VHTR. Following helium depressurization, it is anticipated that unless countermeasures are taken, air will enter the core through the break by molecular diffusion and ultimately by natural convection leading to oxidation of the in-core graphite structure. Thus, without any mitigating features, a LOCA will lead to an air ingress event, which will lead to exothermic chemical reactions of graphite with oxygen resulting in potentially significant increases of the core temperature. Figure 5-1 shows calculated temperature histories in a HTGR (pebble bed) following a LOCA. Calculations were made using MELCOR computer code to computer air ingress after a pipe-break accident.

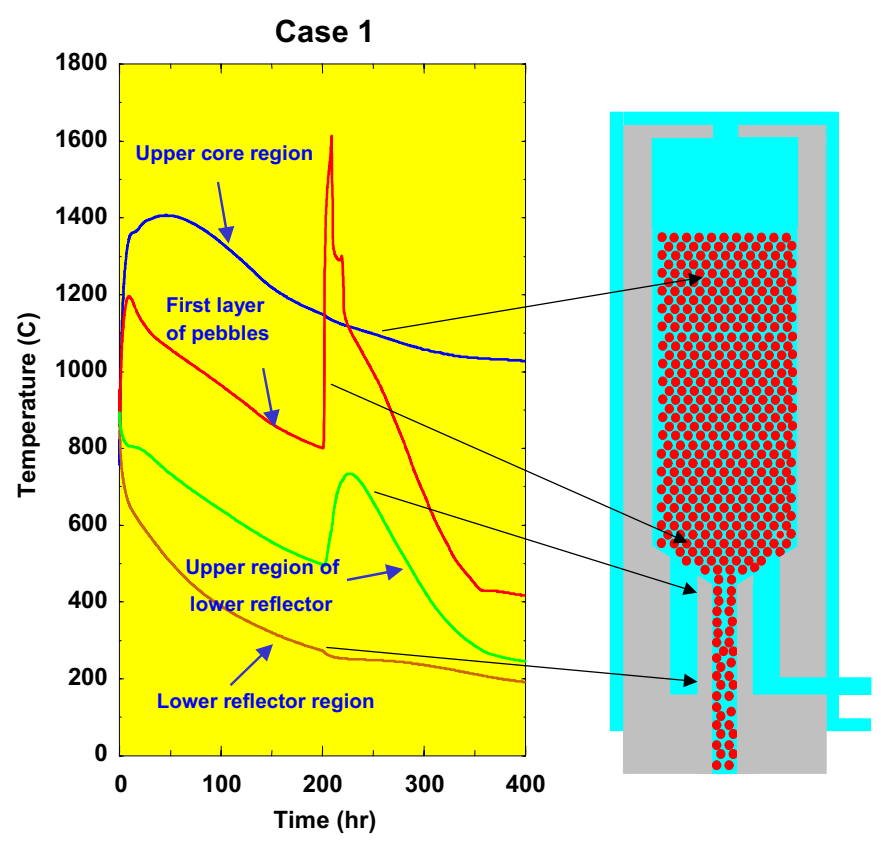

Figure 5-1. Temperature history of the PBR core and lower reflector region (base case).

One of the objectives of the VHTR design is to achieve a higher reactor safety margin. Given the level of maturity associated with today's nuclear designs, this level of safety feature is a challenge and will never be overcome by marginal improvements. Rather, aggressive technical approaches, which recognize constraints of passive safety, are required to meet the world challenge of the power industry. Without adequate numerical tools, the technical challenge of reactor safety cannot be met. For resolving the technical challenges and safety design of the VHTR, the following subtasks were performed to improve the thermal hydraulics safety system codes.

Improvements to the RELAP5/ATHENA and MELCOR computer codes are described in Sections 5.1 and 5.2 respectively. 


\subsection{RELAP5/ATHENA Code Improvements}

The RELAP5/ATHENA code (INEEL 2005) modeling of a VHTR was improved by implementation of models for molecular diffusion and graphite oxidation, as well as the inclusion of the noncondensable gases involved in the oxidation process. The molecular diffusion models calculate the diffusion of several species of gas through a system represented by a general network of control volumes wherein any control volume can be connected to several other control volumes on its inlet and outlet sides. The molecular diffusion modeling can be applied to gas mixtures containing up to five species of gas $\left(\mathrm{He}, \mathrm{N}_{2}, \mathrm{O}_{2}, \mathrm{CO}_{2}\right.$, and $\left.\mathrm{CO}\right)$ and any individual control volume may be connected up to twelve other control volumes on either its inlet or outlet sides. The graphite oxidation models account over a wide range of temperature for the chemical reactions occurring between graphite, $\mathrm{O}_{2}, \mathrm{CO}_{2}$, and $\mathrm{CO}$. The molecular diffusion and graphite oxidation models were assessed using theoretical and experimental results.

\section{Task 5.1-1 Modeling of Molecular Diffusion}

The transient concentrations of the various species of gas diffusing in a general network of control volumes are calculated by applying the finite difference form of the diffusion equation. The changes in gas concentration during a time step are calculated in RELAP5/ATHENA by the equation (Press et al. 1986)

$$
\frac{C_{j i}^{n+1}-C_{j i}^{n}}{\Delta t}=\left[\frac{\sum_{k=1}^{k \max } D_{j i k} A_{i k}\left(\frac{C_{j k}^{n}-C_{j i}^{n}}{\Delta x_{i k}}\right)-\sum_{m=1}^{m \max } D_{j i m} A_{i m}\left(\frac{C_{j i}^{n}-C_{j m}^{n}}{\Delta x_{i m}}\right)}{\Delta x_{i} A_{i}}\right]
$$

where

$C_{j i}^{n}=$ mole-fraction of $\mathrm{j}$-th species of gases in control volume " $\mathrm{i}$ " at time step " $\mathrm{n}$ " (unitless),

$\mathrm{k}=$ index identifying one of the RELAP5 control volumes on outlet side of control volume "i",

$\mathrm{kmax}=$ total number of RELAP5 control volumes connected to outlet side of control volume "i", $\mathrm{m}=$ index identifying one of the RELAP5 control volumes on inlet side of control volume "i", $\operatorname{mmax}=$ total number of RELAP5 control volumes connected to inlet side of control volume " $i$ ",

$D_{j i k}=$ effective binary diffusivity of the $\mathrm{j}$-th species of gas and the gas mixture in the diffusion path between control volumes "i" and " $\mathrm{k}$ " $\left(\mathrm{m}^{2} / \mathrm{s}\right)$,

$$
\begin{aligned}
D_{j i k} & =\left(\Delta x_{i} D_{j i}+\Delta x_{k} D_{j k}\right) /\left(\Delta x_{i}+\Delta x_{k}\right), \\
D_{j i m} & =\left(\Delta x_{i} D_{j i}+\Delta x_{m} D_{j m}\right) /\left(\Delta x_{i}+\Delta x_{m}\right), \\
D_{j i} & =\text { effective binary diffusivity of the } \mathrm{j} \text {-th species of gas and the gas mixture in control volume "i", } \\
A_{i k} & =\text { cross-sectional area for diffusion path between control volumes "i" and "k" }\left(\mathrm{m}^{2}\right), \\
A_{i k} & =\min \left(A_{i}, A_{k}\right) \\
A_{i m} & =\min \left(A_{i}, A_{m}\right), \\
A_{i} & =\operatorname{cross}-\text { sectional area for diffusion path in control volume "i", } \\
\Delta x_{i k} & =\text { length of diffusion path between control volumes "i" and "k" (m), } \\
\Delta x_{i k} & =0.5\left(\Delta x_{i}+\Delta x_{k}\right),
\end{aligned}
$$




$$
\begin{aligned}
& \Delta x_{i m}=0.5\left(\Delta x_{i}+\Delta x_{m}\right) \\
& x_{i}=\text { length of diffusion path in control volume "i”. }
\end{aligned}
$$

The effective binary diffusivity of the $\mathrm{j}$-th species of gas and the gas mixture in control volume "i" is calculated by the equation (Reid et al. 1986)

$$
D_{j i}=\left(\sum_{n=1, n \neq j}^{n \max } \frac{y_{n i}}{D_{j n i}}\right)^{-1}
$$

where

$D_{j i}=$ effective binary diffusivity of the $\mathrm{j}$-th species of gas and the gas mixture in control volume "i" $\left(\mathrm{m}^{2} / \mathrm{s}\right)$,

nmax $=$ number of species of gas in the gas mixture (unitless),

$y_{n i}=$ mole-fraction of $\mathrm{n}$-th species of gas in the gas mixture in control volume " $\mathrm{i}$ " (unitless),

$D_{j n i}=$ binary diffusivity of the $\mathrm{j}$-th and $\mathrm{n}$-th species of gas in control volume "i" $\left(\mathrm{m}^{2} / \mathrm{s}\right)$.

The binary diffusivity for the $\mathrm{j}$-th and $\mathrm{n}$-th species of gas is calculated by the correlation (Reid et al. 1986, INEEL 2005, Davis 2003)

$D_{j n}=\frac{0.0101\left(\frac{1 .}{w_{j}}+\frac{1 .}{w_{n}}\right)^{0.5} T_{i}^{1.75}}{\left[P_{i}\left\{a_{d j}^{0.333}+a_{d n}^{0.333}\right\}^{2.0}\right]}$

where

$T_{i}=$ temperature of gas mixture in control volume with index " $\mathrm{i}$ ” $(\mathrm{K})$,

$P_{i}=$ pressure of gas mixture in control volume with index "i" (Pa),

$w_{j}=$ molecular weight of $\mathrm{j}$-th species of gas in gas mixture,

$w_{n}=$ molecular weight of $\mathrm{n}$-th species of gas in gas mixture,

$a_{d j}=$ atomic diffusion volume for $\mathrm{j}$-th species of gas in gas mixture,

$a_{d n}=$ atomic diffusion volume for $\mathrm{n}$-th species of gas in gas mixture. 2003).

The atomic diffusion volumes for each species of gas are defined as shown in Table 5-1 (Davis

Table 5-1. Atomic diffusion volumes of various species of gas.

$\begin{array}{ll}\text { Species of gas } & \text { Atomic diffusion volume } \\ \text { helium } & 2.67 \\ \text { nitrogen } & 18.5 \\ \text { oxygen } & 16.3 \\ \text { carbon dioxide } & 26.9 \\ \text { carbon monoxide } & 18.0\end{array}$




\section{Task 5.1-2 Addition of Noncondensable Gases into RELAP5/ATHENA}

RELAP5 was developed to contain models for several noncondensable gases, including helium, hydrogen, nitrogen, krypton, xenon, air, and argon. This means that the code assumes that each noncondensable species is an ideal gas whose density is determined by the ideal gas law. Furthermore, the internal energy is assumed to be a function of temperature alone. Therefore, there was a need to add multiple noncondensable gases in RELAP5 accounting for the diffusion process in real fashion. The inclusion of a noncondensable species into RELAP5 requires a gas constant, correlations for the internal energy, thermal conductivity, viscosity, and diffusion coefficients.

Oxygen, carbon dioxide, and carbon monoxide have been added to the RELAP5-3D computer code as noncondensable gases to support analysis of high temperature gas-cooled reactors. Models of these gases are required to simulate the effects of air ingress on graphite oxidation following a loss-of-coolant accident. Correlations were developed for specific internal energy, thermal conductivity, and viscosity for each gas at temperatures up to $3000 \mathrm{~K}$. The existing model for internal energy (a quadratic function of temperature) was not sufficiently accurate at these high temperatures and was replaced by a more general, fourth-order polynomial. The maximum deviation between the correlations and the underlying data was $2.2 \%$ for the specific internal energy and $7 \%$ for the specific heat capacity at constant volume. The maximum deviation in the transport properties was $4 \%$ for oxygen and carbon monoxide and $12 \%$ for carbon dioxide.

The following subsections describe the parameters needed for each noncondensable gas, including the gas constant, correlations for specific internal energy, thermal conductivity, and viscosity, and diffusion parameters.

\section{Gas Constants}

The gas constant for each species, $\mathrm{R}_{\mathrm{ni}}$, of noncondensable gas is calculated as

$$
\mathrm{R}_{\mathrm{ni}}=\frac{\mathrm{R}}{\mathrm{M}_{\mathrm{ni}}}
$$

where $\mathrm{R}$ is the universal gas constant $(8314.3 \mathrm{~J} / \mathrm{kg}-\mathrm{K})$ and $\mathrm{M}_{\mathrm{ni}}$ is the molecular weight. Table 5-2 shows the molecular weight and gas constant for each species. These values were obtained from Appendix $\mathrm{C}$ of Zucrow and Hoffman (1976).

Table 5-2. Gas constants.

\begin{tabular}{|l|l|l|}
\hline Species & $\mathbf{M}_{\mathbf{n i}}$ & $\begin{array}{l}\mathbf{R}_{\mathbf{n i}} \\
(\mathbf{J} / \mathbf{k g}-\mathbf{K})\end{array}$ \\
\hline Oxygen & 32.000 & $8,314.3 / 32.000=259.82$ \\
\hline Carbon dioxide & 44.010 & $8,314.3 / 44.010=188.92$ \\
\hline Carbon monoxide & 28.010 & $8,314.3 / 28.010=296.83$ \\
\hline
\end{tabular}

Specific Internal Energy

RELAP5-3D assumes that the specific internal energy of a noncondensable species, $U_{\text {ni }}$, is (see Equation 3.226 of Volume 1 of the code manual (INEEL 2005))

$$
\begin{array}{ll}
\mathrm{U}_{\mathrm{ni}}=\mathrm{U}_{\mathrm{o}, \mathrm{ni}}+\mathrm{C}_{\mathrm{o}, \mathrm{ni}} \mathrm{T} & \text { for } \mathrm{T}<\mathrm{T}_{\mathrm{o}} \\
\mathrm{U}_{\mathrm{ni}}=\mathrm{U}_{\mathrm{o}, \mathrm{ni}}+\mathrm{C}_{\mathrm{o}, \mathrm{ni}} \mathrm{T}+0.5 \mathrm{D}_{\mathrm{o}, \mathrm{ni}}\left(\mathrm{T}-\mathrm{T}_{\mathrm{o}}\right)^{2} & \text { for } \mathrm{T}>\mathrm{T}_{\mathrm{o}}
\end{array}
$$


where $\mathrm{U}_{\mathrm{o}, \mathrm{ni}}, \mathrm{C}_{\mathrm{o} \text {,ni }}$, and $\mathrm{D}_{\mathrm{o}, \mathrm{ni}}$ are constants that vary between species, $\mathrm{T}$ is the temperature in degrees Kelvin, and $\mathrm{T}_{\mathrm{o}}=250 \mathrm{~K} . \mathrm{U}_{\mathrm{o}, \mathrm{ni}}$ and $\mathrm{D}_{\mathrm{o}, \mathrm{ni}}$ were obtained by least-squares fitting to the data reported by Reynolds (1979) for $250<\mathrm{T}<700 \mathrm{~K}$ for the currently modeled noncondensable gases. For monatomic gases, such as helium and xenon, $\mathrm{C}_{\mathrm{o}, \mathrm{ni}}$ is set to $1.5 \mathrm{R}_{\mathrm{ni}}$. For diatomic gases, such as hydrogen and nitrogen, $\mathrm{C}_{\mathrm{o}, \mathrm{ni}}$ is set to $2.5 \mathrm{R}_{\mathrm{ni}}$.

Differentiating Equations (5-5) and (5-6) with respect to temperature yields the specific heat capacity at constant volume, $\mathrm{C}_{\mathrm{v}, \mathrm{ni}}$,

$$
\begin{array}{lr}
\mathrm{C}_{\mathrm{v}, \text { ni }}=\mathrm{C}_{\mathrm{o}, \mathrm{ni}} & \text { for } \mathrm{T}<\mathrm{T}_{\mathrm{o}} \\
\mathrm{C}_{\mathrm{v}, \mathrm{ni}}=\mathrm{C}_{\mathrm{o}, \mathrm{ni}}+\mathrm{D}_{\mathrm{o}, \mathrm{ni}}\left(\mathrm{T}-\mathrm{T}_{\mathrm{o}}\right) & \text { for } \mathrm{T}>\mathrm{T}_{\mathrm{o}}
\end{array}
$$

Equations (5-7) and (5-8) show that $\mathrm{C}_{\mathrm{v}, \mathrm{ni}}$ is assumed to be constant at low temperatures and linear at higher temperatures. The linear assumption is reasonable for the temperature range between 250 and $700 \mathrm{~K}$, but is not sufficiently accurate when the temperatures are extended to near $2000 \mathrm{~K}$. Consequently, the functional form of the specific internal energy in Equation (5-6) was changed to

$$
\mathrm{U}_{\mathrm{ni}}=\mathrm{U}_{\mathrm{o}, \mathrm{ni}}+\mathrm{C}_{\mathrm{o}, \mathrm{ni}} \mathrm{T}+\mathrm{D}_{\mathrm{o}, \mathrm{ni}}\left(\mathrm{T}-\mathrm{T}_{\mathrm{o}}\right)^{2} / 2+\mathrm{E}_{\mathrm{o}, \mathrm{ni}}\left(\mathrm{T}-\mathrm{T}_{\mathrm{o}}\right)^{3} / 3+\mathrm{F}_{\mathrm{o}, \mathrm{ni}}\left(\mathrm{T}-\mathrm{T}_{\mathrm{o}}\right)^{4} / 4 \text { for } \mathrm{T}>\mathrm{T}_{\mathrm{o}}
$$

The values of the constants were determined by the method of least squares using values from Rivken (1988) for temperatures between 250 and $289 \mathrm{~K}$ and from Avallone (1987) for temperatures between 289 and 3000 $\mathrm{K}$. The values of the coefficients are shown in Table 5-3. The fitted values of $\mathrm{C}_{\mathrm{o}, \mathrm{ni}} / \mathrm{R}_{\mathrm{ni}}$ are 2.46 for oxygen and 2.34 for carbon monoxide, which are close to the 2.5 value derived from kinetic theory for rigid diatomic molecules (Zucrow and Hoffman 1976). The fitted value of $C_{o, n i} / R_{n i}$ for carbon dioxide is 3.49, which is reasonably close to the theoretical value of 3.0 for rigid polyatomic molecules.

Table 5-3. Specific internal energy coefficients.

\begin{tabular}{|l|l|l|l|l|l|}
\hline Gas species & $\begin{array}{l}\mathbf{U}_{\mathbf{o , n i}} \\
(\mathbf{J} / \mathbf{k g})\end{array}$ & $\begin{array}{l}\mathbf{C}_{\mathbf{o , n i}} \\
\mathbf{( J / k g - K )}\end{array}$ & $\begin{array}{l}\mathbf{D}_{\mathbf{o , n i}} \\
\left(\mathbf{J} / \mathbf{k g}-\mathbf{K}^{\mathbf{2}}\right)\end{array}$ & $\begin{array}{l}\mathbf{E}_{\mathbf{o , n i}} \\
\left(\mathbf{J} / \mathbf{k g}-\mathbf{K}^{\mathbf{3}}\right)\end{array}$ & $\begin{array}{l}\mathbf{F}_{\mathbf{o , n i}} \\
\left(\mathbf{J} / \mathbf{k g}-\mathbf{K}^{\mathbf{4}}\right)\end{array}$ \\
\hline Oxygen & $1,641.42$ & 639.8541 & 0.3537302 & $-1.613807 \mathrm{e}-4$ & $2.923424 \mathrm{e}-8$ \\
\hline $\begin{array}{l}\text { Carbon } \\
\text { dioxide }\end{array}$ & $-41,467.2$ & 658.7377 & 0.7563373 & $-3.726885 \mathrm{e}-4$ & $6.513268 \mathrm{e}-8$ \\
\hline $\begin{array}{l}\text { Carbon } \\
\text { monoxide }\end{array}$ & $14,231.1$ & 693.2758 & 0.3421647 & $-1.216078 \mathrm{e}-4$ & $1.503636 \mathrm{e}-8$ \\
\hline
\end{tabular}

Figure 5-2 compares the results of Equation (5-9) with the constants from Table 5-3 and the spliced values reported by Rivken (1988) and Avallone (1987) for oxygen. The results are in excellent agreement with a maximum deviation of $0.7 \%$. Figure 5-3 compares the specific heat capacity at constant volume, $\mathrm{C}_{\mathrm{v}}$, which is obtained by differentiating Equation (5-9) with respect to temperature, for oxygen to reported results from a variety of sources, including Avalone (1987), Rivken (1988), Lemmon et al. (2002), Kayes and Crawford (1980), and Reynolds (1979). The fitted results are generally in excellent agreement. However, the fitted results diverge slightly at the temperature extremes, with maximum deviation of less than $2 \%$. Figure 5-3 shows that the linear model represented by Equation (5-8) would not represent the specific heat very well over the wide temperature range required for simulating graphite oxidation.

Comparisons between the reported and the fitted values for carbon dioxide are presented in Figures 5-4 and 5-5. The results are similar to, but slightly worse than, those presented previously for oxygen. The maximum deviation between the reported and fitted specific internal energy values was $2.2 \%$. The maximum deviation between the reported and fitted specific heat values was $7 \%$. In both cases, the maximum deviation occurred at $250 \mathrm{~K}$. 


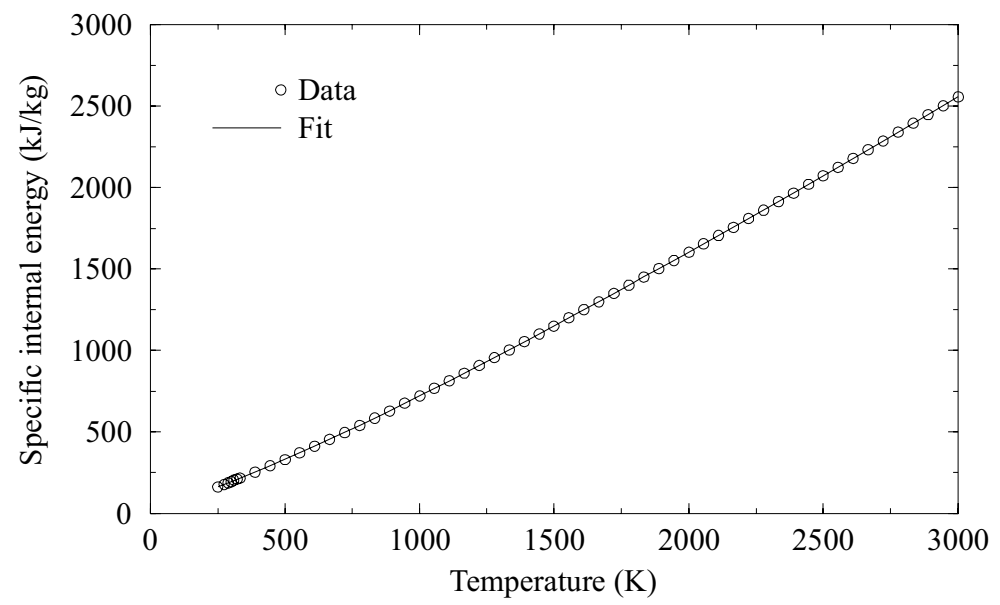

Figure 5-2. Oxygen specific internal energy as a function of temperature.

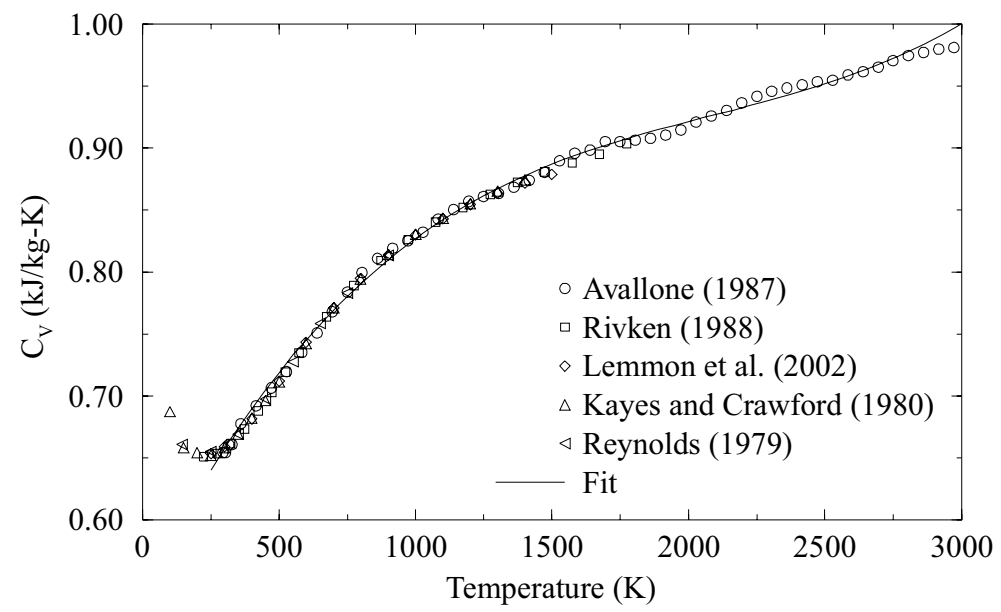

Figure 5-3. Oxygen specific heat capacity at constant volume as a function of temperature.

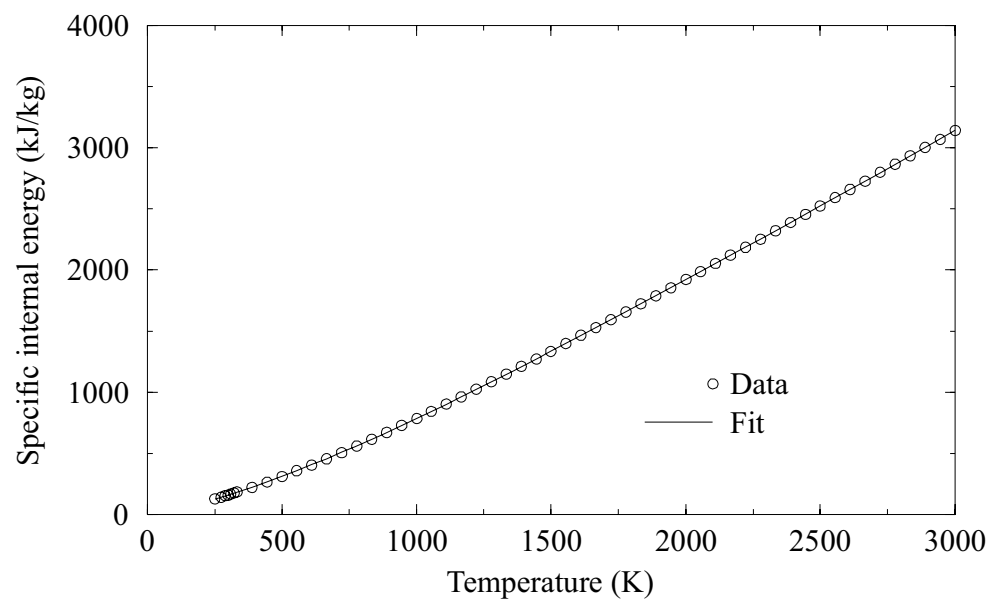

Figure 5-4. Carbon dioxide specific internal energy as a function of temperature. 


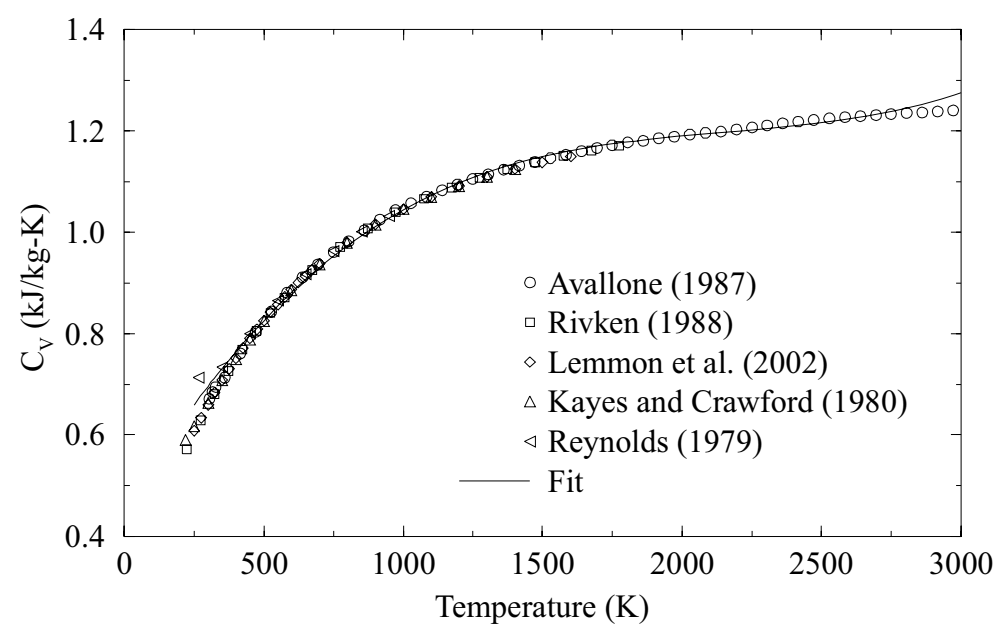

Figure 5-5. Carbon dioxide specific heat capacity at constant volume as a function of temperature.

Comparisons between the reported and the fitted values for carbon monoxide are presented in Figures 5-6 and 5-7. Figure 5-7 shows a discrepancy in the reported values for the specific heat for carbon monoxide between Lemmon et al. (2002) and the other sources of data. The values of Avallone (1987) and Rivken (1988) were consistent with other references, such as Daubert et al. (2000) and Cambel and Jennings (1958), and thus were used in the least-squares method. The comparison between the results reported by Avallone (1987) and Rivken (1988) and the fitted results were generally similar to, but slightly worse than, those described previously for oxygen. The maximum deviation between the reported and fitted specific internal energy values was $1.2 \%$. The maximum deviation between the reported and fitted specific heat values was $7 \%$. In both cases, the maximum deviation occurred at $250 \mathrm{~K}$.

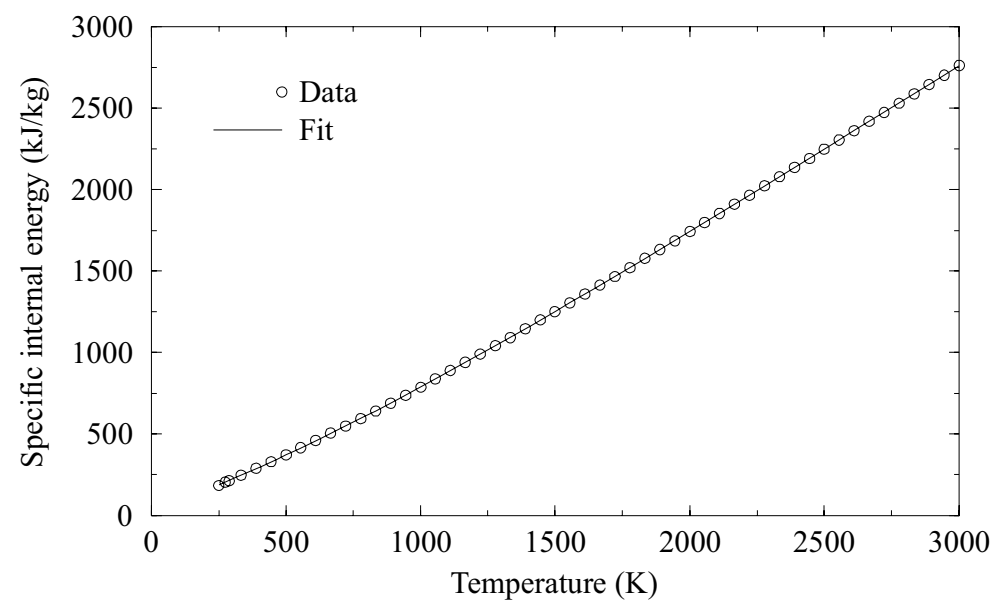

Figure 5-6. Carbon monoxide specific internal energy as a function of temperature. 


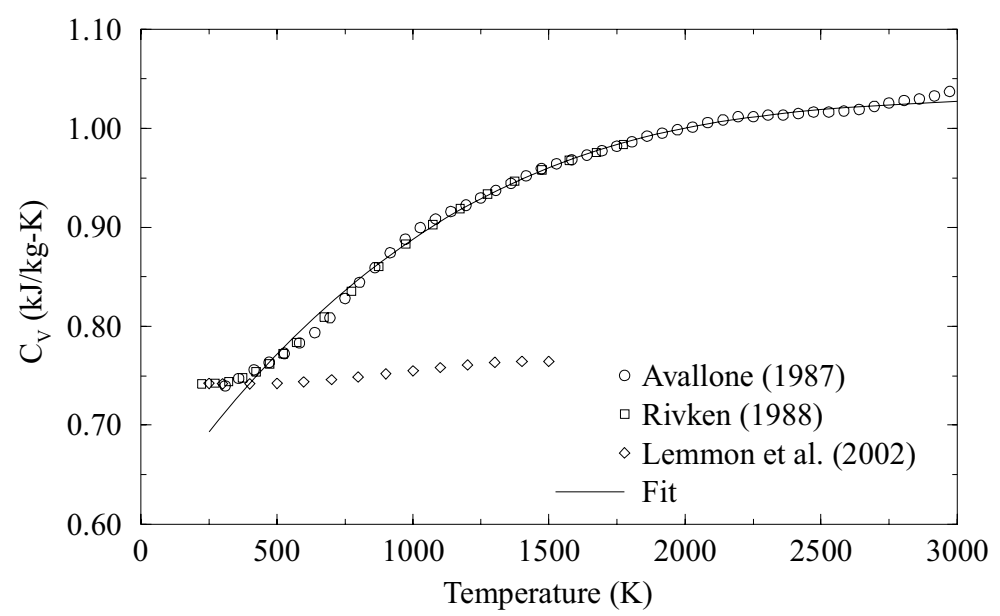

Figure 5-7. Carbon monoxide specific heat capacity at constant volume as a function of temperature.

Another fourth-order least-squares fit was generated for carbon monoxide in which $\mathrm{C}_{\mathrm{o}, \mathrm{ni}}$ was forced to be 2.5 $\mathrm{R}_{\mathrm{o}, \mathrm{ni}}$ based on kinetic theory. The results of this new fit are presented in Figure 5-8. A comparison of Figures 5-7 and 5-8 reveals that the new fit was better near $250 \mathrm{~K}$, but was significantly worse near $500 \mathrm{~K}$ and above $2500 \mathrm{~K}$. Since the higher temperature region is of more concern for graphite oxidation, the original least-squares fit, with the values presented in Table 5-3, was implemented in the code.

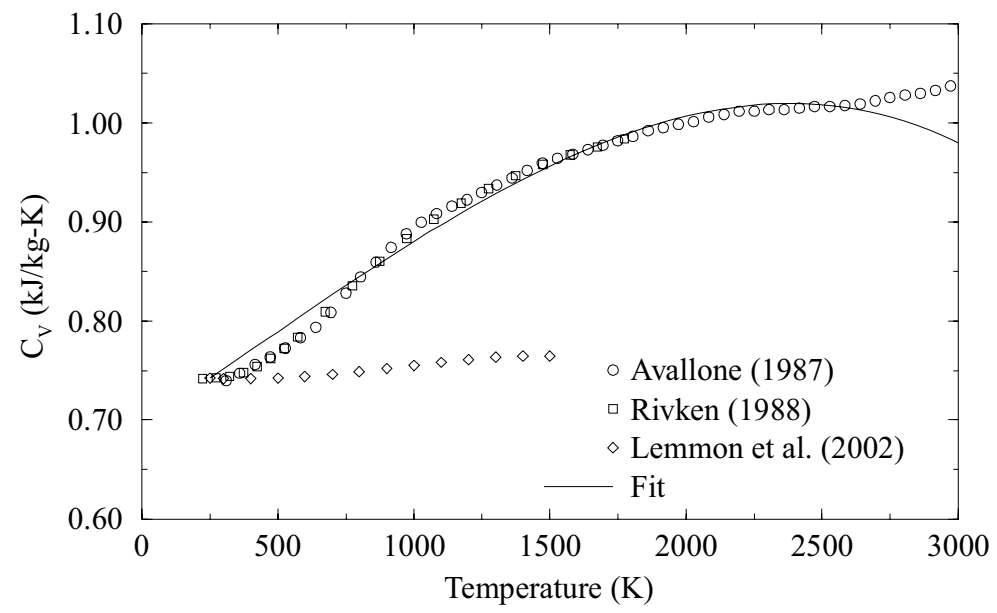

Figure 5-8. Carbon monoxide specific heat capacity at constant volume with the linear coefficient based on kinetic theory.

Equation (5-9) was implemented for all of the noncondensable gases in the code, including previously modeled gases such as helium and nitrogen. The coefficients $\mathrm{E}_{\mathrm{o}, \mathrm{ni}}$ and $\mathrm{F}_{\mathrm{o}, \mathrm{ni}}$ were set to zero for the previously modeled gases since new least squares fits for these gases were not generated during this task. Thus, Equation (5-9) reduces to Equation (5-5) for the previously modeled gases and the revised code will produce identical results to those obtained previously. The more generalized form of Equation (5-9) will allow a more accurate representation of the high-temperature range for the previously modeled gases if new fits are generated in the future.

An extrapolation of Figures 5-3 and 5-5 indicates that the specific heat capacity at constant volume obtained by differentiating Equation (5-9) would eventually become too large if extrapolated to too high of a temperature. For example, using the values from Table 5-2 for carbon dioxide yields a specific heat capacity 
at $5000 \mathrm{~K}$ that is more than two times the value at $3000 \mathrm{~K}$, which significantly exceeds the value expected by extrapolating the curve of Avallone (1987). If the fit illustrated in Figure 5-8 had been used, the specific heat capacity for carbon monoxide would have become negative near $5000 \mathrm{~K}$. In order to prevent grossly unphysical results at extremely high temperatures, the specific heat capacity was set to a constant value based on the computed value at $4000 \mathrm{~K}$. Thus, at very high temperatures,

$\left.\mathrm{C}_{\mathrm{v}}\right|_{\mathrm{T}=4000}=\mathrm{C}_{\mathrm{o}, \mathrm{ni}}+\mathrm{D}_{\mathrm{o}, \mathrm{ni}}\left(4000-\mathrm{T}_{\mathrm{o}}\right)+\mathrm{E}_{\mathrm{o}, \mathrm{ni}}\left(4000-\mathrm{T}_{\mathrm{o}}\right)^{2}+\mathrm{F}_{\mathrm{o}, \mathrm{ni}}\left(4000-\mathrm{T}_{\mathrm{o}}\right)^{3}$

and

$\mathrm{U}_{\mathrm{ni}}=\left.\mathrm{U}\right|_{\mathrm{T}=4000}+\left.\mathrm{C}_{\mathrm{v}}\right|_{\mathrm{T}=4000}(\mathrm{~T}-4000) \quad$ for $\mathrm{T}>4000 \mathrm{~K}$

where $\left.\mathrm{U}\right|_{\mathrm{T}=4000}$ is evaluated from Equation (5-9). Although somewhat arbitrary, the value of $4000 \mathrm{~K}$ prevents the specific heat from exceeding the maximum values shown in the figures by more than $33 \%$.

\section{Thermal Conductivity}

RELAP5-3D assumes that the thermal conductivity of a noncondensable gas, $\mathrm{k}$, can be represented as

$\mathrm{k}=\mathrm{AT}^{\mathrm{B}}$

where $\mathrm{k}$ is in $\mathrm{W} / \mathrm{m}-\mathrm{K}$ and $\mathrm{T}$ is the temperature in degrees $\mathrm{K}$.

The values for A and B for most of the noncondensable gases currently modeled in RELAP-3D are given in Table 12-1 of the MATPRO library used in the SCDAP/RELAP5-3D computer code (SCDAP/RELAP5-3D Development Team 2002). However, the database used in the development of the MATPRO constants for oxygen, carbon dioxide, and carbon monoxide was limited to $700 \mathrm{~K}$, which is considerably below the temperatures of interest relative to graphite oxidation. Consequently, several other sources of data were evaluated for the oxygen, carbon dioxide, and carbon monoxide gases. These sources of data include Lemmon et al. (2002), Daubert et al. (2000), and Kayes and Crawford (1980). Thermal conductivities from the various references are compared in Figures 5-9 through 5-11. All of the references were in reasonable agreement for oxygen, as the deviations between references were generally less than $10 \%$ of the values from Lemmon et al. (2002). For carbon dioxide and carbon monoxide, however, the values from MATPRO were more than $30 \%$ higher than from the other references at $1500 \mathrm{~K}$. This is not entirely unexpected because of the rather limited temperature range in the MATPRO database.

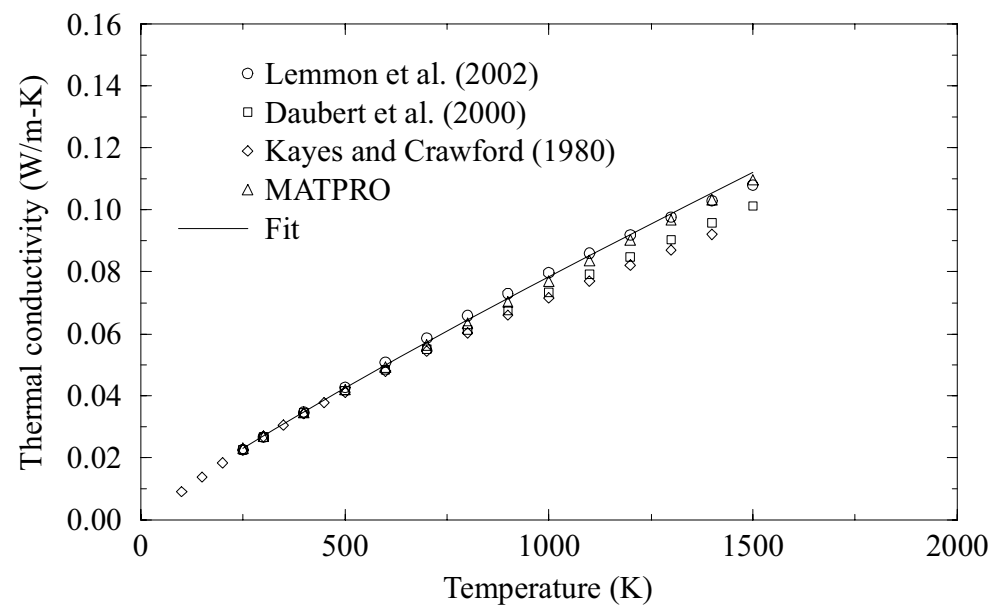

Figure 5-9. Thermal conductivity of oxygen as a function of temperature. 


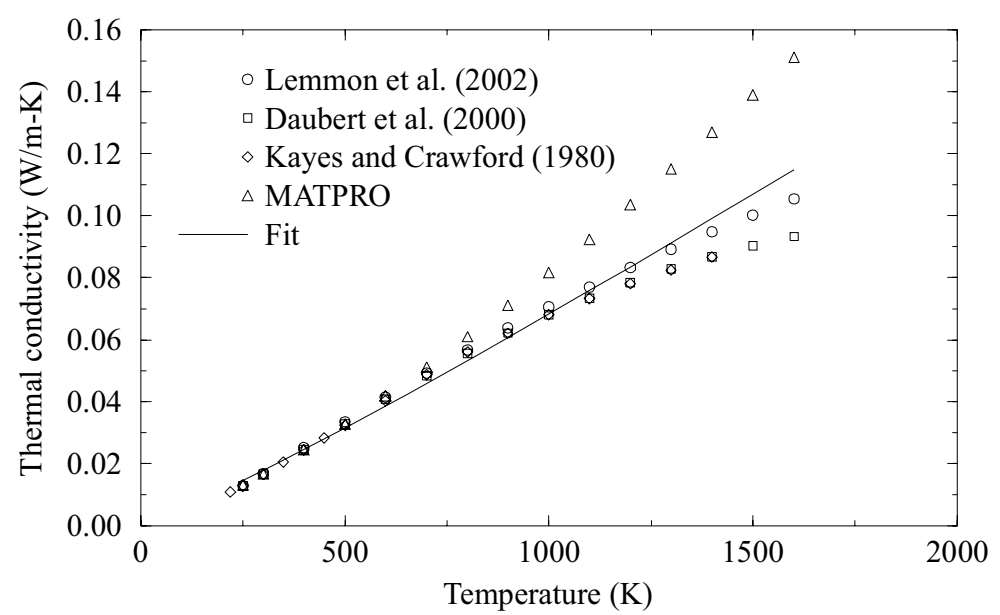

Figure 5-10. Thermal conductivity of carbon dioxide as a function of temperature.

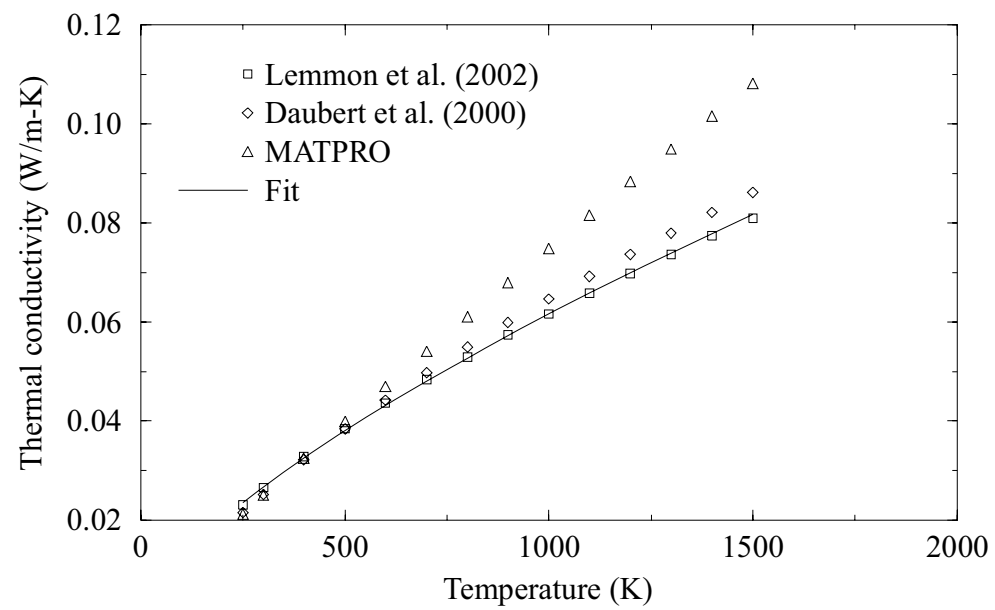

Figure 5-11. Thermal conductivity of carbon monoxide as a function of temperature.

Based on the results shown in Figures 5-9 through 5-11, data from Lemmon et al. (2002) were used to determine the values of $A$ and $B$ using the method of least squares. The results of the least-squares fit are presented in Table 5-4. The results of the least squares fit are also shown in Figures 5-9 through 5-11. The least-squares fit matched the values from Lemmon et al. (2002) to within $4 \%$ for oxygen, $12 \%$ for carbon dioxide, and $2 \%$ for carbon monoxide over the range shown in the figures.

Table 5-4. Thermal conductivity constants.

\begin{tabular}{|l|c|c|}
\hline \multicolumn{1}{|c|}{ Gas species } & $\begin{array}{c}\mathbf{A} \\
\left(\mathbf{W} / \mathbf{m}-\mathbf{K}^{\mathbf{1 + \mathbf { B }}}\right)\end{array}$ & $\mathbf{B}$ \\
\hline Oxygen & $1.766 \mathrm{e}-4$ & 0.8824 \\
\hline Carbon dioxide & $3.110 \mathrm{e}-5$ & 1.1136 \\
\hline Carbon monoxide & $5.050 \mathrm{e}-4$ & 0.6954 \\
\hline
\end{tabular}

$\underline{\text { Viscosity }}$

RELAP5-3D assumes that the viscosity, $\mu$, of a noncondensable gas can be represented as 
$\mu=\frac{\mathrm{AT}^{1.5}}{\mathrm{~T}+\mathrm{B}}$

where $\mu$ is in $\mathrm{kg} / \mathrm{m}-\mathrm{s}, \mathrm{T}$ is the temperature in degrees $\mathrm{K}$, and $\mathrm{A}$ and $\mathrm{B}$ are constants.

The values for A and B were determined using the method of least squares. Because the form of Equation (513) differs from that typically used for least-squares fitting, additional details are provided below. The coefficients $A$ and $B$ are chosen to minimize the function $\mathrm{f}$

$\mathrm{f}(\mathrm{A}, \mathrm{B})=\sum_{\mathrm{i}}\left[\mathrm{y}_{\mathrm{i}}-\frac{\mathrm{AT}_{\mathrm{i}}{ }^{1.5}}{\mathrm{~T}_{\mathrm{i}}+\mathrm{B}}\right]^{2}$

where $y_{i}$ is the value of the viscosity corresponding to a temperature $T_{i}$. Taking the partial derivative of $f$ with respect to A, setting it equal to zero, and solving for A yields

$$
\mathrm{A}=\frac{\sum_{\mathrm{i}} \frac{\mathrm{y}_{\mathrm{i}} \mathrm{T}_{\mathrm{i}} \mathrm{T}_{\mathrm{i}}+\mathrm{B}}{\sum_{\mathrm{i}} \frac{\mathrm{T}_{\mathrm{i}}^{3}}{\left(\mathrm{~T}_{\mathrm{i}}+\mathrm{B}\right)^{2}}}}{{ }^{2}}
$$

Because the partial derivative with respect to B does not lend itself to algebraic solution, an iterative technique was employed. Specifically, a value of B was assumed, Equation (5-15) was used to calculate the optimal value of A consistent with the assumed value of B, and then the sum in Equation (5-14) was determined. The value of B was then varied by hand until the sum in Equation (5-14) was minimized.

Table 5-5 shows the optimal values of A and B that were obtained fitting the data of Lemmon et al. (2002). The results of the fitting process are compared with data from Lemmon et al. (2002) and Daubert et al. (2000) in Figures 5-12 through 5-14. Figures 5-12 and 5-13 also contain values from Kayes and Crawford (1980). The viscosity data from the various references were consistent and agreed to within 5\% over the temperature ranges presented in the figures.

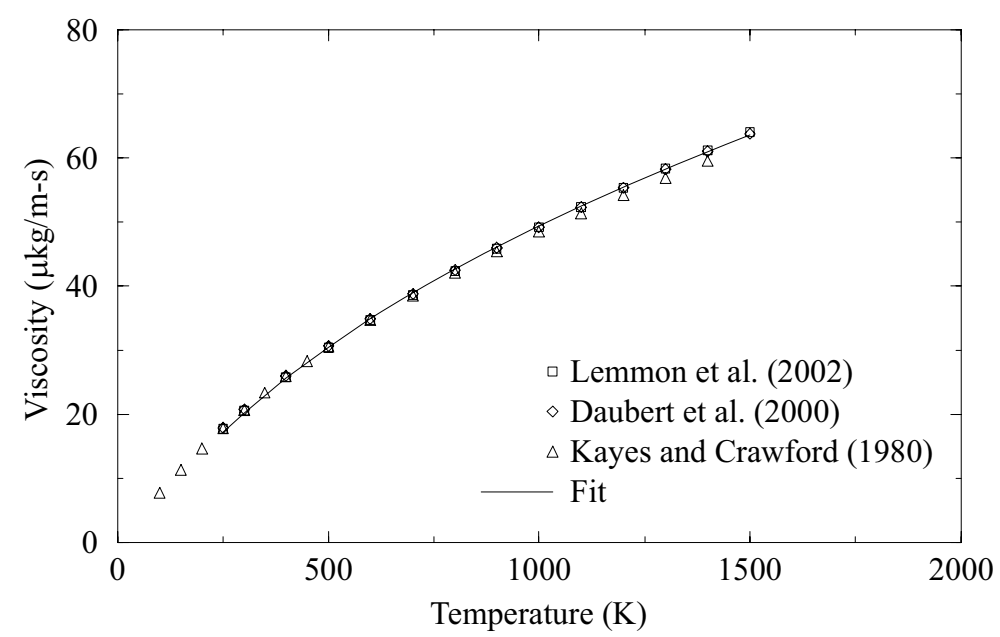

Figure 5-12. Viscosity of oxygen as a function of temperature. 


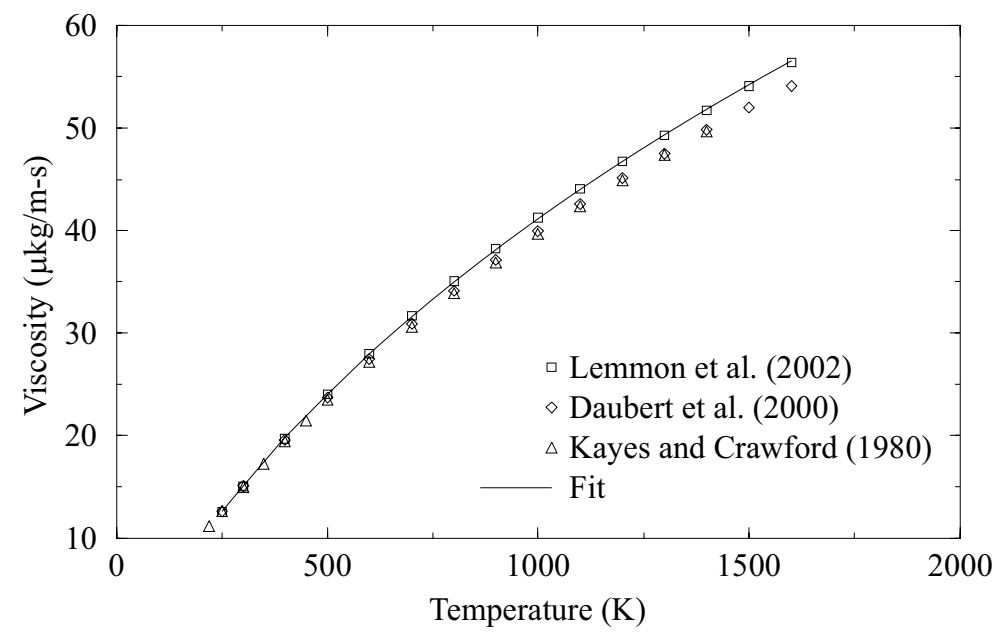

Figure 5-13. Viscosity of carbon dioxide as a function of temperature.

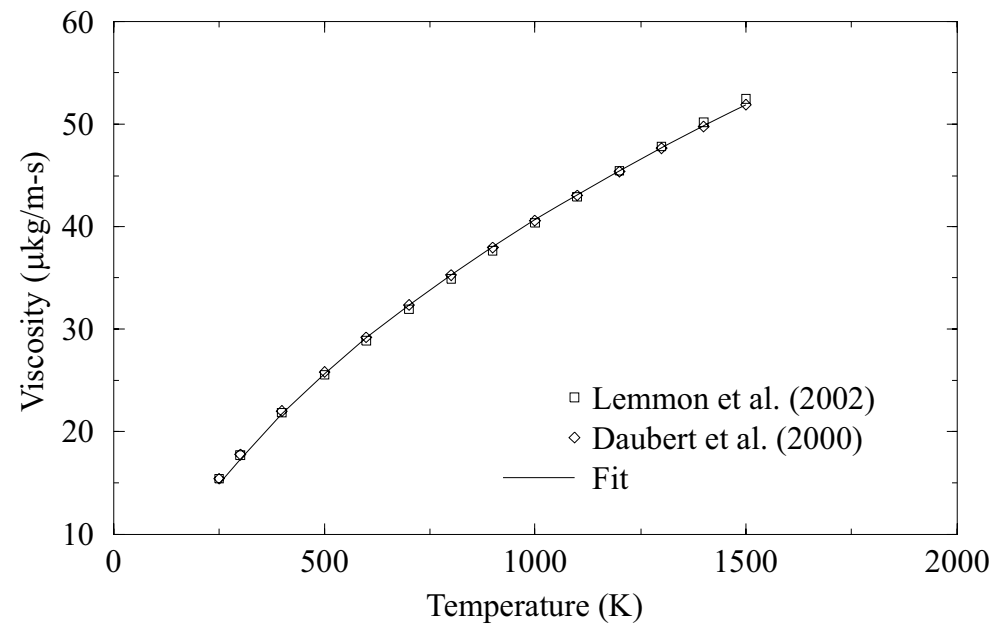

Figure 5-14. Viscosity of carbon monoxide as a function of temperature.

The least-squares fit matched the values from Lemmon et al. (2002) to within $3 \%$ for oxygen, $1 \%$ for carbon dioxide, and $4 \%$ for carbon monoxide over the ranges shown in the figures.

Table 5-5. Viscosity constants.

\begin{tabular}{|l|c|c|}
\hline \multicolumn{1}{|c|}{ Gas species } & $\begin{array}{c}\text { A } \\
\left(\mathbf{k g} / \mathbf{m}-\mathbf{s}-\mathbf{K}^{\mathbf{0 . 5}}\right)\end{array}$ & $\begin{array}{c}\text { B } \\
(\mathbf{K})\end{array}$ \\
\hline Oxygen & $1.826 \mathrm{e}-6$ & 169 \\
\hline Carbon dioxide & $1.651 \mathrm{e}-6$ & 268 \\
\hline Carbon monoxide & $1.463 \mathrm{e}-6$ & 138 \\
\hline
\end{tabular}

\section{Diffusion Parameters}

The presence of a noncondensable gas can significantly affect the heat transfer coefficient during condensation. RELAP5-3D represents this effect by simulating the diffusion of the noncondensable gas from a homogeneous steam/noncondensable mixture to the surface of a water film. The RELAP5-3D model is based on the diffusion coefficient given by Equation (11.4.4) of Reid et al. (1987) 
$\mathrm{D}_{\mathrm{AB}}=\frac{0.00143 \mathrm{~T}^{1.75}}{\mathrm{PM}_{\mathrm{AB}}{ }^{1 / 2}\left[\left(\sum_{\mathrm{v}}\right)_{\mathrm{A}}^{1 / 3}+\left(\sum_{\mathrm{v}}\right)_{\mathrm{B}}^{1 / 3}\right]^{2}}$

where

$\mathrm{D}_{\mathrm{AB}}=$ binary diffusion coefficient $\left(\mathrm{cm}^{2} / \mathrm{s}\right)$

$\mathrm{T}=$ temperature $(\mathrm{K})$

$\mathrm{P}=$ pressure (bar)

$\mathrm{M}_{\mathrm{A}}, \mathrm{M}_{\mathrm{B}}=$ molecular weights of $\mathrm{A}$ and $\mathrm{B}(\mathrm{g} /$ mole) and

$\mathrm{M}_{\mathrm{AB}}=2 /\left[\left(1 / \mathrm{M}_{\mathrm{A}}\right)+\left(1 / \mathrm{M}_{\mathrm{B}}\right)\right]$

$\sum_{\mathrm{v}}=$ atomic diffusion volume given in Table 11-1 of Reid et al. (1987).

Converting to the units used by RELAP5-3D yields

$\mathrm{D}_{\mathrm{AB}}=\frac{\mathrm{D}_{\mathrm{c}} \mathrm{T}^{1.75}}{\mathrm{P}}$

where

$\mathrm{D}_{\mathrm{AB}}=$ binary diffusion coefficient $\left(\mathrm{m}^{2} / \mathrm{s}\right)$

$\mathrm{P}=$ pressure $(\mathrm{Pa})$

and

$$
\mathrm{D}_{\mathrm{C}}=\frac{\frac{0.0143}{\sqrt{2}}\left[\frac{1}{\mathrm{M}_{\mathrm{A}}}+\frac{1}{\mathrm{M}_{\mathrm{B}}}\right]^{1 / 2}}{\left[\left(\sum_{\mathrm{v}}\right)_{\mathrm{A}}^{1 / 3}+\left(\sum_{\mathrm{v}}\right)_{\mathrm{B}}^{1 / 3}\right]^{2}} \frac{\mathrm{m}^{2} \mathrm{~Pa}}{\mathrm{~s} \mathrm{~K}^{1.75}}
$$

The constant $\mathrm{D}_{\mathrm{C}}$ was determined for each noncondensable gas using the values given in Table 5-6, a molecular weight of 18.016 (Zucrow and Hoffman (1976)) for water, and an atomic diffusion volume of 13.1 for water (Reid at al. 1987).

Table 5-6. Diffusion parameters.

\begin{tabular}{|c|c|c|c|}
\hline Gas species & Molecular weight & $\sum_{\mathrm{v}}$ & $\begin{array}{c}\mathbf{D}_{\mathbf{C}} \\
\mathbf{m}^{\mathbf{2}} \mathbf{- P a} / \mathbf{s}-\mathbf{K}^{\mathbf{1 . 7 5}}\end{array}$ \\
\hline Oxygen & 32.000 & 16.3 & $1.2441 \times 10^{-4}$ \\
\hline Carbon dioxide & 44.010 & 26.9 & $9.8678 \times 10^{-5}$ \\
\hline Carbon monoxide & 28.010 & 18.0 & $1.2323 \times 10^{-4}$ \\
\hline
\end{tabular}

\section{Task 5.1-3 Modeling of Graphite Oxidation}

The RELAP5/ATHENA code (INEEL 2005) was extended to model the chemical reactions occurring in a VHTR core in the event of air ingress. Several different chemical reactions involving $\mathrm{C}, \mathrm{O}_{2}, \mathrm{CO}$, and $\mathrm{CO}_{2}$ may occur. These chemical reactions, the rate of these reactions and the heat produced by these chemical reactions vary with the temperature of the reactants. The chemical reactions are modeled taking into account 
five species of gas (1) He, (2) $\mathrm{O}_{2}$, (3) $\mathrm{N}_{2}$, (4) $\mathrm{CO}_{2}$, and (5) CO. The chemical reactions are modeled according to the equation

$\mathrm{C}+\mathrm{zO}_{2} \rightarrow \mathrm{xCO}+\mathrm{yCO}_{2}$

where

$\mathrm{x}, \mathrm{y}$, and $\mathrm{z}$ are the mole fractions of carbon monoxide, carbon dioxide, and oxygen, respectively, of this chemical reaction.

The coefficients $\mathrm{x}, \mathrm{y}$, and $\mathrm{z}$ in the above reaction are a function of temperature of the graphite contacted by the gases involved in the reaction. The values of the coefficients are determined from a correlation for the equilibrium production ratio of $\mathrm{CO}$ to $\mathrm{CO}_{2}$ (Takeda and Hishida 1996, Oh and NO 2004). The correlation for the equilibrium production ratio is

$f_{e}=1995 \exp (-E / R T)$

where

$\mathrm{f}_{\mathrm{e}}=$ equilibrium production ratio of $\mathrm{CO}$ to $\mathrm{CO}_{2}$,

$\mathrm{E}=$ activation energy $\left(59.86 \times 10^{6} \mathrm{~J} / \mathrm{kg}\right.$-mole $)$,

$\mathrm{R}=$ universal gas constant $(8314.3 \mathrm{~J} / \mathrm{kg}$-mole $\cdot \mathrm{K})$,

$\mathrm{T}=$ temperature of the gas mixture $(\mathrm{K})$.

The values of the coefficients $\mathrm{x}, \mathrm{y}$, and $\mathrm{z}$ are determined from a mass balance as follows;

$\mathrm{x}=\mathrm{f}_{\mathrm{e}} /\left(\mathrm{f}_{\mathrm{e}}+1\right)$

$\mathrm{y}=1 /\left(\mathrm{f}_{\mathrm{e}}+1\right)$

$\mathrm{z}=\left(\mathrm{f}_{\mathrm{e}}+2\right) /\left(2 \mathrm{f}_{\mathrm{e}}+2\right)$.

For a gas mixture temperature greater than about $1000 \mathrm{~K}$, the correlation for equilibrium production ratio predicts that the production of $\mathrm{CO}$ exceeds the production of $\mathrm{CO}_{2}$.

The heat of formation for $\mathrm{CO}$ is calculated by the equation

$$
H_{C O}=\left(4.187 \times 10^{3} / M_{C O}\right)\left[2.54 \times 10^{4}+0.89 T+0.27 \times 10^{-3} T^{2}+2.19 \times 10^{5} / T\right]
$$

where

$H_{C O}=$ heat of formation of $\mathrm{CO}(\mathrm{J} / \mathrm{kg}-\mathrm{CO})$,

$M_{\mathrm{CO}}=$ molecular weight of $\mathrm{CO}(28.01 \mathrm{~kg}-$ moles $)$,

$T=$ temperature of $\mathrm{CO}(\mathrm{K})$.

The heat of formation for $\mathrm{CO}_{2}$ is calculated by the equation

$$
H_{C O 2}=\left(4.187 \times 10^{3} / M_{C O 2}\right)\left[9.369 \times 10^{4}+0.78 T-0.7 \times 10^{-4} T^{2}+4.60 \times 10^{4} / T\right]
$$

where

$$
\begin{aligned}
& \mathrm{H}_{\mathrm{CO}_{2}}=\text { heat of formation of } \mathrm{CO}_{2}\left(\mathrm{~J} / \mathrm{kg}-\mathrm{CO}_{2}\right), \\
& M_{\mathrm{CO}_{2}}=\text { molecular weight of } \mathrm{CO}_{2}(44.01 \mathrm{~kg}-\mathrm{moles}) .
\end{aligned}
$$

For graphite in the temperature range of $798 \mathrm{~K}$ to $1448 \mathrm{~K}$, the rate of oxidation is calculated by the equation (Oh et al. 2003) 
$\mathrm{R}=1.57 \times 10^{6} \exp \left(\frac{-1.88 \times 10^{5}}{\mathrm{R}_{\mathrm{g}} \mathrm{T}}\right)\left(\frac{p_{O 2}}{0.209 \times 10^{5}}\right)^{0.75}$

where

$\mathrm{R}=$ rate of burnup of graphite for temperature in range of $395 \mathrm{~K}$ to $1448 \mathrm{~K}$

( $\mathrm{kg}$ of C oxidized $/ \mathrm{m}^{2} \cdot \mathrm{s}$ ),

$R_{g}=\quad$ universal gas constant $(8.3143 \mathrm{~J} / \mathrm{g}-\mathrm{mol} \cdot \mathrm{K})$,

$P_{\mathrm{O} 2}=\quad$ partial pressure of oxygen in gas adjacent to graphite $(\mathrm{Pa})$.

For a temperature greater than $1448 \mathrm{~K}$, the rate of oxidation is equal to the rate governed by mass diffusion or the rate governed by the bulk flow rate of oxygen, which ever is least.

The rate of oxidation governed by mass diffusion is calculated by the equation

$\mathrm{R}_{\mathrm{D}}=\mathrm{M}_{\mathrm{c}} \mathrm{k}_{\mathrm{mt}} \mathrm{C}_{\mathrm{g}} \mathrm{y}_{\mathrm{b}} / \mathrm{z}$

where

$\mathrm{R}_{\mathrm{D}}=$ limit on rate of oxidation imposed by diffusion of oxygen through boundary layer $\left(\mathrm{kg}\right.$ of $\mathrm{C}$ oxidized $\left./ \mathrm{m}^{2} \cdot \mathrm{s}\right)$.

$\mathrm{k}_{\mathrm{mt}}=$ mass transfer coefficient at location at which rate of oxidation being calculated $(\mathrm{m} / \mathrm{s})$,

$\mathrm{C}_{\mathrm{g}}=$ molar density of bulk gas $\left(\mathrm{kg}-\mathrm{mole} / \mathrm{m}^{3}\right)$,

$\mathrm{y}_{\mathrm{b}}=$ mole-fraction of oxygen in bulk gas at location at which rate of oxidation being

calculated,

$\mathrm{M}_{\mathrm{c}} \quad=\quad$ atomic weight of carbon (12.011).

The molar density of the bulk gas is calculated by the equation

$\mathrm{C}_{\mathrm{g}}=\mathrm{P}_{\text {tot }} / \mathrm{RT}$

where

$\mathrm{P}_{\text {tot }}=$ total pressure of bulk gas $(\mathrm{Pa})$,

$\mathrm{R}=$ universal gas constant $\left(8314 . \mathrm{Pa} \cdot \mathrm{m}^{3} / \mathrm{kg}-\mathrm{mole} \cdot \mathrm{K}\right)$,

$\mathrm{T}=\quad$ temperature of bulk gas $(\mathrm{K})$.

The mass transfer coefficient is calculated using the analogy between heat and mass transfer. According to this analogy (Olander 1994),

$\mathrm{k}_{\mathrm{mt}}=\frac{\mathrm{NuD}_{\mathrm{gO} 2}}{\mathrm{~d}_{\mathrm{h}}}$

where

$\begin{array}{lll} & \\ \mathrm{Nu} & = & \text { Nusselt number of the bulk gas } \\ \mathrm{D}_{\mathrm{gO2}}= & \text { diffusivity of oxygen in bulk gas }\left(\mathrm{m}_{\mathrm{h}}^{2} / \mathrm{s}\right), \\ \mathrm{d}_{\mathrm{h}} & = & \text { characteristic length, (equal to diameter of pebbles for PB-HTGR) }(\mathrm{m}), \\ \mathrm{k}_{\mathrm{g}} & = & \text { thermal conductivity of the bulk gas }(\mathrm{W} / \mathrm{m} \cdot \mathrm{K}), \\ \mathrm{h} & = & \text { convective heat transfer coefficient }\left(\mathrm{W} / \mathrm{m}^{2} \cdot \mathrm{K}\right) .\end{array}$

The RELAP5/ATHENA code variables for the production or consumption of each species of gas in the reactor system are updated at each time step to account for the chemical reactions occurring throughout 
the core and reflector regions of a HTGR. The volumetric consumption of oxygen due to reaction with the graphite is calculated for each control volume by the equation

$\gamma_{O 2 i}=-z V_{m k} A_{s} R_{x}\left(\frac{M_{O 2}}{M c}\right)\left(\frac{1}{V_{R i}}\right)$

where

$\gamma_{O 2 i}=$ volumetric consumption of oxygen per unit volume in RELAP5/ATHENA control volume with index $i$ and which represents the gas at radial node $\mathrm{m}$ of axial node $\mathrm{k}$ in the numerical scheme calculating the transient temperature distribution in the reactor core $\left(\mathrm{kg}\right.$ of oxygen consumed $\left./ \mathrm{m}^{3} \cdot \mathrm{s}\right)$,

$V_{m k}=$ volume of radial node $\mathrm{m}$ of axial node $\mathrm{k}$ in numerical scheme used to calculate the transient temperature distribution in the reactor core, and where the RELAP5/ATHENA control volume with index i represents the gas at that location $\left(\mathrm{m}^{3}\right)$,

$A_{s}=$ surface area of reactor core per unit volume at radial node $\mathrm{m}$ of axial node $\mathrm{k}$ in numerical scheme used to calculate the transient temperature distribution in the reactor core $\left(\mathrm{m}^{2} / \mathrm{m}^{3}\right)$,

$R_{x}=$ rate of reaction of oxygen with graphite at radial node $\mathrm{m}$ of axial node $\mathrm{k}$ in numerical scheme used to calculate the transient temperature distribution in the reactor core (minimum of $R$ and $R_{D}$ in above equations) ( $\mathrm{kg}$ of $\mathrm{C}$ oxidized $\left./ \mathrm{m}^{2} \cdot \mathrm{s}\right)$,

$$
\begin{aligned}
& M_{O 2}=\text { molecular weight of oxygen }(32.0), \\
& M_{c}=\text { molecular weight of graphite }(12.011), \\
& V_{R i}=\text { volume of RELAP5/ATHENA control volume with index } \mathrm{i}\left(\mathrm{m}^{3}\right) .
\end{aligned}
$$

The volumetric production of $\mathrm{CO}_{2}$ corresponding with the volumetric consumption of oxygen is calculated by the equation

$\gamma_{c o 2 i}=y V_{m k} A_{s} R_{x}\left(\frac{M_{c o 2}}{M_{c}}\right)\left(\frac{1}{V_{R i}}\right)$

where

$\gamma_{c o 2 i}=$ volumetric rate of production of $\mathrm{CO}_{2}$ in RELAP5/ATHENA control volume with index $\mathrm{i}$ $\left(\mathrm{kg} / \mathrm{m}^{3} \cdot \mathrm{s}\right)$,

$$
M_{c o 2}=\text { molecular weight of } \mathrm{CO}_{2}(44.011) \text {. }
$$

The volumetric production of $\mathrm{CO}$ corresponding with the volumetric consumption of oxygen is calculated by the equation

$$
\gamma_{c o i}=x V_{m k} A_{s} R_{x}\left(\frac{M_{w c o}}{M_{w c}}\right)\left(\frac{1}{V_{R i}}\right)
$$

where

$\gamma_{c o i}=$ volumetric rate of production of CO in RELAP5/ATHENA control volume with index i $\left(\mathrm{kg} / \mathrm{m}^{3} \cdot \mathrm{s}\right)$.

The internal energies of the various gases produced by chemical reactions are required by the RELAP5/ATHENA energy equation. The general form of the internal energy of the produced gases is 
$u_{j}=u_{0 j}+c_{1 j} T+0.5 c_{2 j}(T-250 .)^{2}$

where

$$
\begin{aligned}
& u_{j}=\text { internal energy of } \mathrm{j} \text {-th species of gas added to a RELAP5/ATHENA control volume }(\mathrm{J} / \mathrm{kg}), \\
& u_{0 j}, c_{1 j},{ }^{c_{2 j}}=\text { coefficients in the calculation of internal energy for the } \mathrm{j} \text {-th component of gas, } \\
& T \quad=\text { temperature of surface at which the component of gas was produced }(\mathrm{K}) .
\end{aligned}
$$

The coefficients in the above equation for each species of gas produced or consumed are defined in Table 5-7 (Davis 2003).

Table 5-7. Coefficients in polynomial equation for internal energy of each species of gas produced or consumed.

Species of gas Coefficients

$\begin{array}{llll} & \mathrm{u}_{0} & \mathrm{c}_{1} & \mathrm{c}_{2} \\ \mathrm{O}_{2} & 1641.42 & 639.8541 & 0.3537302 \\ \mathrm{CO}_{2} & -41467.2 & 658.7377 & 0.7563373 \\ \mathrm{CO} & 14231.1 & 693.2758 & 0.3421647\end{array}$

\section{Task 5.1-4 Initial Assessment of Molecular Diffusion Modeling}

Two simple but rigorous theoretical problems were used to assess the molecular diffusion model implemented into RELAP5/ATHENA. The first theoretical problem, named the Pipe Diffusion Test Problem, involved the calculation of the transient concentrations of air ingressing a long straight pipe. The second theoretical problem, named the Multiple Junction Test Problem, compared the diffusion calculated in a pipe for two different nodalizations of the pipe, one which had multiple junctions on some control volumes and the other did not.

A preliminary assessment was made to the model for molecular gas diffusion implemented into ATHENA. The model was applied to calculate the diffusion of air into a semi-infinitely long pipe initially filled with helium. One end of the pipe is suddenly exposed to a large reservoir of air. A theoretical solution is available in the literature for the transient distribution in the concentration of air in the pipe (Holman 1981). A schematic of the system analyzed is shown in Figure 5-15. The pipe is initially filled with helium at a pressure of $0.1 \mathrm{MPa}$ and a temperature of $320 \mathrm{~K}$. The infinitely large reservoir of air is at a pressure of 0.1 $\mathrm{MPa}$ and $320 \mathrm{~K}$. At a time of $0.0 \mathrm{~s}$, a valve at one of the pipe is suddenly opened and air begins to diffuse into the pipe. The binary diffusion coefficient for the air-helium mixture is equal to $0.81 \times 10^{-4} \mathrm{~m}^{2} / \mathrm{s}$. 


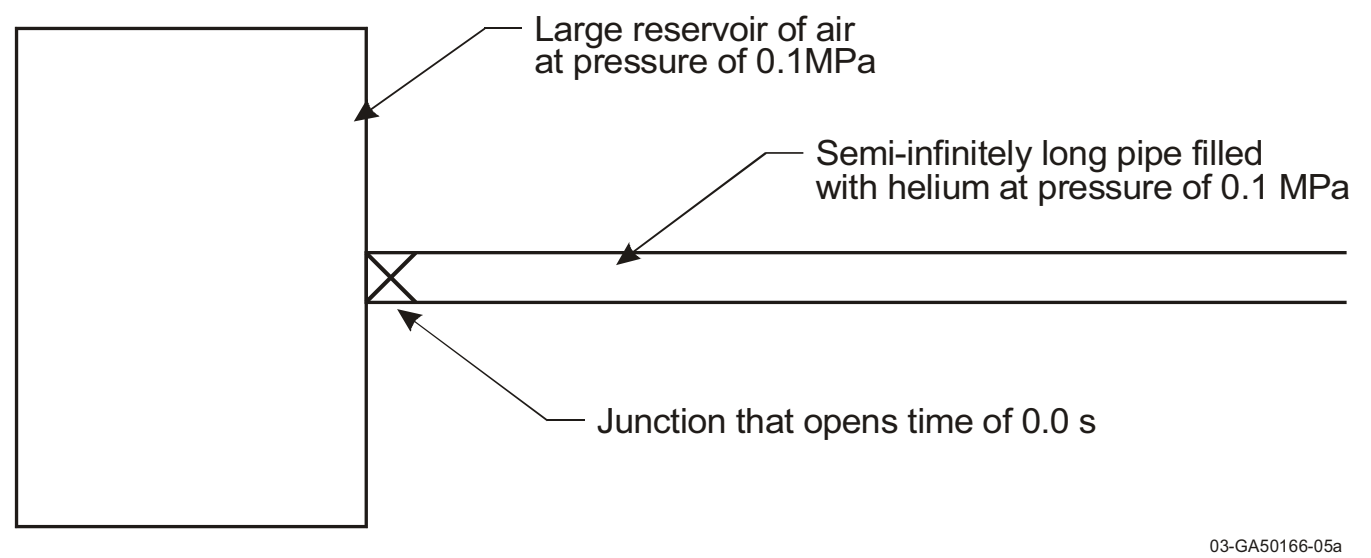

Figure 5-15. Problem analyzed to assess ATHENA modeling of molecular diffusion of air.

The theoretical solution for the transient distribution in the concentration of air in the pipe is expressed by the equation (Holman 1981)

$\mathrm{C}(\mathrm{x}, \mathrm{t})=\mathrm{C}_{\mathrm{atm}}-\frac{2 \mathrm{C}_{\mathrm{atm}}}{\sqrt{\pi}} \int_{0}^{\mathrm{x} / 2 \sqrt{\mathrm{Dat}^{\mathrm{t}}}} \mathrm{e}^{-\eta^{2}} d \eta$

where

$\mathrm{C}(\mathrm{x}, \mathrm{t})=\quad$ concentration of air at spatial coordinate $\mathrm{x}$ at time $\mathrm{t}(\mathrm{x}=0=$ location of break $)\left(\mathrm{kg} / \mathrm{m}^{3}\right)$,

$\mathrm{C}_{\mathrm{atm}}=$ concentration of air in large reservoir of air $\left(1.09 \mathrm{~kg} / \mathrm{m}^{3}\right)$,

$\mathrm{D}_{\mathrm{ah}}=$ binary diffusion coefficient for mixture of helium and air $\left(\mathrm{m}^{2} / \mathrm{s}\right)$.

The ATHENA nodalization of the system being analyzed is described in Figure 5-16. A $50 \mathrm{~m}$ long pipe was divided into 50 control volumes. Each control volume had a length of $1.0 \mathrm{~m}$. Each of the 50 control volumes representing the pipe was initially filled with helium at a pressure of $0.1 \mathrm{MPa}$, a temperature of $320 \mathrm{~K}$, and a density of $0.151 \mathrm{~kg} / \mathrm{m}^{3}$. The large reservoir of air at one end of the pipe was at a pressure of $0.1 \mathrm{MPa}, \mathrm{a}$ temperature of $320 \mathrm{~K}$, and a density of $1.09 \mathrm{~kg} / \mathrm{m}^{3}$. The other end of the pipe was closed.

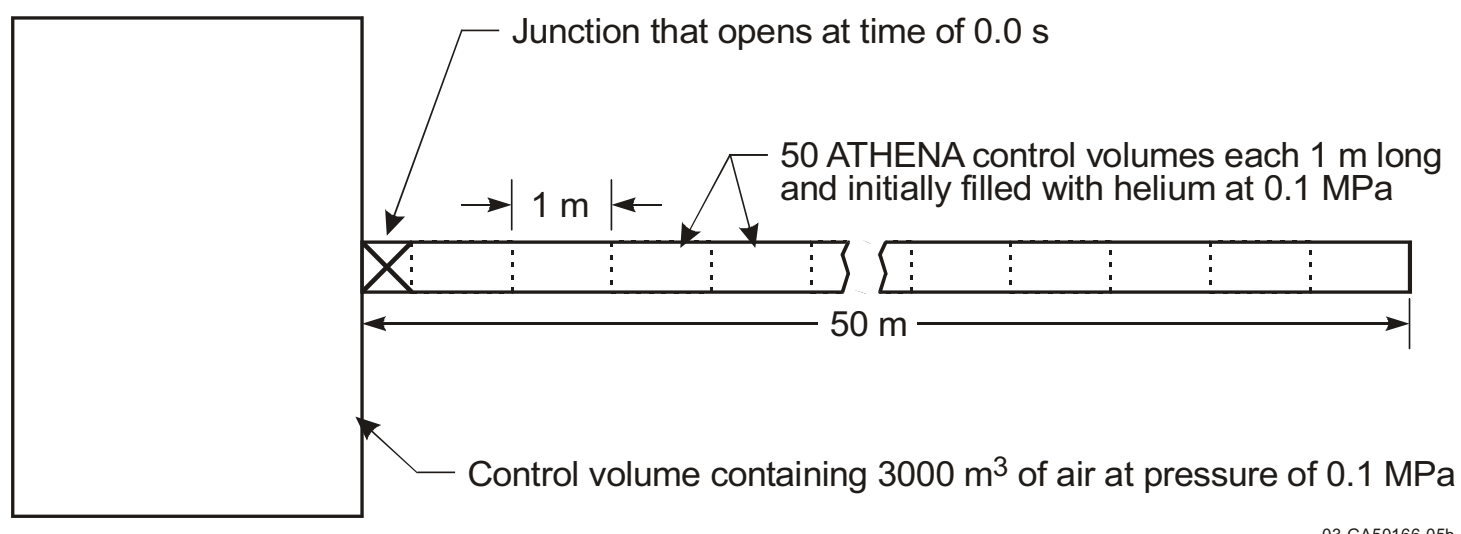

Figure 5-16. ATHENA nodalization of test problem.

The ATHENA calculation of the transient distribution of air concentration in the pipe is compared with the theoretical solution in Figure 5-17. The ATHENA and theoretical solution values for the concentration of air are plotted every $2 \mathrm{~m}$ of distance. The theoretical solution was performed by numerically integrating Equation (5-34). The ATHENA solution and the theoretical solution are in reasonable agreement. The 
ATHENA solution slightly overpredicts the theoretical solution at distances far away from the point of air ingress. An important objective of the modeling of air ingress in ATHENA is the calculation of the transient position of the leading front of air ingress. The results in Figure 5-17 indicate that ATHENA may calculate a somewhat quicker time for air ingress than may actually occur.

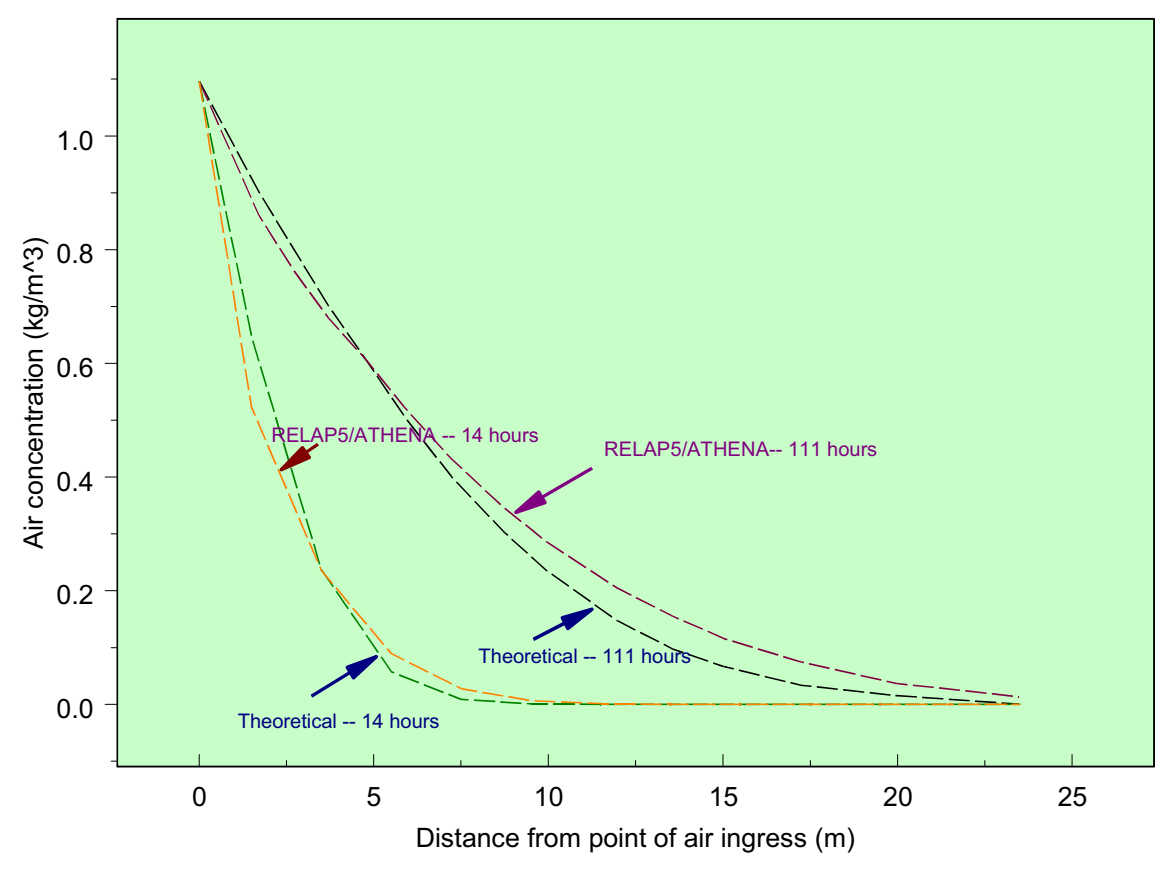

Figure 5-17. Comparison of ATHENA and theoretical spatial-dependent calculations for ingress of air into semi-infinitely long pipe.

The Multiple Junction Test Problem involved the calculation of the transient mixing in a pipe of four species of gas originally separated from each other. The calculation was performed with two different nodalizations that should produce identical results for correct modeling of a general network of control volumes. In the first nodalization, the gas mixture in the pipe is represented by a single row of four equally sized control volumes. This nodalization is shown in Figure 5-18. In the second nodalization, multiple connections were applied to the outlet side of control volume 101 and to the inlet side of control volume 104 shown in Figure 5-18. This nodalization is shown in Figure 5-19. For the nodalization shown in Figure 5-18, control volumes with identification numbers of 101 and 102 initially contain a mixture of $\mathrm{N}_{2}, \mathrm{O}_{2}$, and $\mathrm{CO}_{2}$ at a pressure of 0.1 $\mathrm{MPa}$ and a temperature of $291 \mathrm{~K}$. The initial mass fractions of $\mathrm{N}_{2}, \mathrm{O}_{2}$, and $\mathrm{CO}_{2}$ in these control volumes were $0.769,0.1$, and 0.131 , respectively. These gases are the "non-working" fluid. The control volumes with identification numbers of 103 and 104 initially contain $\mathrm{He}$ (the working fluid) at a pressure of $0.1 \mathrm{MPa}$ and a temperature of $291 \mathrm{~K}$. The length of each of the four control volume is $0.1 \mathrm{~m}$ and the control volumes are equal in cross-sectional area. At the time of zero seconds, the valve isolating control volumes 101 and 102 from control volumes 103 and 104 is fully opened (cross-sectional area of opened valve equal to the crosssectional area of the four control volumes) and diffusion results in the gradual mixing of the various species of gas. In the second nodalization of the pipe, control volumes 102 and 103 in Figure 5-18 were each split into two equally sized control volumes with the sum of the cross-sectional area of each of these two pairs equal to the cross-sectional area of the pipe shown in Figure 5-19. For this nodalization, control volumes 102 
and 104 represent one half of the middle section of the pipe and control volumes 103 and 105 the other half. The outlet side of control volume 101 is connected to both control volumes 102 and 103 and the inlet side of control volume 106 is connected to both control volumes 104 and 105. The cross-sectional area of the pipe is everywhere the same as for the pipe shown in Figure 5-19. Control volumes 101, 102, and 103 initially contain the same mixture of $\mathrm{N}_{2}, \mathrm{O}_{2}$, and $\mathrm{CO}_{2}$ as the control volumes 101 and 102 in Figure 5-18, and control volumes 104, 105, and 106 initially contain $\mathrm{He}$ at the same conditions as that in control volumes 103 and 104 in Figure 5-18.

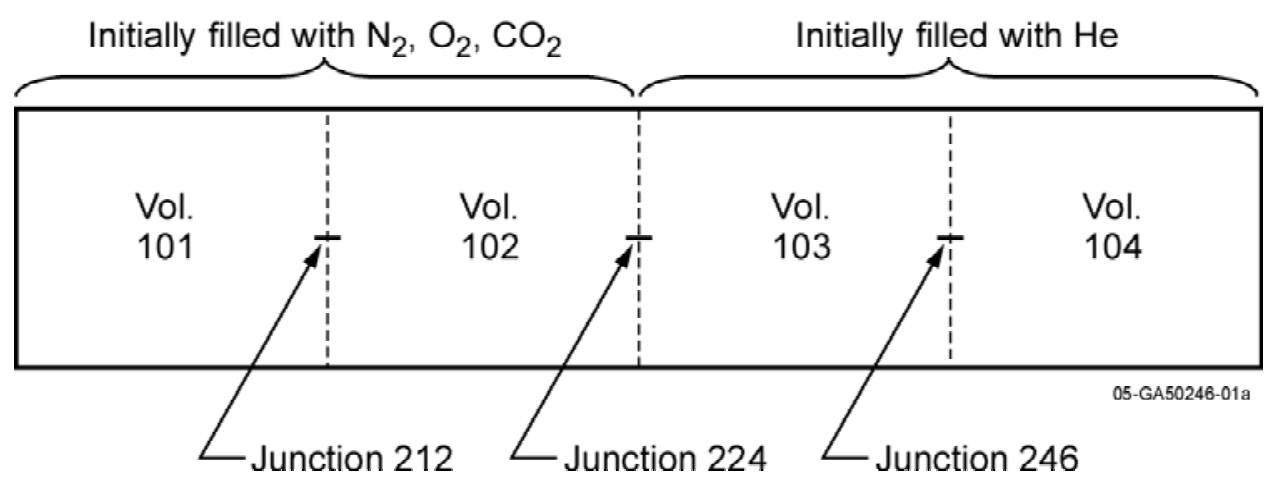

Figure 5-18. Nodalization of pipe containing a mixture of gases as a single row of four equally sized control volumes.

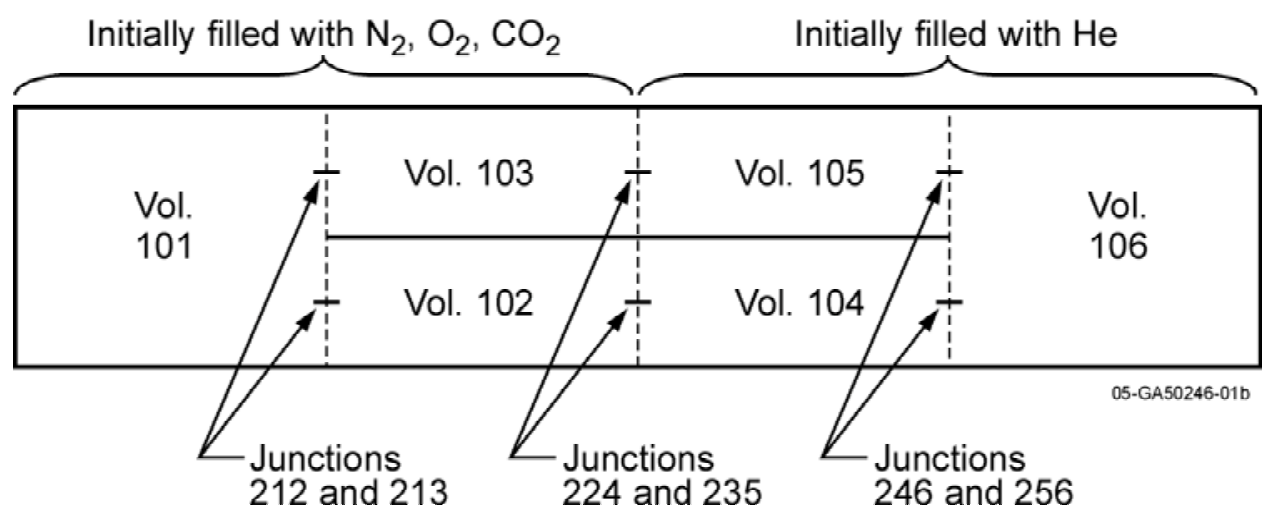

Figure 5-19. Nodalization of pipe with control volumes at ends of pipe having multiple connections to inlet or outlet side.

The calculations performed with the nodalization shown in Figure 5-18 were found to be identical with the calculations performed using the nodalization shown in Figure 5-19. Figure 5-20 compares the transient mass fraction of the non-working gases $\left(\mathrm{N}_{2}, \mathrm{O}_{2}\right.$, and $\left.\mathrm{CO}_{2}\right)$ at the two ends of the pipe as calculated for the case with single connections in the control volumes at the two ends of the pipe and for the case with multiple connections in the control volumes at the two ends of the pipe. As shown in this figure, the results for the two nodalizations are identical. For internally consistent modeling, the calculations should predict that the mass fractions of each species of gas in each control volume asymptotically approach the same value. Figure 5-20 shows that this requirement is also satisfied by the modeling. In summary, the results shown in Figure 5-20 indicate that the extensions made to the RELAP5/ATHENA code for modeling a generalized network of control volumes and gas mixtures with several species of gas have been correctly implemented. 


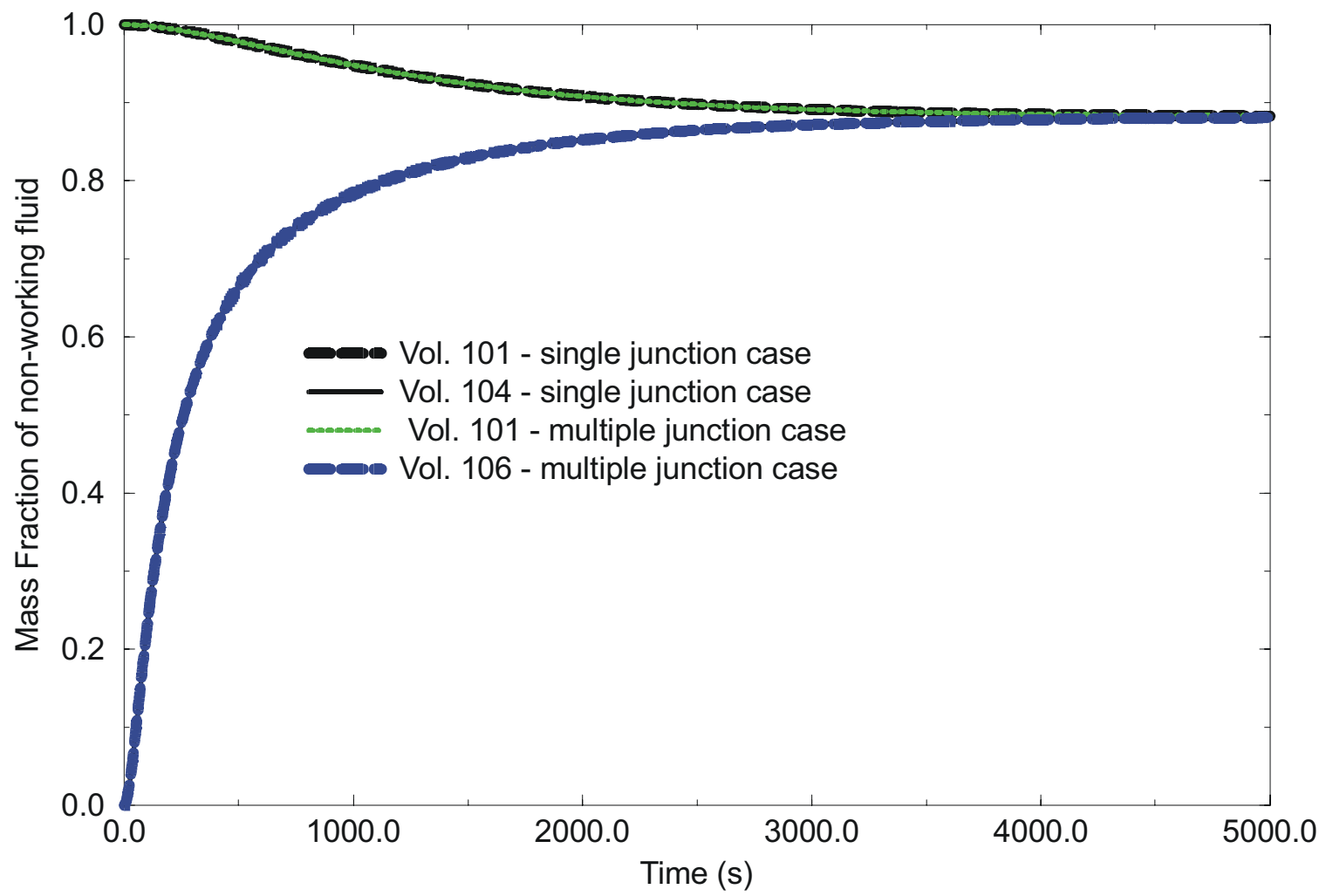

Figure 5-20. Transient concentration of non-working fluid gases $\left(\mathrm{N}_{2}, \mathrm{O}_{2}\right.$, and $\left.\mathrm{CO}_{2}\right)$ at the two ends of pipe.

The calculated transient mole-fractions of gas show that the diffusion modeling in RELAP5/ATHENA is conserving the moles of gas in a system being modeled. For the four control volume system shown in Figure 5-18, the left half of the system contained only helium before opening of the valve isolating the two halves of the system and the right half contained 0.8181 mole-fractions of $\mathrm{N}_{2}, 0.0932$ mole-fractions of $\mathrm{O}_{2}$, and 0.0887 mole fractions of $\mathrm{CO}_{2}$. Each half of the system contained the same moles of gas. Figure 5-21 shows the calculated transient mole-fractions of helium and oxygen in control volumes 101 (left most control volume) and 104 (right most control volume). After the valve has been open for $5000 \mathrm{~s}$, the calculated mole-fractions of gas in the system approach their asymptotic values. The mole-fractions of helium in control volumes 101 and 104 are calculated to approach 0.5 , which is the correct value for conserving the moles of helium in the system. The mole-fractions of $\mathrm{O}_{2}$ in control volumes 101 and 104 are calculated to approach 0.0466 , which is the correct value for conserving $\mathrm{O}_{2}$ in the system. 


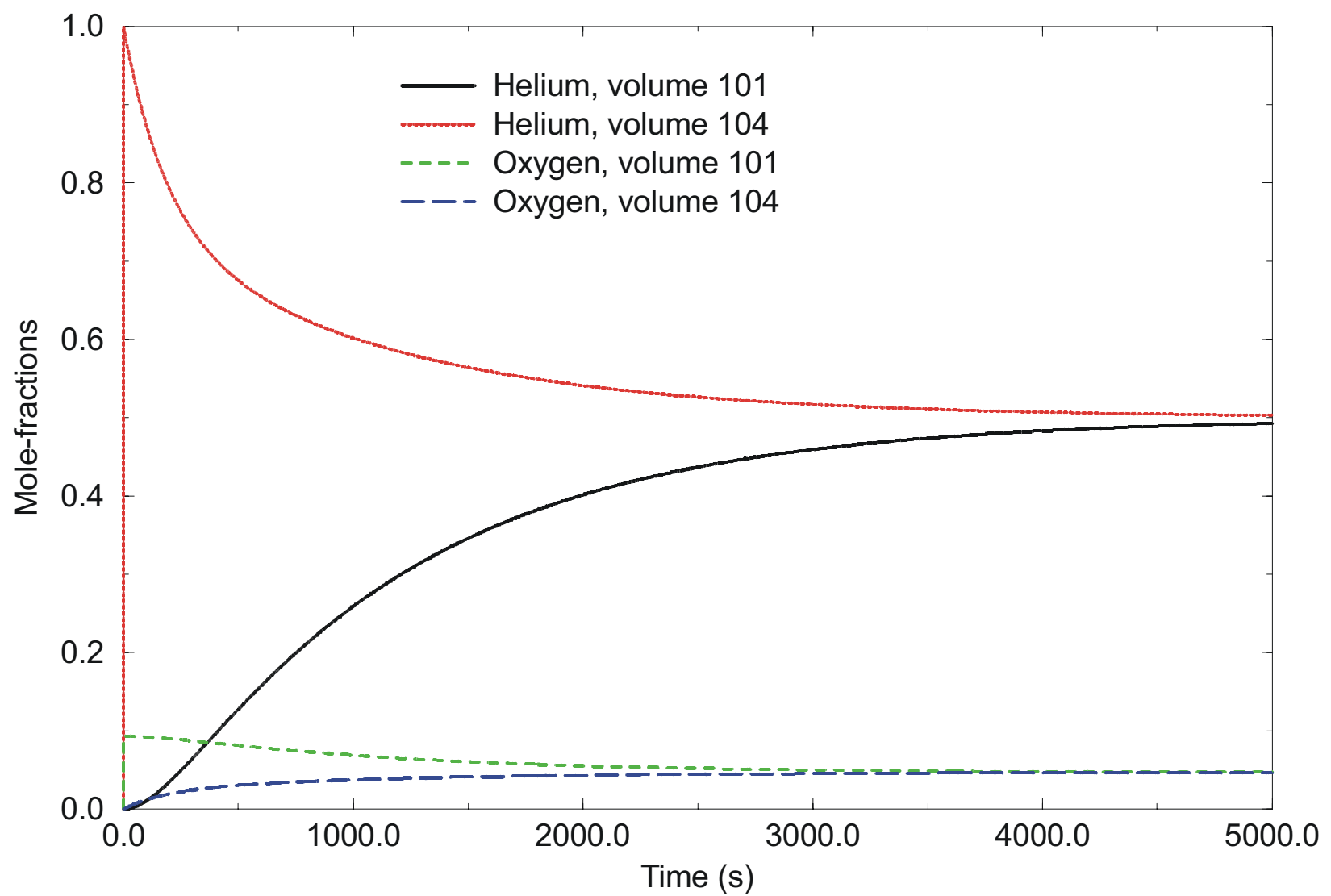

Figure 5-21. Calculated transient mole-fractions of helium and oxygen for four volume control system shown in Figure 5-19.

The presence of helium was calculated to cause the effective binary diffusivities of the three non-working gases $\left(\mathrm{N}_{2}, \mathrm{O}_{2}\right.$, and $\left.\mathrm{CO}_{2}\right)$ to increase by about a factor of two. The effective binary diffusivities of each species of gas in the gas mixture in control volume 101 at the times of $0.0 \mathrm{~s}$ and $5000 \mathrm{~s}$ are shown in Table 58. At the time of $0.0 \mathrm{~s}$, no helium was present in control volume 101 and at the time of $5000 \mathrm{~s}$ about half the moles of gas in the control volume was helium. The effective binary diffusivities of $\mathrm{O}_{2}$ and the gas mixture at the times of $0.0 \mathrm{~s}$ and $5000 \mathrm{~s}$ are $0.1002 \times 10^{-4} \mathrm{~m}^{2} / \mathrm{s}$ and $0.1696 \times 10^{-4} \mathrm{~m}^{2} / \mathrm{s}$, respectively. For $\mathrm{N}_{2}$ and $\mathrm{CO}_{2}$, the effective binary coefficients also increased by similar amounts between $0.0 \mathrm{~s}$ and $5000.0 \mathrm{~s}$.

Table 5-8. Effective binary diffusivities of each gas species in mixture in control volume 101.

$\begin{array}{lllll}\mathrm{Gas} & \text { Time of } 0.0 \mathrm{~s} & & \text { Time of } 5000 \mathrm{~s} & \\ \text { species } & \text { Mole fractions } & \text { Diffusivity }\left(\mathrm{m}^{2} / \mathrm{s}\right) & \text { Mole fractions } & \text { Diffusivity }\left(\mathrm{m}^{2} / \mathrm{s}\right) \\ \mathrm{N}_{2} & 0.8181 & 0.1080 \times 10^{-4} & 0.4113 & 0.1808 \times 10^{-4} \\ \mathrm{O}_{2} & 0.09314 & 0.1002 \times 10^{-4} & 0.04691 & 0.1696 \times 10^{-4} \\ \mathrm{CO}_{2} & 0.08872 & 0.9715 \times 10^{-4} & 0.04473 & 0.1652 \times 10^{-4} \\ \mathrm{He} & 0.0 & - & 0.4971 & -\end{array}$

In summary, the initial assessment of the diffusion modeling in the RELAP5/ATHENA code indicates correct modeling for a general system containing several species of gases. The Multiple Junction Test Problem showed correct modeling of diffusion in a network of control volumes with multiple inlet and outlet junctions. 


\subsection{MELCOR Code Improvements}

MELCOR is a fully integrated, engineering-level computer code that predicts the progression of severe accidents in light water reactor (LWR) nuclear power plants (MELCOR 1997). MELCOR is being developed at the Sandia National Laboratories (SNL) for the U.S. Nuclear Regulatory Commission (NRC). This code calculates a spectrum of accident phenomena, some of which are: reactor cooling system and containment fluid flow, heat transfer, and aerosol transport.

MELCOR solves non-equilibrium conservation equations for mass, momentum, and energy (different phase temperatures and velocities) for the liquid and vapor phase of water. Within user-defined control volumes, the coolant exists as a liquid pool and a vapor atmosphere. Non-condensable gases can be included in the vapor atmosphere. Flow between confinement volumes includes the effect of frictional/momentum form losses and sonically limited (choked) fluid flow. MELCOR addresses a number of plant engineering features that can affect this fluid flow, such as: pressure suppression pools, ice condensers, containment sprays, fan coolers, heat exchangers, valves, pumps, etc. MELCOR treats hydrogen and carbon monoxide combustion, including predictions of ignition conditions and burn rates of hydrogen-carbon monoxide-steam-air-mixtures.

MELCOR solves one-dimensional heat conduction equations for temperatures in various structures of a given facility. Boundary conditions for these equations are either user-specified, or calculated by MELCOR from its heat transfer package. Aerosol transport and deposition is treated by MELCOR to allow the user to track the movement of radioactive-laden aerosol particles (e.g., dust or particulate) within a given facility, and eventually even release to the environment.

\section{Task 5.2-1. Addition of Diffusion Equation to MELCOR}

The INL made modifications to MELCOR (MELCOR 1.8.2) specifically for modeling air ingress accidents in a PBR. In this task, the chemical diffusion equation and chemical equilibrium model were implemented in MELCOR.

The differential equation expressing conservation of mass for the $i^{\text {th }}$ material solved by MELCOR for the atmospheric phase of the fluid flow is:

$$
\frac{\partial \rho_{\mathrm{A}}^{\mathrm{i}}}{\partial \mathrm{t}}+\nabla \bullet\left(\rho_{\mathrm{A}}^{\mathrm{i}} \mathbf{v}_{\mathrm{A}}\right)=\Gamma_{\mathrm{A}}^{\mathrm{i}}
$$

where

$$
\begin{array}{ll}
\rho_{\mathrm{A}}^{\mathrm{i}}- & \text { atmosphere material density }\left(\mathrm{kg} / \mathrm{m}^{3}\right) \\
\mathrm{V}_{\mathrm{A}}- & \text { atmosphere flow velocity }(\mathrm{m} / \mathrm{s}) \\
\Gamma_{\mathrm{A}}^{\mathrm{i}}- & \text { atmosphere material source term }\left(\mathrm{kg} / \mathrm{m}^{3}-\mathrm{s}\right)
\end{array}
$$

Equation (5-35) was modified by adding a gaseous diffusion term that obeys Fick's Law of Diffusion as follows:

$$
\begin{aligned}
& \frac{\partial \rho_{\mathrm{A}}^{\mathrm{i}}}{\partial t}+\nabla \bullet\left(\rho_{\mathrm{A}}^{\mathrm{i}} \mathbf{v}_{\mathrm{A}}\right)=\nabla \bullet \rho_{\mathrm{A}} \mathrm{D}_{\mathrm{A}}^{\mathrm{i}} \nabla \omega_{\mathrm{A}}^{\mathrm{i}}+\Gamma_{\mathrm{A}}^{\mathrm{i}} \\
& \text { where } \\
& \begin{array}{l}
\mathrm{D}_{\mathrm{A}}^{\mathrm{i}}-{ }^{2} \\
\text { mass diffusivity }\left(\mathrm{m}^{2} / \mathrm{s}\right) \text { for the } \mathrm{i}^{\text {th }} \text { material diffusing through the atmosphere phase }(\mathrm{A}) \text { of the } \\
\quad \omega_{\mathrm{A}}^{\mathrm{i}} \quad \text { mass fraction of the } \mathrm{i}^{\text {th }} \text { material }=\rho_{\mathrm{i}} / \rho_{\mathrm{A}}
\end{array}
\end{aligned}
$$


In MELCOR these equations are solved for control volumes (volumes defined by average material properties such as rooms) that are interconnected by flow paths (connections between volumes such as piping). After integrating Equation (5-36) over the $\mathrm{j}^{\text {th }}$ control volume, the result is as follows:

$$
\begin{aligned}
\frac{\partial \mathrm{M}_{\mathrm{j}, \mathrm{A}}^{\mathrm{i}}}{\partial t} & =\sum_{k} \sigma_{\mathrm{j}, \mathrm{k}} \alpha_{\mathrm{k}, \mathrm{A}} \rho_{\mathrm{k}, \mathrm{A}}^{\mathrm{i}, \mathrm{d}} \mathrm{v}_{\mathrm{k}, \mathrm{A}} \mathrm{F}_{\mathrm{k}} \mathrm{A}_{\mathrm{k}} \\
& +\sum_{k} \rho_{\mathrm{k}, \mathrm{A}} \mathrm{D}_{\mathrm{k}, \mathrm{A}}^{\mathrm{i}}\left(\frac{\omega_{\mathrm{m}, \mathrm{A}}^{\mathrm{i}}-\omega_{\mathrm{j}, \mathrm{A}}^{\mathrm{i}}}{\mathrm{L}_{\mathrm{k}}}\right) \alpha_{\mathrm{k}, \mathrm{A}} \mathrm{F}_{\mathrm{k}} \mathrm{A}_{\mathrm{k}}+\stackrel{\mathrm{M}_{\mathrm{j}}, \mathrm{A}}{\mathrm{i}}
\end{aligned}
$$

Here, as described in Reference (MELCOR, 1997), $\mathrm{M}$ is the total mass; subscript k refers to a given flow path, with $\sigma_{\mathrm{j}, \mathrm{k}}$ accounting for the direction of flow in flow path $\mathrm{k}$ with respect to volume $\mathrm{j} ; \alpha_{\mathrm{k}, \mathrm{A}}$ is the volume fraction of the atmospheric phase in flow path k; superscript d denotes "donor", corresponding to the control volume from which the material is flowing; $\mathrm{A}$ is the flow path area; $\mathrm{F}$ is the fraction that the flow area is open; $\mathrm{L}_{\mathrm{k}}$ is the length of flow path $\mathrm{k}$; subscript $\mathrm{m}$ refers to the volume connected to volume $\mathrm{j}$ by flow path $\mathrm{k}$, and $\dot{\mathrm{M}}$ includes all sources of mass. The diffusion coefficients for Equation (5-37) are calculated by MELCOR as described in Reference (MELCOR code manual, 1997).

The equation expressing conservation of energy for the atmospheric phase in the $\mathrm{j}^{\text {th }}$ control volume is derived in a similar manner. The conservation equation, written neglecting potential and kinetic energy, is as follows:

$$
\begin{aligned}
\frac{\partial \mathrm{E}_{\mathrm{j}, \mathrm{A}}}{\partial t}=\sum_{k} \sigma_{\mathrm{j}, \mathrm{k}} & \alpha_{\mathrm{k}, \mathrm{A}}\left(\sum_{i} \rho_{\mathrm{k}, \mathrm{A}}^{\mathrm{i}, \mathrm{d}} \mathrm{h}_{\mathrm{k}, \mathrm{A}}^{\mathrm{i}, \mathrm{d}}\right) \mathrm{v}_{\mathrm{k}, \mathrm{A}} \mathrm{F}_{\mathrm{k}} \mathrm{A}_{\mathrm{k}} \\
& +\sum_{\mathrm{k}} \rho_{\mathrm{k}, \mathrm{A}}\left(\sum_{\mathrm{i}} \mathrm{D}_{\mathrm{k}, \mathrm{A}}^{\mathrm{i}}\left(\frac{\omega_{\mathrm{m}, \mathrm{A}}^{\mathrm{i}}-\omega_{\mathrm{j}, \mathrm{A}}^{\mathrm{i}}}{\mathrm{L}_{\mathrm{k}}}\right)\right) h_{\mathrm{k}, \mathrm{A}}^{\mathrm{d}} \alpha_{\mathrm{k}, \mathrm{A}} \mathrm{F}_{\mathrm{k}} \mathrm{A}_{\mathrm{k}}+\dot{\mathrm{H}}_{\mathrm{j}, \mathrm{A}}
\end{aligned}
$$

where, $\mathrm{E}$ is the total internal energy, " $\mathrm{i}$ " represents the summation over all species in the atmosphere phase, $\mathrm{h}^{\mathrm{i}}$ is the specific enthalpy of specie "i", $h$ is the total specific enthalpy for the atmosphere in the volume attached

to the $\mathrm{j}^{\text {th }}$ control volume by the $\mathrm{k}^{\text {th }}$ flow path, and $\dot{\mathrm{H}}$ is the non-flow energy sources related to the mass sources of Equation (5-37).

\section{Task 5.2-2. Addition of Chemical Equilibrium Model}

The objective of this task is to calculate the chemical equilibrium between $\mathrm{CO}$ and $\mathrm{CO}_{2}$ gases. Modifications to the INL graphite oxidation model of the MELCOR code (Version 1.8.2) were completed that implement the graphite oxidation correlations proposed by Lim and NO (2003). These correlations include a new graphite oxidation rate equation, a kinetics oxidation limit based on diffusion of oxygen through the gaseous boundary layer that develops above an oxidizing surface, and the production ratio of $\mathrm{CO}$ to $\mathrm{CO}_{2}$ at this oxidizing surface. This section describes these modifications.

When oxygen from air comes into contact with a hot graphite surface, $\mathrm{CO}$ and $\mathrm{CO}_{2}$ will form according to the following general chemical equation (Takeda and Hishida, 1996): 


$$
\mathrm{C}+\mathrm{zO}_{2} \rightarrow \mathrm{xCO}+\mathrm{yCO}_{2}
$$

where $x, y$, and $z$ are the mole fractions of carbon monoxide, carbon dioxide and oxygen, respectively, of this chemical reaction. Graphite oxidation data often exhibit an exponential increase with temperature and the rate ( $\mathrm{kg}$-carbon $/ \mathrm{m}^{2} \mathrm{~s}$ ) at which the oxidation process of Equation (5-39) proceeds can be represented by an Arrhenius type rate relationship that takes the following general form:

A chemical reaction rate of the graphite oxidation is described as

$$
\mathrm{r}_{\mathrm{C}-\mathrm{O}_{2}}=\mathrm{K}_{\mathrm{o}} \exp \left(-\frac{\mathrm{E}_{\mathrm{o}}}{\mathrm{RT}}\right)\left(\frac{\mathrm{P}_{\mathrm{O}_{2}}}{\mathrm{P}_{\mathrm{o}}}\right)^{\mathrm{n}}
$$

where $\mathrm{K}_{\mathrm{o}}$ is a pre-exponent constant $\left(\mathrm{kg} / \mathrm{m}^{2} \mathrm{~s}\right)$, the reaction constant, $\mathrm{E}_{\mathrm{o}}$ is the activation energy $(\mathrm{J} / \mathrm{g}-\mathrm{mol})$, $\mathrm{R}$ is the universal gas constant $(8.134 \mathrm{~J} / \mathrm{g}-\mathrm{mol}-\mathrm{K}), T$ is the absolute surface temperature, and $\mathrm{P}_{\mathrm{o}_{2}}$ is the oxygen partial pressure $(\mathrm{Pa}), \mathrm{P}_{\mathrm{o}}$ is the oxygen partial pressure at which the oxidation experiments were conducted, and the exponent " $n$ " represents the order of the reaction. Those constants are based on experimental data using IG-110 graphite (H. Kawakami, 1986): $\mathrm{K}_{0}=3.60 \times 10^{2}$ and $\mathrm{E}_{\mathrm{o}}=2.09 \times 10^{2} \mathrm{~kJ} / \mathrm{mol}$.

The oxidation of graphite has been extensively studied and it is generally accepted that different mechanisms control the oxidation process depending on the temperature of the graphite. At low temperatures, the rate of oxidation will be limited by the chemical reactivity of the graphite and the rate at which oxygen can diffuse into the bulk of the graphite (Regime I). At intermediate temperatures, the rate is controlled by the combined effect of oxygen diffusion through the gaseous boundary layer developing above the oxidizing surface, diffusion of oxygen into the bulk graphite located near the surface, and the chemical reactivity of the graphite (Regime II). At high temperatures, the graphite surface becomes so reactive that the only rate limiting mechanism becomes the rate at which oxygen can diffuse through the boundary layer to the graphite surface (Regime III). Because these three regimes exist, the application of an Arrhenius type rate relationship to graphite oxidation data requires three individual sets of constants for this rate relationship (i.e., one set per regime).

The new correlation added to the INL graphite oxidation model is for IG-110 nuclear-grade graphite. The correlation was developed by Lim and NO (2003) based on data obtained by Fuller and Okoh (1997), and is as follows

$$
\mathrm{r}_{\mathrm{C}-\mathrm{O}_{2}}=1.57 \times 10^{6} \exp (-188000 / \mathrm{RT})\left(\frac{\mathrm{P}_{\mathrm{O}_{2}}}{0.209 \times 10^{5}}\right)^{\mathrm{n}}
$$

where " $\mathrm{n}$ " is allowed to vary from $1 / 2$ to 1 based on user preference. This correlation is applicable for oxidation Regime II, or the intermediate temperature regime. This correlation is also used by Lim and NO (2003) to extrapolate to lower temperatures of Regime I, and as such should be a conservative upper estimate of the oxidation rate for Regime I. For the high temperature Regime III, Lima and NO (2003) apply a rate limit based on the diffusion of oxygen through the surface gaseous boundary layer. This limit is based on a 
boundary layer analogy between mass and heat transfer known as the Sherwood number $\left(\mathrm{Sh}=\mathrm{k}_{\mathrm{m}} \mathrm{L} / \mathrm{D}_{\mathrm{AB}}\right)$ correlation, defined as follows (Incropera, 2002):

$$
\frac{\mathrm{Sh}}{\mathrm{Sc}^{\mathrm{m}}}=\frac{\mathrm{Nu}}{\operatorname{Pr}^{\mathrm{m}}}
$$

where $\mathrm{k}_{\mathrm{m}}$ is a mass transfer coefficient $(\mathrm{m} / \mathrm{s}), \mathrm{L}$ is a characteristic length $(\mathrm{m}), \mathrm{D}_{\mathrm{AB}}$ is the binary diffusion coefficient for specie A in specie B $\left(\mathrm{m}^{2} / \mathrm{s}\right)$, Sc is the mass transfer Schmidt number $\left(\mu / \rho_{\mathrm{AB}}\right)$, Nu is the heat transfer Nusselt number $(\mathrm{Nu}=\mathrm{hL} / \mathrm{k})$, Pr is the heat transfer Prandlt number $\left(\mathrm{c}_{\mathrm{p}} \mu / \mathrm{k}\right)$, ' $\mathrm{m}$ ' is a correlation parameter, $\mu$ is the fluid viscosity $(\mathrm{kg} / \mathrm{m}-\mathrm{s}), \rho$ fluid density $\left(\mathrm{kg} / \mathrm{m}^{3}\right), \mathrm{h}$ is a convective heat transfer coefficient $\left(\mathrm{W} / \mathrm{m}^{2}-\mathrm{k}\right), \mathrm{k}$ is the fluid thermal conductivity $(\mathrm{W} / \mathrm{m}-\mathrm{K})$, and $\mathrm{c}_{\mathrm{p}}$ is the fluid specific heat $(\mathrm{J} / \mathrm{kg}-\mathrm{K})$. The Sherwood number correlation can be use to determine a mass transfer coefficient provided a $\mathrm{Nu}$ can be defined. Lim and NO (2003) have chosen a Nu for fully developed laminar flow in a circular tube at a constant temperature, which has been found analytically to be a constant equal to 3.66 (Incropera, 2002). The result for the mass transfer coefficient becomes:

$$
\mathrm{k}_{\mathrm{m}}=3.66\left(\frac{\mathrm{D}_{\mathrm{AB}}}{\mathrm{L}}\right)\left(\frac{\mathrm{Sc}}{\mathrm{Pr}}\right)^{1 / 3}
$$

With this mass transfer coefficient, the graphite oxidation rate for all three oxidation regimes can be limited to the rate at which oxygen diffuses through the boundary layer $\left(\mathrm{kg}-\mathrm{O}_{2} / \mathrm{m}^{2} \mathrm{~s}\right)$, which is:

$$
\mathrm{r}_{\mathrm{O}_{2}}=\mathrm{k}_{\mathrm{m}} \rho_{\mathrm{O}_{2}}
$$

where $\rho_{\mathrm{O} 2}$ is the oxygen density in the bulk of the fluid. An implicit assumption for Equation (5-44) is that the oxygen density at the surface of the graphite (e.g., at the opposite side of the boundary layer) equals zero. Given this limit, the graphite oxidation rate for this correlation is as follows

$$
\mathrm{r}_{\mathrm{C}-\mathrm{O}_{2}}=\min \left(\mathrm{r}_{\mathrm{Eqn4}-41}, \mathrm{k}_{\mathrm{m}} \rho_{\mathrm{O}_{2}} \mathrm{M}_{\mathrm{C}} /\left(\mathrm{zM}_{\mathrm{O}_{2}}\right), \mathrm{r}_{\mathrm{O} 2} \mathrm{M}_{\mathrm{C}} / \mathrm{M}_{\mathrm{O} 2}\right)
$$

where $z$ is the $\mathrm{O}_{2}$ mole fraction of Equation (5-39), and $\mathrm{M}_{\mathrm{c}}$ and $\mathrm{M}_{\mathrm{O} 2}$ are the molecular weights $(\mathrm{g} / \mathrm{mol})$ of carbon and oxygen, respectively.

The remaining mechanism to be defined regarding this correlation is the production rate of $\mathrm{CO}$ and $\mathrm{CO}_{2}$ as the graphite surface oxidizes. Lim and NO (2003) adopted the following Arrhenius type relationship investigated by Takahashi, et al. (1956), which defines the equilibrium production ratio of $\mathrm{CO}$ to $\mathrm{CO}_{2}$ as:

$$
\mathrm{f}_{\mathrm{CO} / \mathrm{CO}_{2}}=\mathrm{x} / \mathrm{y}=\mathrm{K}_{1} \exp \left(-\mathrm{E}_{1} / \mathrm{RT}\right)
$$

where $\mathrm{K}_{1}$ is a pre-exponential constant, and $\mathrm{E}_{1}$ is an activation energy. Values for these constants adopted by Lim and NO (2003) are those that best represent the oxidation data of Takahashi et al. (1956) and are those developed by Rossberg (1956): $\mathrm{K}_{1}=1995$, and $\mathrm{E}_{1}=59860(\mathrm{~J} / \mathrm{gmol})$. 
The documented values for $\mathrm{K}_{1}$ and $\mathrm{E}_{1}$ are various over a wide range of parameters and the catalytic effect of the impurities contained in the graphite and the grade of graphite depending on graphite properties as investigated by Takeda and Hishida (1996). Among several sets of the reported values (Takeda and Hishida (1996)), we compared two data sets: one data set with $\mathrm{K}_{1}=1995$ and $\mathrm{E}_{1}=59860 \mathrm{~J} / \mathrm{g}-\mathrm{mol}$. and the other data set with $\mathrm{K}_{2}=7,943$ and $\mathrm{E}_{2}=78300 \mathrm{~J} / \mathrm{g}-\mathrm{mol}$. As shown in Figure 5-22, the equimolar fraction between $\mathrm{CO}$ and $\mathrm{CO}_{2}$ appear at temperatures of $950 \mathrm{~K}$ and $1050 \mathrm{~K}$, respectively.

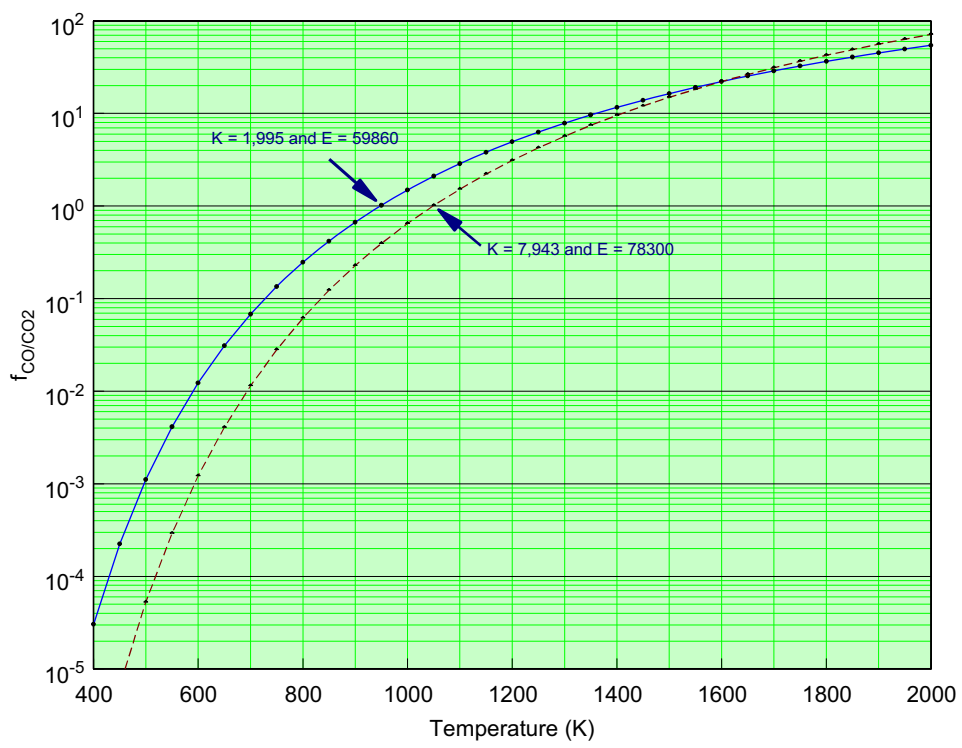

Figure 5-22. Mole ratio of $\mathrm{CO}$ over $\mathrm{CO}_{2}$ as a function of surface temperature.

The stoichiometric mole numbers for the partial oxidation equation above can be calculated from the mass balance of carbon and oxygen to be

$$
\mathrm{z}=\mathrm{N}_{\mathrm{o}_{2}}=\frac{\mathrm{f}_{{\mathrm{co} / \mathrm{co}_{2}}}+2}{2 \mathrm{f}_{\mathrm{co} / \mathrm{co}_{2}}+2}, \quad \mathrm{x}=\mathrm{N}_{\mathrm{co}}=\frac{\mathrm{f}_{\mathrm{CO} / \mathrm{CO}_{2}}}{\mathrm{f}_{\mathrm{co} / \mathrm{co}_{2}}+1} \text {, and } \mathrm{y}=\mathrm{N}_{\mathrm{co}_{2}}=\frac{1}{\mathrm{f}_{{\mathrm{co} / \mathrm{co}_{2}}+1}}
$$

Using $\mathrm{f}_{\mathrm{CO} / \mathrm{CO}_{2}}$ values calculated from Figure 5-22, the number of chemical species moles based on 1 mole of carbon are plotted as shown in Figure 5-23 which is based on $\mathrm{k}_{2}=7,943$ and $\mathrm{E}_{2}=78300 \mathrm{~J} / \mathrm{g}-\mathrm{mol}$ among two different sets. 


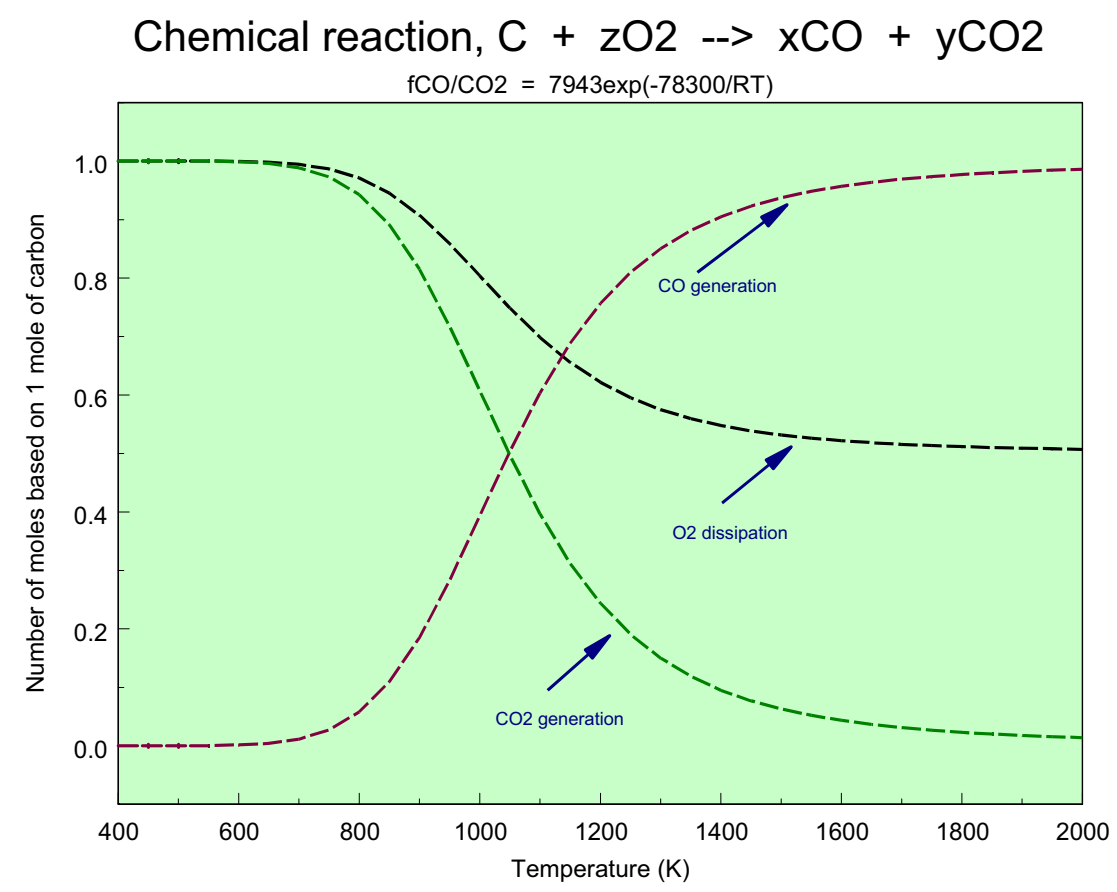

Figure 5-23. Chemical equilibrium using $\mathrm{K}=7943$ and $\mathrm{E}=78300 \mathrm{~J} / \mathrm{g}-\mathrm{mol}$.

These calculations indicate that at temperature higher than $1050 \mathrm{~K}$, the chemical reaction of graphite oxidation proceeds favorably towards to the generation $\mathrm{CO}$ gas as opposed to temperatures less than $1050 \mathrm{~K}$ where $\mathrm{CO} 2$ is more dominant.

Similar results were obtained from using $\mathrm{k}_{1}=1995$ and $\mathrm{E}_{1}=59860 \mathrm{~J} / \mathrm{g}$-mol as shown in Figure 5-24 below. 


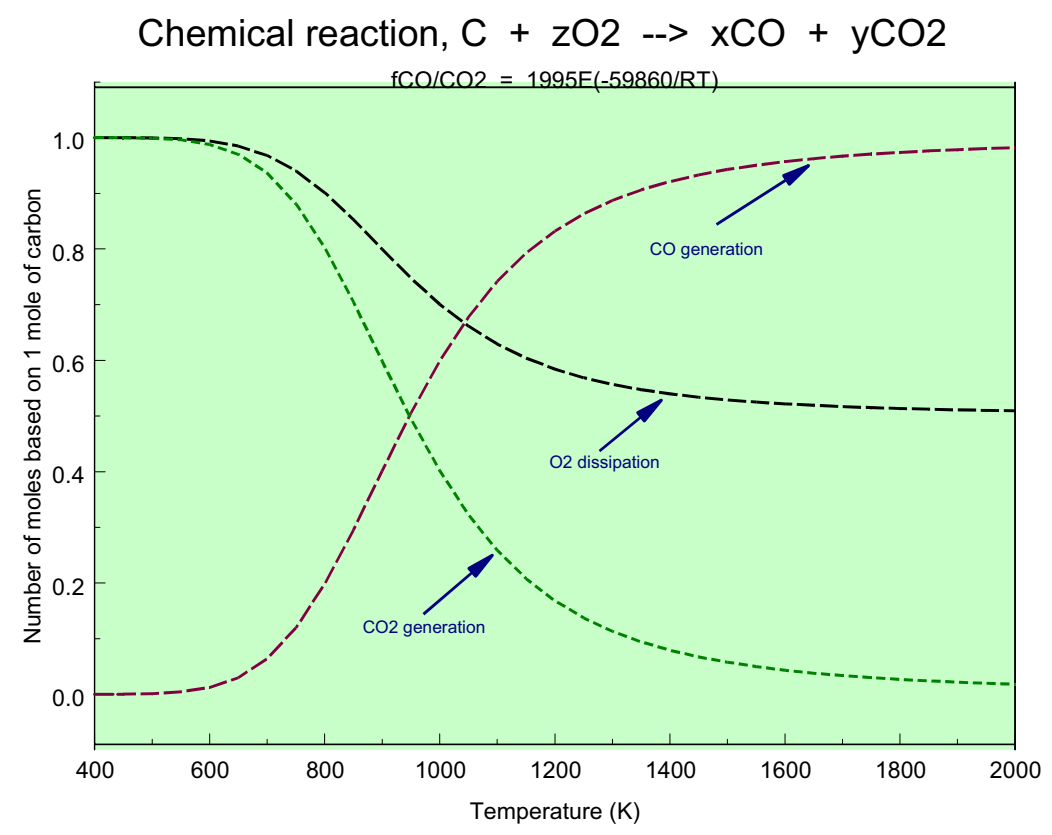

Figure 5-24. Chemical equilibrium using $\mathrm{K}=1995$ and $\mathrm{E}=59860 \mathrm{~J} / \mathrm{g}-\mathrm{mol}$.

Given these mole fractions, the mass of $\mathrm{O}_{2}$ consumed, and the mass of $\mathrm{CO}$ and $\mathrm{CO}_{2}$ produced by the oxidizing graphite surface can be determined as follows:

$r_{\mathrm{O}_{2}}=-\mathrm{z}\left(\frac{\mathrm{M}_{\mathrm{O}_{2}}}{\mathrm{M}_{\mathrm{c}}}\right) \mathrm{r}_{\mathrm{Equ4}-18}, \mathrm{r}_{\mathrm{CO}}=\mathrm{x}\left(\frac{\mathrm{M}_{\mathrm{CO}}}{\mathrm{M}_{\mathrm{c}}}\right) \mathrm{r}_{\mathrm{Eqn4-18}}$, and $\mathrm{r}_{\mathrm{CO}_{2}}=\mathrm{y}\left(\frac{\mathrm{M}_{\mathrm{CO}_{2}}}{\mathrm{M}_{\mathrm{c}}}\right) \mathrm{r}_{\mathrm{Eqn4-18}}$

The chemical heating generated by this oxidation reaction at the surface of the graphite is the product of the heat of reaction for $\mathrm{CO}(\mathrm{J} / \mathrm{kg}-\mathrm{CO})$ and $\mathrm{CO}_{2}\left(\mathrm{~J} / \mathrm{kg}-\mathrm{CO}_{2}\right)$ times the production rate of these gases. In the INL MELCOR oxidation model the following formulas are used for the heat of reaction as obtained from Weast (1979):

$\mathrm{h}_{\mathrm{R}, \mathrm{CO}}=\frac{4187}{\mathrm{M}_{\mathrm{CO}}}\left(25400+0.89 \mathrm{~T}+0.00027 \mathrm{~T}^{2}+219000 / \mathrm{T}\right)$

$\mathrm{h}_{\mathrm{R}, \mathrm{CO}_{2}}=\frac{4187}{\mathrm{M}_{\mathrm{CO} 2}}\left(93690+0.708 \mathrm{~T}-0.00007 \mathrm{~T}^{2}+46000 / \mathrm{T}\right)$

The resulting surface heat flux becomes:

$\mathrm{q}=\mathrm{h}_{\mathrm{R}, \mathrm{CO}} \Gamma_{\mathrm{CO}}+\mathrm{h}_{\mathrm{R}, \mathrm{CO}_{2}} \Gamma_{\mathrm{CO}_{2}}$

In addition to the new oxidation correlation of Lim and NO (2003), the INL graphite oxidation model already has two oxidation correlations, which are based on bulk graphite data from O'Brien, et al. (1988), designated as INL-1988, and on carbon fiber composite graphite data from Marshall, et al., (2002), designated as INL- 
2002. The INL-1988 correlation gives oxidation rate relationships for Regimes II and III, but extrapolates the Regime II correlation down to temperatures that represent oxidation Regime I. The INL-2002 correlation gives oxidation rate relationships for Regimes I and II, but uses the rate relationship of INL-1988 for oxidation Regime III. Both oxidation correlations assume that the oxidation reaction goes completely to $\mathrm{CO}_{2}$ at the oxidizing surface regardless of surface temperature, that is $y$ equals one and $x$ equals zero for Equation (5-39). Because of this assumption, one additional modification to the INL MELCOR oxidation model was completed for the INL-2002 correlation. This modification limits the rate of surface oxidation predicted by the INL-2002 correlation by applying Equation 5-45 and employs Equation 5-46 to predict the production ratio of $\mathrm{CO}$ to $\mathrm{CO}_{2}$ as a function of graphite surface temperature. The MELCOR user, through MELCOR user input, can access these correlations individually per heat structure. By defining the material of a given heat structure as carbon01, carbon02, carbon03, carbon04, or carbon05, the user will apply INEL-1988, INL2002, INL-2002 modified, KAERI (Lim and NO, 2003) with pressure dependence of $1 / 2$, and KAERI with a pressure dependence of 1 , respectively, to that heat structure.

It should be pointed out that the oxidation correlations of the INL graphite oxidation model should give reliable results as long as the selected correlation is prototypical of the system being analyzed, that is similar geometries, flow conditions, etc. For example, the data on which the Arrhenius type relationships (Equation 5-40) are based inherently contain the mechanism of boundary layer diffusion. If the adopted correlation is to be used for a non-prototypical application, then an attempt should be made to correct for this fact. As an example, it can be shown that by making use of Equation (5-41), and the fact that in oxidation Regime III the oxygen concentration near the oxidizing surface is approximately zero, that the Arrhenius type relationship can be modified for different heat transfer conditions as follows:

$$
\Gamma_{\mathrm{C}-\mathrm{O}_{2}}=\Gamma_{\mathrm{o}} \exp \left(-\mathrm{E}_{\mathrm{o}} / \mathrm{RT}_{\mathrm{s}}\right)\left(\frac{\mathrm{p}_{\mathrm{O}_{2}}}{\mathrm{p}_{\mathrm{o}}}\right)^{\mathrm{n}}\left(\frac{\mathrm{Nu}}{\mathrm{Nu}_{\mathrm{o}}}\right)
$$

where, $\mathrm{Nu}_{\mathrm{o}}$ is the Nusselt number associated with the experimental conditions during which the test data were obtained. Equations (4-42) through (4-44) represent a good attempt at accomplishing this correction, but the result is limited to fully developed laminar flow in a constant temperature tube. However, this may not be the condition inside of a pebble bed.

For combustion of carbon monoxide in air, the reaction rate equation of Dreyer and Glassman (1973), as developed in Lim and NO (2003), was added to the INL graphite oxidation model. The general chemical equation for this reaction is as follows:

$$
\mathrm{CO}+\frac{1}{2} \mathrm{O}_{2}=\mathrm{CO}_{2}
$$

The adopted rate equation $\left(\mathrm{mol}-\mathrm{CO} / \mathrm{cm}^{3}-\mathrm{s}\right)$ for this reaction is:

$$
-\frac{\mathrm{dc}_{\mathrm{CO}}}{\mathrm{dt}}=\mathrm{K}_{\mathrm{o}} \mathrm{c}_{\mathrm{CO}} \mathrm{c}_{\mathrm{O}_{2}}^{1 / 4} \mathrm{c}_{\mathrm{H}_{2} \mathrm{O}}^{1 / 2} \exp \left(-\mathrm{E}_{\mathrm{o}} / \mathrm{RT}\right)
$$

where $\mathrm{K}_{\mathrm{o}}$ is a constant $\left(10^{4.6}\right)$, $\mathrm{c}$ is specie molar concentration $\left(\mathrm{mol} / \mathrm{cm}^{3}\right)$, and $\mathrm{E}_{\mathrm{o}}$ the activation energy (40 $\mathrm{kcal} / \mathrm{mol}$ ). By substituting densities for the specie molar concentration, and multiplying by the molecular mass of $\mathrm{CO}$ to convert from moles $\mathrm{CO}$ to mass $\mathrm{CO}$, gives the following rate equation $\left(\mathrm{kg} / \mathrm{m}^{3}-\mathrm{s}\right)$ as implemented in the INL graphite oxidation model: 


$$
\frac{\mathrm{d} \rho_{\mathrm{CO}}}{\mathrm{dt}}=-2.24 \times 10^{12} \rho_{\mathrm{CO}}\left(\frac{\rho_{\mathrm{O}_{2}}}{\mathrm{M}_{\mathrm{O}_{2}}}\right)^{1 / 4}\left(\frac{\rho_{\mathrm{H}_{2} \mathrm{O}}}{\mathrm{M}_{\mathrm{H}_{2} \mathrm{O}}}\right)^{1 / 2} \exp (-167400 / \mathrm{RT})
$$

The production of $\mathrm{CO}_{2}$ and dissipation of $\mathrm{O}_{2}$ are determined from Equation 5-55 as follows:

$$
\frac{\mathrm{d} \rho_{\mathrm{CO}_{2}}}{\mathrm{dt}}=-\frac{\mathrm{d} \rho_{\mathrm{CO}}}{\mathrm{dt}} \frac{\mathrm{M}_{\mathrm{CO}_{2}}}{\mathrm{M}_{\mathrm{CO}}} ; \quad \frac{\mathrm{d} \rho_{\mathrm{O}_{2}}}{\mathrm{dt}}=\frac{1}{2} \frac{\mathrm{d} \rho_{\mathrm{CO}}}{\mathrm{dt}} \frac{\mathrm{M}_{\mathrm{O}_{2}}}{\mathrm{M}_{\mathrm{CO}}}
$$

To activate this portion of the oxidation model, the user must specify a surface oxidation correlation that produces carbon monoxide and specify the water vapor (air humidity) as the non-condensable gas in MELCOR input designated as 'GASA'. 


\section{TASK 5: Neutronic modeling (UM)}

\subsection{Objectives and Sub-tasks}

The main objective for this task was the development of neutronics modeling capability for the reactor physics and safety analysis of the VHTR. The original task structure included the following three sub-tasks:

○ Task 5-1: Global MCNP Model. Development of a global Monte Carlo model using MCNP5, based on a homogenized fuel mixture.

○ Task 5-2: Combined Deterministic-Monte Carlo Methodology. Development of a new coupled deterministic-Monte Carlo methodology for MCNP assembly-level lattice physics calculations, where fine-group microscopic or macroscopic cross sections are generated, fully accounting for the double heterogeneities inherent in the VHTR lattice.

○ Task 5-3: MCNP5 Development and Benchmarking. Verification and validation of MCNP5 for VHTR configurations.

At the time these tasks were determined (2002), the MCNP5 code [Brown 2003] had not been released and the status of global Monte Carlo methods with depletion was in question. However, since that time the MCNP5 code was released with a number of features that allowed it to be used for VHTR analysis, including improved $S(\alpha, \beta)$ treatment, Doppler-broadened resonance cross sections. In addition, the use of Monteburns and other Monte Carlo-based depletion codes was becoming more prevalent for global depletion calculations. As the project progressed, it became clear that MCNP5 could be used for the entire VHTR calculation, including the global calculation with depletion and temperature feedback, thus eliminating the need to perform deterministic transport analyses or global diffusion-depletion analyses for flux-power distributions.

This focus on MCNP5 as the primary neutronics analysis method obviated the need to develop a coupled deterministic-Monte Carlo capability; therefore, Task 5-2: Combined Deterministic-Monte Carlo Methodology, was changed to Task 5-2: Coupled Nuclear-Thermal-Hydraulic Calculations. A consequence of this realignment of our effort was that most of the activities originally proposed for Task 5-3: MCNP Development and Benchmarking, were subsumed into Task 5-1. Task 5-3 was renamed to Fission Product Decay Heat Calculations. The revised task structure became:

○ Task 5-1: Global MCNP Model. Development of a global Monte Carlo model using MCNP5, based on explicit representation and analysis of the particle fuel, including fuel depletion and thermal-hydraulic feedback.

○ Task 5-2: Coupled Nuclear-Thermal-Hydraulic Calculations. Development of the methodology to couple MCNP5 and RELAP-Athena, allowing global Monte Carlo simulation of VHTR cores with temperature feedback.

○ Task 5-3: Fission Product Decay Heat Calculations. Prediction of the global decay heat source distribution as a function of depletion and temperature feedback.

\subsection{Task 5-1: Global MCNP Model}

Effort has been focused on analyzing the double heterogeneity posed by the TRISO fuel by analyzing static reactor configurations for $\mathrm{k}_{\mathrm{eff}}$ and flux/power distributions as well as accounting for depletion effects. The predictions of the full core VHTR power distributions with thermal/hydraulic feedback and depletion were performed have been performed with Monte Carlo codes. Effort has been focused on developing MCNP5 (Brown, 2003) models with both homogeneous and heterogeneous microsphere fuel particle (TRISO), 
including (a) single microsphere models, (b) fuel compact models, and (c) full-core models. Results have been obtained for all levels of analysis, including preliminary results for a full-core model with reflectors that included explicit modeling of the TRISO fuel, and accounted for temperature feedback by using a temperature distribution obtained from a thermal-hydraulic calculation. These efforts are described below.

\subsubsection{Single microsphere model}

A heterogeneous microsphere fuel particle model has been created with MCNP consistent with the NGNP Point Design [MacDonald, 2003]. This single microsphere cell consists of a $10.36 \%$ enriched fuel microsphere and graphite matrix to yield a packing fraction $=0.289$ and explicitly models all regions: fuel kernel, carbon buffer, SiC, and inner/outer pyrolytic carbon shells and graphite matrix. Figure 6-1 depicts the geometry.

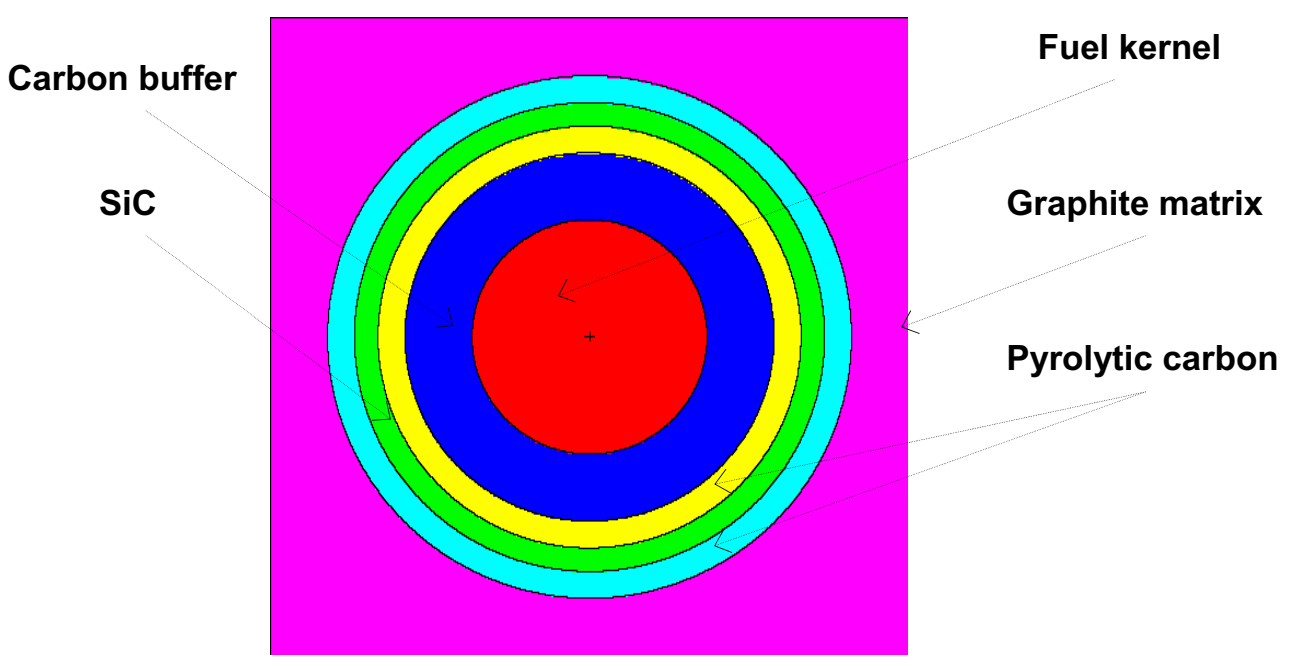

Figure 6-1. Heterogeneous Microsphere Cell for TRISO Fuel

Neutronic analyses were performed for a single microsphere fuel cell for three different models: homogeneous model (homogenized mixture of microsphere and graphite matrix), two-region model (fuel kernel and remainder of microsphere/graphite matrix), and a six-region model that explicitly represents the geometry shown in Figure 6-1. Figure 6-2 shows the MCNP5 geometries for these three models.

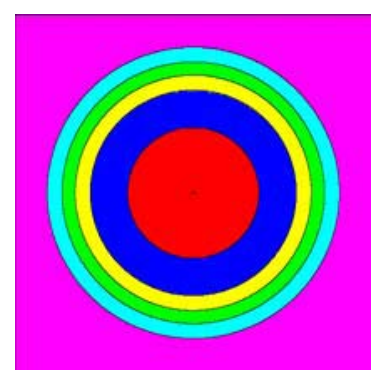

Six-region Heterogeneous

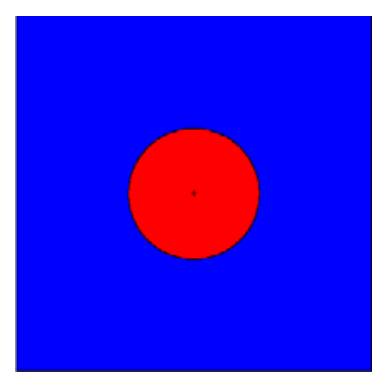

Two-Region Heterogeneous

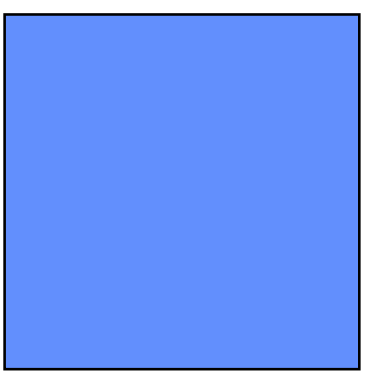

Homogeneous

Reflecting b.c. on all sides of cubes

Figure 6-2. Single Microsphere Cells 
For each of the models shown in Fig. 6-2, criticality calculations were performed and the results are given in Table 6-1 [Ji 2004]. It is clear that the fuel kernel heterogeneity must be included in the neutronic analysis as it represents a 5\% reactivity effect, and this does not include the other portion of the "double heterogeneity", the fuel compact heterogeneity.

The LANL calculation [Brown, 2004a; Brown, 2004b)] was performed by Dr. Forrest Brown of the MCNP

Table 6-1. MCNP5 Simulations of Microsphere Cells

\begin{tabular}{|l|c|c|c|}
\hline \multicolumn{1}{|c|}{ Configuration } & $\begin{array}{l}\text { Kernel } \\
\text { location }\end{array}$ & $\mathrm{k}_{\text {eff }}$ & Sigma \\
\hline Homogeneous cell & --- & 1.0995 & .0004 \\
\hline Two-region heterogeneous cell & Centered & 1.1535 & .0004 \\
\hline Six-region heterogeneous cell & Centered & 1.1533 & .0003 \\
\hline Six-region heterogeneous cell (LANL) & Random & 1.1515 & .0004 \\
\hline
\end{tabular}

Group in X-5 division. In Table 6-1, "Kernel location" refers to the location of the microsphere in the enclosing cube. "Centered" means the microsphere is centered in the cube (hence comprising a simple cubic lattice), whereas "Random" means the location of the microsphere was "jiggled" randomly every time the neutron entered (or re-entered) the cubical cell, with the constraint that the sphere could not overlap the boundary of the enclosing cube. Table 6-1 shows that the random location of the sphere within the cubical cell results in a $.2 \%$ decrease in $\mathrm{k}_{\mathrm{eff}}$ compared with the sphere centered in the cell.

The two-region cell gave nearly the same results as the six-region cell and since the latter case takes twice the computational time, this may warrant the use of the two-region cell for neutronic analyses of VHTR fuel. This is discussed further in the next paragraph.

To analyze the two-region model, we calculated the multi-group radial flux profiles for both two-region and six-region microsphere cells. Figure 6-3 shows the tally region divisions for the two models and Figure 6-4 depicts the scalar flux profiles for the fully heterogeneous model. Figure 6-5 compares the radial profiles for the two-region cell versus the heterogeneous cell for resonance energy neutrons in the energy range 6.57-6.77 $\mathrm{eV}$. This energy range was chosen to represent neutrons near a typical resonance, in this case the $6.67 \mathrm{eV}$ resonance in U-238.

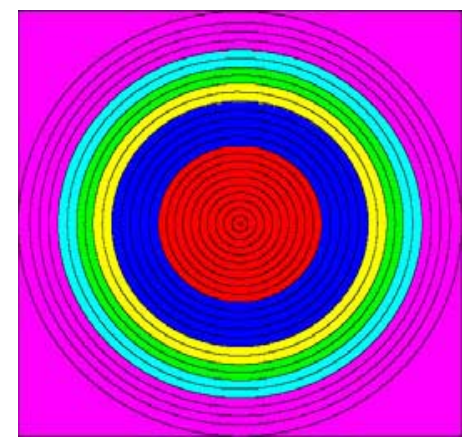

Six-Region Heterogeneous

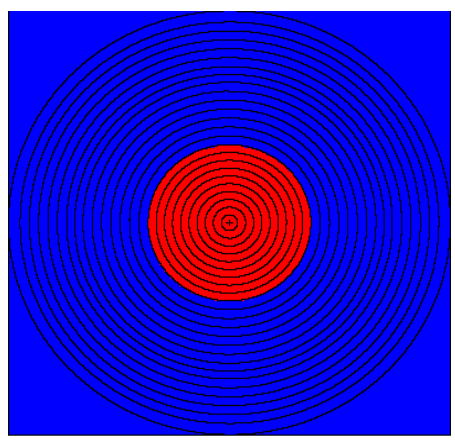

Two-Region Heterogeneous

Reflecting b.c. on all sides of cubes

Figure 6-3. Tally Regions for Two-Region Microsphere Models 


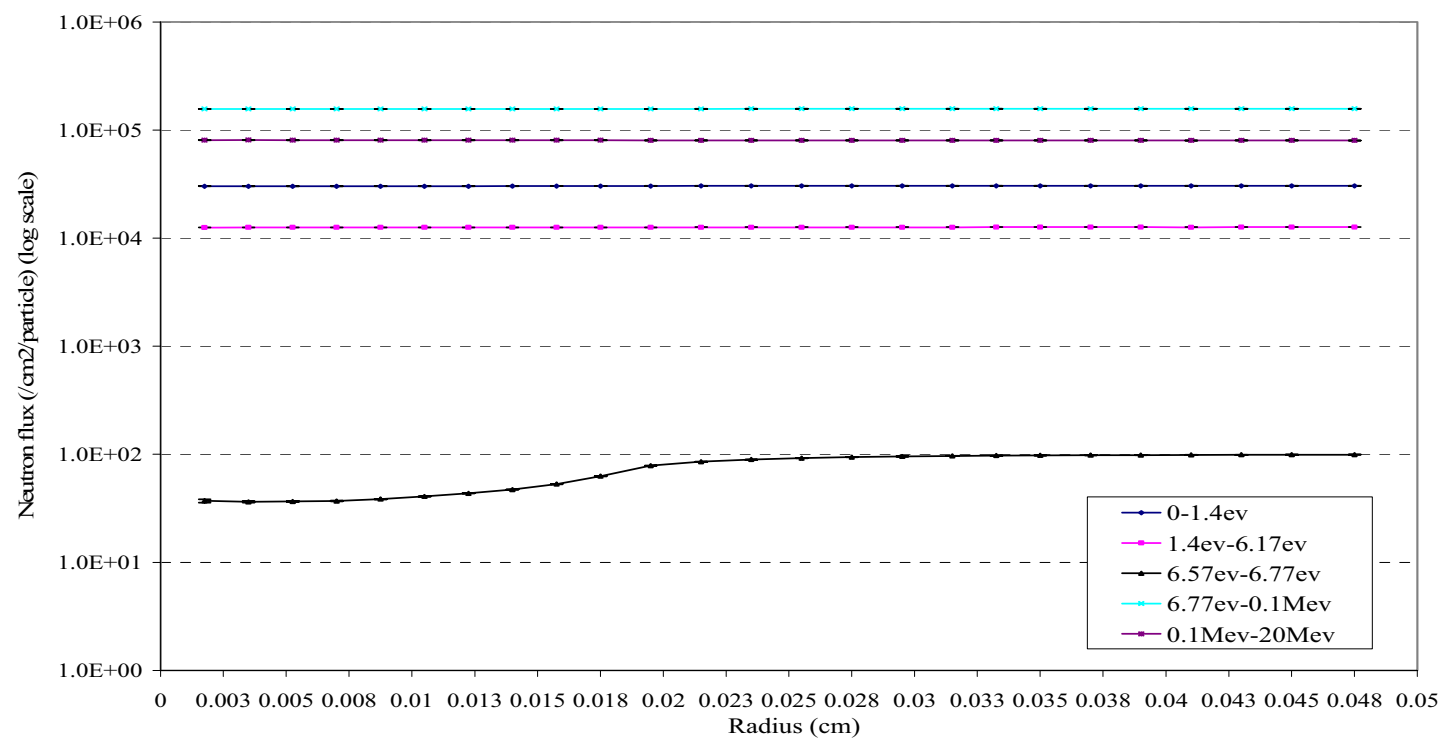

Figure 6-4. Energy-Dependent Radial Flux Profiles for Heterogeneous Microsphere Cell

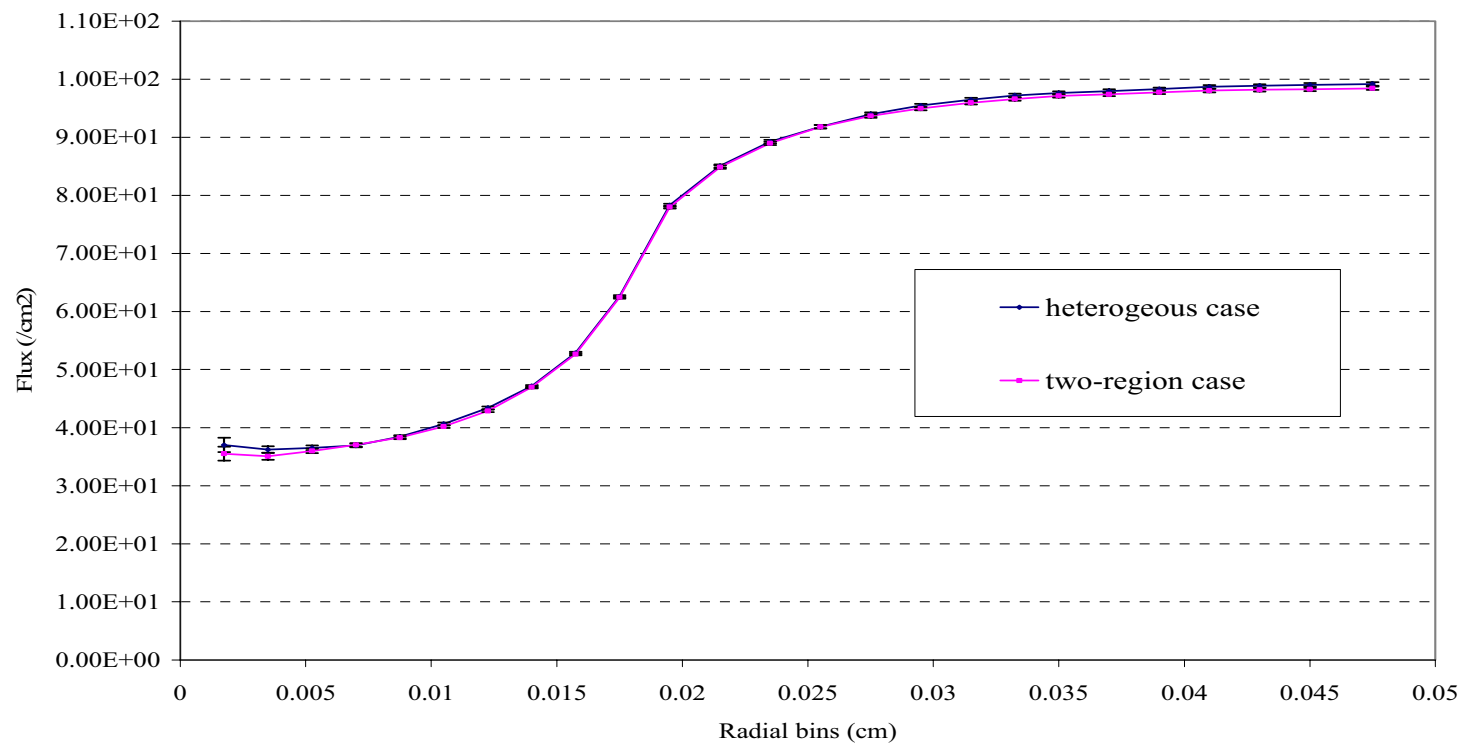

Figure 6-5. Comparison of 6.57-6.77 eV Flux Profiles

Energy spectra in the fully heterogeneous microsphere cell model were calculated. The calculations were done separately for all six regions of the cell depicted in Figure 6-1. Figure 6-6 shows the results and it can be seen that except for the neutron energies close to the resonance peaks, which Figure 6-6 does not resolve, the spectra are essentially the same in all regions of the microsphere cell. Together, Figures 6-4 and 6-6 indicate that the TRISO fuel is homogeneous for all neutrons except resonance energy neutrons. 


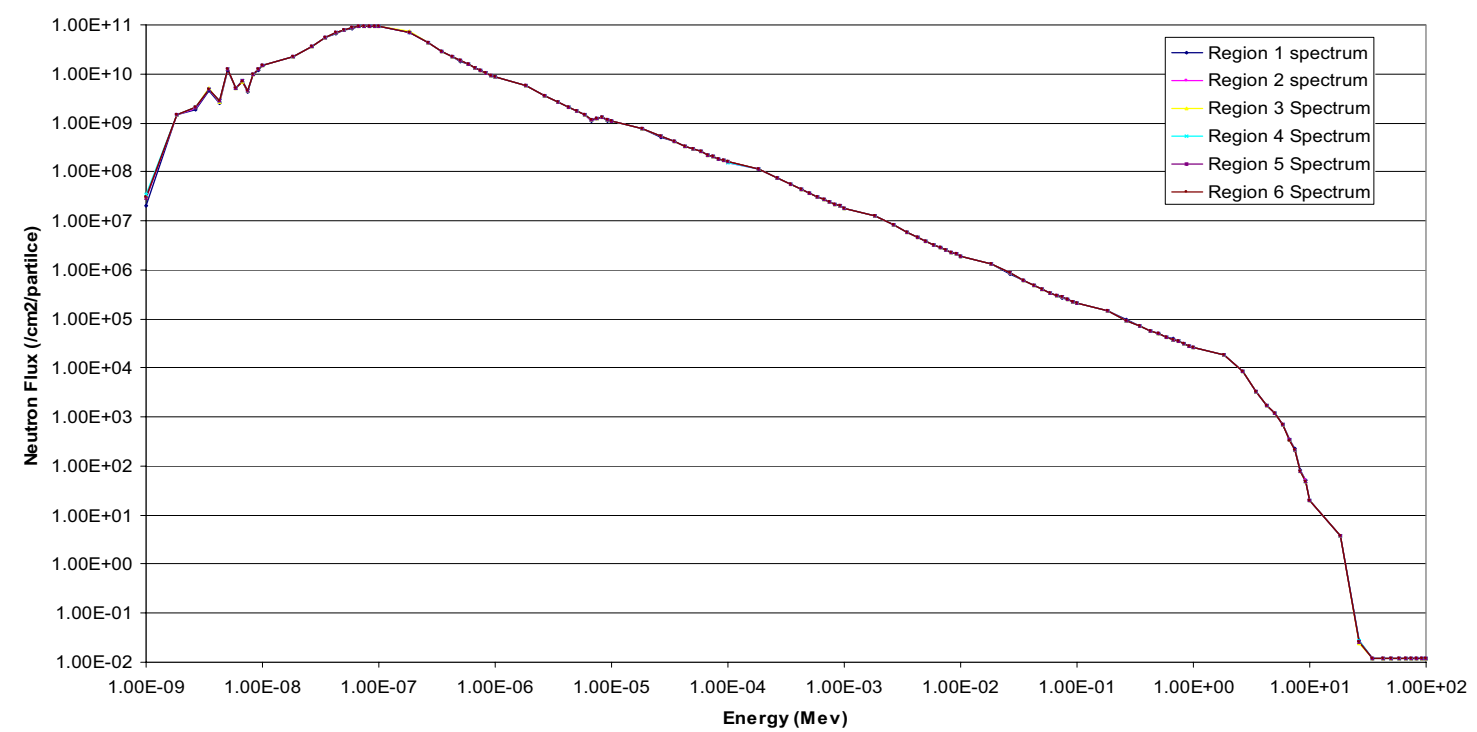

Figure 6-6. Energy-dependent Scalar Flux for the Six-region Microsphere Cell

\subsubsection{Fuel compact model}

The fuel compact geometry was modeled in three different ways: homogeneous, two-region heterogeneous and six-region heterogeneous. Fig. 6-7 shows the three different MCNP5 fuel compact models. The graphite region surrounding the fuel compact is the proportional share of the graphite in a hexagonal block that "belongs" to a fuel compact. Table 6-2 is the summary of the MCNP5 criticality calculations for these fuel compact cells. The results show that the fuel kernel heterogeneity is important at the fuel compact level, yielding a $4 \%$ increase in reactivity compared to a homogenized fuel region.

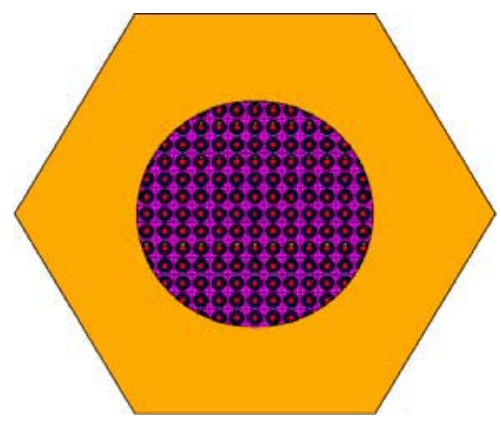

Six-reaion Heteroqeneous

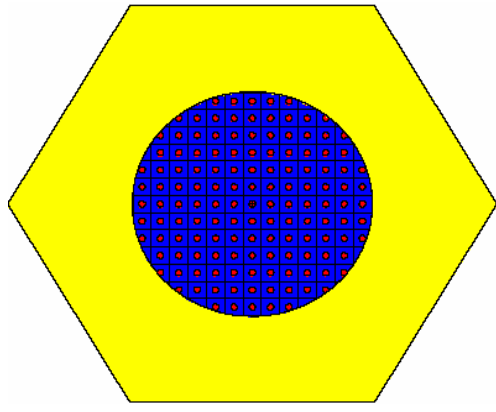

Two-Region Heterogeneous

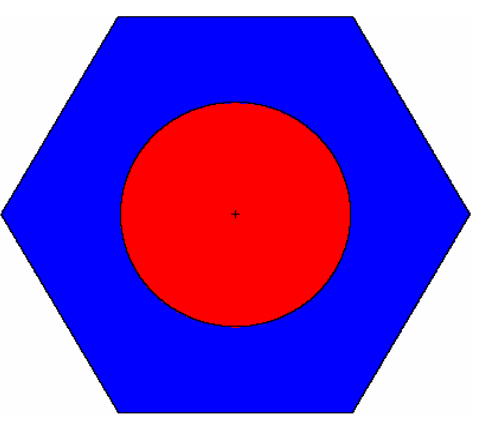

Homogeneous

Reflecting b.c. on all surfaces of fuel compact cell

Figure 6-7. Fuel Compact Cells 
Table 6-2. MCNP5 Simulations of Fuel Compact Cells

\begin{tabular}{|l|c|l|}
\hline \multicolumn{1}{|c|}{ Fuel Compact } & $\mathrm{k}_{\text {eff }}$ & Sigma \\
\hline Homogeneous microsphere cells & 1.2885 & .0004 \\
\hline Two-region heterogeneous microsphere cells & 1.3408 & .0004 \\
\hline Six-region heterogeneous microsphere cells & 1.3401 & .0004 \\
\hline
\end{tabular}

However, a closer look at the MCNP5 models of the heterogeneous microsphere cells in the fuel compact shows "clipped" or partial microspheres at the cylindrical boundary of the fuel compact. This is an artifact of the MCNP5 geometry handling routines for imbedding a universe (the lattice of microspheres) in an enclosing body (the outer diameter of the fuel compact). This unphysical anomaly is illustrated in Figure 6-8. Clipping may change the packing fraction; however, a more important consequence is the reduction in the Dancoff factor, since the escape probability from a partial fuel kernel will be significantly larger than for a full kernel. This will reduce the self-shielding of the fuel kernel and increase the resonance absorption, resulting in a decrease in $\mathrm{k}_{\text {eff. }}$ Even though this effect is due to only those kernels on the boundary of the fuel compact, this reactivity effect is noticeable as shown in Table 6-3.

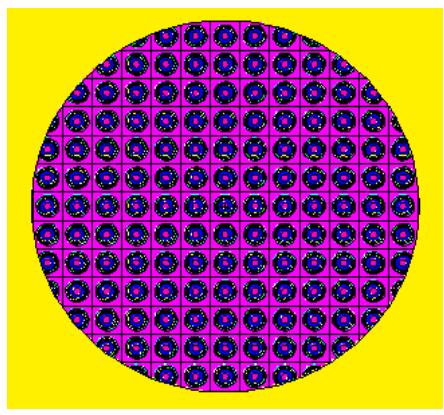

Figure 6-8. Clipped Model

In order to correct this error caused by clipping while keeping the total packing fraction constant, where the packing fraction is defined as the ratio of the total microsphere volume to the total volume of the fuel compact, two models were investigated. These models are depicted in Figure 6-9.

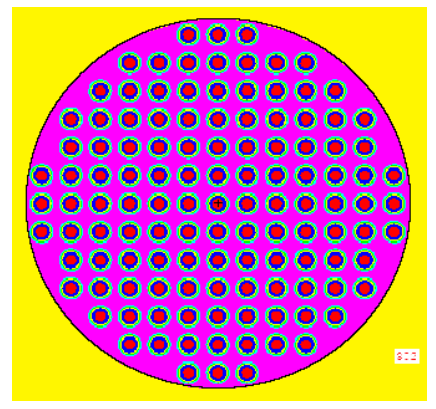

Unclipped Model (1)

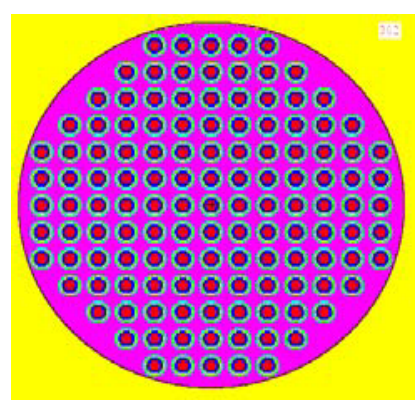

Unclipped Model (2)

Figure 6-9. Unclipped Models 
Both models still use simple cubic lattice structures but the lattice cells in the x-y plane are adjusted to avoid clipping. However, the cubic cells are different in the two models, although the packing fraction is preserved. In model 1, the $\mathrm{z}$-dimension of the "cubical" cell is reduced to preserve the overall packing fraction, but the $\mathrm{x}$ and y dimensions are left unchanged, resulting in a non-cubical cell (a cuboid), which is then repeated throughout the fuel compact cylinder to form a finite lattice of microspheres. For a packing fraction of 0.289 , this results in 121 microspheres in the $\mathrm{x}-\mathrm{y}$ plane for model 1. In model 2, the cube is uniformly squeezed in three dimensions to yield the desired packing fraction, and this cubical cell is then repeated throughout the fuel compact. There are 129 microspheres in the x-y plane for model 2. Neutronic results are shown in Table 6-3 for clipped cells and for unclipped cells using both models 1 and 2. Both models 1 and 2 yield $k_{\text {eff }}$ higher than with the clipped model, a consequence of the artificial reduction in the Dancoff factor for the clipped fuel kernels as noted above. Model 2 is preferred because it preserves a simple cubic lattice while maintaining the correct packing fraction.

Table 6-3. Clipped vs. Unclipped Fuel Compact Cells

\begin{tabular}{|l|l|l|}
\hline \multicolumn{1}{|c|}{ Fuel region modeled as } & $\mathrm{k}_{\text {eff }}$ & Sigma \\
\hline Six-region microsphere cells (clipped) & 1.3401 & .0004 \\
\hline Six-region microsphere cells (not clipped using model 1) & 1.3438 & .0002 \\
\hline Six-region microsphere cells (not clipped using model 2) & 1.3445 & .0002 \\
\hline & & \\
\hline Two-region microsphere cells (clipped) & 1.3408 & .0004 \\
\hline Two-region microsphere cells (not clipped using model 1) & 1.3435 & .0002 \\
\hline Two-region microsphere cells (not clipped using model 2) & 1.3447 & .0002 \\
\hline
\end{tabular}

A more realistic model of the stochastic mixture of microsphere particles has been examined, whereby microspheres are randomly distributed in the fuel compact. This was studied by using the RSA (Random Sequential Addition) method [Widom 1966] for both the six-region and two-region fuel compact models. This was done by taking a single fuel compact, which has an average of 6050 microspheres using a packing fraction of .289 , and subdividing it into 50 axial layers, each containing 121 microspheres. RSA was then used to insert the 121 microspheres randomly within each layer. Each layer was statistically different, so this may be described as a "stratified" RSA approach. An MCNP5 input deck was then written for the compact cell, explicitly accounting for the 6050 randomly placed microspheres. Fig. 6-10 illustrates the "stratified" RSA models and Table 6-4 compares two realizations of each of the two-region and six-region RSA models. 

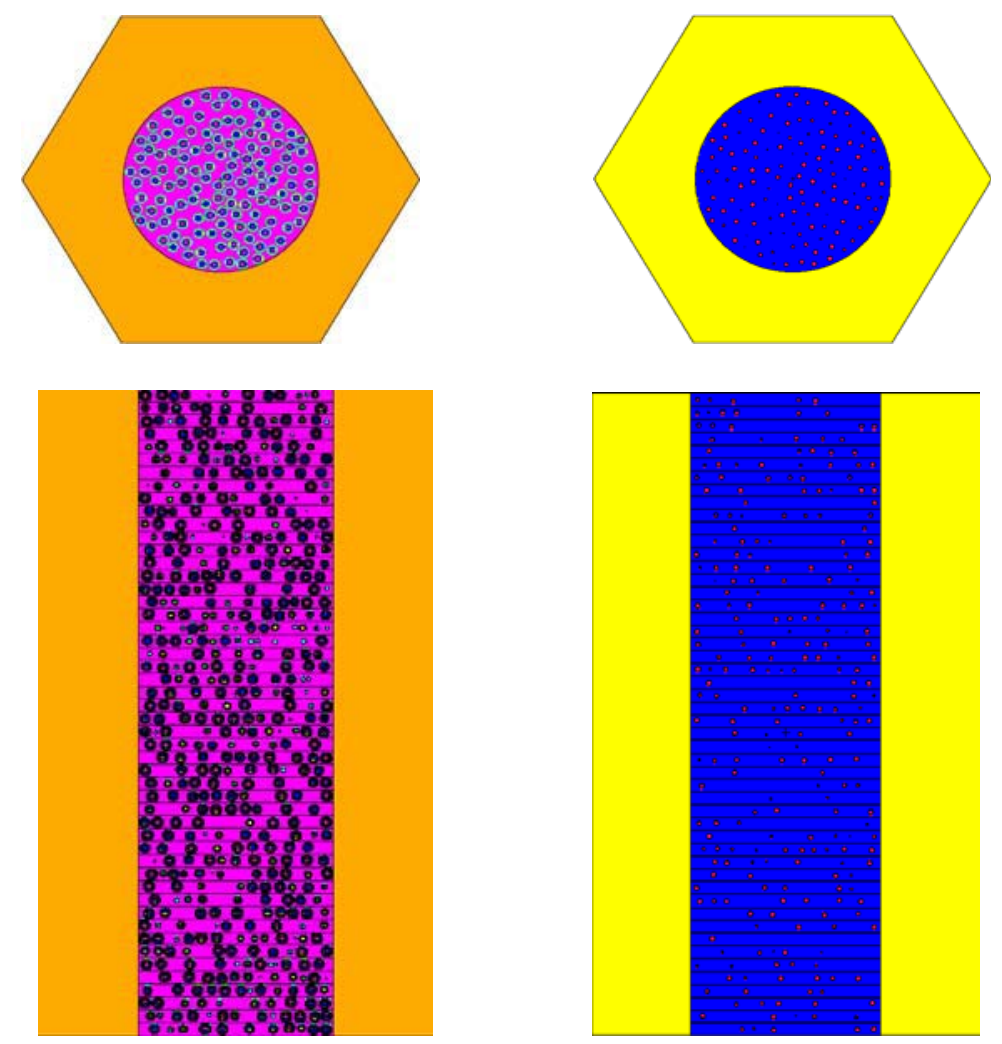

Figure 6-10. Two-region and Six-region RSA Models

Table 6-4. Fuel Compact Cells with Stratified RSA Models

\begin{tabular}{|c|c|c|}
\hline Fuel region modeled as 50 layers with & $\mathrm{k}_{\text {eff }}$ & Sigma \\
\hline Two-region RSA model (realization 1) & 1.34232 & .00023 \\
\hline Six-region RSA model (realization 1) & 1.34262 & .00022 \\
\hline & & \\
\hline Two-region RSA model (realization 2) & 1.34243 & .00022 \\
\hline Six-region RSA model (realization 2) & 1.34275 & .00022 \\
\hline
\end{tabular}

Table 6-4 shows the equivalence of the two-region and six-region RSA models. A comparison of Tables 6-3 and 6-4 show that the stochastic effect (RSA versus a lattice of kernels) is small: either two-region or sixregion RSA models yield $\sim 0.15 \%$ decreases in $\mathrm{k}_{\mathrm{eff}}$ compared to the corresponding lattice models. In order to investigate the axial effect of this stratified model, RSA was used to generate a more random distribution of two-region microsphere cells by using 10 axial layers rather than 50 layers, as shown in Fig. 6-11. The results are compared in Table 6-5, where it is seen that the stratified model with 10 layers yields essentially the same results (within $.02 \% \mathrm{k}_{\text {eff }}$ ) as the 50 layer case. 

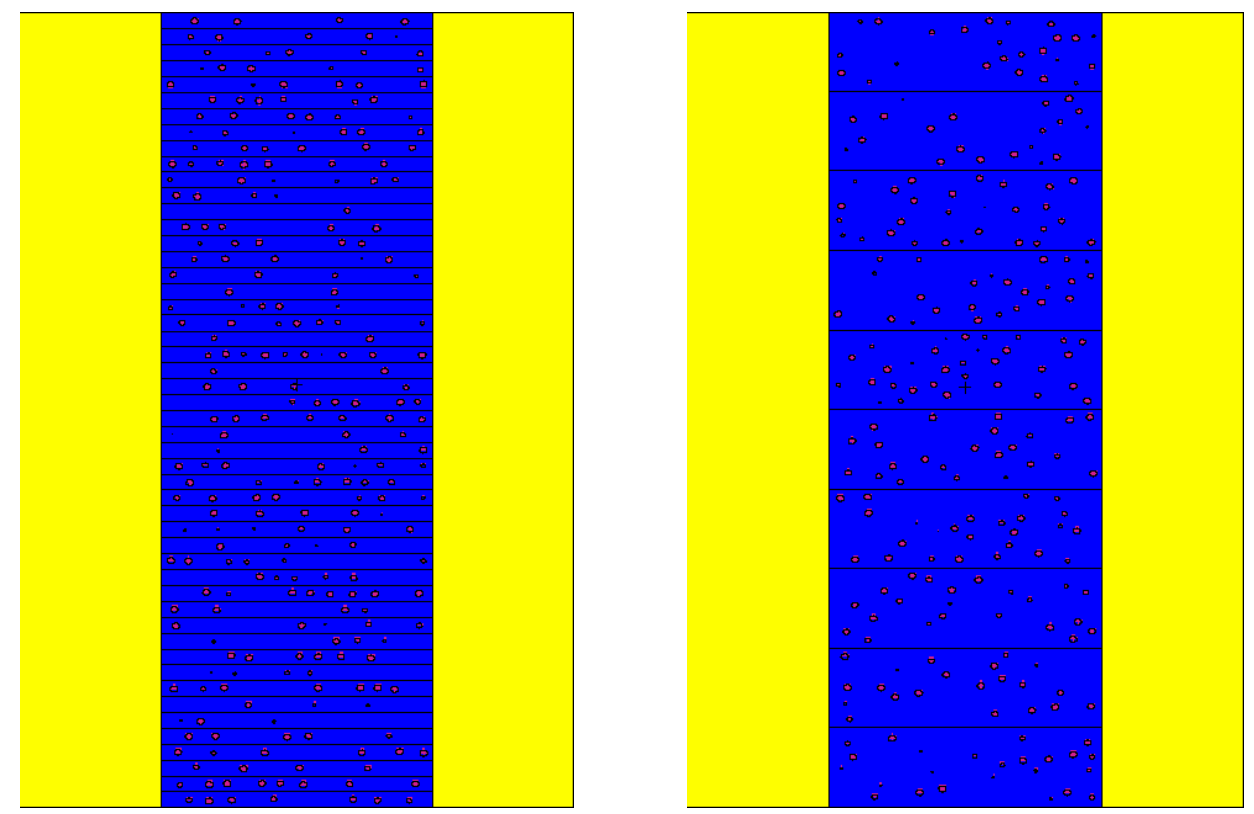

Figure 6-11. Fuel Compacts Cells Modeled with 50 RSA Layers and Ten RSA Layers

Table 6-5. Fuel Compacts Modeled with 50 RSA Layers vs. 10 RSA Layers

\begin{tabular}{|c|c|c|}
\hline Fuel region modeled as & $\mathrm{k}_{\mathrm{eff}}$ & Sigma \\
\hline $\begin{array}{c}\text { Two-region fifty-layer randomly distributed microsphere cells } \\
\text { (average over 19 realizations) }\end{array}$ & 1.34228 & .00019 \\
\hline $\begin{array}{c}\text { Two-region ten-layer randomly distributed microsphere cells (average } \\
\text { over 2 realizations) }\end{array}$ & 1.34258 & .00022 \\
\hline
\end{tabular}

We have also modeled hexagonal fuel block geometry, where coolant holes and fuel compact cells are modeled explicitly. These calculations have been done with homogeneous and heterogeneous fuel compacts but the results are not included in this report, since they are consistent with the fuel compact results and we also have full-core results to present.

\subsubsection{Full core model}

Based on the microsphere cell and fuel compact cell models, we have modeled the full core geometry with MCNP5. Figure 6-12 shows the model progression from microsphere cell to fuel compact cell to fuel block cell to full core geometry. 


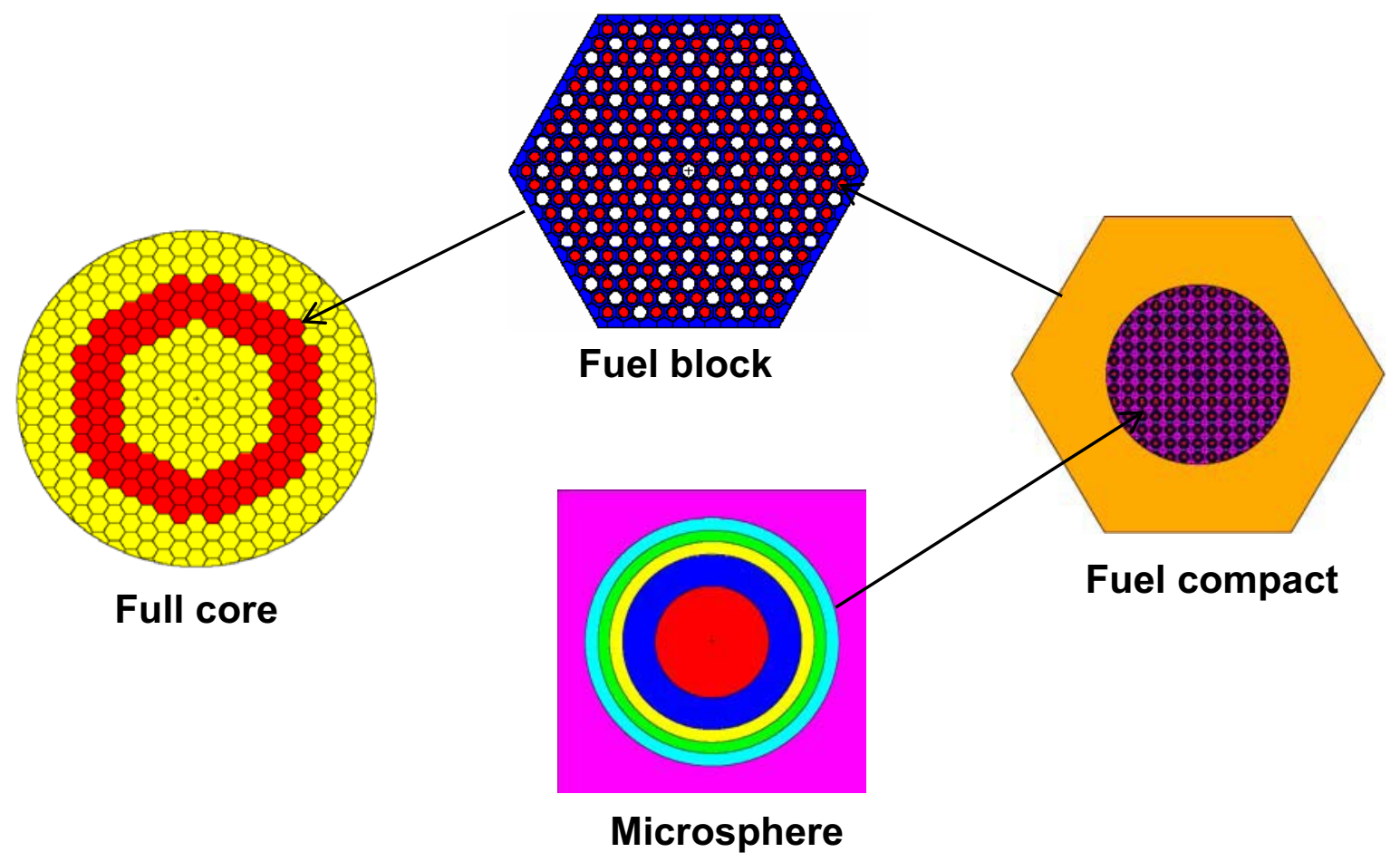

Figure 6-12. Modeling the Double Heterogeneity from Microsphere Cell to Full Core

Early calculations were performed with MCNP5 for four different full core configurations, modeling all fuel blocks and all axial and radial reflector blocks. These simulations assumed $6.69 \mathrm{ppm}$ boron-10 in the graphite fuel blocks and reflector blocks in accordance with the NGNP Point Design Report [MacDonald 2003]. This has since been determined to be $6.69 \mathrm{ppm}$ natural boron but we will present these results anyway, since the relative changes in $k_{\text {eff }}$ are of interest, not the actual value of $k_{\text {eff. }}$ For these cases, the fuel blocks were modeled as:

(1) homogeneous fuel blocks

(2) heterogeneous fuel blocks with homogeneous fuel

(3) heterogeneous fuel blocks with two-region heterogeneous fuel

(4) heterogeneous fuel blocks with six-region heterogeneous fuel .

Case (1) does not account for either portion of the double heterogeneity, while Case (2) only accounts for the fuel compact portion and Case (3) and (4) account for both the fuel compact and fuel kernel heterogeneities. Table 6-6 presents the results. These are preliminary calculations and need to be compared with other results, but the relative changes are interesting. 
Table 6-6. MCNP5 Simulations of Full Core Configurations

\begin{tabular}{|c|l|c|c|}
\hline Case & \multicolumn{1}{|c|}{ Full core modeled with: } & $\mathbf{k}_{\text {eff }}$ & Sigma \\
\hline 1 & Homogeneous fuel blocks & 1.0153 & .0002 \\
\hline 2 & Heterogeneous fuel blocks with homogeneous fuel regions & 1.0583 & .0001 \\
\hline & & & \\
\hline & Heterogeneous fuel blocks with fuel regions modeled as: & & \\
\hline 3 & Six-region microsphere cells (clipped) & 1.0949 & .0002 \\
\hline 4 & Six-region microsphere cells (not clipped using model 1) & 1.0957 & .0002 \\
\hline 5 & Six-region microsphere cells (not clipped using model 2) & 1.0966 & .0002 \\
\hline & & & .0002 \\
\hline 6 & Two-region microsphere cells (clipped) & 1.0952 & .0002 \\
\hline 7 & Two-region microsphere cells (not clipped using model 1) & 1.0959 & .0002 \\
\hline 8 & Two-region microsphere cells (not clipped using model 2) & 1.0965 & .0002 \\
\hline 9 & Two-region randomly distributed microsphere cells (RSA) & 1.0948 & .0002 \\
\hline
\end{tabular}

On the basis of the results, it is evident that modeling the double heterogeneity is a necessity for full core VHTR analysis. The effect of the first heterogeneity (fuel compact heterogeneity) is seen to be $4 \%$ by comparing Cases 1 and 2 in Table 6-6. The effect of the second heterogeneity (particle fuel heterogeneity) is seen to be another $4 \%$ by comparing Cases 2 and 4 .

The results given in Table 6-6 corroborate the results for the fuel compact cell cases given in Table 6-3, showing that the two-region cells are adequate for full-core configurations. This can be seen by comparison of Cases 4 and 7 or 5 and 8 . Therefore, the two-region microsphere cell gives acceptable results $(<.02 \%)$ for all comparisons, from microsphere cell to full core. In addition to speeding up the MCNP5 calculations by a factor of two, this allows the analysis of larger systems due to the MCNP5 limit on the number of regions. This may be a limiting factor for full core depletion cases.

A comparison of Cases 3 and 4 or 6 and 7 show that clipping the microsphere cells is less significant for full core $(\sim 0.1 \%)$ than for the fuel compact cell $(\sim 0.3 \%)$. This is consistent with previous observations regarding the effect of the double heterogeneity for finite geometries versus infinite geometries, because the finite geometries include the graphite reflectors and the increased moderation reduces the impact of the change in resonance absorption due to the particle fuel. Since it is easy to do so, clipping the cells should be avoided to eliminate the resultant unphysical geometry.

A comparison of Cases 7 or 8 with Case 9 shows that the effect of modeling the stochastic distribution of fuel particles with a lattice of fuel particles results in $\sim 0.15 \%$ reactivity effect for a full core simulation. This is consistent with the fuel compact results, where the reactivity effect was $\sim 0.17 \%$ from a comparison of the unclipped and RSA two-region models given in Tables 6-3 and 6-4.

The effect of fuel temperature feedback was also examined, which is important for the VHTR since it is operated at very high temperatures. Using the coupled thermal/hydraulic calculation results discussed in Section 6-3 below, the temperature distribution throughout the reactor core was obtained and shown in Table 6-7. MCNP5 was then run with the fuel regions modeled at the temperatures given in Table 6-7 and the results are in Table 6-8. For these cases, the boron impurity in graphite was assumed to be $6.69 \mathrm{ppm}$ of natural boron in graphite. 
Table 6-7. Temperature Distribution in the VHTR

\begin{tabular}{|c|c|c|c|c|}
\hline Block \# & Height (m) & Inner Ring (K) & Middle Ring (K) & Outer Ring (K) \\
\hline 11 & 9.12 & 785 & 788 & 788 \\
\hline 10 & 8.33 & 895 & 890 & 882 \\
\hline 9 & 7.53 & 992 & 980 & 965 \\
\hline 8 & 6.74 & 1076 & 1058 & 1036 \\
\hline 7 & 5.95 & 1146 & 1122 & 1096 \\
\hline 6 & 5.15 & 1204 & 1175 & 1145 \\
\hline 5 & 4.36 & 1248 & 1215 & 1182 \\
\hline 4 & 3.57 & 1279 & 1243 & 1208 \\
\hline 3 & 2.78 & 1297 & 1258 & 1222 \\
\hline 2 & 1.98 & 1302 & 1260 & 1225 \\
\hline 1 & 1.19 & 1294 & 1251 & 1216 \\
\hline 0 & 0.40 & 1273 & 1228 & 1196 \\
\hline
\end{tabular}

Table 6-8. MCNP5 Full Core Simulations with Heterogeneous Fuel Blocks

\begin{tabular}{|l|c|c|}
\hline $\begin{array}{c}\text { Heterogeneous fuel blocks with fuel regions modeled as } \\
\text { six-region microsphere cells (not clipped using model 2) }\end{array}$ & $\mathbf{k}_{\text {eff }}$ & Sigma \\
\hline Room temperature & 1.3190 & .0002 \\
\hline Uniform temperature at 1020K & 1.2714 & .0001 \\
\hline Distributed temperature in active core and 1083K in reflectors & 1.2608 & .0001 \\
\hline Distributed temperature in active core and in reflectors & 1.2606 & .0001 \\
\hline & & \\
\hline $\begin{array}{c}\text { Heterogeneous fuel blocks with fuel regions modeled as } \\
\text { two -region microsphere cells (not clipped using model 2) }\end{array}$ & & \\
\hline Room temperature & 1.3187 & .0002 \\
\hline Distributed temperature in active core and in reflectors & 1.2622 & .0001 \\
\hline
\end{tabular}

From room temperature to high temperature calculations, there is a $5 \%$ change in $\mathrm{k}_{\text {eff. }}$ Both six-region and two-region models consistently reflect this large effect. This is due to the Doppler broadening of the resonance capture cross sections of the fuel. Also, Table 6-8 indicates that a uniform temperature distribution in the fuel region yields a $\mathrm{k}_{\text {eff }}$ that is higher by $1 \%$ than the distributed temperature case with the same average temperature. Therefore, it is important to predict an accurate temperature distribution in the VHTR to get accurate simulation results.

Depletion analysis is another important issue that needs to be accounted for in the full core simulation. Depletion calculations have been performed for full core models at room temperature and elevated temperatures. Figure 6-13 compares the predicted $\mathrm{k}_{\mathrm{eff}}$ rundown for room temperature and elevated temperature cases with heterogeneous fuel blocks with six-region microsphere cells. Figure 6-14 compares the reactivity rundown for six-region vs. two-region full-core models at room temperature, and the difference is negligible. 


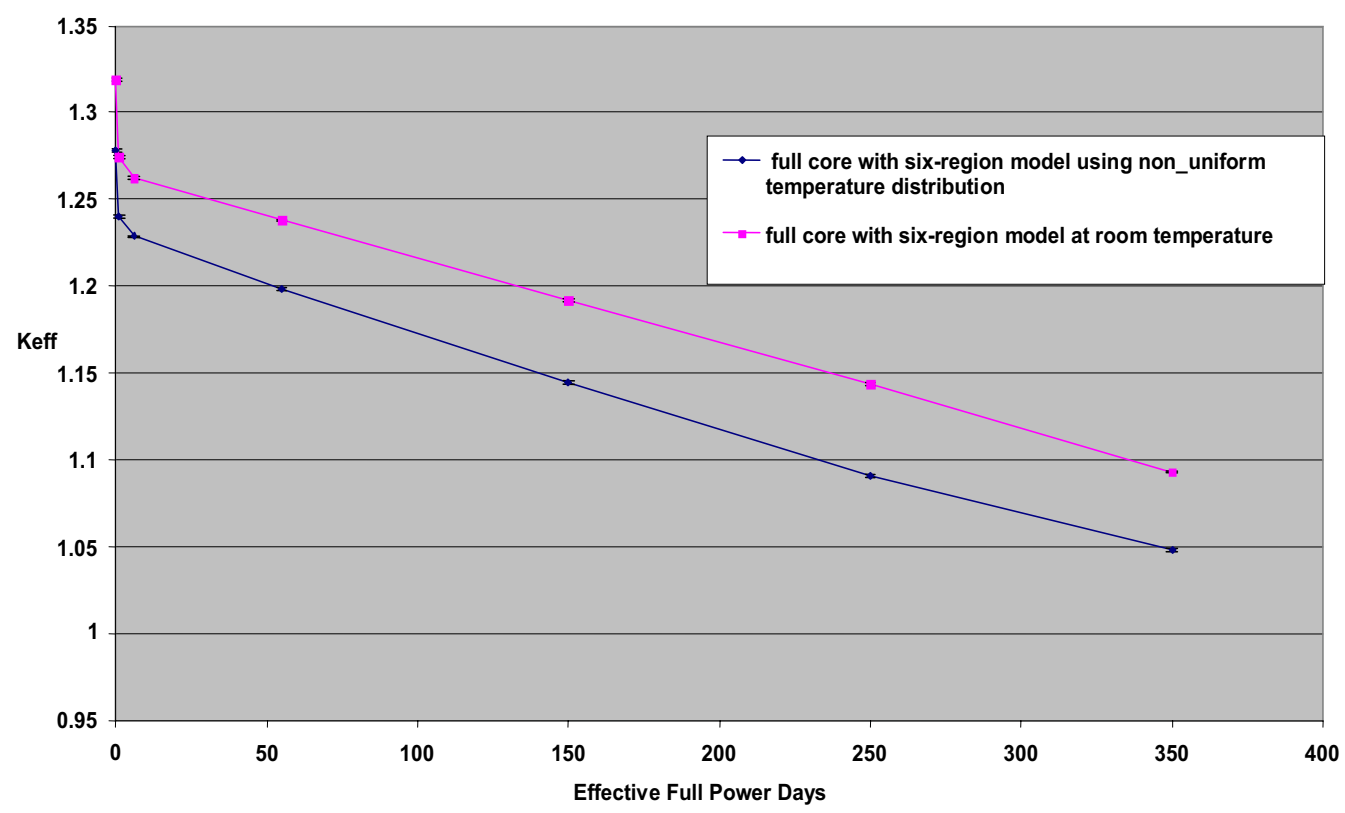

Figure 6-13. Depletion Comparison: Room Temperature vs. Distributed Temperature

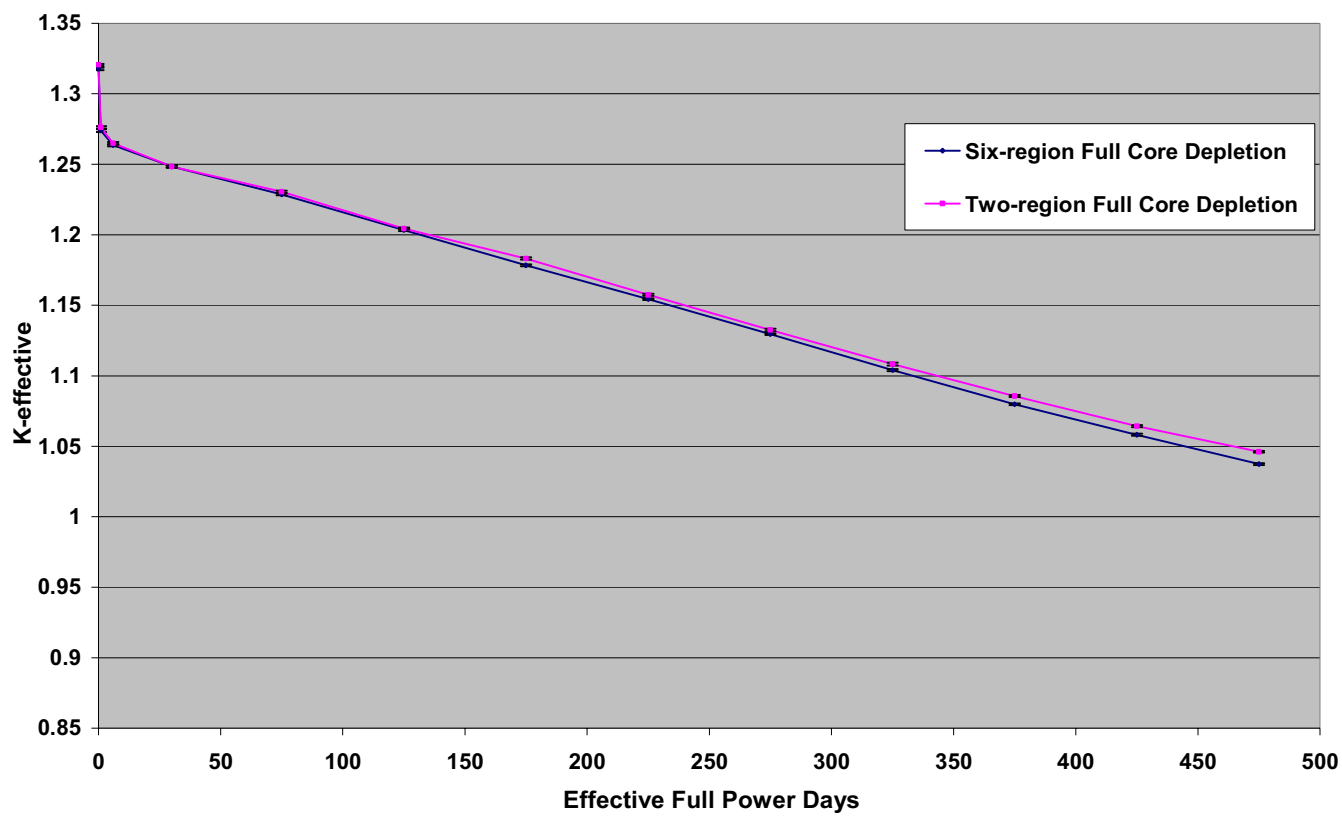

Figure 6-14. Depletion Comparison: Two-region vs. Six-region Models 


\subsection{Task 5-2: Coupled Nuclear-Thermal-Hydraulic Calculations}

Coupled nuclear-thermal-hydraulic (NTH) calculations have been performed representing the effects of temperature feedback on global power distributions. This was done by coupling MCNP5 power distribution calculations with thermal-hydraulic calculations performed with the RELAP5-3D code [Athena 2003] in an iterative scheme.

The MCNP5 calculation used 102 homogenized prismatic fuel assemblies to represent the VHTR core. These were grouped into inner, middle, and outer rings comprising 30, 36, and 36 assemblies, respectively. The VHTR core with an active core height of $7.93 \mathrm{~m}$ was divided into 12 temperature regions for each of the three radial rings for a total of 36 segments. For the purpose of MCNP5 calculations, axial temperature distributions for the inner and outer reflector are assumed the same as the inner and outer fuel ring, respectively.

The RELAP5-3D model [Athena 2003] for the VHTR represents each of the three radial rings of the core as a cylindrical assembly comprising four annular fuel regions surrounded by three inner graphite rings and one outer graphite ring, with a He coolant channel at the center of the assembly. The varying numbers of fuel assemblies in the three core rings are represented through properly scaling the heat structure lengths for the rings. The outer surface of the outer graphite region of the cylindrical assembly is represented as an adiabatic surface. The radial temperatures calculated for the eight radial rings in each of the 36 axial segments are volume-averaged to determine the temperature distribution for the 36 homogenized core volumes in the MCNP5 representation of the VHTR.

MCNP5 cases were set up to calculate a power distribution given a temperature distribution for the core. The RELAP5-3D model accepts a power distribution and outputs a temperature distribution. A cosine shaped power distribution was used as the initial input to RELAP5-3D. The coupling of MCNP5 and RELAP5-3D was achieved through the use of a Python script. The RELAP5-3D temperature distributions for the 36 fuel segments were read by the script, which created an MCNP5 input file representing the distributed temperatures for the core. The MCNP5 power distributions were then input to RELAP5-3D and the NTH iteration repeated until convergence on power and temperature distributions was achieved.

Plots of the power and temperature distributions for each coupled NTH iteration are shown in Figs. 6-15 and 6-16, for the middle core ring. It is clear that, after four NTH iterations, the power and temperature distributions for the middle ring nearly converged, with similar results for the inner and outer rings. The iteration 3 temperature distributions were used for the fuel cycle analysis for Task 5-3. 


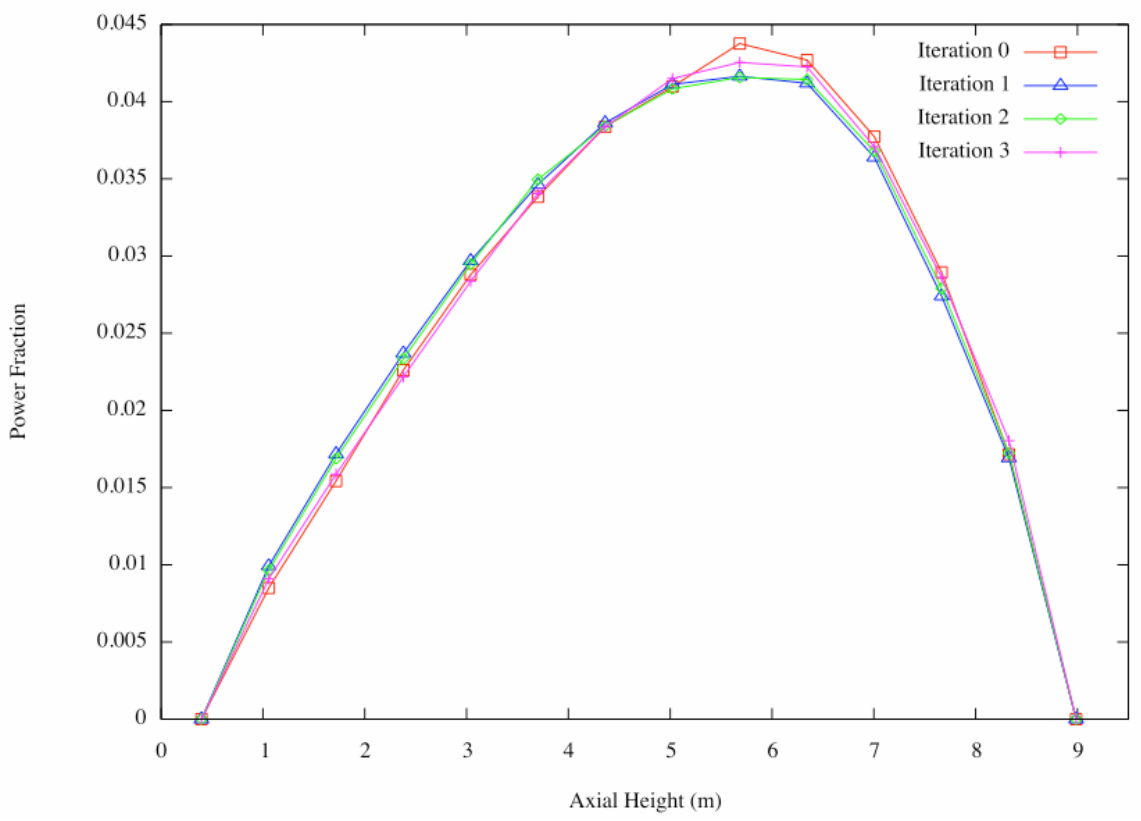

Figure 6-15. Iterations on Axial Power Distribution in the Middle Core Ring

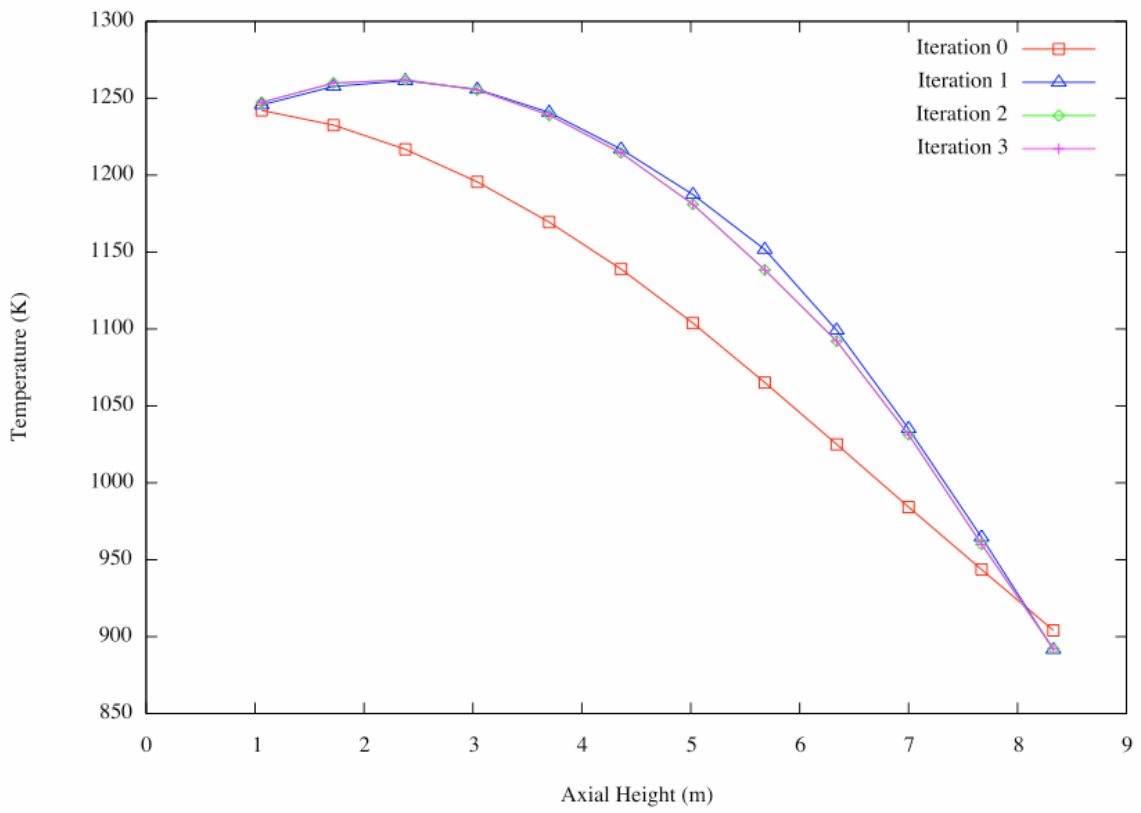

Figure 6-16. Iterations on Temperature Distribution in the Middle Core Ring

The MCNP5 cross section data are usually limited in the range of temperatures provided by the standard MCNP5 distribution. Even for key uranium isotopes, ${ }^{235} \mathrm{U}$ and ${ }^{238} \mathrm{U}$, the cross section library is provided for only 12 discrete temperatures and for most other nuclides the cross section data are provided only at room temperature. In order to represent distributed temperatures explicitly in our coupled NTH calculations, we have developed a pseudo material construct [Conlin 2005] that allows us to use a cross section library at any desired temperature without the need to generate numerous new cross section libraries. 
A pseudo material uses two cross section libraries for a nuclide with two different temperatures, $T_{L}$ and $T_{H}$, bracketing temperature $T$ for which a cross section library is desired. The MCNP5 cross section library for $T$ is obtained as a weighted average of two libraries at $T_{L}$ and $T_{H}$. Since, for each nuclide defined in an MCNP5 material, a number density is required, we assign, in the pseudo material construct, a fraction of this number density to each of the two libraries. The actual interpolation of the cross section libraries is performed with the observation that the resonance integral for fuel material typically exhibits the Doppler broadening effects proportional to $\sqrt{T}$. Thus the weighting factor for the library at $T_{L}$ is calculated as

$$
f_{L}=\frac{\sqrt{T_{H}}-\sqrt{T}}{\sqrt{T_{H}}-\sqrt{T_{L}}},
$$

with the corresponding weighting factor for $T_{H}$

$$
f_{H}=1-f_{L} .
$$

The fractions $f_{L}$ and $f_{H}$ are used, with the libraries generated for $T_{L}$ and $T_{H}$, in lieu of a single number density and library to represent the temperature $T$ of interest. We have tested the pseudo material construct by running MCNP5 simulations of the VHTR at different temperatures for the whole core, with normal materials and pseudo materials. A comparison of effective multiplication factor $k_{\text {eff calculated }}$ with both approaches in Fig. 6-17 indicates that the pseudo material construct provides sufficiently accurate representation of Doppler broadening effects for VHTR calculations in general.

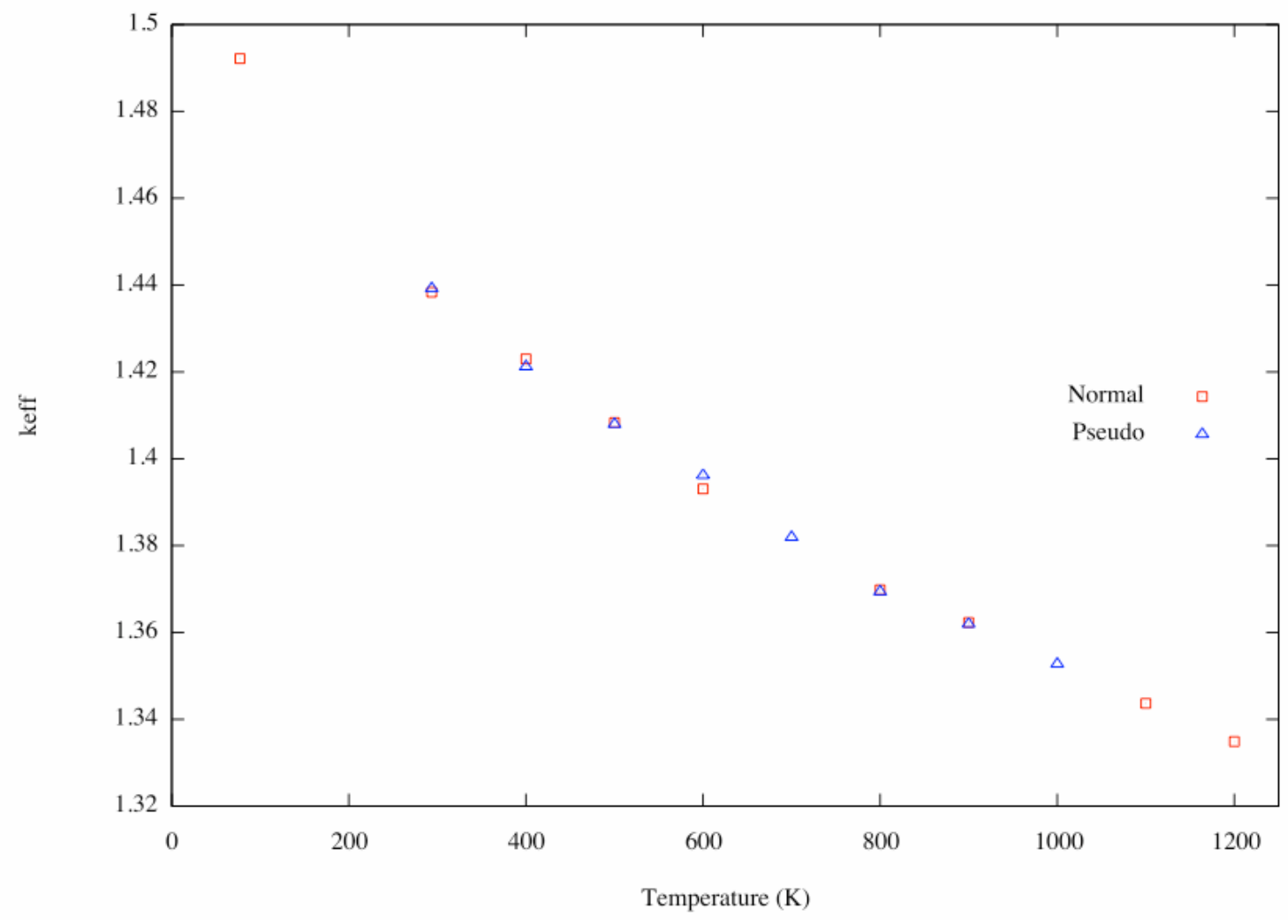

Figure 6-17. Comparison of $k_{\text {eff }}$ Calculated with Normal Materials and Pseudo Materials 


\subsection{Task 5-3: Fission Product Decay Heat Calculations}

As our final task to determine the fission product (FP) decay heat deposition rates in the VHTR core following shutdown, we have performed Monteburns calculations representing the VHTR operation at rated power of $600 \mathrm{MWt}$ for a fuel cycle length of 12 months. The Monteburns code [Poston 1999] links MCNP5 with the ORIGEN2.2 point isotopic depletion code [Ludwig 2002] to perform Monte Carlo fuel depletion calculations. The Monteburns code passes space-dependent MCNP5 flux and power tallies to ORIGEN2.2, which then performs isotopic depletion calculations for each point or region as appropriate and returns the isotopic concentrations for fuel and FP nuclides for subsequent MCNP5 calculations. Through an iterative use of MCNP5 and ORIGEN2.2, full-core fuel depletion and FP buildup are determined.

For our Monteburns calculations, a MCNP5 input deck was set up to represent the VHTR core with homogenized fuel assemblies, grouped into three annular rings, each ring comprising 10 axial fuel segments. The radial rings represent clusters of 30, 36, and 36 fuel assemblies, respectively, for the inner, middle, and outer core rings. Axial temperature distributions were determined for 12 axial zones for each of the three rings through RELAP5-3D calculations at the beginning of cycle (BOC) as part of Task 5-2. The temperature distributions are interpolated here for the configuration of 10 axial segments per ring, plus two reflector segments, and are tabulated in Fig. 6-18. For a simplified representation of graphite reflectors surrounding the core, we have used a core-average temperature of $1020 \mathrm{~K}$ for the entire reflector region. Furthermore, although the power distribution evolves as a function of fuel burnup, we have assumed the same BOC temperature distributions throughout the cycle. To represent distributed temperatures for the core and reflectors, we used the pseudo material construct developed in Task 5-2 and performed interpolations of cross section libraries generated at a few temperature points by the DOPPLER code [MacFarlane 2003]. We also made a modification to the ORIGEN2.2 code to be able to read in nuclide identification numbers corresponding to the distributed temperatures. For a full representation of fuel depletion and FP buildup, we have tracked 18 fuel nuclides and 81 fission products, tabulated in Table 6-9, in each of the 30 fuel regions.

\begin{tabular}{|c|c|c|c|c|}
\multicolumn{1}{|c}{ Inner Reflector } & Inner Ring & \multicolumn{1}{c}{ Middle Ring } & Outer Ring & Outer Reflector \\
\hline $1020 \mathrm{~K}$ & $1020 \mathrm{~K}$ & $1020 \mathrm{~K}$ & $1020 \mathrm{~K}$ & $1020 \mathrm{~K}$ \\
\hline $1020 \mathrm{~K}$ & $889 \mathrm{~K}$ & $891 \mathrm{~K}$ & $882 \mathrm{~K}$ & $1020 \mathrm{~K}$ \\
\hline $1020 \mathrm{~K}$ & $993 \mathrm{~K}$ & $981 \mathrm{~K}$ & $965 \mathrm{~K}$ & $1020 \mathrm{~K}$ \\
\hline $1020 \mathrm{~K}$ & $1076 \mathrm{~K}$ & $1058 \mathrm{~K}$ & $1037 \mathrm{~K}$ & $1020 \mathrm{~K}$ \\
\hline $1020 \mathrm{~K}$ & $1147 \mathrm{~K}$ & $1123 \mathrm{~K}$ & $1097 \mathrm{~K}$ & $1020 \mathrm{~K}$ \\
\hline $1020 \mathrm{~K}$ & $1204 \mathrm{~K}$ & $1175 \mathrm{~K}$ & $1145 \mathrm{~K}$ & $1020 \mathrm{~K}$ \\
\hline $1020 \mathrm{~K}$ & $1248 \mathrm{~K}$ & $1215 \mathrm{~K}$ & $1183 \mathrm{~K}$ & $1020 \mathrm{~K}$ \\
\hline $1020 \mathrm{~K}$ & $1280 \mathrm{~K}$ & $1243 \mathrm{~K}$ & $1208 \mathrm{~K}$ & $1020 \mathrm{~K}$ \\
\hline $1020 \mathrm{~K}$ & $1298 \mathrm{~K}$ & $1258 \mathrm{~K}$ & $1222 \mathrm{~K}$ & $1020 \mathrm{~K}$ \\
\hline $1020 \mathrm{~K}$ & $1303 \mathrm{~K}$ & $1261 \mathrm{~K}$ & $1225 \mathrm{~K}$ & $1020 \mathrm{~K}$ \\
\hline $1020 \mathrm{~K}$ & $1020 \mathrm{~K}$ & $1251 \mathrm{~K}$ & $1216 \mathrm{~K}$ & $1020 \mathrm{~K}$ \\
\hline & & $1020 \mathrm{~K}$ & $1020 \mathrm{~K}$ & $1020 \mathrm{~K}$ \\
\hline
\end{tabular}

Figure 6-18. Temperature Distribution for 30 Fuel Regions and Reflectors

We performed Monteburns calculations at rated power of $600 \mathrm{MWt}$ for a 12-month cycle using 12 fuel depletion steps. Assembly-wise axial power distributions for the three fuel rings plotted for BOC in Fig. 6-20 and for the end of cycle (EOC) in Fig. 6-21 indicate a significant evolution of the power distributions during the cycle. Beginning with a slight asymmetry in the axial power distributions due to the distributed axial temperatures at BOC, space-dependent fuel depletion and FP buildup causes a substantial flattening of the axial power distributions. 
This depletion run was a preliminary calculation to get an approximate estimate of the decay heat distribution in the VHTR at EOC for the purpose of this report. It utilized a non-uniform temperature distribution from the BOC thermal-hydraulic analysis discussed in Section 6.3 and this temperature distribution was used for the entire depletion. Also, the neutronic model assumed homogeneous fuel blocks, hence did not account for the double heterogeneity of the TRISO fuel. As we have seen, the double heterogeneity has a reactivity effect on the order of $8 \%$, which is consistent with the results discussed below. These approximations will be removed in our final full-core depletion runs that will be used for the final prediction of the decay heat deposition in the VHTR.

Table 6-9. List of Fuel and FP Nuclides Tracked in Monteburns Calculations

\begin{tabular}{|c|c|c|c|c|c|c|c|c|c|}
\hline \multicolumn{10}{|c|}{ Fission products } \\
\hline $36-\mathrm{Kr}-78$ & 80 & & & & & & & & \\
\hline $37-R b-85$ & 87 & & & & & & & & \\
\hline $38-\mathrm{Sr}-84$ & 86 & 87 & 88 & 89 & 90 & & & & \\
\hline $39-Y-89$ & & & & & & & & & \\
\hline $40-Z r-90$ & 91 & 92 & 93 & 94 & 96 & & & & \\
\hline $41-\mathrm{Nb}-93$ & & & & & & & & & \\
\hline 42-Mo- 95 & & & & & & & & & \\
\hline 43-Тc- 99 & & & & & & & & & \\
\hline 44-Ru- 101 & 103 & & & & & & & & \\
\hline 45-Rh-103 & 105 & & & & & & & & \\
\hline $46-\mathrm{Pd}-105$ & 108 & & & & & & & & \\
\hline $47-\mathrm{Ag}-107$ & 109 & & & & & & & & \\
\hline $48-\mathrm{Cd}-106$ & 108 & 110 & 111 & 112 & 113 & 114 & & & \\
\hline 53-I -127 & 129 & 135 & & & & & & & \\
\hline $54-X e-124$ & 126 & 128 & 129 & 130 & 131 & 132 & 134 & 135 & 136 \\
\hline $55-C s-133$ & 134 & 135 & 136 & 137 & & & & & \\
\hline 56-Ba-138 & & & & & & & & & \\
\hline 59-Pr-141 & & & & & & & & & \\
\hline $60-\mathrm{Nd}-143$ & 145 & 147 & 148 & & & & & & \\
\hline 61-Pm-147 & 148 & 149 & & & & & & & \\
\hline 62-Sm-147 & 149 & 150 & 151 & 15 & & & & & \\
\hline 63-Eu-151 & 152 & 153 & 154 & 15 & & & & & \\
\hline 64-Gd-152 & 154 & 155 & 156 & 15 & 158 & 160 & & & \\
\hline 67-Нo-165 & & & & & & & & & \\
\hline
\end{tabular}

\begin{tabular}{|l|l|l|l|l|}
\hline \multicolumn{5}{|c|}{ Fuel nuclides } \\
\hline 92-U-234 & 235 & 237 & 238 & 239 \\
\hline 93-Np-237 & 238 & 239 & & \\
\hline 94-Pu-238 & 239 & 240 & 241 & 242 \\
\hline 95-Am-241 & 243 & & & \\
\hline 96-Cm243 & 244 & 245 & & \\
\hline
\end{tabular}

Our preliminary prediction of the decay heat deposition distribution is based on this approximate full core depletion run. Another simplifying assumption we are making is that the decay heat deposition has the same spatial distribution as the EOC power distribution, modulated in time by a time-dependent factor that accounts for the beta and gamma decay of the fission products and fuel isotopes following shutdown. This is tantamount to assuming the decay gammas and betas deposit their energy locally in accordance with the EOC power distribution. Local deposition is reasonable for the decay betas but is certainly not valid for the decay gammas. On the other hand, the decay beta source distribution is not necessarily in accordance with the EOC power distribution since it is due to the accumulated inventories of the fission products and transuranics. These issues are under investigation and will be addressed in the near future. 


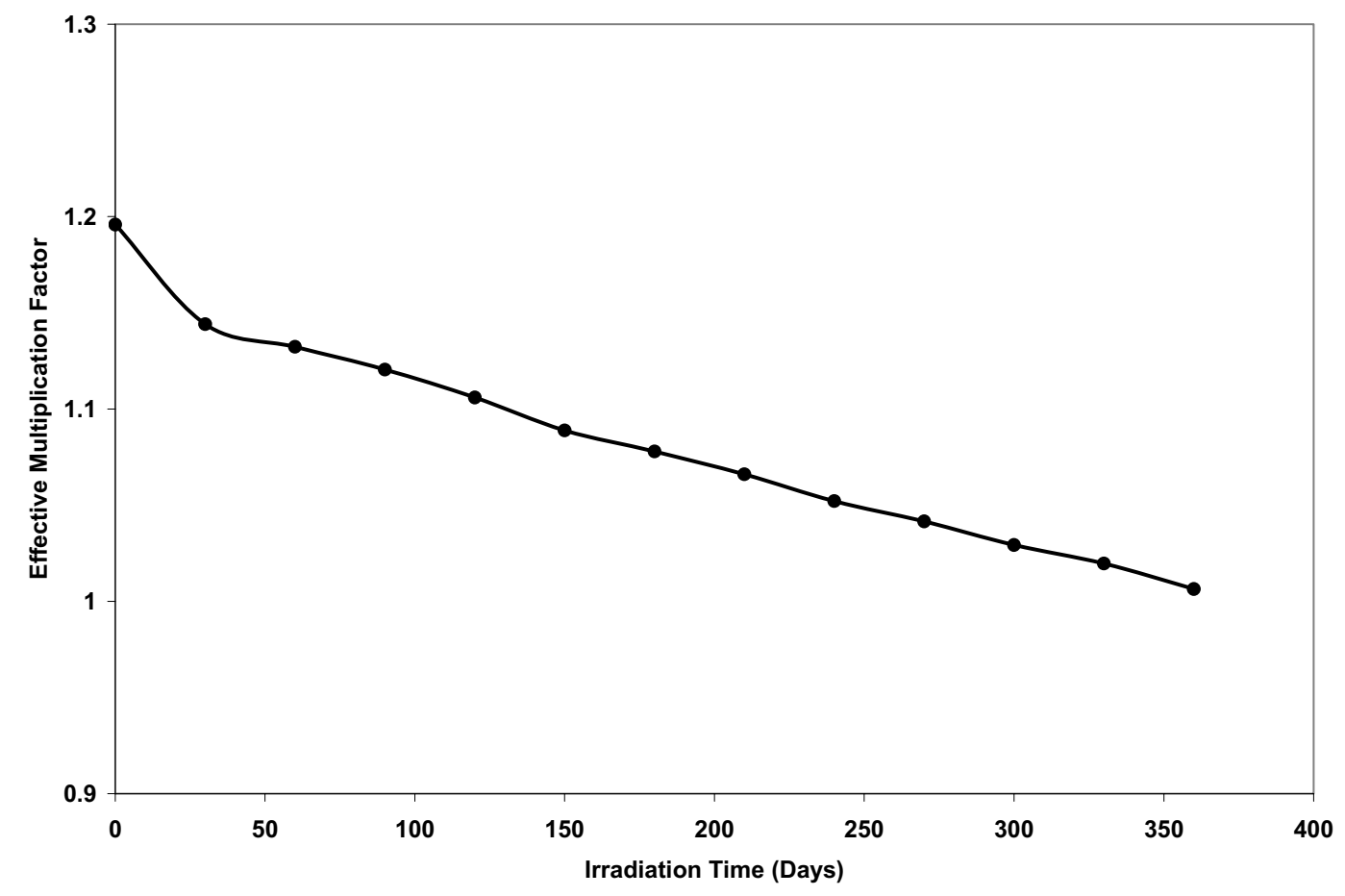

Figure 6-19. Effective Multiplication Factor as a Function of Irradiation Time

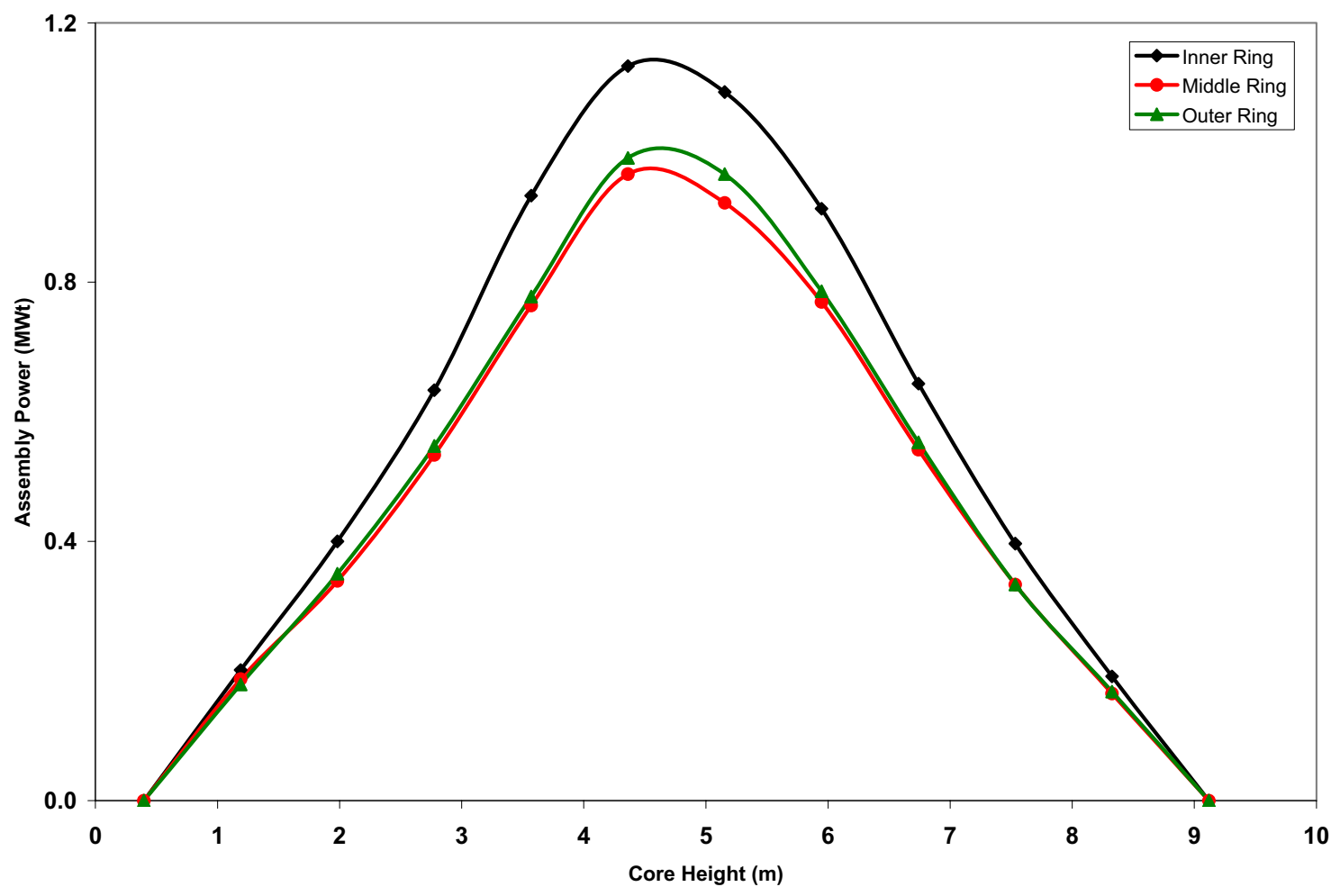

Figure 6-20. BOC Axial Power Distribution per Fuel Assembly 


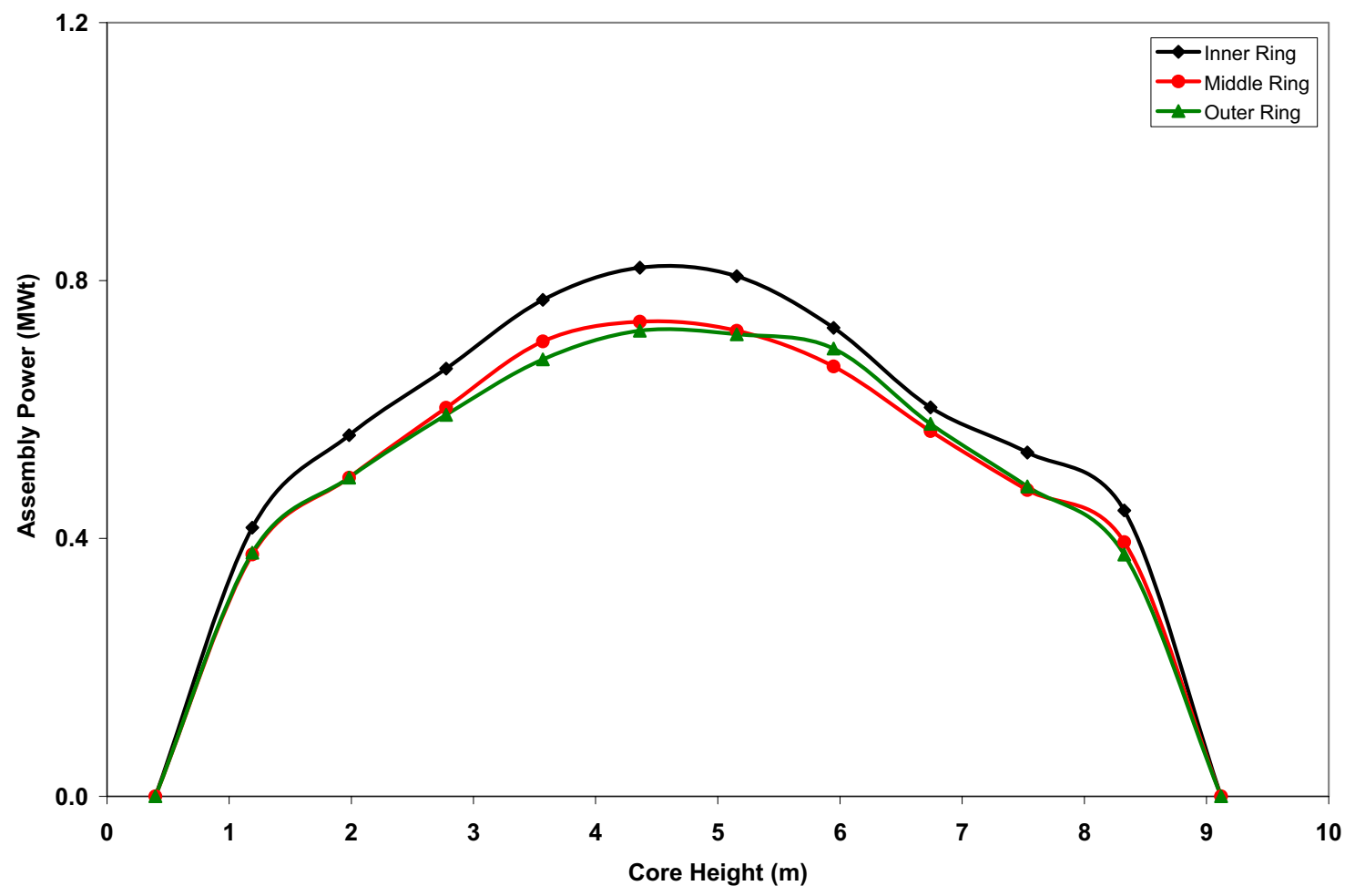

Figure 6-21. EOC Axial Power Distribution per Fuel Assembly

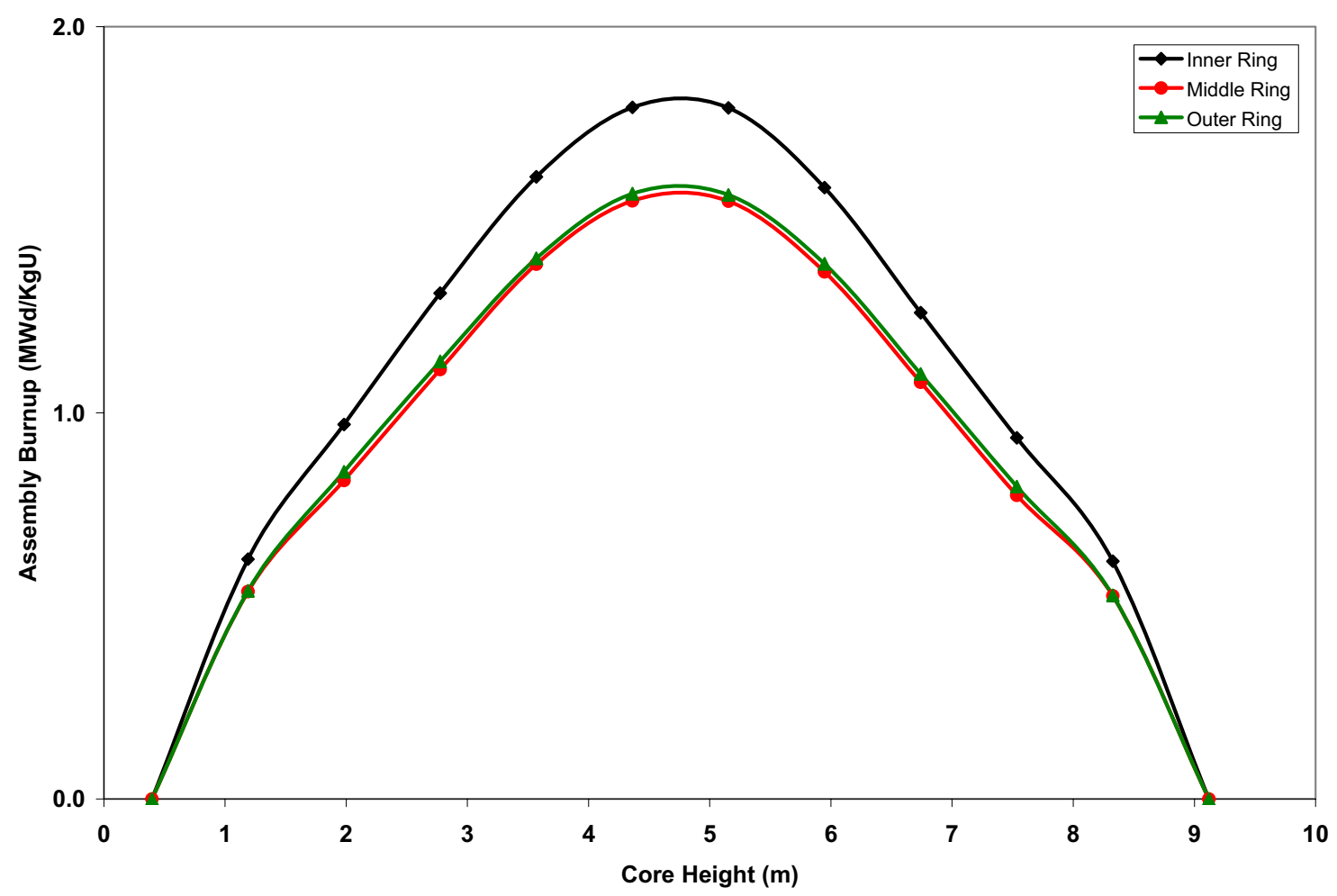

Figure 6-22. EOC Axial Fuel Burnup Distribution 
Table 6-10 is the resultant EOC power distribution from the Monte Carlo simulation, normalized to a full core power level of $600 \mathrm{MWt}$. As discussed above, the decay heat deposition rates at time $t(\mathrm{~s})$ following reactor shutdown are obtained by multiplying the power for each assembly in Table 6-10 with the fraction of rated power due to decay heat at time $t$ from Table 6-11 which was constructed with the help of ANS-5.1 [ANS 1995], corresponding to ${ }^{235} \mathrm{U}$ fissions for an indefinitely long reactor operation time.

Table 6-10. EOC Assembly Powers (600 MWt total)

\begin{tabular}{|c|c|c|c|}
\hline Axial Zone \# & Inner Ring & Middle Ring & Outer Ring \\
\hline & MWt/Assembly & MWt/Assembly & MWt/Assembly \\
\hline $\mathbf{1}$ & 0.409 & 0.356 & 0.358 \\
\hline $\mathbf{2}$ & 0.578 & 0.495 & 0.505 \\
\hline $\mathbf{3}$ & 0.694 & 0.621 & 0.620 \\
\hline $\mathbf{4}$ & 0.766 & 0.718 & 0.706 \\
\hline $\mathbf{5}$ & 0.851 & 0.790 & 0.768 \\
\hline $\mathbf{6}$ & 0.832 & 0.799 & 0.755 \\
\hline $\mathbf{7}$ & 0.713 & 0.654 & 0.632 \\
\hline $\mathbf{8}$ & 0.595 & 0.550 & 0.548 \\
\hline $\mathbf{9}$ & 0.493 & 0.445 & 0.461 \\
\hline $\mathbf{1 0}$ & 0.359 & 0.321 & 0.323 \\
\hline Total & $\mathbf{6 . 2 9 0}$ & $\mathbf{5 . 7 5 0}$ & $\mathbf{5 . 6 7 5}$ \\
\hline
\end{tabular}

Table 6-11. Fraction of Rated Power Deposited as Decay Heat Following Shutdown

\begin{tabular}{|c|r|r|r|r|r|r|}
\hline Time $(\mathrm{s})$ & $1.00 \mathrm{E}+00$ & $1.50 \mathrm{E}+00$ & $2.00 \mathrm{E}+00$ & $4.00 \mathrm{E}+00$ & $6.00 \mathrm{E}+00$ & $8.00 \mathrm{E}+00$ \\
\hline Fraction & $6.13 \mathrm{E}-02$ & $5.95 \mathrm{E}-02$ & $5.79 \mathrm{E}-02$ & $5.37 \mathrm{E}-02$ & $5.08 \mathrm{E}-02$ & $4.87 \mathrm{E}-02$ \\
\hline Time $(\mathrm{s})$ & $1.00 \mathrm{E}+01$ & $1.50 \mathrm{E}+01$ & $2.00 \mathrm{E}+01$ & $4.00 \mathrm{E}+01$ & $6.00 \mathrm{E}+01$ & $8.00 \mathrm{E}+01$ \\
\hline Fraction & $4.70 \mathrm{E}-02$ & $4.40 \mathrm{E}-02$ & $4.19 \mathrm{E}-02$ & $3.69 \mathrm{E}-02$ & $3.41 \mathrm{E}-02$ & $3.22 \mathrm{E}-02$ \\
\hline Time $(\mathrm{s})$ & $1.00 \mathrm{E}+02$ & $1.50 \mathrm{E}+02$ & $2.00 \mathrm{E}+02$ & $4.00 \mathrm{E}+02$ & $6.00 \mathrm{E}+02$ & $8.00 \mathrm{E}+02$ \\
\hline Fraction & $3.07 \mathrm{E}-02$ & $2.82 \mathrm{E}-02$ & $2.66 \mathrm{E}-02$ & $2.31 \mathrm{E}-02$ & $2.12 \mathrm{E}-02$ & $1.99 \mathrm{E}-02$ \\
\hline Time $(\mathrm{s})$ & $1.00 \mathrm{E}+03$ & $1.50 \mathrm{E}+03$ & $2.00 \mathrm{E}+03$ & $4.00 \mathrm{E}+03$ & $6.00 \mathrm{E}+03$ & $8.00 \mathrm{E}+03$ \\
\hline Fraction & $1.88 \mathrm{E}-02$ & $1.69 \mathrm{E}-02$ & $1.55 \mathrm{E}-02$ & $1.26 \mathrm{E}-02$ & $1.11 \mathrm{E}-02$ & $1.01 \mathrm{E}-02$ \\
\hline Time $(\mathrm{s})$ & $1.00 \mathrm{E}+04$ & $1.50 \mathrm{E}+04$ & $2.00 \mathrm{E}+04$ & $4.00 \mathrm{E}+04$ & $6.00 \mathrm{E}+04$ & $8.00 \mathrm{E}+04$ \\
\hline Fraction & $9.47 \mathrm{E}-03$ & $8.36 \mathrm{E}-03$ & $7.67 \mathrm{E}-03$ & $6.25 \mathrm{E}-03$ & $5.55 \mathrm{E}-03$ & $5.11 \mathrm{E}-03$ \\
\hline Time $(\mathrm{s})$ & $1.00 \mathrm{E}+05$ & $1.50 \mathrm{E}+05$ & $2.00 \mathrm{E}+05$ & $4.00 \mathrm{E}+05$ & $6.00 \mathrm{E}+05$ & $8.00 \mathrm{E}+05$ \\
\hline Fraction & $4.82 \mathrm{E}-03$ & $4.34 \mathrm{E}-03$ & $4.05 \mathrm{E}-03$ & $3.47 \mathrm{E}-03$ & $3.15 \mathrm{E}-03$ & $2.92 \mathrm{E}-03$ \\
\hline Time $(\mathrm{s})$ & $1.00 \mathrm{E}+06$ & $1.50 \mathrm{E}+06$ & $2.00 \mathrm{E}+06$ & & & \\
\cline { 1 - 4 } Fraction & $2.75 \mathrm{E}-03$ & $2.43 \mathrm{E}-03$ & $2.21 \mathrm{E}-03$ & &
\end{tabular}

In continuation of our effort to accurately determine the decay heat deposition rates, we will perform additional Monteburns calculations representing fuel and fission product decays following reactor shutdown and perform photon transport calculations with MCNP5. With more accurate accounting of beta energy deposition rates coupled to MCNP5 calculations for the gamma deposition rates, we plan to determine an improved decay heat deposition rates following reactor shutdown for the VHTR configuration. 


\section{Task 6: Code Validation and Verification (V\&V) (All)}

\subsection{Task 6-1 KAIST V\&V}

\subsubsection{Large Scale Air Ingress Analysis}

\section{HTTR-Simulated Air Ingress Experiment}

For V\&V with large scale air ingress experiment we chose the HTTR-simulated experimental facility. Figure 7-1-1 shows the HTTR-simulated experimental facility (Takeda 1997), which consisted of a reactor core simulator, a high-temperature plenum, and a water-cooled jacket corresponding to the reactor vessel and the simulated inlet and outlet pipes corresponding to the coaxial pipe. The reactor core simulator had four graphite tubes (one central and three peripherals) and a ceramic plenum. The graphite tube had an inner diameter of $40 \mathrm{~mm}$ and a height of $800 \mathrm{~mm}$. Density of gas mixture and concentrations of $\mathrm{O}_{2}, \mathrm{CO}$, and $\mathrm{CO}_{2}$ were measured at five sampling points indicated by the symbol, $(\odot)$, in Figure 7-1-2.

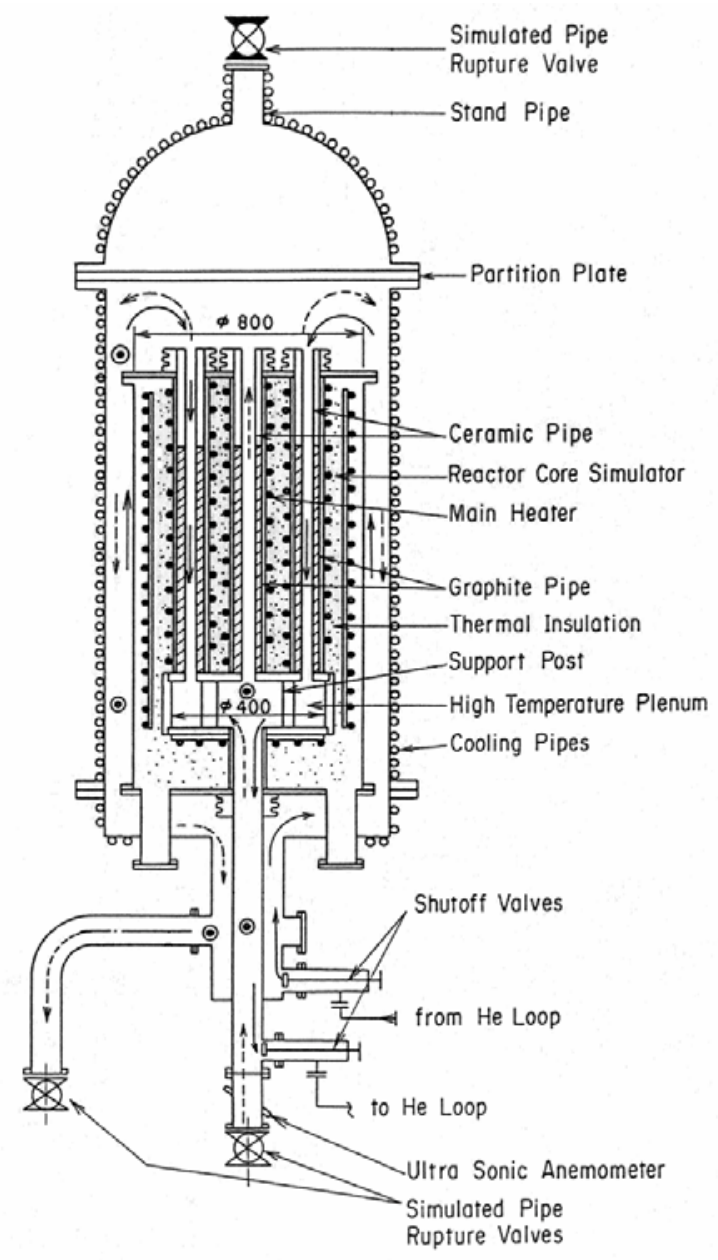

Figure 7-1-1 Schematic diagram of the HTTR-simulated system 

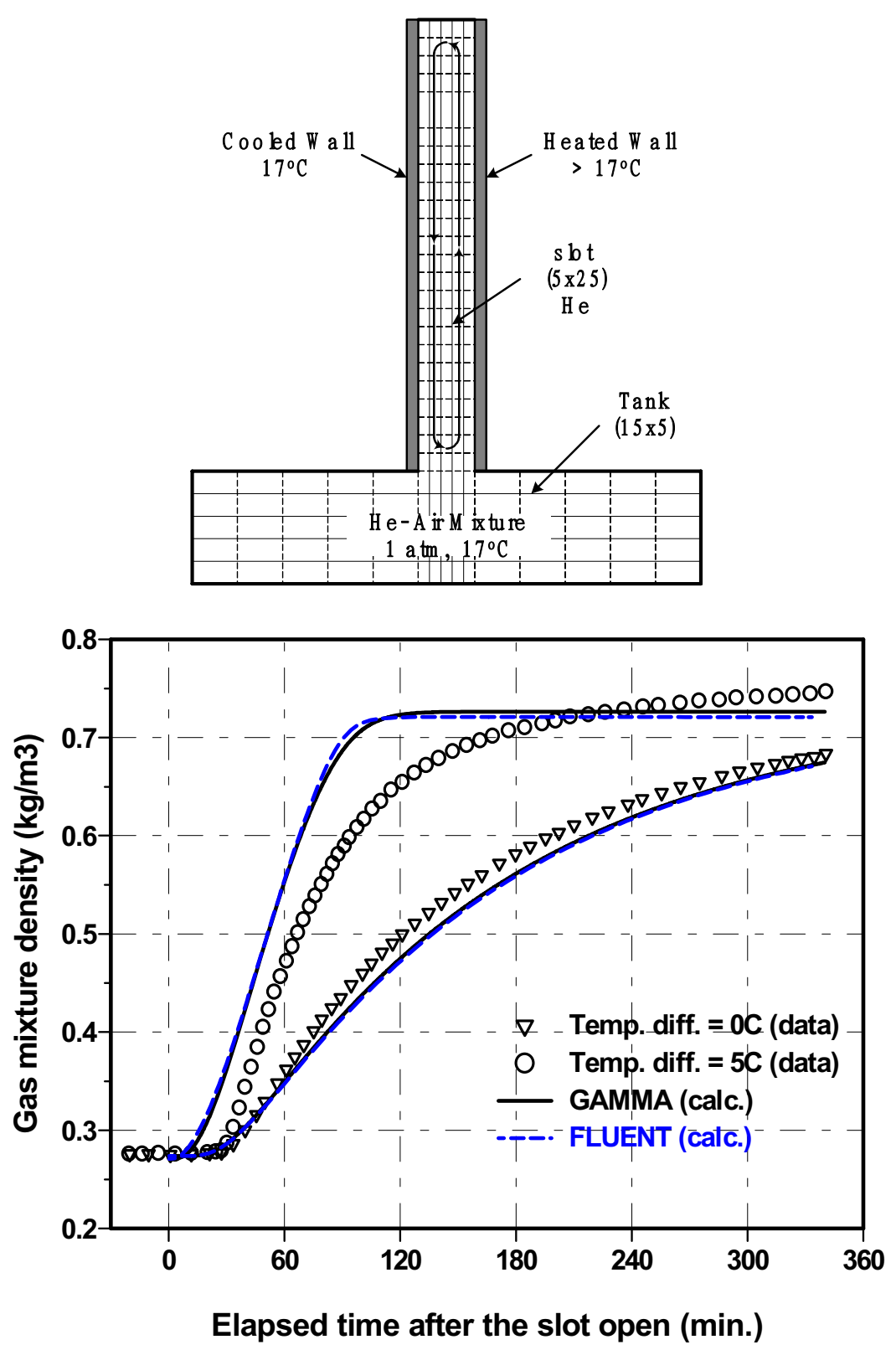

Figure 7-1-2 Predicted densities of gas mixture at the top of a vertical slot

First of all, a vertical slot test (Takeda 1997) has been simulated to investigate the effect of local circulation on molecular diffusion, which is expected to occur in an annular passage of the HTTR-simulated system and thus affects the consequence of air ingress. The vertical slot system consisted of a rectangular dimension of $720 \times 290 \times 820 \mathrm{~mm}$ in a bottom tank and $20 \times 956 \times 590 \mathrm{~mm}$ in a thin slot, each initially filled with a He-air mixture and He only, respectively. Since the width of the slot is relatively very thin compared to that of the tank, a 2-D geometry model can be used. Figure 7-1-3 shows that air ingress for the temperature difference of $5^{\circ} \mathrm{C}$ is greatly promoted by local natural circulation compared to the pure diffusion case. The range of the Rayleigh number calculated based on the width of the slot is about $350<R a<550$. Compared to the ideal calculation result, it seems that the flow field is distorted by non-uniform slot temperature distribution and rough surface conditions due to thermocouples attached on the inner surface of the slot thus lowering the air transport rate during the experiment. 


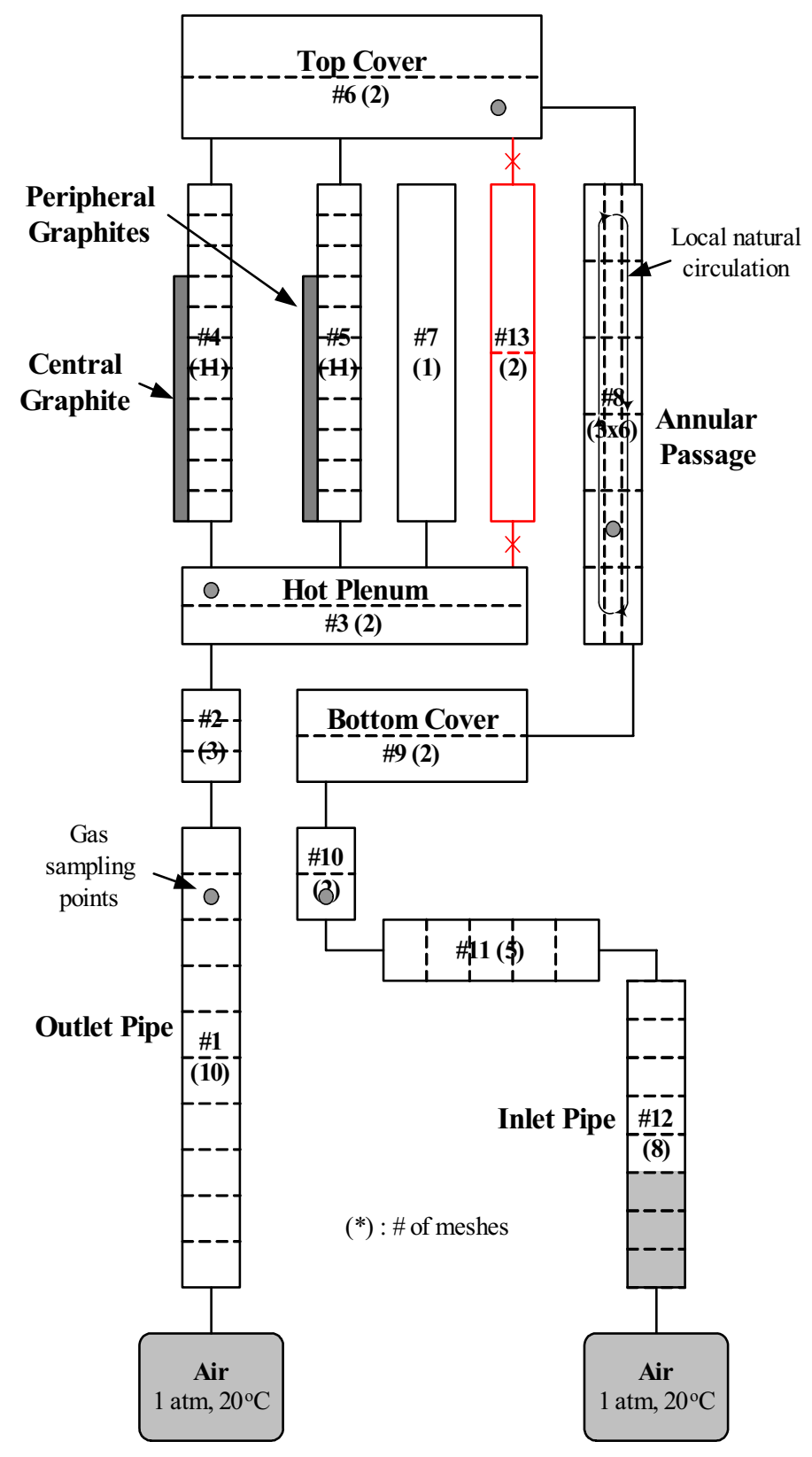

Figure 7-1-3 Nodalization diagram for the HTTR-simulated system

The GAMMA nodal scheme for the HTTR-simulated system is shown in Figure 7-1-4. On the basis of the previous investigation, an annular passage in the HTTR-simulated system is modeled by 2-D geometry to consider the effect of local natural circulation. A local natural circulation in other regions is much less important because it mixes gas components quickly. Among all the test cases listed at Table 7-1-1, the predicted results for the non-equal temperature case $\mathrm{B} 8575\left(850^{\circ} \mathrm{C}\right.$ and $750^{\circ} \mathrm{C}$ for central and peripheral graphites) are described in detail. As time passes, as shown in Figure 7-1-5, oxygen is transported by molecular diffusion and weak natural convection toward both hot and cold sides. In particular, since a local natural circulation in the annular passage promotes an air transport into the top cover, graphite oxidation takes place in both the bottom and top portions of the graphite tubes. We estimate that the range of the Rayleigh number is about $2 \times 10^{4}<R a<2 \times 10^{5}$ based on the width of an annular passage, and the local velocity 
is about $0.6 \mathrm{~m} / \mathrm{s}$, which is comparable to the velocity of a global natural convection. As oxygen is consumed in the graphite tubes and the amount of heavy gases, $\mathrm{CO}$ and $\mathrm{CO}_{2}$, produced by the chemical reactions increases gradually, the buoyancy force induced by the distribution of the gas mixture becomes substantial enough to overcome a gravitational force. Eventually a global natural convection is initiated through the entire test apparatus several days after the test initiates.

Table 7-1-1 Test cases conducted in the HTTR-simulated test facility

\begin{tabular}{|c|c|c|c||}
\hline \multicolumn{2}{|c|}{ Equal temperature condition } & \multicolumn{2}{c||}{ Non-equal temperature condition } \\
\hline $\begin{array}{c}\text { Run } \\
\text { No. }\end{array}$ & $\begin{array}{c}\text { Central and peripheral } \\
\text { graphite temperatures }\left({ }^{\circ} \mathrm{C}\right)\end{array}$ & $\begin{array}{c}\text { Run } \\
\text { No. }\end{array}$ & $\begin{array}{c}\text { Central and peripheral } \\
\text { graphite temperatures }\left({ }^{\circ} \mathrm{C}\right)\end{array}$ \\
\hline A40 & $405.4(108.8 / 20.3)^{\mathrm{a}}$ & B1090 & $994.1,907.7(214.4 / 25.0)$ \\
\hline A60 & $607.8(159.3 / 28.0)$ & B8575 & $847.8,765.6(180.9 / 26.6)$ \\
\hline A70 & $708.2(181.2 / 26.4)$ & B7060 & $697.6,613.2(136.3 / 15.2)$ \\
\hline A75 & $756.9(193.2 / 29.5)$ & B7060b & $697.6,619.2(142.7 / 27.7)$ \\
\hline A80 & $806.7(195.9 / 29.6)$ & B4030 & $396.3,329.6(90.0 / 27.8)$ \\
\hline A85 & $857.7(220.5 / 37.2)$ & & \\
\hline A90 & $908.0(225.4 / 33.2)$ & & \\
\hline A95 & $955.7(247.0 / 44.6)$ & & \\
\hline A100 & $1002.7(247.7 / 46.1)$ & & \\
\hline A105 & $1045.7(270.1 / 53.6)$ & & \\
\hline \hline
\end{tabular}

Note: $\quad$ a. Core barrel and pressure vessel wall temperatures 


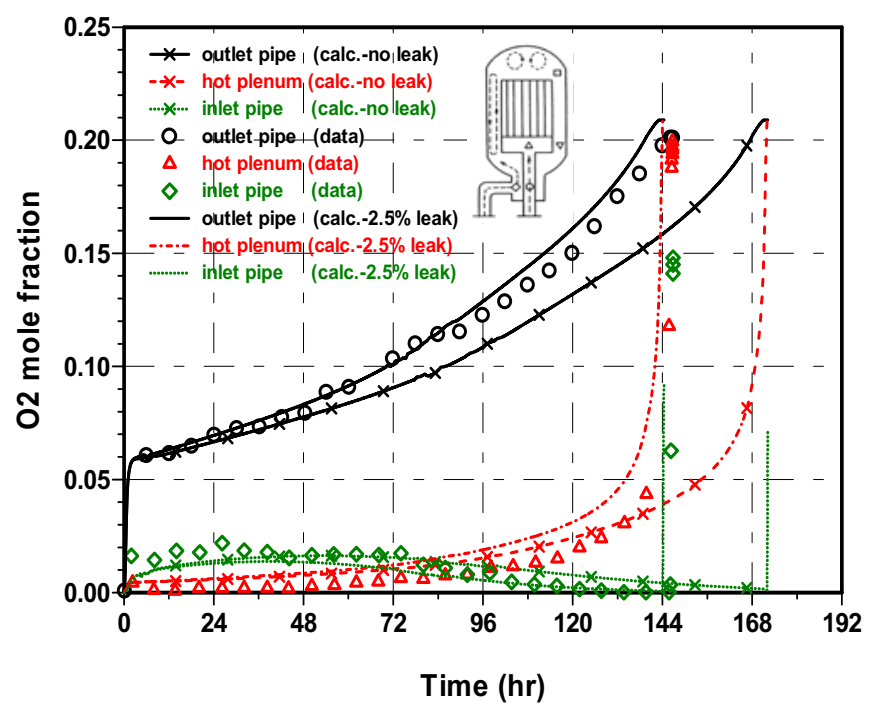

Figure 7-1-4 Predicted mole fractions of oxygen for the case B8575

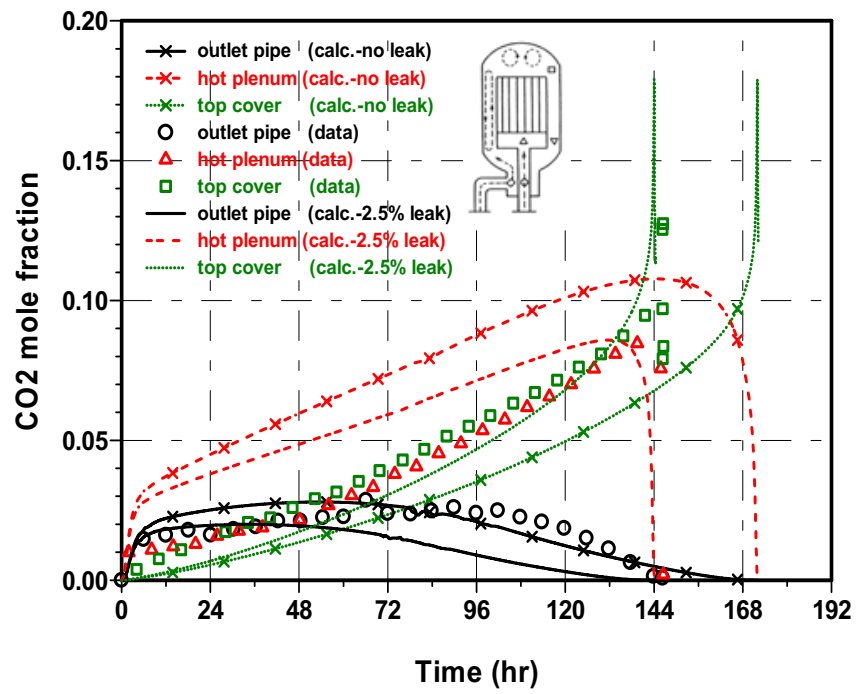

Figure 7-1-5 Predicted mole fractions of dioxide for the case B8575

In the first calculation, we found that the $\mathrm{CO}_{2}$ produced in the bottom of graphite tubes is trapped in the hot plenum. Therefore, as shown in Figure 7-1-6, the predicted mole fractions of $\mathrm{CO}_{2}$ are higher in the hot plenum but lower in the top cover and annular regions compared to those of the measured data, thus delaying the consequence of air ingress. The onset time of natural convection is delayed by around one day, corresponding to a deviation greater than $10 \%$. It is presumed that its delay is caused by several leak paths, which are established from the hot side to the cold side directly as well as indirectly through the thermal insulator. In the present calculation, $2.5 \%$ leak area (100\% leak area is equal to the flow area of one graphite tube with a diameter of $4 \mathrm{~cm}$ ) is found to well predict the experimental data. Figure 7-1-7 shows that the onset times of natural convection for all the test cases are predicted well within a $10 \%$ deviation. 


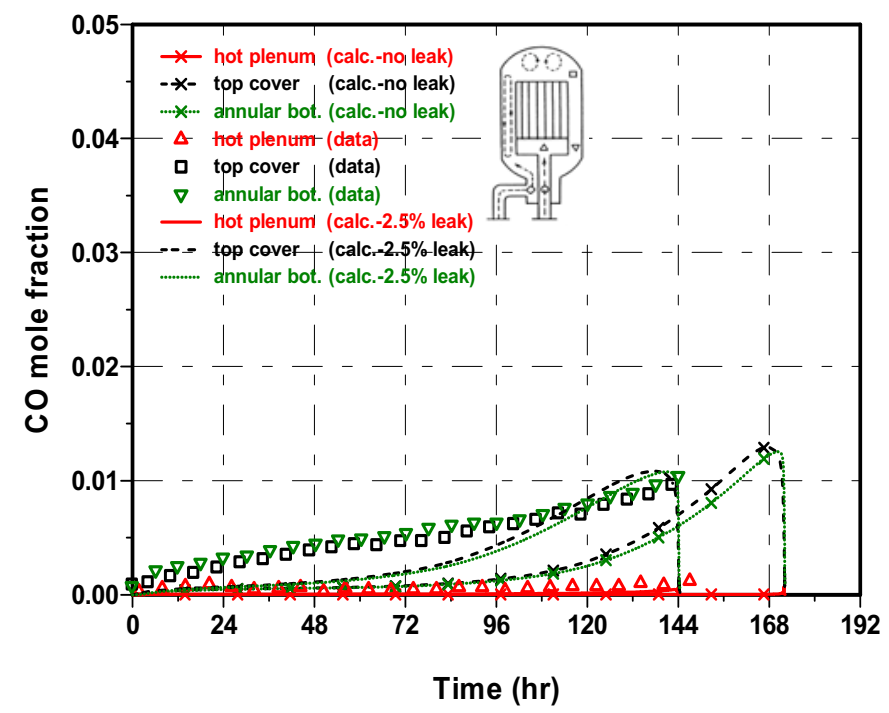

Figure 7-1-6 Predicted mole fractions of monoxide for the case B8575

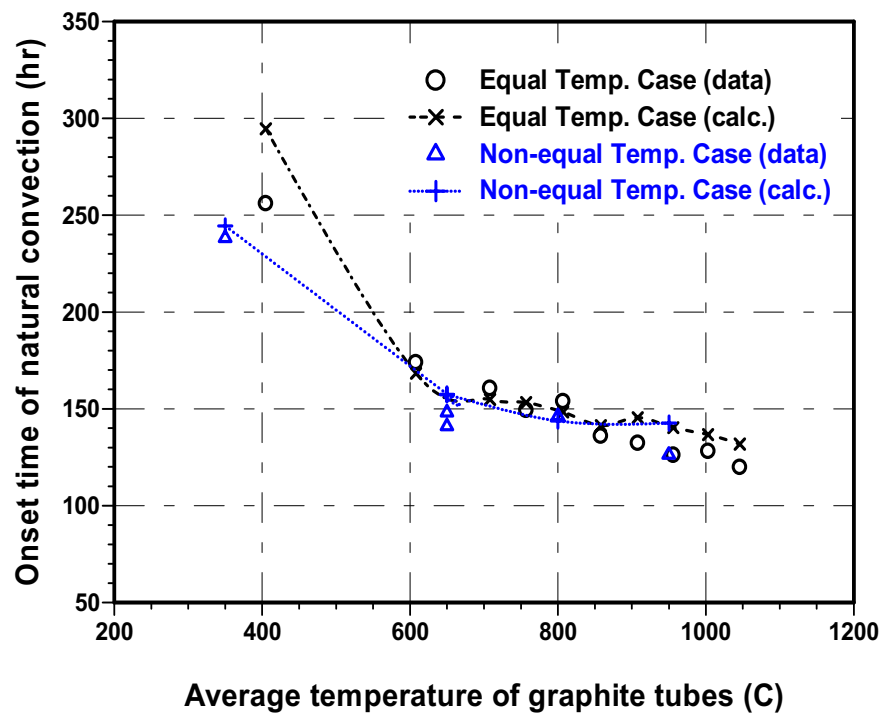

Figure 7-1-7 Predicted onset times of natural convection (OTNC) for the HTTR-simulated air ingress experiment

\subsubsection{Large Scale System V\&V Simulation}

\section{SANA-1 Afterheat Removal Test (IAEA Benchmark)}

The SANA-1 self-acting afterheat removal tests (IAEA-TECDOC-1163 2000), one of the IAEA benchmark problems, have been simulated to validate the porous media model that is incorporated into the GAMMA code. The SANA-1 test apparatus shown in Figure 7-1-8 consisted of a cylindrical pebble bed having a diameter of $1.5 \mathrm{~m}$ and a height of $1 \mathrm{~m}$, a central heating element, and bottom and top insulators. The pebble bed was filled with approximately 9500 graphite pebbles with diameters of $6 \mathrm{~cm}$ in an irregular arrangement. In this experiment, various configurations and conditions given at Table 7-1-2 were investigated: different kinds of fluids (nitrogen and helium), pebble sizes $(3 \mathrm{~cm}$ and $6 \mathrm{~cm}$ ), heating conditions (long and short heater elements), and configurations with and without a gas plenum above the pebble bed. For the tests conducted 
with the $6 \mathrm{~cm}$ diameter pebbles, measurements were taken of the pebble temperatures at different radial positions close to the bottom of the pebble bed (height $9 \mathrm{~cm}$ ), at the center (height $50 \mathrm{~cm}$ ), and close to the top (height $90 \mathrm{~cm}$ ).
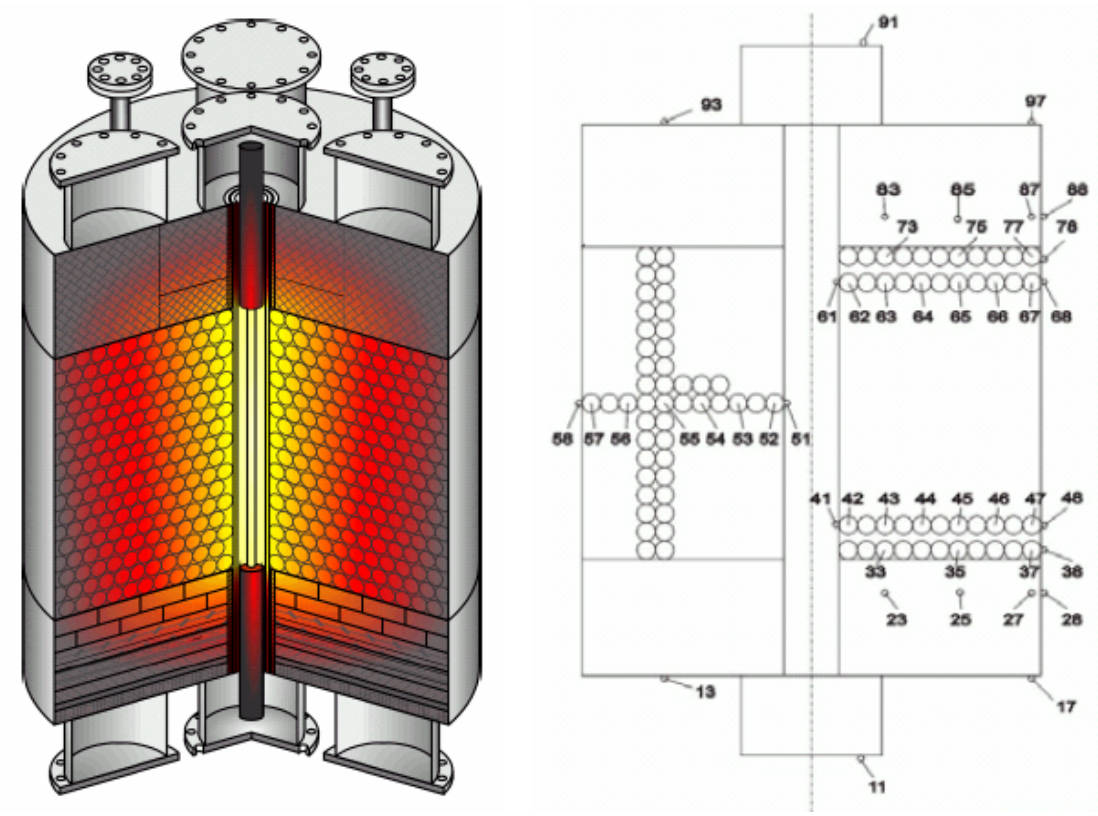

Figure 7-1-8 SANA-1 test facility and the temperature measuring points

Table 7-1-2 Test cases conducted in the SANA-1 test facility

\begin{tabular}{||c|c|c|c|c||}
\hline $\begin{array}{c}\text { Configu- } \\
\text { rations }\end{array}$ & $\begin{array}{c}\text { Pebble } \\
\text { diameter } \\
(\mathrm{mm})\end{array}$ & Heating tube/pebble bed geometry & Gas & $\begin{array}{c}\text { Heating power } \\
\text { (kW) }\end{array}$ \\
\hline 1 & 60 & long heating element & $\mathrm{N}_{2}, \mathrm{He}$ & 10,30 \\
\hline 2 & 60 & short heating element at the top & $\mathrm{N}_{2}, \mathrm{He}$ & 20 \\
\hline 3 & 60 & short heating element at the bottom & $\mathrm{N}_{2}, \mathrm{He}$ & 20 \\
\hline 4 & 60 & $\begin{array}{c}\text { short heating element at the bottom } \\
\text { with gas plenum above the pebble bed }\end{array}$ & $\mathrm{N}_{2}, \mathrm{He}$ & 20 \\
\hline 5 & 30 & long heating element & $\mathrm{N}_{2}, \mathrm{He}$ & 10,30 \\
\hline 6 & 60 & long heating element & $\mathrm{N}_{2}, \mathrm{He}$ & $\begin{array}{c}30 \text { to } 10 \text { ramp } \\
10 \text { to 25 step } \\
\text { change }\end{array}$ \\
\hline
\end{tabular}

Figure 7-1-9 shows the GAMMA axi-symmetric mesh scheme with 782 meshes for the fluid region and 1242 meshes for the solid region including a pebble bed. Due to the rotational symmetry of the SANA-1 facility, the 2-D geometry model can be used and, since the amount of heat generated or removed from the heater element and the top and bottom coolers is known, heat flux can be used as a boundary condition at the heater side. On the surface of the enclosing shell, the heat exchange with the surrounding environment by the convection and the radiation is considered using the convective heat transfer coefficient of $5 \mathrm{~W} / \mathrm{m}^{2}-\mathrm{K}$ and the environment temperature of $25^{\circ} \mathrm{C}$. For the flow resistances in a packed bed, Forchheimer-extended Darcy's law (Nield and Bejan 1999) with the parameters recommended by the German safety guide KTA3102.3 1981 is used. 

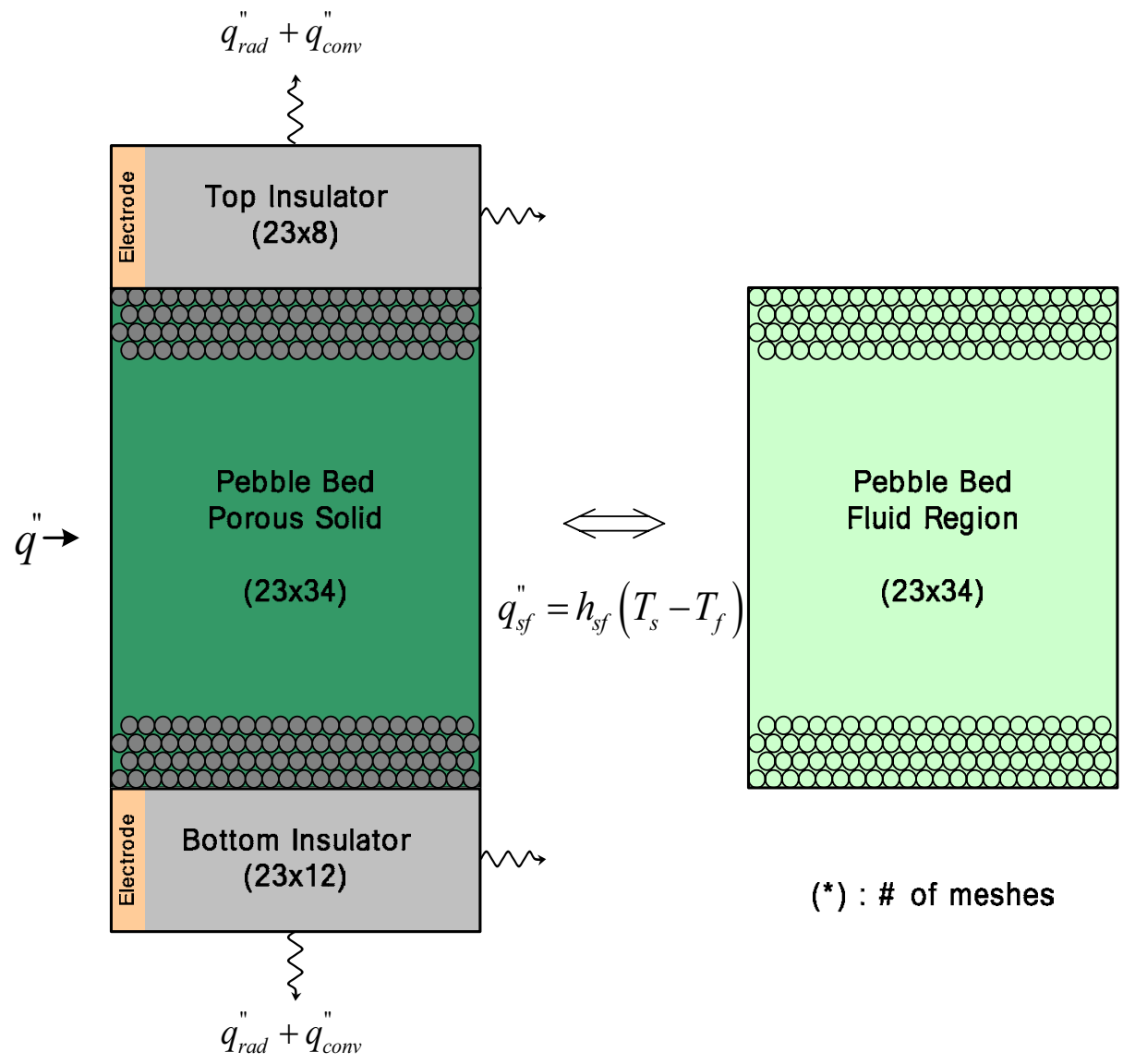

Figure 7-1-9 GAMMA 2-D mesh scheme for the SANA-1 test apparatus

$K=\frac{d_{p}^{2} \varphi^{3}}{160\left(1-\varphi^{2}\right)}$ and $C_{F}=\frac{3}{\sqrt{160} \varphi^{3 / 2}}\left(\frac{1-\varphi}{\operatorname{Re}_{p}}\right)^{0.1}$

The effective thermal conductivity of a pebble bed is calculated from the cell model of Zehner/Bauer/Schlünder described in the IAEA technical document (IAEA-TECDOC-1163 2000); it considers the radiation in void region, the conduction of gas, and the contact conduction of spherical pebbles.

$\lambda_{\text {eff }}=\lambda_{\text {eff }}^{\text {voidradiation }}+\lambda_{\text {eff }}^{\text {gas_conduction }}+\lambda_{\text {eff }}^{\text {contact_conduction }}$

where

$$
\begin{aligned}
& \lambda_{\text {eff }}^{\text {vid_radiation }}=\left\{[1-\sqrt{1-\varphi}] \varphi+\frac{\sqrt{1-\varphi}}{2 / \varepsilon_{r}-1} \frac{B+1}{B}\left[1+\frac{1}{\left(2 / \varepsilon_{r}-1\right) \Lambda}\right]^{-1}\right\} 4 \sigma T^{3} d_{p} \\
& \lambda_{e f f}^{\text {gas_conduction }}=\lambda_{f}\left\{1-\sqrt{1-\varphi}+\frac{2 \sqrt{1-\varphi}}{1-\lambda_{r} B}\left[\frac{\left(1-\lambda_{r}\right) B}{\left(1-\lambda_{r} B\right)^{2}} \ln \left(\frac{1}{\lambda_{r} B}\right)-\frac{B+1}{2}-\frac{B-1}{1-\lambda_{r} B}\right]\right\} \\
& \lambda_{\text {eff }}^{\text {contact_conduction }}=\lambda_{s}\left[\frac{3\left(1-\mu_{p}^{2}\right)}{4 E_{s}} \frac{P_{e} S_{F} d_{p}}{2 N_{A}}\right]^{1 / 3} \frac{1}{0.531 S}\left(\frac{N_{A}}{N_{L}}\right)
\end{aligned}
$$




$$
\begin{aligned}
& B=1.25\left(\frac{1-\varphi}{\varphi}\right)^{10 / 9}, \Lambda=\frac{\lambda_{s}}{4 \sigma T^{3} d_{p}}, \lambda_{r}=\frac{\lambda_{f}}{\lambda_{s}}, S=1, S_{F}=1, N_{A}=\frac{1}{d_{p}{ }^{2}}, N_{L}=\frac{1}{d_{p}}, \\
& \mu_{p}=0.136 \text { (Poisson ratio), } E_{s}=9 \times 10^{9}\left(\text { Young modulus, } N / m^{2}\right), \\
& P_{e}=\text { External pressure estimated by the weight of particles, } \varepsilon_{r}=\text { pebble emissivity }
\end{aligned}
$$

The boundary effects due to the variation of porosity near the wall, channeling and thermal dispersion (Nield and Bejan 1999), are considered as follows:

$$
\begin{aligned}
& \varphi=\varphi_{\infty}\left[1+1.4 \exp \left(-5 y / d_{p}\right)\right] \quad(6-1-3) \\
& \left(\lambda_{\text {disp }}\right)_{y y}=0.12(\rho C)_{f} d_{p}\left(V_{z}\right)\left[1-\exp \left(-y / d_{p}\right)\right] \text { and } \\
& \left(\lambda_{\text {disp }}\right)_{x x}=\left(\lambda_{\text {disp }}\right)_{z z}=\left(\lambda_{\text {disp }}\right)_{y y} / 3
\end{aligned}
$$

where $\varphi_{\infty}$ is the bulk porosity of a pebble bed $(=0.41)$ and $y$ is the distance from the wall boundary. For the convection of gas, the pebble-to-fluid heat transfer coefficient recommended by the German safety guide KTA3102.2 1981 and the specific interfacial area relation are used as follows:

$$
\begin{aligned}
& h_{s f}=\frac{\lambda_{f}}{d_{p}}\left(1.27 \frac{\operatorname{Pr}^{1 / 3}}{\varphi^{1.18}} \operatorname{Re}_{p}^{0.36}+0.033 \frac{\operatorname{Pr}^{1 / 2}}{\varphi^{1.07}} \operatorname{Re}_{p}^{0.86}\right) \\
& a_{s f}=6(1-\varphi) / d_{p}
\end{aligned}
$$

Steady Power Tests

All the kinds of test cases for the configurations 1 through 5 conducted in a SANA-1 test facility have been simulated and the predicted results have been compared with those of other codes (TINTE,

THERMIX/DIREKT, and TRIO-EF). Among all the predictions, we would like to describe two representative cases, the high-powered long-element heating case (Configuration 1) and the bottom-element heating case with a gas plenum (Configuration 4).

Figure 7-1-10 shows the predicted temperature profiles at three layers from the bottom of the pebble bed for the long element heating case at $30 \mathrm{~kW}$ power. The radial temperature distributions are the highest in the upper layer and the lowest in the lower layer due to the free convection flow. The relative temperature difference between the layers is larger with nitrogen as a filled-gas than with helium, due to larger free convection flow caused by difference in gas density. 

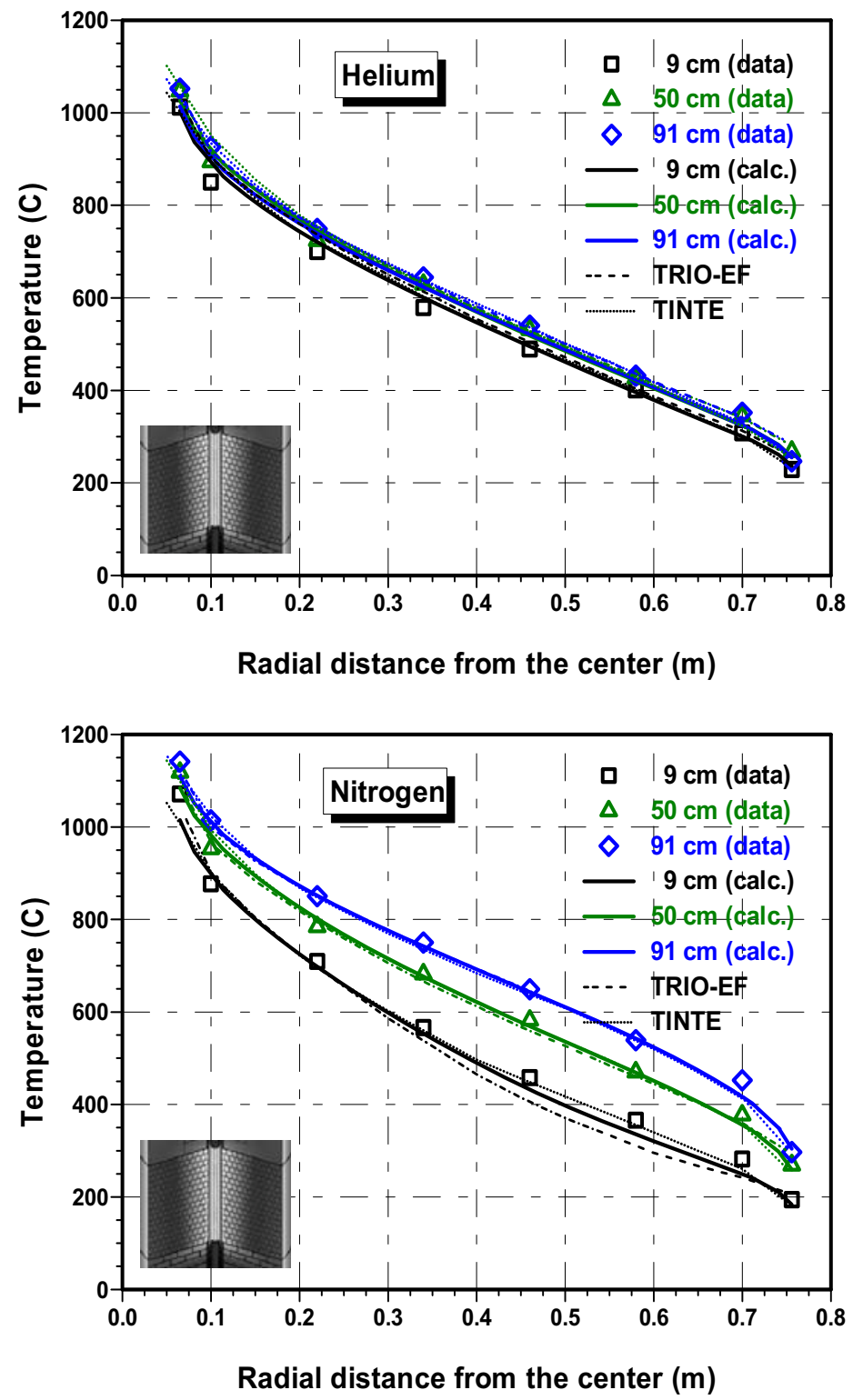

Figure 7-1-10 Measured and calculated temperature distributions: long heating element, $30 \mathrm{~kW}$ heating power, and $6 \mathrm{~cm}$ pebble

Figure 6-1-11 shows the predicted and measured temperature profiles in three layers from the bottom of the pebble bed for the bottom-element heating case at $20 \mathrm{~kW}$ power with a gas plenum. The radial temperatures are the highest in the lower layer and the lowest in the upper layer in all the radial positions for the He-filled test, but these are reversed in the cold region away from the heating rod for the $\mathrm{N}_{2}$-filled test because of a large free convection flow. We considered the radiation exchange in the gas plenum. Therefore, the calculated temperature distribution near the radiating surface at $63 \mathrm{~cm}$ shows a satisfactory level of agreement with the measured data. Figure 7-1-12 shows the calculated local velocity and gas temperature fields for the test with helium as a filled gas. The circulating velocity is much lower in the pebble region due to the corresponding large flow resistance than that in the gas plenum. The heat transport is governed by heat conduction in the pebble region since the isothermal lines of the gas are almost stratified in the radial direction, while the radiation heat transfer is dominant in the gas plenum since the gas temperature is the 
lowest. For the test with nitrogen as the gaseous medium with the same experimental setup, Figure 7-1-13 shows a much larger contribution of the convective heat transport to the total heat transport because of larger variations of the gas density and lower thermal conductivity of the gas when compared to the He-filled test.
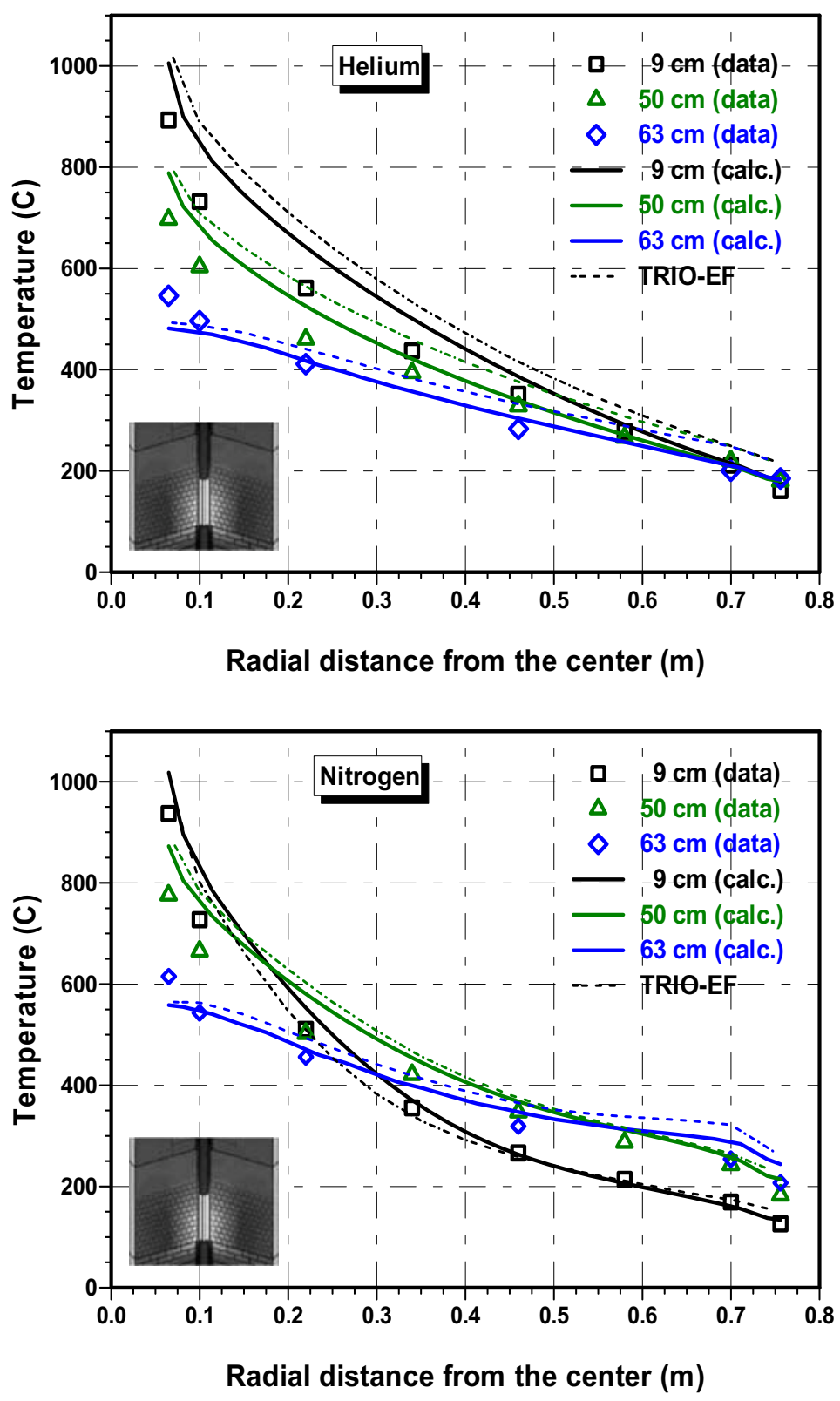

Figure 7-1-11 Measured and calculated temperature distributions: short heating element at the bottom and a gas plenum, $20 \mathrm{~kW}$ heating power, and $6 \mathrm{~cm}$ pebble 

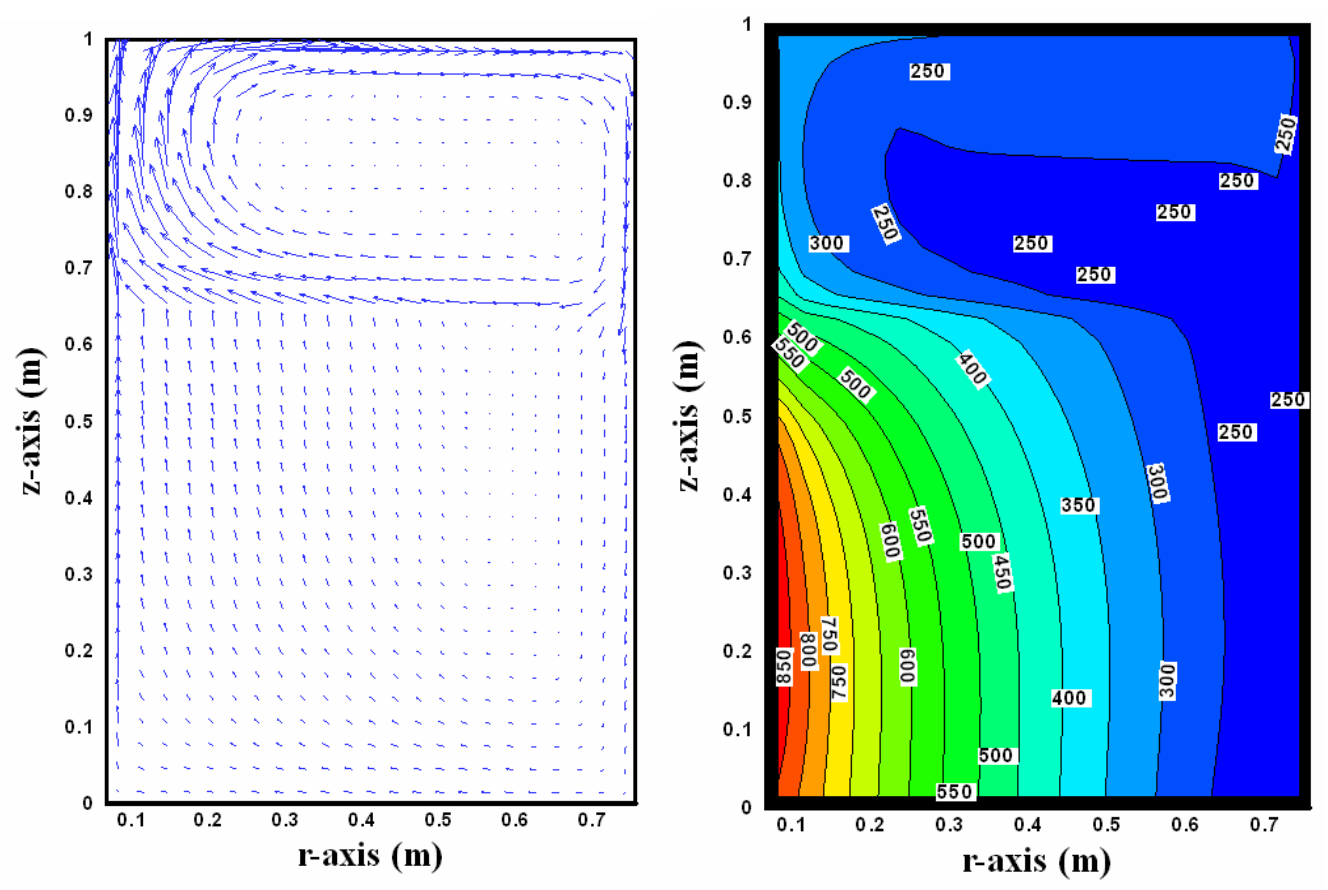

Figure 7-1-12. Calculated velocity vector and gas temperature fields for the He-filled test
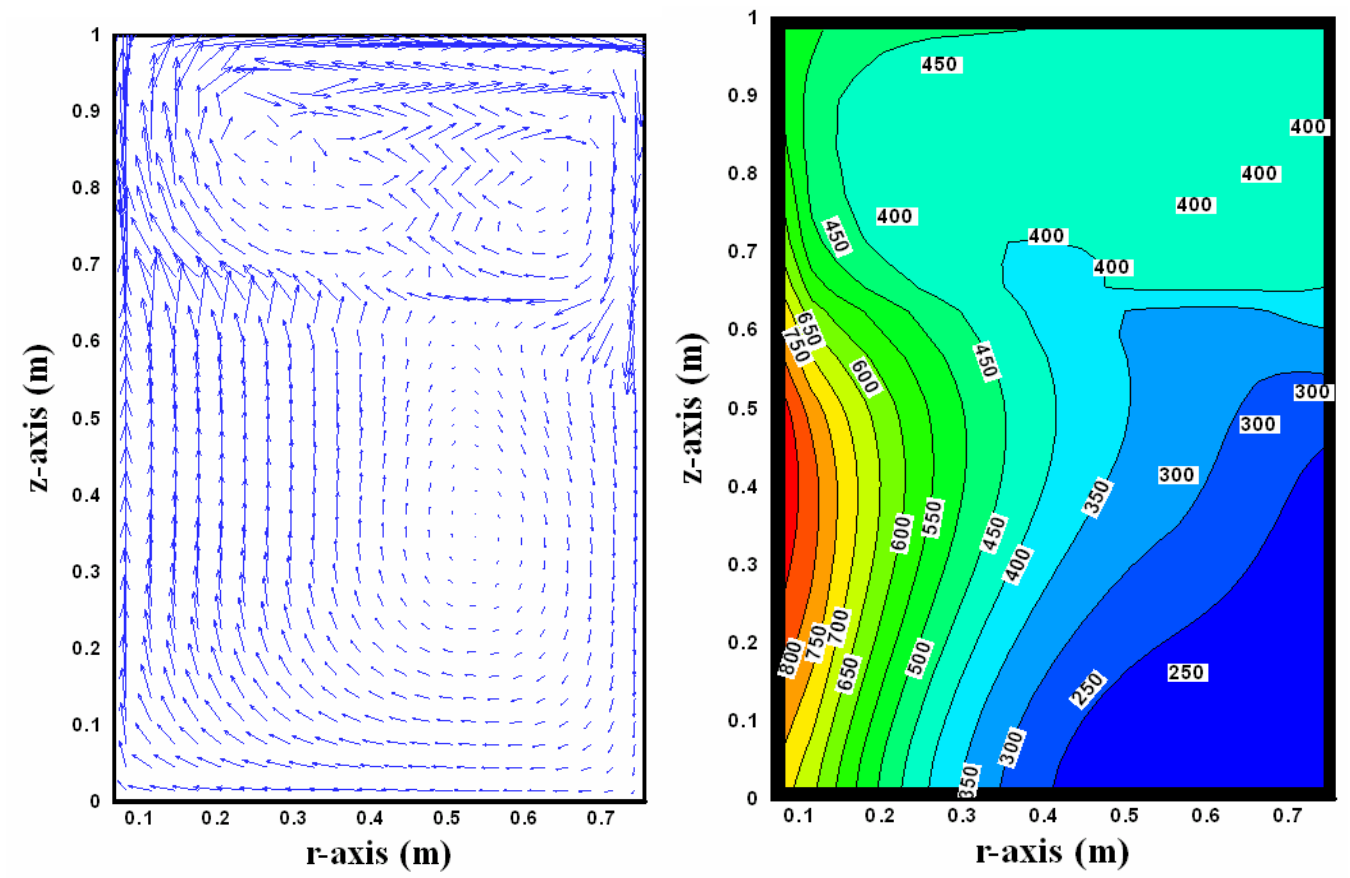

Figure 7-1-13. Calculated velocity vector and gas temperature fields for the $\mathrm{N}_{2}$-filled test

In all the simulated cases for the steady power tests, the prediction results of GAMMA agree closely with the measured data and are comparable to those of other analysis codes (TINTE, THERMIX/DIREKT, and TRIO$\mathrm{EF})$. The simulated results show that the heat transport by conduction, convection, and radiation in a pebble bed, which acts a major role to remove the afterheat during the accident conditions, is well predicted using 
the effective thermal conductivity by Zehner/Bauer/Schlünder and the selected correlations for the interfacial convective heat transfer and the flow resistance.

\section{Power Ramp-Down and Step-Up Tests}

The GAMMA simulation results for the SANA transient tests (Configuration 6 with helium as a coolant) are shown in Figures 7-1-14 and 7-1-15. In the power ramp-down test, the heater power changes slowly from 30 $\mathrm{kW}$ to $10 \mathrm{~kW}$ during 50 hours. In the power step-up test, the heater power changes rapidly from $10 \mathrm{~kW}$ to 25 $\mathrm{kW}$ by a step.

In both the simulations of the SANA power ramp-down and step-up transient tests, a little deviation in the spatial distribution of pebble temperatures is observed near the surface of heater element $(6.5 \mathrm{~cm})$. This difference seems to be caused by the near-wall effect. Except near the heater rod, however, the predicted trends of the pebble temperatures are well in accordance with the measured data (Rouaaeau et al. 2004) and those of other analysis codes (THERMIX and FLOWNEX). Therefore, the obtained results demonstrate that the GAMMA code is capable to predict the heatup and cooling processes of the pebble-bed core during the transients.

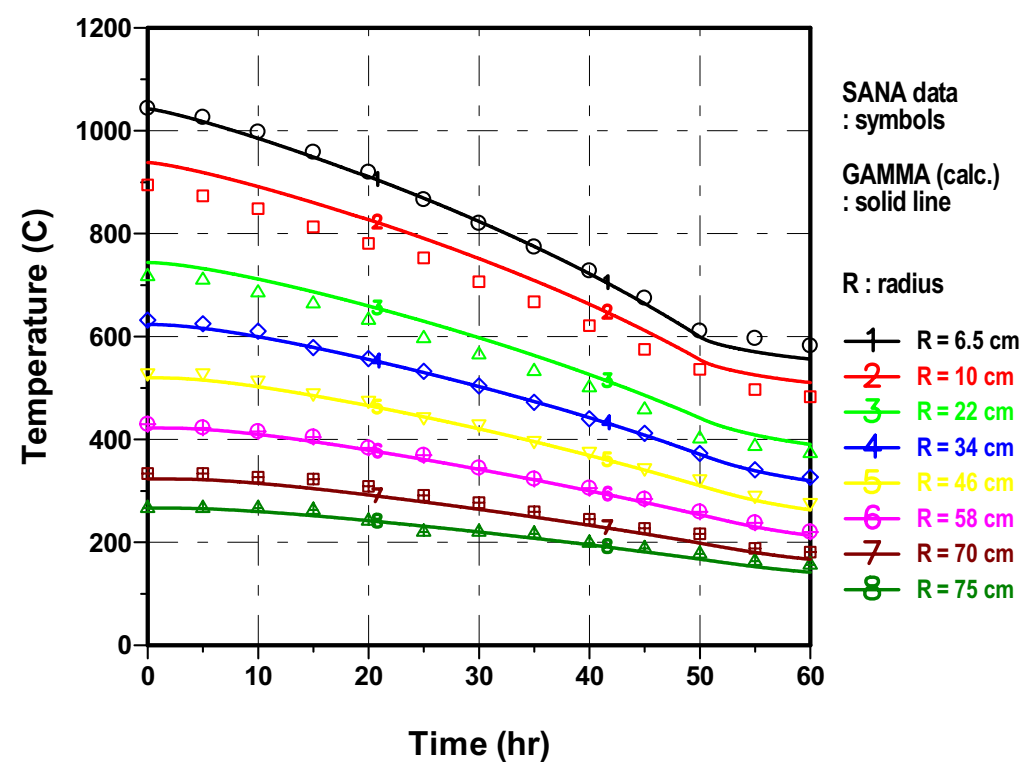




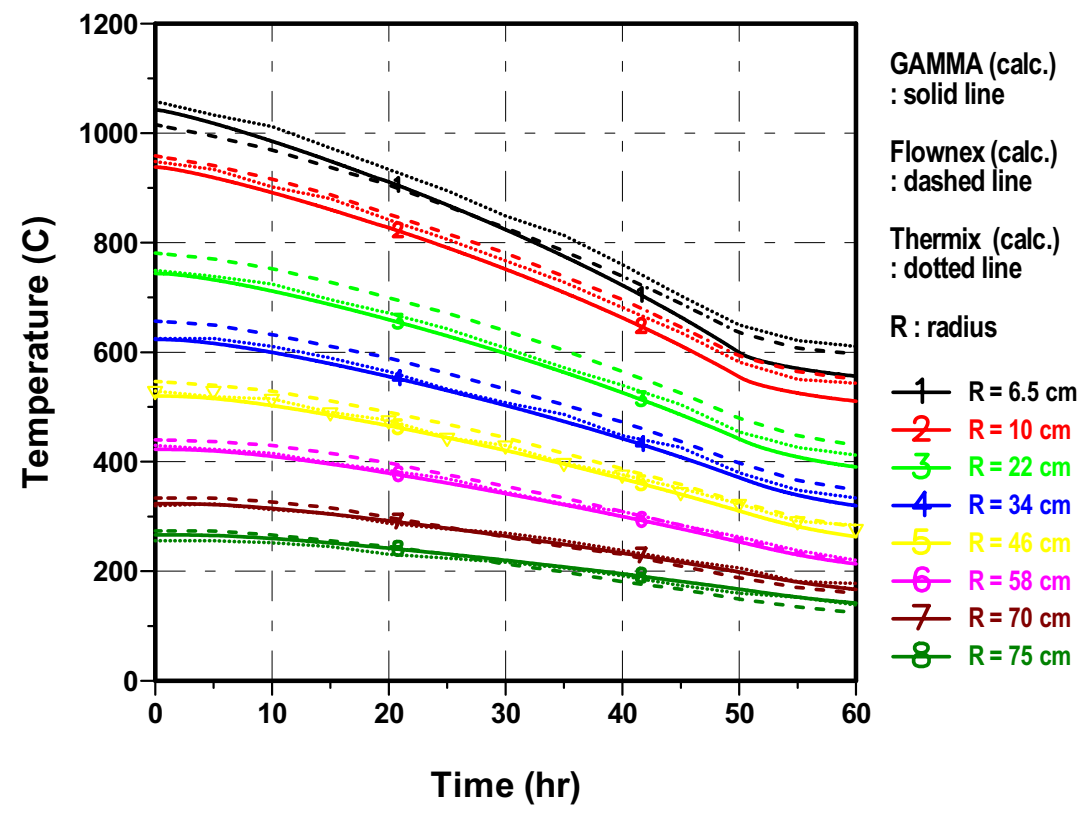

Figure 7-1-14 Predicted pebble temperatures for power ramp-down test: comparison with SANA data (above) and other analysis codes' results (below)

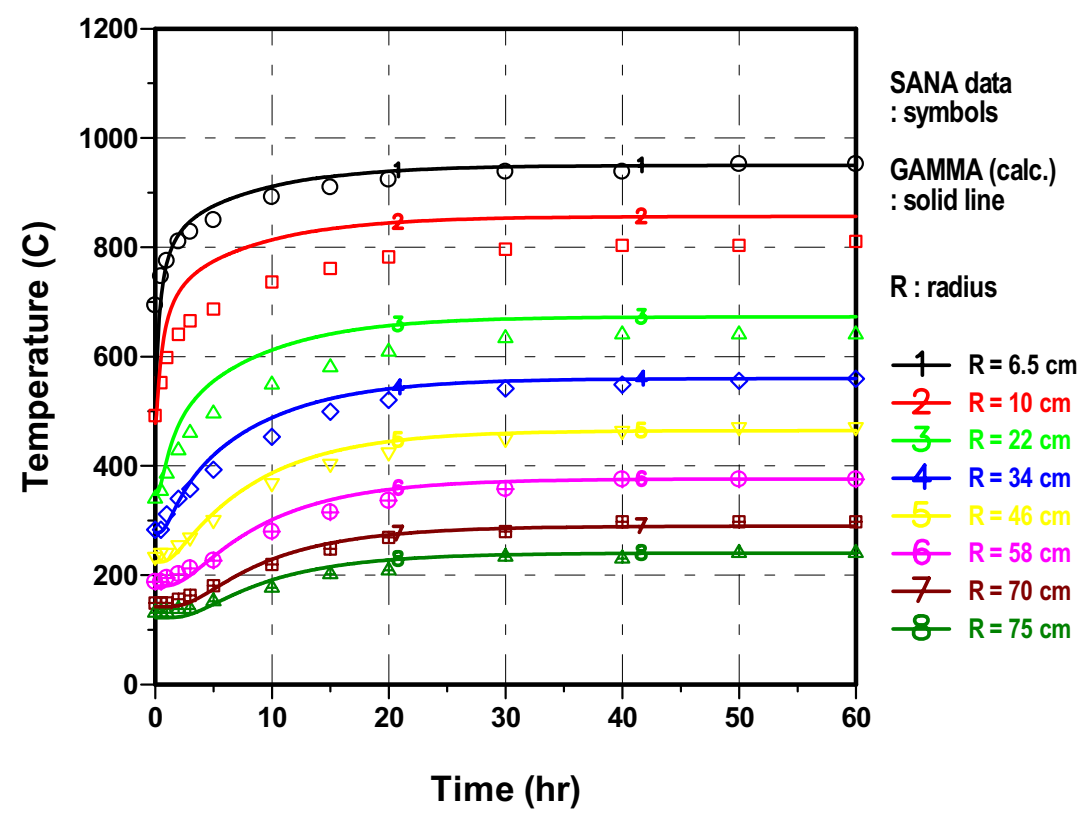




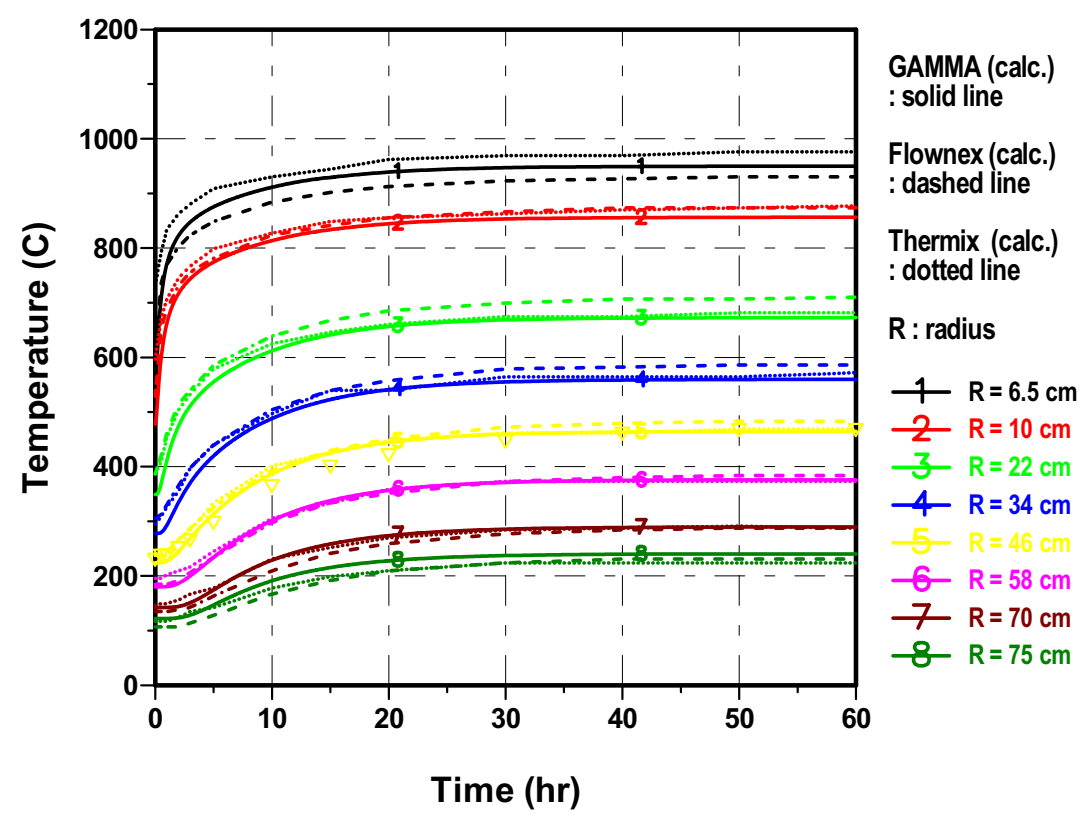

Figure 7-1-15 Predicted pebble temperatures for power step-up test: comparison with SANA data (above) and other analysis codes' results (below)

\subsubsection{RCCS $V \& V$}

Validation with Reactor Cavity Cooling System Heat Removal Tests

The ultimate decay heat removal in HTGRs is achieved by the reactor cavity cooling system, water-cooled or air-cooled. On the outside surface of the reactor vessel, the heat transferred from the core to the reactor vessel is passively removed by thermal radiation and free convection. The GAMMA code is applied to the assessment of the heat removal characteristics in the reactor cavity cooling system (RCCS), which performs the passive safe function to remove the afterheat under accident conditions. We selected two kinds of the RCCS heat removal experiments, SNU-RCCS and HTTR RCCS Mockup. We performed different simulation approaches: fine-mesh approach for the small-size and simple-designed SNU RCCS facility and coarse-mesh approach for the large-size and realistic-designed HTTR RCCS mockup facility.

\section{SNU RCCS Experiment}

The SNU RCCS experimental facility (IAEA-TECHDOC-1163 2000) shown in Figure 7-1-16 was designed to assess the effectiveness of the water-pool type RCCS. The facility consisted of the reactor vessel (RV) simulator containing six heater rods, the reactor cavity filled with air, and the water pool tanks surrounding the reactor vessel simulator. 

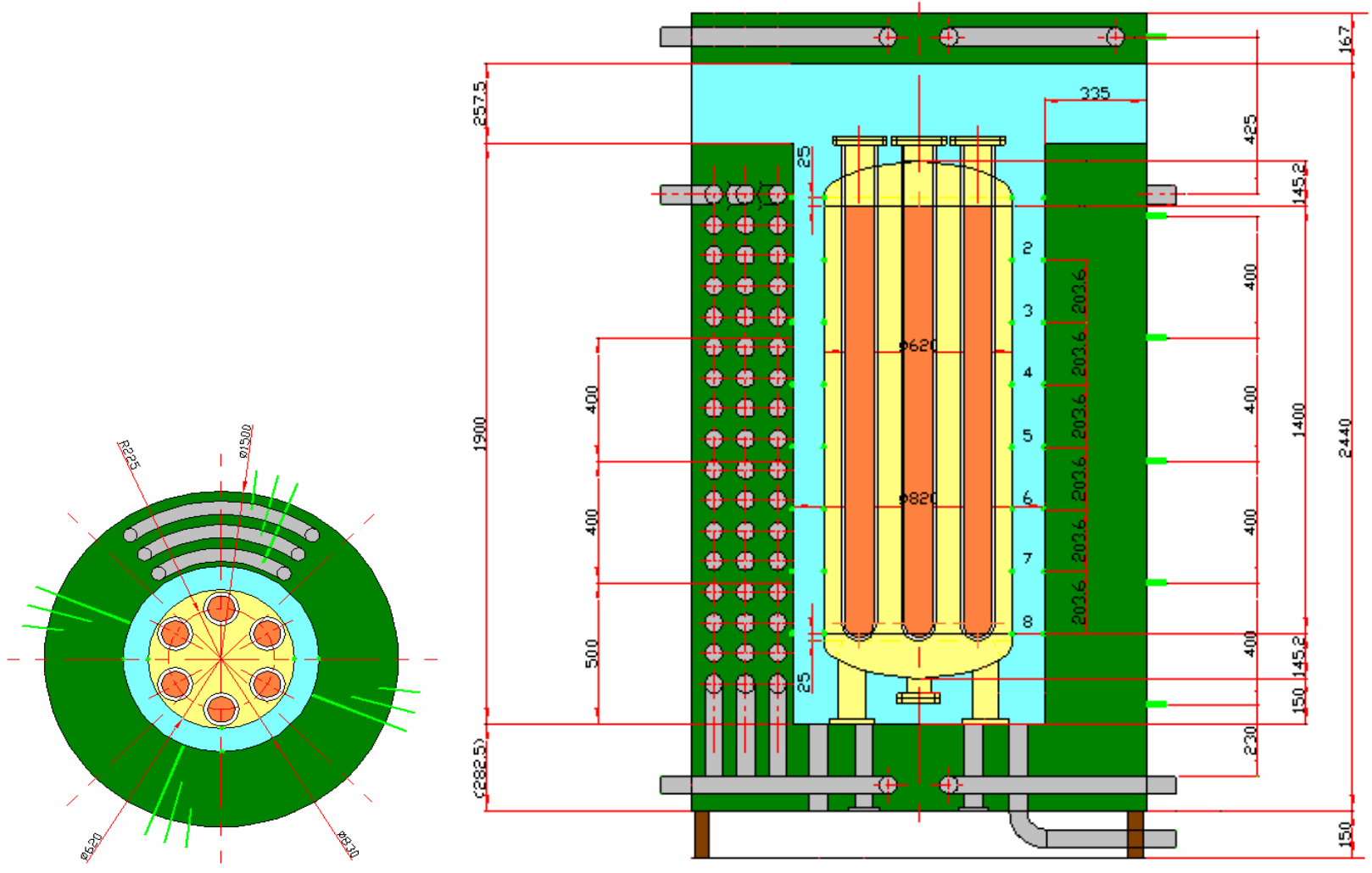

Figure 7-1-16 Schematics of the SNU RCCS Facility (top and side views)

We used the axi-symmetric mesh layout by assuming the rotational symmetry of the apparatus, as shown in Figure 7-1-17. And we considered the radiation heat transfer in each enclosure by using the irradiation/radiosity method of Eq. (2-7) for the gray and diffuse surface. In the simulation, the measured temperature distribution (Ts) of the cavity walls (CW) is used as a boundary condition. The contribution of the RV support legs to the heat removal is investigated using the axial heat conductor model shown in Figure 7-1-17. The axial conductor model considers the heat transport by conduction, radiation, and convection with the surrounding solid surfaces and the fluid. 

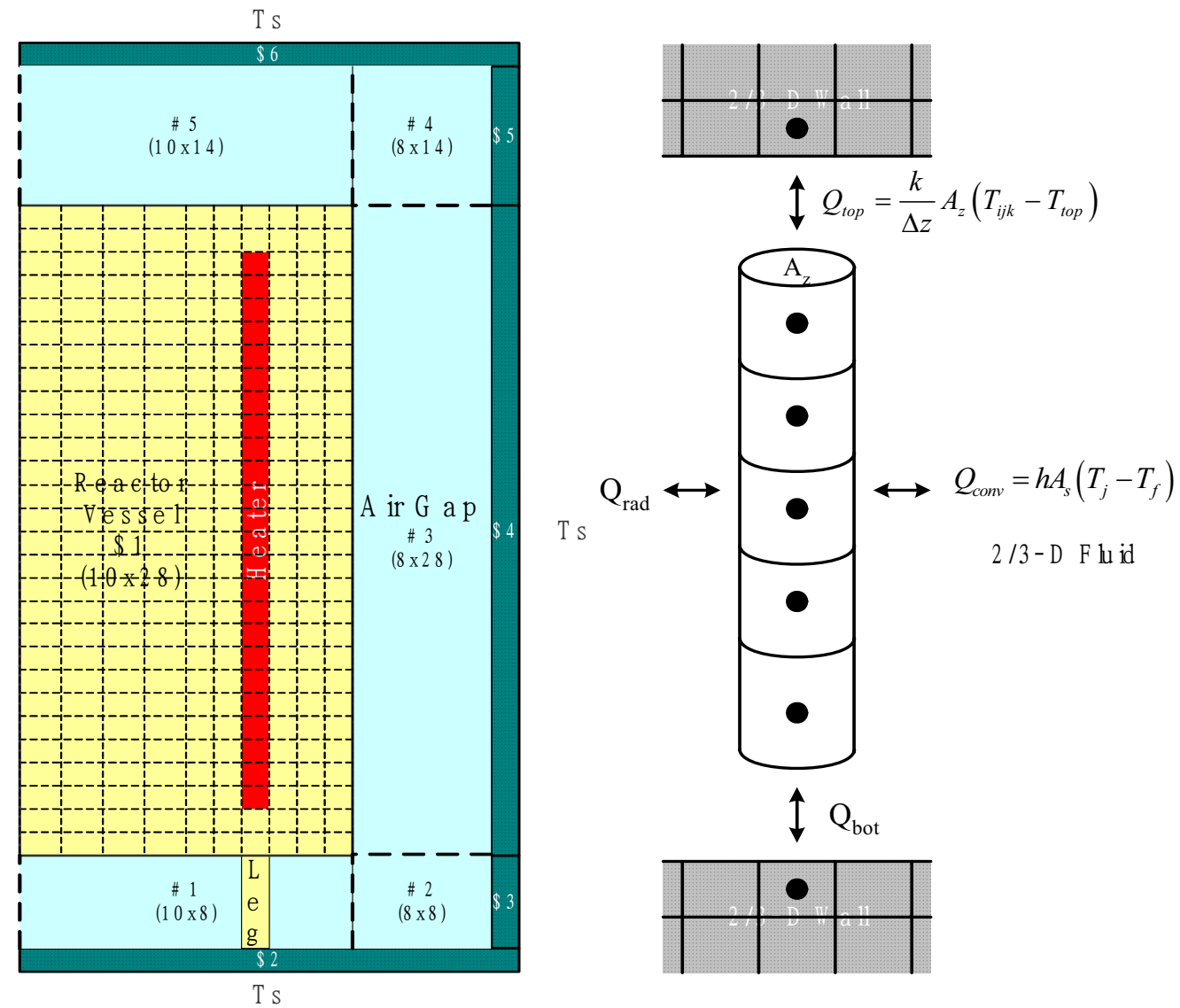

Figure 7-1-17 GAMMA Nodal scheme for the SNU RCCS simulation (left) and axial conductor model for the RV support legs (right)

The simulation is performed for the case with a total heater power of $25 \mathrm{~kW}$ and with the measured cavity wall temperature profile, the left-most curve shown in Figure 7-1-18. As shown in the figure, the RV surface temperatures are well predicted except at the bottom two measuring points. When the effect of the RV support legs is considered, the calculated temperatures become closer to the measured data. For this simulation case, the portions of total heat removed by radiation, convection, and conduction through the support legs on the RV surface are estimated to be $80 \%, 15 \%$, and 5\%, respectively. Figure 7-1-19 shows that the magnitude of the RV surface temperature changes linearly with the emissivity. 


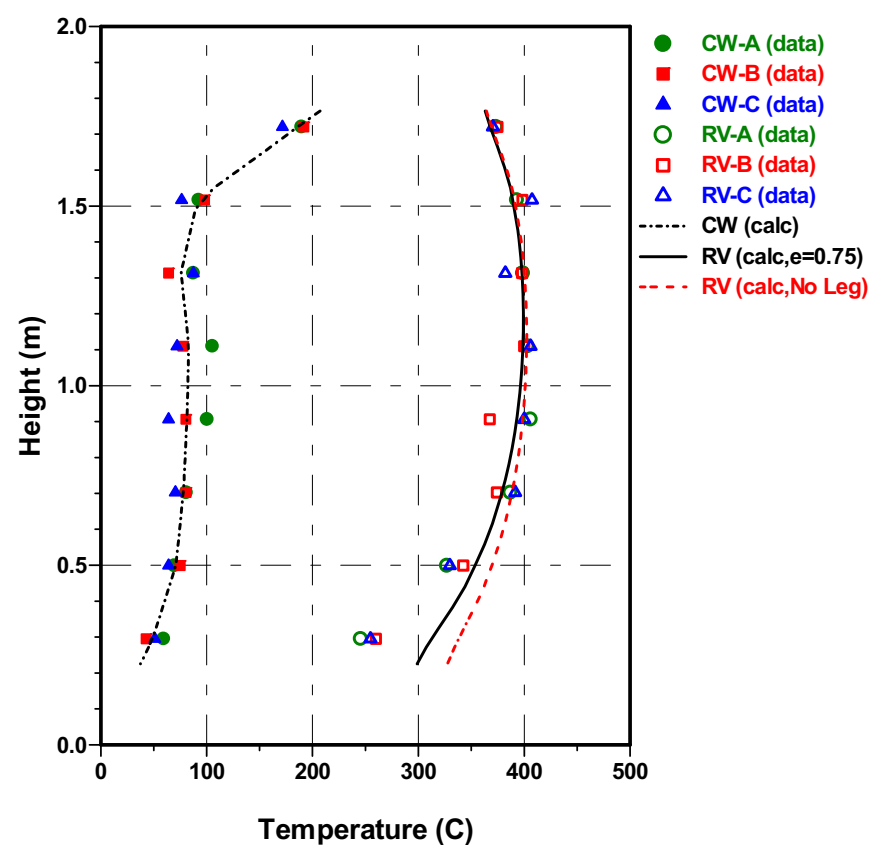

Figure 7-1-18 Predicted RV surface temperatures with/without RV support legs

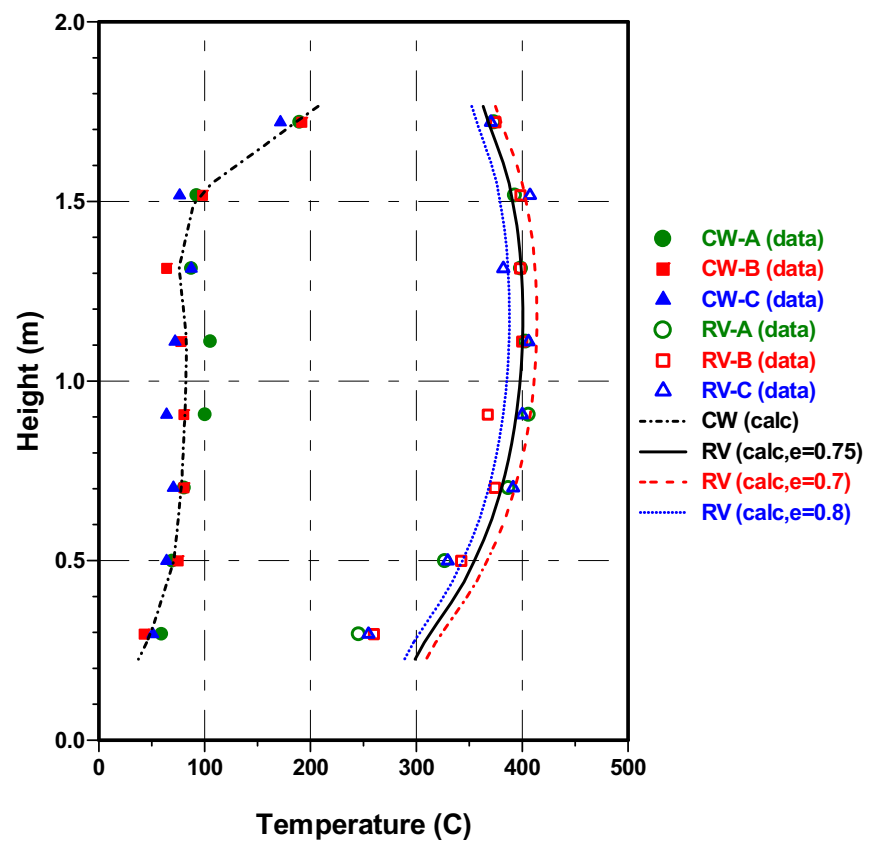

Figure 7-1-19 Predicted RV surface temperatures for different emissivities on the RV and CW surfaces 


\section{HTTR RCCS Mockup Experiment (IAEA Benchmark)}

Figure 7-1-20 shows the HTTR RCCS mockup test facility (IAEA 2000) consisted of a pressure vessel with 1 $\mathrm{m}$ in diameter and $3 \mathrm{~m}$ high, a heater block with $0.6 \mathrm{~m}$ in diameter and $2 \mathrm{~m}$ high simulating decay heat, the tube-type three cooling panels surrounding the pressure vessel, and the cavity wall with $2 \mathrm{~m}$ in diameter and 4 $\mathrm{m}$ high occupied by air at the atmospheric pressure. Table 7-1-3 shows all the tests conducted with different kinds of fluids in a pressure vessel (vacuum, $\mathrm{He}$, or $\mathrm{N}_{2}$ ) and cooling panels (air or water), total heating powers, axial power shapes, and pressure vessel top head with/without the stand pipes simulating the control element driving assembles.

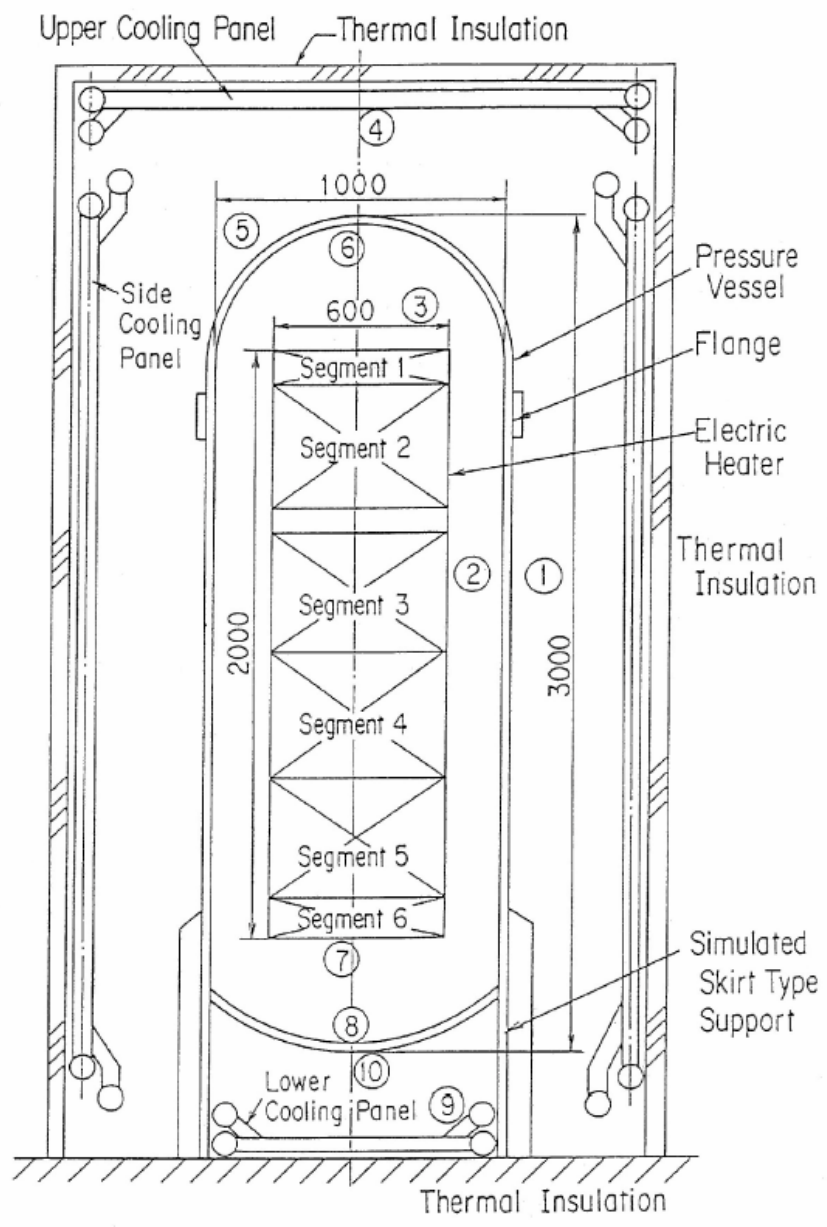

Figure 7-1-20 Schematic diagram of the HTTR RCCS mockup test facility

We used the axi-symmetric mesh layout by assuming the rotational symmetry of the apparatus, as shown in Figure 7-1-21, and considered the radiation heat transfer in each enclosure by using the irradiation/radiosity method of Eq. (2-7) for the gray and diffuse surface. In the simulation, the measured temperature distribution of cooling panels is used as a boundary condition. Since the heat removal contribution by natural convection is not negligible, the heat flux between the wall and the adjacent fluid cell are calculated by 


$$
\begin{aligned}
& q^{\prime \prime}=N u \frac{k_{f}}{\Delta x}\left(T_{w}-T_{f}\right) \text { and } \\
& N u=f(R a) \text { where } R a=\frac{\rho^{2} C p \Delta x^{3}\left(T_{w}-T_{f}\right)}{T_{f} k_{f} \mu_{f}}
\end{aligned}
$$

Table 7-1-3 Experimental cases conducted in the HTTR RCCS mockup test facility

\begin{tabular}{|l|l|l|l|l|l|l|l|}
\hline Test Cases & 1 & 2 & 3 & 4 & 5 & 6 & 7 \\
\hline Item of gas & Vacuum & $\mathrm{He}$ & $\mathrm{N}_{2}$ & $\mathrm{He}$ & $\mathrm{He}$ & $\mathrm{He}$ & $\mathrm{He}$ \\
\hline Pressure, MPa & $1.3 \mathrm{e}-6$ & 0.73 & 1.1 & 0.47 & 0.64 & 0.96 & 0.98 \\
\hline Heat input, kW & 13.14 & 28.79 & 93.93 & 77.54 & 29.71 & 2.58 & 7.99 \\
\hline Heater segment, & & & & & & & \\
$\mathrm{kW}$ & 1.01 & 1.16 & 5.90 & 5.63 & 1.80 & 0 & 0 \\
No. 1 & 2.31 & 3.11 & 16.05 & 19.60 & 5.23 & 0 & 0 \\
No. 2 & 2.64 & 3.52 & 19.88 & 2.59 & 5.68 & 0 & 0 \\
No. 3 & 2.46 & 5.10 & 22.24 & 22.70 & 11.26 & 0 & 0 \\
No. 4 & 3.76 & 10.42 & 22.13 & 0 & 0 & 0 & 0 \\
No. 5 & 0.96 & 5.49 & 7.72 & 8.00 & 5.74 & 2.58 & 7.99 \\
No. 6 & & & & & & & \\
& & & & & & \\
\hline Cooling panel & Water & Water & Water & Water & Air & Air & Air \\
\hline Stand pipes & No & No & No & With & With & With & With \\
\hline \hline
\end{tabular}

In the GAMMA mesh scheme, since the coarse meshes are used near the wall surface, proper empirical correlations are selected for the prediction of the convective heat transfer and applied to specific surface types as follows: 


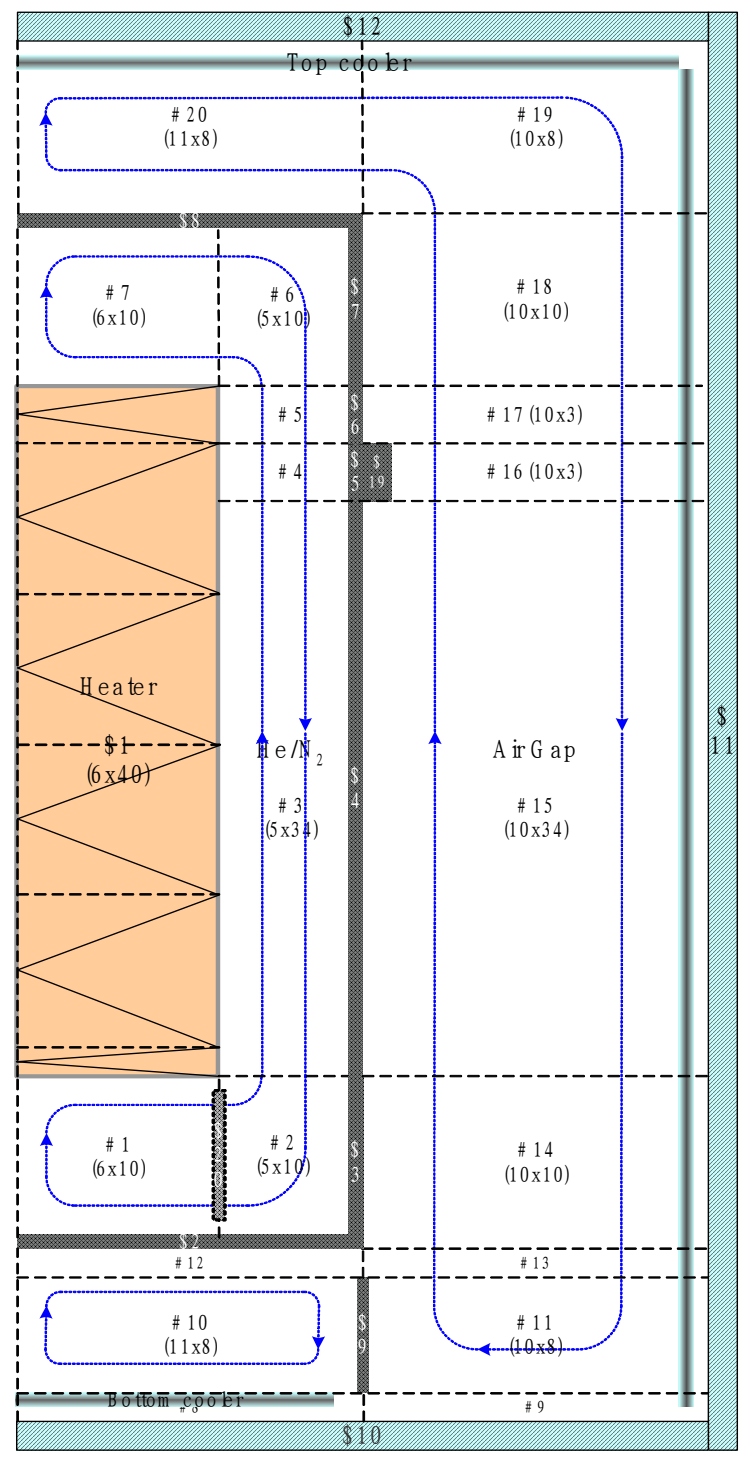

Figure 7-1-21 Nodalization diagram of the HTTR RCCS mockup test facility

(a) Thomas and de Vahl Davis's correlation (Keyhani et al. 1983) for the annular cavities between the outside surface of the heater and the inside surface of the pressure vessel and between the outside surface of the pressure vessel and the inside surface of the cavity wall.

$N u=0.286 R a^{0.258} H^{-0.238} K^{0.442}$ where $K=r_{o} / r_{i}, H=L /\left(r_{o}-r_{i}\right)$

(b) Fishenden and Saunders's correlation (Incropera and DeWitt 2003) at the bottom and top surfaces of the heaters and at the bottom and top head surfaces of the pressure vessel.

For the bottom-faced cold and top-faced hot,

$N u=0.54 R a^{0.25}(\mathrm{Ra} \leq 1 \mathrm{e} 7)$
$N u=0.15 R a^{0.33}(\mathrm{Ra}>1 \mathrm{e} 7)$

For the bottom-faced hot and top-faced cold,

$N u=0.27 R a^{0.25}$ 
(c) Churchill and Chu's correlation (Incropera and DeWitt 2003) for the external surface of the cooling panel tubes

$$
N u=\left\{0.825+\frac{0.387 R a^{1 / 6}}{\left[1+(0.492 / \mathrm{Pr})^{9 / 16}\right]^{8 / 27}}\right\}^{2}
$$

(d) An assumed value of $\mathrm{Nu}=10$ for the pin-type pressure vessel flange.

Figure 7-1-22 shows the calculated surface temperature profiles for one representative case (vacuum in a pressure vessel). The temperatures of the pressure vessel are well predicted by GAMMA while there are considerable scatters in the results of other analysis codes, as shown in Figure 7-1-23. Therefore it can be demonstrated that the GAMMA code is applicable to evaluate the hot spots on the pressure vessel and heat removal by thermal radiation and natural convection.

Figure 7-1-24 shows the relative contribution of thermal radiation to total heat removed from the outside of the pressure vessel. The calculated ratio of heat transferred by thermal radiation to the total heat input is about $68 \%-97 \%$, comparable to that of the other analysis codes. It has been observed that radiation heat increases with the increase in temperature and pressure. The GAMMA code predicts well the heat removal by free convection while some of other analysis codes slightly underestimate it.
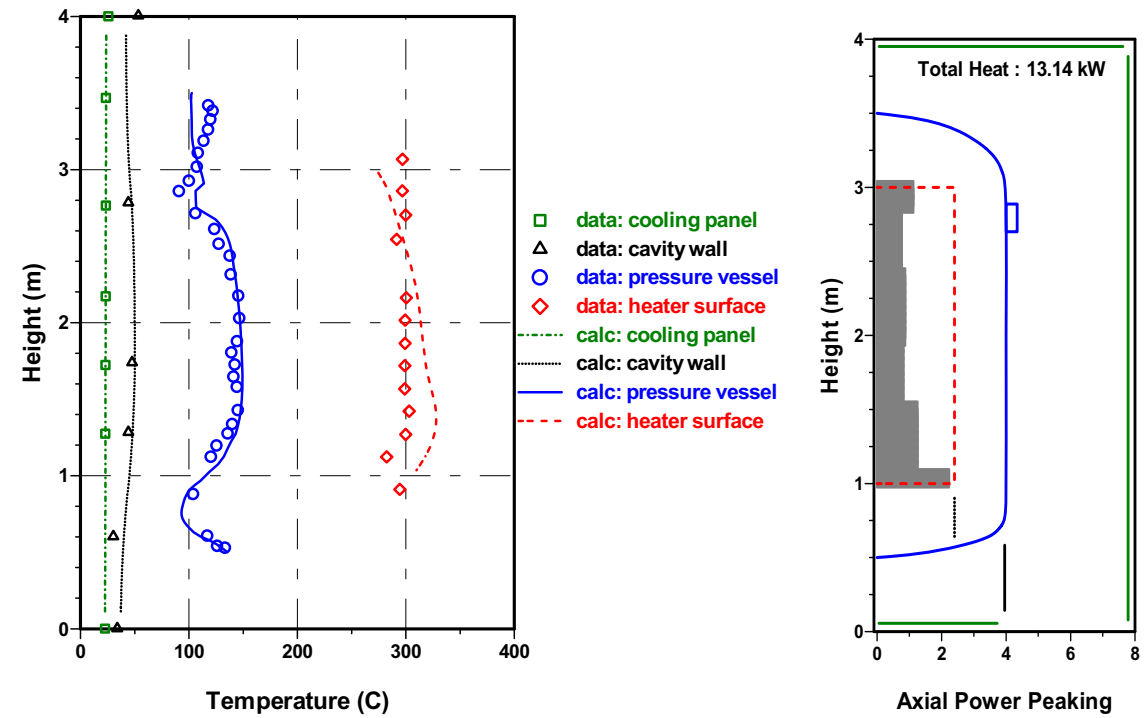

Figure 7-1-22 Calculated results of surface temperature profiles of heater, pressure vessel, and cavity wall for the vacuum case 1 


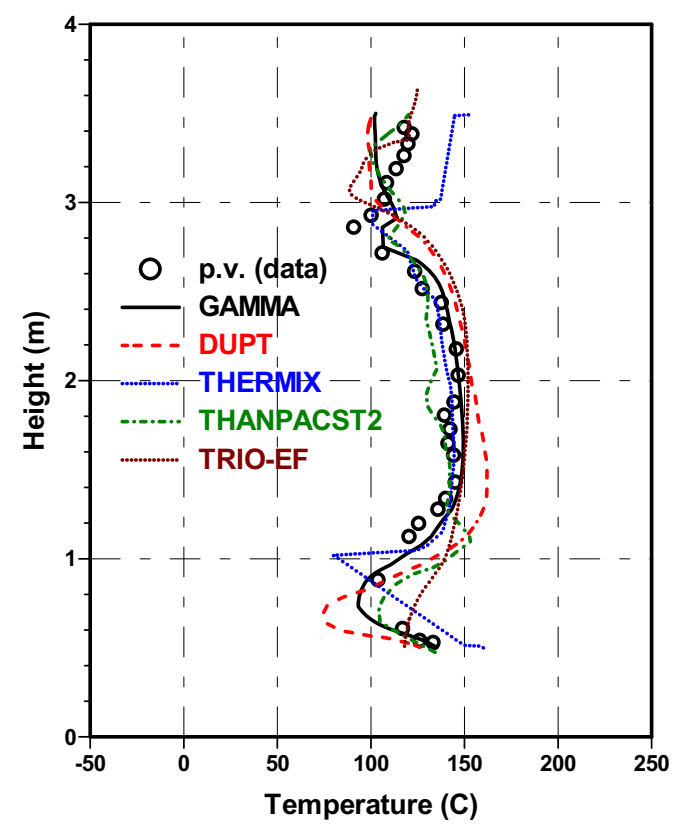

Figure 7-1-23 Comparison of the predicted pressure vessel temperature with the results of other analysis codes for the vacuum case 1

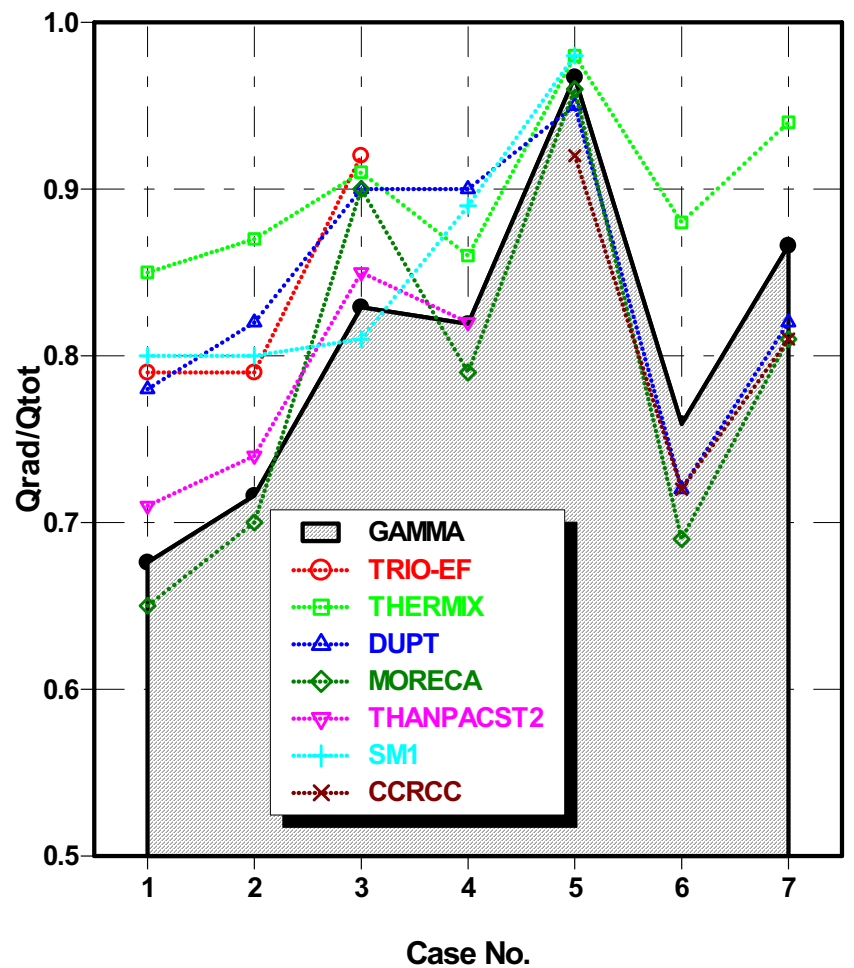

Figure 7-1-24 Calculated relative contributions of thermal radiation to total heat removal for all the test cases 


\subsubsection{Full-Plant Simulation}

\section{Consequences of Air Ingress}

An air ingress accident is one of the most common modes of accidents related to the basic design regarding an HTGR. The accident is initiated by a guillotine-ended break of the main co-axial pipes of the connecting part between the reactor vessel and the heat extraction or power conversion system. After the break, the flow passages inside the reactor vessel and the pressure and species distributions are illustrated in Figure 7-1-25. Based on the phenomenological characteristics, the air ingress accident can be categorized as 3 phases: blowdown phase, molecular diffusion phase, and natural convection phase. Table 7-1-4 lists the major processes in each phase.

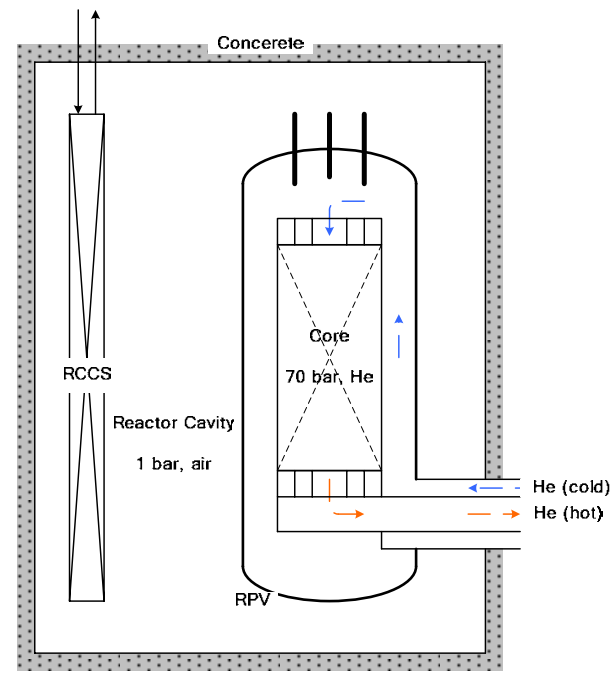

(a) Normal Operation

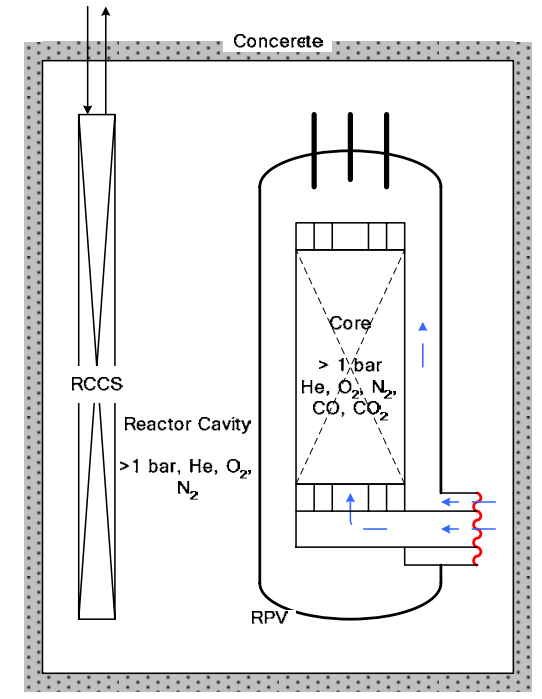

(c) Molecular Diffusion Phase

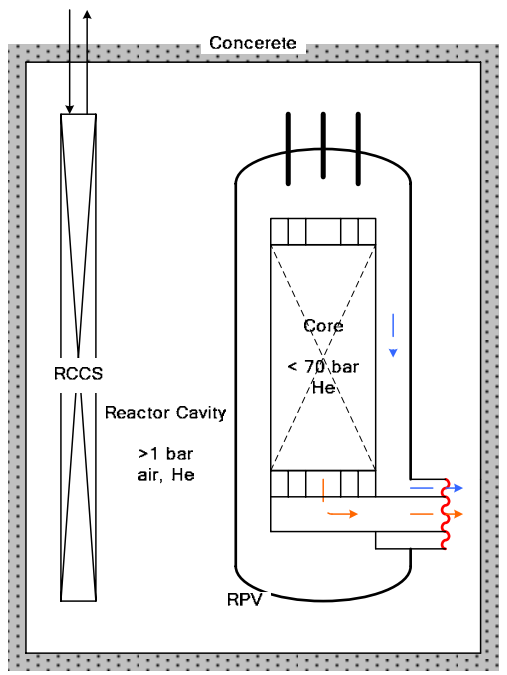

(b) Blowdown Phase

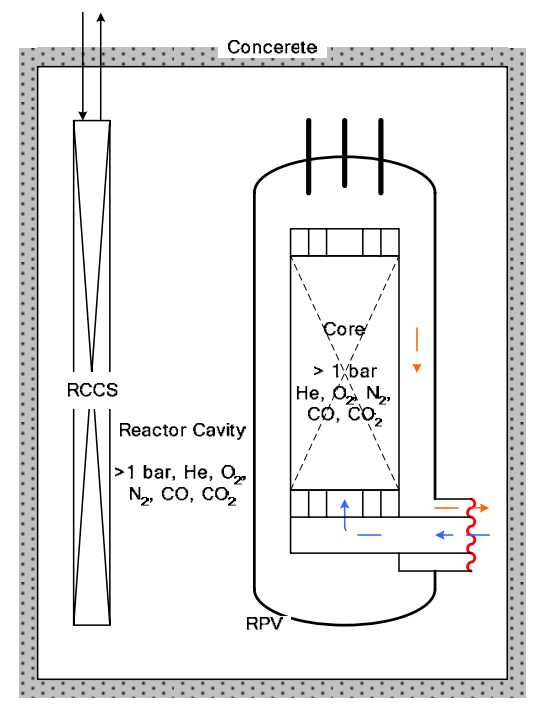

(d) Natural Convection Phase

Figure 7-1-25 Air ingress behaviors during the three phases following the guillotine break of co-axial pipes

Table 7-1-4 Major processes of each phase during an air ingress accident 


\begin{tabular}{|c|c|}
\hline Phases & Major Processes \\
\hline Blowdown & $\begin{array}{l}\text { - Event initiated by double ended break of co-axial pipes } \\
\text { - System depressurizes and reactor trips immediately } \\
\text { - He discharge into reactor cavity stops when pressure equalizes } \\
\text { - Rapid heat-up of core }\end{array}$ \\
\hline $\begin{array}{l}\text { Molecular } \\
\text { diffusion }\end{array}$ & $\begin{array}{l}\text { - Air in reactor cavity enters reactor vessel by molecular diffusion, } \\
\text { thus, graphite chemical reaction rate is very slow } \\
\bullet \text { Very weak natural circulation inside core } \\
\text { - First peak of fuel temperature, then it starts to decrease by the } \\
\text { conduction cooling and radiation to RCCS }\end{array}$ \\
\hline $\begin{array}{c}\text { Natural } \\
\text { convection }\end{array}$ & $\begin{array}{l}\text { - Large amount of air ingress at onset of natural circulation by } \\
\text { density difference of air } \\
\text { - Second peak of fuel temperature by active graphite reaction } \\
\text { - When air is depleted, graphite chemical reaction stops } \\
\text { - Core cools down to safe shutdown state, by heat removal by } \\
\text { conduction cooling and radiation to RCCS }\end{array}$ \\
\hline
\end{tabular}

When the postulated guillotine break of the coaxial pipes happens, a reactor trips immediately and, as the high-pressure helium gas is discharged into the reactor container through the breach, the reactor vessel depressurizes rapidly. After a few minutes, the gas pressure is balanced between the inside and outside of the reactor vessel. During this blowdown phase, air is unable to enter the reactor core from the breach.

After the helium depressurization, air in the reactor cavity enters the reactor vessel through the breach, due to molecular diffusion and a weak natural convection induced by the non-uniform temperature distribution. During this molecular diffusion phase, the air transport rate is very low; therefore, this process continues for a very long time. It is possible that carbon monoxide $(\mathrm{CO})$ and dioxide $\left(\mathrm{CO}_{2}\right)$ are produced in the reactor, because the oxygen $\left(\mathrm{O}_{2}\right)$ contained in air chemically reacts with the high temperature graphite structures. During this stage, fuel is heated up due to the mismatch between the decay heat and the heat removal through the reactor cavity coolers. After reaching the first peak, the fuel temperature begins to decrease slowly, because of continuous heat removal by passive cooling mechanisms.

As air ingresses into the reactor core, the density of the gas mixture in the reactor core gradually increases. When the buoyancy force becomes large enough to overcome the gravitational force, global natural convection eventually takes place in the reverse direction of a normal-operating flow path and the natural convection phase begins. Since the graphite oxidation is very active due to high rate of air inflow during this phase, the graphite fuel temperature rapidly increases and the graphite components are rapidly gasified. As a result, several days following the break, as shown in Figure 7-1-26, a fuel re-heatup may take place due to the exothermic heat generated by the graphite oxidation accompanied by air ingress though the breach. As oxygen is rapidly depleted due to high natural convection flow and active chemical reactions, the graphite reaction stops and then the decay heat continues to be removed by conduction and radiation heat transfer to the reactor cavity cooling system (RCCS). Eventually, the long-term core cooldown stage is established. 


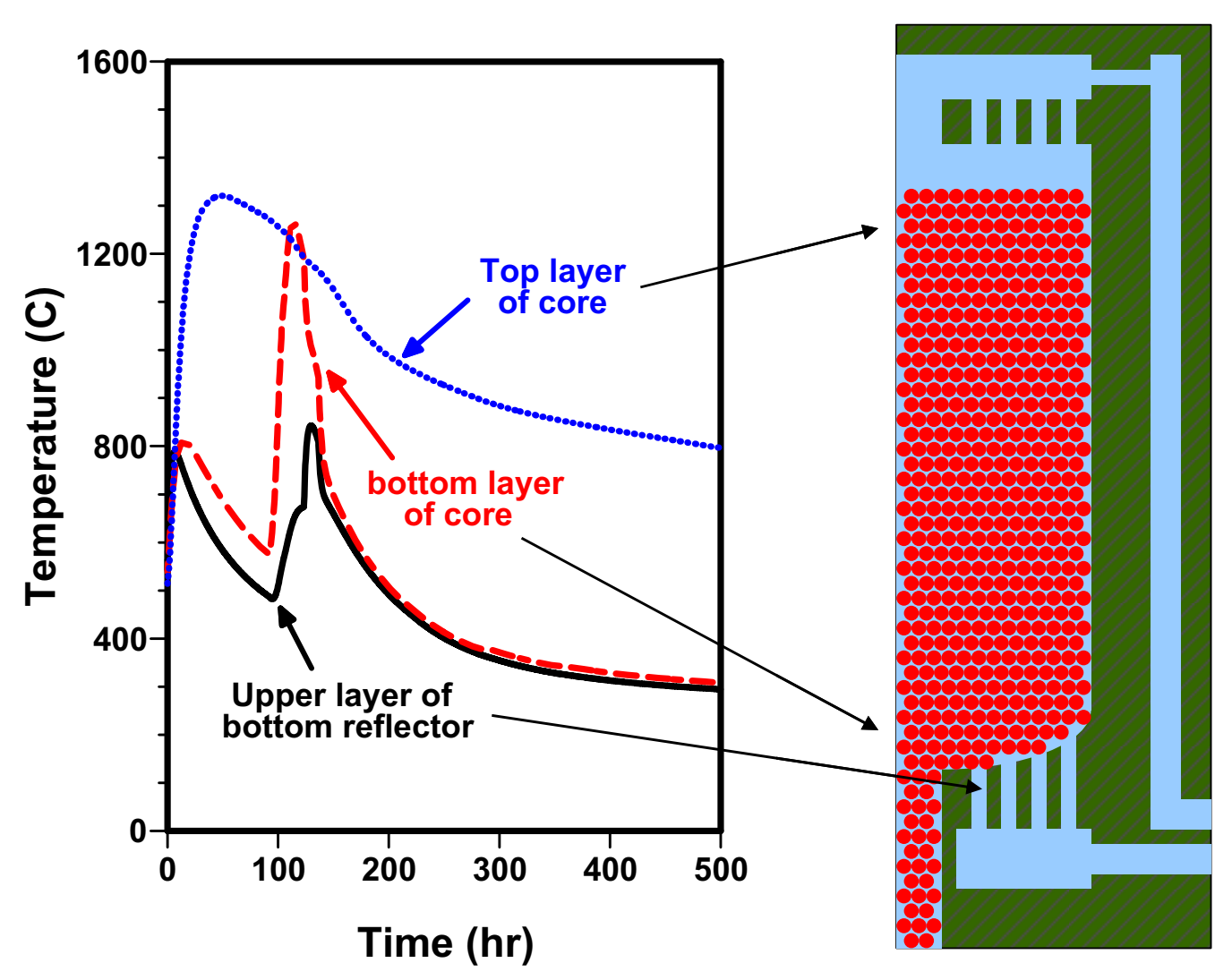

Figure 7-1-26 Core and reflector temperatures expected during the air ingress accident

\section{Pebble-Bed Core Gas-Cooled Reactor}

The GAMMA code is applied to the analysis of the air ingress accident following a double-ended break of the coaxial pipes for a pebble-bed gas-cooled reactor. We selected PBMR $268 \mathrm{MWt}$ (Reitsma et al. 2004) as a reference reactor and performed the sensitivity analyses on the air volumes in a vault, the onset timings of natural convection, and the coupling of a vault and the reactor coolant system.

System Modeling and Assumptions

In the PBMR $268 \mathrm{MWt}$, helium at $500^{\circ} \mathrm{C}$ and $7 \mathrm{MPa}$ enters the pebble core through the riser holes and exits at $900^{\circ} \mathrm{C}$ and a flow rate of $129 \mathrm{~kg} / \mathrm{s}$. Figure 7-1-27 shows the schematic diagram of the PBMR and its arrangement in a reactor cavity. The circular pebble-bed core consists of approximately 333,000 fuel spheres and 110,000 graphite spheres in the central reflector zone. The water-cooling RCCS installed in a reactor cavity removes passively the core decay heat. 


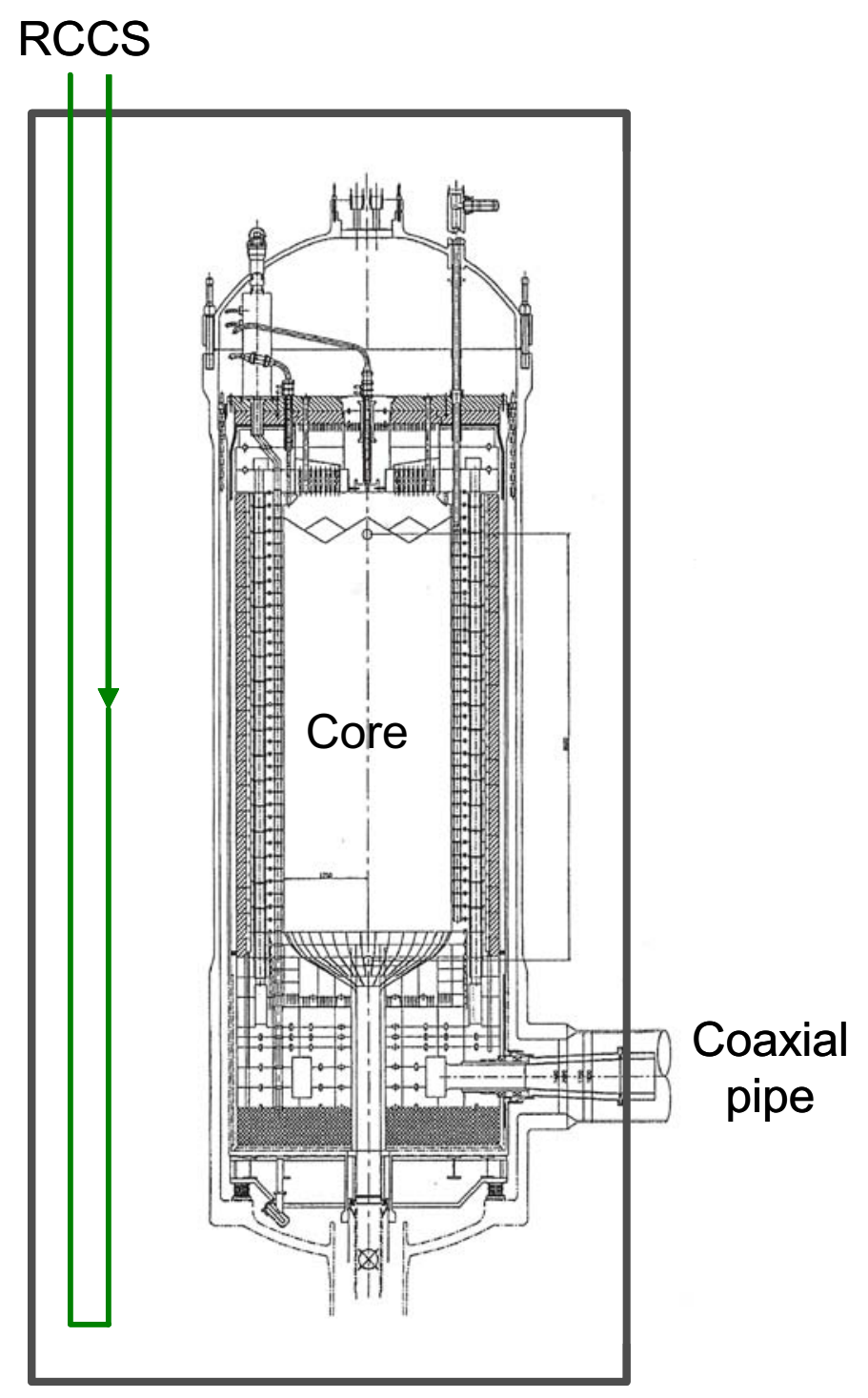

Figure 7-1-27 Schematic diagram of PBMR $268 \mathrm{MWt}$

Figure 7-1-28 shows the GAMMA modeling of PBMR where 2-D geometry models are used for the pebble core and the reactor cavity to consider heat removal by natural convection flow, and for all the solid structures including the pebbles to consider multi-dimensional heat conduction. In all the cavities or plenums, the radiation heat exchanges are considered. For the water cooling RCCS, constant temperature of $80^{\circ} \mathrm{C}$ is assumed to be maintained during the transient. Following the accident, since a reactor trips immediately, the core power is determined directly from the German decay heat curve. 

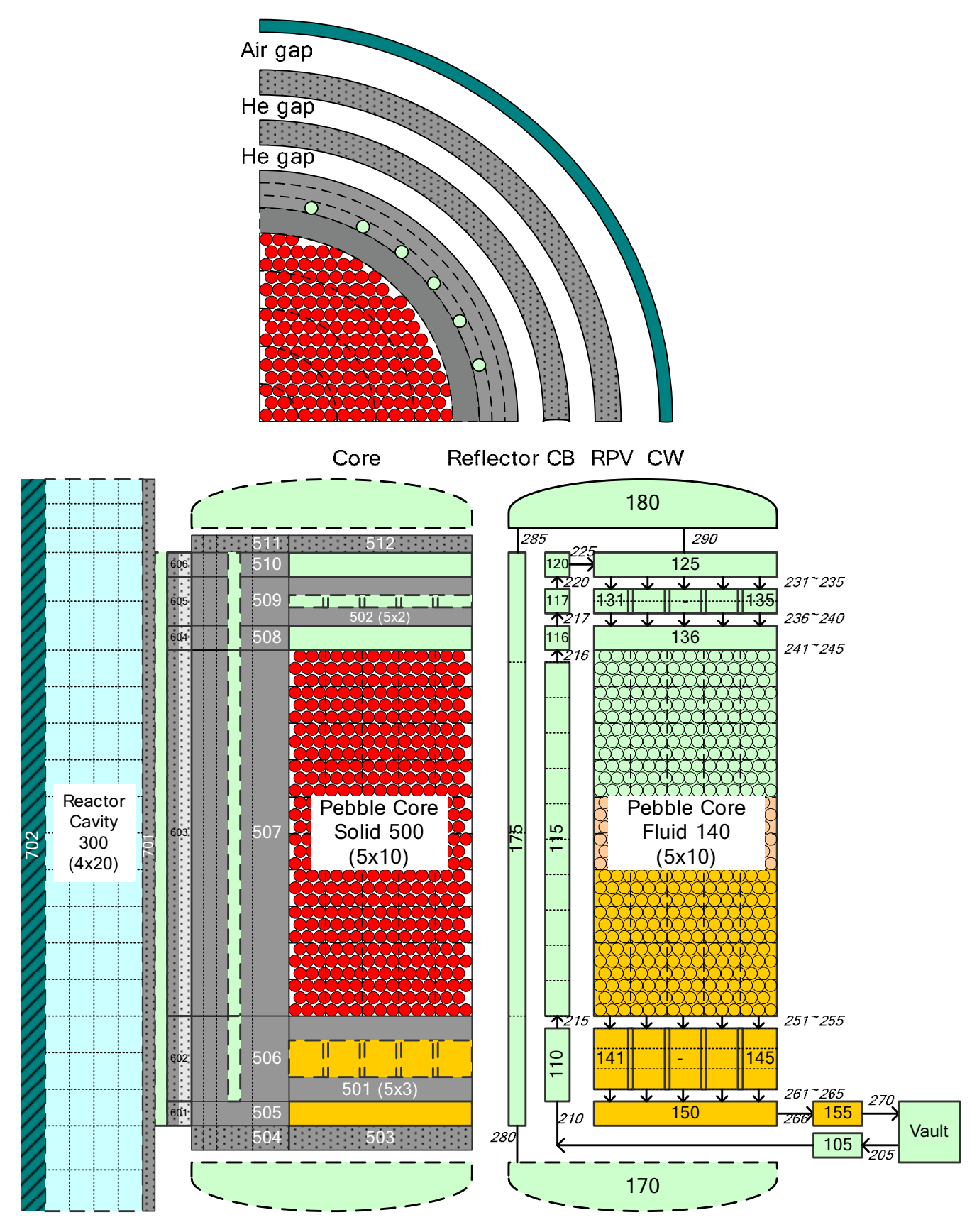

Figure 7-1-28 GAMMA nodal scheme for PBMR $268 \mathrm{MWt}$

Analysis Results and Discussions

The base case run has been performed with the air volume of $50,000 \mathrm{~m}^{3}$ (German HTR-module data) in a vault. Following the break, the discharged helium mixes with air in a vault and therefore, as shown in Figure 7-1-29, the oxygen concentration in a vault drops quickly and then decreases very slowly during the molecular diffusion phase. When the global natural convection occurs at about $70 \mathrm{hrs}$, the air ingress rate rapidly increases. As the oxygen is continuously consumed by the chemical reactions within the core and reflectors, the oxygen concentration in a vault begins to decrease. Hence the $\mathrm{CO}$ and $\mathrm{CO}_{2}$ transported out of the reactor begin to accumulate in a vault. Due to the large air ingress rate at about 70 hours, the graphite 
oxidation becomes active, producing the additional heat. Therefore, the bottom reflector and the pebbles in the core bottom region undergo a rapid increase in temperature as shown in Figure 7-1-30. However, the peak fuel temperature remains below the fuel failure criterion $\left(1600^{\circ} \mathrm{C}\right)$, as shown in Figure 7-1-31. As air is depleted by chemical reactions, heat generation by the graphite oxidation stops and then temperature continues to decrease by the conduction cooling and thermal radiation to RCCS.

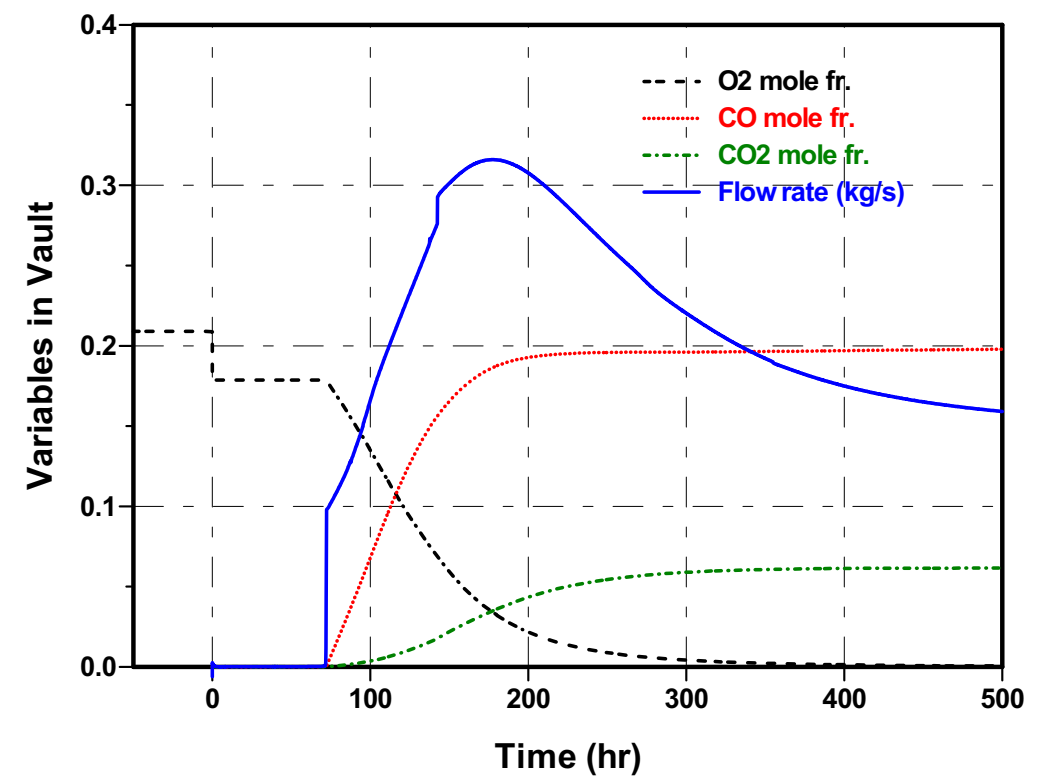

Figure 7-1-29 Air ingress rate and species concentrations in a vault for the PBMR $\left(\mathrm{V}_{\text {air }}=50,000 \mathrm{~m}^{3}\right)$

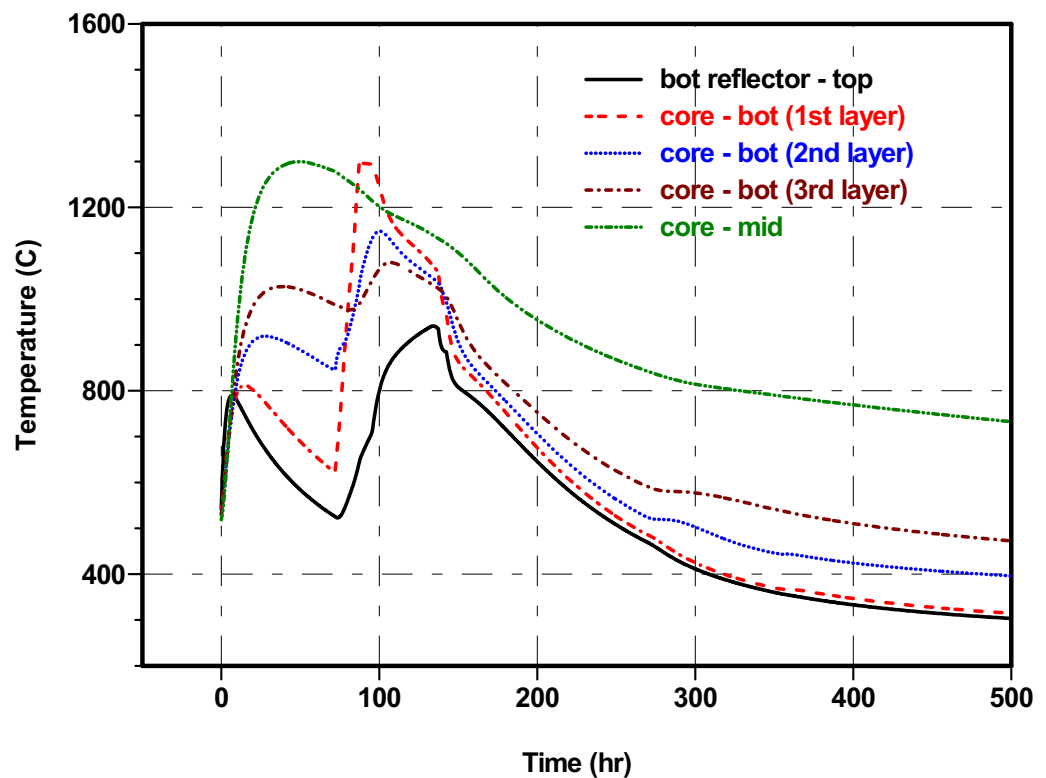

Figure 7-1-30 Core and reflector temperatures for the PBMR $\left(\mathrm{V}_{\text {air }}=50,000 \mathrm{~m}^{3}\right)$ 


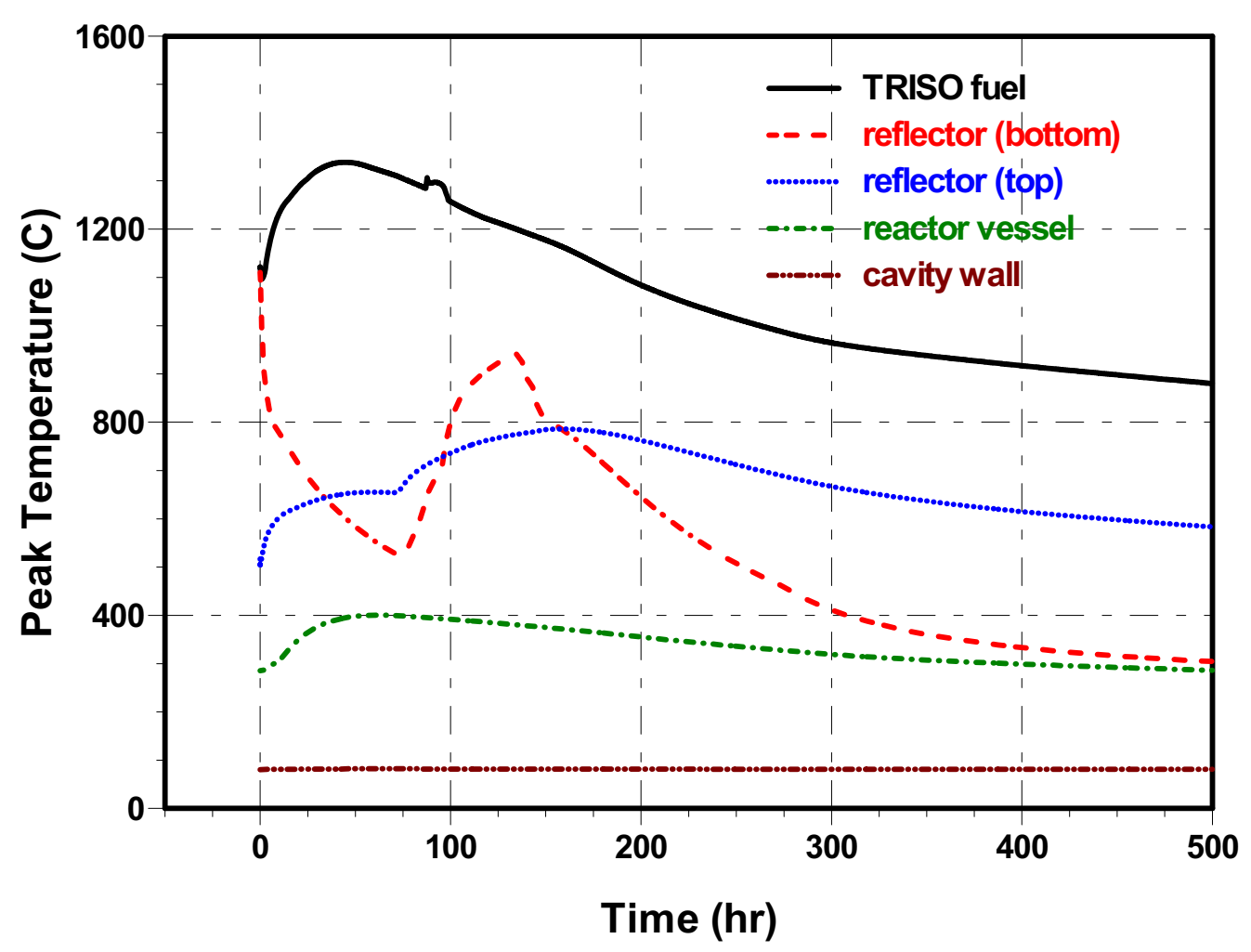

Figure 7-1-31 Peak temperatures for the PBMR (Vair=50,000 m3)

The sensitivity analysis on the air volumes, as shown in Figures 7-1-32 and 7-1-33, shows that, as the air volume in a vault decreases, the onset time of natural convection is delayed gradually because of lower air concentration in a vault during the molecular diffusion phase. The air ingress rate highly depends upon the vault mixture density determined by pressure, temperature, and concentrations of each species. Meanwhile, in the infinite air volume case, the air ingress rate depends upon the temperature distribution only within the reactor vessel. It explains why the air flow rate transient of the infinite air volume case is much different from those of the finite air volume cases. As shown in Figures 7-1-34 and 7-1-35, it is believed that the temperature rise in the fuel pebbles and the bottom reflector are mainly determined by the air ingress flow rate. The temperature rise is slowed down by the decreasing oxygen concentration in a vault. 


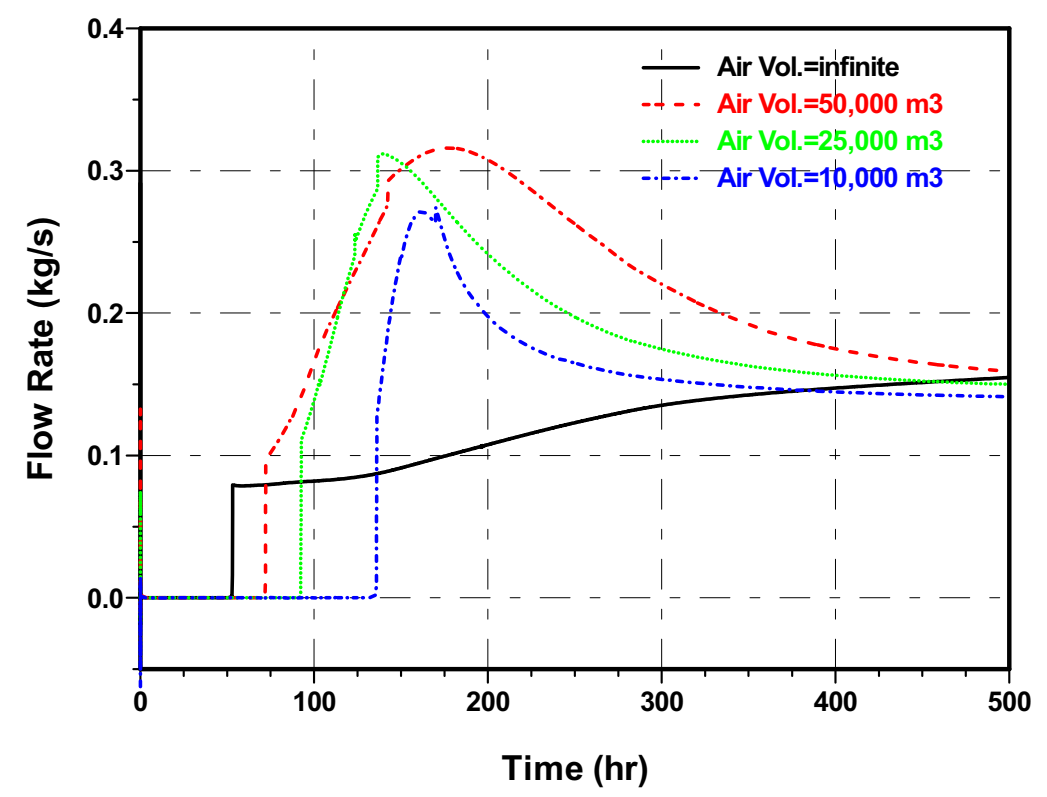

Figure 7-1-32 Air ingress rates for the sensitivity on the air volumes in a vault

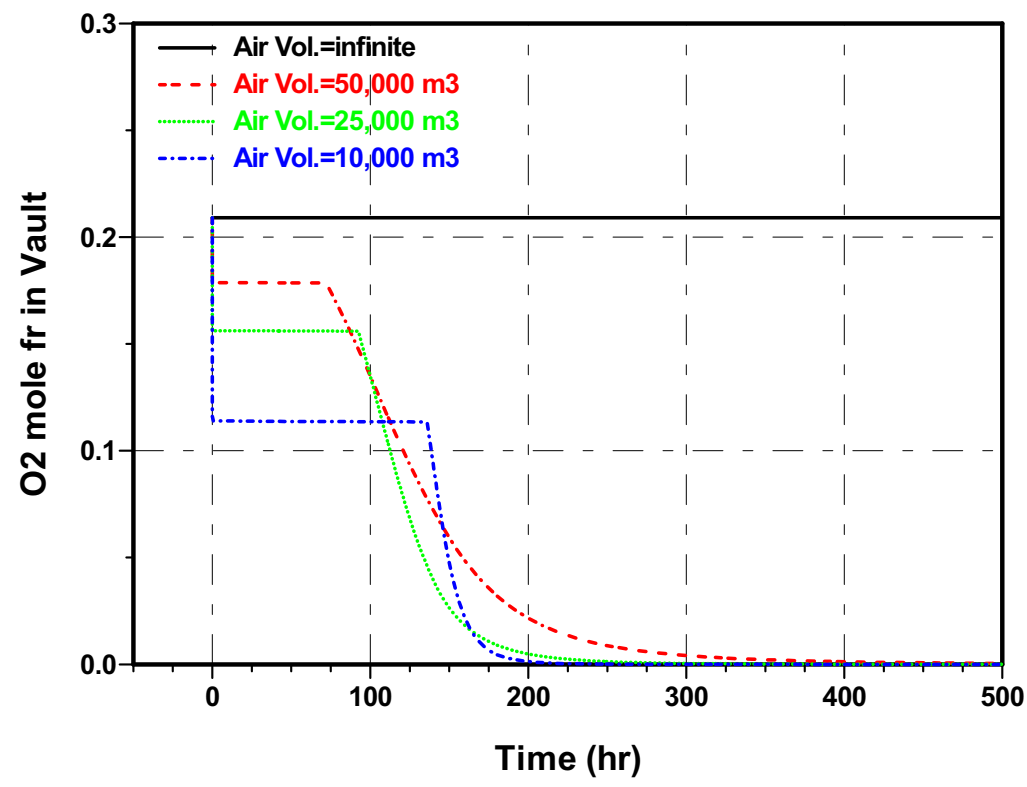

Figure 7-1-33 Oxygen concentrations for the sensitivity on the air volumes in a vault

Here a question arises: what happens if the natural convection occurs earlier than the predicted OTNC due to a background noise, an unintended pressure pulse, internal bypass flow path, or local circulation? The earlier OTNC can be simulated by applying a pressure pulse at the specified time, as shown in Figures 7-1-36 and 71-37. From the figures, it is found that the earlier OTNC is less serious from the view of peak fuel temperature. However, it is believed that the earlier OTNC is more serious from the view of bottom reflector corrosion. If the OTNC occurs early, since the bottom reflector is hotter, more oxygen is consumed through the bottom reflector and therefore the less amount of oxygen reaches the graphite fuel. Meanwhile, the later OTNC case simulated by reducing the molecular diffusion coefficient shows the highest peak in fuel temperature. When the bottom reflector temperature is low at the initiation time of natural convection, the 
temperature rise in the core is more significant. It is because more oxygen survives through the bottom reflector by increasing the graphite oxidation rate of fuel pebbles and eventually the fuel temperature.

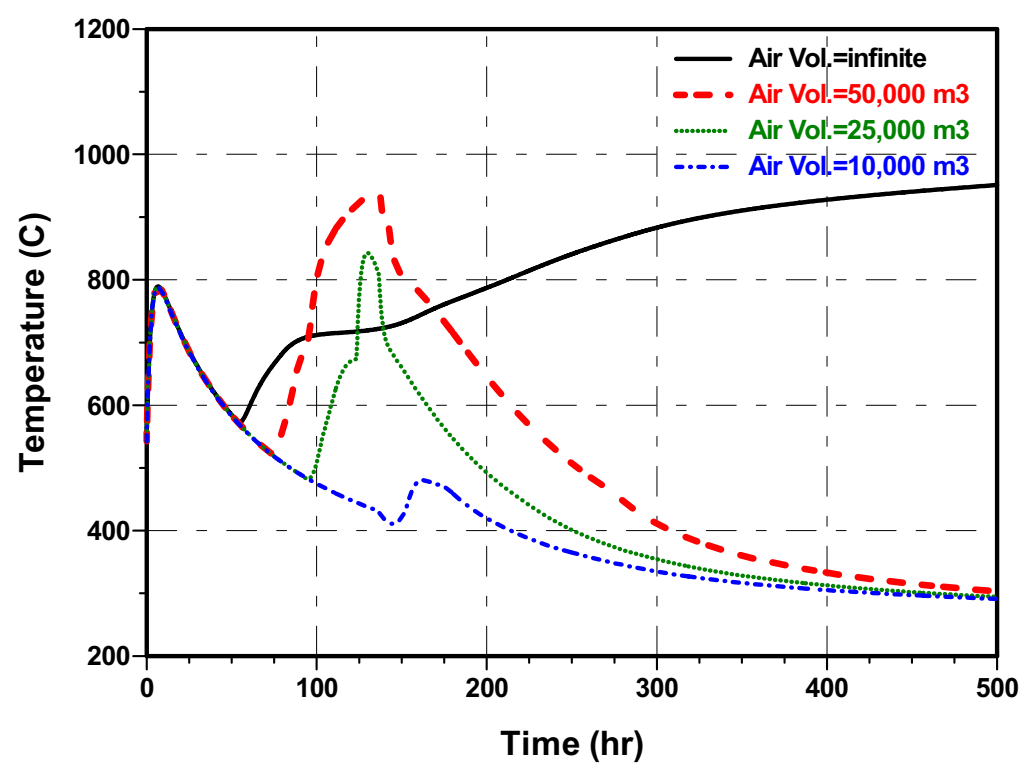

Figure 7-1-34 Top layer temperatures of bottom reflector for the sensitivity on the air volumes in a vault

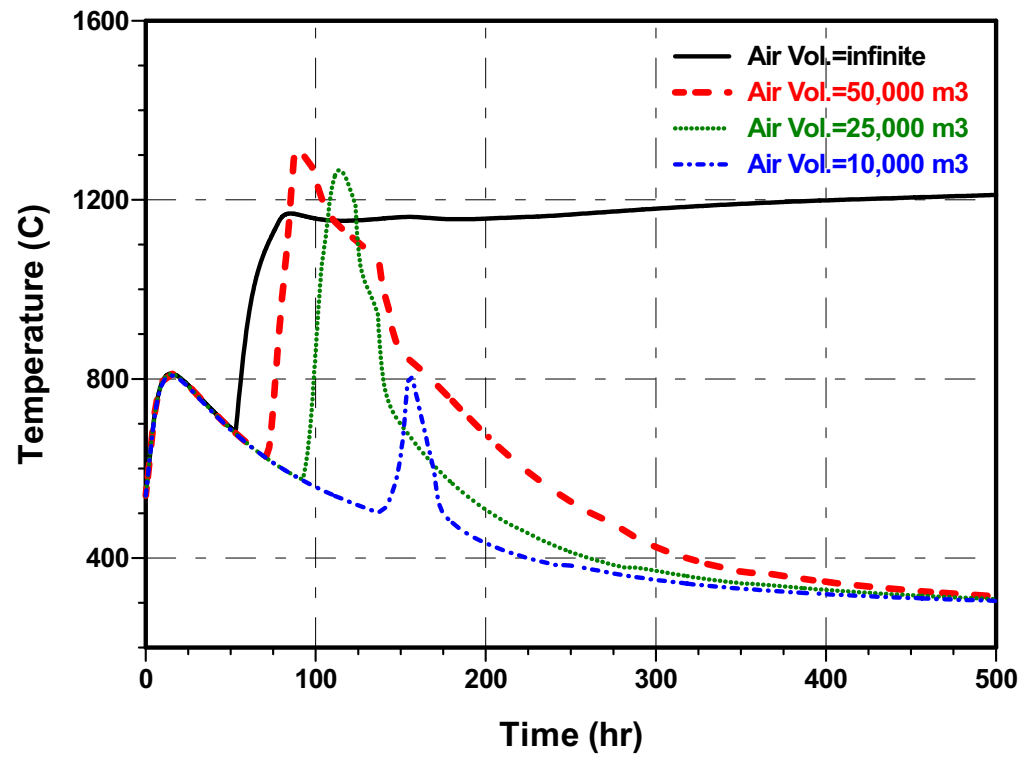

Figure 7-1-35 Bottom layer temperatures of pebble core for the sensitivity on the air volumes in a vault

In the previous cases, the vault is assumed to be decoupled from the reactor coolant system. In the real design, after the break, the reactor opens to the reactor cavity and also the reactor cavity opens to other compartments in a vault. Also the volume of the reactor cavity is just about $2,000 \mathrm{~m}^{3}\left(50,000 \mathrm{~m}^{3}\right.$ for the total volume of the confinement) and other compartments occupy most of the air volume. Therefore, by distributing the total air volume to the three compartments, as shown in Figure 7-1-38, the case of the coupled RCS/vault system model is simulated. Figures 7-1-39 and 7-1-40 show that, like the previous case with small vault volume, there are a delay in the OTNC and also no significant rise in bottom reflector and fuel 
temperatures. It demonstrates that more realistic system modeling is necessary to investigate the air ingress consequence particularly for the reactor building.

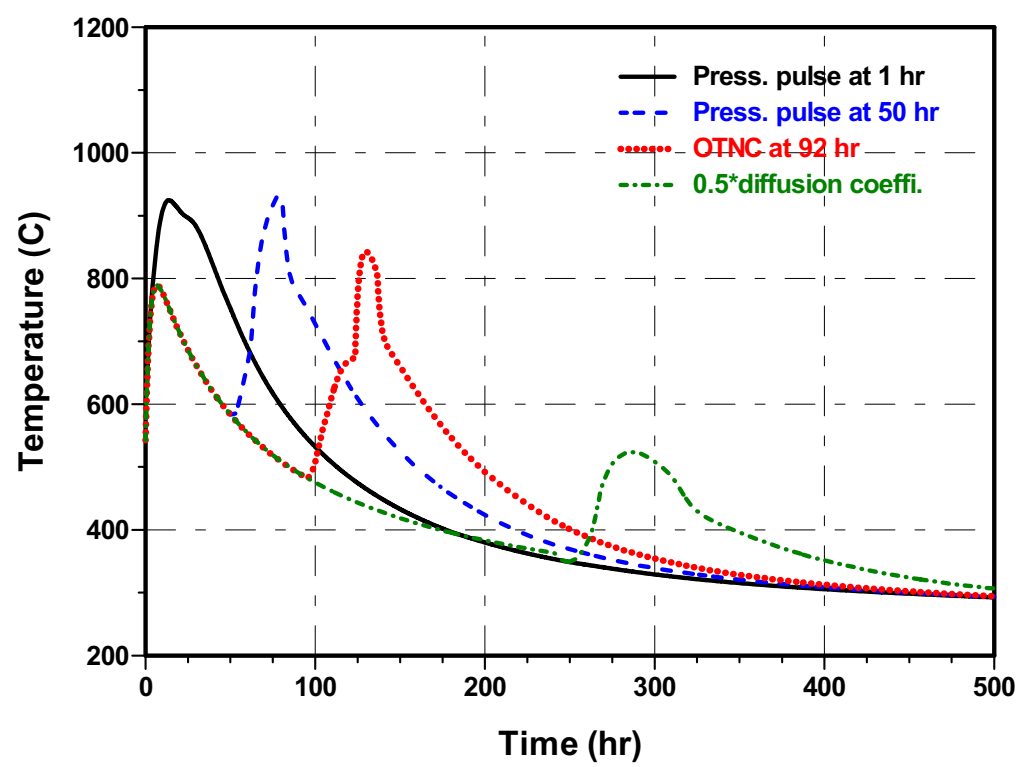

Figure 7-1-36 Top layer temperatures of bottom reflector for the sensitivity on the OTNC $\left(\mathrm{V}_{\text {air }}=25,000 \mathrm{~m}^{3}\right)$

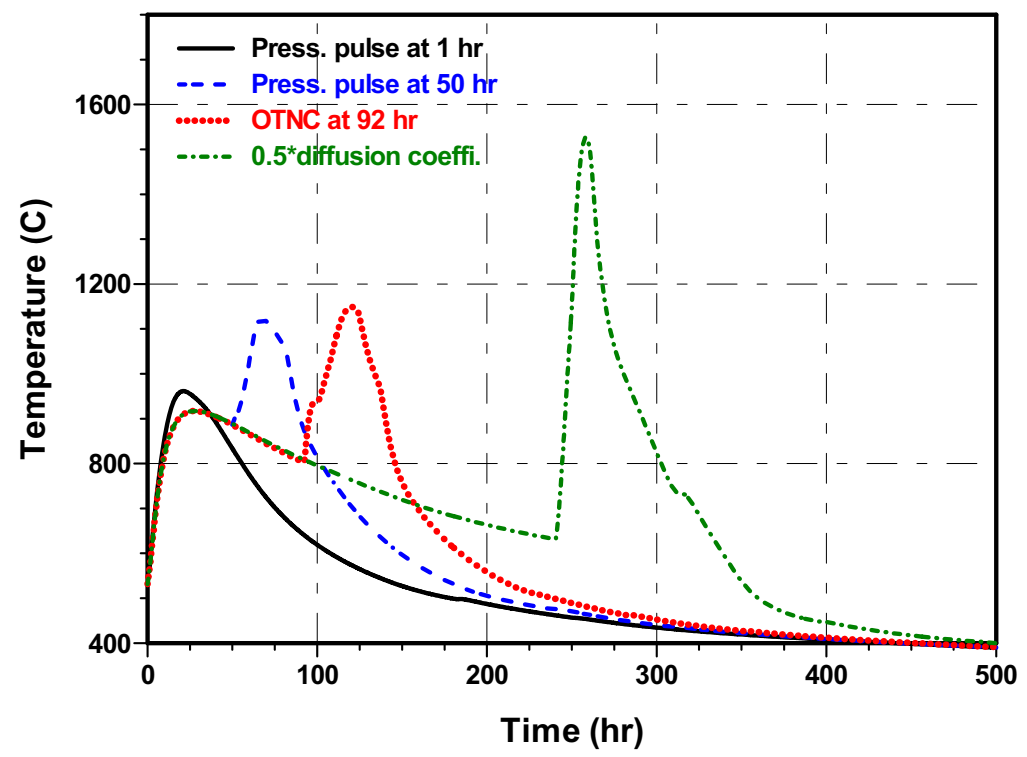

Figure 7-1-37 Bottom layer temperatures of pebble core for the sensitivity on the OTNC $\left(\mathrm{V}_{\text {air }}=25,000 \mathrm{~m}^{3}\right)$ 


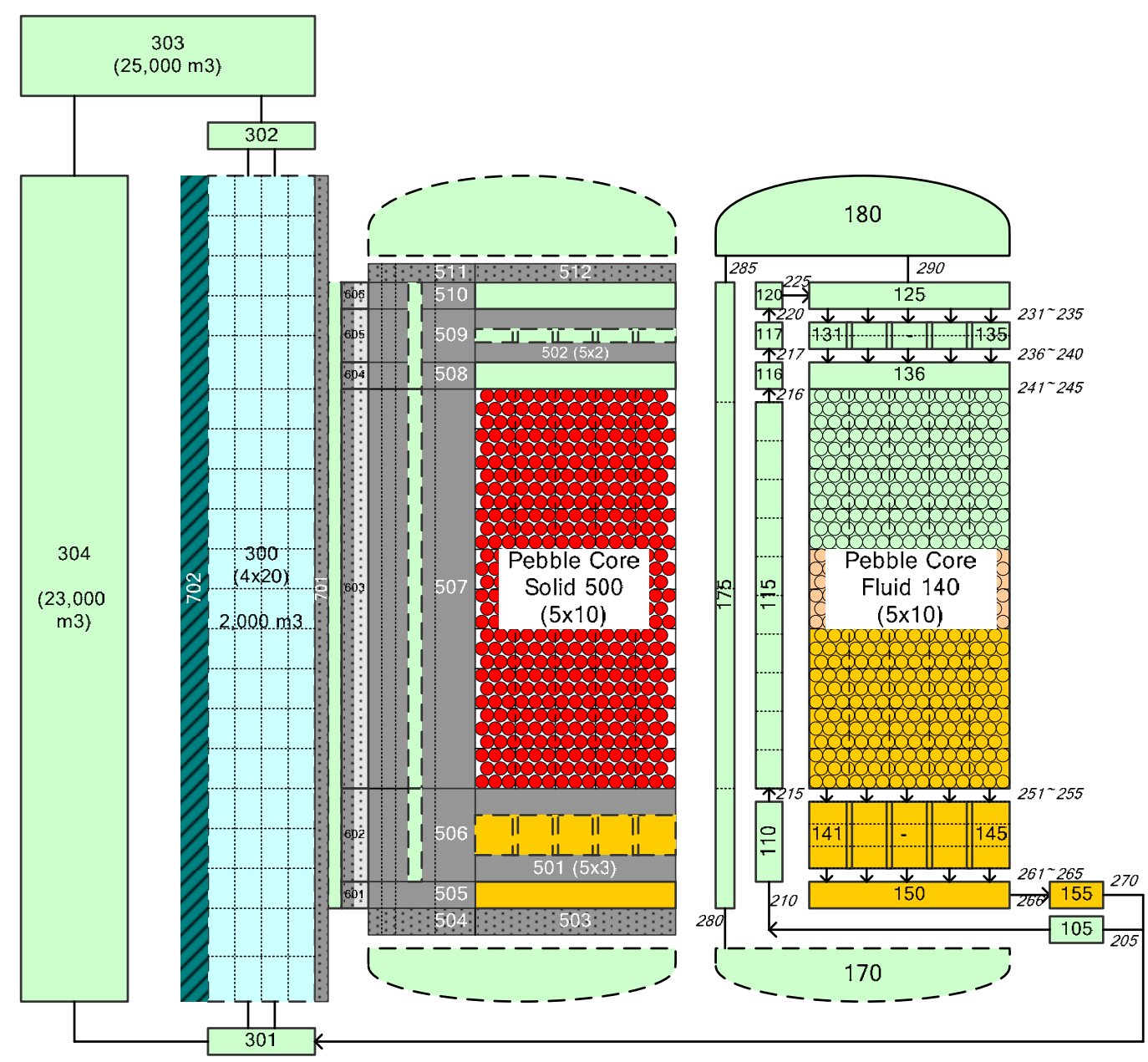

Figure 7-1-38 Nodal scheme for the coupling of a vault and the reactor coolant system 


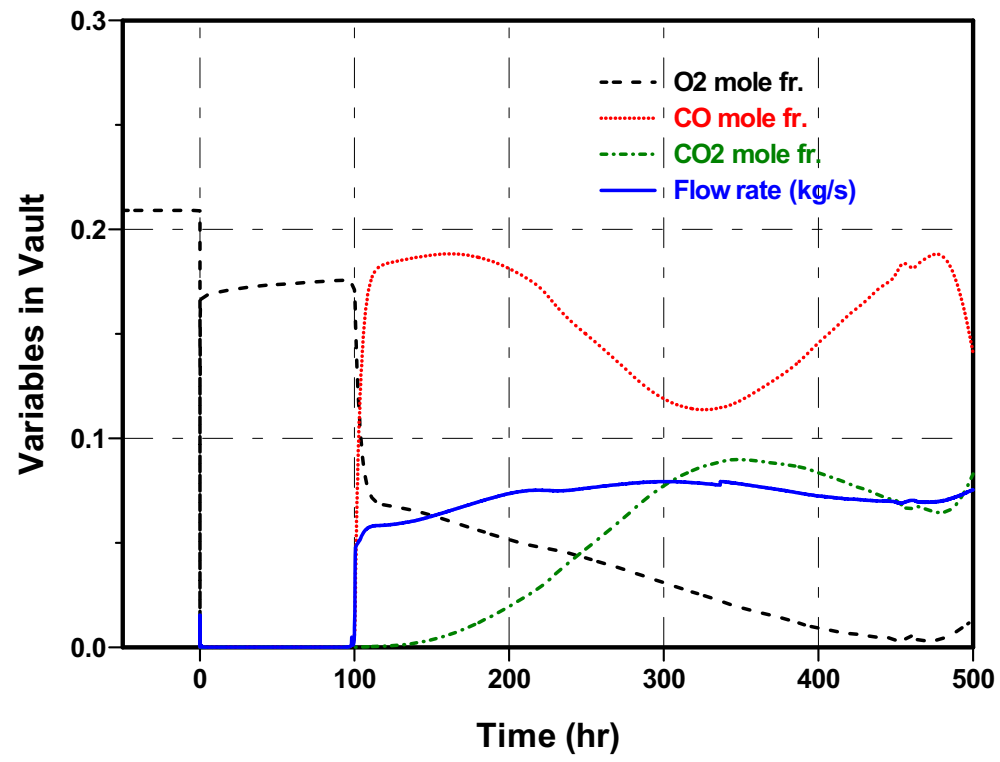

Figure 7-1-39 Air ingress rate and species concentrations in a vault for the coupling of a vault and the reactor coolant system $\left(\mathrm{V}_{\text {air }}=50,000 \mathrm{~m}^{3}\right)$

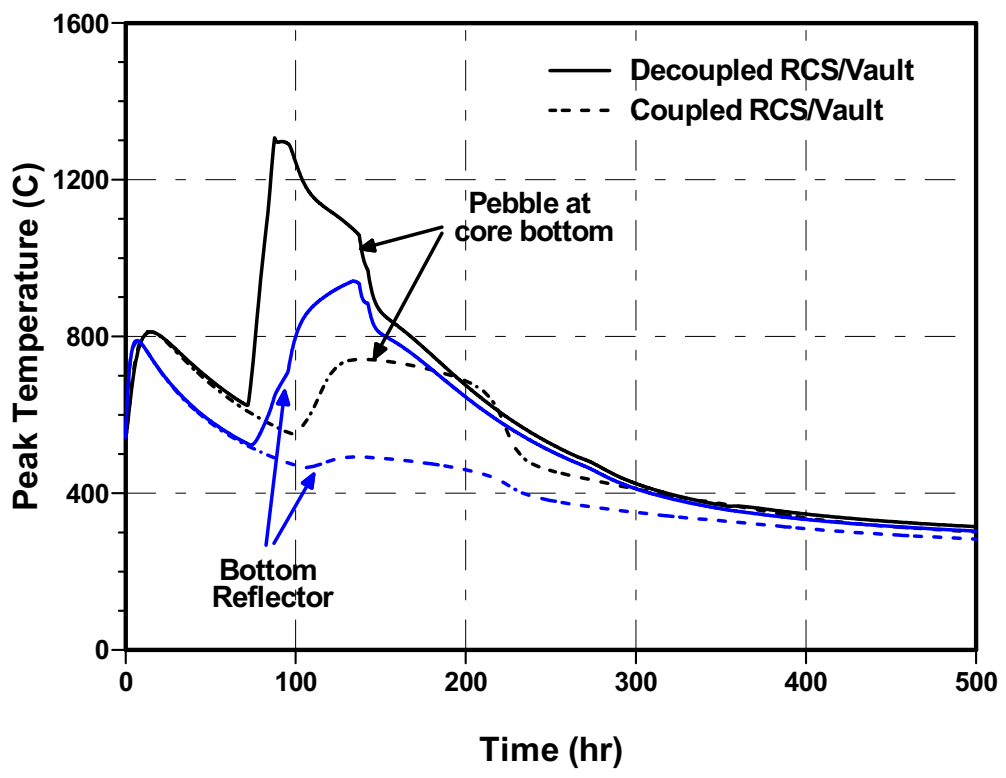

Figure 7-1-40 Core and reflector temperatures at hot spot for the coupling of a vault and the reactor coolant system $\left(\mathrm{V}_{\text {air }}=50,000 \mathrm{~m}^{3}\right)$ 


\section{Prismatic Core Gas-Cooled Reactor}

For the GAMMA air ingress analysis for a prismatic core gas-cooled reactor, we selected GT-MHR $600 \mathrm{MWt}$ (GA/NRC-337-02 2002) as a reference reactor.

a. System Modeling and Assumptions
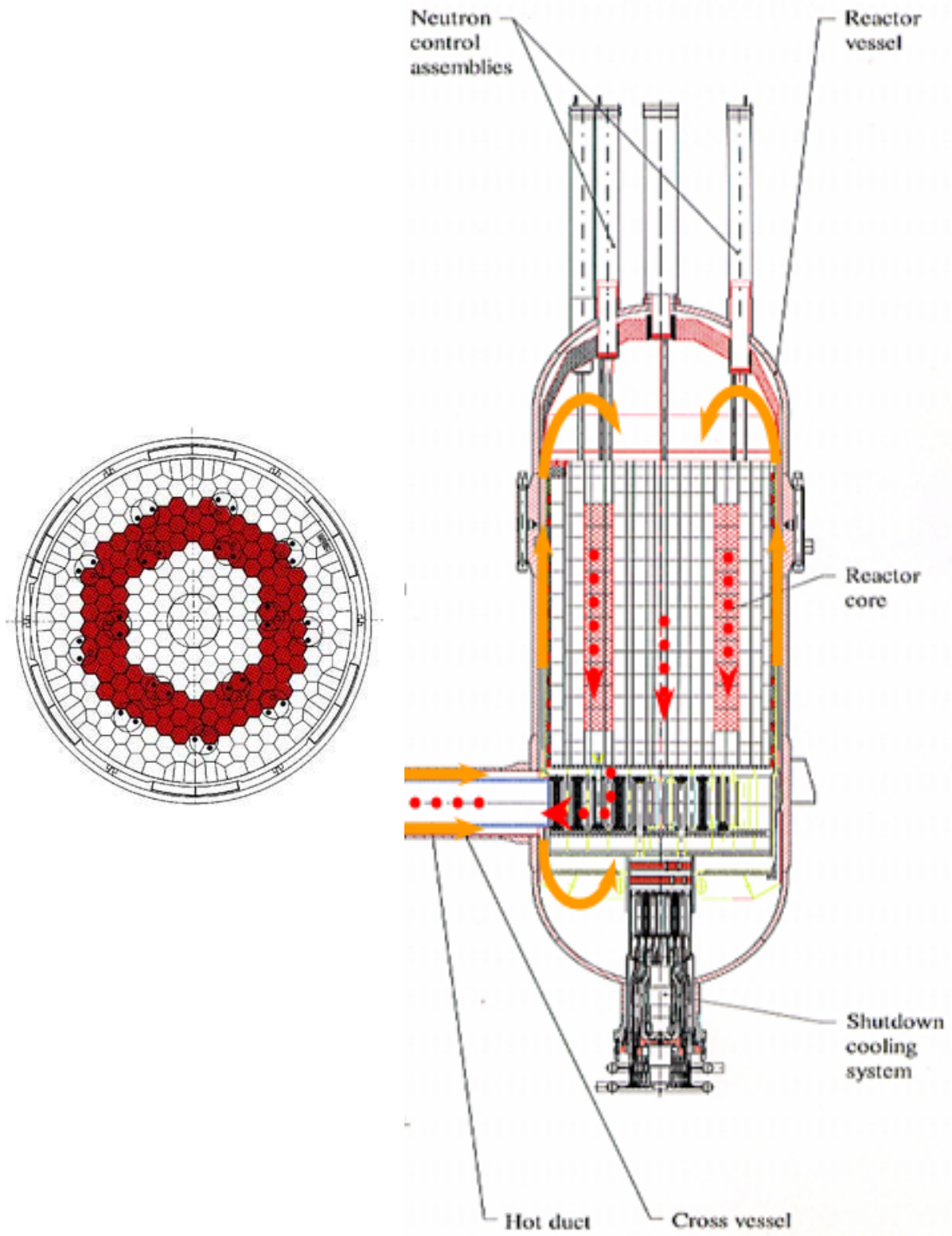

Figure 7-1-41 Schematic diagram of GT-HMR $600 \mathrm{MWt}$

In the GT-MHR $600 \mathrm{MWt}$, helium at $490^{\circ} \mathrm{C}$ and $7 \mathrm{MPa}$ enters the prismatic core through the riser and exits at $850^{\circ} \mathrm{C}$ and a flow rate of $320 \mathrm{~kg} / \mathrm{s}$. Figure 7-1-41 shows the schematic diagram of the GT-MHR reactor vessel and its cross-sectional view of the core at the mid plane. One hundred and two columns of the hexagonal fuel elements are stacked 10 elements high to form an annular core. Reflector graphite blocks are provided inside and outside of the active core. The air-cooling RCCS installed in a reactor cavity removes passively the core decay heat. 


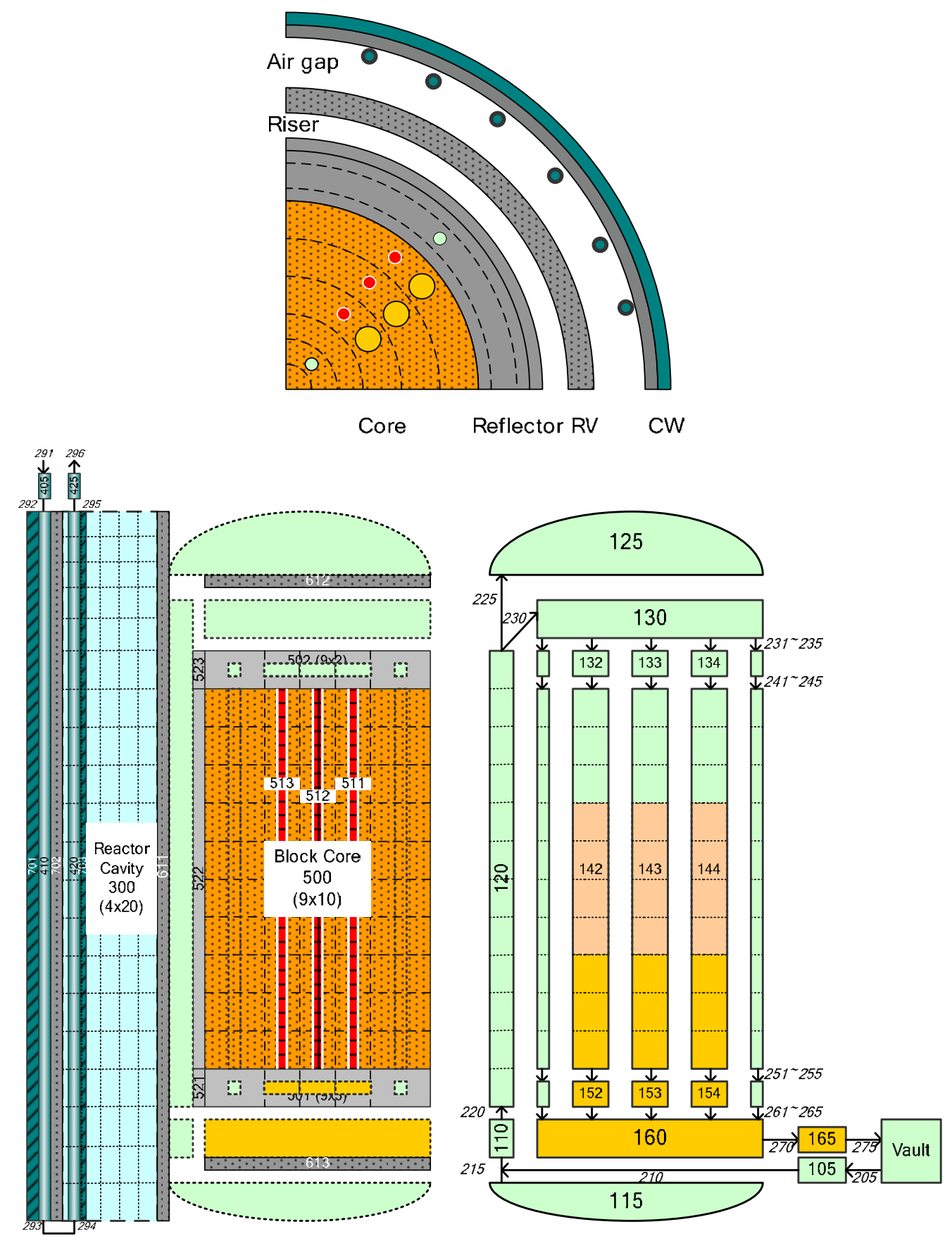

Figure 7-1-42 GAMMA nodal scheme of GT-HMR $600 \mathrm{MWt}$

Figure 7-1-42 shows the GAMMA modeling of GT-MHR where 2-D geometry models are used for the reactor cavity to consider the heat removal by natural convection flow, and for the solid structures including the core and reflector blocks to consider multi-dimensional heat conduction. For the three fuel-loaded zones, fuel compacts in each zone are grouped to one representative fuel rod. The 2-D heat conduction model is used to calculate the temperature distribution of each fuel rod. The heat transport in the prismatic core is much complicated by the combined effect of solid conduction in the fuel and the graphite matrix as well as gas and contact conduction and radiation in the fuel and fuel block gaps. As well, the heat transport becomes 
anisotropic due to the negligible effect of radiation in the axial direction. In the GAMMA code, the coolant channel and the fuel compact are separately treated by 1-D fluid equations and 2-D heat conduction equation from the graphite matrix, respectively. A porous media approach is used to obtain the effective volumetric heat capacity ( $\left.\rho C p_{e f f}^{\text {core }}\right)$ and thermal conductivity $\left(\lambda_{\text {eff }}^{\text {core }}\right)$ in the prismatic core.

$\rho C p_{\text {eff }}^{\text {core }}=\left(1-\varphi_{c}-\varphi_{f}\right) \rho C p_{g m}(7-1-12)$

where

$\varphi_{c} \quad=$ volume fraction of the coolant channel

$\varphi_{f} \quad=$ volume fraction of the fuel compact

$\rho C p_{g m}=$ volumetric heat capacity of the graphite matrix

First, the effective thermal conductivity for the fuel assembly (FA) is evaluated considering the coolant channel, fuel compact, and fuel gap:

$$
\begin{aligned}
& \lambda_{\text {eff } \_z}^{F A}=\left(1-\varphi_{c}-\varphi_{f}\right) \lambda_{g m}+\varphi_{c}\left(\lambda_{\text {gas }}^{c}\right)+\varphi_{f}\left[\left(1-\varphi_{f g}\right) \lambda_{f c}+\varphi_{f g}\left(\lambda_{\text {gas }+ \text { cont }}^{f g}\right)\right] \\
& \lambda_{\text {eff_- }}^{F A}=\lambda_{\text {eff_z }}^{F A}+\varphi_{c}\left(\lambda_{\text {rad }}^{c}\right)+\varphi_{f}\left[\varphi_{f g}\left(\lambda_{\text {rad }}^{f g}\right)\right]
\end{aligned}
$$

where

$\varphi_{f g} \quad=$ volume fraction of the fuel gap

$\lambda_{g m}=$ thermal conductivity of the graphite matrix

$\lambda_{\text {gas }}^{c}=$ gas conductivity in the coolant channel

$\lambda_{\text {rad }}^{c} \quad=$ radiation-equivalent conductivity in the coolant channel

$\lambda_{f c}=$ thermal conductivity of the fuel compact

$\lambda_{\text {gas }+ \text { cont }}^{f g}=$ gas and contact conductivity in the fuel gap

$\lambda_{\text {rad }}^{f g}=$ radiation-equivalent conductivity in the fuel gap

Next, the effective thermal conductivity for the prismatic core is evaluated considering the FA gaps:

$\lambda_{\text {eff_z }}^{\text {core }}=\left(1-\varphi_{\text {gap }}^{F A}\right) \lambda_{\text {eff_z }}^{F A}+\varphi_{\text {gap }}^{F A}\left(\lambda_{\text {gas }+ \text { cont }}^{F A}\right)$

$\lambda_{\text {eff_ } x}^{\text {core }}=\left(1-\varphi_{\text {gap }}^{F A}\right) \lambda_{\text {eff_ } x}^{F A}+\varphi_{\text {gap }}^{F A}\left(\lambda_{\text {gas }+ \text { cont }}^{F A}+\lambda_{\text {rad }}^{F A}\right)$

where

$\varphi_{\text {gap }}^{F A} \quad=$ volume fraction of the FA gap

$\lambda_{\text {gas }+ \text { cont }}^{F A}=$ gas and contact conductivity in the FA gap

$\lambda_{\text {rad }}^{F A}=$ radiation-equivalent conductivity in the FA gap

In all the cavities or plenums, the radiation heat exchanges are considered. The air cooling RCCS system is modeled using the 1-D pipe network for the air flow loop and the 3-D tube model for the cooling tubes. Following the accident, since a reactor trips immediately, the core power is determined directly from the General Atomics (GA) decay heat curve.

b. Analysis Results and Discussions

The case with the air volume of $50,000 \mathrm{~m}^{3}$ in a vault has been performed. As shown in Figure 7-1-43, the onset time of natural convection occurs at 570 hours, much delayed compared to that of PBMR. It is because of the lower equilibrium air concentration at the end of blowdown caused by the larger fluid volume ratio of the reactor coolant system to the vault. In addition, due to the large fluid volume inside the reactor vessel, the 
molecular diffusion process proceeds slowly and therefore the density of a gas mixture increases slowly, by delaying the onset time of a natural convection. There is no significant rise in the core and reflector temperatures, as shown in Figure 7-1-44. It is mainly due to the higher ratio of the graphite volume to the surface area contacting with the oxygen. If the surface area contacting with the oxygen is small, the graphite oxidation rate becomes low eventually by lowering the produced heat. Also if the graphite volume is large, the temperature rise becomes small by absorbing the produced heat.

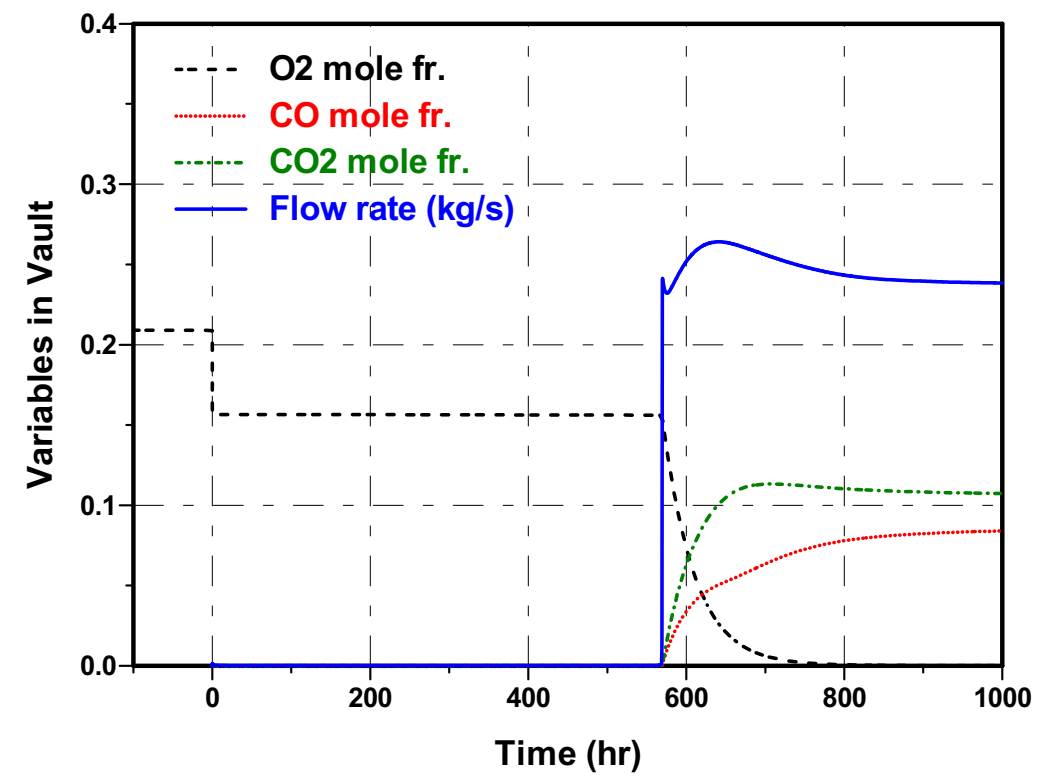

Figure 7-1-43 Air ingress rate and oxygen concentration in a vault for the GT-MHR $\left(\mathrm{V}_{\mathrm{air}}=50,000 \mathrm{~m}^{3}\right)$

\section{7-2 Task 6-2 SNU V\&V}

(Described in Section 3.)

\section{7-3 Task 6-3 INL V\&V}

\subsubsection{V\&V of RELAP5}

Diffusion, natural circulation, and heat transfer are important phenomena during a LOCA in a VHTR. The capability of RELAP5-3D to represent these phenomena was assessed using four experiments. The diffusion model was assessed using data from inverted U-tube experiments (Hishida and Takeda 1991) and bulb experiments (Duncan and Toor 1962). The code capability to simulate the natural circulation of air through a pebble bed was assessed using data from the NACOK facility (Kuhlmann 2002). The code capability to represent heat transfer (conduction, convection, and radiation) from a simulated vessel was assessed using RCCS experiments from SNU.

\section{Assessment of the Molecular Diffusion Model (Inverted U-Tube)}

The experimental apparatus of Hishida and Takeda (1991) is shown in Figure 7-3-1. The apparatus consisted of an inverted U-tube, ball valves, and a tank. The inner diameters of the U-tube and the tank were 0.0527 and $1.0 \mathrm{~m}$, respectively. The heights of the U-tube and tank were 1.45 and $0.5 \mathrm{~m}$, respectively. 


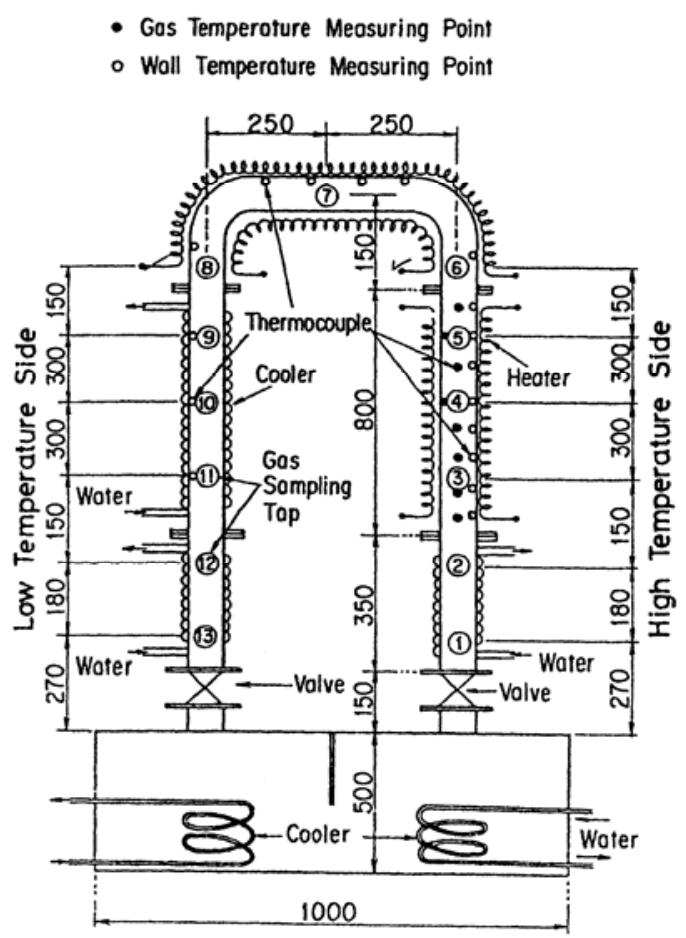

Figure 7-3-1 Inverted U-tube experimental apparatus (from Hishida and Takeda (1991)).

The ball valves that separated the inverted U-tube from the tank were closed before the start of the test. The tank and the inverted U-tube were then evacuated and filled with nitrogen and helium, respectively. Electrical heaters controlled the fluid temperatures of one vertical leg and the horizontal leg at the top of the inverted U-tube. The temperature of the other vertical leg was controlled by external cooling with water. After the temperatures had stabilized, the pressures in the tank and inverted U-tube were adjusted to match atmospheric pressure. The test was initiated by opening the ball valves, which allowed nitrogen to diffuse from the tank upwards through the U-tube. The mole fraction of nitrogen was measured at several locations in both legs of the inverted U-tube. The uncertainty in the mole fraction measurement was 5\%. Two tests were conducted; one utilized isothermal conditions at room temperature, while the other utilized a nonisothermal profile with values varying between 18 and $256^{\circ} \mathrm{C}$.

A RELAP5 model of the inverted U-tube was developed as illustrated in Figure 7-3-2. The model represented the inverted U-tube, ball valves, and tank components. The tank was divided into two halves, with a connecting junction at the bottom, because the experimental version of the diffusion model does not currently allow more than one junction to be connected at each face of a control volume. Heat structures were used to simulate the walls of the inverted U-tube and the tank. The temperatures of the outer surface of the heat structures were set at the measured values. The RELAP5 model shown in Figure 7-3-2 is much more detailed than typical reactor models and consists of 144 control volumes, most of which are $2.45 \mathrm{~cm}$ long. The nodalization is similar to that used previously by Hishida and Takeda (1991) and Lim and No (2003). 


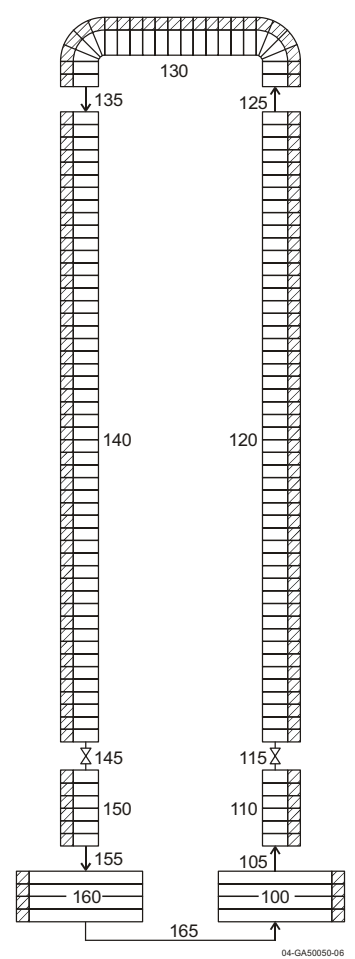

Figure 7-3-2 RELAP5 model of the inverted U-tube experiment.

RELAP5 calculations were performed for both the isothermal and non-isothermal experiments. Results for the isothermal experiment are presented in Figure 7-3-3. The figure shows measured and calculated mole fractions of nitrogen versus time at four elevations, ranging from 0.6 to $1.35 \mathrm{~m}$ above the top of the tank. The measured results are represented with symbols, while the calculated results are represented with solid lines containing symbols.

The calculation reasonably represented the trends observed in the isothermal experiment. First, the mole fraction of nitrogen initially increased more rapidly at the lowest elevation, due to the shorter distance from the tank, which was initially full of nitrogen, and more slowly at the higher elevations. Second, because there were no buoyancy differences between the two vertical legs of the U-tube in this experiment, the mole fractions in both legs increased symmetrically. The calculated results were also generally in reasonable quantitative agreement with the measured values. The calculated results were slightly outside the uncertainty of the measurements at the elevation of $0.6 \mathrm{~m}$, but within the uncertainty at the higher elevations. Similar results were obtained by Lim and No (2003), which indicates that possible errors in the RELAP5 code or input model are not the likely causes of the differences. 


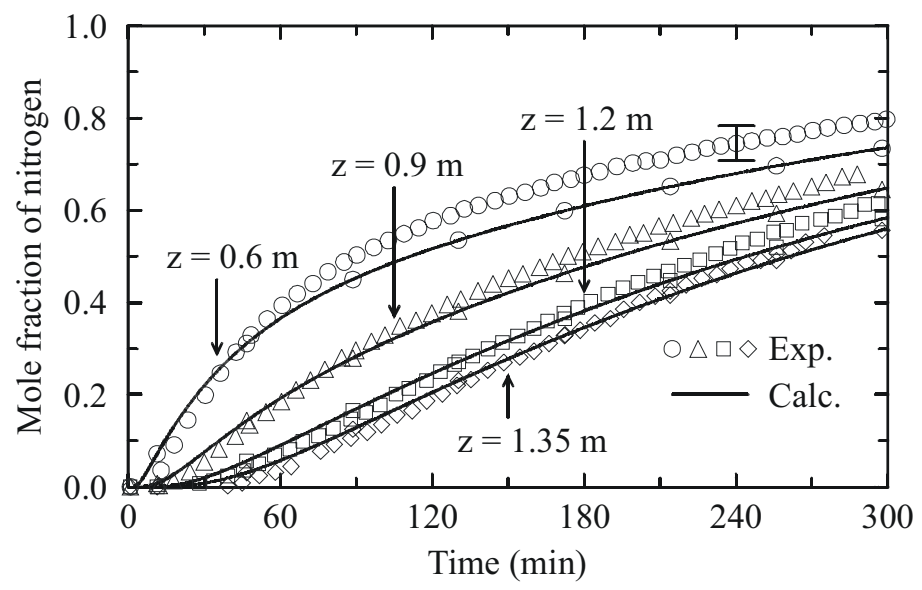

Figure 7-3-3. Measured and calculated results for the isothermal test.

A sensitivity calculation was performed in which the number of control volumes was doubled from that shown in Figure 7-3-2. As shown in Figure 7-3-4, the calculated results were slightly better with the more detailed nodalization. The calculated results are not expected to be as accurate using a coarser nodalization that is typical of most reactor system models, where the core is generally modeled with about 10 control volumes. However, the more coarsely nodalized system models are expected to show correct trends.

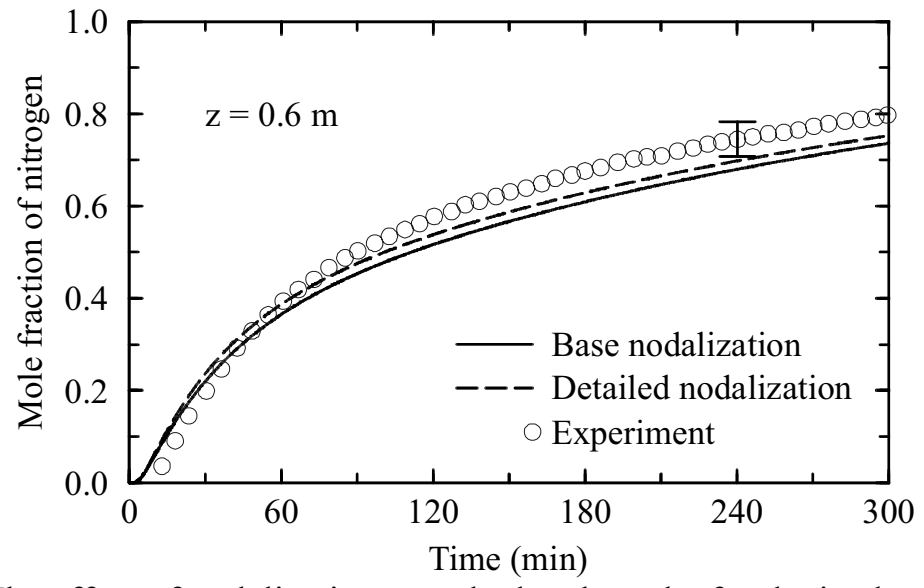

Figure 7-3-4. The effect of nodalization on calculated results for the isothermal experiment.

Calculated and measured results for the non-isothermal experiment are shown in Figures 7-3-4, 7-3-5, 7-3-6, and 7-3-7, which correspond to elevations $0.6,0.9$, and $1.35 \mathrm{~m}$ above the top of the tank, respectively. Each figure shows results for both the hot and cold legs of the inverted U-tube. In both the calculation and the test, the mole fraction of nitrogen increased more rapidly on the hot side of the U-tube than on the cold side due to a larger diffusion coefficient, which increases with temperature, and buoyancy effects, which aided the movement of nitrogen on the hot side of the U-tube and opposed it on the cold side. The rapid increase in mole fraction near 220 min was caused by the onset of natural circulation. 


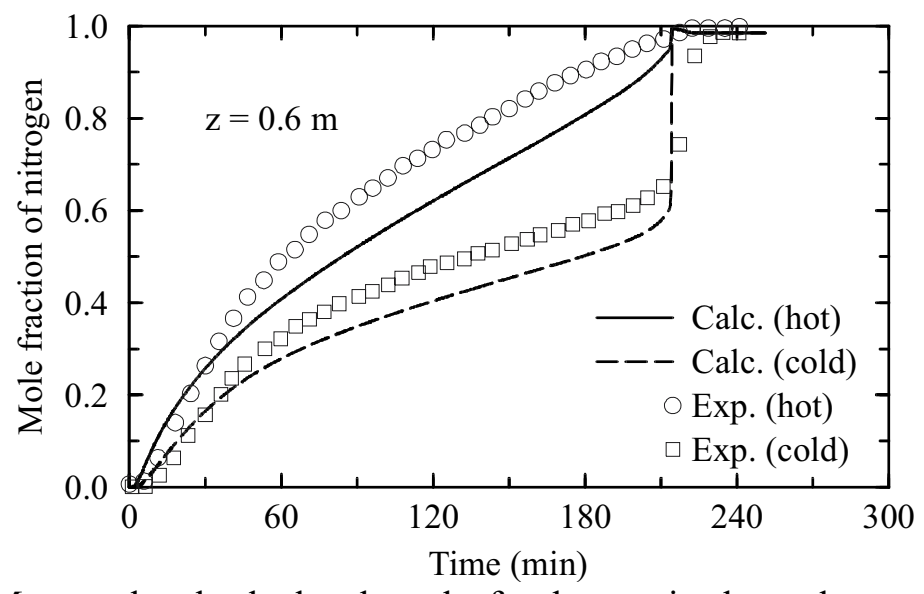

Figure 7-3-5. Measured and calculated results for the non-isothermal experiment at $0.6 \mathrm{~m}$.

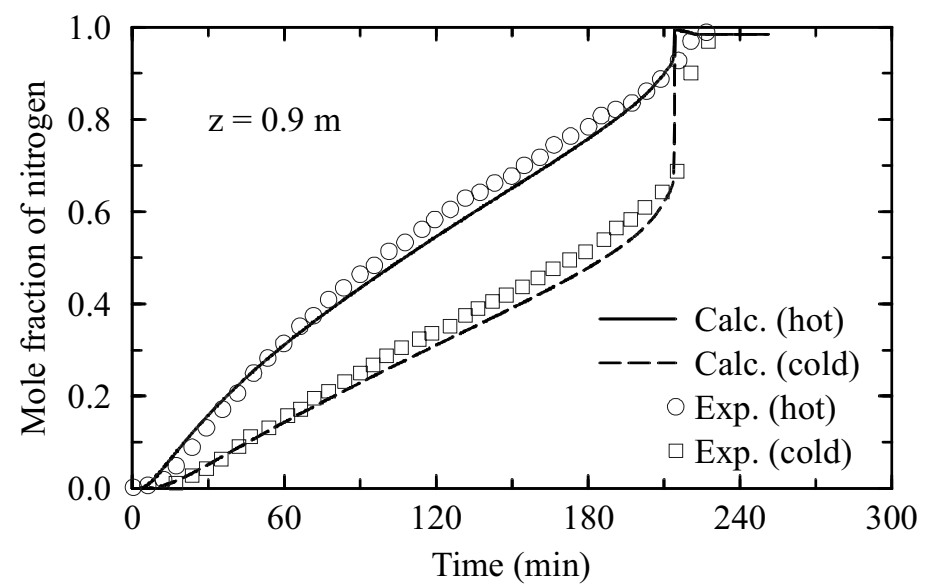

Figure 7-3-6. Measured and calculated results for the non-isothermal experiment at $0.9 \mathrm{~m}$.

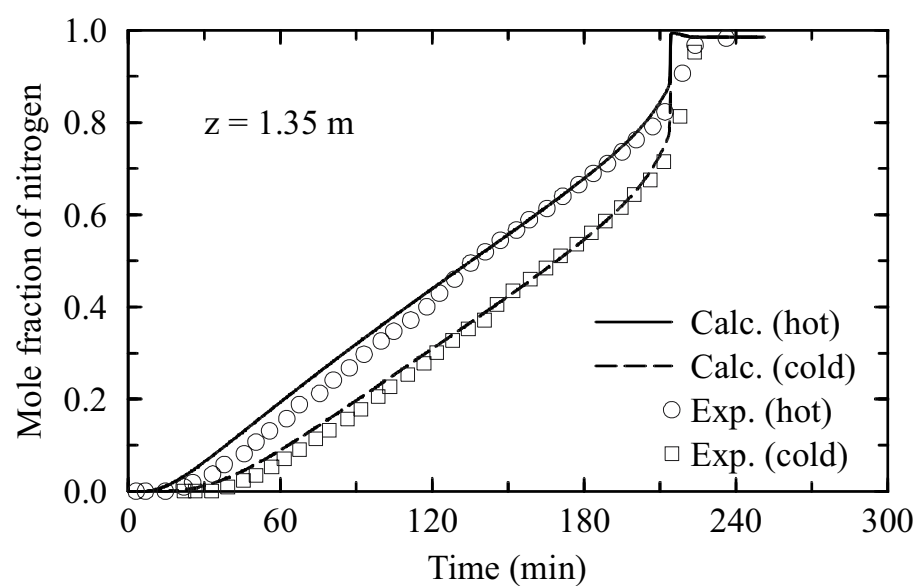

Figure 7-3-7. Measured and calculated results for the non-isothermal experiment at $1.35 \mathrm{~m}$.

The calculated results were in reasonable quantitative agreement with the measured values. The differences between the calculated and measured results were generally within the reported uncertainty at all measurement locations except for the lowest one. The calculated results at the lowest locations are similar to those obtained by Lim and No (2003). The timing of the onset of natural circulation, which introduces 
relatively large amounts of air into the core and therefore could initiate significant graphite oxidation in a reactor, was calculated to within a few minutes.

\section{Assessment of Natural Circulation through a Pebble Bed}

The RELAP5-3D computer code was assessed using natural circulation data generated in the NACOK experimental apparatus (Kuhlmann 2002). The NACOK experiments were designed to investigate the effects of air ingress into the core of a high-temperature reactor following a loss-of-coolant accident. The experiments investigated the effects of molecular diffusion, natural circulation, and oxidation. The natural circulation experiments were used for this assessment.

The NACOK experiments simulated natural circulation of air through a scaled model of a high-temperature reactor containing a pebble bed core as shown in Figure 7-3-8. The experimental apparatus consisted of an experimental channel, a coaxial duct, supply and return tubes, and heating elements. The experimental channel had a square $300 \times 300 \mathrm{~mm}$ cross-section and a total height of $7.3 \mathrm{~m}$. The experimental channel consisted of three axial sections including a bottom reflector, a 5.0-m long section containing packed spheres, and an empty $1.7-\mathrm{m}$ long section hereafter called the top reflector. The $60-\mathrm{mm}$ diameter spheres were packed in a regular arrangement of 25 spheres per layer. Every other layer used half spheres along two of the four channel walls. The resulting porosity of the packing was 0.395 . The inner diameter of the supply and return tubes was $125 \mathrm{~mm}$. The coaxial duct was a horizontal annulus with both the inner and outer tubes connected to the atmosphere.

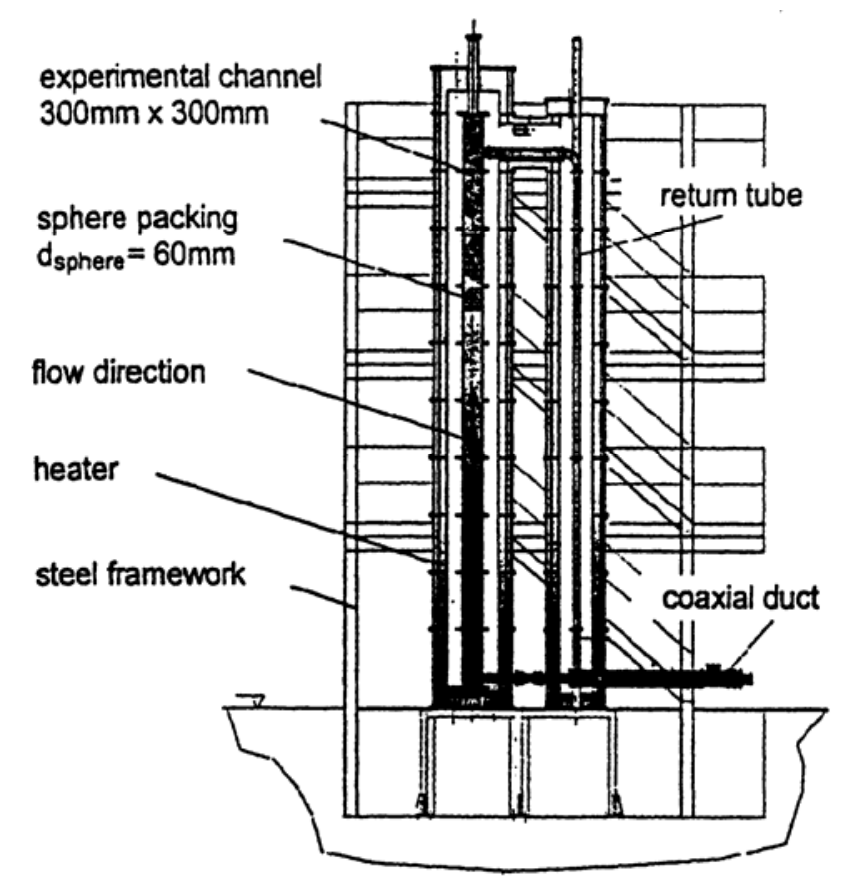

Figure 7-3-8. Schematic of the NACOK experimental apparatus (from Schaaf et al. 1998).

Heating elements were used to control the temperature of the walls in the experimental channel and the return tube during the experiments. The wall temperature of the return tube was set at $200,400,600$ or $800^{\circ} \mathrm{C}$. The temperature of the experimental channel was controlled between a minimum value that was $50^{\circ} \mathrm{C}$ higher than the temperature of the return tube and a maximum value of $1000^{\circ} \mathrm{C}$. The difference in temperature between the experimental channel and the return tube induced air to naturally circulate through the supply tube, up through the experimental channel, down through the return tube to the outer tube in the coaxial duct, and back 
to the atmosphere. The packed spheres in the pebble bed represented the dominant hydraulic resistance in the flow circuit. Thus, the experiments were used to test the calculation of friction factors in a pebble bed.

The RELAP5 model of the NACOK facility is shown in Figure 7-3-9. The model represented all the hydraulic components of the experimental apparatus, including the coaxial duct, supply tube, bottom reflector, packed spheres, top reflector, and return tube. Boundary conditions of atmospheric pressure and $20^{\circ} \mathrm{C}$ were applied in Components 100 and 170, which were time-dependent volumes. The thickness of the supply and return tubes was taken as $4 \mathrm{~mm}$ based on Schaaf et al. (1997). The inner diameter of the outer tube in the coaxial duct was then calculated from the area of $0.0080 \mathrm{~m}^{2}$ reported by Kuhlmann (2002).

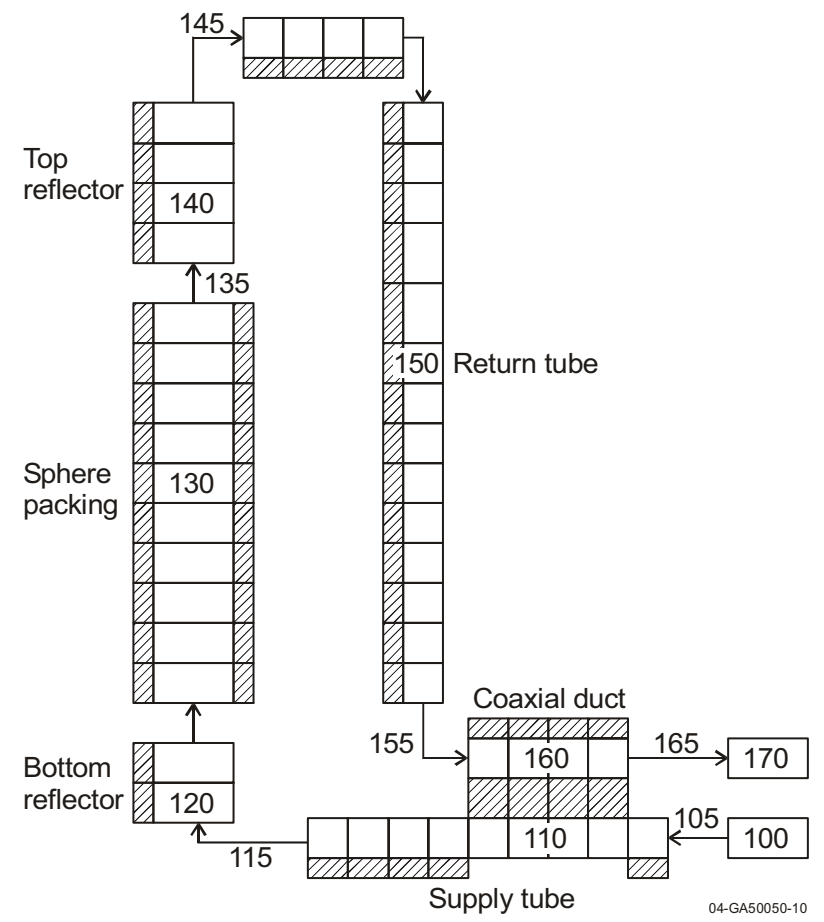

Figure 7-3-9. RELAP5 model of the NACOK natural circulation experiment.

Heat structures were used to represent the walls of the coaxial duct, the supply and return tubes, and the experimental channel. The packed spheres were also modeled with a heat structure. The wall temperature of the bottom reflector, spheres, top reflector, and the horizontal portion of the return tube were set at the measured value of the experimental channel. The wall temperature of the vertical portion of the return tube was set at the measured value of the return tube. The code calculated the temperature drop due to conduction across each heat structure and the heat flux to the fluid in the adjacent control volume. Because of the small mass flow rates involved, the heat transfer coefficients were generally calculated using natural convection or laminar correlations.

The inner wall of the coaxial duct was modeled to preheat the air entering the experimental channel. The surface temperature of the first heat structure in the supply tube downstream of the coaxial duct was set at the measured temperature of the return tube to represent the portion of the tube within the heating vessel of the return tube. Similarly, the surface temperature of the last heat structure in the supply tube was set at the temperature of the experimental channel. The remaining heat structures in the supply tube were set at the ambient temperature. As shown later, the calculated air flow rates were relatively sensitive to the preheating of the air entering the experimental channel. 
The pressure loss across the packed spheres was calculated using the model from SCDAP/RELAP5 (SCDAP/RELAP5-3D Code Development Team 2003). For single-phase flow, the model reduces to the Ergun equation given by Bird et al. (1960). The Ergun equation is

$$
\Delta \mathrm{P}=\rho \mathrm{V}_{\mathrm{o}}^{2}\left(\frac{150(1-\varepsilon)^{2}}{\operatorname{Re}_{\mathrm{o}} \varepsilon^{3}}+\frac{1.75(1-\varepsilon)}{\varepsilon^{3}}\right) \frac{\mathrm{L}}{\mathrm{D}_{\mathrm{p}}},
$$

where

$\Delta \mathrm{P}=$ pressure drop due to friction

$\rho=$ fluid density

$\mathrm{V}_{\mathrm{o}}=\varepsilon \mathrm{V}=$ superficial velocity, where $\mathrm{V}$ is the actual fluid velocity

$\varepsilon=$ porosity or void fraction

$\operatorname{Re}_{\mathrm{o}}=\frac{\rho \mathrm{V}_{\mathrm{o}} \mathrm{D}_{\mathrm{p}}}{\mu}=$ Reynolds number based on superficial velocity

$\mu=$ fluid dynamic viscosity

$\mathrm{L}=$ length of the pebble bed

$\mathrm{D}_{\mathrm{p}}=$ diameter of a pebble.

The RELAP5 model shown in Figure 7-3-9 was used to perform a series of calculations in which the wall temperatures were held constant at the measured values until a steady state was achieved. The results of the calculations are illustrated in Figure 7-3-10, which shows mass flow rate of air as a function of the temperature in the experimental channel for temperatures of the return tube, $T_{R}$ that varied between 200 and $800^{\circ} \mathrm{C}$. The calculated results were in reasonable agreement with the measured values. The important trends observed in the experiments were predicted by the code. In particular, the shape of the curve at $\mathrm{T}_{\mathrm{R}}=200^{\circ} \mathrm{C}$ was similar in the calculations and the experiment. The mass flow rate initially increased sharply with increasing experimental channel temperature, reached a maximum value near $550^{\circ} \mathrm{C}$, and then gradually decreased. The volumetric flow increased monotonically with experimental channel temperature because the increased temperature difference between the channel and the supply tube caused an increased driving head for natural circulation. However, the mass flow decreased at higher temperatures because the density decreased at a faster rate than the volumetric flow increased, and, to a lesser extent, because the Reynolds number was decreasing, which caused increased hydraulic resistance as indicated by Equation 1. The code also correctly predicted the trend of decreasing mass flow as $T_{R}$ increased at a given experimental channel temperature. This trend was primarily caused by the decreased temperature difference between the experimental channel and the return tube, which decreased the driving head for natural circulation. The RELAP5 model was used to simulate all 40 data points reported by Kuhlmann (2002). The root-mean-square error in the calculated flow rate was $0.21 \mathrm{~g} / \mathrm{s}$, which corresponds to about $5 \%$ of the maximum measured value. The value of $\operatorname{Re}_{\mathrm{o}} /(1-\varepsilon)$ varied between 9 and 120 in the calculated results shown in Figure 7-3-10. 


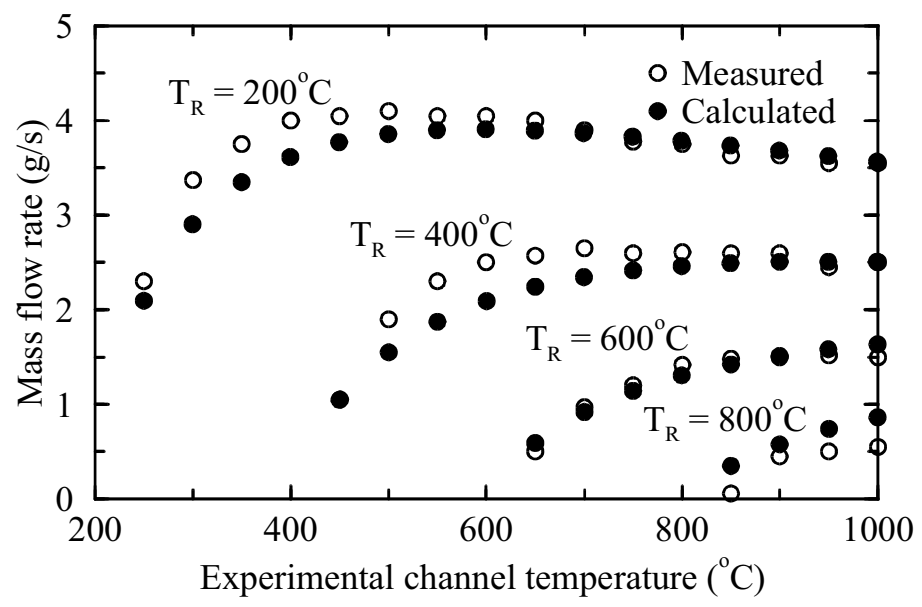

Figure 7-3-10. Measured and calculated air flow rates for the NACOK natural circulation experiments.

The average calculated fluid temperature in the experimental channel was less than the reported temperature because a certain distance was required to heat the fluid in the experimental channel up to the temperature of the wall. Similarly, the average calculated fluid temperature in the return tube was less than the imposed wall temperature of the return tube. As a result, the actual driving head for natural circulation was less than that obtained from the difference in fluid densities corresponding to the difference in wall temperatures between the experimental channel and the return tube. The calculated driving head for natural circulation varied between 60 and $90 \%$ of the value calculated from the difference in wall temperatures.

The calculated mass flow rates were sensitive to the temperature boundary conditions applied to the walls of the heat structures, which affected the relative fluid temperatures and densities in the experimental channel and the return tube and thus affected the driving head for natural circulation. For example, a sensitivity calculation was performed in which the boundary condition in the horizontal leg at the top of the return tube was changed from the temperature of the experimental channel to the temperature of the return tube.

Although Kuhlmann (2002) did not report the average wall temperature in this portion of the return tube, the actual temperature is expected to be between the reported values for the experimental channel and the return tube, and probably nearer to that of the experimental channel as assumed in the original calculation.

Applying a lower wall temperature in this relatively short region resulted in a lower average fluid temperature in the return tube, which increased the driving head for natural circulation and resulted in an increase in the calculated mass flow rate of $0.26 \mathrm{~g} / \mathrm{s}$ averaged over all 40 data points. A second sensitivity calculation was performed in which the wall temperatures of the last heat structure in the supply pipe and of the two heat structures in the bottom reflector were changed from the reported temperature of the experimental channel to a value halfway between the ambient and experimental channel temperatures. Applying a lower wall temperature in this portion of the experimental channel decreased the average fluid temperature in the channel, which decreased the driving head for natural circulation and resulted in an average decrease in the calculated mass flow rate of $0.23 \mathrm{~g} / \mathrm{s}$. The average change in the flow rate for each sensitivity calculation is significant compared to the root-mean-square error of $0.21 \mathrm{~g} / \mathrm{s}$ reported earlier.

\section{Assessment of RCCS Test}

The RELAP5-3D code (INEEL 2005) was assessed using an experiment from Seoul National University (SNU) (V30Q25) that simulated multi-dimensional heat conduction through a reactor vessel (RV) and heat transfer to a surrounding cavity wall $(\mathrm{CW})$. 
The RELAP5-3D model of the SNU experiment is illustrated in Figure 7-3-11. The RV was modeled as a solid circular cylinder with a radius and height of 410 and $1585 \mathrm{~mm}$, respectively. The RV contained six 90$\mathrm{mm}$ diameter heater rods centered at a radius of $225 \mathrm{~mm}$ and spaced uniformly in the azimuthal direction. The power applied to the heater rods was $25 \mathrm{~kW}$. The power was conducted from the heater rods to the various surfaces of the $\mathrm{RV}$, where it was transferred to the $\mathrm{CW}$ through radiation and convection. Some of the power was also conducted through the support legs of the reactor vessel to the lower wall of the cavity. Most of the outer surface of the $\mathrm{CW}$ was cooled with water. Temperature measurements were taken at various locations on the cavity and vessel walls.

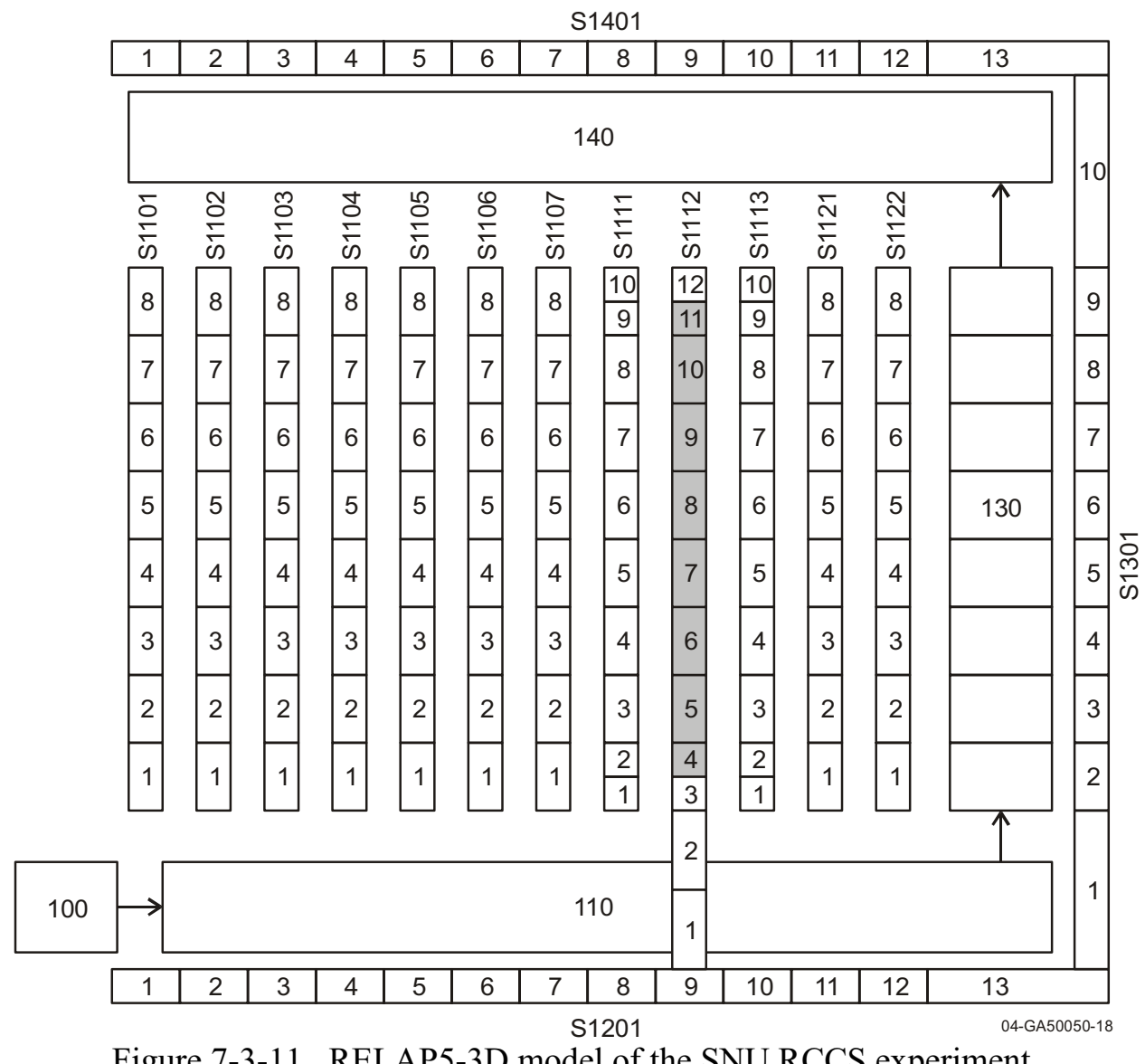

Figure 7-3-11. RELAP5-3D model of the SNU RCCS experiment.

The RV was modeled with a two-dimensional mesh with 12 radial rings and at least 8 axial segments. The inner and outer rings were represented with Structures 1101 and 1122, respectively. Structure 1112 represented the heater rods. The thickness of this structure preserved the volume of the heater rods. A uniform volumetric heat generation rate was applied to the active section of the heater rods (Segments 4 through 11). The RV was modeled as CA508 carbon steel. The CW was modeled as SS304 stainless steel.

The multi-dimensional heat conduction through the RV was approximated using a conduction enclosure model. Each heat structure communicated thermally with the adjacent heat structures in both the radial and axial directions. The support legs were modeled below the heater rods and were assumed to have the same thickness. The outer ring of the RV was coupled to the vertical $\mathrm{CW}$ through a radiation enclosure model. Free convection heat transfer coefficients were applied to the upper, lower, and vertical surfaces of the RV and the inner walls of the cavity. The code's default correlations were applied to the vertical surfaces.

Correlations for isothermal heated and cooled plates (Holman 1986) were applied at the top and bottom of the reactor vessel and the horizontal surfaces of the $\mathrm{CW}$. Temperature boundary conditions were applied to the 
outside surfaces of the CW heat structures (S1201, S1301, and S1401). These temperatures were 28 and 207 ${ }^{\circ} \mathrm{C}$ for Structures 1201 and 1401, respectively. The temperatures applied to the outside surface of the Structure 1301 were a function of elevation.

A simple, one-dimensional hydrodynamic model was used to simulate the air spaces surrounding the RV. The gaps represents by Components 110,130, and 140 were 200, 90, and $370 \mathrm{~mm}$ thick, respectively. A time-dependent volume (Component 100) was used to maintain the pressure at $0.13 \mathrm{MPa}$. The temperature profiles in the experiment would induce multi-dimensional natural circulation patterns within the horizontal and vertical sections, but such patterns cannot be predicted with the simple, one-dimensional model used here. The multi-dimensional flow patterns were neglected for this analysis, and the free convection heat transfer correlations were relied upon to calculate the heat transfer between the walls and the fluid.

The code's enclosure models have several limitations for modeling a complicated geometry such as involved in the SNU experiment. First, the gap conductance in the conduction model and the emissivity in the radiation model are treated as constants for each surface, whereas they actually depend on temperature. For this analysis, the gap conductances in the axial direction were based on a thermal conductivity of $45 \mathrm{~W} / \mathrm{m}-\mathrm{K}$. The gap conductances in the radial direction were set to a large value because the thermal resistance within each heat structure was already accounted for with the code's one-dimensional heat conduction model. The emissivities of the RV and CW were set to 0.8 and 0.6 , respectively, based on measurements. Second, and more serious, the heat conduction model is based on a one-dimensional formulation in which each structure has only two surfaces, instead of the six surfaces actually present. Each surface can be included in only one enclosure model. For this analysis, all the RV surfaces were utilized for radial and axial conduction except for the outer surface of S1122, which was utilized for radiation to the vertical CW. Consequently, no surfaces were available to account for radiation between the upper and lower faces of the RV and the horizontal walls of the cavity. The radiation from these faces was simulated through the use of enhanced convection to the fluid. The radiation heat transfer between walls was converted to an equivalent heat transfer coefficient to the fluid and then added to the coefficient obtained for free convection as described previously. A combined heat transfer coefficient was then applied to both the RV and CW surfaces that preserved the total heat transfer between surfaces. Finally, the input required for the conduction enclosure model is more complicated to generate than would be required with a true two-dimensional heat conduction model.

The results of the assessment using the SNU RCCS experiment are shown in Figure 7-3-12. The figure presents calculated and measured temperatures of the $\mathrm{CW}$ and $\mathrm{RV}$ as a function of height above the lower $\mathrm{CW}$. Scatter in the measured values reflects azimuthal temperature variation at a given height. There is no corresponding scatter in the calculated results because the heat conduction model was two-dimensional rather than three-dimensional. The increase in the $\mathrm{CW}$ temperature near $1.6 \mathrm{~m}$ reflects the location of the liquid level on the outside of the wall. CW temperatures were input to the model as boundary conditions. 


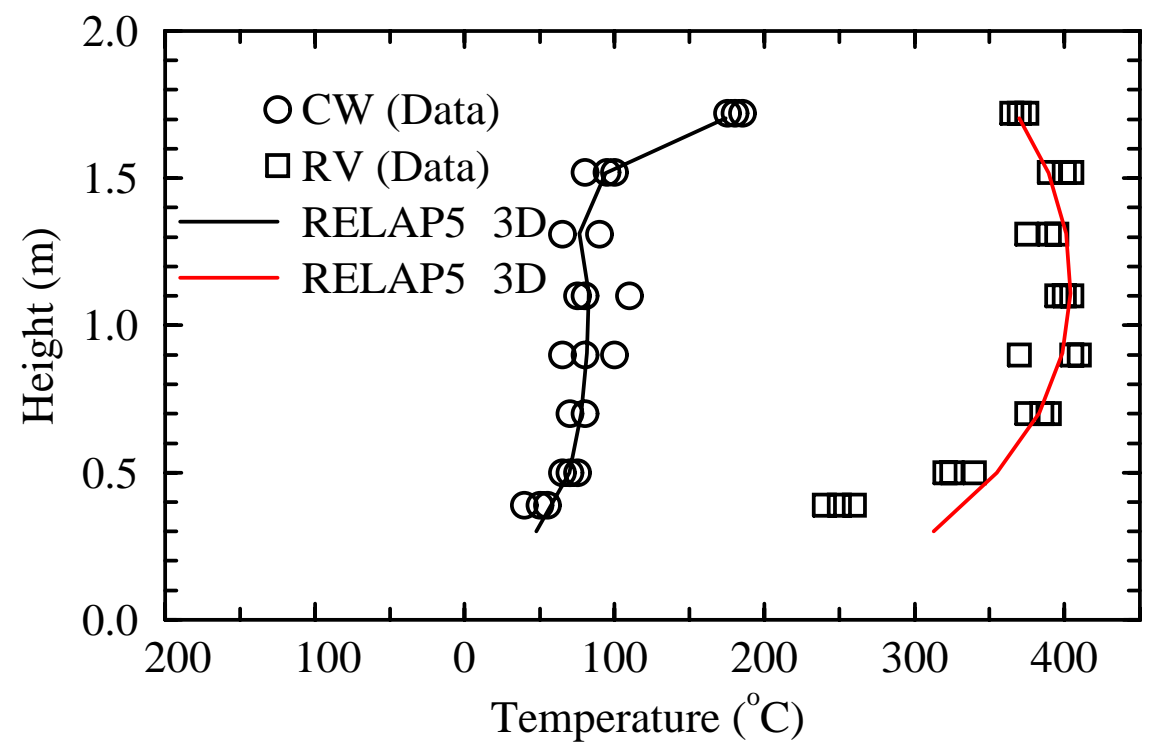

Figure 7-3-12. A comparison of calculated and measured temperatures for the SNU RCCS experiment.

The calculated RV wall temperatures were in reasonable agreement with the measured values. The maximum predicted value occurred slightly above the centerline of the heated length and was in excellent agreement with the maximum measured value. The code's prediction of the temperature decrease near the top of the vessel was also in excellent agreement with the test. A larger decrease occurred near the bottom of the RV in both the calculation and the experiment because of the heat loss through the support legs of the vessel and because of the smaller sink temperature applied to the lower $\mathrm{CW}$. The magnitude of the temperature decrease near the bottom of the RV was larger in the experiment than in the calculation. The results obtained with RELAP5-3D are similar to those obtained previously with the GAMMA code. Possible causes for the discrepancy include the lack of modeling of the bottom insulation plug of the heater element and the lack of detailed information about the geometry of the support legs.

An evaluation of the calculated results showed that radiation from the vertical wall of the RV to the CW accounted for about $60 \%$ of the total power. Convection between the vertical walls accounted for an additional $20 \%$ of the power. Conduction through the support legs accounted for about $10 \%$ of the power while radiation and convection from the lower and upper faces accounted for the remainder.

The RV temperature profile calculated by RELAP5-3D was in reasonable agreement with the measurements from the SNU RCCS experiment, even with the simple, one-dimensional hydrodynamic model and the noted limitations in the code's enclosure models.

\section{Assessment of Molecular Diffusion Model (Bulb Experiment)}

The RELAP5 diffusion model was assessed against the experimental data of Duncan and Toor (1962). The apparatus for the experiment consisted of bulbs on the left and right ends of a diffusion path $85.9 \mathrm{~mm}$ long and $2.08 \mathrm{~mm}$ in diameter. The volume of the left bulb was $77.99 \times 10^{-6} \mathrm{~m}^{3}$ and the volume of the right bulb was $78.63 \times 10^{-6} \mathrm{~m}^{3}$. The left bulb was filled with a gas mixture consisting of 0.5 mole fractions of $\mathrm{N}_{2}$ and 0.5 mole fractions of $\mathrm{CO}_{2}$. The right bulb was filled with a gas mixture consisting of 0.499 mole-fractions of $\mathrm{N}_{2}$ and 0.501 mole-fractions of $\mathrm{H}_{2}$. The gases in the bulbs and the diffusion path were at a pressure of $0.1 \mathrm{MPa}$ and a temperature of $308 \mathrm{~K}$ through out the experiment. A stopcock was located in the middle of the diffusion path and opened at the experiment time of zero seconds to start the experiment. A measurement was performed of the transient mole-fractions of each species of gas in the left and right bulbs. 
RELAP5/ATHENA calculations of the bulb experiment were performed using a network of twelve equal length control volumes in a horizontal line. The left and right control volumes in the network represented the left and right bulbs in the experiment; their volumes were equal to the volumes of the left and right bulbs in the experiment, respectively. The ten control volumes between the left and right control volumes had cross sectional areas corresponding with a diffusion path with a diameter of $2.08 \mathrm{~mm}$ and the combined lengths of these ten control volumes was equal to $85.9 \mathrm{~mm}$.

The RELAP5/ATHENA calculations of the bulb experiment are generally in fair to good agreement with the measured results. Comparisons of the RELAP5/ATHENA calculations of the mole-fractions of gas in the left and right bulbs with the measured results are shown in Figures 7-3-13 and 7-4-13. The figures show the transient mole-fractions calculated by the RELAP5/ATHENA code and the measured results at the earliest time at which measurements were made, namely 14,400 s (4 hours). The gas species $\mathrm{H}_{2}$ is the fastest diffusing of the three species of gas in the experiment. As shown in Figure 7-3-13 the calculated and measured values of the mole-fractions of $\mathrm{H}_{2}$ in the left and right bulbs at the time of 14,400 $\mathrm{s}$ are in good agreement. As shown in Figure 7-3-14, the calculated and measured values of $\mathrm{CO}_{2}$ in the left bulb at the time of 14,400 s are in good agreement, while the calculated value of $\mathrm{CO}_{2}$ for the right bulb under-predicts the measured value by a factor of two. As shown in Figure 7-3-15, the calculated and measured values of $\mathrm{N}_{2}$ for the right bulb at the time of 14,400 s are in good agreement, while the calculated value for the left bulb underpredicts the measured value by about $30 \%$.

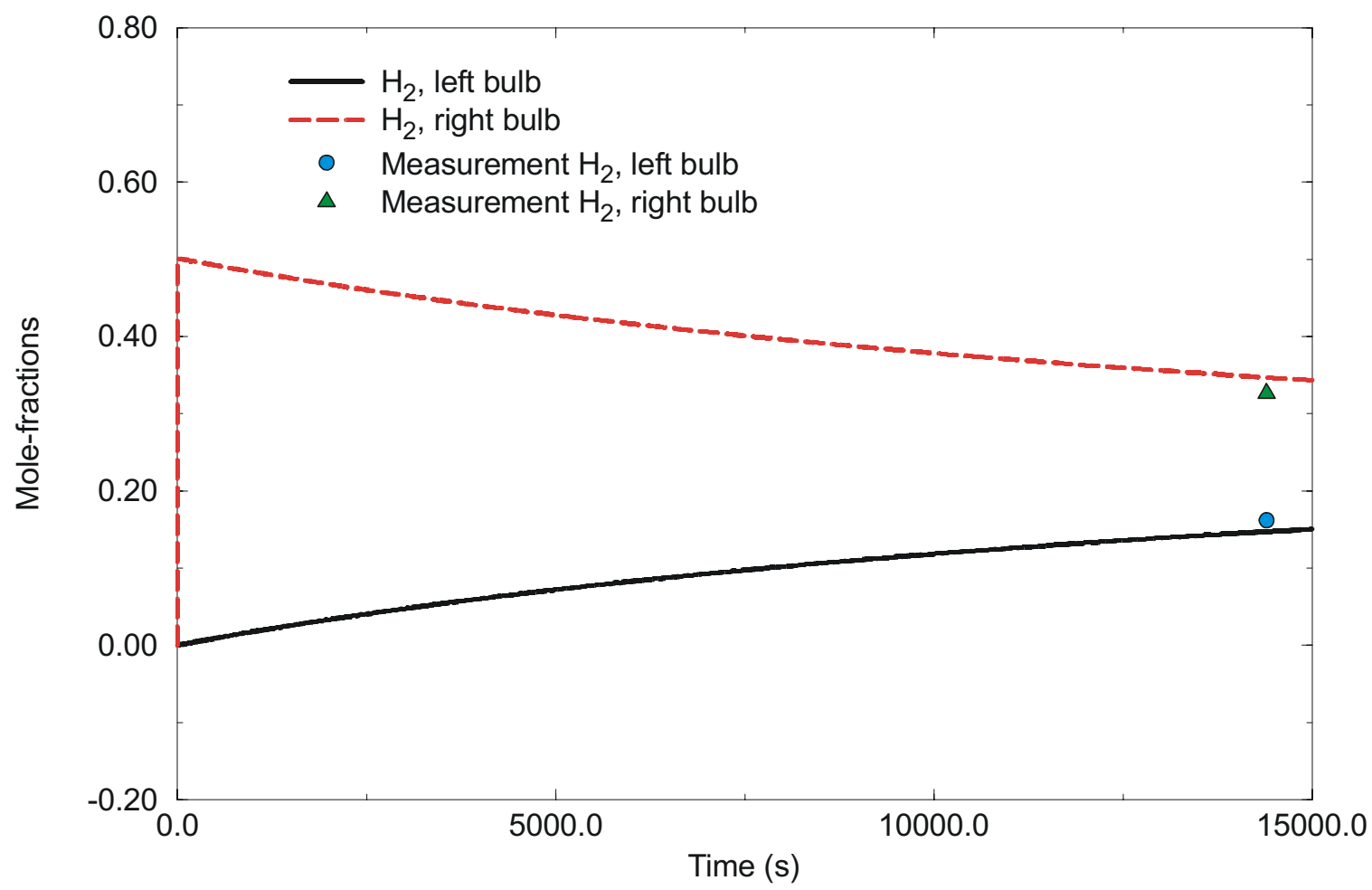

Figure 7-3-13. Comparison of calculated and measured mole-fractions of $\mathrm{H}_{2}$ in left and right bulbs of the bulb experiment. 


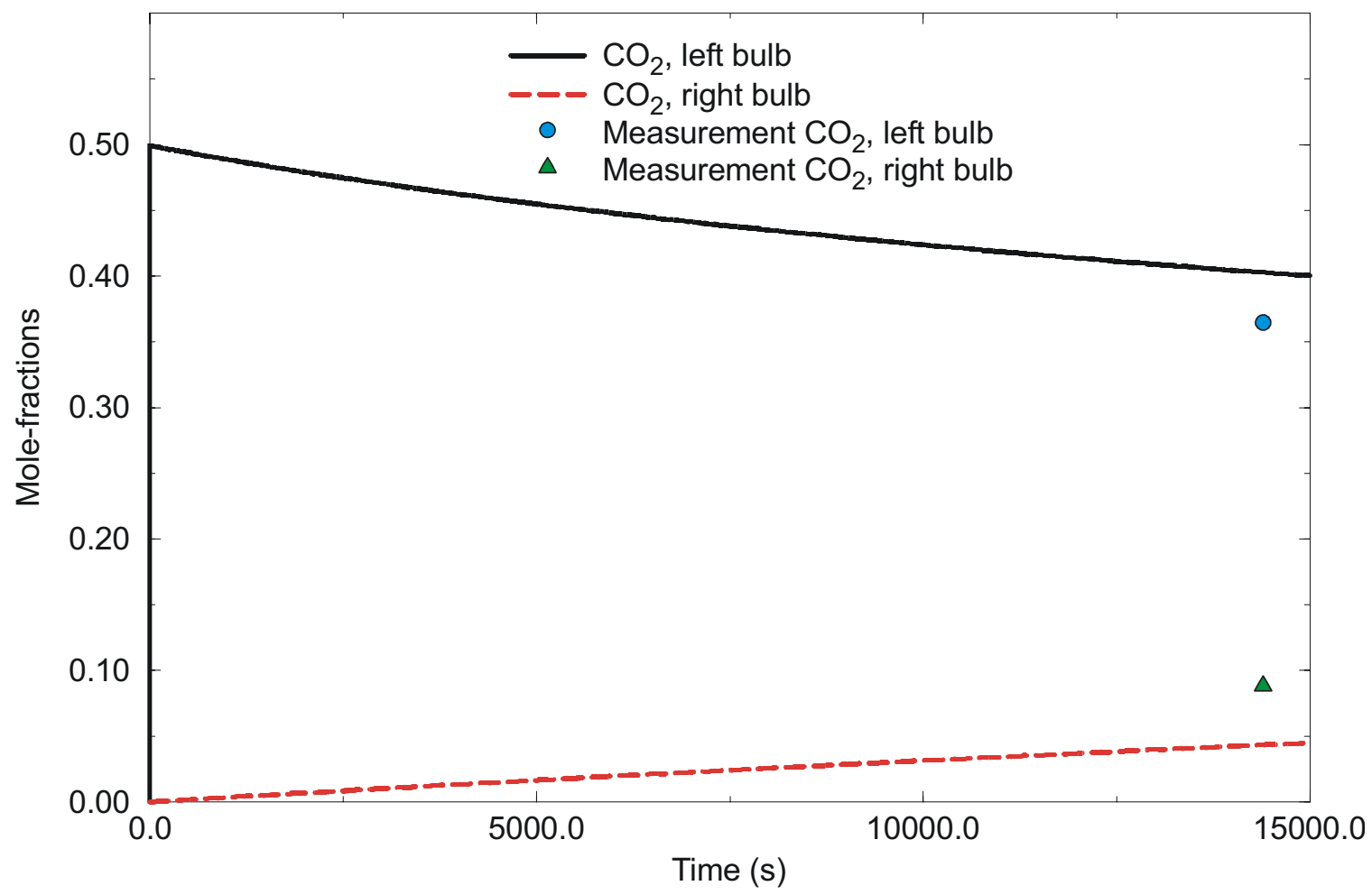

Figure 7-3-14. Comparison of calculated and measured mole-fractions of $\mathrm{CO}_{2}$ in left and right bulbs of the bulb experiment.

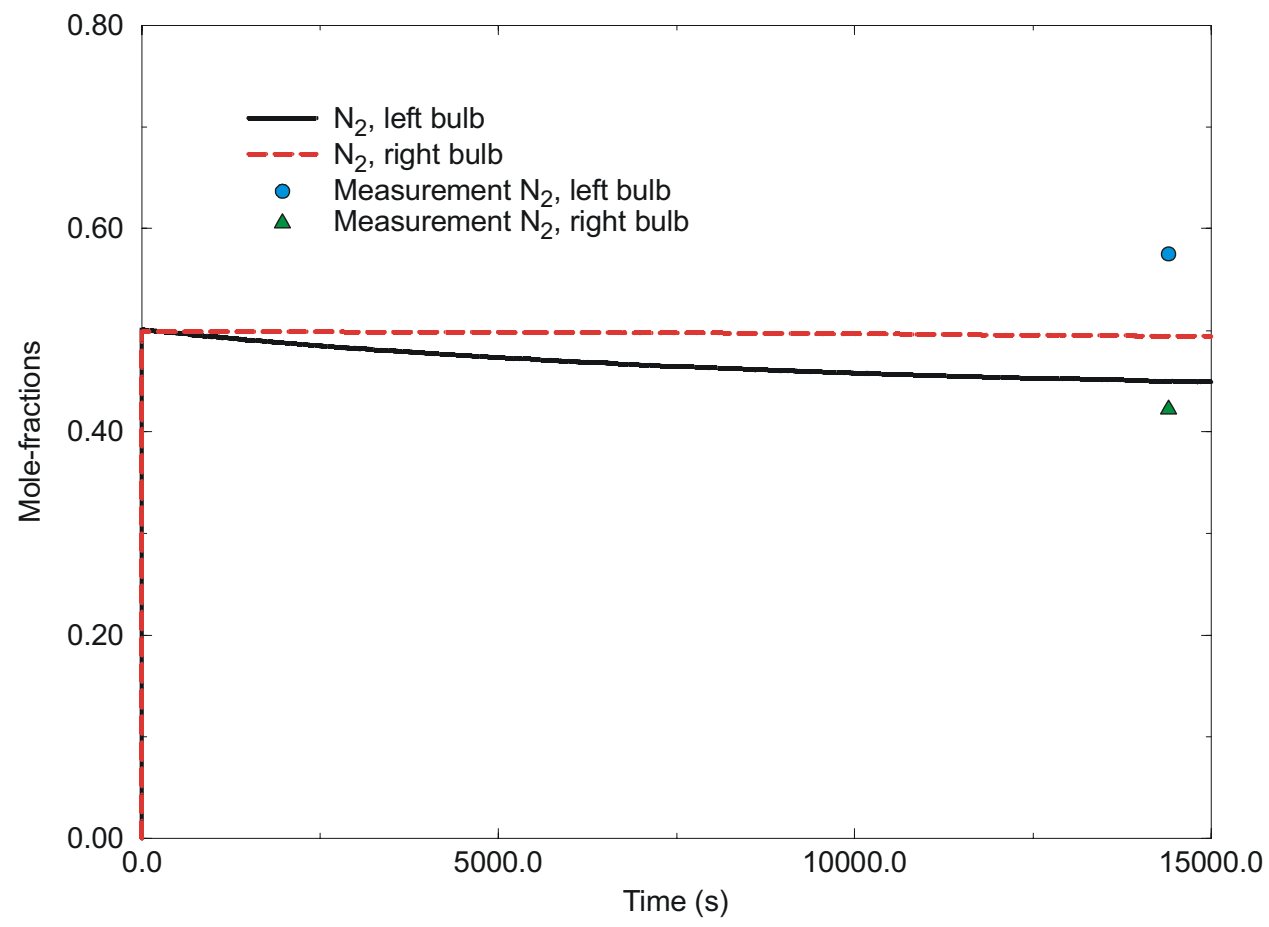

Figure 7-3-15. Comparison of calculated and measured mole-fractions of $\mathrm{N}_{2}$ in left and right bulbs of the bulb experiment. 
The under-prediction of the concentration of $\mathrm{N}_{2}$ in the left bulb and the under-prediction of the amount of $\mathrm{CO}_{2}$ in the right bulb were due to the special case of reverse diffusion occurring for a period of time in the vicinity of the left bulb (Duncan and Toor 1962). RELAP5/ATHENA is not capable of modeling reverse diffusion, which requires the calculation of a negative value of the effective diffusion coefficient so as to calculate diffusion against the concentration gradient.

\section{$\underline{7.3 .2 V \& V \text { of MELCOR }}$}

The MELCOR diffusion model was validated using the data from Takeda (1996) and Duncan and Toor (1962). MELCOR was also validated using the natural circulation data from Kuhlmann (2002).

\section{Assessment of Molecular Diffusion Model (U-Tube Experiment)}

In order to validate the diffusion model incorporated into MELCOR, MELCOR predictions (Merrill et al., 2004) were compared with data from the Takeda (1996) inverse U-tube experiment. The Takeda experiment consisted of a reverse U-shaped Inconel tube containing a $450 \mathrm{~mm}$ length graphite sleeve. Both ends of the tube were attached to the top of a tank containing helium and air. The tube was initially isolated from the tank by valves located at the ends of the tube. One side of the U-shaped tube was heated and the other side is cooled.

A MELCOR model of the experiment was constructed and analyzed, a comparison of the preliminary MELCOR results and the experiment data is shown in Figures 7-3-16 and 7-3-17. Figure 7-3-16 is a plot of the mixture $\left(\mathrm{N}_{2}, \mathrm{O}_{2}, \mathrm{He}, \mathrm{CO}_{2}\right.$, and $\left.\mathrm{CO}\right)$ density as a function of time and location within the pipe. The solid lines represent MELCOR results while the symbols represent experiment data. In Figure 7-3-17, the mole fraction of $\mathrm{O}_{2}$ is shown for the same pipe locations as in Figure 7-3-16. The MELCOR results appear to have good agreement with the experiment data.

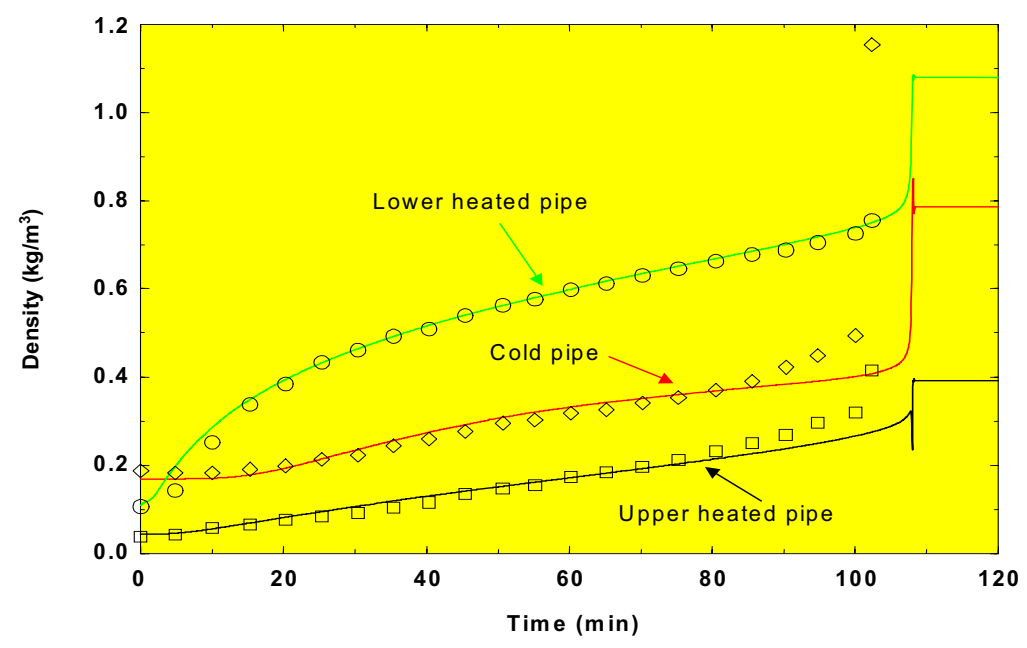

Figure 7-3-16. Comparison of MELCOR-predicted (solid lines) and the inverse U-tube experiment density change of mixtures (symbols). 


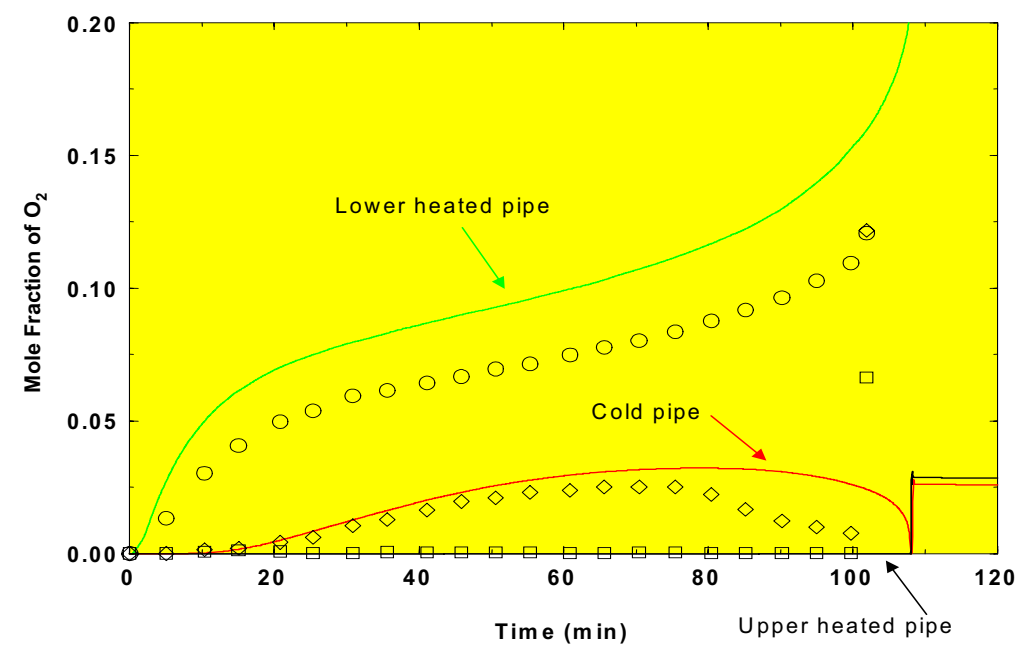

Figure 7-3-17. Comparison of MELCOR-predicted (solid lines) and the inverse U-tube experiment oxygen mole fractions (symbols).

\section{Assessment of Molecular Diffusion Model (Bulb Experiment)}

The molecular diffusion model was validated using the data of Duncan and Toor (1962). The experiment, shown in Figure 7-3-18, consisted of two volumes connected by a short capillary diffusion line. The two volumes are referred to in the figure as Bulb \#1 and Bulb \#2. Bulbs \#1 and \#2 have volumes of $77.99 \mathrm{~cm}^{3}$ and $78.63 \mathrm{~cm}^{3}$, respectively. The diffusion line connecting the two bulbs is $85.9 \mathrm{~mm}$ long with an internal diameter of $2.08 \mathrm{~mm}$. The diffusion line contains a stopcock, which is used to separate the gas mixture contained in bulb \#1 from the gas mixture contained in bulb \#2. The gas contained in bulb \#1 is a mixture of nitrogen and carbon dioxide with a molar composition of 50.086 percent nitrogen and 49.914 percent carbon dioxide. The gas mixture in bulb \#2 contains nitrogen and hydrogen with a molar composition of 49.879 percent nitrogen and 50.121 percent hydrogen. When thermal $\left(32.5^{\circ} \mathrm{C}\right)$ and mechanical $(1 \mathrm{~atm})$ equilibrium was established between the two bulbs, the stopcock was opened allowing the diffusion process to begin.

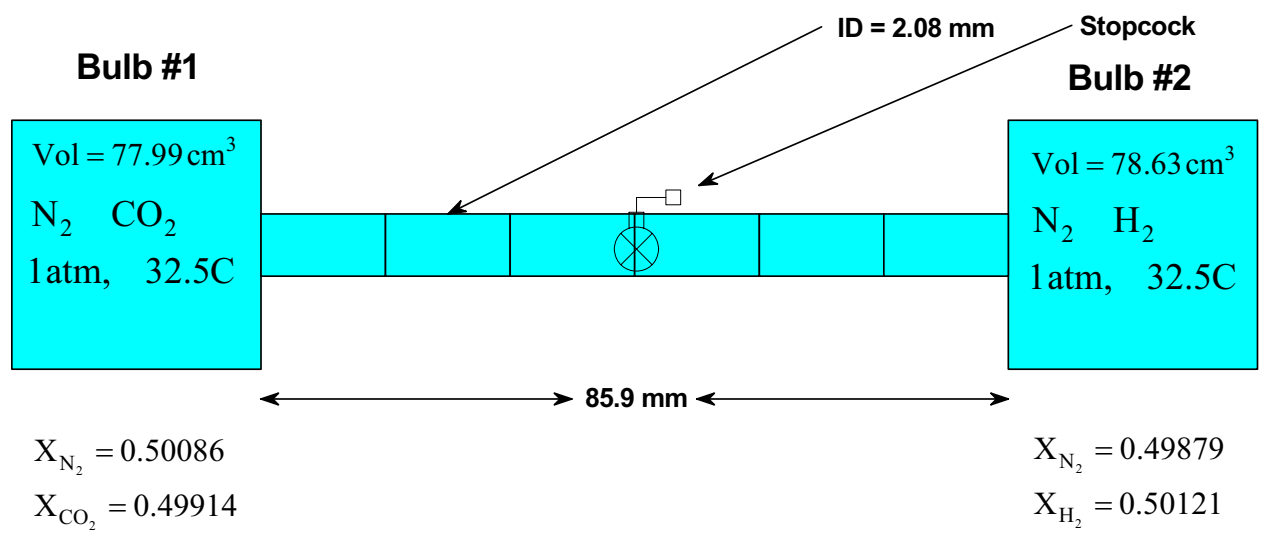

Figure 7-3-18. Schematic of the two-bulb experiment. 
The results from the experiment show that the nitrogen in bulb \#2, which has a slightly higher concentration of $\mathrm{N} 2$, begins to diffuse toward bulb $\# 1$. In a short time $(\approx 130 \mathrm{sec})$ the mole fraction of $\mathrm{N} 2 \mathrm{in}$ bulb \#2 drop below the mole fraction of $\mathrm{N} 2$ in bulb \#1, however the mole fraction of $\mathrm{N} 2$ in bulb \#2 continues to decrease while the mole fraction of $\mathrm{N} 2$ in bulb \#1 increases. This trend continues until approximately $6 \mathrm{hr}$ at which time the mole fraction in bulb \#1 stops increasing and starts to decrease. At this time the mole fraction in bulb \#2 stops decreasing and starts to increase. The period between $130 \mathrm{sec}$ and $6 \mathrm{hr}$ is referred to as the reverse diffusion of nitrogen.

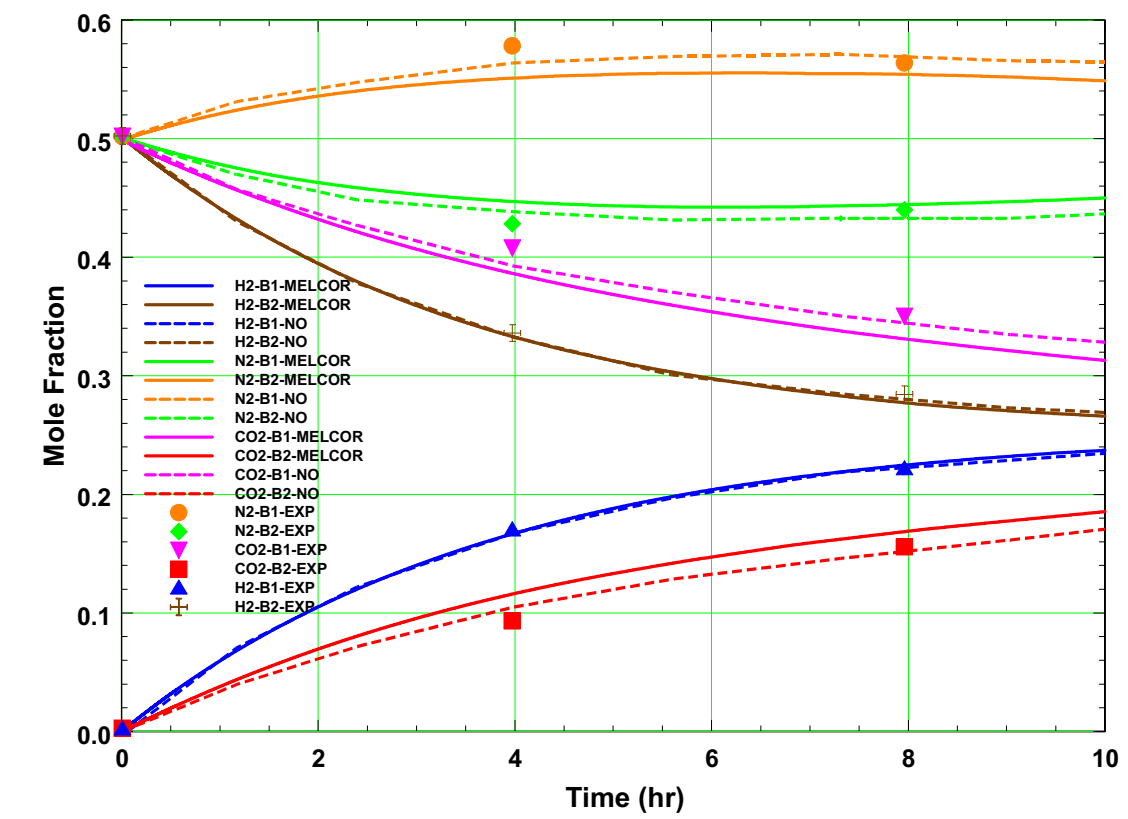

Figure 7-3-19. Multi-component diffusion results.

Viewing the results in Figure 7-3-20, we see that the MELCOR results compare very well with the experimental results for the diffusion of a ternary gas mixture.

\section{Assessment with Natural Circulation Data}

The MELCOR computer code (version 1.8.5) was assessed using natural circulation data generated in the NACOK experimental apparatus (Kuhlmann 2002). The MELCOR model contained the same number of control volumes and heat structures as used in the RELAP5 model shown previously in as shown in Figure 73-9, thus a direct comparison of the RELAP5 and MELCOR 1.8.5 results could be made. The boundary geometry and boundary conditions are as described previously. Results of the comparison are show in Figure 7-3-20. 


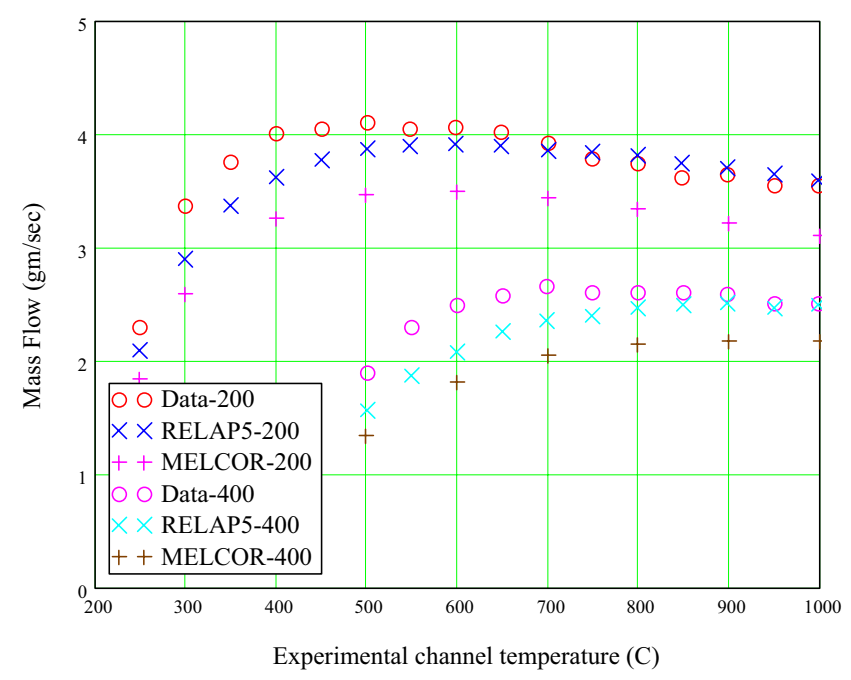

Figure 7-3-20. Measured and calculated air flow rates for the NACOK natural circulation experiment corresponding to return tube temperatures of $200^{\circ} \mathrm{C}$ and $400{ }^{\circ} \mathrm{C}$.

Preliminary assessment of the results indicate that the convective heat transfer coefficients calculated by MELCOR in the return tube are slightly lower than the ones calculated by the RELAP5 code thus, resulting in lower mass flow rates as seen in the figure. The lower heat transfer coefficients result in less heat loss from the air, resulting in higher gas temperatures in the return tube which in turn affects the net buoyancy force driving the experimental flow rate.

\section{7-4 Task 6-4 UM V\&V}

(Described in Section 6.) 


\section{CONCLUSIONS}

\section{$\underline{\text { Task } 1}$}

A multi-dimensional gas multi-component mixture analysis code (GAMMA) has been developed to predict the thermo-fluid and chemical reaction behaviors in a multi-component mixture system related to an air/water ingress accident in a HTGR. GAMMA has the capability to handle the multi-dimensional convection and conduction behaviors as well as heat transfer within the solid components, free and forced convection between a solid and a fluid, and radiative heat transfer between the solid surfaces.

\section{Task 2}

A new kind of RCCS, water pool type RCCS was proposed to overcome the disadvantages of the weak cooling ability of air-cooled RCCS and the complex structure of water-cooled RCCS. The feasibility of the system was estimated by a series of experiment. In both the normal operation test and the active cooling failure tests, it was found that the maximum temperature of the reactor vessel wall was kept below the design limitation of PBMR. The experimental results were also used to validate MARS-GCR and GAMMA.

\section{$\underline{\text { Task } 3}$}

Regarding the air-ingress accident, graphite oxidation has been experimentally investigated. Effects of temperature, oxygen concentration, flow rate, graphite shape and size, moisture and degree of burn-off were considered. Finally, a graphite oxidation model that covers the whole ranges of experimental conditions was developed and successfully validated by experimental data.

\section{$\underline{\text { Task } 4}$}

The RELAP5 and MELCOR codes have been improved significantly for the analysis of the VHTR. The improvements now allow the codes to simulate air ingress following LOCAs, including the effects of molecular diffusion, graphite oxidation, and chemical equilibrium between $\mathrm{CO}_{2}$ and $\mathrm{CO}$. The INL performed a number of $\mathrm{V} \& \mathrm{~V}$ using experimental data of NACOK natural circulation, inverse U-tube experiments and others. The results from numerical calculations agreed very well with those test data.

\section{$\underline{\text { Task } 5}$}

A global Monte Carlo model using MCNP5 has been developed based on explicit representation and analysis of the particle fuel, including fuel depletion and thermal-hydraulic feedback. To couple MCNP5 and RELAPAthena, a methodology was developed allowing global Monte Carlo simulation of VHTR cores with temperature feedback. Finally, global decay heat source distribution was predicted as a function of depletion and temperature feedback.

\section{$\underline{\text { Task } 6}$}

In KAIST, GAMMA code was applied to assess the system behaviors during the air ingress accident following the complete break of main pipes. The analysis of air-ingress was performed for PBMR $268 \mathrm{MWt}$. In the analysis, the significant rise in pebble temperature was observed in the bottom of the core due to graphite oxidation. Since the air ingress process depends on the vault conditions, further analysis coupled with more detailed vault or containment modeling would be necessary as future study. In the other analyses: IAEA GT-MHR benchmark calculation for LPCC and air ingress analysis for PMR $600 \mathrm{MWt}$, comparable peak fuel temperature trend was observed compared with those of other country codes. The analysis result for 
air ingress shows much different trend form that of previous PBR analysis: later onset of natural circulation and less significant rise in graphite temperature.

In INL, the RELAP5 and MELCOR codes have been validated against several experiments that simulated phenomena related to LOCAs in the VHTR. These phenomena included molecular diffusion, graphite oxidation, natural circulation, and heat transfer. The calculated results from both codes were generally in reasonable agreement with measurements. Thus, the codes are ready to be used for initial calculations that investigate safety issues related to LOCAs in the VHTR. Additional work would have to be performed before either code could be considered to be adequately validated from a licensing point of view. 


\section{References}

A.I.Ch. E. Journal, March, 1962, pages 38-41.

ANS, 1995, "American National Standard for Decay Heat Power in Light Water Reactors," ANSI/ANS-5.11994, American Nuclear Society (1995).

Aleksander Sala, 1986, Radiant Properties of Materials, ELSEVIER SCIENCE PUBLISHING CO., INC. New York, pp. 341-354.

Aurthur J., The reaction between carbon and oxygen, Trans. Faraday Soc. 47 (1951) 164-178

Avallone, E. A, 1987, Marks' Standard Handbook for Mechanical Engineers, Ninth Edition, McGraw-Hill Book Company, New York.

Bird, R. B., W. E. Stewart, and E. N. Lightfoot, 1960, Transport Phenomena, John Wiley \& Sons, Inc., New York.

Brown, F. B. and W. R. Martin, "Stochastic Geometry Capability in MCNP5 for the Analysis of Particle Fuel," Annals of Nuclear Energy 31, (November 2004).

Brown, F. B. and W. R. Martin, "Stochastic Geometry for MCNP5," Trans. Am. Nucl. Soc. 91, 171-173, Washington, DC (November 2004).

Brown, F. B., et al., 2003, "MCNP - A General Monte Carlo N-Particle Transport Code, Version 5 Volume I: Overview and Theory," LA-UR-03-1987, Los Alamos National Laboratory.

Buchanan F. J. and Little J. A., Journal of Material Science,1994, vol. 28, pp. 2324

Cambel, A. B. and B. H. Jennings, 1958, Gas Dynamics, McGraw-Hill Book Company, New York.

Carbiener W.A. and Cudnik R.A., 1969, "Similitude Considerations for Modeling Nuclear Reactor Blowdowns," Trans. Am. Nucl. Soc., Vol. 12, p.361.

Chen L., Yang B. T. , Hu X. R. , 1990, "Design Principle for Simultaneous Emissivity and Temperature and Emissivity Measurement," Opt. Eng., 29, 1445-1448.

Chunhe T. and Guan Jie, Improvement in oxidation resistance of the nuclear graphite by reaction-coated SiC coating

Churchill S. W. and Chu H. H. S., 1975, "Correlating Equations for Laminar and Turbulent Free Convection from a Vertical Plate," International Journal of Heat and Mass Transfer, Vol. 18, pp. 1323-1329

Conlin, J. L., W. Ji, J. C. Lee, and W. R. Martin, "Pseudo Material Construct for Coupled NeutronicThermal-Hydraulic Analysis of VHTR," Trans. Am. Nucl. Soc., 92, 225 (2005).

Daubert, T. E., R. P. Danner, H. M. Sibul, C. C. Stebbins, R. L. Rowley, W. V. Wilding, J. L. Oscarson, M. E. Adams, T. L. Marshall, and N. A. Zundel, 2000, Physical and Thermodynamic Properties of Pure 
Chemicals, DIPPR Project 801 Evaluated Process Design Data, American Institute of Chemical Engineers and Design Institute for Physical Property Data.

Davis, C. B., 2003, The Addition of Oxygen, Carbon Dioxide, and Carbon Monoxide

Dilling D.A. et al., 1982, Passive Decay and Residual Heat Removal in the MHTGR, IAEA-TECDOC-757, Juelich.

Dryer F. L. and Glassman I., "High-Temperature Oxidation of CO and H2," The Fourteenth Symposium (International) on Combustion, Pittsburgh, p.987, The Combustion Institute (1973).

Duncan J.B. and Toor H.L., "An experimental study of three component gas diffusion," A.I.Ch.E. Journal, 8, $1,38(1962)$

Duncan, J.B. and H. L. Toor, 1962, "An Experimental Study of Three Component Gas Diffusion,"

Dyer, F. L., and I. Glassman, 1973, "High-temperature oxidation of CO and H2," 14th Symposium on Combustion, The Combustion Institute, Pittsburgh, PA, pp. 987-1003, 1973.

Edition, McGraw-Hill Book Company, New York.

FLUENT 6.0 User's Guide, FLUENT Inc., 2002.

Frank P. Incopera, 1996, Introduction to Heat Transfer, John Wiley \& Sons, USA .

Fuller E.L., Okoh J.M., 1997, Kinetics and mechanisms of the reaction of air with nuclear grade graphites: IG-110, J. Nuclear Materials, Vol. 240, pp. 241-250

Fuller, E. L., and J.. M. Okoh, 1997, "Kinetics mechanisms of the reaction of air with nuclear grade graphites: IG-110," Journal of Nuclear Materials, 240, 1997, pp. 241-250.

General Atomics, "GT-MHR Conceptual Design Description Report," GA/NRC-337-02, August 2002.

German Safety Guide KTA3102.2 \& KTA3102.3, German Nuclear Safety Standards Commission (1981).

Gerwin H., Scherer W., and Teuchert E., "The TINTE Modular Code System for Computational Simulation of Transient Processes in the Primary Circuit of a Pebble Bed High Temperature Gas-Cooled Reactor," Nucl. Sci. Eng., 103, 302 (1989).

Harlow F. H. and Amsden A. A., "A Numerical Fluid Dynamics Calculation Method for All Flow Speeds," J. Chem. Phys., 8, 197 (1971).

Hinsen H.K., Katscher W., Moorman R., Kinetics of the graphite/oxygen reaction in the in-pore diffusion controlled regime, KFA, ISSN (1983) 0366-0885

Hirschfelder J. O., Curtiss C. F., and Bird R. B., Molecular Theory of Gases and Liquids, John Wiley \& Sons, New York (1964). 
Hishida M. and Takeda T., "Study on air ingress during an early stage of a primary-pipe rupture accident of a high-temperature gas-cooled reactor," Nucl. Eng. \& Des., 126, 175 (1991)

Hishida. M. and T. Takeda, 1991, "Study of Air Ingress During an Early Stage of a Primary-Pipe Rupture Accident of a High-Temperature Gas-Cooled Reactor," Nucl. Eng. and Des., 126, 1991, pp. 175-197.

Hogg G. W. , The Effect of Secondary Flow on Point Heat Transfer Coefficients for Turbulent Flow inside Curved Tubes, Ph.D. Thesis, Univ. of Idaho, 1968.

Holaman J.P., Heat Transfer, McGraw-Hill, 1986.

Holman, J. P., 1986, Heat Transfer, Sixth Edition, McGraw-Hill Book Company, New York.

Hurtado A.M. ,Schoeder B. and Gottaut H., Presented at IAEA Technical Committee Meeting on Response of Fuel Elements and Gas-cooled Reactor Cores under Accidental Air or Water Ingress Conditions, Tsinghua University, Beijing, China, 25-27 October 1933

IAEA, 2000, Heat Transport and Afterheat Removal for Gas Cooled Reactors under Accident Conditions, IAEA-TECDOC-1163, Vienna.

IAEA, 2001, Current Status and Future Development of Modular High Temperature Gas Cooled Reactor Technology, IAEA-TECDOC-1198.

IAEA-TECDOC-1163, "Heat Transport and Afterheat Removal for Gas Cooled Reactors under Accident Conditions," International Atomic Energy Agency (2000).

INEEL, 1999, Strategic Nuclear Reactor Collaboration, INEEL/EXT-99-00694.

INEEL, 2000, Modular Pebble Bed Reactor Project University Research Consortium Annual Report, INEEL/EXT-2000-01034, MIT-ANP-PR-75, July 2000.

INEEL, 2001, Modular Pebble-Bed Reactor Project Laboratory-Directed Research and Development Program FY 2001 Annual Report, December 2001.

INEEL, 2005, RELAP5-3D? Code Manual, INEEL-EXT-98-00834, Revision 2.3, April 2005.

Incropera F. P. and DeWitt D. P., Fundamentals of Heat and Mass Transfer, John Wiley \& Sons, New York, 2003.

Incropera, F. P. and D. P. DeWitt, 2002, Fundamentals of Heat and Mass Transfer, 5th Edition, John Wiley and Sons, New York, 2002, pp. 357.

Ji, W., J. L. Conlin, W. R. Martin, and J. C. Lee, "Reactor Physics Analysis of the VHTGR Core," Trans. Am. Nucl. Soc. 91, 556-558, Washington, DC (November 2004).

KAERI (Korea Atomic Energy Research Institute), 2004, MARS3.0 code manual, KAERI, Korea, pp. 239240. 
Kakac S., Yener Y., Convective Heat Transfer, CRC Press, 1994, pp. 147

Kawakami H., 1986, Air oxidation behavior of carbon and graphite materials for HTGR, JEARI, No. 124, pp. 26-33

Kawakami, H., 1986, "Air oxidation behavior of carbon and graphite material for HTGR," TANSO 124, 1986, pp. 26-33.

Kays, W. M. and M. E. Crawford, 1980, Convective Heat and Mass Transfer, Second Edition, McGraw-Hill Book Company, New York.

Keyhani M., Kulacki F. A., and Christensen R. N., "Free Convection in a Vertical Annulus with Constant Heat Flux on the Inner Wall", ASME, Vol.105, p.454, 1983.

Kuhlmann, Mario B., 2002, Experimente zu Gastransport und Graphitkorrosion bei Lufteinbruchstorfallen im Hocktemperaturereaktor, Berichte des Forshungzaentrums, Julich 4003, ISSN 0944-2952.

Lee W.J. et al., 2003, "Development of MARS-GCR for Gas-Cooled Reactor Analysis - Incorporation of Gas Properties," Proceeding of NURETH-10, Seoul, Korea, October 5-9.

Lemmon, E. W., M. O. McLinden, and M. L. Huber, 2002, REFPROP Reference Fluid Thermodynamic and Transport Properties, NIST Standard Reference Database 23, Version 7.0 Beta Version, National Institute of Standards and Technology.

Lim, H. S. and H. C. No, 2003, "Transient multicomponent mixture analysis based on ICE numerical scheme for predicting an air ingress phenomena in an HTGR," 10th International Topical Meeting on Nuclear Reactor Thermal Hydraulics (NURETH-10), Seoul, Korea, October 5-9, 2003.

Ludwig, S., 2002, "Revision to ORIGEN2 - Version 2.2," transmittal memorandum.

MELCOR Computer Code Manuals, 1997, Material Properties (MP) Package Reference Manual, NUREG/CR-6119, Vol. 2, SAND-97-2398.

MELCOR Computer Code Manuals, 1997, Thermal Hydraulic (CVH and FL) Packages Reference Manual, NUREG/CR-6119, Vol. 2, SAND-97-2398.

MacDonald, P. E., et al., "NGNP Preliminary Point Design - Results of the Initial Neutronics and ThermalHydraulic Assessments During FY-03", INEEL/EXT-03-00870 Rev. 1, Idaho National Engineering and Environmental Laboratory (2003).

MacFarlane, R. E. and P. Talou, 2003, "DOPPLER: A Utility code for Preparing Customized TemperatureDependent Data Libraries for the MCNP Monte Carlo Transport Code," Internal Memorandum, Los Alamos National Laboratory.

MacGregor et al., 1969, "Free Convection Through Vertical Plane Layers: Moderate and High Prandtl Number Fluids", J. Heat Transfer, Vol. 91, pp. 391.

Magnaud J. P. and Goldstein S., "The Finite Element Version of TRIO Code," 7th International Conference in Fluid Mechanics, Huntsville, USA (1989). 
Marshall, T. D., R. J. Pawelko, R. Anderl, G. Smolik, B. Merrill, and R. Moore, 2002, Air Chemical Reactivity Measurements of the Carbon Fiber Composite NB31, INEEL/EXT-02-00745, May, 2002.

Merrill, B. J., R. L. Moore, and C. H. Oh, 2004, "Addition of Diffusion Model to MELCOR and Comparison with Data," ANS Annual Meeting, Pittsburgh, PA, June 13 - 17, 2004.

Mills A.F., Mass Transfer, Prentice Hall, Upper Saddle River NJ, 2001.

Moormann R., "Effect of Delays in Afterheat Removal on Consequences of Massive Air Ingress Accidents in High-Temperature Gas Cooled Reactors," J. Nucl. Sci. Tech., 21, 11, 824 (1984).

Mori Y. and Nakayama W. , 1967, "Study on Forced convective Heat Transfer in Curved Pipes," Int. J. Mass Transfer, 10, pp. 37-59.

Moshfeghian M. and Bell K.J., 1979, "Local Heat Transfer Measurements in and Downstream from a Ubend," ASME paper No. 79-HT-82.

NO H. C., Kim E. S., and Lim H. S., "Multi-Dimensional Multi-component Mixture Air-Ingress Analysis and Air-Ingress Experiment in an HTGR," The Sixth International Conference on Nuclear Thermal Hydraulics, Operation and Safety (NUTHOS-6), Paper ID:KN-01, Nara, , Japan, October 4-8, 2004.

Nield D. A. and Bejan A., Convection in Porous Media, Springer-Verlag, New York (1999).

Nino M. et al., Japan Patent 60-297042 (1985)

Nixon T. T. and Cawley J. D. , Journal of American Ceramic Society, 1992, vol. 75, pp. 703

Noncondensable Gases into RELAP5-3D, INL Report R5/3D-03-05.

O'Brien, M. H., B. Merrill, and S. Ugaki, 1988, Combustion testing and Thermal Modeling of proposed CIT Graphite Tile materials, EGG-FSP-8255, September, 1988.

Ogawa M., "Mass Transfer with Graphite Oxidation in Gas Mixture Laminar Flow through Circular Tube," J. At. Energy Soc. Jpn., 35, 3, 245 (1993).

Oh, C. H., L. J. Siefken, R. Moore, C. Davis, J. Lee, W. Martin, J. Holloway, H. C. No, J. H. Kim, and G. C. Park, 2002, "Development of Safety Analysis Codes and Experimental Validation for a Very High Temperature Gas-Cooled Reactor," International Nuclear Energy Research Initiative Proposal, Program Announcement, NE-INERI-2002-001.

Poinsot T. and Veynante D., Theoretical and Numerical Combustion, R.T. Edwards Inc., 2001

Poling B. E., Prausnitz J. M., and O'Connell J. P., The Properties of Gases and Liquids, fifth ed., McGrawHill, New York (2001).

Popper P. , Special Ceramics 5 (1970) 1 
Poston, D. L. and H. R. Trellue, 1999, "User's Manual, Version 2.0 for Monteburns Version 1.0," LA-UR-994999, Los Alamos National Laboratory.

Press, W. H., et al., 1986, Numerical Recipes, Cambridge University Press, New York, NY.

Raznjevic K., Handbook of Thermodynamic Tables and Charts, Hemisphere, Washington (1976).

Reid, R. C., J. M. Prausnitz, and B. E. Poling, 1986, The Properties of Gases and Liquids, Fourth

Reid, R. C., J. M. Prausnitz, and B. E. Poling, 1987, The Properties of Gases and Liquids, Fourth Edition, McGraw-Hill Book Company, New York.

Reitsma F. et. al., "The PBMR Steady State and Coupled Kinetics Core Thermal-Hydraulics Benchmark Test Problems," 2nd international Topical Meeting on High Temperature Reactor Technology, Paper C17, Beijing, China, September 2004.

Reynolds, W. C., 1979, Thermodynamic Properties in SI, Stanford University.

Rivken, S. L, 1988, Thermodynamic Properties of Gases, Fourth Edition (Revised), Hemisphere Publishing Corporation.

Roes J., "Experimental Investigations of Graphite Corrosion and Aerosol Formation during Air Ingress into the Core of a High Temperature Pebble Bed Reactor," Juel-2956, 1994

Rossberg M.Z., Elektrochem. 60 (1956) 952

Rouaaeau P.G., Toit S.G., and Landman W.A., "Validation of a Transient Thermal-Fluid Systems CFD Model for a Packed Bed High Temperature Gas-Cooled Nuclear Reactor," 2nd international Topical Meeting on High Temperature Reactor Technology, Paper C07, Beijing, China, September 2004.

SCDAP-RELAP5-3D Development Team, 2002, Volume 4: MATPRO-A Library of Materials Properties for Light-Water-Reactor Accident Analysis, INEEL-EXT-02-00589, May 2002.

SCDAP/RELAP5-3D Code Development Team, 2003, SCDAP/RELAP5-3D? Code Manual, Volume 2: Modeling of Reactor Core and Vessel Behavior during Severe Accidents, INEEL-EXT-02-00589, Revision 2.2, October 2003.

Saito et al., 2000, Design and Safety Consideration in the High-Temperature Engineering Test Reactor (HTTR), IAEA-TC-389, Dimitrovgrad.

Schaaf, J., W. Frohling, and H. Hohn, 1997, "Status of the Experiment NACOK for Investigations on the Ingress of Air into the Core of an HTR-Module," Nuclear Energy Agency, "Workshop on High Temperature Engineering Research Facilities and Experiments, Petten, Netherlands, 12-14 November 1997.

Seban R.A. and Mclaughlin E.F., 1963, "Heat Transfer in Tube Coils with Laminar and Turbulent Flow," International Journal of Heat and Mass Transfer, Vol. 6, pp. 387-395.

Shuford DM, Prairie G. US Patent 4465777, 1984 
Shurford D.M. , Prairie G. , US Patent, 4471023, 1984

Struth S., "DIREKT - A Computer Programme for Non-Steady, Two-Dimensional Simulation of ThermoHydraulic Transients," JUL-1702, Research Centre Julich (1999).

TAC Technologies, 2000, NEVADA Software Package Quick Reference Series, TAC Technologies, USA.

Tailby S. R. and Staddon P.W., 1970, "The Influence of 90o and 180o Pipe Bends on Heat Transfer from an Internally Flowing Gas Stream," Heat Transfer, Vol.2 paper No. FC 4.5.

Takahashi M., Kotaka M., Sekimoto H., Burn-off and production of CO and CO2 in the oxidation of nuclear reactor grade graphite in a flow system, J. Nuclear Science and Technology, 31(12) (1994) 1275-1286

Takahashi, M., M. Kotaka, and H. Sekimoto, 1994, "Burn-off and production of CO and CO2 in the oxidation of nuclear-grade graphites in a flow system," Journal of Nuclear Science and Technology, 31, December, 1994, pp. 1275-1286.

Takeda T., "Air Ingress Behavior during a Primary-pipe Rupture Accident of HTGR," JAERI-1338, Japan Atomic Energy Research Institute (1997).

Takeda T., "Mixing Process of a Binary Gas in a Density Stratified Layer," JAERI-Research97-061, Japan Atomic Energy Research Institute (1997).

Takeda, T. and M. Hishida, 1996, "Studies on Molecular Diffusion and Natural Convection in a Multicomponent Gas System," International Journal of Heat and Mass Transfer, 39, Vol. 3, 1996, pp. 527-536.

Tang C. and Guan J., Improvement in oxidation resistance of the nuclear graphite by reaction-coated $\mathrm{SiC}$ coating, J. Nucl. Mater. 224 (1995) 103-108

The RELAP5-3D Code Development Team, "ATHENA Code Manual," INEEL-EXT-98-00834, Rev. 2.2, Idaho National Engineering and Environmental Laboratory (2003).

Van Der Merwe, J. \& Van Ravenswaay, and J.P., Flownex Version 6.4 User Manual, M-Tech Industrial, Potchefstroom, South Africa.

Welty J.R., Wicks C.E., Wilson R.E., Fundamentals of Momentum, Heat and Mass Transfer, John Wiley and Sons

Widom, S., 1966, "Random sequential addition of hard spheres to a volume," J. Chem. Phys. 44, 3888-3894.

Workshop on High Temperature Gas Cooled Reactor and Hydrogen Production, Korean Nuclear Society, Yongpyong, Korea, Oct. 2004.

Wu et al., 2002, "The Design Features of the HTR-10," Nuclear Engineering and Design, Vol. 218, pp. 25-32.

Yamamoto O., Sasammoto T. and Inagaki M. , Carbon, 1995, vol. 33. Pp. 359 
Yun B. J. et al., 2004, "Measurement of the Two-phase Mass Flow Rate Using an Average Bidirectional Flow Tube," Proceeding of Two-Phase Flow Modeling and Experimentation, Pisa, Italy, September.

Zhu Q., Qiu X. and Ma C., Oxidation resistance SiC coating for graphite materials, 1999, Carbon, Vol. 37, pp. 1475-1484

Zhu Q. and Qiu X., Ma C., The oxidation resistance improvement of matrix graphite of spherical fuel element by slip-gelation process, J. Nucl. Mater. 254 (1998) 221-225

Zucrow, M. J. and J. D. Hoffman, 1976, Gas Dynamics, Volume 1, John Wiley and Sons, New York. 